\title{
AN EVALUATION OF MARKET RESPONSES TO CORPORATE DISCLOSURES IN A CONTINUOUS DISCLOSURE ENVIRONMENT
}

BY

HANQIAO LI

\author{
A thesis \\ submitted to the Victoria University of Wellington \\ in fulfilment of the requirements for the degree of \\ Doctor of Philosophy
}

Victoria University of Wellington

2018 



\section{ACKNOWLEDGEMENTS}

I would like to express my appreciation to everybody who has helped and supported me as I completed this $\mathrm{PhD}$ thesis. This includes my primary supervisor Dr Thu Phuong Truong and my secondary supervisor Ms Trish Keeper. Their comments and insights guided me throughout the long and many stages of the $\mathrm{PhD}$ work and helped me achieve the depth of understanding presented in the thesis. Phuong also gave me the opportunity to be a research assistant for her research projects, which has supported my life in New Zealand.

I would also like to express my gratitude to other Professors who have helped me. In particular Professor Rachel Baskerville, as she introduced me to the VUW PhD programme when we met in China and supported me during my PhD study. Professor Tony van Zijl provided constructive advice on my research topic, and Professor Michael Bradbury provided valuable feedback on my PhD study at the AFAANZ symposium and conference. I also express my gratitude to Dr Rodney Dormer and the late Kevin Simpkins who gave me the opportunity to tutor in ACCY111.

I would like to thank all my friends and colleagues at Victoria University of Wellington for the support and advice they have given me. This includes, but is not limited to Asma Jahan, Rubeena Tashfeen, Ryan Kerr, Hien Hoang, Kathleen Makale, Sendirella George, Clare Markham, Farzana Tanima and Matthew Sorola. To every other member of academic and $\mathrm{PhD}$ colleagues, who there are too many to include, I express thanks.

I would like to thank the scholarships which I got from Victoria University of Wellington, the CSC Scholarship and the Victoria Doctoral Submission Scholarship.

I would like to acknowledge the emotional support from my fiancée, Alexander McConnochie. Without this support I would not have made it to the end.

Finally I would like to extend a special thank to my parents for their love when they raised me. Without their sacrifices I would not be where I am today. 


\section{STATEMENT OF ORIGINALITY}

I declare that the work presented in this thesis is my own original work carried out through School of Accounting and Commercial Law at Victoria University of Wellington. I completed my Thesis as a PhD student. I declare that the material of this thesis has not been submitted either in whole or in part for the award of any other degree and/or diploma at this or any other university. Any contribution made to the research by others, with whom I have worked at Victoria University of Wellington or elsewhere, is explicitly acknowledged.

To the best of my knowledge, this thesis contains no material previously published or written by other persons or institutions except where due reference has been made. I declare that the intellectual content of this thesis is the product of my own work except the acknowledged assistance from others in the project's design and conception or in style, expression and presentation.

Signature:

Date: 


\begin{abstract}
Corporate disclosure has attracted the attention of researchers from the accounting and capital markets. Researchers have been trying to better understand how capital markets respond to corporate disclosures. This study explores the short- and the long- term market effects of corporate disclosures in a continuous disclosure environment. This study aims to find the answers to the following research questions. (1) How market (price/volume) sensitive is the information contained in each disclosure category in a continuous disclosure environment? (2) How quickly is the value-relevant information incorporated into share prices before the annual earnings announcement is released, and how can company announcements promote this timeliness of price discovery? Specifically, this study examines the extent to which the Australian share market reacts to new public information immediately after the information is released, and the impacts of announcement frequency as to different continuous disclosure categories on the timeliness of price discovery (also known as the speed of price discovery).
\end{abstract}

Prior research has mostly concentrated on a single type of information event to examine the information's market consequences by monthly, weekly or daily data. Instead, by covering all public announcements and using tick (transaction) data to calculate intraday abnormal returns and abnormal trading volumes, this study examines which type of announcement has the largest immediate market responses, and compares the magnitude (with positive/negative signs) of market reactions to different announcement categories on the Australian Securities Exchange (ASX). This study is more attractive under the Australian Continuous Disclosure Regime (CDR) because of the classification system regarding the market-sensitive announcements. Whether or not an announcement is market-sensitive is predetermined by ASX 'specialist staff' before the announcement is released on the market. This study investigates how the market interprets 
the announcement, examining market sensitivity from both the share price and trading volume movements after the announcement is available to the market. In other words, to some extent, the 'market sensitivity tag' shows the announcement that the market should react to, and this study reveals the announcement that the market does react to.

Focusing on market-sensitive announcements, except for the ASX Query, the significant results show a positive association between announcement categories and intraday Cumulative Abnormal Returns (CARs). Share prices react the most to Progress Reports and to Asset Acquisition \& Disposal, and the least to Periodic Reports and Issued Capital. Share price reactions to Distribution Announcements and Other are in the middle. There are at least two possible reasons for the different market reaction magnitudes. One is the information materiality nature: if one type of information is more important to investors than other information, this may cause higher abnormal returns on the market. The other is the information predictability: if there has been a lot of relevant information available in previous days or months, share prices could adjust to the information more smoothly. In terms of the trading volume reactions, intraday Abnormal Trading Volumes (ATVs) are observed after the release of Progress Reports, Asset Acquisition \& Disposal, Issued Capital and Periodic Reports. After separating good and bad news subsamples, the results show that Progress Reports and Asset Acquisition \& Disposal tend to indicate good signs to the market and cause larger trading volumes. Periodic Reports and Issued Capital could contain either good or bad news, but the trading volumes are driven by bad news. The results also reveal that company size is an important factor. Large companies tend to have relatively small market responses after information is released, which confirms that the larger companies are more stable. 
This study also evaluates long-term market effects of corporate disclosures by examining the timeliness of price discovery (also known as the speed of price discovery). It is the timeliness with which the full value relevant information is priced over a year before the annual earnings announcement is released. Previous literature has revealed that earnings announcements convey information, because significant stock returns or increased trading volumes are observed around the time that earnings announcements are released. However, it has also been suggested that there are other more timely information sources available that contain essentially similar or even the same value-relevant information. By the time preliminary final reports become available to the market, any potential value-relevant information has been included in the share price. The CDR again provides an attractive environment to examine the impact of corporate disclosures on the timeliness of price discovery, because listed companies have to disclose to the market before any other media sources. This enables an exploration of the relationship between the timeliness and the frequency of continuous disclosures. If the CDR is efficient in keeping investors fully informed, there should be no stock price surprise after the earnings announcement is released.

Given the importance of the Preliminary Final Reports to the timeliness metric and the fact that the immediate market reaction tests only cover announcements released during trading hours, this study investigates in depth the Preliminary Final Reports and finds some evidence that companies tend to release bad news after the market closes and/or on Fridays. In terms of the impacts of announcement frequency on the timeliness of price discovery, following the step 'All announcements - Periodic/Non-periodic announcements - Detailed categories of Nonperiodic announcements', the findings reveal that only earnings-related and cash flow announcements (Periodic Reports, Distribution Announcements and Quarterly Cash Flow Reports) can accelerate the timeliness of price discovery. Unfortunately, the results do not 
confirm that more announcements on the market could promote the timeliness with which the value-relevant (i.e. earning/income) information is incorporated into share prices. In addition to announcement frequency influences, the price discovery process is faster for companies with better share performance, are larger or have greater profitability. Financial companies have a timelier price discovery process than other companies; the price discovery process of financial companies was affected more by the $2008-2010$ Global Financial Crisis than that of other companies.

This study contributes to the market reaction literature by covering all categories of company announcements and comparing immediate market consequences for each type of announcement, and builds upon the timeliness of the price discovery literature by linking the announcement frequency of each continuous disclosure category to the timeliness with which annual earnings information is incorporated into share prices.

Keywords: Immediate Market Reactions, Timeliness of Price Discovery, Continuous Disclosure Regime, Market Sensitivity, Announcement Categories, Announcement Frequency 


\section{TABLE OF CONTENT}

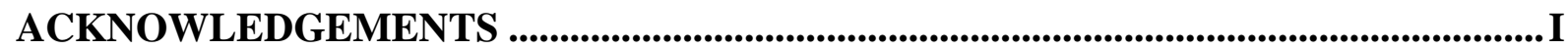

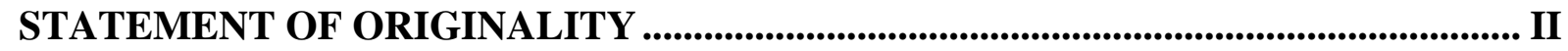

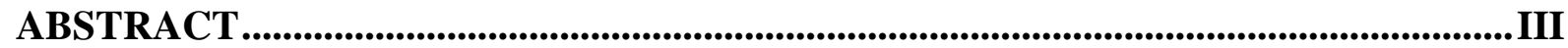

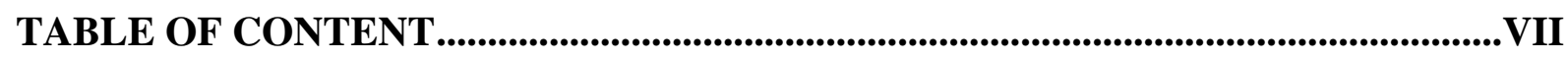

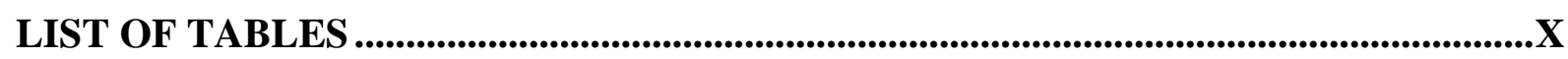

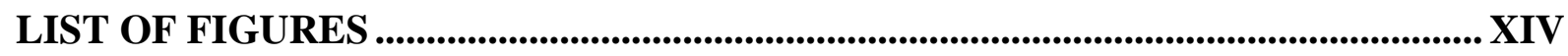

CHAPTER ONE: INTRODUCTION

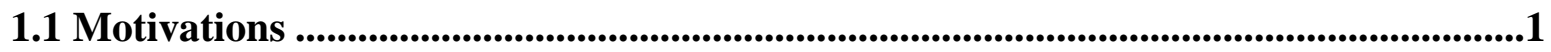

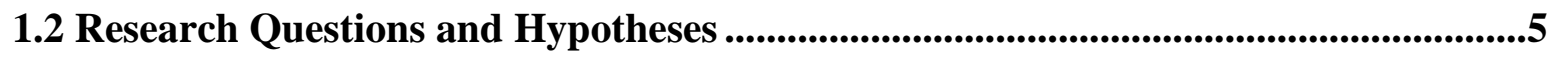

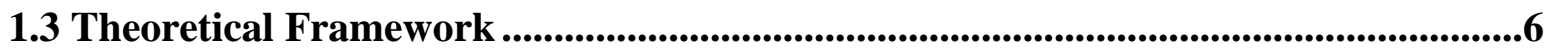

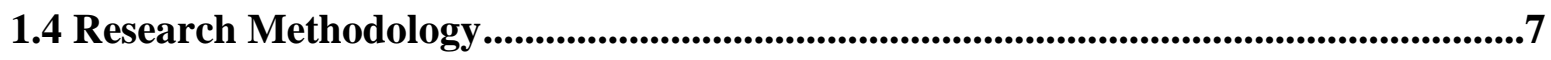

1.5 Key Research Findings ..........................................................................................................10

1.6 Research Contributions ............................................................................................12

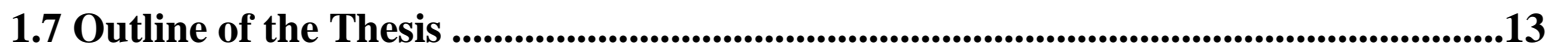

CHAPTER TWO: THE AUSTRALIAN CONTINUOUS DISCLOSURE REGIME .....15

2.1 Continuous Disclosure Requirements in ASX Listing Rules ................................16

2.2 Continuous Disclosure Provisions in the Corporations Act 2001 ............................19

2.3 CDR Public Enforcement ...............................................................................................................23

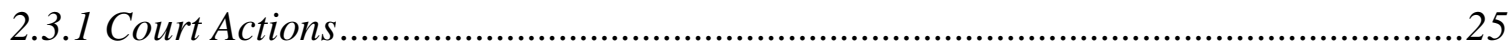

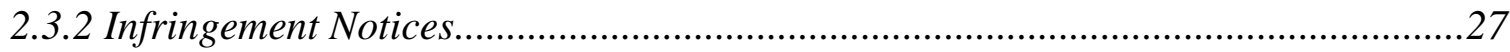

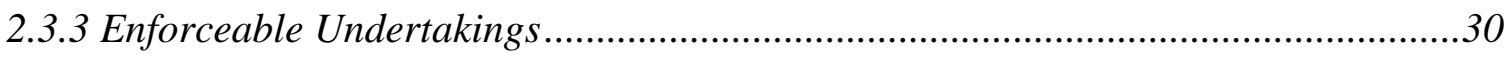

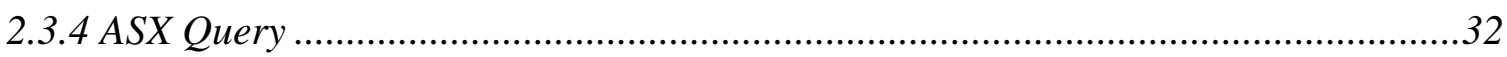

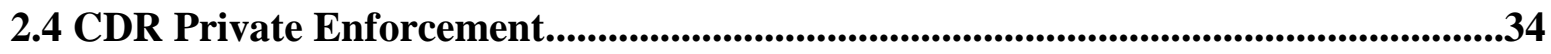

2.5 Summary

CHAPTER THREE: LITERATURE REVIEW ..........................................................37

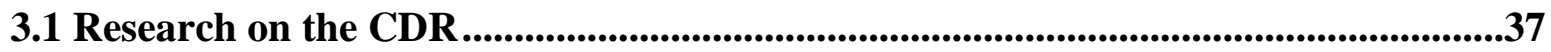

3.1.1 Legal Research on Continuous Disclosure Regulations .........................................38

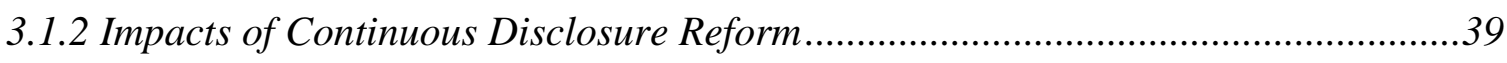

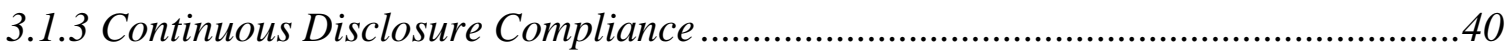

3.1.4 Prior Research on the Microstructure of ASX......................................................41

3.2 Literature on Market Reactions to Information Disclosure ....................................48

3.2.1 Market Reactions to Earnings Information .................................................................... 48 


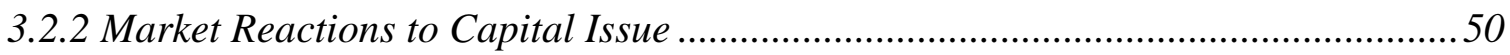

3.2.3 Market Reactions to Dividend and Other Types of Information .............................52

3.3 Literature on the Timeliness of Price Discovery ......................................................54

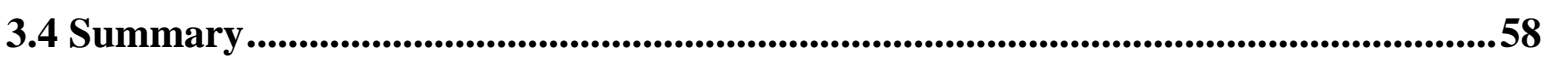

CHAPTER FOUR: HYPOTHESES DEVELOPMENT ...........................................59

4.1 Intraday Market Reactions to Announcements ....................................................................59

4.2 The Timeliness of Price Discovery ..........................................................................................67

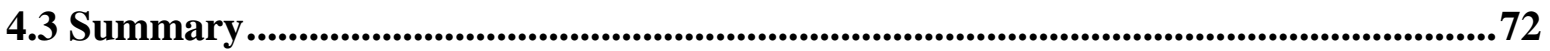

CHAPTER FIVE: RESEARCH METHODOLOGY ...............................................................73

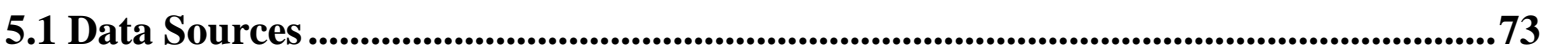

5.2 Sample Selection..........................................................................................................74

5.3 The Variables and Models of the Immediate Market Reaction Tests.......................75

5.3.1 Intraday Cumulative Abnormal Returns (CARs) and Abnormal Trading Volumes

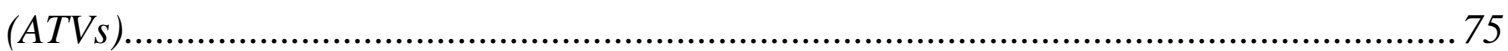

5.3.2 The Regression Models of the Immediate Market Reaction Tests .......................... 79

5.4 The Variables and Models of the Timeliness of Price Discovery Test ........................81

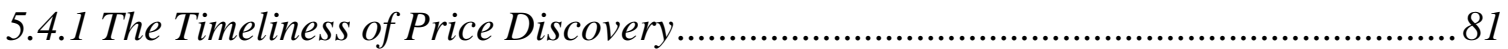

5.4.2 The Regression Model of the Timeliness of Price Discovery Test ...........................84

5.5 Summary ............................................................................................................................................8 87

CHAPTER SIX: RESEARCH ANALYSIS ON THE IMMEDIATE MARKET

REACTIONS TO DIFFERENT ANNOUNCEMENTS ............................................89

6.1 Original Announcement Distribution ...................................................................................91

6.2 Immediate Price and Volume Reaction $t$-test Results .............................................106

6.2.1 Investment/project-related Announcements ........................................................ 121

6.2.2 Earnings-related Announcements ................................................................... 123

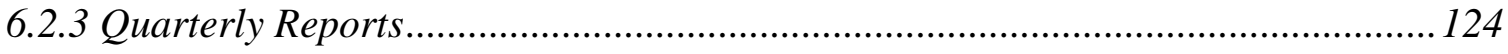

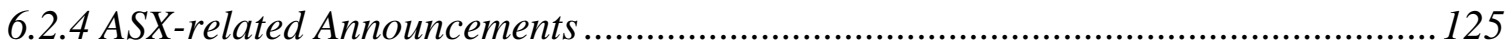

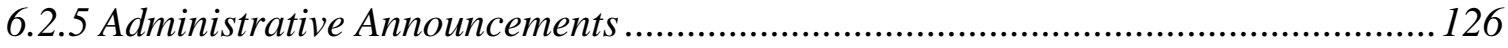

6.3 Immediate Price and Volume Reaction Regressions ..................................................127

6.3.1 Descriptive Analysis for the Regression Samples ............................................... 127

6.3.2 Share Price Reactions to Different Announcements ............................................. 132

6.3.3 Share Price Reactions to Good and Bad News as to Each Announcement Category

6.3.4 Regression Results of Trading Volume Reactions to Different Announcements.... 181 
CHAPTER SEVEN: RESEARCH ANALYSIS ON THE TIMELINESS OF PRICE

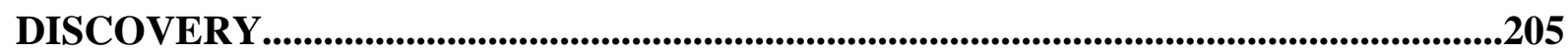

7.1 Analysis on Preliminary Final Reports ................................................................205

7.2 Regressions on the Timeliness of Price Discovery ................................................213

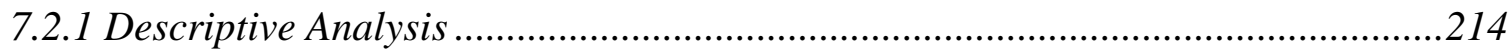

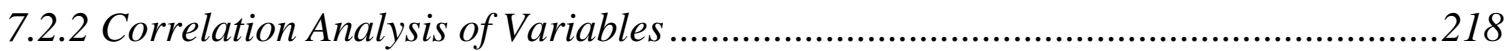

7.2.3 The Regression Results on the Timeliness of Price Discovery ..............................225

7.3 Robustness Tests on the Timeliness of Price Discovery .............................................233

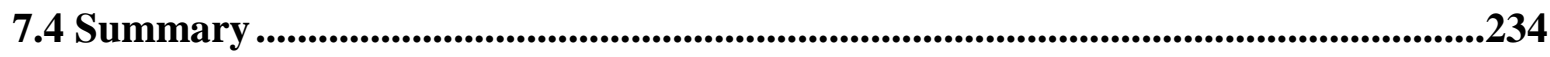

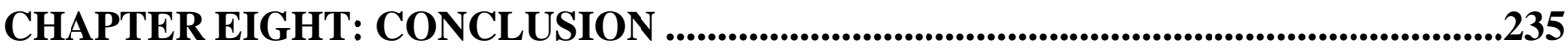

8.1 Summary of the Thesis ............................................................................................235

8.1.1 Immediate Market Reaction to Different Announcements ......................................236

8.1.2 The Impacts of Announcement Frequency on the Timeliness of Price Discovery..238

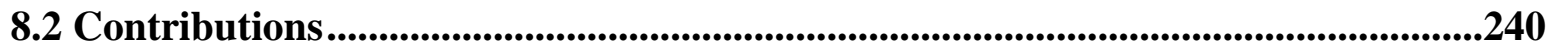

8.3 Limitations and Future Research Suggestions ...................................................................242

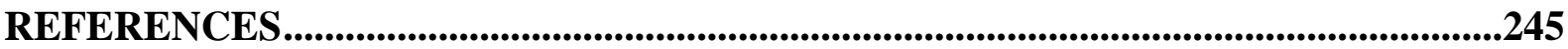

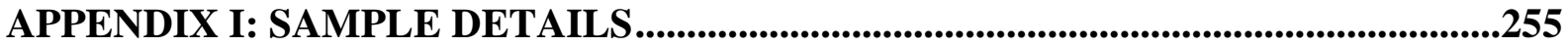

APPENDIX II: ANNOUNCEMENT DISTRIBUTION OF EACH CATEGORY OVER

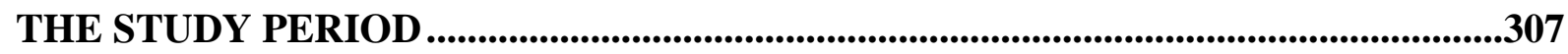

APPENDIX III: THE $T$-TEST RESULTS OF NON-MARKET-SENSITIVE

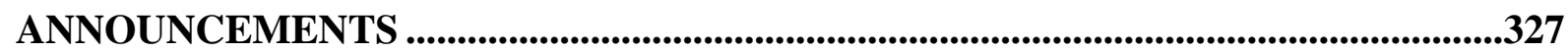

APPENDIX IV: THE CORRELATION MATRIX FOR VARIABLES IN IMMEDIATE

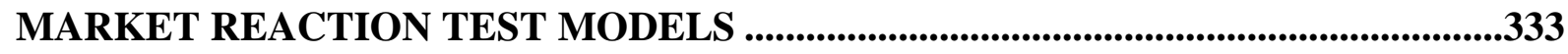

APPENDIX V: STATISTIC SUMMARY OF MARKET-SENSITIVE ANNOUNCEMENTS RELEASED AT DIFFERENT TIMES ....................................337

APPENDIX VI: ROBUSTNESS TESTS ON THE TIMELINESS OF PRICE DISCOVERY 


\section{LIST OF TABLES}

Table 1-1. Company Announcements: Main Categorisations ............................................

Table 2-1. Court Actions Relating to Continuous Disclosure Contraventions up to 31/12/2014

Table 2-2. Infringement Notices Concerning Continuous Disclosure Breaches up to $31 / 12 / 2014$ .28

Table 2-3. Enforceable Undertakings in Terms of Continuous Disclosure Failures up to $31 / 12 / 2014$

Table 2-4. Shareholder/Class Actions in Respect of Continuous Disclosure Failures. .34

Table 4-1. ASX Announcement Types/Subtypes 61

Table 5-1. Variable Description for the Immediate Market Reaction Tests. .80

Table 5-2. Variable Description for the Timeliness of Price Discovery Test .85

Table 5-3. Company Announcements: Main Categorisations .86

Table 6-1. All Announcements' Distribution as to Each Category. . .92

Table 6-2. Market-sensitive Announcements' Distribution as to Each Category ... .96

Table 6-3. Non-market-sensitive Announcements' Distribution as to Each Category 101

Table 6-4. Announcement Distribution Pattern from 2004 to 2014 105

Table 6-5. Intraday Cumulative Abnormal Returns of All Announcements 108

Table 6-6. Intraday Cumulative Abnormal Returns of Market-sensitive Announcements ....110

Table 6-7. The Difference between Intraday Cumulative Abnormal Returns before and after the Announcements

Table 6-8. The Difference between Intraday Cumulative Abnormal Returns before and after the Market-sensitive Announcements

Table 6-9. Intraday Abnormal Trading Volumes of All Announcements............................116

Table 6-10. Intraday Abnormal Trading Volumes of Market-sensitive Announcements ......118

Table 6-11. Statistic Summary of the Regressions for the Market Immediate Reactions ......129

Table 6-12. The Regression Results of Market-sensitive Announcements - Intraday CARs before the GFC

Table 6-13. The Regression Results of Market-sensitive Announcements - Intraday CARs during the GFC 
Table 6-14. The Regression Results of Market-sensitive Announcements - Intraday CARs after the GFC.

Table 6-15. The Regression Results of Market-sensitive Announcements - Intraday $\triangle$ CARs before the GFC

Table 6-16. The Regression Results of Market-sensitive Announcements - Intraday $\triangle$ CARs during the GFC

Table 6-17. The Regression Results of Market-sensitive Announcements - Intraday $\triangle$ CARs after the GFC.....

Table 6-18. The Regression Results of Market-sensitive Announcements with Bad News Intraday CARs before the GFC.

Table 6-19. The Regression Results of Market-sensitive Announcements with Bad News Intraday CARs during the GFC

Table 6-20. The Regression Results of Market-sensitive Announcements with Bad News Intraday CARs after the GFC

Table 6-21. The Regression Results of Market-sensitive Announcements with Bad News Intraday $\triangle$ CARs before the GFC.

Table 6-22. The Regression Results of Market-sensitive Announcements with Bad News Intraday $\triangle$ CARs during the GFC.

Table 6-23. The Regression Results of Market-sensitive Announcements with Bad News Intraday $\triangle$ CARs after the GFC....

Table 6-24. The Regression Results of Market-sensitive Announcements with Good News Intraday CARs before the GFC.

Table 6-25. The Regression Results of Market-sensitive Announcements with Good News Intraday CARs during the GFC

Table 6-26. The Regression Results of Market-sensitive Announcements with Good News Intraday CARs after the GFC

Table 6-27. The Regression Results of Market-sensitive Announcements with Good News Intraday $\triangle$ CARs before the GFC

Table 6-28. The Regression Results of Market-sensitive Announcements with Good News Intraday $\triangle \mathrm{CARs}$ during the GFC

Table 6-29. The Regression Results of Market-sensitive Announcements with Good News Intraday $\triangle$ CARs after the GFC.....

Table 6-30. The Regression Results of Market-sensitive Announcements - Intraday ATVs before the GFC 
Table 6-31. The Regression Results of Market-sensitive Announcements - Intraday ATVs during the GFC

Table 6-32. The Regression Results of Market-sensitive Announcements - Intraday ATVs after the GFC

Table 6-33. The Regression Results of Market-sensitive Announcements with Bad News Intraday ATVs before the GFC

Table 6-34. The Regression Results of Market-sensitive Announcements with Bad News Intraday ATVs during the GFC

Table 6-35. The Regression Results of Market-sensitive Announcements with Bad News Intraday ATVs after the GFC

Table 6-36. The Regression Results of Market-sensitive Announcements with Good News -

Intraday ATVs before the GFC

Table 6-37. The Regression Results of Market-sensitive Announcements with Good News -

Intraday ATVs during the GFC

Table 6-38. The Regression Results of Market-sensitive Announcements with Good News -

Intraday ATVs after the GFC

Table 7-1. Preliminary Final Report Distribution by Month 206

Table 7-2. One-Way ANOVA on the Market-adjusted Returns of Pre/During/Post Trading hours Preliminary Final Reports.

Table 7-3. One-Way ANOVA on the Market-adjusted Returns of Preliminary Final Reports on Different Weekdays

Table 7-4. Statistic Summary of the Timeliness of Price Discovery Regressions - Total, Periodic and Non-periodic Announcements.

Table 7-5. Statistic Summary of the Timeliness of Price Discovery Regressions - Different Types of Non-periodic Announcements

Table 7-6. Correlation Matrix for Deflated Timeliness Metric, Announcement Frequency, Market-sensitive Announcement Frequency and Control Variables

Table 7-7. Correlation Matrix for Deflated Timeliness Metric, Periodic/Non-periodic Report Frequency and Control Variables

Table 7-8. Correlation Matrix for Deflated Timeliness Metric, Market-sensitive Periodic/Nonperiodic Report Frequency and Control Variables

Table 7-9. Correlation Matrix for Deflated Timeliness Metric, Announcement Frequency as to Each Category and Control Variables

Table 7-10. Correlation Matrix for Deflated Timeliness Metric, Market-sensitive Announcement Frequency as to Each Category and Control Variables 
Table 7-11. The Impact of Announcement and Periodic/Non-periodic Report Frequency on

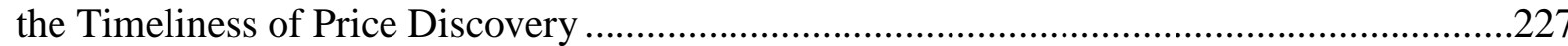

Table 7-12. The Impact of Different Non-periodic Announcement Frequency on the Timeliness of Price Discovery .228 


\section{LIST OF FIGURES}

Figure 2-1. The Public CDR Enforcement Pyramid, modified from Boros (2009) .................25

Figure 4-1. Abnormal Performance Indexes - from Ball and Brown (1968).........................68

Figure 5-1. The Timeline for the Release of a Market-sensitive Announcement ................... 77

Figure 6-1. Trading Hour Announcements Distribution by Time........................................95

Figure 6-2. Trading Hour Market-sensitive Announcements Distribution by Time ............... 99

Figure 6-3. Trading Hour Non-market-sensitive Announcements Distribution by Time ......102

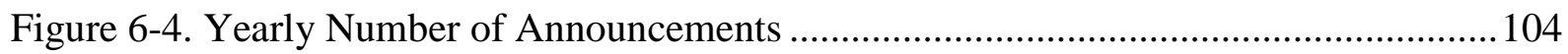

Figure 6-5. Yearly Proportion of Announcements Released Pre, During and Post Trading

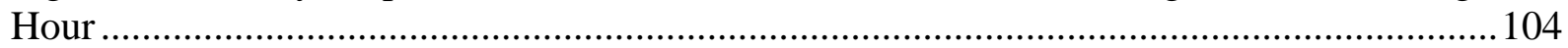

Figure 7-1. Preliminary Final Reports Distribution throughout the Day ..............................207

Figure 7-2. The Pre/During/Post Trading Hours Preliminary Final Reports by Year............208

Figure 7-3. Preliminary Final Reports Distribution by Weekdays .......................................208

Figure 7-4. Preliminary Final Reports Distribution throughout the Day - Good/Bad News..209

Figure 7-5. Preliminary Final Reports Distribution by Weekdays - Good/Bad News ...........210

Figure 7-6. The Average Deflated Timeliness Metric for Different Sectors by Year............233 


\section{CHAPTER ONE: INTRODUCTION}

The cornerstone of financial market research is the Efficient Market Hypothesis (EMH), which states that prices should fully reflect all information available in an ideal efficient market (Fama, 1970). Corporate disclosures can reduce information asymmetry and promote investor confidence, thereby improving market efficiency and integrity. Corporate disclosure has attracted the attention of accounting and capital market researchers. A lot of research has been conducted to understand with growing confidence how capital markets respond to corporate disclosures. Prior studies have examined the market reaction to public information using the event study approach although most research limits its concentration to a single type of information event.

Despite the numerous studies supporting market efficiency, it is often in the interest of companies to be less than transparent. The Continuous Disclosure Regime (CDR) in Australia plays an important role in improving market transparency, which provides a unique setting to analyse corporate disclosures in depth. This study focuses on the market effects of company announcements under the CDR over both the short and long terms. Specifically, this study aims to explore which type of information leads to more significant immediate price and volume movement, and which type of announcement assists share prices to more accurately reflect a company's performance (i.e. earnings information) within a financial year.

\subsection{Motivations}

This study is motivated by the contradiction between efficient market and a company's opportunistic disclosure behaviour. It is possible to boost stock prices or avoid sudden price 
decreases by disclosing certain information at particular times. Evidence supports the concept that companies tend to 'hide' bad news by releasing it after the trading hours when market attention is low, or to 'highlight' good news by releasing it before or during trading hours when market attention is high (deHaan, Shevlin, \& Thornock, 2015). The CDR provides an appropriate setting to examine the relationship between market efficiency and corporate disclosures, because the CDR should constrain this opportunistic disclosure behaviour. Unlike US stock markets, in which the Regulation Fair Disclosure (Reg FD) merely reduces asymmetric information among investors, the Australian stock market provides an information environment in which the CDR reduces the asymmetric information between internal managers and external traders to a large extent (Matolcsy, Tyler, \& Wells, 2012). The CDR requires listed companies to continuously release all information which is likely to materially impact the share price as soon as they become aware of the information. In Australia, the CDR aims to keep investors fully and equally informed, maintaining market efficiency. Under the CDR, it is possible to evaluate in depth the market effects of corporate disclosures in the short and long terms.

To evaluate short term market reactions to corporate disclosures, this study combines research techniques found in the market reaction literature with those in the microstructure literature. This allows further exploration of the immediate market reactions to corporate disclosures, especially market-sensitive (also known as price-sensitive) announcements. Most prior studies on market reactions are based on daily data, focusing on a single disclosure type but ignoring the intraday impact of announcements on share prices (or returns) and trading volumes. Such studies are less likely to capture a comprehensive view in terms of market responses to different information. By using tick (transaction) data to calculate intraday abnormal returns and abnormal trading volumes, this study aims to examine which type of announcement has the 
largest immediate market response, and to compare the magnitude (with positive/negative sign) of market reactions to different announcement categories on the Australian Securities Exchange (ASX). This study is possible under the CDR because of the classification system for marketsensitive announcements. Whether or not an announcement is market-sensitive is pre-decided by the ASX 'specialist staff' before the announcement is released on the market. This study investigates how the market interprets the announcement, examining the market sensitivity from the share price and trading volume movements after the announcement is available to the market. Therefore, this study enhances the understanding of the immediate effects of stock prices and trading volumes on public information disclosure.

In Australia, the time stamps of companies' announcements make precise market reaction tests possible. Some stock markets do not record the release time of company announcements, such as the New York Stock Exchange (NYSE), London Stock Exchange (LSE) and Toronto Stock Exchange (TSX). There are other stock markets that provide announcement time stamps [such as Hong Kong Stock Exchange (HKEx), Singapore Exchange (SGX) and New Zealand Exchange (NZX)], but the Australian Securities Exchange (ASX) is an attractive stock market to conduct research on intraday market efficiency. Due to the CDR, companies have fewer opportunities to combine several kinds of information in one announcement. Announcements on the ASX are categorised in detail (19 main types and 152 subtypes), and provide relatively "clean" information, contributing to more accurate market efficiency tests. In contrast, Hong Kong did not introduce a statutory backing to the Listing Rules until $2010^{1}$ (Chan \& Ho, 2011). HKEx is one of the most volatile stock markets in the world and is dominated by small companies (Lam \& Tam, 2011). In addition, a number of listed companies on both HKEx and SGX have family ownerships and/or state ownerships. NZX is a small market with fewer than

\footnotetext{
1 "In March 2010, the HK government issued another consultation paper proposing statutory codification for disclosing PSI [price sensitive information] by listed companies" (Chan \& Ho, 2011, pp. 87-88)
} 
150 listed companies in 2014. Therefore, ASX provides more comprehensive information to study how the market reacts to different information immediately after the information is released.

To evaluate long term market effects of corporate disclosures, this study examines the timeliness of price discovery (also known as the speed of price discovery). It is the timeliness with which the full value relevant information is priced over a year before the annual earnings announcement is released. Earnings announcements may convey information, but it has been suggested that there are other more timely information sources available that contain essentially similar or even the same value-relevant information (Ball \& Brown, 1968; Beaver, 1968). By the time preliminary final reports become available to the market, any potentially value-relevant information has been impounded in the share price. If there is more value-relevant information available to the market in a timely manner, the price discovery process should be faster. In an extreme case, if a firm did not release any other information except for its annual financial report, investors would have no idea rather overstates the situation. As a result, the firm's share price would not be able to reflect the firm's true value during the year before the release of its financial report, leading to an extremely slow price discovery process. The CDR again provides an attractive environment to examine the impact of corporate disclosures on the timeliness of price discovery, because the ASX-listed companies have to disclose information to the market before any other media sources. By taking public announcements into account, this study explores the finer relationship between the timeliness and the frequency of continuous disclosures, contributing to the literature on the timeliness of price discovery. 


\subsection{Research Questions and Hypotheses}

This study aims to find the answers to the following questions.

(1) How market (price/volume) sensitive is the information contained in each disclosure category in a continuous disclosure environment?

(2) How quickly is the value-relevant information incorporated into share prices before the annual earnings announcement is released, and how can company announcements promote this timeliness of price discovery?

Specifically, this study examines immediate market reactions to different types of announcements, and examines the impacts of announcement frequency as to different continuous disclosure categories on the timeliness of price discovery, using the model developed by Beekes and Brown (2006). Accordingly, two main hypotheses are developed in a null form

H1 a0: There is no price reactions to company announcements on the ASX.

H1 bo: There is no trading volume reactions to company announcements on the ASX.

H20: The announcement frequency regarding each category has no impact on the timeliness with which the annual earnings information is priced before the earnings announcement is made available to the market. 


\subsection{Theoretical Framework}

This study employs the EMH theory to evaluate the market effects of disclosures for both short and long terms under the Australian CDR. Fama (1970) pointed out three forms of efficient markets: weak, semi-strong, and strong. Each represents one information subset. In weak market tests, research focuses on whether current prices reflect the historical prices (or past information); in semi-strong market tests, the research question is whether share prices are able to efficiently adjust to new public information (e.g. earnings and other announcements on stock markets); in strong market tests, the relevant research is concerned with whether or not the participants, including individual investors and groups, have equal access to all information relative to prices. In a strong market, prices are supposed to instantly incorporate both public and private (i.e. insider) information. The CDR promotes stock market efficiency by reducing information asymmetry between external investors and internal managers to a large extent, so share prices can efficiently reflect the information contained in different announcements.

Market microstructure theory deals with "the process and outcomes of exchanging assets under explicit trading rules" (O'Hara, 1995, p. 1), enhancing the understanding of how prices emerge and investors (i.e. buyers and sellers) trade on the market, and how the market becomes efficient. Due to the availability of detailed market information such as real-time, prices, volumes and orders on the ASX, this study can empirically investigate how investors use different information to make trading decisions and adjust their trading prices and volumes at the intraday level, as reflected in the immediate market reactions to disclosures. In addition, the ASX market microstructure study can provide some insights into the classification system of announcement 'market sensitivity' under the CDR. 
If the market is efficient, share prices should not only reflect information in the short-term, but also reflect a firm's total value or performance in the long term (typically a financial year). The timeliness of price discovery reveals the timeliness (or speed) with which share prices incorporate value-relevant (i.e. earnings) information before the earnings announcement is available to the market. It is posited that the everyday share price adjustment to information should be getting closer to the 'terminal value', the agreement on share price when the investors have been informed about the firm's annual performance. There would be a positive impact of disclosures on the timeliness of price discovery.

\subsection{Research Methodology}

This study encompasses all available market announcements issued by companies on the ASX between 1 July 2004 and 31 December 2014. This study period could avoid any potential impacts from the regulation change, as the current provisions regarding continuous disclosure in the Corporations Act 2001 came into effect on 1 July 2004. The final sample contains 2,893 companies. For immediate market reaction tests, there are 427,899 observations $^{2}: 122,853$ market-sensitive and 305,046 non-market-sensitive announcements. For the timeliness of price discovery tests, this study manually worked out 20,122 Preliminary Final Report dates for 2,893 companies during the same period and identifies 17,188 firm-years. ${ }^{3}$

For immediate market reaction tests, intraday Cumulative Abnormal Returns (CARs) and Abnormal Trading Volumes (ATVs) are calculated over eight time windows: [0, 10min], [0,

\footnotetext{
${ }^{2}$ Some observations have missing share price or financial data. After deleting the observations with missing data, 257,951 observations remained: 67,620 market-sensitive and 190,331 non-market-sensitive announcements. For each of the eight time windows, if there is one CAR, $\triangle \mathrm{CAR}$ or ATV available, that observation is kept.

${ }^{3}$ Some firm-years have missing share price (for timeliness metric calculation) or financial data. After deleting the missing data, 11,517 firm-years remained.
} 
$15 \mathrm{~min}],[0,30 \mathrm{~min}],[0,60 \mathrm{~min}],[0,90 \mathrm{~min}],[0,120 \mathrm{~min}],[0,150 \mathrm{~min}]$, and $[0,180 \mathrm{~min}]$. The $t-$ tests and linear regression models are employed to test Hypothesis one. The Variance Inflation Factor (VIF) is used to examine the multicollinearity problems among the announcement category indicators and control variables. Due to the serious multicollinearity problems when including non-market-sensitive announcements, the regressions models only examine marketsensitive announcements.

For the timeliness of price discovery tests, this study uses a model slightly modified from Beekes and Brown's (2006) timeliness metric to measure the timeliness with which valuerelevant information is incorporated into share prices over a year before the earnings announcements are available. With respect to the announcement frequency, this study first examines the association between the timeliness of price discovery and the number of all announcements released over the timeliness metric time window. Then, this study further divides the announcements into two groups: Periodic Reports and Non-periodic Reports. Lastly, this study focuses on the Non-periodic Reports to test whether different types of announcements influence the timeliness of price discovery in different ways. This study explores the effects of both the total announcements and the market-sensitive announcements on the timeliness of price discovery. ANOVA tests, VIF (Variance Inflation Factor) and linear regression models are used.

Company announcements are categorised according to the ASX Primary Report Code. All tests and results are discussed concerning the 19 announcement categories. A brief explanation of the announcement categories is outlined in Table $1-1 .{ }^{4}$

\footnotetext{
${ }^{4}$ The explanations of announcement categories are based on google search, Investopedia, ASX ComNews ${ }^{\mathrm{TM}}$ Manual, and ASX Listing Rules Guidance Notes.
} 
Table 1-1. Company Announcements: Main Categorisations

\begin{tabular}{ll}
\hline Type & Announcements \\
\hline 1 & $\begin{array}{l}\text { Takeover / Schem } \\
\text { Announcements }\end{array}$ \\
2 & $\begin{array}{l}\text { Security Holder } \\
\text { Details } \\
3\end{array}$ \\
Periodic Reports
\end{tabular}

Description

Announcements that are related to a takeover event from either bidder or target companies, such as bidder's statement, target's statement, directors' statement reply takeover.

Announcements that provide shareholder or ownership information, such as becoming/ceasing to be a substantial holder, directors' interests.

Periodic Reports

Financial reports that are released at a certain time of a year, referring to preliminary final report, half yearly report and annual report; it may also include other announcements that are related to financial reports such as profit guidance and debt facility.

$4 \quad$ Quarterly

Activities Report

$5 \quad$ Quarterly Cash

Flow Report

Quarterly reports of mining activities; they could be provided either by mining entities or by other entities that have mining projects.

Quarterly reports of cash flow provided by investment entities, mining exploration entities, and oil and gas exploration entities. Commitments Test Entities are also required to provide quarterly cash flow reports, but these reports are under Category 19 Commitments Test Entity Quarterly Reports.

$6 \quad$ Issued Capital

$7 \quad$ Asset Acquisition

\& Disposal

$8 \quad$ Notice of Meeting

Announcements that relate to issuing shares to shareholders, or buying back shares from shareholders.

Announcements relating to buying or disposing assets. This is a vague category with only three subcategories asset acquisition, disposal and other.

The notice to shareholders regarding the date, time and place of shareholder meetings; the results of such meetings are also included in this announcement category.

$9 \quad$ ASX

Announcements that are sent from ASX or relate to communications between a

Announcement

10 Distribution

Announcement

11 Progress Reports

12 Company

Administration

$13 \quad$ Notice of Call

14 Other

15 Chairman's

Address

16 Letter to

Shareholders

ASX Query listed company and ASX, such as trading halt notices, suspension from official quotation.

Dividend related announcements, such as dividend rate, pay date.

A report of the progress regarding certain project, and release time is unknown. This is also a vague category with only two subcategories progress report and other.

Announcements relating to company profile or company management, such as Director Appointment/Resignation, company address, company name change. When there are unpaid or partly paid shares issued, a call notice can be sent by the company requiring particular shareholders to pay the amount outstanding. Announcements that cannot be classified into any other categories, such as open briefings, legal proceedings, overseas listing.

Chairman's addresses or presentations on the company's financial performance, market position, and invested projects.

A letter that is written by top management to shareholders, providing an overview of the company's operations over the year (could be half-year, quarter or month).

A query letter sent by ASX. It is part of ASX's enforcement practices regarding a company' compliance with its continuous disclosure obligations. There are two types of ASX queries, price query letters and aware letter. If ASX notices abnormal trading in a company's securities, a price query will be sent to verify if any undisclosed information is causing this abnormal trading; if ASX has concerns about whether the company has fully and promptly disclosed any market-sensitive information, an aware letter will be sent.

$18 \quad$ Structured

Products

Announcements that are related to the company's structured products. A structured product is a financial derivative package, including securities, options, bonds and foreign currencies.

19 CTE Quarterly Reports

The entities that, under the 'commitments test', are required to provide quarterly cash flow reports. To list on the ASX, an entity must satisfy either the 'profit test' or the 'assets test' in the listing requirements, and the 'commitments test' is under the 'assets test'. If an entity's main (half or more) tangible asset is cash or readily convertible to cash after raising funds from an Initial Public Offering (IPO), the entity is required to makes commitments to spend at least half of the cash or equivalent assets consistently with its objectives. 


\subsection{Key Research Findings}

To address Research Question One, the results obtained from the immediate market reaction tests provide evidence that the market reacts to different types of announcements differently, revealing the different price and sensitivity to different types of information. Focusing on the market-sensitive announcements, the ASX Query is the only announcement category that causes immediate negative market responses. Intraday CARs are positive for the release of six types of announcements. Share prices react the most to Progress Reports and Asset Acquisition \& Disposal, and the least to Periodic Reports and Issued Capital. Share price reactions to Distribution Announcements and Other are in the middle.

After taking good and bad news into account, this study further explores and compares the magnitude of positive/negative share price reactions to different announcement categories. In the bad news subsamples, the CARs are significantly negatively associated with the release of ASX Query, Issued Capital, Periodic Reports, Progress Reports and Acquisition \& Disposal. In the good news subsamples, share prices react positively to Progress Reports, Asset Acquisition \& Disposal, Periodic Reports, Distribution Announcements, Issued Capital, Other and ASX Query. The announcements are ranked according to the magnitude of price responses. Combining the results of good and bad news, the immediate market reactions show the information nature conveyed in different types of announcements. For example, Progress Reports Asset Acquisition \& Disposal, Distribution Announcements, and Other indicate good signs for the market during the trading hours. There are consistent positive price reactions to good news but small or no significant negative price reactions to bad news after these announcements are released. 
In terms of the trading volume reactions, significant ATVs are observed after the release of four types of announcements: Progress Reports, Asset Acquisition \& Disposal, Issued Capital and Periodic Reports, ranking from the largest to the smallest trading volume reactions. After separating good and bad news subsamples, the results show that Progress Reports and Asset Acquisition \& Disposal tend to indicate good signs in the market and cause large trading volumes. Periodic Reports and Issued Capital could contain either good or bad news, but trading volumes are driven by bad news.

The immediate market reaction tests can only cover announcements released during trading hours. To further explore and understand the association between corporate disclosure behaviour and its market consequences, this study investigates the Preliminary Final Reports in depth. The results from the ANOVA tests reveal that the market-adjusted returns around Preliminary Final Reports released outside trading hours are significantly less than those released pre- and during trading hours, which may indicate that companies tend to release bad news after the market has closed.

In terms of the Research Question Two, this study shows that financial companies have timelier price discovery, while resource companies have less timely price discovery. The results from the timeliness of price discovery test do not confirm that more announcements on the market could promote the timeliness with which the value-relevant (i.e. earning/income) information is incorporated into share prices. Only earnings-related and cash flow announcements (Periodic Reports, Distribution Announcements and Quarterly Cash Flow Reports) can help stock prices anticipate companies' annual income information before the Preliminary Final Reports are available to the market. Other types of announcements may influence investors' expectations 
(either optimistic or pessimistic) on firm value. As a result, when the earnings announcements become available, investors may find the earnings information different from their expectations.

\subsection{Research Contributions}

The intraday market reaction examination reveals the extent to which share prices react to different corporate disclosures within three hours. It contributes to the market reaction literature by covering all categories of company announcements, and by comparing the market consequences for each type of announcement. It also contributes to the literature on both market efficiency and market microstructure by exploring the role of public information in price formation and trading behaviour changes over a short time (minutes and hours) around the information release.

To some extent, the timeliness of price discovery test suggests which type of announcement promotes the timeliness of price discovery, and which type does not. It contributes to the timeliness of price discovery literature by linking the announcement frequency of each continuous disclosure category to the timeliness with which annual earnings information is incorporated into share prices.

This study shows how well the ASX 'market sensitivity tag' operates, building upon the empirical literature on continuous disclosure. The results from the immediate market reaction tests show that the market reacts strongly to market-sensitive announcements in project- or earnings-related announcement categories but not in the administrative announcement categories. This suggests that the CDR applies the market-sensitive label conservatively; that 
is, the ASX is more likely to label a non-market-sensitive disclosure as market-sensitive than to label a market-sensitive document non-market-sensitive.

\subsection{Outline of the Thesis}

The following chapters develop the basis of this study. Chapter Two introduces the continuous disclosure regime in Australia. Chapter Three reviews the prior related literature. Chapter Four presents hypotheses development, followed by research design in Chapter Five. The empirical results and research analysis are reported in Chapters Six and Seven. Finally, Chapter Eight presents the conclusions, including contributions and limitations of this study. 


\section{CHAPTER TWO: THE AUSTRALIAN CONTINUOUS DISCLOSURE REGIME}

The Continuous Disclosure Regime (CDR) in Australia consists of Listing Rule 3.1 (Continuous Disclosure) of the Australian Stock Exchange (ASX), the statutory regime in the Australian Corporations Act 2001 (Cth) that reinforces Listing Rule 3.1. The Australian Securities and Investments Commission (ASIC) $)^{5}$ also plays an important role in the CDR, as it regulates Australian corporations, financial markets and other financial services. Under the Corporations Act 2001, ASX is obliged to ensure that its financial markets are fair, orderly and transparent. ASIC describes its role as "ensuring that Australia's financial markets are fair and transparent, supported by confident and informed investors and consumers" (ASIC official website), which is also reflected in its strategic priorities. The purpose of the CDR is to ensure sufficient and timely disclosures and to prevent unfairness between public investors and insiders, thereby promoting investor confidence in decision-making and ultimately promoting capital market integrity (Bloch, Weatherhead, \& Webster, 2011).

Because there are continuing improvements in information technology, timely disclosure is increasingly important. Although in many other countries, listed companies are required to disclose their information to stock markets, 'immediate' disclosure is not strictly required. However, in Australia, the CDR set out in ASX Listing Rule 3.1 requires listed companies to immediately disclose any potential 'market-sensitive' information. This provides a strictly mandatory disclosure environment and reduces information asymmetry to a large extent. The CDR was introduced into the ASX Listing Rules in 1994 and also into the Corporations Law (later replaced by the Corporations Act 2001), as discussed below. To match the research

\footnotetext{
${ }^{5}$ ASIC is an independent Commonwealth Government body and carries out most of its work under the Corporations Act 2001 (ASIC official website).
} 
sample period (July 2004-December 2014), the following review of the development of CDR in Australia is up to 31 December $2014 .^{6}$

\subsection{Continuous Disclosure Requirements in ASX Listing Rules}

Under Listing Rule 3.1, unless an exception arises, an Australian listed company must keep investors fully informed of any material (or potential market-sensitive) information as soon as the company becomes aware of it. For ASX-listed companies, while the CDR was introduced in 1994, the current primary obligation regarding continuous disclosure was amended in July 1996, followed by some further key amendments in January 2003.

In 1996, ASX Listing Rule 3.1 stated that:

\section{“General Rule}

3.1 Once an entity is or becomes aware of any information concerning it that a reasonable person would expect to have a material effect on the price or value of the entity's securities, the entity must immediately tell the ASX that information. This rule does not apply to particular information while each of the following applies.

3.1.1 A reasonable person would not expect the information to be disclosed.

3.1.2 The information is confidential.

3.1.3 One or more of the following applies.

(a) It would be a breach of a law to disclose the information.

(b) The information concerns an incomplete proposal or negotiation.

(c) The information comprises matters of supposition or is insufficiently definite to warrant disclosure.

(d) The information is generated for the internal management purposes of the entity.

\footnotetext{
${ }^{6}$ At the time of writing, there has been no change in the listing rules and provisions regarding the CDR since 2014. There are some alleged continuous disclosure breaches after 2014, but they are not included in this chapter.
} 
(e) The information is a trade secret."

In 2003, amendments were made for the "enhanced disclosure reforms" (Bloch et al., 2011). At that time, ASX Listing Rule 3.1 was amended to:

“General Rule

3.1 Once an entity is or becomes aware of any information concerning it that a reasonable person would expect to have a material effect on the price or value of the entity's securities, the entity must immediately tell the ASX that information.

Exception to rule 3.1

3.1A Listing Rule does not apply to particular information while all of the following are satisfied.

3.1A.1 A reasonable person would not expect the information to be disclosed.

3.1A.2 The information is confidential and ASX has not formed the view that the information has ceased to be confidential.

3.1A.3 One or more of the following 5 situations applies:

- It would be a breach of a law to disclose the information.

- The information concerns an incomplete proposal or negotiation.

- The information comprises matters of supposition or is insufficiently definite to warrant disclosure.

- The information is generated for the internal management purposes of the entity.

- The information is a trade secret.

False market

3.1B If ASX considers that there is or is likely to be a false market in an entity's securities and asks the entity to give it information to correct or prevent a false market, the entity must give ASX the information needed to correct or prevent the false market."

One impact of these amendments is to separate the exceptions to the continuous disclosure obligation (the Listing Rule 3.1A). It was also clarified that a listed entity has to meet all three 
exceptions to withhold the information from immediate disclosure. The most important amendment was the 'false market rule', namely Listing Rule 3.1B, according to which ASX has the right to require additional information to prevent a false market. ${ }^{7}$ This 'false market' situation may be created by market speculation or media comment, which may affect the share price (North, 2013a, pp. 69-70).

Since 2003, only a few minor amendments have been made to the Listing Rule 3.1. For example, the order of Listing Rule 3.1A.1 and 3.1A.3 was exchanged. Furthermore, the original Listing Rule 3.1B (cited on the previous page) merely required the company to provide the information, but the amended wording of Listing Rule 3.1B has been slightly changed to emphasise the 'immediate' nature of the disclosure obligations.

"3.1B If ASX considers there is or is likely to be a false market in an entity's securities and asks the entity to give it information to correct or prevent a false market, the entity must immediately give ASX that information."

If a company is not able to disclose certain information immediately, a trading halt (or a further voluntary suspension) can be requested to help the company comply with continuous disclosure provisions under the ASX Listing Rule 17.1 and 17.2 (ASX Guidance Note 8, Continuous Disclosure).

ASX plays an important role in monitoring and regulating listed companies' disclosure under the CDR. Compared with ASX's powers in 1996, ASX is now able to form the view that some particular information is no longer confidential, based on its knowledge (Listing Rule 3.1A.2). In addition, ASX is able to apply a false market test (Listing Rule 3.1B). These changes have

\footnotetext{
7 'False Market' definition: “A market where prices are manipulated and impacted by erroneous information, preventing the efficient negotiation of prices. These types of markets will often be marred by volatile swings because the true value of the market is clouded by the misinformation." - From Investopedia http://www.investopedia.com/terms/f/falsemarket.asp.
} 
increased ASX's powers to monitor and enforce disclosure. If ASX believes that there might have been a false market, and/or that some information might have lost the confidentiality, ASX can require the relevant listed companies to provide immediate information disclosure.

\subsection{Continuous Disclosure Provisions in the Corporations Act 2001}

The earliest statutory provisions on continuous disclosure were introduced by the Corporate Law Reform Act 1994. Section 1001A of the Corporations Law ${ }^{8}$ conferred statutory support on ASX Listing Rule 3.1, which required disclosing entities ${ }^{9}$ to disclose any "material" information immediately. This legislative framework was later provided in the Corporations Act 2001 .

In the Corporations Act 2001 (Act No. 50, 2001), as originally enacted, Continuous Disclosure rules were enacted in Section 1001. Particularly, Section 1001A:

"(1) This section applies to a listed disclosing entity if provisions of the listing rules of a securities exchange:

(a) apply to the entity; and

(b) require the entity to notify the securities exchange of information about specified events or matters as they arise for the purpose of the securities exchange making that information available to a stock market conducted by the securities exchange.

(2) The disclosing entity must not contravene those provisions by intentionally, recklessly or negligently failing to notify the securities exchange of information:

(a) that is not generally available; and

(b) that a reasonable person would expect, if it were generally available, to have a material effect on the price or value of ED securities ${ }^{10}$ of the entity.

\footnotetext{
${ }^{8}$ In 2001, the Corporations Law changed to Corporations Act 2001. Since then the Corporations Law has been ceased.

${ }^{9}$ A 'disclosing entity' is defined in s111 AC of the Corporations Act 2001: “(1) If any securities of a body (except interests in a managed investment scheme) are ED securities, the body is a disclosing entity for the purposes of this Act. (2) If any interests in a managed investment scheme are ED securities, the undertaking to which the interests relate is a disclosing entity for the purposes of this Act".

10 'ED securities' are defined in s111AD: “(1) Securities of a body are ED securities (short for "enhanced disclosure
} 
(3) A contravention of subsection (2) is only an offence if the failure concerned is intentional or reckless."

The Corporations Act 2001 prohibited disclosing entities from intentionally breaking the continuous disclosure provisions. In other words, disclosing entities failed to comply with their continuous disclosure obligations only when they were intentional or reckless.

Further amendments were made as part of the Financial Services Reform Act 2001, which came into effect on 11 March 2002. These changes to the Corporations Act 2001 explained that "a disclosing entity is required to disclose information about its securities that is material and not generally available" (Corporations Act 2001, Chapter 6CA). Moreover, Sections 1001A and 1001B were deleted from the Corporations Act 2001, and Sections 674 and 675 were introduced. These sections are generally similar. The difference is that Section 675 is about other disclosing entities that are unlisted or listed on markets without "provisions of a kind referred to in subsection 674(1)".11

\section{“674 Continuous disclosure — listed disclosing entity bound by a disclosure requirement in market listing rules}

Obligation to disclose in accordance with listing rules

(1) Subsection (2) applies to a listed disclosing entity if provisions of the listing rules of a listing market in relation to that entity require the entity to notify the market operator of information about specified events or matters as they arise for the purpose of the operator making that information available to participants in the market.

securities") for the purposes of this Act if, and only if: (a) they are ED securities under section 111AE, 111AF, 111AFA, $111 \mathrm{AG}$ or $111 \mathrm{AI}$; and (b) they are not declared under section 111AJ not to be ED securities. (2) For the purposes of sections $111 \mathrm{AE}, 111 \mathrm{AF}, 111 \mathrm{AG}$ and $111 \mathrm{AI}$, a class of shares or debentures is taken to include units of shares or debentures in that class".

${ }^{11}$ Corporations Act 2001 section "675 Continuous disclosure—other disclosing entities

(1) This section applies to:

(a) a listed disclosing entity if:

(i) there is only one listing market in relation to the entity and the listing rules of that market do not contain provisions of a kind referred to in subsection 674(1); or (ii) there is more than one listing market in relation to the entity and none of those markets have listing rules that contain provisions of a kind referred to in subsection 674(1); or

(b) an unlisted disclosing entity". 
(2) If:

(a) this subsection applies to a listed disclosing entity; and

(b) the entity has information that those provisions require the entity to notify to the market operator; and

(c) that information:

(i) is not generally available; and

(ii) is information that a reasonable person would expect, if it were generally available, to have a material effect on the price or value of ED securities of the entity;

the entity must notify the market operator of that information in accordance with those provisions.

Note 1: Failure to comply with this subsection is an offence (see subsection 1311(1)).

Note 2: This subsection is also a civil penalty provision (see section 1317E). For relief from liability to a civil penalty relating to this subsection, see section $1317 \mathrm{~S}$.

(3) For the purposes of the application of subsection (2) to a listed disclosing entity that is an undertaking to which interests in a registered scheme relate, the obligation of the entity to notify the market operator of information is an obligation of the responsible entity.

(4) Nothing in subsection (2) is intended to affect or limit the situations in which action can be taken (otherwise than by way of a prosecution for an offence based on subsection (2)) in respect of a failure to comply with provisions referred to in subsection (1).

\section{Obligation to make provisions of listing rules available}

(5) If the listing rules of a listing market in relation to a listed disclosing entity contain provisions of a kind referred to in subsection (1), the market operator must ensure that those provisions are available, on reasonable terms, to:

(a) the entity; or

(b) if the entity is an undertaking to which interests in a registered scheme relate - the undertaking's responsible entity.

Note: Failure to comply with this subsection is an offence (see subsection 1311(1))."

Compared to the prior provision in the Corporations Act 2001, the current provisions are more rigorous and detailed. A contravention is no longer defined as "only an offence if the failure concerned is intentional or reckless", which means the offence does not require proof of intention. According to Section 674(2), a contravention of Listing Rule 3.1 is a contravention 
of the statutory provision. Whether particular information is 'material' should be determined by the statutory test laid down under Section 674(2)(c)(ii), and also under Section 677:

\section{“677 Sections 674 and 675 -material effect on price or value}

For the purposes of sections 674 and 675, a reasonable person would be taken to expect information to have a material effect on the price or value of ED securities of a disclosing entity if the information would, or would be likely to, influence persons who commonly invest in securities in deciding whether to acquire or dispose of the ED securities".

ASX applies this test by interpreting "the reference to persons who commonly invest in securities as a reference to persons who commonly buy and hold securities for a period of time, based on their view of the inherent value of the security" (ASX Guidance Note 8, Continuous Disclosure). Furthermore, the continuous disclosure provisions are included in the civil penalty regime. As a result, a court may make declarations, pecuniary penalty orders or compensation orders for contraventions of the regime.

The most recent amendments to the statutory CDR were introduced in the Corporate Law Economic Reform Program (Audit Reform and Corporate Disclosure) Act 2004. These amendments to the Corporations Act 2001 were implemented as from 1 July 2004, and extended the list of those who are potentially liable for a CDR breach beyond directors to others involved in the breach. Sections 674(2A), (2B)/ and 675(2A), (2B) were inserted.

“(2A) A person who is involved in a listed disclosing entity's contravention of subsection (2) contravenes this subsection.

Note 1: This subsection is a civil penalty provision (see section 1317E). For relief from liability to a civil penalty relating to this subsection, see section $1317 \mathrm{~S}$.

Note 2: Section 79 defines involved. 
(2B) A person does not contravene subsection (2A) if the person proves that they:

(a) took all steps (if any) that were reasonable in the circumstances to ensure that the listed disclosing entity complied with its obligations under subsection (2); and

(b) after doing so, believed on reasonable grounds that the listed disclosing entity was complying with its obligations under that subsection."

Since its inception, the CDR has become increasingly strict. Based on Section 674(2A)(2B), not only a disclosing entity but also a person 'involved' in a contravention of continuous disclosure may be liable to civil and/or criminal penalties. Further, the powers of ASIC in respect of CDR have also increased, which are discussed in the following section.

\subsection{CDR Public Enforcement}

In Australia, public and private enforcement strategies are combined to punish companies that fail to disclose material information in a timely fashion, thus ensuring that companies better understand and comply with their continuous disclosure obligations, and to protect investors/shareholders who suffered loss or damage due to the company's breaches of continuous disclosure (Chan \& Ho, 2011).

A failure to disclose material information immediately could lead to a breach of the continuous disclosure rules. In the ASX Guidance Note 8, Continuous Disclosure, the meaning of 'immediately' is stated as 'promptly and without delay', which depends on just how quickly a company can go through the disclosure process. Unlike the 'materiality', which is defined in Section 677 of the Corporations Act 2001, 'immediately' is not identified. However, based on the infringement notices to Promina Group Ltd and Rio Tinto Ltd, probably 'immediately' is less than one hour. Promina paid $\$ 100,000$ as penalty due to a half-trading day delay of a 
takeover offer; Rio Tinto also paid the same amount as penalty for a delay of just over one hour in terms of a merger offer (Bloch et al., 2011). This shows the continuous disclosure obligations are strict for listed companies.

The Australian stock market is co-regulated by both ASIC and ASX. Matters under the Corporations Act 2001 are regulated by ASIC, and matters under the Listing Rules are governed by ASX. A breach of ASX Listing Rule 3.1 (continuous disclosure) is a breach of continuous disclosure provisions under the Corporations Act 2001. However, unlike ASIC, ASX does not have the power to impose any criminal or civil penalties on a disclosing entity for breaching its continuous disclosure obligations. In addition to asking the entity to take corrective actions (e.g. making a corrective announcement), ASX can suspend trading in the entity's securities, or even terminate its listing. Therefore, only ASIC can initiate public enforcement actions in relation to CDR.

Under Section 13 of the Australian Securities and Investments Commission Act 2001 (ASIC Act), ASIC is authorised to conduct an investigation if it is concerned that there may have been a breach of provisions in an entity under the Corporations Act 2001 or other relevant corporations legislation. As to the public enforcement of continuous disclosure contraventions, currently ASIC has a range of enforcement options to deal with the non-compliance with continuous disclosure, including court actions, infringement notices and enforceable undertakings. Figure 2-1 shows the continuous disclosure enforcement pyramid, which is based on ever-increasing intrusiveness from the bottom to top; it is also argued that the CDR is effective as the pyramid is matched with ever-decreasing frequency of use from the bottom-totop enforcement option (Ayres \& Braithwaite, 1992; Boros, 2009). The ASX Query is sent by the ASX asking a company to confirm that it is compliant with the CDR when the ASX has 
concerns on its disclosures. All the enforcement outcomes discussed below are for ASX-listed companies up to 31 December 2014.

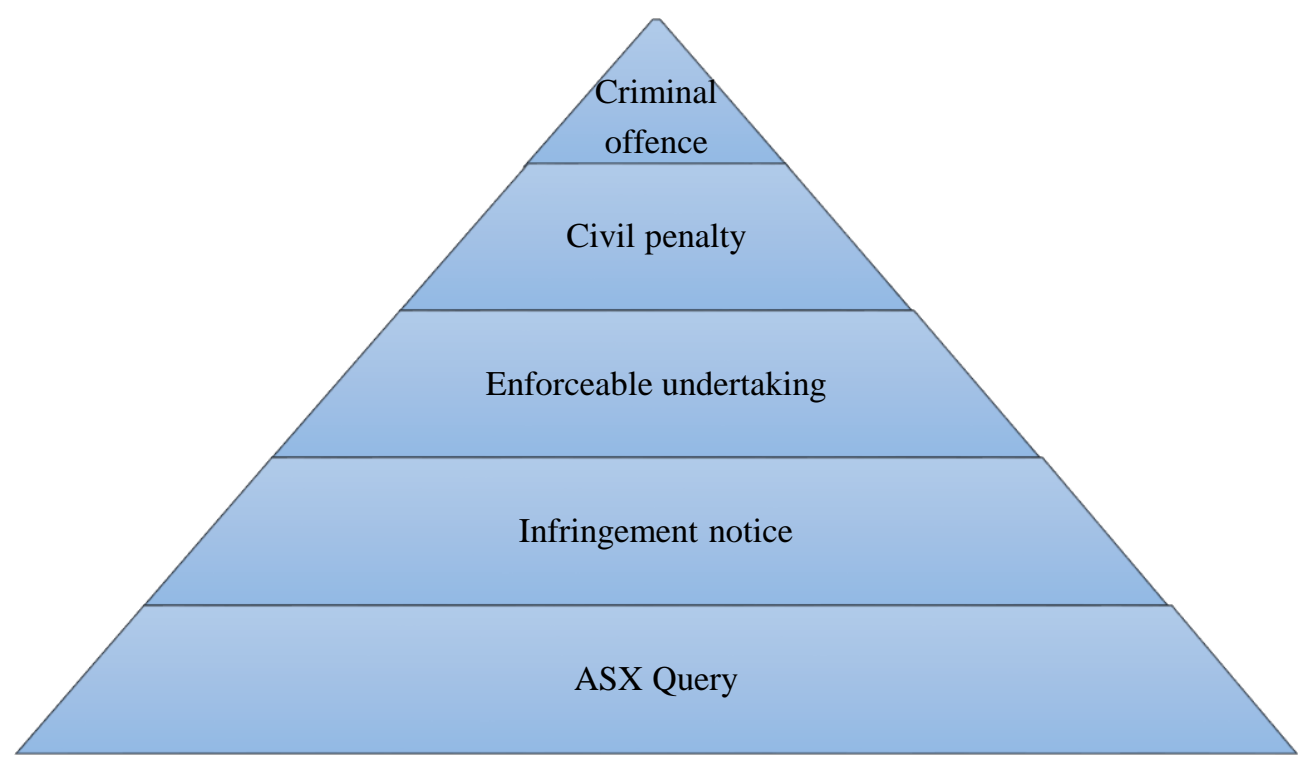

Figure 2-1. The Public CDR Enforcement Pyramid, modified from Boros (2009)

\subsubsection{Court Actions}

Court actions are applied to severe contraventions of the continuous disclosure regime. In the

Financial Services Reform Act 2001, which came into effect on 11 March 2002, continuous disclosure provisions were included in the civil penalty provisions (s.1317E Declarations of Contravention), and the Criminal Code was also inserted into the Continuous Disclosure Chapter (s.678). In other words, from March 2002, a contravention of continuous disclosure was regulated in both civil penalty provisions and the Criminal Code. The criminal sanction is the most severe punishment of all enforcement options. Due to the difficulty of meeting the high criminal standard of proof, there were no successful criminal prosecution ${ }^{12}$ as to breaches

\footnotetext{
${ }^{12}$ ASIC announced a criminal prosecution of two former Harts executives regarding continuous disclosure in 2006 and 2007 , Mr. Richard Melvyn Hayter and Mr. Steven Irvine Hart. Mr. Hayter was found not guilty on 25 September 2008; the charges against Mr. Hart were withdrawn on 19 January 2009 (ASIC Media Release 06-333, 07-110, AD08-26, AD 09-06 and AD0927).
} 
of continuous disclosure (Chan \& Ho, 2011) in the study period. This study focuses on the civil penalty provisions as to continuous disclosure. According to Section 1317E (civil penalty provisions) and Section $1317 \mathrm{G}$ (pecuniary penalty orders) in the period March 2002-July 2004, the maximum penalty for a non-disclosing company was $\$ 200,000$. Even if a company failed to notify the market unintentionally, it may still face civil liability.

In the Corporate Law Economic Reform Program (Audit Reform and Corporate Disclosure) Act 2004, which came into effect on 1 July 2004, the maximum penalty for a corporate breach of the civil penalty provisions (s.1317G) was amended. This amendment clarified the maximum amounts of civil penalty for a person and a disclosing entity respectively. Directors, officers or other involved persons are included in the civil penalty provisions (s.1317E), and the maximum penalty (per person) is $\$ 200,000$. Further, from July 2004 , for the disclosing firm, the maximum penalty boomed to $\$ 1$ million, five times as much as the previous amount.

There have been a limited number of civil prosecutions for continuous disclosure. Only three successful prosecutions were initiated by ASIC under the civil penalty provisions before 2012 (North, 2013a, p. 75). More recently, ASIC has brought one more successful action for continuous disclosure contravention, resulting in a listed company paying the highest civil penalties so far (AU\$1.2 million). In order to better understand the court actions regarding continuous disclosure, Table 2-1 shows the details of the four successful prosecutions under the civil penalty provisions. Two cases relate to earnings or profit information disclosure; that is earnings forecast, construction costs, and the commercial impact of a patent. One case is based on the important agreements in terms of a deed of covenant and indemnity, and the ABN 60 Foundation information. The most recent case concerned the delayed disclosure of management expectations on gold production and capital expenditure. 
Table 2-1. Court Actions Relating to Continuous Disclosure Contraventions up to 31/12/2014

\begin{tabular}{|c|c|c|c|c|}
\hline Cases & Year & Admitted Contraventions & Penalty & Maximum Penalty \\
\hline $\begin{array}{l}\text { ASIC v Southcorp } \\
\text { Limited* }^{*}\end{array}$ & 2003 & $\begin{array}{l}\text { A failure to disclose its earnings } \\
\text { expectation of } \$ 30 \text { million profit } \\
\text { decrease. }\end{array}$ & $\$ 100,000$ & $\$ 200,000$ \\
\hline \multirow[t]{2}{*}{ ASIC v Chemeq Limited* } & 2003 & $\begin{array}{l}\text { A failure to disclose the increased } \\
\text { costs of the construction of } \\
\text { manufacturing facility. }\end{array}$ & $\$ 150,000$ & $\$ 200,000$ \\
\hline & 2004 & $\begin{array}{l}\text { A failure to disclose the commercial } \\
\text { impact of a patent granted in the } \\
\text { United States. }\end{array}$ & $\$ 350,000$ & $\$ 1,000,000$ \\
\hline \multirow[t]{2}{*}{ ASIC v Macdonald* } & 2001 & $\begin{array}{l}\text { A failure to disclose the information } \\
\text { relating to a deed of covenant and } \\
\text { indemnity. }\end{array}$ & - & - \\
\hline & 2003 & $\begin{array}{l}\text { A failure to disclose the } \mathrm{ABN} 60 \\
\text { Foundation information }\end{array}$ & $\$ 80,000$ & $\$ 200,000$ \\
\hline \multirow[t]{2}{*}{$\begin{array}{l}\text { ASIC v Newcrest Mining } \\
\text { Limited }^{\#}\end{array}$} & 2014 & $\begin{array}{l}\text { A failure to disclose its expectation } \\
\text { of } 2.2-2.3 \text { ounces gold production. }\end{array}$ & $\$ 800,000$ & $\$ 1,000,000$ \\
\hline & 2014 & $\begin{array}{l}\text { A failure to disclose its expectation } \\
\text { of around AU } \$ 1 \text { billion capital } \\
\text { expenditure. }\end{array}$ & $\$ 400,000$ & $\$ 1,000,000$ \\
\hline
\end{tabular}

Source: * North (2013a). Company disclosure in Australia. pp. 75-77.

\# ASIC Media Release 14-148MR. "Newcrest ordered to pay \$1.2 million for breaching continuous disclosure laws", 2 July 2014.

\subsubsection{Infringement Notices}

In 2004 the Corporate Law Economic Reform Program (Audit Reform and Corporate Disclosure) Act 2004 further strengthened continuous disclosure regulations. An infringement notice procedure was introduced by adding a new subject at the end of Section 674(2): "Note 3: An infringement notice may be issued [by ASIC] for an alleged contravention of this subsection, see section 1317DAC."

This infringement notice procedure is used by ASIC for relatively minor or less serious violations of continuous disclosure obligations, as it is a timely enforcement process compared with court actions. As shown in Table 2-2, there were 24 notices issued to 31 December 2014, including alleged breaches of not timely and/or accurately releasing study/trial/analysis results, fund raising/rights issue, expected production/earnings/expenses/financial results, estimation of 
mineral resource or investment, takeover proposals, acquisitions, and key information in terms of essential agreements/contracts (North, 2013, pp. 80-83; ASIC Media Release) .

Table 2-2. Infringement Notices Concerning Continuous Disclosure Breaches up to 31/12/2014

\begin{tabular}{|c|c|c|c|}
\hline Company & $\begin{array}{l}\text { Press Release } \\
\text { Time } \\
\end{array}$ & Breaches of Continuous Disclosure Obligations & Penalty \\
\hline $\begin{array}{l}\text { Solbec } \\
\text { Pharmaceuticals } \\
\text { Limited }^{*}\end{array}$ & $\begin{array}{l}1 \text { August } \\
2005\end{array}$ & $\begin{array}{l}\text { An alleged failure to disclose the nature of the } \\
\text { animal study results relating to the company's } \\
\text { cancer drug. }\end{array}$ & $\$ 33,000$ \\
\hline $\begin{array}{l}\text { QRSciences } \\
\text { Holdings Limited }^{*}\end{array}$ & $\begin{array}{l}17 \text { February } \\
2006\end{array}$ & $\begin{array}{l}\text { An alleged failure to disclose the information that } \\
\text { an underwriter withdrew its fund raising. }\end{array}$ & $\$ 33,000$ \\
\hline SDI Limited* & 21 April 2006 & $\begin{array}{l}\text { An alleged failure to update the company's } \\
\text { earnings forecast. }\end{array}$ & $\$ 33,000$ \\
\hline Avastra Limited* & 15 May 2006 & $\begin{array}{l}\text { An alleged failure to disclose the significant delay } \\
\text { of publishing the results regarding a clinical trial. }\end{array}$ & $\$ 33,000$ \\
\hline Astron Limited* & 18 July 2006 & $\begin{array}{l}\text { An alleged failure to disclose that the estimation of } \\
\text { the company's mineral resource increased } \\
\text { considerably. }\end{array}$ & $\$ 66,000$ \\
\hline Avantogen Limited* & $\begin{array}{l}8 \text { December } \\
2006\end{array}$ & $\begin{array}{l}\text { An alleged failure to disclose the unsuccessful } \\
\text { results of a phase II clinical trial in respect of the } \\
\text { company's Pentrys anti-cancer vaccine. }\end{array}$ & $\$ 33,000$ \\
\hline $\begin{array}{l}\text { Promina Group } \\
\text { Limited }^{*}\end{array}$ & $\begin{array}{l}20 \text { March } \\
2007\end{array}$ & $\begin{array}{l}\text { An alleged failure to disclose a takeover proposal } \\
\text { from Suncorp Metway Limited }\end{array}$ & $\$ 100,000$ \\
\hline $\begin{array}{l}\text { Raw Capital Partners } \\
\text { Limited* }^{*}\end{array}$ & $\begin{array}{l}1 \text { August } \\
2007\end{array}$ & $\begin{array}{l}\text { An alleged failure to disclose the loss of an } \\
\text { important IT service contract. }\end{array}$ & $\$ 33,000$ \\
\hline $\begin{array}{l}\text { Centrex Metals } \\
\text { Limited }^{*}\end{array}$ & $\begin{array}{l}12 \text { March } \\
2008\end{array}$ & $\begin{array}{l}\text { An alleged failure to disclose the information of } \\
\text { signing a binding Heads of Agreement in terms of } \\
\text { the hematite supply with Baotou Iron and Steel } \\
\text { Company Limited. }\end{array}$ & $\$ 33,000$ \\
\hline $\begin{array}{l}\text { Sub-Sahara } \\
\text { Resources NL* }\end{array}$ & $\begin{array}{l}29 \text { March } \\
2008\end{array}$ & $\begin{array}{l}\text { An alleged failure to disclose metallurgical test } \\
\text { results. }\end{array}$ & $\$ 33,000$ \\
\hline Rio Tinto Limited* & 5 June 2008 & $\begin{array}{l}\text { An alleged failure to disclose an acquisition of } \\
\text { Alcan Inc. }\end{array}$ & $\$ 100,000$ \\
\hline $\begin{array}{l}\text { The Commonwealth } \\
\text { Bank of Australia } \\
\text { Limited }^{*}\end{array}$ & $\begin{array}{l}14 \text { October } \\
2009\end{array}$ & $\begin{array}{l}\text { An alleged failure to disclose the company’s } \\
\text { expected loan impairment expense. }\end{array}$ & $\$ 100,000$ \\
\hline $\begin{array}{l}\text { Citigold } \\
\text { Corporation }\end{array}$ & $\begin{array}{l}22 \text { September } \\
2010\end{array}$ & $\begin{array}{l}\text { An alleged failure to disclose revisions to the } \\
\text { company's expected gold production at the } \\
\text { Charters Towers goldfield in Queensland. }\end{array}$ & $\$ 33,000$ \\
\hline Nufarm Limited* & $\begin{array}{l}1 \text { December } \\
2010\end{array}$ & $\begin{array}{l}\text { An alleged failure to disclose the company's } \\
\text { expected financial results. }\end{array}$ & $\$ 66,000$ \\
\hline Nexbis Limited* & $\begin{array}{l}12 \text { August } \\
2011\end{array}$ & $\begin{array}{l}\text { An alleged failure to disclose a completed } \\
\text { agreement that gave the company the rights to } \\
\text { supply the Nexcode security suite to the General } \\
\text { Administration of Quality Supervision, and } \\
\text { Inspection and Quarantine of the People's } \\
\text { Republic of China. }\end{array}$ & $\$ 33,000$ \\
\hline $\begin{array}{l}\text { BioProspect } \\
\text { Limited }^{*}\end{array}$ & 8 March 2012 & $\begin{array}{l}\text { Alleged misleading statements in terms of the } \\
\text { interests of Frontier Gasfields Pty Ltd. }\end{array}$ & $\$ 33,000$ \\
\hline BC Iron Limited* & 8 March 2012 & $\begin{array}{l}\text { An alleged failure to fully disclose the information } \\
\text { of the termination rights in terms of an } \\
\text { arrangement scheme with Regent Pacific Group } \\
\text { Limited. }\end{array}$ & $\$ 66,000$ \\
\hline $\begin{array}{l}\text { Leighton Holdings } \\
\text { Limited* }^{*}\end{array}$ & $\begin{array}{l}18 \text { March } \\
2012\end{array}$ & $\begin{array}{l}\text { An alleged failure to timely disclose the } \\
\text { deterioration of the financial position of the Airport } \\
\text { Link Project. }\end{array}$ & $\$ 100,000$ \\
\hline
\end{tabular}




\begin{tabular}{|c|c|c|c|}
\hline Company & $\begin{array}{l}\text { Press Release } \\
\text { Time }\end{array}$ & Breaches of Continuous Disclosure Obligations & Penalty \\
\hline $\begin{array}{l}\text { Leighton Holdings } \\
\text { Limited }^{*}\end{array}$ & $\begin{array}{l}18 \text { March } \\
2012\end{array}$ & $\begin{array}{l}\text { An alleged failure to timely disclose information } \\
\text { concerned with the increased costs of the Victorian } \\
\text { Desalination Project. }\end{array}$ & $\$ 100,000$ \\
\hline $\begin{array}{l}\text { Leighton Holdings } \\
\text { Limited* }^{*}\end{array}$ & $\begin{array}{l}18 \text { March } \\
2012\end{array}$ & $\begin{array}{l}\text { An alleged failure to timely disclose the decreased } \\
\text { evaluation of the Al Habtoor Leighton Group } \\
\text { investment. }\end{array}$ & $\$ 100,000$ \\
\hline $\begin{array}{l}\text { Navigator Resources } \\
\text { Limited* }^{*}\end{array}$ & 15 June 2012 & $\begin{array}{l}\text { Alleged misleading statements which implied that } \\
\text { Patersons Securities Limited was legally bound to } \\
\text { underwrite Navigator's renounceable rights issue. }\end{array}$ & $\$ 33,000$ \\
\hline $\begin{array}{l}\text { Northern Iron } \\
\text { Limited }^{*}\end{array}$ & $\begin{array}{l}19 \text { December } \\
2012\end{array}$ & $\begin{array}{l}\text { An alleged failure to immediately disclose the non- } \\
\text { binding indicative offer from Essel to acquire } \\
100 \% \text { equity in Northern Iron Limited. }\end{array}$ & $\$ 66,000$ \\
\hline ZYL Ltd & $\begin{array}{l}20 \text { September } \\
2013\end{array}$ & $\begin{array}{l}\text { An alleged failure to timely disclose the earlier } \\
\text { repayment date of a } \$ 2 \text { million bridging facility as } \\
\text { to a convertible note agreement; } \\
\text { And an alleged failure to disclose the results of the } \\
\text { confirmed decrease in reserves from mining } \\
\text { ratification workshop. }\end{array}$ & $\$ 66,000$ \\
\hline $\begin{array}{l}\text { Central Asia } \\
\text { Resources Limited }^{\#}\end{array}$ & $\begin{array}{c}14 \text { October } \\
2013\end{array}$ & $\begin{array}{l}\text { An alleged failure to immediately disclose its } \\
\text { actual gold equivalent production figures at the } \\
\text { Dalabai operations. }\end{array}$ & $\$ 33,000$ \\
\hline $\begin{array}{l}\text { Stone Resources } \\
\text { Australia Limited }^{\#}\end{array}$ & $\begin{array}{l}3 \text { December } \\
2013\end{array}$ & $\begin{array}{l}\text { An alleged failure to immediately disclose the } \\
\text { increased gold resource. }\end{array}$ & $\$ 33,000$ \\
\hline Diploma Group Ltd & $\begin{array}{l}10 \text { February } \\
2014\end{array}$ & $\begin{array}{l}\text { An alleged failure to disclose its entry into the } \\
\text { contract for the sale of } 69 \text { Adelaide Terrace, East } \\
\text { Perth subject to two conditions. }\end{array}$ & $\$ 33,000$ \\
\hline $\begin{array}{l}\text { Reward Minerals } \\
\text { Ltd }^{\#}\end{array}$ & 13 June 2014 & $\begin{array}{l}\text { An alleged failure to immediately disclose the } \\
\text { analysis results showing samples of near-surface } \\
\text { brines contained encouraging potassium levels. }\end{array}$ & $\$ 33,000$ \\
\hline
\end{tabular}

Source: * North (2013a). Company disclosure in Australia. pp. 80-83.

\# ASIC Media Release: 13-262MR, 13-274MR, 13-323MR, 14-025MR and 14-126MR.

ASIC is able to issue an infringement notice to a company if ASIC has reasonable grounds to believe that the company has breached the continuous disclosure provisions. Once a company receives an infringement notice, it has to comply with the notice and pay the penalty within 28 days from the issuing date of the notice. The amount of this penalty is fixed depending on the company's market capitalisation and whether it has received a notice previously. If it is the first time it has received the notice, the penalty would be $\$ 100,000$ for companies whose market capitalisation exceeds $\$ 1,000$ million; $\$ 66,000$ for companies whose market capitalisation exceeds $\$ 100$ million, and $\$ 33,000$ for companies of which the market capitalisation is below \$100 million (North, 2013a, pp. 82-83). 
The company can ask ASIC to withdraw the notice. After taking whatever steps against the company it sees fit, ASIC will withdraw if it is satisfied the evidence provided by the company supports its compliance with continuous disclosure obligations. The infringement notice is a tool to remedy an alleged contravention of continuous disclosure. The main advantage of the infringement notice is that, compared with other enforcement mechanisms, it is fast and less expensive.

\subsubsection{Enforceable Undertakings}

In order to encourage compliance with the continuous disclosure obligations, ASIC may seek enforceable undertakings concerning alleged failures of continuous disclosure under Section 93AA of the ASIC Act 2001. Although the undertakings may be flexible in expression, ASIC can enforce them against the relevant company.

Table 2-3 provides the cases of enforceable undertakings in terms of continuous disclosure failures (North, 2013, pp. 83-84). These enforceable undertakings are driven by alleged failures to continuously disclose changes of revenues or profits/losses, breach of an important covenant and expected trading results. The enforceable undertaking also can be relegated to potential continuous disclosure breaches. 
Table 2-3. Enforceable Undertakings in Terms of Continuous Disclosure Failures up to 31/12/2014

\begin{tabular}{|c|c|c|}
\hline Company & Press Release Time & Enforceable Undertakings \\
\hline Crown Limited* & 11 September 1998 & $\begin{array}{l}\text { An alleged failure to disclose accumulated operating } \\
\text { losses in } 1998 \text { and the fact that the company received } \\
\text { a notice regarding its breach of a debt to equity } \\
\text { covenant in its casino licence. }\end{array}$ \\
\hline $\begin{array}{l}\text { Pahth Telecommunications } \\
\text { Limited* }^{*}\end{array}$ & 2 February 2001 & $\begin{array}{l}\text { An alleged failure to disclose revenue and profit } \\
\text { changes, and the company agreed to the undertaking } \\
\text { which required audits of the disclosure. }\end{array}$ \\
\hline Plexus International Limited ${ }^{*}$ & 5 April 2001 & $\begin{array}{l}\text { An alleged failure to disclose the variation of the } \\
\text { company's revenue and profit, and the company } \\
\text { agreed to the undertaking which required disclosure } \\
\text { audits. }\end{array}$ \\
\hline Uecomm Limited* & 17 October 2002 & $\begin{array}{l}\text { An alleged failure to continuously disclose the } \\
\text { company's expectation of trading results, and the } \\
\text { company agreed to review its proceedings in respect } \\
\text { of continuous disclosure, which was stated in the } \\
\text { undertaking. }\end{array}$ \\
\hline Multiplex Group* & 20 December 2006 & $\begin{array}{l}\text { An alleged failure to immediately disclose a } \\
\text { substantial change in profits, and the undertaking } \\
\text { secured a } \$ 32 \text { million compensation fund for affected } \\
\text { investors. }\end{array}$ \\
\hline TZ Limited* & 4 July 2008 & $\begin{array}{l}\text { An alleged failure to disclose material information, } \\
\text { and the undertaking required the company to engage } \\
\text { an external consultant to ensure proper disclosure. }\end{array}$ \\
\hline Leighton Holdings Limited* & 18 March 2012 & $\begin{array}{l}\text { An alleged failure to immediately disclose the } \\
\text { considerable decreases in the profitability of the } \\
\text { Airport Link Project, the Victorian Desalination } \\
\text { Project, and the investment in the Al Habtoor } \\
\text { Leighton Group. The company agreed to engage an } \\
\text { independent consultant to reinforce its continuous } \\
\text { disclosure procedures. }\end{array}$ \\
\hline NuSep Holdings Limited ${ }^{\#}$ & 19 December 2014 & $\begin{array}{l}\text { Corporate governance issues with potential breaches } \\
\text { of continuous disclosure laws. ASIC concerns on the } \\
\text { release of inaccurate information. The company } \\
\text { agreed to have its continuous disclosure and corporate } \\
\text { governance procedures reviewed and rectified by an } \\
\text { independent consultant. }\end{array}$ \\
\hline
\end{tabular}

Source: * North (2013a). Company disclosure in Australia. pp. 83-84.

\# ASIC Media Release 14-341MR “ASIC investigation prompts NuSep into enforceable undertaking”.

An enforceable undertaking does not necessarily impose a penalty, unless it is combined with an infringement notice or a court action. The main purpose of enforceable undertakings is to help companies comply with continuous disclosure provisions by, for example, requiring them to review and improve their continuous disclosure procedures, and ultimately to reduce the likelihood of future continuous disclosure contraventions. 


\subsubsection{ASX Query}

The Listing Rules are governed by the ASX, so the ASX is obliged to monitor and enforce companies' compliance with the Listing Rule 3.1 (continuous disclosure). Although the ASX has no power to impose any criminal or civil penalties on a firm, it can conduct monitoring activities to detect companies' possible breaches of, and to confirm their compliance with, the continuous disclosure rules. The ASX Query plays a key role in its enforcement practices of the CDR. ASX Query is a query letter sent by ASX to a company to verify its compliance with the continuous disclosure obligations, and the company needs to reply promptly to the ASX by the deadline specified in the Query. The market will not be notified until the company's response to its ASX Query is ready to disclose after a listings adviser's review. Both the response and the Query letter will be released together on the ASX Market Announcements Platform. There are two types of ASX queries: price query letters and aware letter (ASX Guidance Note 8, Continuous Disclosure). ${ }^{13}$

If ASX notices abnormal trading in a company's securities, a price query will be sent to discover if any undisclosed information is causing this abnormal trading. The abnormal trading activities are identified by the ASX's Surveillance Group. The group "uses sophisticated computer technology to monitor trading in ASX quoted securities on a real-time basis across all licensed markets in Australia, seeking to identify abnormal trading which could indicate that there has been a leak of market-sensitive information yet to be announced under Listing Rule 3.1" (ASX Guidance Note 8, Continuous Disclosure, p. 55). Some studies have provided evidence to support the concept that the ASX price query is able to correct the abnormal trading to some extent (Drienko \& Sault, 2013; Drienko, Sault, \& von Reibnitz, 2016).

\footnotetext{
${ }^{13}$ The explanations on the ASX Query refer to the ASX Guidance Note 8, Continuous Disclosure Listing Rule 3.1-3.1B.
} 
If ASX has concerns about whether a company has fully and promptly disclosed any marketsensitive information, an aware letter will be sent based on a specific company announcement or company news. On every trading day before the market opens, the ASX reviews all major newspapers to identify whether there is any potentially material information released in any article which may not have been disclosed on the ASX market. If an article has attracted ASX's attention, the 'relevant listings adviser' ${ }^{14}$ will either discuss the issue with the company or send an aware letter to it. The aware letter could also be based on many other information sources as the ASX, especially the Surveillance Group, also reviews news media including, but not limited to, investor forums/chat sites and broker research. Once the ASX has reason to believe there may be a continuous disclosure issue (not triggered by abnormal trading), an aware letter will be sent to the company, which needs to respond promptly to confirm its compliance with the CDR.

If a significant contravention of the CDR is suspected by the ASX through the ASX Query or other approaches (such as complaints), the issue will be referred to the ASIC for further enforcement actions. The ASX can only suspend trading of a suspect company's securities or terminate its listing in an extreme case. The ASX has no power to decide whether or not a company has breached the CDR, or to impose any penalties. The ASX and ASIC may hold different views on the same potential breach of the CDR. The ASIC needs to investigate the issue through using its enforcement powers.

\footnotetext{
${ }^{14}$ Each ASX-listed entity has an assigned listing adviser, who assists the entity on any questions or concerns between the company and the ASX.
} 


\subsection{CDR Private Enforcement}

In addition to the public enforcement methods mentioned above, individual shareholders can take private actions to seek compensation from a company or an involved person for losses or damages suffered from the alleged continuous disclosure failures under Section 1317HA and/or Section 1325 of the Corporations Act 2001. In recent years, there has been an increasing number of litigation-funded shareholder class actions. Australian shareholders have received considerable compensation through class actions in terms of alleged continuous disclosure breaches and/or failures. Table 2-4 indicates the company's name, the year when class action took place and the amount that companies paid for investor compensation.

Table 2-4. Shareholder/Class Actions in Respect of Continuous Disclosure Failures

\begin{tabular}{|c|c|c|}
\hline Company & Year & Payment for Investor Compensation \\
\hline Concept Sports ${ }^{\#}$ & 2006 & $\$ 3$ million including costs \\
\hline Harris Scarfe ${ }^{\sharp \wedge}$ & 2006 & $\$ 3$ million including costs \\
\hline Telstra $^{\#}$ & 2007 & $\$ 5$ million including costs \\
\hline Downer EDI ${ }^{\#}$ & 2008 & Approximately $\$ 20$ million (confidential) \\
\hline Aristocrat Leisure Limited* & 2008 & $\$ 144.5$ million including costs \\
\hline Village Life (Fig Tree Developments) $\#^{\# \wedge}$ & 2009 & $\$ 3$ million including costs \\
\hline Sons of Gwalia ${ }^{\#}$ & 2009 & Approximately $\$ 70$ million \\
\hline Bookfield Multiplex Limited* & 2010 & $\$ 110$ million including costs \\
\hline AWB Limited ${ }^{*}$ & 2010 & $\$ 39.5$ million including interest and costs \\
\hline Oz Minerals Limited* & 2011 & $\$ 60$ million including costs \\
\hline Centro Group entities* & 2012 & $\$ 200$ million including costs \\
\hline Credit Corp Group ${ }^{\#}$ & 2012 & $\$ 6.5$ million including costs \\
\hline Nufarm $^{\#}$ & 2012 & $\$ 46.6$ million including costs \\
\hline $\mathrm{NAB}^{\#}$ & 2012 & $\$ 115$ million including costs \\
\hline Sigma Pharmaceuticals" & 2012 & $\$ 57.5$ million including costs \\
\hline Transpacific Industries Group ${ }^{\#}$ & 2012 & $\$ 35$ million including costs \\
\hline $\mathrm{GPT}^{\# \wedge}$ & 2013 & $\$ 75$ million including costs \\
\hline Leighton Holdings ${ }^{\# \wedge}$ & 2014 & $\$ 69.45$ million including costs \\
\hline
\end{tabular}

Source: *North (2013a). Company disclosure in Australia. pp. 85-89.

\# King \& Wood Mallesons (KWM). The review - class actions in Australia 2015/2016. p. 9.

${ }^{\wedge}$ The allegation includes continuous disclosure and misleading statements/misleading or deceptive conduct. 
As shown in Table 2-4, the amount of compensation through class actions is substantial. It is suggested that it is too early to assess the effects of class actions on companies' disclosure practices (North, 2013a, p. 87). However, the class actions could improve continuous disclosure procedures of listed companies, since they protect investors' interests, and motivate investors to monitor the companies that they invest in.

\subsection{Summary}

This chapter has reviewed the development of the continuous disclosure listing rules and provisions. It summarises the CDR's key aspects and discusses its enforcement mechanisms. The CDR appears to be now at a stable stage as no amendments have been introduced since 2004. Furthermore, the fact that not many public court actions or private class actions occurred over the ten years between 2004 and 2014 reinforces this conclusion. Also, infringement Notices, Enforceable Undertakings and ASX Queries are able to prevent significant contraventions of the CDR to some extent. On the other hand, the CDR breach cases (Table 2$1-2-4)$ indicate concerns that the level of penalties may be 'too low' as the 'cost' for companies that are non-compliant with the CDR. Although the CDR effectiveness (especially enforcement) is controversial, with twenty years development, the Australian CDR "is generally regarded as being at the forefront of world's best practice" (Bloch et al., 2011, p. 256).

Researchers have realised the importance of the CDR, but there is a limited amount of literature empirically testing its effects, which is discussed in the next chapter: Literature Review. The next chapter also reviews prior studies on the market reactions and the timeliness of price discovery. This study contributes to the CDR empirical literature by examining the actual 
market effects of continuous disclosure over both short- and long- terms. It also provides insights into CDR's effectiveness in terms of announcement 'market sensitivity' and continuous disclosure categorisation. 


\section{CHAPTER THREE: LITERATURE REVIEW}

The policy objective of the Australian CDR is "to enhance the integrity and efficiency of Australian capital markets by ensuring that the market is fully informed. The timely disclosure of market-sensitive information is essential to maintaining and increasing the confidence of investors in Australian markets, and to improving the accountability of company management. It is also integral to minimising incidences of insider trading and other market distortions" 15 (ASX Guidance Note 8, Continuous Disclosure, pp. 6-7). Researchers have been interested in the CDR, exploring whether or not it is achieving its goals from various perspectives, but little work has been done to provide a comprehensive understanding of the market effects of continuous disclosures. This study builds upon prior literature by evaluating in depth the market responses to continuous disclosure categories, as reflected in both the short-term share price and trading volume movements immediately after the announcement release and the long-term timeliness of price discovery over one year. This chapter reviews the literature relating to the CDR, market reactions to information disclosure and the timeliness of price discovery.

\subsection{Research on the CDR}

Literature relating to the CDR has focused on the following areas: (1) continuous disclosure regulation, (2) impacts of continuous disclosure reform, and (3) factors influencing companies' compliance with continuous disclosure provisions. More recently, there have been a few studies related to the microstructure of the ASX, focusing on the investor behaviour and price effects around disclosures. These areas are separately discussed below.

\footnotetext{
15 The NSW Court of Appeal in James Hardie Industries NV v ASIC [2010] NSWCA 332, at paragraph 355.
} 


\subsubsection{Legal Research on Continuous Disclosure Regulations}

As continuous disclosure in Australia is also regulated under the Corporations Act 2001, many studies have focused on the regulations per se, tending to undertake analytical research instead of empirical tests.

Some researchers have focused on specific disclosure provisions and/or rules under the CDR. Low, Burdon, and von Nessen (2010) compared the CDR and data breach notification laws by discussing the rationales behind the two different types of laws when a disclosure is needed to explain data breaches of consumers' personal information (e.g. computer hacking). They concluded that both the CDR and data breach notification laws are needed for the aggregate and individual consequences of a data breach. Fisse and Beaton-Wells (2011) analysed the continuous disclosure exception in the Competition and Consumer Amendment Bill (No 1) 2011. The Bill states, "Corporation[s] must not make private disclosure of pricing information etc. to competitors" (s.44ZZW), and "Corporation[s] must not make disclosure of pricing information etc. for purpose of substantially lessening competition" (s.44ZZX). But these provisions do not apply to disclosures required by the CDR. Fisse and Beaton-Wells (2011) revealed the Bill's potential traps and loopholes by practical examples, and argued that continuous disclosure compliance becomes more complex for ASX-listed companies due to this law change. Focusing on the ASX Guidance Note 8, Continuous Disclosure Listing Rule 3.1-3.1B amended in 2013, North (2013b) argued that the amendments relating to earnings information disclosure are misconceived, which may mislead investors to make uninformed and unconfident decisions.

Furthermore, some prior studies sought to find whether or not the enforcement can promote companies' compliance with their continuous disclosure obligations. Welsh (2009) found a 
clear positive association between the enforcement activity undertaken by ASIC and continuous disclosure compliance. Additionally, Mayanja (2010) emphasised the importance to the Australian CDR of private enforcement and suggested that laws and/or reforms normally should avoid creating barriers to private enforcement.

In summary, the regulatory studies promote the understanding of continuous disclosure regulations, and provide some suggestions for policy makers to further improve the regulations.

\subsubsection{Impacts of Continuous Disclosure Reform}

Researchers have pointed out the importance of continuous disclosure reform by comparing the market consequences of disclosures, especially the information asymmetry level, before and after a specific reform. For a sample of 250 ASX-listed companies from September 1993 to September 1995, one year either side of the Corporations Law Reform Act 1994, Poskitt (2005) found an initial increased level of information-based trading after the reform by using the probability of informed trading (PIN) model. Frijns, Gilbert, and Tourani-Rad (2008) indicated a significant decrease in the bid-ask spread after the Securities Markets Amendment Act 2002 was enacted in New Zealand. This enactment introduced CDR into New Zealand law. It is shown that after enactment, the level of information asymmetry reduced considerably. The New Zealand CDR is closely modelled on the Australian regime.

Brown, Taylor, and Walter (1999) found increased disclosures in relatively small and poorperforming ASX-listed firms after the introduction of civil and criminal sanctions for noncompliance. In the New Zealand context, Huang, Marsden, and Poskitt (2009) concluded that the statutory-backed disclosure regime promotes the amount of earnings-related information 
disclosures, and that the half/full year earnings announcements are released on a timelier basis under the disclosure regime. Research has shown positive impacts of the continuous disclosure reform on information disclosure and, in particular, the increased frequency and timeliness of price-sensitive disclosures in the post-reform period.

Additionally, some researchers have focused on how earnings forecasts behaviour changes along with the legal environment changes relating to continuous disclosure. Chan, Faff, Ho, and Ramsay (2007) concluded that, as a result of legislative environment changes in Australia, companies disclose much more non-routine earnings forecasts of bad news. Similarly, using a sample of NZX-listed companies, Dunstan, Gallery, and Truong (2011) stated that after the 2002 reform, the likelihood and frequency of both routine and non-routine forecasts have increased significantly regardless of good or bad news, and the precision and accuracy of earnings forecasts improved considerably. The literature supports the view that the continuous disclosure reform enhances both market efficiency and corporate disclosure behaviour.

\subsubsection{Continuous Disclosure Compliance}

Continuous disclosure is a mandatory requirement for companies listed on the ASX; companies cannot selectively release information or delay the disclosure of material information. An ASXlisted company has to disclose any information that potentially has a significant impact on stock price immediately after it becomes aware of the information; otherwise the company is in breach of its continuous disclosure obligations. As a result, the disclosure should be independent from all other factors. But corporate governance and company financial performance are likely to influence the way companies comply with those obligations. 
Although Matolcsy et al. (2012) failed to find any association between board composition and continuous disclosures, Beekes and Brown (2006) suggested that Australian firms with better corporate governance release more informative and quicker announcements. Similarly, Seamer (2014) revealed that a company is less likely to breach its continuous disclosure obligations if it has larger proportion of independent directors and a segregation of CEO and Board chair. Chapple and Truong (2015) suggested that Board size exerts a positive influence on the quality of continuous disclosure compliance, but failed to find supporting evidence on audit committee independence, Board gender diversity and Board independence. By examining the association between a company's earnings and the amount of its price-sensitive announcements over a year, Hsu (2009) suggested that companies with bad financial performance (decreased earnings) tend to release more continuous disclosure than those with good financial performance (increased earnings). The above studies focused on disclosure behaviour under CDR instead of market reactions to the continuous disclosures.

\subsubsection{Prior Research on the Microstructure of ASX}

Market microstructure concerns the trading process and its outcomes on financial markets. O'Hara (1995, p. 1) defined market microstructure as "the study of the process and outcomes of exchanging assets under a specific set of rules... [T]he microstructure literature analyses how specific trading mechanisms affect the price formation process." An improvement in the market microstructure can bring about improved liquidity and price appreciation (Amihud, Mendelson, \& Lauterbach, 1997). 
Many studies have shown the implications of market microstructure for asset pricing. Researchers have focused on two important microstructure variables: market liquidity and imbalanced order flows.

Liquidity is often hard to measure: several methods have been utilised. However, what is known is that liquidity is a priced factor. Illiquidity has been seen as one of the most important market factors that affect asset prices (Florackis, Gregoriou, \& Kostakis, 2011). Numerous researchers have supported the positive (negative) relationship between illiquidity (liquidity) and stock returns by various liquidity measures (Amihud \& Mendelson, 1986; Brennan, Chordia, Subrahmanyam, \& Tong, 2012; Eleswarapu, 1997; Florackis et al., 2011). ${ }^{16}$

Studies regarding effects of liquidity (or liquidity costs) on asset prices include Amihud and Mendelson (1986) who studied the effect of the bid-ask spread, a proxy of liquidity, on asset pricing. Based on companies listed on NYSE from 1960 to 1980, they found that marketobserved cross-sectional expected return (risk-adjusted) is positively related to the spread. The results are interpreted by a theoretical model in which investors demand a premium for less liquid (larger bid-ask spread) stocks depending on their expected holding periods, indicating the role of liquidity as a determinant of expected returns. Since then, their argument on the relationship between liquidity and stock prices/returns has been expanded and supported by many studies. For instance, Eleswarapu (1997) re-examined Amihud and Mendelson (1986)'s liquidity premium using Nasdaq (instead of NYSE) data over the 1973-1990 period, and found a much stronger relation between expected returns and the cost of transacting (demanding

\footnotetext{
${ }^{16}$ Many other studies have focused on the relationship between liquidity and stock returns and include Brennan and Subrahmanyam (1996), Chordia, Subrahmanyam, and Anshuman (2001), Amihud (2002), Chordia, Huh, and Subrahmanyam (2009), and Lam and Tam (2011).
} 
liquidity), suggesting the dealers' inside spreads could be a better proxy for liquidity than the quoted spreads.

More recently, using daily data for listed companies on the London Stock Exchange over the period 1991-2008, Florackis et al. (2011) proposed an alternative, more appropriate price impact ratio, Return-to-Turnover ratio, which could overcome the size bias and control the impacts of both trading costs and trading frequency on asset pricing. Brennan et al. (2012) developed an asymmetric relationship between trading costs and price changes by using intraday transactions data to separate buy and sell measures of illiquidity ("lambdas"). Based on a large cross-section sample of NYSE listed common stocks from 1983 to 2008, they found that sell-order liquidity is priced more strongly than buy-order liquidity in the equity returns, and even suggested that the liquidity premium is generated predominantly from the sell-order side. Overall, the effects of liquidity/illiquidity on the asset prices or returns have been extensively studied.

Order imbalance is another important microstructure variable. Researchers have not only studied the positive return-order imbalance relation (Chordia, Roll, \& Subrahmanyam, 2002; Chordia \& Subrahmanyam, 2004), but have also expanded their scope to the liquidity-market efficiency relationships. Chordia, Roll, and Subrahmanyam (2008) regarded short-horizon return predictability from order flows as an inverse indicator of market efficiency, and suggested that liquidity (the reduction of minimum tick size and narrower bid-ask spreads) can enhance market efficiency. Chung and Hrazdil (2010) extended and supported Chordia et al. (2008) by testing the relationships among information, liquidity and market efficiency. They found a positive association between a continuous measure of liquidity (the effective bid-ask 
spread) and market efficiency, and this effect is magnified during periods that contain new information (periods of high adverse selection).

Research on market microstructure provides a new perspective and approach to examine the price formation process, thereby further exploring how information is incorporated into asset prices (Chordia et al., 2008). However, most microstructure literature has not taken corporate disclosures into account, and the research samples mainly focused on the U.S. stock market. There are few studies exploring the microstructure of the ASX.

The ASX has its own microstructure features due to the CDR. The most important characteristic is the 'market sensitivity' tag along with each announcement release. The release of a marketsensitive announcement is followed by a complex process. Brown, $\mathrm{Ng}$, and Wee (2003, p. 1) described this process as having four steps: “(1) a halt to trading, (2) the release of a summary headline of the announcement, (3) the subsequent release of the announcement details (which may be in segments) and (4) the resumption of trading". They examined investor trading behaviour before and after each step when market-sensitive earnings and takeover announcements are released. Their results showed some evidence of price protective strategies due to the increased information asymmetry before the release of announcement headline. Following the headline release, each step can cause significantly increased trade activities (order revisions and order imbalance); especially after trading resumes, all investor trade activities increase significantly, including new orders and trades.

Brown, Howitt, and Wee (2005) and Brown, Kwan, and Wee (2006) investigated the instant investor trading behaviour and stock price movements around company announcements (both market-sensitive and non-market-sensitive) on the ASX at the market microstructure level. 
Brown et al. (2006) extended the Brown et al. (2005) study by covering more announcement types. They distinguished between the trading behaviours of uninformed and informed traders, and applied a more powerful statistical test. The 2006 study found increased abnormal trade activities (the number of trades, new and revised orders) around both market-sensitive and nonmarket-sensitive announcements, but the absolute share price change around market-sensitive announcements is significantly greater than non-market-sensitive ones. It also supported uninformed traders' price protection from informed traders before the release of marketsensitive announcements and during trading halts.

Some studies have focused on the ASX microstructures that can positively influence market liquidity, such as the reduction in the minimum tick size (Aitken \& Comerton-Forde, 2005) and the introduction of the closing call auction (Aitken, Comerton-Forde, \& Frino, 2005). In addition, Frino, Segara, and Zheng (2009) investigated the effects of trade characteristics (e.g. trading frequency, trade size and trade direction) on stock return volatility. They found that the number of medium size trades affects the stock return volatility more significantly than the trading frequency of smaller and larger trades. They also concluded that the number of buyerinitiated trades has a larger effect on stock return volatility than those of seller-initiated trades as the number of buyer-initiated trades is more informative. Frino, Lecce, and Segara (2011) examined the trading halt effects on both liquidity and price volatility, and concluded that trading halts cannot improve either liquidity or price volatility, as abnormally wide bid-ask spreads and abnormally high price volatility are observed immediately after trading halts.

The literature identified above explores the microstructure factors of the ASX, providing insights into whether the ASX correctly classified the market-sensitive information, and how the market or trade characteristics influence liquidity and/or price volatility. However, those 
studies failed to examine the impacts of different announcement types. The tests were limited to the absolute price effects or price volatilities without taking good/bad news into account. This study analyses in depth the trading activity and price effects of different announcements with good/bad news.

Another characteristic of the ASX is the detailed announcement categorisations. A few recent studies have estimated market reactions to a specific announcement type on the ASX at the microstructure level (intraday event studies). Two announcement types have been investigated: one is the ASX Query, and the other is Opening Briefings.

An ASX Query is a query sent to a company if ASX requires additional information. For example, if the company's share price changes significantly over a short period, an ASX Price Query is applied to ask if the company is aware of any new information that is not available on the market which may explain the share price movement. The Query is released when the company responds to it. Drienko and Sault (2013) found significant stock price reversals and increased trading volumes after disclosing ASX Query responses, especially those that provided no further information. They also suggested the market takes about 60 minutes to incorporate the information contained in Query responses into stock prices. Drienko et al. (2016) further confirmed the price reversals after the release of price query responses that indicate no undisclosed material information other than the information already available to the market. They suggested that the information contained in the unexpected price query responses can be incorporated into prices within 20 minutes.

An Open Briefing is a type of continuous disclosure subcategory, under the Main Category 14 “Other - Open Briefing”. An investor relations consultant, Orient Capital, helps a company 
draft an Open Briefing, using question-and-answer transcripts from mock analyst interviews to update the investors about the company's status (Ferguson \& Scott, 2016). Ferguson and Scott (2016) found positive market reactions to the Open Briefings: positive abnormal returns, and increased trading activities (turnover) after the announcement release.

Based on the above literature analysis, in the continuous disclosure research area, empirical literature focuses on the market event study of the continuous disclosure reform, and the factors influencing company disclosure behaviour under the CDR. There are a few studies working on the market responses to disclosures under the CDR, most of which focused on ASX Price Query research. The lack of empirical literature on the market effects of continuous disclosure might be caused by either of the following. First, it is time-consuming to investigate each disclosure under the CDR. Given the number of companies listed on the ASX, and the number of announcements per company each year, it is difficult to cover all the announcements in one study. Second, it is difficult to measure the market reaction to each ASX announcement. It is essential to distinguish the market (price/volume) effect between different types of disclosures. To some extent, this study overcomes these difficulties by developing a new approach of tick (transaction) data analysis which will contribute to the continuous disclosure literature.

The short horizon created by tick data can provide precise tests of market reactions (price and volume) to disclosures without external noise, and thereby make the examination of immediate effects induced by announcements possible. In addition, the response time of some trading in Australia is measured by milliseconds (North, 2013a). Given the nature of the Australian stock market, it is not appropriate to examine the processes of price discovery and trading reaction by daily or monthly data; tick data is more advantageous. In addition, tick data can be applied 
to examine how long the market takes to adjust to new information, or how long the market reactions can last.

This study contributes to market microstructure literature by exploring the role of public information on price formation over a short time. Specifically, this study explores and compares the immediate market (price and volume) reactions to different disclosure categories by using microstructure data (e.g. intraday transaction price, trading volume and bid-ask spread).

\subsection{Literature on Market Reactions to Information Disclosure}

Researchers in accounting literature have paid attention to the way that the market reacts to information released by companies (Kothari, 2001), especially earnings-related information.

\subsubsection{Market Reactions to Earnings Information}

Starting with Ball and Brown (1968) and Beaver (1968), over the last fifty years there has been a significant amount of accounting research on the valuation implications of earnings information. The literature has suggested that earnings announcements significantly influence both stock prices and trading volumes. Beaver (1968) investigated the weekly share price and trading volume changes surrounding the week when the announcement of annual earnings was released, and found higher levels of abnormal price and volume activities in that week than at other times. Ball and Brown (1968) compared annual income with the 'expected' (market average) income, and showed that the stock price positively reacts to a positive forecast error (annual income better than expected income), and negatively reacts to a negative forecast error (annual income worse than expected income). Beaver, Clarke, and Wright (1979) extended that 
study by examining the share price reactions to the magnitude, not the (positive/negative) sign, of income forecast errors. They suggested there is a significant positive correlation between abnormal stock price returns and the forecast errors.

Numerous empirical studies have confirmed the market reactions to earnings information, especially the observed abnormal stock returns around the earnings announcement release (Lev \& Ohlson, 1982). Nevertheless, the earnings announcement may not be the only reason for this abnormal stock price activity. The additional disclosures concurrent with earnings releases play a pivotal role in assessing the abnormal returns in the announcement period. Hoskin, Hughes, and Ricks (1986) examined the non-earnings disclosures on the Dow Jones News Retrieval Service on the earnings release day and the day after. They found excess returns are also caused by disclosures regarding the earnings component, the dividend component and prospective management comments.

Francis, Schipper, and Vincent (2002) and Kohlbeck and Magilke (2002) attempted to find the reason for increased market reactions on earnings announcement dates over time. But neither study thought it is because accounting earnings convey more information as time passes. Francis et al. (2002) suggested it is because of the increased concurrent disclosures in the earnings press releases, while Kohlbeck and Magilke (2002) argued it is due to increased use of concurrent conference calls. Atiase, Li, Supattarakul, and Tse (2005) investigated the concurrent earnings forecast guidance, and suggested there is no impact of earnings guidance on the market reactions to earnings announcements. More recently, literature has focused on the factors affecting market reactions to earnings information, such as announcement timing (Doyle \& Magilke, 2009) and company-specific characteristics (Truong, 2010). 
The huge number of empirical studies related to the impact of earnings on stock returns or trading volumes means it is impossible to review them all. But this type of research is consistent with the efficient market hypothesis, arguing that stock prices and/or trading volumes can reflect the information content in the earnings and/or concurrent disclosures.

\subsubsection{Market Reactions to Capital Issue}

Capital issue is another major type of event studies. Many empirical studies in this area have revealed a negative impact of new capital issue announcements on stock prices, and the generally accepted explanation is the information asymmetry between internal managers and external investors (Eckbo, Masulis, \& Norli, 2007). However, other possible explanations could be temporary price pressure and underwriter pricing practices (Corwin, 2003). Based on the information hypothesis, Myers and Majluf (1984) indicated that the negative market reaction to a new capital issue is due to the asymmetric information regarding the inherent value of the firm's assets. Particularly in the accounting literature, among the various measures on the information asymmetry, empirical studies have supported the concept that lower accounting information quality is associated with larger negative SEO announcement effects (Lee \& Masulis, 2009; Shivakumar, 2000). Kim, Li, Pan, and Zuo (2013) suggested that accounting conservatism can reduce negative market consequences of SEO announcements by monitoring managers and by providing 'less overstated' financial performances.

In contrast, a number of studies have found a positive announcement effect in respect of private placements, a way of raising capital by issuing new stock to a few, certain investors. Wruck (1989) attributed this positive return on the private placement announcement to the increased monitoring effect from the private investors. But for small companies, it is suggested that the 
monitoring effect is less important than information effects; this positive market reaction can be viewed as a resolution of information asymmetry, which reflects compensation to investors' information costs of certifying the company's prospects, signalling the market that the company is undervalued (Hertzel \& Smith, 1993). Barclay, Holderness, and Sheehan (2007) argued that the monitoring effect exists only when private investors are active, but the proportion of such active placements is small (12\%). Most of placements are sold to passive investors, reinforcing management control rather than monitoring management.

Based on the ASX continuous disclosure categories, On-Market and Off-Market Buy-Backs (also known as share repurchases) are subcategories under the announcement main category, Issued Capital. Since Dann (1981) and Vermaelen (1981), the market reaction to the announcement related to share repurchases has attracted the attention of researchers. To date, all empirical studies have shown positive market reactions to share repurchase announcements (Au Yong, Brown, \& Ho, 2014). Early studies using US data have supported the signalling/undervaluation hypothesis, namely that share repurchases signal the market that the firm's share is undervalued (Comment \& Jarrell, 1991; D'mello \& Shroff, 2000; Dann, 1981; Vermaelen, 1981). Studies using Australian data have also supported the undervaluation motives of on-market buybacks, and the positive announcement effects (Akyol \& Foo, 2013; Brown \& Norman, 2010; Lamba \& Ramsay, 2005; Mitchell, Izan, \& Lim, 2006; Mitchell \& Dharmawan, 2007; Reddy Yarram, 2014). Off-market share buybacks in Australia are motivated by tax benefits (i.e. franking credits), and the level of tax benefits is positively associated with the market reactions to off-market buyback announcements (Au Yong et al., 2014; Brown, 2007; Brown \& Davis, 2012; Brown \& Norman, 2010). 
Equity issuance is a complex research question involving methods of raising capital and the information asymmetry underlying the relevant financing decisions. The announcements related to Issued Capital can send a signal to the market about firm value. As a result, market consequences of announcements related to Issued Capital can be either positive or negative on the ASX. Without looking at the detailed subcategories, it is impossible to compare the different market consequence patterns to the various methods of capital issue. However, this study can explore the price and trading behaviour around Issued Capital announcements in depth by taking good/bad news into account.

\subsubsection{Market Reactions to Dividend and Other Types of Information}

In addition to information generated by earnings announcements and capital issues, many other information events have been related to stock prices, including research on dividends, takeovers and other events.

The nature of dividends is similar to share repurchases, as both can be interpreted as "negative outside financing" (Miller \& Rock, 1985). But under the ASX classification, Issued Capital and Distribution Announcement are two different types of continuous disclosure main categories. Theoretical and empirical literature has suggested there is a positive price reaction to dividend change, arguing that dividend increase (decrease) can be viewed as a positive (negative) signal to the market (Bhattacharya, 1979; Foster III \& Vickrey, 1978; Impson \& Karafiath, 1992; John \& Williams, 1985; Lee, 1995; Miller \& Rock, 1985). This information-effect of dividends has also been supported by Australian evidence (Brown, Finn, \& Hancock, 1977). Due to the Australian "unique imputation tax system", more recent studies focus on the determinants of firms' dividend pay-out decisions (Akhtar, 2015; Pattenden \& Twite, 2008). 
Takeover activity is a complicated event because it involves a target firm and an acquiring firm (i.e. bidder, acquirer or buyer). It has been observed that there are consistent and large positive market reactions to target firms across studies in various countries, but mixed (negative, zero and small positive) bidder returns around takeovers in different studies, although the combined shareholder returns are consistently positive. Takeovers can create value for shareholders of target firms due to improved efficiency and/or redistributed gains. But there is no conclusive evidence to support the value creation hypothesis for acquiring firms. For bidders, it could be because the gains from takeovers are equal to the shareholders' required returns (namely, not above normal returns) (Bruner, 2002; Burkart \& Panunzi, 2006). The market response to takeovers could also be impacted by factors such as managerial self-interest, takeover timing, firm size, industry, toehold, target firm type (private or listed) and payment method (Bruner, 2002; Burkart \& Panunzi, 2006; Campa \& Hernando, 2004; Chen, Chou, \& Lee, 2011; da Silva Rosa, Limmack, Supriadi, \& Woodliff, 2004; Le \& Schultz, 2007). Contrary to insignificant or negative abnormal returns for acquirers from takeovers using U.S. data, Australian evidence is that asset acquisitions can add value to acquirers (da Silva Rosa, Nguyen, \& Walter, 2004).

Studies have tried to link various events to share prices. Ritter and Wells (2006) found a positive association between stock prices and disclosed intangible assets on the Australian stock market, and Dedman, Mouselli, Shen, and Stark (2009) supported this positive association by evidence from the U.K. Based on U.S. data, Hammersley, Myers, and Shakespeare (2008) found negative price reactions to internal control weakness disclosures. Similarly, Rezaee, Espahbodi, Espahbodi, and Espahbodi (2012) showed a positive (negative) relationship between stock returns and effective (ineffective) internal controls. Menon and Williams (2010) indicated negative excess returns after disclosing modified audit reports due to going concern problems. 
Adams, Gray, and Nowland (2011) found positive share price reactions to announcements of new female director appointments.

There have been numerous event studies in the literature. Researchers attribute the share price and/or trading volume movements around company announcements to its information content, which signals the market about the company's value. In other words, if an announcement has a significant market response, especially share price reactions, it is value-relevant. However, the available empirical evidence is limited to a single information generating event. Little research has been done to answer the question of how the stock price reacts to different types of announcements. Based on the Australian stock market, this study attempts to reveal the different patterns of immediate market responses to new information, building on market reaction literature by investigating all types of announcements.

\subsection{Literature on the Timeliness of Price Discovery}

This study explores the long-term market reactions to value-relevant information by testing the timeliness of price discovery. Value-relevant information provides investors with better understanding about the firm's value and performance. Therefore investors can use this information to anticipate the firm's profitability before the earnings announcement is available to the market, which is reflected in the share prices. The timeliness of price discovery (also known as speed of price discovery) reveals how timely (quickly) share prices reflect a company's overall income in a fiscal year.

Ball and Brown (1968) first examined the timeliness of the annual earnings report. As the difference between the realised and expected return can indicate the value of new information, 
they used the stock's monthly return residual to measure the value of the income information at a portfolio level (three portfolios: firm years with positive residuals, firm years with negative residuals, and all firm years ). Their empirical results suggested that by the month when the annual report is released, 85 to $90 \%$ of income information has been anticipated by other, more prompt, media. Therefore, the annual earnings report is not timely. Beaver (1968) also found abnormal price movements in the week before the earnings announcement was released, suggesting that there may be "information leakage" or more prompt sources of the earnings information.

Based on Ball and Brown (1968)'s concept and estimates of earnings timeliness, some studies have provided supporting evidence by using updated data in different countries including the U.S. (Alford, Jones, Leftwich, \& Zmijewski, 1993; Nichols \& Wahlen, 2004). Ball and Shivakumar (2008) conducted a regression of stock annual returns on three day returns (Day 1 to Day +1) around four quarterly earnings reports, and the low R square (5-9\%) indicate that on average each quarterly earnings report conveys only $1-2 \%$ of the total information over one year. These studies have proven that the earnings announcement is not a timely medium, and share prices can reflect or anticipate the earnings information in advance. The earlier valuerelevant (i.e. earnings information) is priced, the timelier the price discovery process is (also known as faster speed of the price discovery).

Some researchers have tried to extend the estimation of the timeliness of price discovery. They have explored the elements that can affect this timeliness to better understand the process of price discovery. Brown et al. (1999) investigated the regulative effects of the statutorily sanctioned CDR in Australia in 1994. They employed a similar approach to Ball and Brown (1968) and Alford et al. (1993) to estimate earnings timeliness, concluding that only the share 
prices of smaller firms have anticipated earlier the value-relevant accounting information in the post-reform period.

Butler, Kraft, and Weiss (2007) focused on American listed firms that voluntarily or mandatorily increased their earnings reports from half-yearly to quarterly, in order to examine whether or not the increased financial reports frequency can accelerate the timeliness with which the earnings information is reflected in stock returns over the year. They used monthly buy and hold returns over a year to estimate the earnings timeliness at both portfolio and individual firm level (two portfolios: the companies released financial reports half-yearly and those released financial reports quarterly ${ }^{17}$ ). But only at the portfolio level, Butler et al. (2007) found evidence for voluntary firms that the earnings timeliness improved after switching to quarterly reports. Bushman, Smith, and Wittenberg-Moerman (2010) modified Butler et al. (2007)'s portfolio timeliness measure using daily data instead of monthly data to examine whether 'early private information' from the loan market could accelerate timeliness of price discovery. They found timelier price discovery for the portfolio where companies could disseminate private information to the syndicate; for example, the companies have loans with financial covenants.

Because the analyst forecast keeps revising and getting closer to the actual earnings over time, Donelson, McInnis, Mergenthaler, and Yu (2012) developed a timeliness measure of earnings news by using analyst forecast errors of a firm to calculate the average percentage of earnings information revealed by the analyst forecasts. The higher percentage indicates the firm's analyst forecast is a more timely revelation. Donelson et al. (2012) suggested that if bad news is made

\footnotetext{
17 The two portfolios also can be seen as firm years before switching to quarterly reports, and firm years after switching to quarterly reports.
} 
available to investors more promptly by the analyst forecasts, the firm has a lower risk of litigation.

While most of the literature on the timeliness of earnings information (or price discovery) focuses on the US stock market, Beekes and Brown (2006) developed a metric to measure the timeliness of price discovery in Australia at the individual firm level based on stock returns. They examined the impact of corporate governance on timeliness, suggesting that the better corporate governance a company has, the more rapidly the value-relevant information is priced. Beekes, Brown, and Zhang (2015) re-examined the timeliness of price discovery by taking good and bad news into account. With an extended research sample, they further affirmed the positive association between corporate governance and the timeliness of price discovery, although good news price discovery is timelier for firms with poorer corporate governance. Applying a similar measure to Beekes et al. (2015), Coulton, Dinh, and Jackson (2016) modified the timeliness metric to fit U.S. companies, and found timelier price discovery for companies that are more sensitive to investor sentiment during periods when the sentiment is high for the economy in general.

The above review on the existing published articles shows there is limited research exploring the link between the timeliness and publicly available corporate disclosures on the market. This study extends the literature on the timeliness of price discovery by testing whether increased announcement frequency as to different categories can help value-relevant information be anticipated more quickly by share prices. This study also modified the Beekes and Brown's (2006) timeliness metric in order to match it with the announcement frequency over the same measurement period. Detailed explanations on the modified timeliness metric are highlighted in Section 5.4.1. 


\subsection{Summary}

This chapter has reviewed the literature on the CDR, the market reactions to a single information event and the timeliness of price discovery. In terms of the CDR literature, there are a limited number of empirical studies focusing on the market reactions to ASX-listed companies' announcements, especially market-sensitive announcements. The market reaction literature is limited to a single type of information, and fails to advise investors which type of information could be more important or to induce higher share prices or trading volume changes. Using tick data analysis, this study contributes to the literature on both the CDR and market reactions by exploring the magnitude of immediate market reactions to different types of announcements, especially market-sensitive announcements.

In addition to the immediate market effects of company announcements, this study examines the long term market effect of announcements by the timeliness of price discovery under the CDR. There are few published articles examining the relationship between the corporate disclosures and the timeliness of price discovery. The CDR is designed to keep investors fully informed and to improve investors' confidence in trading. The continuously disclosed announcements should help investors to anticipate companies' performance, i.e. the income or profit status. Therefore, the increased number of announcements under the CDR should be able to promote the timeliness with which value-relevant information is impounded in the share prices before the earnings announcement is released to the market. This study contributes to the timeliness of price discovery literature by taking the public company announcements into account. The next chapter develops hypotheses to fill the gap identified in the literature review, and to specify the areas that this study focuses on. 


\section{CHAPTER FOUR: HYPOTHESES DEVELOPMENT}

This study examines the process by which the Australian stock market absorbs new information contained in different announcements into its prices. In particular, by examining the magnitude of market reactions and the timeliness of price discovery under CDR, this study aims to find the answers to the following two questions. (1) How market (price/volume) sensitive is the information contained in each disclosure category in a continuous disclosure environment? (2) How quickly is the value-relevant information incorporated into share prices before the annual earnings announcement is released, and how can company announcements promote this timeliness of price discovery? Accordingly, this chapter develops two hypotheses to address the Research Questions according to the Efficient Market Hypothesis (EMH) and market microstructure theories. Specifically, this chapter discusses the influences of different types of announcements regarding the share prices and trading volumes, and identifies the link between the announcement frequency and the timeliness of price discovery.

\subsection{Intraday Market Reactions to Announcements}

According to the EMH, share prices are able to efficiently adjust to new public information (Fama, 1970). Prior studies have indicated that if an announcement conveys new information, it can send signals to the market about the company's value, as reflected in the share price and trading volume movements around the information release. However, as reviewed in the previous chapter, most market reaction studies focus on a single information event, such as the release of earnings, equity issue or dividend announcements. A few studies have attempted to cover a wider range of public company specific news, but the way they have classified the information is varies. For example, the information is classified as earnings/dividend/capital 
budgeting/other (Nofsinger, 2001), or earnings/non-earnings announcements (Ranaldo, 2006).

This study explores in depth the market reactions to different information. The CDR provides an appropriate setting to conduct the research, as it promotes stock market efficiency by reducing information asymmetry between external investors and internal managers to a large extent. How the market reacts to corporate disclosures depends on the nature of the information contained in the disclosures. The CDR not only guarantees prompt information release to a large extent, but also classifies information into detailed categories, both of which enable assessments to be made of immediate market reactions to different announcements.

An immediate disclosure is required if the information is "material" as defined by the Australian CDR, though exceptions or trading halts may apply in certain cases. The materiality in the Corporations Act 2001 means "a reasonable person would be taken to expect information to have a material effect on the price or value of ED securities ${ }^{18}$ of a disclosing entity if the information would, or would be likely to, influence persons who commonly invest in securities in deciding whether to acquire or dispose of the ED securities" (Corporations Act 2001, Section 677). Therefore, the 'reasonable investor' test is used to test the effectiveness of disclosures, to judge conduct objectively, and to assess whether the representations would mislead shareholders or affect shareholders' trading behaviour (Duffy, 2012).

From the legal perspective, the materiality test is an objective test, which is used to determine ex ante if the event requires disclosure rather than to examine ex post facto the market reaction to the disclosure. Duffy (2012) supported the reasonable investor test (the information's impact on the investor's trading decision) as preferable to the market test (the information's effect on

\footnotetext{
18 'ED securities' are defined in s.111AD of Corporations Act 2001 (Cth): "(1) Securities of a body are ED securities (short for "enhanced disclosure securities") for the purposes of this Act if, and only if: (a) they are ED securities under section $111 \mathrm{AE}, 111 \mathrm{AF}, 111 \mathrm{AFA}, 111 \mathrm{AG}$ or $111 \mathrm{AI}$; and (b) they are not declared under section $111 \mathrm{AJ}$ not to be ED securities. (2) For the purposes of sections $111 \mathrm{AE}, 111 \mathrm{AF}, 111 \mathrm{AG}$ and $111 \mathrm{AI}$, a class of shares or debentures is taken to include units of shares or debentures in that class".
} 
share prices and/or trading volumes), because the reasonable investor test is more flexible. However, for academics, the reasonable investor test and the market test are not seen as substitutes for one another, but rather as complementary to one another. In other words, the presence of one test may not be sufficient to capture a comprehensive understanding of the materiality or market sensitivity of corporate disclosures.

Theoretically, disclosures under the CDR are supposed to provide material information. However, in the real market, it is difficult or unnecessary to judge whether an announcement is material (required by CDR) or non-material (not required by CDR). On the ASX, disclosures are categorised into 19 main types ${ }^{19}$ and over 152 subtypes. Announcement types are marked by ASX as "always sensitive" (*), "may be sensitive" (\#) or "never sensitive" (blank) (Brown et al., 2006). ${ }^{20}$ Details regarding the announcement category are shown in Table 4-1.

Table 4-1. ASX Announcement Types/Subtypes

\begin{tabular}{|c|c|c|c|}
\hline $\begin{array}{l}\text { Primary } \\
\text { Code }\end{array}$ & Description & $\begin{array}{l}\text { Sub } \\
\text { Code }\end{array}$ & Description \\
\hline \multirow[t]{12}{*}{ 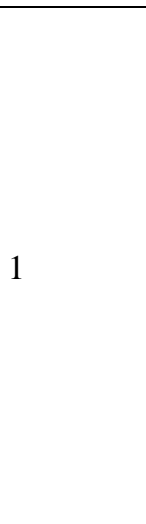 } & \multirow{12}{*}{$\begin{array}{l}\text { Takeover } \\
\text { Announcement }\end{array}$} & $1 *$ & Intention to Make Takeover Bid \\
\hline & & 2\# & Bidder's Statement - Off-market bid \\
\hline & & $3 *$ & Target's Statement - Off-market bid \\
\hline & & 4\# & Bidder's Statement - Market bid \\
\hline & & $5^{*}$ & Target's Statement - Market bid \\
\hline & & $6 *$ & Off-market bid offer document to bid class \\
\hline & & $7 *$ & $\begin{array}{l}\text { holders } \\
\text { Directors' Statement re Takeover }\end{array}$ \\
\hline & & $8^{*}$ & Variation of Takeover Bid \\
\hline & & 9\# & Takeover - Other \\
\hline & & $10 *$ & Supplementary Bidder's Statement \\
\hline & & $11 *$ & Supplementary Target's Statement \\
\hline & & 12 & Scheme of Agreement \\
\hline \multirow{10}{*}{2} & \multirow{10}{*}{$\begin{array}{l}\text { Security Holder } \\
\text { Details }\end{array}$} & 1 & Becoming a substantial holder \\
\hline & & 2 & Change in substantial holding \\
\hline & & 3 & Ceasing to be a substantial holder \\
\hline & & 4 & Beneficial ownership - Part 6C.2 \\
\hline & & 5 & Takeover update - Section 689 Notice \\
\hline & & $6 \#$ & Security holder details - Other \\
\hline & & 7 & Section $205 G$ Notice - Director's Interests \\
\hline & & 8 & Initial Director's Interest Notice \\
\hline & & 9 & Change of Director's Interest Notice \\
\hline & & 10 & Final Director's Interest Notice \\
\hline
\end{tabular}

\footnotetext{
${ }^{19}$ There are 20 main types according to the ASX ComNews ${ }^{\mathrm{TM}}$ Manual. But mFund is not a listed company and does not issue ordinary shares on the market, and is thus excluded from the analysis.

${ }^{20}$ The detailed market sensitivity information is from Morningstar Database.
} 


\begin{tabular}{|c|c|c|c|}
\hline $\begin{array}{l}\text { Primary } \\
\text { Code }\end{array}$ & Description & $\begin{array}{l}\text { Sub } \\
\text { Code }\end{array}$ & Description \\
\hline \multirow{23}{*}{3} & \multirow{23}{*}{ Periodic Reports } & 1 & Annual Report \\
\hline & & 2 & Top 20 shareholders \\
\hline & & $3 *$ & Preliminary Final Report \\
\hline & & $4 *$ & Half Yearly Report \\
\hline & & 5 & Confirmation that Annual Report was sent to \\
\hline & & 5 & Security Holders \\
\hline & & 6 & Trust 6 month accounts \\
\hline & & 7 & Trust 12 month accounts \\
\hline & & 8 & Loan securities on issue \\
\hline & & 9 & Half Year Audit Review \\
\hline & & 10 & Half Year Directors' Statement \\
\hline & & 11 & Full Year Accounts \\
\hline & & 12 & Full Year Audit Review \\
\hline & & 13 & Full Year Directors' Statement \\
\hline & & $14 \#$ & Periodic Reports - Other \\
\hline & & 15 & Half Year Accounts \\
\hline & & 16 & Net Tangible Asset Backing \\
\hline & & 17 & Concise Financial Report \\
\hline & & 18 & Daily Fund Update \\
\hline & & 19 & Half Year Directors' Report \\
\hline & & 20 & Full Year Directors' Report \\
\hline & & 21 & Profit Guidance \\
\hline & & 22 & Debt Facility \\
\hline \multirow{5}{*}{4} & \multirow{5}{*}{$\begin{array}{l}\text { Quarterly } \\
\text { Activities Report }\end{array}$} & $1 *$ & First Quarter Activities Report \\
\hline & & $2 *$ & Second Quarter Activities Report \\
\hline & & $3 *$ & Third Quarter Activities Report \\
\hline & & $4 *$ & Fourth Quarter Activities Report \\
\hline & & $5 \#$ & Quarterly Activities Report - Other \\
\hline \multirow{5}{*}{5} & \multirow{5}{*}{$\begin{array}{l}\text { Quarterly Cash } \\
\text { Flow Report }\end{array}$} & $1 *$ & First Quarter Cash Flow Report \\
\hline & & $2 *$ & Second Quarter Cash Flow Report \\
\hline & & $3 *$ & Third Quarter Cash Flow Report \\
\hline & & $4 *$ & Fourth Quarter Cash Flow Report \\
\hline & & $5 \#$ & Quarter Cash Flow Report - Other \\
\hline \multirow{18}{*}{6} & \multirow{18}{*}{ Issued Capital } & $1 *$ & Renounceable Issue \\
\hline & & $2 *$ & Bonus Issue/In-Specie Issue \\
\hline & & $3 *$ & Placement \\
\hline & & 4 & Issues to the Public \\
\hline & & $5 *$ & Capital Reconstruction \\
\hline & & 6 & New Issue Letter of Offer \& Acc. Form \\
\hline & & 7 & Alteration to issued capital \\
\hline & & $8 *$ & Non-Renounceable Issue \\
\hline & & 9 & Issued Capital - Other \\
\hline & & $10 \#$ & Disclosure Document \\
\hline & & $11 *$ & Market Buy-Back \\
\hline & & 12 & Daily Share Buy-Back Notice \\
\hline & & $13 \#$ & Appendix 3B \\
\hline & & 14 & ASX BookBuild - Upcoming/Commenced \\
\hline & & 15 & ASX BookBuild - Change in Public Parameter \\
\hline & & 16 & ASX BookBuild - Close/Cancel \\
\hline & & 17 & Security Purchase Plan \\
\hline & & 18 & Cleansing Notice \\
\hline \multirow{3}{*}{7} & \multirow{3}{*}{$\begin{array}{l}\text { Asset Acquisition } \\
\& \text { Disposal }\end{array}$} & $1 \#$ & Asset Acquisition \\
\hline & & $2 \#$ & Asset Disposal \\
\hline & & 3 & Other \\
\hline \multirow{4}{*}{8} & \multirow{4}{*}{ Notice Of Meeting } & $1 \#$ & Notice of Annual General Meeting \\
\hline & & $2 \#$ & Notice of Extraordinary General Meeting \\
\hline & & $3 \#$ & Results of Meeting \\
\hline & & 4 & Proxy Form \\
\hline
\end{tabular}




\begin{tabular}{|c|c|c|c|}
\hline $\begin{array}{l}\text { Primary } \\
\text { Code }\end{array}$ & Description & $\begin{array}{l}\text { Sub } \\
\text { Code }\end{array}$ & Description \\
\hline & & $5 \#$ & Alteration to Notice of Meeting \\
\hline & & $6 \#$ & Notice of Meeting - Other \\
\hline & & $7 \#$ & Notice of General Meeting \\
\hline \multirow{15}{*}{9} & \multirow{15}{*}{$\begin{array}{l}\text { ASX } \\
\text { Announcement }\end{array}$} & $1 *$ & Suspension from Official Quotation \\
\hline & & $2 *$ & Reinstatement to Official Quotation \\
\hline & & 3 & Removal from Official List \\
\hline & & 4 & ASX Query \\
\hline & & 5 & Notice Pending \\
\hline & & $6^{*}$ & Change in Basis of Quotation \\
\hline & & $7 *$ & Trading Halt \\
\hline & & $8^{*}$ & Admission to Official List \\
\hline & & $9 *$ & Commencement of Official Quotation \\
\hline & & $10 \#$ & ASX Announcement - Other \\
\hline & & 11 & CAP Cancellation \\
\hline & & 12 & CAP Correction \\
\hline & & 13 & End of Day \\
\hline & & $14^{*}$ & Trading Halt Lifted \\
\hline & & 15 & ASX Circulars \\
\hline \multirow{5}{*}{10} & \multirow{5}{*}{$\begin{array}{l}\text { Distribution } \\
\text { Announcement }\end{array}$} & $1 *$ & Dividend Books Closing \\
\hline & & 2 & Dividend Pay Date \\
\hline & & $3 *$ & Dividend Rate \\
\hline & & $4 \#$ & Dividend Alteration \\
\hline & & $5 \#$ & Dividend - Other \\
\hline \multirow{2}{*}{11} & \multirow{2}{*}{ Progress Report } & $1 \#$ & Progress Report \\
\hline & & $2 \#$ & Progress Report - Other \\
\hline \multirow{15}{*}{12} & \multirow{15}{*}{$\begin{array}{l}\text { Company } \\
\text { Administration }\end{array}$} & $1 \#$ & Director Appointment/Resignation \\
\hline & & 2 & Details of Company Address \\
\hline & & 3 & Details of Registered Office address \\
\hline & & 4 & Details of Share Registry address \\
\hline & & 5 & Trustee Appointment/Resignation \\
\hline & & $6 \#$ & Trust Manager Appointment/Resignation \\
\hline & & 7 & Company Secretary Appointment/Resignation \\
\hline & & $8 \#$ & Company Administration - Other \\
\hline & & 9 & Change of Balance Date \\
\hline & & 10 & Trust Deed \\
\hline & & 11 & Articles of Association \\
\hline & & 12 & Constitution \\
\hline & & $13 \#$ & Responsible Entity Appointment/Resignation \\
\hline & & 14 & Change of Company Name \\
\hline & & 15 & Administrator/Receiver - Appointed/Removed \\
\hline \multirow{3}{*}{13} & Notice of Call & $1 \#$ & Announcement of call \\
\hline & (Contributing & 2 & Notice of call to shareholders \\
\hline & Shares) & 3 & Notice of Call - Other \\
\hline \multirow{10}{*}{14} & \multirow{10}{*}{ Other } & $1 \#$ & Other \\
\hline & & 2 & Internal \\
\hline & & 3 & Legal Proceedings \\
\hline & & 4 & Appendix 16A \\
\hline & & 5 & Year 2000 Advice \\
\hline & & $6 \#$ & Open Briefing \\
\hline & & 7 & Overseas Listing \\
\hline & & $8 \#$ & Standard and Poor's Announcement \\
\hline & & 9 & Trading Policy \\
\hline & & 10 & MAP Test \\
\hline \multirow{2}{*}{15} & Chairman's & 1 & Chairman's Address - Other \\
\hline & Address & $2 \#$ & Chairman's Address to Shareholders \\
\hline \multirow{2}{*}{16} & Letter To & 1 & Letter to Shareholders - Other \\
\hline & Shareholders & 2\# & Letter to Shareholders \\
\hline 17 & ASX Query & 1 & ASX Query - Other \\
\hline
\end{tabular}




\begin{tabular}{|c|c|c|c|}
\hline $\begin{array}{l}\text { Primary } \\
\text { Code }\end{array}$ & Description & $\begin{array}{l}\text { Sub } \\
\text { Code }\end{array}$ & Description \\
\hline & & 2 & ASX Query \\
\hline & & 3\# & Response to ASX Query \\
\hline \multirow{8}{*}{18} & \multirow{8}{*}{$\begin{array}{l}\text { Structured } \\
\text { Products }\end{array}$} & 1 & Other \\
\hline & & 2 & Structured Products Issuer Report \\
\hline & & 3 & $\begin{array}{l}\text { Structured Products Warrant Disclosure } \\
\text { Document }\end{array}$ \\
\hline & & 4 & Structured Products Warrant Acceptance \\
\hline & & 5 & Structured Products Warrant Trust Deed \\
\hline & & 6 & Structured Products Warrant Distribution \\
\hline & & 7 & Structured Products Warrant Adjustment \\
\hline & & 8 & $\begin{array}{l}\text { Structured Products Supplementary Disclosure } \\
\text { Document }\end{array}$ \\
\hline \multirow{6}{*}{19} & \multirow{6}{*}{$\begin{array}{l}\text { Commitments Test } \\
\text { Entity (CTE) } \\
\text { Quarterly Reports }\end{array}$} & $1 *$ & Commitments Test Entity - First Quarter Report \\
\hline & & $2 *$ & $\begin{array}{l}\text { Commitments Test Entity - Second Quarter } \\
\text { Report }\end{array}$ \\
\hline & & $3 *$ & Commitments Test Entity - Third Quarter \\
\hline & & $3^{\prime \prime}$ & Report \\
\hline & & $4 *$ & $\begin{array}{l}\text { Commitments Test Entity - Fourth Quarter } \\
\text { Report }\end{array}$ \\
\hline & & 5\# & Other \\
\hline
\end{tabular}

Market Sensitivity: * Always sensitive; \# May be sensitive.

Under the CDR, the market sensitivity is regarded as a technical description, which is given to the ASX announcement accompanied by approximately a 10 minute trading halt, unless a special case applies (e.g. some takeover announcements) then a longer trading halt may apply (Brown et al., 2006; Brown et al., 2003; Matolcsy et al., 2012). The number of market-sensitive disclosures is widely used as a proxy to measure a company's continuous disclosure behaviour or its quality of continuous disclosure compliance (Chapple \& Truong, 2015; Matolcsy et al., 2012). ${ }^{21}$ To some extent, Table 4-1 reveals which type of announcement may contain more important information. The main announcement subcategories with an 'always sensitive' tag are Takeover Announcement, Periodic Report, Quarterly Activities Report, Quarterly Cash Flow Report, Issued Capital, ASX Announcement, Distribution Announcement and Commitments Test Entity (CTE) Quarterly Report. But it does not necessarily mean that those announcements may cause larger market reactions. Research to date has seldom examined whether the market-sensitive disclosures can cause significant market responses on the stock

\footnotetext{
21 'Market-sensitive' is also known as 'price-sensitive', Chapple and Truong (2015) and Matolcsy et al. (2012) used the terminology 'price-sensitive'.
} 
market. This study builds upon the prior literature regarding continuous disclosure by examining the magnitude of immediate market reactions to different types of company announcements, to reveal how the market interprets the announcements, especially the marketsensitive ones.

For disclosures under the CDR, the market response patterns should be different in terms of various types of announcements, as the CDR provides an information environment for investors to obtain new information on a continuous basis. Listed companies have to meet high requirements regarding information disclosure: companies have to release material information immediately on becoming aware of it. If an announcement conveys information that is material and clear, the adjustment of share prices to this information is significant and efficient. However, if an announcement conveys no new information to the market (non-material), the price adjustment would not be observed. In addition, different types of information may send different signals to the market. It is a 'black box' on how the market interprets information and reaches the equilibrium value of the stock price. However, because of the increased availability of market information such as real-time, prices, volumes and orders, market microstructure literature has started to empirically investigate the price formation process at a detailed level. So far, no literature has used tick data to measure and compare the price formation of all announcements. This study empirically investigates how investors use different information to make trading decisions and adjust their trading prices and volumes at the intraday level, as reflected in the immediate market reactions to disclosures.

As discussed in Chapter Three, researchers have suggested that both price and volume effects are of great importance, as they reflect information materiality from different perspectives. Share price changes reflect the expectations of the market as a whole on the information, while 
volume effects of disclosures show the information materiality from individual trading behaviour, that is the individual investor's expectations (Beaver, 1968). It is possible that the trading volume changes significantly around an information release although there might be no significant price change. It is also highly possible that the volume effect of an announcement is opposite to its price effect (e.g. increased trading volume but decreased or zero stock return). Researchers have shown interest in investors' trade size and trading volume. For instance, by investigating investors' net buying behaviour, Battalio, Lerman, Livnat, and Mendenhall (2012) found that sophisticated investors trade almost immediately after accrual information is available.

This study aims to find whether certain types of announcement are market (price/volume) sensitive, and which type of announcement has the largest magnitude (with sign) of market response, especially for the announcements with the market sensitivity tag. This study examines whether the market sensitivity tag is correctly assigned to announcements by the ASX, and whether the categorisation under the CDR is advantageous. It also provides insights into how different types of information affects price formation and individual investor's trading behaviour. Therefore, the hypothesis is presented in a null form as follows.

$H 1_{a 0}$ : There are no price reactions to company announcements on the ASX.

$H 1_{b 0:}$ There are no trading volume reactions to company announcements on the ASX. 


\subsection{The Timeliness of Price Discovery}

The intraday price and trading volume changes can clearly show how the market reflects the information immediately after the release of different announcements, but do not reveal how well share prices can reflect a company's total value or performance within a period (typically a financial year). If the market is efficient, share prices should not only reflect information in the short term, but also reflect a company's total value or performance in the long term (typically a financial year). This study explores the market reactions to value-relevant information over a long term (a year) by testing the timeliness of price discovery, and links this timeliness to the frequency of company announcements. In accounting literature, price discovery is defined as the process by which stock prices can incorporate value-relevant information and in particular, earnings-based information. The timeliness of price discovery ${ }^{22}$ reveals how quickly the stock price can reflect the "end-of-period terminal value" (Coulton et al., 2016, p. 670) when the market is fully informed about the company's earnings information, which in this study is the date when the annual earnings announcement is released.

As mentioned in Chapter Three, there is limited literature exploring the association between timeliness and corporate disclosures, although the timeliness concept has a long history originating in the seminal article by Ball and Brown (1968).

Figure 4-1 shows the basic idea of the timeliness concept. The earnings announcement is not a timely information source as most of the information (known as value-relevant, income or earnings information) ${ }^{23}$ has been reflected in the share prices by the time the earnings

\footnotetext{
${ }^{22}$ This timeliness of price discovery is also regarded as the timeliness of earnings, the timeliness of price formation, the speed of price discovery, or the speed of information discovery.

${ }^{23}$ It is also interpreted as the information contained in income/earnings numbers.
} 
announcement is released. If share prices could anticipate the 'end-of-period terminal value' earlier (shown as the blue lines in Figure 4-1), it would suggest a timelier price discovery process in the period. In other words, the closer the share prices are to the 'terminal value', the better.

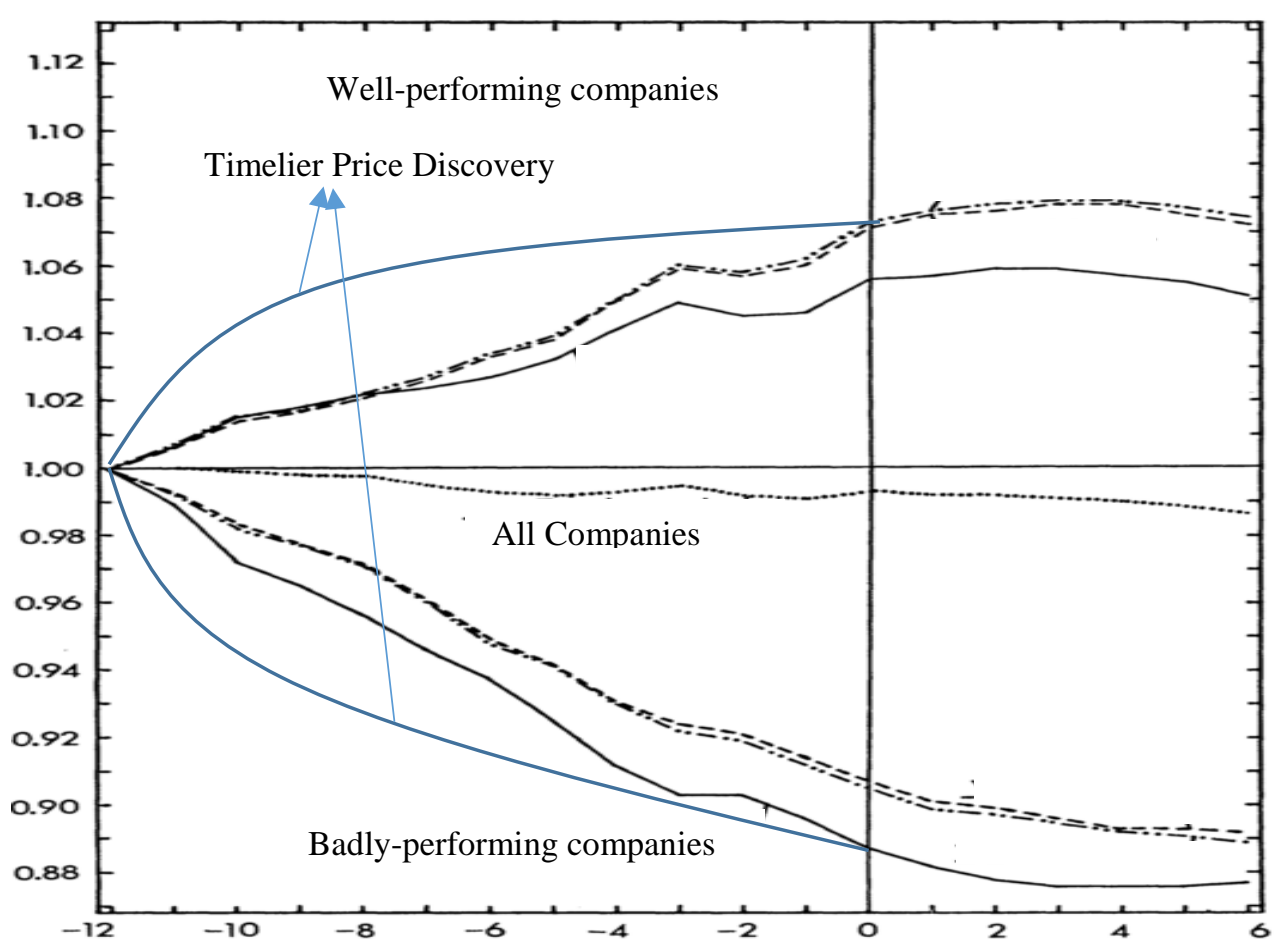

Figure 4-1. Abnormal Performance Indexes - from Ball and Brown (1968) ${ }^{24}$

Butler et al. (2007, p. 182) claimed their study was "the first to directly test the effect of disclosure frequency on timeliness". However, their research sample focused on the impact of financial report frequency (quarterly or half-yearly) on timeliness during the period 1950-1973, because the quarterly reporting was mandated in 1970. Butler et al. (2007) could not find evidence to support the concept that companies with quarterly financial reports have timelier price discovery than those with half-yearly financial reports. But companies that voluntarily

\footnotetext{
${ }^{24}$ Abnormal Performance Indexes were calculated from market-adjust stock price returns. Well (badly) performing companies were those with positive (negative) abnormal incomes. Every company's abnormal income was the difference between the actual income and the expected income which was calculated from the average income of all companies in the market. Blue lines are drawn to interpret the timeliness concept, which are not from the 1968 study.
} 
increased their reporting frequency (the year switching to quarterly reports before 1967) did experience timelier price discovery than prior to the increase in reporting. Using a similar timeliness measure to Butler et al. (2007), McMullin, Miller, and Twedt (2015) directly investigate how the timeliness of price discovery is impacted by the increased mandatory disclosures in 8-K forms after the SEC regulation change. McMullin et al. (2015) found a timelier price discovery after the regulation change, and the timeliness improves the most in companies with the largest disclosure increases.

Lerman and Livnat (2010) investigated the Securities and Exchange Commission's (SEC) regulation change of $8-\mathrm{K}$ requirements in 2004 , which expanded disclosure scope in 8 -K reports and shortened the disclosure preparation time. Their paper was not a direct examination on timeliness. It assumed that if the increased disclosures by 8 -K reports could provide the market with more and timelier earnings-related information than financial reports, the financial reports after the regulation change would be less informative than before. But they found no change in the information content in financial reports $(10-\mathrm{K} / 10-\mathrm{Q})$ under the new disclosure regime, as reflected in the similarity of market reactions before and after the regulation change. They imply that the expansive and timelier $8-\mathrm{K}$ disclosures do not help the market anticipate earnings information in advance.

When investigating the association between the timeliness of price discovery (or the timeliness of earnings) and information disclosures, U.S. studies have focused on one particular disclosure type (10-K/10-Q or $8-\mathrm{K}$ reports) to explore the influences of increased mandatory disclosures due to changes in disclosure regulations. In the Australian continuous disclosure environment, immediate disclosure is required if the information is material. In other words, it is mandatory for ASX-listed companies to disclose any important information. In this context, there has been 
little literature which has looked directly at the association between the timeliness of price discovery and the continuous disclosure frequency.

Brown et al. (1999) studied the effects of the introduction of continuous disclosure rules in Australia. For relatively small and poorly-performed companies, it was revealed that after the law change, the value-relevant information is anticipated by share prices earlier, and the number of price-sensitive announcements is also increased. Although no direct evidence was found, those results may imply a positive association between the timeliness and the price-sensitive disclosure frequency.

Beekes and Brown (2006, p. 423) regarded both the timeliness of price discovery and the disclosure frequency as two "indicators of informativeness". They concluded that better corporate governance is associated with more total/price-sensitive announcements and timelier price discovery. This positive association was also re-examined and supported by Beekes et al. (2015).

In research on continuous disclosures in Australia, Chang, Lim, and Wee (2015, p. 7) described their contribution as "the first to study the relationship between disclosure informativeness and price discovery". Based on non-period announcements with future prospect information, they applied textual analysis to measure the informativeness by disclosure readability, quantifiability and forward-looking ability; they found readability and quantifiability can accelerate the speed of price discovery but not forward-looking ability.

Fernández (2012) was the first to directly test the association between the timeliness of price discovery and the market-sensitive announcement frequency, although he found mixed results. 
He focused on six (out of nineteen) announcement categories: Takeover Announcement, Issued Capital, Asset Acquisition \& Disposal, Other and Chairman's Address. Based on the entire sample, he found that the timelier price discovery is associated with more frequent announcements in three categories: Asset Acquisition and Disposal, Other, and Chairman's Address. In addition, the results are inconsistent across different industry sectors. This may suggest that more disclosure is not always favourable, because it may distract investors' attention (Hirshleifer, Lim, \& Teoh, 2009; Hirshleifer, Lim, \& Teoh, 2004).

The above analysis shows that little research has been done to directly test the relationship between the timeliness of price discovery and the corporate disclosures. Irrespective of the paucity of research, researchers have held the belief that increased disclosure frequency should help the value-relevant information to be reflected faster in share prices, namely timelier or faster price discovery. It could be argued that there might be more prompt information sources such as social media, but under the Australian CDR all information is required to be released on the ASX first. The CDR therefore provides a valuable context to explore the long-term market effects of increased/decreased disclosure frequency on the timeliness of price discovery. This study extends literature on the timeliness of price discovery by covering all available announcements on the market, and taking both the industry effects and the macroeconomic environment into account. This study provides insights into whether increased announcement frequency as to different categories can help value-relevant information to be anticipated by share prices more quickly. Therefore, Hypothesis 2 (in null form) is:

H20: The announcement frequency regarding each category has no impact on the timeliness with which the annual earnings information is priced before the earnings announcement is made available to the market. 


\subsection{Summary}

Based on the prior literature and the EMH and market microstructure theoretical framework, this chapter has developed the hypotheses that evaluate market responses to different corporate disclosures under the CDR over both short- and long- terms. It is posited that the market would immediately react to announcements, and the price/volume reaction patterns would be different depending on the nature of the information contained in different types of announcements. Furthermore, it is also posited that the increased announcement frequency would accelerate the timeliness of price discovery under the CDR. The next chapter presents the research methodology designed to examine the developed hypotheses. 


\section{CHAPTER FIVE: RESEARCH METHODOLOGY}

In addition to descriptive analysis and $t$-tests, this study mainly builds regression models to examine the share price and trading volume movements within the three hours surrounding an announcement release time, and to test the impacts of different types of disclosures on the timeliness of price discovery. This chapter details the data sources sample and selection process, provides the methods employed to measure the dependent and independent variables and describes the regression models that test the research hypotheses. The immediate market reactions are measured by intraday Cumulative Abnormal Returns (CARs) and intraday Abnormal Trading Volumes (ATVs). The timeliness of price discovery is measured by a modified model from Beekes and Brown (2006). The models designed by this study are able to test in depth the market effects of company announcements.

\subsection{Data Sources}

As the current provisions regarding continuous disclosure in the Corporations Act 2001 came into effect on 1 July 2004, this study selects all companies that were listed on the ASX during the period from 01/07/2004 to 31/12/2014 as the research sample. Companies' announcement data (company code, release date, time, headline, market sensitivity tag, announcement categories etc.) and daily share price and market index information (for the timeliness test) are extracted from the SIRCA (Securities Industry Research Centre of Asia Pacific) database. The stock intraday transaction information (i.e. tick data, e.g. trading prices, trading volumes, market index and bid/ask information) is taken from the TRTH (Thomson Reuters Tick History) database. Companies' names, track and industry information are provided by the Morningstar 
DatAnalysis Premium database. Companies' financial data are collected from the Datastream database. SAS statistical software is used to conduct analysis.

\subsection{Sample Selection}

This study attempts to use as much information as possible, but missing data is unavoidable. As the data is collected and matched across different databases, a company is included in the sample only when it can be found in all databases although possibly using different identifiers. In so doing, 2,893 companies remain in the research sample. Details of these companies appear in Appendix I. But not all variables are completed for the 2,893 companies, because of data missing from the database. Even though one particular company is recorded in the database, some specific information about it could be missing either due to the nature of the information or that it is simply missing from the database records. For example, it is possible that the stock price or trading volume information is missing when there is no trading activity at specific times or on particular dates. It is also possible that in a specific year, a company's leverage information is available but ROA (return on assets) information is missing (i.e. blank cells in the original data file downloaded). The observations with missing data for specific variables are automatically omitted by SAS in the statistical tests. Detailed descriptive analysis is provided in the analysis chapters (Chapters Six and Seven). The basic information on the sample size is shown as follows.

Originally, there were 1,142,534 announcement observations, of which 286,227 were marketsensitive. The immediate market reaction tests can only be applied to announcements released during trading hours, which originally totalled 541,585 announcements and 140,662 market- 
sensitive ones. After merging companies from different datasets, 427,899 observations ${ }^{25}$ remained: 122,853 market-sensitive and 305,046 non-market-sensitive announcements. The timeliness of price discovery tests require two adjacent Preliminary Final Report dates of a company to identify one research firm-year. This study has manually calculated 20,122 Preliminary Final Report dates for the 2,893 companies during the research period and identified 17,188 firm-years. ${ }^{26}$

\subsection{The Variables and Models of the Immediate Market Reaction Tests}

\subsubsection{Intraday Cumulative Abnormal Returns (CARs) and Abnormal Trading Volumes (ATVs)}

This study employs intraday event study approaches to test the immediate market reactions to announcements. The first step for an event study is to set time windows. The time intervals of 10, 15, and 30 minutes were widely used in previous literature (Drienko \& Sault, 2013), and market-sensitive announcements marked by ASX are typically accompanied by a 10-minute trading halt (Brown et al., 2006; Brown et al., 2003; Matolcsy et al., 2012). Therefore, this study selects eight event windows for each announcement: [0, 10min], [0, 15min], [0,30min], [0, $60 \mathrm{~min}],[0,90 \mathrm{~min}],[0,120 \mathrm{~min}],[0,150 \mathrm{~min}]$, and $[0,180 \mathrm{~min}]: \mathrm{t}=0$ is the time when the announcement is released to the public, rather than when ASX receives the announcement. The difference between receive time and release time is discussed below.

Figure 5-1 shows the timeline of the release process according to Brown et al. (2003)'s fourstep release for a market-sensitive announcement. As this study was conducted in 2003, there

\footnotetext{
${ }^{25}$ Some observations have missing share price or financial data. After deleting the observations with missing data, 257,951 observations remained: 67,620 market-sensitive and 190,331 non-market-sensitive announcements. For each of the eight time windows, if there is one CAR, $\triangle \mathrm{CAR}$ or ATV available, that observation is kept.

${ }^{26}$ Some firm-years have missing share price (for timeliness metric calculation) or financial data. After deleting the missing data, 11,517 firm-years remained.
} 
have been a few changes to the ASX's announcement office and its trading system. The original diagram involved two ASX business units: Company Announcements Office $(\mathrm{CAO})^{27}$, and SEATS Market Control (SMC). SEATS was the abbreviation for the Stock Exchange Automated Trading System, the network platform where most trading activities took place on the ASX. The CAO has since changed its name to the Market Announcement Office (MAO). ${ }^{28}$ The ASX removed SEATS and implemented an Integrated Trading System (ITS) in 2006. But there have been no significant changes to the process of releasing an announcement. The diagram by Brown et al. (2003) still can help one to understand the announcement release process on the ASX. As SMC no longer exists, Figure 5-1 uses Market Control (MC) to identify the ASX unit that can control the trading system.

Once an announcement is transmitted to the MAO through ASX Online, the 'specialist staff' need to decide whether it is market-sensitive or not. If it is market-sensitive and it is also during trading hours $(10 \mathrm{am}-4 \mathrm{pm})$, a trading halt is requested by the MAO and then triggered by the MC (Step 1). The status of the security shifts to 'PREOPENING' from 'NORMAL', immediately (within one minute) followed by the headline release (Step 2). Immediately thereafter, the completed PDF announcement document is released by the MAO; then the announcement summary is also released (Step 3). About ten minutes after the headline, the trading halt ends, marked by the security status becoming 'OPENING', and then instantly to 'NORMAL' (Step 4). As to non-market-sensitive announcement releases, the process is almost the same, except there is no trading halt (i.e. no Steps 1 and 4 in Figure 5-1).

\footnotetext{
${ }^{27}$ The previous Company Announcements Office (CAO) has changed its name to the Market Announcement Office (MAO). ${ }^{28}$ I am not sure when this name change occurred, but the MAO is used on the ASX website and ASX Listing Rules Guidance Note 14, ASX Market Announcements Platform.
} 
$\begin{array}{llllll}\text { Step } 0 & \text { Step } 1 & \text { Step } 2 & \text { Step } 3 & \text { Step } 3 & \text { Step } 4\end{array}$

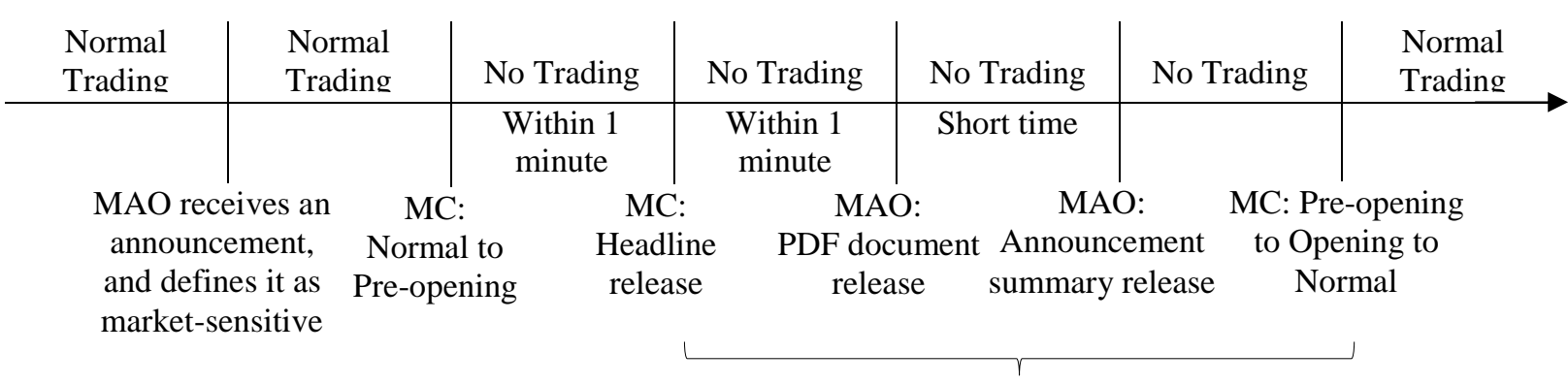

About 10 minutes

Figure 5-1. The Timeline for the Release of a Market-sensitive Announcement

This study uses share price and trading volume at every minute to calculate the intraday CARs and ATVs over the eight time windows. But investors do not trade every minute. It is common that no trading occurs during certain times even though no trading halt then exists. If there is no trading in a specific minute, no trading price or volume information is available. It is also possible that even within one minute there are a few trades occurring at slightly different prices. It is easy to measure the trading volumes, because if there is no trading, the volume is zero $(0)$. It is inappropriate to measure a share price as zero (0) even though there is no trading at that time. In order to measure the share price when there is no trade or more than one trade within a minute, this study applies a method similar to that used by Drienko and Sault (2013): the share price is estimated by the last trading price preceding the end of the minute. ${ }^{29}$ This study employs the natural logarithm return and the $(0,1)$ model to measure intraday cumulative returns, so the abnormal return and cumulative abnormal return are as follows:

$$
\begin{aligned}
& R_{i, t}=\operatorname{Ln}\left(P_{i, t} / P_{i, t-1}\right) \\
& R_{m, t}=\operatorname{Ln}\left(I_{t} / I_{t-1}\right)
\end{aligned}
$$

\footnotetext{
${ }^{29}$ If there is no trading at the minute, the share price is measured by the latest trading price; if there are a few trading prices within the same minute (for example, $\$ 1.00$ at 10:10:20am, and $\$ 1.01$ at 10:10:51am), the share price is estimated by the closing price within that minute $(\$ 1.01)$.
} 


$$
A R_{i, t}=R_{i, t}-R_{m, t}
$$

$C A R_{i,[a, b]}=\sum_{t=a}^{t=b} A R_{i, t}$

where $R_{i, t}$ and $P_{i, t}$ are the stock return and stock price of firm $i$ at minute $t$ respectively; $R_{m, t}$ and $I_{t}$ are the market return and ASX market index (All Ordinaries) at minute $t$ respectively; $A R_{i, t}$ is the abnormal return of firm $i$ at minute $t$, which is computed for each minute over the trading hours on every trading day during the sample period, and for each of the sample's trading hour announcements (originally 541,585 announcements); $R_{m, t}$ is the market return at minute $t$; and $C A R_{i,[a, b]}$ is the cumulative abnormal return for firm $i$ over the event window $[a, b]$ after each announcement release.

As a robustness test, this study also tests the differences between CARs before and after the announcements released over the equivalent time window ( $\triangle \mathrm{CAR})$.

$\Delta C A R_{i,[a, b]}=\sum_{t=a}^{t=b} C A R_{i, t}-\sum_{t=-b}^{t=-a} C A R_{i, t}$

The ATV is estimated by the difference between the numbers of shares traded before and after the announcement over equivalent event window lengths. For example, the volume traded over $[0,+10 \mathrm{~min}]$ is compared with the volume traded over [-10 $\mathrm{min}, 0]$. Thus the calculation of ATV is as follows:

$A T V_{i,[a, b]}=\sum_{t=a}^{t=b} T V_{i, t}-\sum_{t=-b}^{t=-a} T V_{i, t}$ 
where $A T V_{i,[a, b]}$ is the abnormal trading volume of firm $i$ over the event window $[a, b]$ around the announcement release; $T V_{i, t}$ is the trading volume of firm $i$ at minute $t$, which is also calculated for each minute over the trading hours on every trading day, and for each of the sample's trading hour announcements (originally 541,585 announcements).

\subsubsection{The Regression Models of the Immediate Market Reaction Tests}

The regression models for $\mathrm{H} 1$ are expressed as:

$$
\begin{aligned}
C A R / \triangle C A R / A T V= & \alpha_{0}+\sum_{i=1}^{i=17} \alpha_{i} \text { Announcement }+\alpha_{18} \text { BASpread }+\alpha_{19} \text { Size }+\alpha_{20} \text { BTM } \\
& +\alpha_{21} \text { Lev }+\alpha_{22} \text { ROA }+\alpha_{23} \text { RSector }+\alpha_{24} \text { FSector }+\varepsilon
\end{aligned}
$$

where $C A R$ is intraday cumulative abnormal returns in different time windows, $\triangle C A R$ is the change of $C A R$ before and after the announcement over certain time windows, ATV is intraday abnormal trading volumes in different time windows and Announcement is a dummy variable used to measure different types of ASX-listed company announcements according to the main category codes. There are 19 announcement categories in total (details shown in Table 5-3). However, Quarterly Activities Report (Type 4) and Quarterly Cash Flow Report (Type 5) induce serious multicollinearity problems; therefore, these two categories are omitted from the regression models, and 17 dummy variables remain.

Control variables include liquidity (bid-ask spread - BASpread), risk and some companies' financial factors. Liquidity (or illiquidity) is generally accepted as a priced factor (Amihud \& 
Mendelson, 1986; Brennan et al., 2012; Chordia et al., 2009). It is also indicated that intraday stock returns are affected by microstructure factors such as the BASpread (Mucklow, 1994). The advantages of tick data could be different from actively traded stocks to less-actively traded ones (Starks, 1994). More importantly, Chordia et al. (2008) and Chung and Hrazdil (2010) have supported the premise that liquidity enhances market efficiency over an intra-daily horizon. This study uses the BASpread to control the impact of liquidity on intraday market tests. Furthermore, this study controls Fama and French (1993) common risk factors: firm size (Size) and book-to-market ratio (BTM) factors, and financial factors such as leverage (Lev), the return on asset ratio (ROA) prior to the announcement (Drienko \& Sault, 2013). Resource sector (RSector) and financial sector (FSector) are applied to control industry impacts due to their high risk level, low liquidity and special disclosure requirements. For example, mining, gas exploration and investment companies are required to release Quarterly Cash Flow Reports to keep investors informed in a timely manner, whereas other companies are only required to provide half-yearly reports. A detailed description of the variables is shown in Table 5-1.

Table 5-1. Variable Description for the Immediate Market Reaction Tests

\begin{tabular}{ll}
\hline Variables & Description \\
\hline$C A R$ & Intraday cumulative abnormal return after the announcement release, Equation 5-4. \\
& $\begin{array}{l}\text { Change of intraday cumulative abnormal return before and after the announcement release, } \\
\text { Equation 5-5. } \\
\text { Abnormal trading volume, calculated by the change of volume traded before and after } \\
\text { announcement release, Equation 5-6. }\end{array}$ \\
Announcement & $\begin{array}{l}17 \text { dummy variables for } 17 \text { announcement categories: equal to } 1 \text { if the announcement belongs } \\
\text { to the category }{ }^{30} \text { and } 0 \text { otherwise. }\end{array}$ \\
BASpread & Time-weighted bid-ask spread. \\
Size & The natural logarithm of the company's market capitalisation. \\
BTM & The book-to-market ratio: book value of equity divided by market value of equity. \\
Lev & The debt to equity ratio: total debt divided by total equity. \\
ROA & Return on assets: earnings before interest and tax divided by average total assets. \\
RSector & A dummy variable of 1 if the company is in a resource sector, and 0 otherwise. \\
FSector & A dummy variable of 1 if the company is in a financial sector, and 0 otherwise.
\end{tabular}

\footnotetext{
${ }^{30}$ Multi-categorisations exist. If an announcement is classified into several categories, this study applies the first category shown in the SIRCA database file. The same announcement is not repetitively counted in different categories.
} 


\subsection{The Variables and Models of the Timeliness of Price Discovery Test}

\subsubsection{The Timeliness of Price Discovery}

This study refers to Beekes and Brown's (2006) approach regarding the speed of price discovery to uncover how fast the annual earnings information is incorporated into share prices. This approach indicates the net price effect of all value-relevant information over a year. Beekes and Brown's (2006) timeliness metric (shown in Equation 5-8) is the first measure of earnings timeliness at the company-year level instead of portfolio level. It also has been acknowledged as a valid measure by some other studies (e.g. Ball \& Shivakumar, 2008; Bushman et al., 2010; Coulton et al., 2016).

$M^{c}=\left(\sum_{t=-249}^{t=0}\left|\ln \left(P_{0}\right)-\ln \left(P_{t}\right)\right|\right) / 250$

where $P_{t}$ is market-adjusted share price on day $\mathrm{t} ; \mathrm{t}=0$ is 10 trading days after the release of preliminary final reports. This timeliness of price discovery reveals how quickly the stock price can reflect the "end-of-period terminal value" (Coulton et al., 2016, p. 670), which in this equation is the date occurring 10 trading days after the preliminary final report is released. If the share price changed to the terminal value $P_{0}$ on the trading day -249 and later the price moves along with the market index, the speed of price adjustment is fast and $\mathrm{M}^{\mathrm{c}}$ is identically zero (0). By contrast, if the share price changed to the terminal value $P_{0}$ gradually from trading day -249 to day 0 , the speed of price adjustment is slow. Therefore, on average, the closer the share price is to the target terminal value $P_{0}$, the better and faster the share price can reflect 
value-relevant information. In other words, the smaller the value of the timeliness metric, the faster is the timeliness of price discovery.

Building upon Beekes and Brown's (2006) study, Beekes et al. (2015) further constructed additional timeliness metrics of price discovery after taking good and bad news into account. This study does not apply the updated timeliness metric for two main reasons. First, how the missing price information is treated can make a big difference to the metric. Beekes et al. (2015) deal with missing share prices by a forward-filled method over 365 calendar days ending 14 days after the preliminary final report date; that is if there is no trading on one day (e.g. Saturday or Sunday), the share price is filled by the price from the previous day (e.g. Friday). This study does not count the days when there is no trading. Second, when measuring the timeliness of good (bad) news, Beekes et al. (2015) applied the positive (negative) daily raw returns (not adjusted to the market index) to define good (bad) news. In linking the corporate disclosure frequency to the Beekes et al.'s (2015) timeliness of good/bad news, it is necessary to determine if an announcement is good (bad) news. However, it is inappropriate to count an announcement as a good (bad) news disclosure according to whether the announcement is released on a day with positive (negative) returns. This is because the daily return (calculated by the stock closing prices on two adjacent business days) reflects the overall effects of all information released between the market closing times $(4.00 \mathrm{pm})$ of the two trading days, not to mention the announcement that is released on days during trading halts. With this condition, the typical timeliness metric (Beekes \& Brown, 2006) is more appropriate for this study.

Not exactly the same as Beekes and Brown's (2006) timeliness metric, this study assumes that the share price can reflect the value of the company on the day ${ }^{31}$ when the preliminary final

\footnotetext{
${ }^{31}$ If the earnings report is released after trading hours, the next trading day price is used.
} 
report is released, and there is no assumption about a price 'settle down' period (i.e. 10 trading days). It is difficult to conclude when the price discovery process is complete, because it could be argued that a company's value (reflected in its share price) is always under revision and estimated with noise (Coulton et al., 2016). This study's metric aims to capture how fast the market is able to reach the agreement on a share price when the investors have been informed about the company's annual performance.

Moreover, this study does not limit the measurement window to 250 trading days, but uses the actual number of trading days between when two adjacent preliminary final reports are released: this is a more precise way to 'cut off' the year. For a company, the release date of its preliminary final report is not always exactly the same date every year. If the release dates were 30/08/2011 and $01 / 08 / 2012$, the fixed ' 250 trading day' period for year 2012 is between $17 / 08 / 2011^{32}$ and 01/08/2012, which contains an overlap period. When calculating the timeliness of price discovery or the announcement frequency, the share prices and announcements are doublecounted in the overlapping period. Because this study aims to explore whether the company announcements on the market can help share prices anticipate the total value-relevant information (i.e. the terminal value) in advance, the timeliness metric and the announcements need to be matched over exactly the same time window between when two adjacent preliminary final reports are released. Therefore, the modified timeliness metric is:

$M^{c \prime}=\left(\sum_{t=-n}^{t=-1}\left|\ln \left(P_{0}^{\prime}\right)-\ln \left(P^{\prime}{ }_{t}\right)\right|\right) / n$

\footnotetext{
32250 trading days are exactly 50 weeks' work days, or 350 calendar days. 350 calendar days before 30/08/2012 is $17 / 08 / 2011$.
} 
where $M^{c \prime}$ is the modified timeliness metric used in this study, which is measured by every day's market adjusted price over the period between two adjacent preliminary final reports for original 11,517 firm-years. $P^{\prime}{ }_{t}$ is the market-adjusted share price on day $\mathrm{t}$; $\mathrm{t}=0$ is the day of preliminary final reports; $\mathrm{n}$ is the number of trading days between two adjacent preliminary final reports. To control the stock price unit influences (e.g. 10 dollar per share, or 10 cents per share), this study uses the deflated timeliness metric ${ }^{33}$, which is the original timeliness metric divided by one plus the absolute market adjusted return on the Preliminary Final Report release date (Chang et al., 2015). So, the modified deflated timeliness metric is:

Deflated_M $M^{c^{\prime}}=\frac{1}{1+\left|\ln P_{0}^{\prime}{ }_{0}\right|}\left[\left(\sum_{t=-n}^{t=-1}\left|\ln \left(P_{0}^{\prime}\right)-\ln \left(P^{\prime}{ }_{t}\right)\right|\right) / n\right]$

\subsubsection{The Regression Model of the Timeliness of Price Discovery Test}

To further explore how timely the annual value-relevant information is priced, this study examines $\mathrm{H} 2$ by the following regression model:

$$
\begin{aligned}
\text { Deflated } M^{c^{\prime}} & =\beta_{0}+\sum_{i=1}^{i=19} \beta_{i} \text { AnnFre }+\beta_{20} \text { Vol }+W / \text { BCompany }+\beta_{22} \text { Size }+\beta_{23} \text { BTM } \\
& +\beta_{24} \text { Lev }+\beta_{25} \text { ROA }+\beta_{26} \text { RSector }+\beta_{27} \text { FSector }+\varepsilon
\end{aligned}
$$

where Deflated $M^{c \prime}$ is the modified deflated timeliness metric (Equation 5-10); AnnFre is the company announcement frequency as to each announcement group or category (19 categories in total) over the period between two adjacent preliminary final reports (the same time widow

\footnotetext{
${ }^{33}$ The advantage of deflated timeliness metric is further discussed in Chapter Seven.
} 
as the timeliness metric calculation); control variables include the volatility of daily marketadjusted returns over the timeliness specific period ( $\mathrm{Vol}$ ), well-/badly-performing company (W/BCompany), firm size (Size), the book-to-market ratio (BTM), the leverage (Lev), and return on asset ratio (ROA) (Beekes \& Brown, 2006; Chang et al., 2015; Coulton et al., 2016). Resource sector (RSector) and financial sector (FSector) are again controlled for industry impacts. Detailed variable description is shown in Table 5-2.

With respect to the timeliness of price discovery tests, this study first examines the association between the timeliness and the number of all announcements released over the timeliness metric time window. Then, the announcements are further divided into two groups: Periodic Reports (Type 3) and Non-periodic Reports (other types of announcements). Lastly, focusing on the Non-periodic Reports, this study examines whether different types of announcements influence the timeliness of price discovery in different ways. According to the ASX Primary Report Code, the 19 Main Types of announcements are shown in Table 5-3. ${ }^{34}$

Table 5-2. Variable Description for the Timeliness of Price Discovery Test

\begin{tabular}{|c|c|}
\hline Variables & Description \\
\hline Deflated $M^{c^{\prime}}$ & The modified deflated timeliness metric of price discovery: Equation 5-10. \\
\hline AnnFre & $\begin{array}{l}\text { Announcement frequency, the number of company announcements as to each announcement } \\
\text { group or category over the timeliness specific period, and there are } 19 \text { categories in total. }\end{array}$ \\
\hline Vol & The standard deviation of daily market-adjusted returns over the timeliness specific period. \\
\hline W/BCompany & $\begin{array}{l}\text { Well-/badly-performing company. A dummy variable of } 1(0) \text { if the company performs better } \\
\text { (worse) than the market index over the timeliness specific period. }\end{array}$ \\
\hline Size & Natural logarithm of the company's market capitalisation. \\
\hline$B T M$ & The book-to-market ratio: book value of equity divided by market value of equity. \\
\hline Lev & The debt-to-equity ratio: total debt divided by total equity. \\
\hline ROA & Return on asset: earnings before interest and tax divided by average total assets. \\
\hline RSector & A dummy variable of 1 if the company is in a resource sector, and 0 otherwise. \\
\hline FSector & A dummy variable of 1 if the company is in a financial sector, and 0 otherwise. \\
\hline
\end{tabular}

\footnotetext{
${ }^{34}$ Table 5-3 has the same content as Table 1-1, which shows a brief explanation of the different announcement categories.
} 
Table 5-3. Company Announcements: Main Categorisations

\begin{tabular}{ll}
\hline Type & Announcements \\
\hline 1 & $\begin{array}{l}\text { Takeover / Schem } \\
\text { Announcements }\end{array}$ \\
2 & $\begin{array}{l}\text { Security Holder } \\
\text { Details } \\
3\end{array}$ \\
Periodic Reports
\end{tabular}

Description

Announcements that are related to a takeover event from either bidder or target companies, such as bidder's statement, target's statement, directors' statement reply takeover.

Announcements that provide shareholder or ownership information, such as becoming/ceasing to be a substantial holder, directors' interests.

Periodic Reports

Financial reports that are released at a certain time of a year, referring to preliminary final report, half yearly report and annual report; it may also include other announcements that are related to financial reports such as profit guidance and debt facility.

$4 \quad$ Quarterly

Activities Report

5 Quarterly Cash

Quarterly reports of mining activities; they could be provided either by mining entities or by other entities that have mining projects.

Flow Report

$6 \quad$ Issued Capital

$7 \quad$ Asset Acquisition

\& Disposal

$8 \quad$ Notice of Meeting

Quarterly reports of cash flow provided by investment entities, mining exploration entities, and oil and gas exploration entities. Commitments Test Entities are also required to provide quarterly cash flow reports, but these reports are under Category 19 Commitments Test Entity Quarterly Reports.

Announcements that relate to issuing shares to shareholders, or buying back shares from shareholders.

Announcements relating to buying or disposing assets. This is a vague category with only three subcategories asset acquisition, disposal and other.

The notice to shareholders regarding the date, time and place of shareholder meetings; the results of such meetings are also included in this announcement category.

$9 \quad$ ASX

Announcement

Announcements that are sent from ASX or relate to communications between a

10 Distribution

Announcement

11 Progress Reports listed company and ASX, such as trading halt notices, suspension from official quotation.

Dividend related announcements, such as dividend rate, pay date.

A report of the progress regarding certain project, and release time is unknown. This is also a vague category with only two subcategories progress report and other.

12 Company

Administration

$13 \quad$ Notice of Call

14 Other

15 Chairman's

Address

16 Letter to

Shareholders

17 ASX Query

Announcements relating to company profile or company management, such as Director Appointment/Resignation, company address, company name change. When there are unpaid or partly paid shares issued, a call notice can be sent by the company requiring particular shareholders to pay the amount outstanding. Announcements that cannot be classified into any other categories, such as open briefings, legal proceedings, overseas listing.

Chairman's addresses or presentations on the company's financial performance, market position, and invested projects.

A letter that is written by top management to shareholders, providing an overview of the company's operations over the year (could be half-year, quarter or month).

A query letter sent by ASX. It is part of ASX's enforcement practices regarding a company' compliance with its continuous disclosure obligations. There are two types of ASX queries, price query letters and aware letter. If ASX notices abnormal trading in a company's securities, a price query will be sent to verify if any undisclosed information is causing this abnormal trading; if ASX has concerns about whether the company has fully and promptly disclosed any market-sensitive information, an aware letter will be sent.

$18 \quad$ Structured

Products

Announcements that are related to the company's structured products. A structured product is a financial derivative package, including securities, options, bonds and foreign currencies.

19 CTE Quarterly Reports

The entities that, under the 'commitments test', are required to provide quarterly cash flow reports. To list on the ASX, an entity must satisfy either the 'profit test' or the 'assets test' in the listing requirements, and the 'commitments test' is under the 'assets test'. If an entity's main (half or more) tangible asset is cash or readily convertible to cash after raising funds from an Initial Public Offering (IPO), the entity is required to makes commitments to spend at least half of the cash or equivalent assets consistently with its objectives. 


\subsection{Summary}

This chapter has outlined the research methodology designed for hypothesis tests. It has described the data collected from various databases, and has detailed the sample selection process. The final sample contains 2,893 ASX-listed companies from 01/07/2004 to 31/12/2014 The measurements of immediate market reactions and the timeliness of price discovery have been presented; other variables have been defined and linear regression models have been established to test the Research Hypotheses. The results for Hypotheses 1 and 2 are discussed in Chapters Six and Seven respectively. The next chapter provides the descriptive statistics regarding the announcement distributions, the $t$-test results of the average market consequences as to each type of announcement, and the regression results relating to Hypothesis 1 for marketsensitive announcements. 


\section{CHAPTER SIX: RESEARCH ANALYSIS ON THE IMMEDIATE MARKET REACTIONS TO DIFFERENT ANNOUNCEMENTS}

To evaluate the market effects of disclosures under the CDR, this study conducts two examinations: one is the immediate market reaction test and the other is the long-term timeliness of price discovery test. As this study explores whether the detailed announcement type classification could make a difference to immediate market responses under the CDR, all tests and results are discussed surrounding the 19 announcement categories according to the Primary Report Code by ASX (a detailed subcategory description is shown in Table 4-1 in Chapter Four). mFund is excluded from this analysis because mFund is the unlisted managed fund settled through the ASX settlement service, which does not issue ordinary shares on the ASX. For detailed descriptions of the announcement categories, refer to Tables 4-1 and 5-3.

This chapter presents the results of the immediate market reaction tests. It includes a consideration of the following: extensive descriptive analysis on companies' disclosure distribution at different times and days and in different years; a detailed $t$-test analysis of the share price and trading volume reactions to different types of continuous disclosures in the three hours surrounding the information release time, and comprehensive regression analysis after taking good/bad news and the 2008 - 2010 Global Financial Crisis (GFC) into account.

The descriptive analysis presents how the distribution of company announcements changes with time. The immediate market reaction tests focus on the trading hour announcements, but it is also important to understand how the non-trading hour announcements distribution pattern changes with time. It could be argued that if an announcement is released during non-trading hours, investors have more time to read the information and make more rational investment 
decisions. However, for investors in companies listed on the ASX, there should not be a big difference between trading and non-trading hour announcements. The CDR requires material information to be disclosed promptly, and each market-sensitive (also known as price-sensitive) announcement is accompanied by a minimum 10 minute trading halt. ${ }^{35} \mathrm{~A}$ company can also request a trading halt if an event is expected to happen but it is not yet ready to inform the market, which to some extent guarantees rational trading. It is impossible to predict which announcement would be released at exactly what time, but the descriptive analysis can show a bigger picture of companies' disclosure behaviour patterns and time preferences.

As more important announcements are potentially accompanied by a longer trading halt, this study's three-hour time window can only capture the immediate market responses to 'normal' announcements that do not induce long time trading halts. But 'normal' is not equal to 'not important', which is shown in the $t$-tests on CARs (Cumulative Abnormal Returns), $\Delta$ CARs (Changes of Cumulative Abnormal Returns) and ATVs (Abnormal Trading Volumes). CAR $t$ tests show whether there are abnormal returns existing after the release of a particular type of announcement. $\Delta \mathrm{CAR} t$-tests are robust tests to control other factors that may influence share prices. For example, if it is a bull (bear) market day, or if a market-wide incident has just happened, the $\triangle \mathrm{CAR}$ measure can counteract the price effects from the macroeconomic environment. ATV $t$-tests can reveal whether or not trading volumes are increased on average after a particular type of announcement is released.

After presenting the CARs, $\triangle \mathrm{CARs}$ and ATVs for different types of announcements, this study applies regression models to further compare the magnitude of immediate market reactions to different announcements after taking good/bad news and GFC into account. The regression

\footnotetext{
35 The normal procedure is 10 minutes for a market-sensitive announcement, and 60 minutes for a market-sensitive takeover announcement. But the trading halt could be longer depending on the information materiality.
} 
analysis provides insights into which announcement category has the largest immediate price and volume responses, increases the understanding of the information nature (good or bad news) as to different announcement types and reveals the GFC impacts on the immediate market consequences of different types of announcements.

\subsection{Original Announcement Distribution}

This study collects announcement information from the SIRCA database, a record of every announcement released on the ASX. The original announcement distribution shows the corporate disclosure behaviour patterns. This was merged with share prices and other financial data. After merging, the sample size was reduced due to missing data, especially missing share price information. For example, sometimes when listed companies have long-term trading halts or suspensions, there is no share price information available. The original announcement distribution helps to shed light on the companies' disclosure behaviour on when to release their announcements.

Table 6-1 shows the announcement distribution for each category during trading hours and nontrading hours from $01 / 07 / 2004$ to $31 / 12 / 2014$. Tables 6-2 and 6-3 provide the same information

as Table 6-1, but for market-sensitive and non-market-sensitive announcement sub-samples. Table 6-4 presents the announcement summary statistics from 2004 to 2014 year by year (the statistics for 2004 are only half a year: from 01/07/2004 to 31/12/2004). Due to the big sample size, the detailed yearly distribution for each announcement category is displayed in Appendix II. 
Table 6-1. All Announcements' Distribution as to Each Category

\begin{tabular}{|c|c|c|c|c|c|c|c|c|c|c|c|}
\hline $\begin{array}{l}\text { Announcement } \\
\text { Type }\end{array}$ & $\begin{array}{c}\text { Takeover } \\
\text { /Scheme } \\
\text { Announcements }\end{array}$ & $\begin{array}{l}\text { Security } \\
\text { Holder } \\
\text { Details }\end{array}$ & $\begin{array}{l}\text { Periodic } \\
\text { Reports }\end{array}$ & $\begin{array}{c}\text { Quarterly } \\
\text { Activities } \\
\text { Report }\end{array}$ & $\begin{array}{l}\text { Quarterly } \\
\text { Cash Flow } \\
\text { Report }\end{array}$ & $\begin{array}{l}\text { Issued } \\
\text { Capital }\end{array}$ & $\begin{array}{c}\text { Asset } \\
\text { Acquisition } \\
\text { \& Disposal }\end{array}$ & $\begin{array}{l}\text { Notice of } \\
\text { Meeting }\end{array}$ & $\begin{array}{c}\text { ASX } \\
\text { Announce- } \\
\text { ment }\end{array}$ & $\begin{array}{c}\text { Distribution } \\
\text { Announce- } \\
\text { ment }\end{array}$ & $\begin{array}{l}\text { Progress } \\
\text { Reports }\end{array}$ \\
\hline Pre trading & 4,249 & 46,016 & 46,360 & 6,837 & 2,385 & 62,454 & 8,215 & 7,920 & 10,949 & 4,392 & 46,981 \\
\hline $10.00-11.00^{*}$ & 2,086 & 16,419 & 15,628 & 2,826 & 932 & 18,426 & 3,583 & 3,854 & 5,775 & 1,786 & 24,976 \\
\hline $11.00-12.00^{*}$ & 1,208 & 16,693 & 12,167 & 2,790 & 1,131 & 15,962 & 2,263 & 4,868 & 2,318 & 1,686 & 17,773 \\
\hline $12.00-13.00^{*}$ & 1,338 & 16,387 & 9,889 & 2,885 & 1,224 & 15,316 & 1,975 & 6,070 & 1,875 & 1,521 & 14,280 \\
\hline $13.00-14.00^{*}$ & 1,115 & 14,228 & 7,843 & 2,677 & 1,207 & 13,185 & 1,532 & 5,749 & 1,631 & 1,094 & 10,382 \\
\hline $14.00-15.00^{*}$ & 1,267 & 19,073 & 9,512 & 2,940 & 1,250 & 15,477 & 1,756 & 6,480 & 1,729 & 1,908 & 9,601 \\
\hline $15.00-16.00$ & 1,577 & 24,032 & 11,446 & 3,067 & 1,351 & 18,844 & 1,744 & 6,917 & 1,642 & 2,147 & 8,216 \\
\hline Post trading & 5,735 & 81,603 & 46,683 & 9,947 & 4,907 & 68,541 & 4,136 & 18,761 & 7,130 & 4,051 & 15,386 \\
\hline Weekends & 1 & 3 & 2 & 1 & 0 & 3 & 0 & 0 & 0 & 48 & 2 \\
\hline Trading hours & $\begin{array}{c}8,591 \\
46.25 \%\end{array}$ & $\begin{array}{l}106,832 \\
45.57 \%\end{array}$ & $\begin{array}{c}66,485 \\
41.68 \%\end{array}$ & $\begin{array}{c}17,185 \\
50.59 \%\end{array}$ & $\begin{array}{c}7,095 \\
49.32 \%\end{array}$ & $\begin{array}{l}97,210 \\
42.60 \%\end{array}$ & $\begin{array}{c}12,853 \\
51.00 \%\end{array}$ & $\begin{array}{c}33,938 \\
55.99 \%\end{array}$ & $\begin{array}{l}14,970 \\
45.30 \%\end{array}$ & $\begin{array}{c}10,142 \\
54.43 \%\end{array}$ & $\begin{array}{c}85,228 \\
57.74 \%\end{array}$ \\
\hline Off-trading & 9,985 & 127,622 & 93,045 & 16,785 & 7,292 & 130,998 & 12,351 & 26,681 & 18,079 & 8,491 & 62,369 \\
\hline hours & $53.75 \%$ & $54.43 \%$ & $58.32 \%$ & $49.41 \%$ & $50.68 \%$ & 57.40 & $49.00 \%$ & $44.01 \%$ & $54.70 \%$ & $45.57 \%$ & $42.26 \%$ \\
\hline Total & 18,576 & 234,454 & 159,530 & 33,970 & 14,387 & 228,208 & 25,204 & 60,619 & 33,049 & 18,633 & 147,597 \\
\hline (\% No.) & $1.63 \%$ & $20.52 \%$ & $13.96 \%$ & $2.97 \%$ & $1.26 \%$ & $19.97 \%$ & $2.21 \%$ & $5.31 \%$ & $2.89 \%$ & $1.63 \%$ & $12.92 \%$ \\
\hline $\begin{array}{l}\text { Announcement } \\
\text { Type }\end{array}$ & $\begin{array}{c}\text { Company } \\
\text { Administration }\end{array}$ & $\begin{array}{c}\text { Notice of } \\
\text { Call }\end{array}$ & Other & $\begin{array}{l}\text { Chairman's } \\
\text { Address } \\
\end{array}$ & $\begin{array}{c}\text { Letter to } \\
\text { Shareholders }\end{array}$ & ASX Query & $\begin{array}{c}\text { Structured } \\
\text { Products }\end{array}$ & $\begin{array}{c}\text { CTE Quarterly } \\
\text { Reports }\end{array}$ & mFund & $\mathbf{N}$ & Missing N \\
\hline Pre trading & 11,602 & 11 & 7,574 & 4,033 & 643 & 1,906 & 13,045 & 2,379 & 195 & 288,146 & 2 \\
\hline $10.00-11.00^{*}$ & 5,120 & 3 & 2,246 & 3,522 & 341 & 682 & 4,835 & 1,020 & 80 & 114,140 & 0 \\
\hline $11.00-12.00^{*}$ & 4,405 & 2 & 1,873 & 2,410 & 308 & 303 & 3,709 & 930 & 58 & 92,857 & 1 \\
\hline $12.00-13.00^{*}$ & 4,182 & 5 & 1,783 & 1,750 & 325 & 426 & 2,782 & 1,093 & 36 & 85,142 & 0 \\
\hline $13.00-14.00^{*}$ & 3,544 & 1 & 1,440 & 1,540 & 246 & 658 & 1,780 & 1,068 & 10 & 70,930 & 0 \\
\hline $14.00-15.00^{*}$ & 4,225 & 3 & 1,329 & 1,422 & 260 & 704 & 3,129 & 1,341 & 92 & 83,498 & 1 \\
\hline $15.00-16.00$ & 4,730 & 4 & 1,363 & 968 & 344 & 677 & 4,411 & 1,456 & 82 & 95,018 & 1 \\
\hline Post trading & 13,784 & 18 & 3,923 & 1,702 & 1,156 & 2,364 & 17,664 & 4,843 & 214 & 312,548 & 8 \\
\hline Weekends & 0 & 0 & 195 & 0 & 0 & 0 & 0 & 0 & 0 & 255 & 0 \\
\hline Trading hours & $\begin{array}{c}26,206 \\
50.79 \%\end{array}$ & $\begin{array}{c}18 \\
38.30 \%\end{array}$ & $\begin{array}{l}10,034 \\
46.18 \%\end{array}$ & $\begin{array}{c}11,612 \\
66.94 \%\end{array}$ & $\begin{array}{c}1,824 \\
50.35 \%\end{array}$ & $\begin{array}{c}3,450 \\
44.69 \%\end{array}$ & $\begin{array}{l}20,646 \\
40.20 \%\end{array}$ & $\begin{array}{c}6,908 \\
48.89 \%\end{array}$ & $\begin{array}{c}358 \\
46.68 \%\end{array}$ & $\begin{array}{l}541,585 \\
47.40 \%\end{array}$ & 3 \\
\hline $\begin{array}{l}\text { Off-trading } \\
\text { hours }\end{array}$ & $\begin{array}{l}25,386 \\
49.21 \%\end{array}$ & $\begin{array}{c}29 \\
61.70 \%\end{array}$ & $\begin{array}{c}11,692 \\
53.82 \%\end{array}$ & $\begin{array}{c}5,735 \\
33.06 \%\end{array}$ & $\begin{array}{c}1,799 \\
49.65 \%\end{array}$ & $\begin{array}{c}4,270 \\
55.31 \%\end{array}$ & $\begin{array}{l}30,709 \\
59.80 \%\end{array}$ & $\begin{array}{c}7,222 \\
55.11 \%\end{array}$ & $\begin{array}{c}409 \\
53.32 \%\end{array}$ & $\begin{array}{l}600,949 \\
52.60 \%\end{array}$ & 10 \\
\hline Total & 51,592 & 47 & 21,726 & 17,347 & 3,623 & $\begin{array}{l}7,720 \\
7.720\end{array}$ & 51,355 & 14,130 & 767 & $1,142,534$ & \\
\hline (\% No.) & $4.52 \%$ & $0.00 \%$ & $1.90 \%$ & $1.52 \%$ & $0.32 \%$ & $0.68 \%$ & $4.49 \%$ & $1.24 \%$ & $0.07 \%$ & $100 \%$ & 13 \\
\hline
\end{tabular}


Table 6-1 shows that there are 1,142,534 announcements in total in the original announcement sample. There is an uneven amount in each announcement category. Security Holder Details has the most announcements, 234,454 (20.52\%). Issued Capital has the second highest number of announcements, 228,208 (19.97\%). Periodic Reports and Progress Reports are the third and fourth biggest categories, having 159,530 (13.96\%) and 147,597 (12.92\%) announcements respectively. Other categories have about $5 \%$ or fewer of the total number of company announcements, and in particular, Notice of Call has the smallest number of announcements, $47(0.00 \%)$.

Based on the full sample, Table 6-1 shows there is not a big difference between the number of announcements released during the trading hours and the non-trading hours (including weekend announcements): 541,585 and 600,949 (47.4\% and 52.6\%) respectively. Companies release more announcements during non-trading hours for most categories. There are some exceptions with more trading hour announcements: Quarterly Activities Reports (17,185 trading hour and 16,785 non-trading hour), Asset Acquisition \& Disposal announcements (12,853 and 12,351 respectively), Notice of Meeting (33,938 and 26,681), Distribution Announcements $(10,142$ and 8,491), Progress Reports (85,228 and 62,369), Company Administration Announcements $(26,206$ and 25,386) and Chairman's Address (11,612 and 5,735). However, the proportion of trading hour announcements has declined over time from 2004 to 2014 (for details, see Appendix II) for almost all announcement categories except for Notice of Meeting. This indicates that companies tend to release fewer announcements during trading hours. One explanation could be to reduce trading interruptions. A trading hour market-sensitive announcement is accompanied by a minimum 10 minutes trading halt, and longer trading halts may apply depending on the nature of the information disclosed. 
The overall distribution for trading hour announcements is a ' $U$ ' shape. In total, 114,140 announcements were released at $10.00-11.00$; then fewer and fewer announcements are released until 13.00-14.00 when 70,930 were released. After 14.00, the number of announcements increased, reaching 95,018 from 15.00 to 16.00. However, some categories have different distribution patterns (see Figure 6-1). Most announcement categories follow the 'U' shape pattern, showing fewer announcements released around midday. This may indicate that companies have no preference to release certain information at certain times, because the pattern for each category is similar. There are only a few exceptions: Progress Reports, Other and Chairman's Address. More announcements of these categories are released in the morning, reaching the lowest point at 16.00 . This may be because companies try to release information as early as possible. Notice of Meeting is the only category with the fewest announcements in the morning, but most announcements in this category are unlikely to be material, especially those notices which inform shareholders only of a meeting's time, date, venue and purpose. 


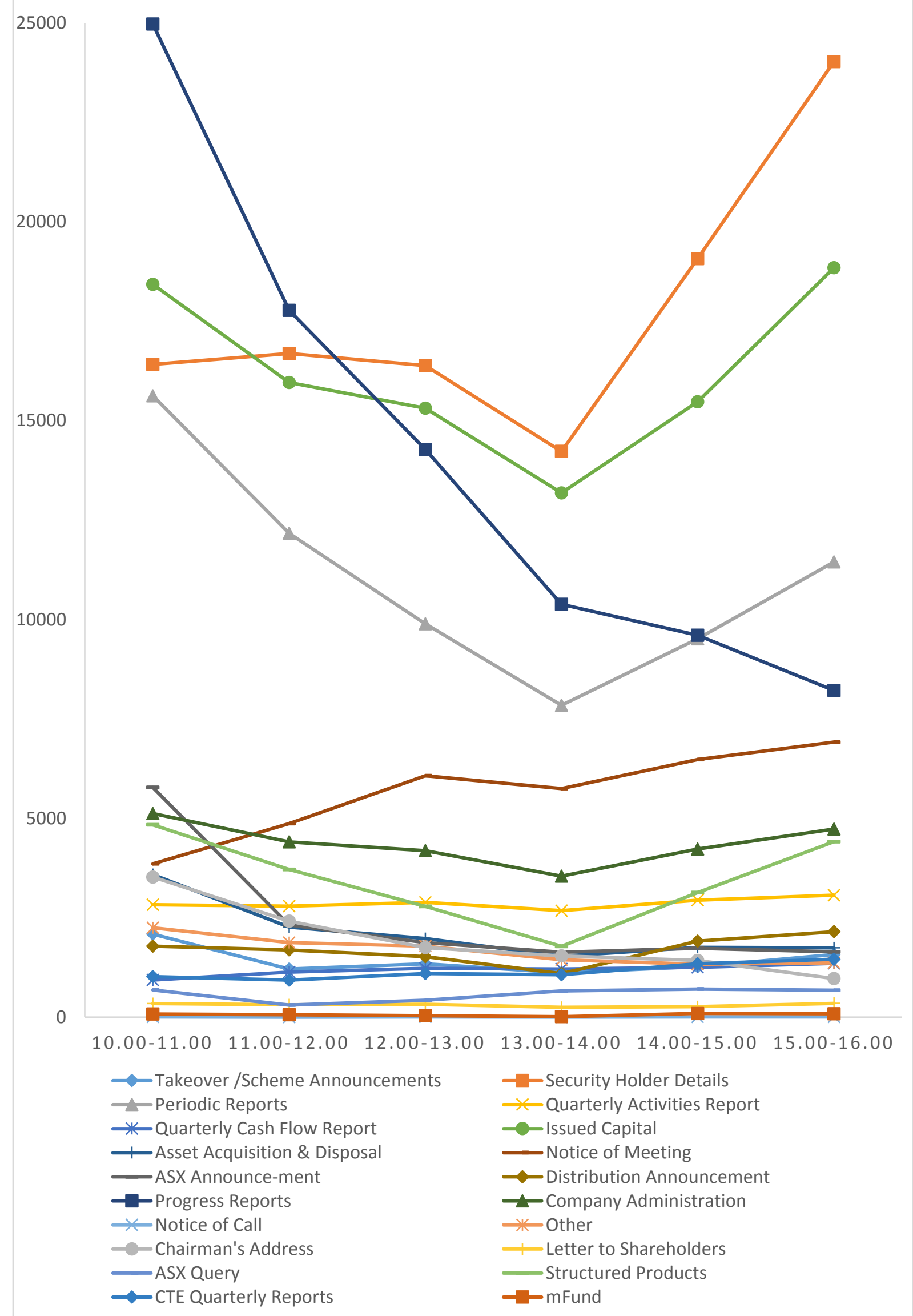

Figure 6-1. Trading Hour Announcements Distribution by Time 
Table 6-2. Market-sensitive Announcements' Distribution as to Each Category

\begin{tabular}{|c|c|c|c|c|c|c|c|c|c|c|c|}
\hline $\begin{array}{l}\text { Market-sensitive } \\
\text { Announcements }\end{array}$ & $\begin{array}{c}\text { Takeover } \\
\text { /Scheme } \\
\text { Announcements }\end{array}$ & $\begin{array}{c}\text { Security } \\
\text { Holder } \\
\text { Details } \\
\end{array}$ & $\begin{array}{l}\text { Periodic } \\
\text { Reports }\end{array}$ & $\begin{array}{c}\text { Quarterly } \\
\text { Activities } \\
\text { Report }\end{array}$ & $\begin{array}{c}\text { Quarterly } \\
\text { Cash Flow } \\
\text { Report }\end{array}$ & $\begin{array}{l}\text { Issued } \\
\text { Capital }\end{array}$ & $\begin{array}{c}\text { Asset } \\
\text { Acquisition \& } \\
\text { Disposal } \\
\end{array}$ & $\begin{array}{l}\text { Notice of } \\
\text { Meeting }\end{array}$ & $\begin{array}{c}\text { ASX } \\
\text { Announce- } \\
\text { ment }\end{array}$ & $\begin{array}{c}\text { Distribution } \\
\text { Announce- } \\
\text { ment }\end{array}$ & $\begin{array}{l}\text { Progress } \\
\text { Reports }\end{array}$ \\
\hline Pre trading & 2,131 & 687 & 14,927 & 6,383 & 2,295 & 9,596 & 6,242 & 83 & 9,755 & 1,866 & 31,757 \\
\hline $10.00-11.00^{*}$ & 1,060 & 289 & 4,255 & 2,669 & 889 & 3,442 & 2,677 & 56 & 4,303 & 633 & 16,634 \\
\hline 11.00-12.00* & 518 & 113 & 2,472 & 2,655 & 1,091 & 1,551 & 1,655 & 38 & 1,420 & 436 & 10,884 \\
\hline $12.00-13.00^{*}$ & 577 & 122 & 2,412 & 2,731 & 1,173 & 1,399 & 1,349 & 36 & 1,169 & 407 & 8,319 \\
\hline $13.00-14.00^{*}$ & 506 & 99 & 2,053 & 2,566 & 1,172 & 1,202 & 980 & 43 & 1,031 & 313 & 5,975 \\
\hline $14.00-15.00^{*}$ & 587 & 100 & 2,389 & 2,801 & 1,199 & 1,373 & 1,110 & 38 & 1,009 & 375 & 5,211 \\
\hline $15.00-16.00$ & 675 & 98 & 2,961 & 2,927 & 1,296 & 1,631 & 1,052 & 36 & 955 & 515 & 4,199 \\
\hline Post trading & 2,605 & 316 & 9,515 & 9,341 & 4,644 & 6,047 & 2,424 & 100 & 3,468 & 1,157 & 7,142 \\
\hline Weekends & 1 & 0 & 1 & 1 & 0 & 1 & 0 & 0 & 0 & 15 & 2 \\
\hline Trading hours & $\begin{array}{c}3,923 \\
45.30 \%\end{array}$ & $\begin{array}{c}821 \\
45.01 \%\end{array}$ & $\begin{array}{l}16,542 \\
40.36 \%\end{array}$ & $\begin{array}{r}16,349 \\
50.97 \%\end{array}$ & $\begin{array}{c}6,820 \\
49.57 \%\end{array}$ & $\begin{array}{l}10,598 \\
40.39 \%\end{array}$ & $\begin{array}{c}8,823 \\
50.54 \%\end{array}$ & $\begin{array}{c}247 \\
57.44 \%\end{array}$ & $\begin{array}{c}9,887 \\
42.78 \%\end{array}$ & $\begin{array}{c}2,679 \\
46.86 \%\end{array}$ & $\begin{array}{l}51,222 \\
56.84 \%\end{array}$ \\
\hline Off-trading hours & $\begin{array}{c}4,737 \\
5470 \%\end{array}$ & $\begin{array}{l}1,003 \\
54,99 \%\end{array}$ & 24,443 & 15,725 & 6,939 & 15,644 & 8,666 & 183 & 13,223 & 3,038 & 38,901 \\
\hline $\begin{array}{l}\text { Market-sensitive total } \\
\text { \% Each Category }\end{array}$ & $\begin{array}{c}54.70 \% \\
8,660 \\
46.62 \%\end{array}$ & $\begin{array}{c}54.99 \% \\
1,824 \\
0.78 \%\end{array}$ & $\begin{array}{l}59.64 \% \\
40,985\end{array}$ & $\begin{array}{c}49.03 \% \\
32,074 \\
94.92 \%\end{array}$ & $\begin{array}{c}50.43 \% \\
13,759 \\
95.63 \%\end{array}$ & $\begin{array}{l}59.61 \% \\
26,242 \\
1150 \%\end{array}$ & $\begin{array}{l}49.55 \% \\
17,489\end{array}$ & $\begin{array}{c}42.56 \% \\
430\end{array}$ & $\begin{array}{l}57.22 \% \\
23,110\end{array}$ & $\begin{array}{c}53.14 \% \\
5,717\end{array}$ & $\begin{array}{l}43.16 \% \\
90,123 \\
6106 \%\end{array}$ \\
\hline \% Total MS & $3.03 \%$ & $0.64 \%$ & $14.32 \%$ & $11.21 \%$ & $4.81 \%$ & $9.17 \%$ & $6.11 \%$ & $0.15 \%$ & $\begin{array}{l}09.95 \% \\
8.07 \%\end{array}$ & $\begin{array}{l}50.00 \% \\
2.00 \%\end{array}$ & $31.49 \%$ \\
\hline $\begin{array}{l}\text { Market-sensitive } \\
\text { Announcements }\end{array}$ & $\begin{array}{c}\text { Company } \\
\text { Administration }\end{array}$ & $\begin{array}{l}\text { Notice of } \\
\text { Call }\end{array}$ & Other & $\begin{array}{l}\text { Chairman's } \\
\text { Address }\end{array}$ & $\begin{array}{c}\text { Letter to } \\
\text { Shareholders }\end{array}$ & $\begin{array}{l}\text { ASX } \\
\text { Query }\end{array}$ & $\begin{array}{c}\text { Structured } \\
\text { Products }\end{array}$ & $\begin{array}{l}\text { CTE Quarterly } \\
\text { Reports }\end{array}$ & mFund & $\mathbf{N}$ & Missing $\mathbf{N}$ \\
\hline Pre trading & 1,209 & 4 & 789 & 317 & 7 & 1,537 & 1 & 2,058 & 0 & 91,644 & 1 \\
\hline $10.00-11.00^{*}$ & 381 & 0 & 515 & 355 & 7 & 493 & 1 & 929 & 0 & 39,588 & 0 \\
\hline $11.00-12.00^{*}$ & 192 & 0 & 341 & 179 & 4 & 184 & 0 & 831 & 0 & 24,564 & 0 \\
\hline $12.00-13.00^{*}$ & 240 & 0 & 291 & 91 & 7 & 296 & 0 & 977 & 0 & 21,596 & 0 \\
\hline $13.00-14.00^{*}$ & 176 & 0 & 200 & 101 & 1 & 507 & 0 & 948 & 0 & 17,873 & 0 \\
\hline $14.00-15.00^{*}$ & 171 & 1 & 262 & 116 & 3 & 547 & 0 & 1,158 & 0 & 18,450 & 0 \\
\hline $15.00-16.00$ & 184 & 0 & 212 & 55 & 4 & 494 & 1 & 1,296 & 0 & 18,591 & 0 \\
\hline Post trading & 631 & 4 & 415 & 73 & 11 & 1,685 & 0 & 4,283 & 0 & 53,861 & 0 \\
\hline Weekends & 0 & 0 & 39 & 0 & 0 & 0 & 0 & 0 & 0 & 60 & 0 \\
\hline Trading hours & $\begin{array}{c}1,344 \\
42.21 \%\end{array}$ & $\begin{array}{c}1 \\
11.11 \%\end{array}$ & $\begin{array}{c}1,821 \\
59.43 \%\end{array}$ & $\begin{array}{c}897 \\
69.70 \%\end{array}$ & $\begin{array}{c}26 \\
59.09 \%\end{array}$ & $\begin{array}{c}2,521 \\
43.90 \%\end{array}$ & $\begin{array}{c}2 \\
66.67 \%\end{array}$ & $\begin{array}{c}6,139 \\
49.19 \%\end{array}$ & 0 & $\begin{array}{l}140,662 \\
49.14 \%\end{array}$ & 0 \\
\hline Off-trading hours & $\begin{array}{c}1,840 \\
57.79 \%\end{array}$ & $\begin{array}{c}8 \\
88.89 \%\end{array}$ & $\begin{array}{c}1,243 \\
40.57 \%\end{array}$ & $\begin{array}{c}390 \\
30.30 \%\end{array}$ & $\begin{array}{c}18 \\
40.91 \%\end{array}$ & $\begin{array}{c}3,222 \\
56.10 \%\end{array}$ & $\begin{array}{c}1 \\
33.33 \%\end{array}$ & $\begin{array}{c}6,341 \\
50.81 \%\end{array}$ & 0 & $\begin{array}{l}145,565 \\
50.86 \%\end{array}$ & 1 \\
\hline $\begin{array}{l}\text { Market-sensitive total } \\
\text { (\% Each Category) }\end{array}$ & $\begin{array}{l}3,184 \\
6.17 \%\end{array}$ & $\begin{array}{c}9 \\
9 \\
19.15 \%\end{array}$ & $\begin{array}{c}3,064 \\
14.10 \%\end{array}$ & $\begin{array}{c}1,287 \\
7.42 \%\end{array}$ & $\begin{array}{c}44 \\
1.21 \%\end{array}$ & $\begin{array}{c}5,743 \\
74.39 \%\end{array}$ & $\begin{array}{c}3 \\
0.01 \%\end{array}$ & $\begin{array}{r}12,480 \\
88.32 \%\end{array}$ & 0 & $\begin{array}{l}286,227 \\
25.05 \%\end{array}$ & 1 \\
\hline$\%$ Total MS & $1.11 \%$ & $0.00 \%$ & $1.07 \%$ & $0.45 \%$ & $0.02 \%$ & $2.01 \%$ & $0.00 \%$ & $4.36 \%$ & $0.00 \%$ & $100.00 \%$ & \\
\hline
\end{tabular}

Note: * excluding the minute in the time window. Pre trading: 00.00-9.59; Post trading: 16.01-23.59. The announcement release time is rounded up to one minute. 
As shown in Table 6-2, there are 286,227 market-sensitive announcements from 1 July 2004 to 31 December 2014, one quarter (25.05\%) of overall announcements. Letter to Shareholders, and Structured Products have minimal market-sensitive announcements: only $44(1.21 \%)$ and $3(0.01 \%)$ respectively. These two categories are less likely to be market-sensitive. Letter to Shareholders is an overview of the company's operation status, which contains information already available to the market, so it is a summary of the important information that has already been released. From Table 4-1 in Chapter Four, Structured Products are classified by ASX as 'never' market-sensitive, which may be because Structured Products are not directly related to the company's shares. As for Quarterly Activities Report, Quarterly Cash Flow Report, and Commitments Test Entity Quarterly Reports, over $88 \%$ of the announcements in those categories are market-sensitive $(94.42 \%, 95.63 \%$ and $88.32 \%)$. Quarterly reports are not required for all listed companies but only for some special companies which are either in a special industry such as mining, oil and gas, and investment companies, or under the 'commitment test' which requires a newly-listed company to spend its cash assets according to an agreement with ASX.

ASX Announcements and ASX Queries have more than 60\% market-sensitive announcements (69.93\% and $74.39 \%$ respectively) because they can indicate that ASX may have concerns about the company. ASX Announcements are more likely to be trading halt-related, while ASX Queries require the company to clarify some information and to ensure it is compliant with the CDR. Other categories that contain around half or more market-sensitive announcements are Asset Acquisition \& Disposal, Progress Reports and Takeover/Scheme Announcements $(69.39 \%, 61.06 \%$, and $46.62 \%$ respectively). These announcements tend to be important because they are related to the company's investment decisions or the progress of projects. Periodic Reports and Distribution Announcements are directly earnings-related announcements, 
and over a quarter of these two categories are market-sensitive $(25.69 \%$ and $30.68 \%$ respectively). It seems that a relatively small proportion of earnings-related announcements are market-sensitive, but Periodic Report is the second biggest category when looking only at the market-sensitive announcements; 40,985 (14.32\% of the total market-sensitive announcements). From the perspective of total market-sensitive announcements, Progress Reports have the highest number of market-sensitive announcements: 90,123 (31.49\%). Quarterly Activities Report, Issued Capital and ASX Announcements cover around 10\% of the total number of market-sensitive announcements: $11.21 \%, 9.17 \%$ and $8.07 \%$ respectively. Other categories have about $6 \%$ or fewer announcements of the total number of market-sensitive announcements.

Table 6-2 shows that there is a small difference between the number of trading hour announcements and non-trading hour announcements in the market-sensitive announcement sub-sample: 140,662 and 145,565 respectively (49.19\% and 50.81\%). But this difference increased for all categories from 2004 to 2014. For many announcement categories, in 2014 more than $60 \%$ of market-sensitive announcements were released during off hours (details are in Appendix II). In terms of the trading hour market-sensitive announcements in Table 6-2, although the distribution is still a ' $U$ ' shape, there are more market-sensitive announcements released during the morning trading hours (i.e. 10.00-13.00) except for the three types of quarterly reports: Quarterly Activities Reports, Quarterly Cash Flow Reports and Commitments Test Entity Quarterly Reports (see Figure 6-2). 


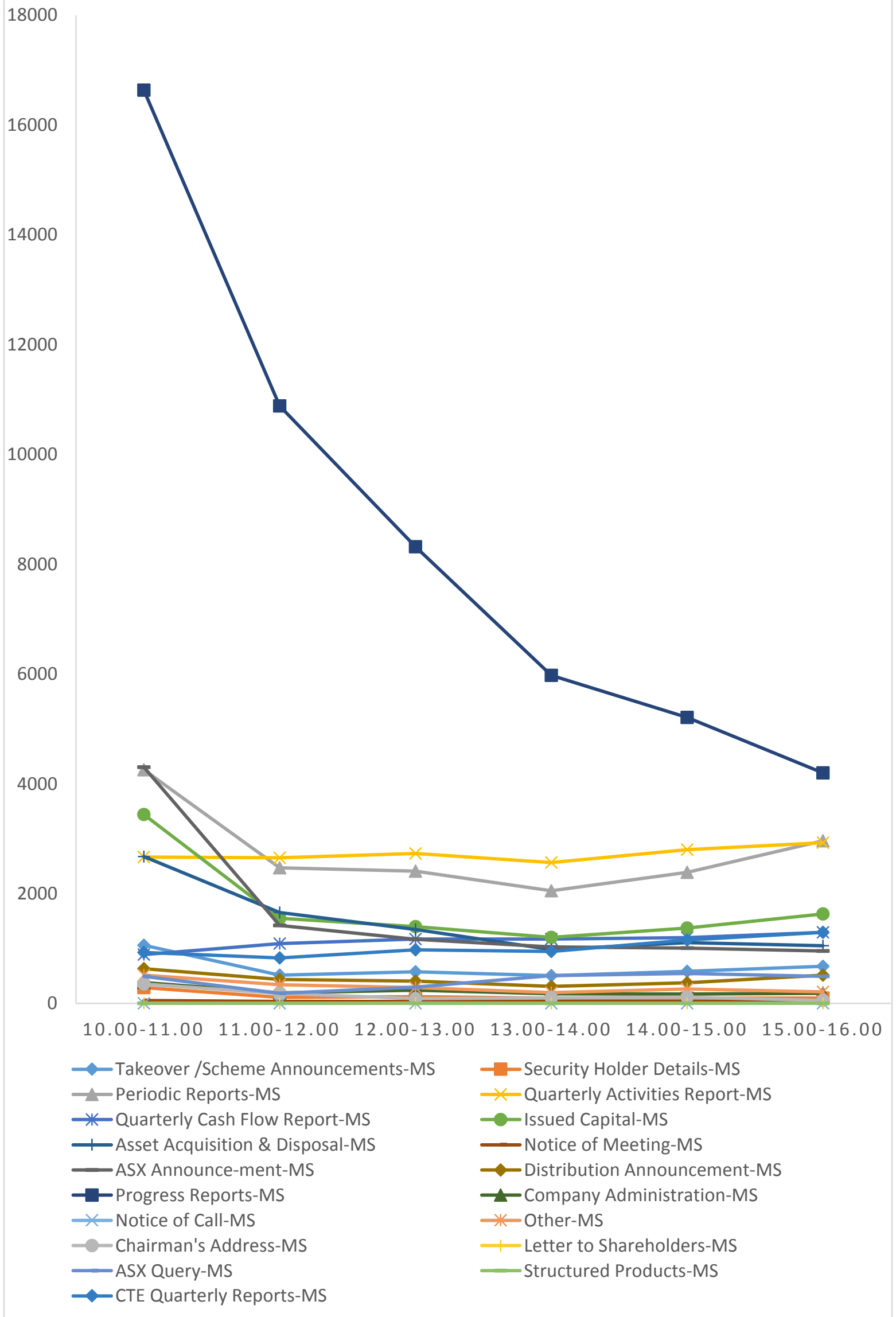

Figure 6-2. Trading Hour Market-sensitive Announcements Distribution by Time 
Table 6-3 shows that there were 856,307 non-market-sensitive announcements, about three quarters $(74.95 \%)$ of overall announcements. Security Holder Details has the most non-marketsensitive announcements: 232,630 (27.17\% of the total non-market-sensitive announcements). Issued Capital is the second biggest category: 201,966 (23.59\%). Periodic Reports is the third biggest category: 118,545 (13.84\%). Notice of Meeting is the fourth biggest category: 60,189 $(7.03 \%)$. Other categories have about $6 \%$ or fewer announcements of the total number of nonmarket-sensitive announcements. Security Holder Details and Notice of Meeting are two announcement categories related to companies' administrative information which are less likely to be market-sensitive. Periodic Reports and Issued Capital have a relatively big amount of both market-sensitive and non-market-sensitive announcements. Periodic Reports refer to financial reports such as half-yearly reports, preliminary final reports and annual reports, and Issued Capital is related to the company's financing activities. The non-market-sensitive announcements in these two categories could be summary or explanatory (not essential) information relating to financial reports or equity issues.

There were more non-market-sensitive announcements released during off hours: 400,923 and $455,384(46.82 \%$ and $53.18 \%)$ respectively for all announcement categories. With regard to trading hour non-market-sensitive announcements, the distribution is more symmetric and has a slightly flatter ' $U$ ' shape than market-sensitive announcements. Because many announcements were released in the afternoon, the left side of the ' $U$ ' shape is longer (see Figure 6-3). This pattern may indicate that companies release market-sensitive information as early as possible, which is reflected by the fact that more market-sensitive announcements are released in the first trading hour while more non-market-sensitive announcements are released in the afternoon. 
Table 6-3. Non-market-sensitive Announcements' Distribution as to Each Category

\begin{tabular}{|c|c|c|c|c|c|c|c|c|c|c|c|}
\hline $\begin{array}{l}\text { Non-market-sensitive } \\
\text { Announcements }\end{array}$ & $\begin{array}{c}\text { Takeover } \\
\text { /Scheme } \\
\text { Announcements }\end{array}$ & $\begin{array}{l}\text { Security } \\
\text { Holder } \\
\text { Details }\end{array}$ & $\begin{array}{l}\text { Periodic } \\
\text { Reports }\end{array}$ & $\begin{array}{c}\text { Quarterly } \\
\text { Activities } \\
\text { Report }\end{array}$ & $\begin{array}{c}\text { Quarterly } \\
\text { Cash Flow } \\
\text { Report }\end{array}$ & $\begin{array}{l}\text { Issued } \\
\text { Capital }\end{array}$ & $\begin{array}{c}\text { Asset } \\
\text { Acquisition \& } \\
\text { Disposal }\end{array}$ & $\begin{array}{l}\text { Notice of } \\
\text { Meeting }\end{array}$ & $\begin{array}{c}\text { ASX } \\
\text { Announce- } \\
\text { ment }\end{array}$ & $\begin{array}{c}\text { Distribution } \\
\text { Announce- } \\
\text { ment }\end{array}$ & $\begin{array}{l}\text { Progress } \\
\text { Reports }\end{array}$ \\
\hline Pre trading & 2,118 & 45,329 & 31,433 & 454 & 90 & 52,858 & 1,973 & 7,837 & 1,194 & 2,526 & 15,224 \\
\hline $10.00-11.00^{*}$ & 1,026 & 16,130 & 11,373 & 157 & 43 & 14,984 & 906 & 3,798 & 1,472 & 1,153 & 8,342 \\
\hline $11.00-12.00^{*}$ & 690 & 16,580 & 9,695 & 135 & 40 & 14,411 & 608 & 4,830 & 898 & 1,250 & 6,889 \\
\hline $12.00-13.00^{*}$ & 761 & 16,265 & 7,477 & 154 & 51 & 13,917 & 626 & 6,034 & 706 & 1,114 & 5,961 \\
\hline $13.00-14.00^{*}$ & 609 & 14,129 & 5,790 & 111 & 35 & 11,983 & 552 & 5,706 & 600 & 781 & 4,407 \\
\hline $14.00-15.00^{*}$ & 680 & 18,973 & 7,123 & 139 & 51 & 14,104 & 646 & 6,442 & 720 & 1,533 & 4,390 \\
\hline $15.00-16.00$ & 902 & 23,934 & 8,485 & 140 & 55 & 17,213 & 692 & 6,881 & 687 & 1,632 & 4,017 \\
\hline Post trading & 3,130 & 81,287 & 37,168 & 606 & 263 & 62,494 & 1,712 & 18,661 & 3,662 & 2,894 & 8,244 \\
\hline Weekends & 0 & 3 & 1 & 0 & 0 & 2 & 0 & 0 & 0 & 33 & 0 \\
\hline Trading hours & 4,668 & 106,011 & 49,943 & 836 & 275 & 86,612 & 4,030 & 33,691 & 5,083 & 7,463 & 34,006 \\
\hline Trading hours & $47.08 \%$ & $45.57 \%$ & $42.13 \%$ & $44.09 \%$ & $43.79 \%$ & $42.88 \%$ & $52.24 \%$ & $(55.98 \%)$ & $51.14 \%$ & $57.78 \%$ & $59.17 \%$ \\
\hline Off-trading hours & 5,248 & 126,619 & 68,602 & 1,060 & 353 & 115,354 & 3,685 & 26,498 & 4856 & 5,453 & 23,468 \\
\hline Off-trading hours & $52.92 \%$ & $54.43 \%$ & $57.87 \%$ & $55.91 \%$ & $56.21 \%$ & $57.12 \%$ & $47.76 \%$ & $(44.02 \%)$ & $48.86 \%$ & $42.22 \%$ & $40.83 \%$ \\
\hline Non-market-sensitive total & 9,916 & 232,630 & 118,545 & 1,896 & 628 & 201,966 & 7,715 & 60,189 & 9,939 & 12,916 & 57,474 \\
\hline$\%$ Each Category & $53.38 \%$ & $99.22 \%$ & $74.31 \%$ & $5.58 \%$ & $4.37 \%$ & $88.50 \%$ & $30.61 \%$ & $(99.29 \%)$ & $30.07 \%$ & $69.32 \%$ & $38.94 \%$ \\
\hline \% Total NMS & $1.16 \%$ & $27.17 \%$ & $13.84 \%$ & $0.22 \%$ & $0.07 \%$ & $23.59 \%$ & $0.90 \%$ & $7.03 \%$ & $1.16 \%$ & $1.51 \%$ & $6.71 \%$ \\
\hline $\begin{array}{l}\text { Non-market-sensitive } \\
\text { Announcements }\end{array}$ & $\begin{array}{c}\text { Company } \\
\text { Administration }\end{array}$ & $\begin{array}{l}\text { Notice of } \\
\text { Call }\end{array}$ & Other & $\begin{array}{l}\text { Chairman's } \\
\text { Address }\end{array}$ & $\begin{array}{c}\text { Letter to } \\
\text { Shareholders }\end{array}$ & ASX Query & $\begin{array}{l}\text { Structured } \\
\text { Products }\end{array}$ & $\begin{array}{l}\text { CTE Quarterly } \\
\text { Reports }\end{array}$ & mFund & $\mathbf{N}$ & Missing $\mathbf{N}$ \\
\hline Pre trading & 10,393 & 7 & 6,785 & 3,716 & 636 & 369 & 13,044 & 321 & 195 & 196,502 & 1 \\
\hline $10.00-11.00^{*}$ & 4,739 & 3 & 1,731 & 3,167 & 334 & 189 & 4,834 & 91 & 80 & 74,552 & 0 \\
\hline $11.00-12.00^{*}$ & 4,213 & 2 & 1,532 & 2,231 & 304 & 119 & 3,709 & 99 & 58 & 68,293 & 1 \\
\hline $12.00-13.00^{*}$ & 3,942 & 5 & 1,492 & 1,659 & 318 & 130 & 2,782 & 116 & 36 & 63,546 & 0 \\
\hline $13.00-14.00^{*}$ & 3,368 & 1 & 1,240 & 1,439 & 245 & 151 & 1,780 & 120 & 10 & 53,057 & 0 \\
\hline $14.00-15.00^{*}$ & 4,054 & 2 & 1,067 & 1,306 & 257 & 157 & 3,129 & 183 & 92 & 65,048 & 1 \\
\hline $15.00-16.00$ & 4,546 & 4 & 1,151 & 913 & 340 & 183 & 4,410 & 160 & 82 & 76,427 & 1 \\
\hline Post trading & 13,153 & 14 & 3,508 & 1,629 & 1,145 & 679 & 17,664 & 560 & 214 & 258,687 & 8 \\
\hline Weekends & 0 & 0 & 156 & 0 & 0 & 0 & 0 & 0 & 0 & 195 & 0 \\
\hline Trading hours & $\begin{array}{c}24,862 \\
51.36 \%\end{array}$ & $\begin{array}{c}17 \\
44.74 \%\end{array}$ & $\begin{array}{c}8,213 \\
44.01 \%\end{array}$ & $\begin{array}{l}10,715 \\
66.72 \%\end{array}$ & $\begin{array}{c}1,798 \\
50.24 \%\end{array}$ & $\begin{array}{c}929 \\
46.99 \%\end{array}$ & $\begin{array}{c}20,644 \\
40.20 \%\end{array}$ & $\begin{array}{c}769 \\
46.61 \%\end{array}$ & $\begin{array}{c}358 \\
46.68 \%\end{array}$ & $\begin{array}{l}400,923 \\
46.82 \%\end{array}$ & 3 \\
\hline Off-trading hours & $\begin{array}{l}23,546 \\
48.64 \%\end{array}$ & $\begin{array}{c}21 \\
55.26 \%\end{array}$ & $\begin{array}{r}10,449 \\
55.99 \%\end{array}$ & $\begin{array}{c}5,345 \\
33.28 \%\end{array}$ & $\begin{array}{c}1,781 \\
49.76 \%\end{array}$ & $\begin{array}{l}1,048 \\
53.01 \%\end{array}$ & $\begin{array}{l}30,708 \\
59.80 \%\end{array}$ & $\begin{array}{c}881 \\
53.39 \%\end{array}$ & $\begin{array}{c}409 \\
53.32 \%\end{array}$ & $\begin{array}{l}455,384 \\
53.18 \%\end{array}$ & 9 \\
\hline $\begin{array}{l}\text { Non-market-sensitive total } \\
\% \text { Each Category }\end{array}$ & $\begin{array}{l}48,408 \\
93.83 \%\end{array}$ & $\begin{array}{c}38 \\
80.85 \%\end{array}$ & $\begin{array}{l}18,662 \\
85.90 \%\end{array}$ & $\begin{array}{l}16,060 \\
92.58 \%\end{array}$ & $\begin{array}{c}3,579 \\
98.79 \%\end{array}$ & $\begin{array}{c}1,977 \\
25.61 \%\end{array}$ & $\begin{array}{l}51,352 \\
99.99 \%\end{array}$ & $\begin{array}{c}1,650 \\
11.68 \%\end{array}$ & $\begin{array}{c}767 \\
100 \%\end{array}$ & $\begin{array}{l}856,307 \\
74.95 \%\end{array}$ & 12 \\
\hline$\%$ Total NMS & $5.65 \%$ & $0.00 \%$ & $2.18 \%$ & $1.88 \%$ & $0.42 \%$ & $0.23 \%$ & $6.00 \%$ & $0.19 \%$ & $0.09 \%$ & $100.00 \%$ & \\
\hline
\end{tabular}

Note: * excluding the minute in the time window. Pre trading: 00.00-9.59; Post trading: 16.01-23.59. The announcement release time is rounded up to one minute. 


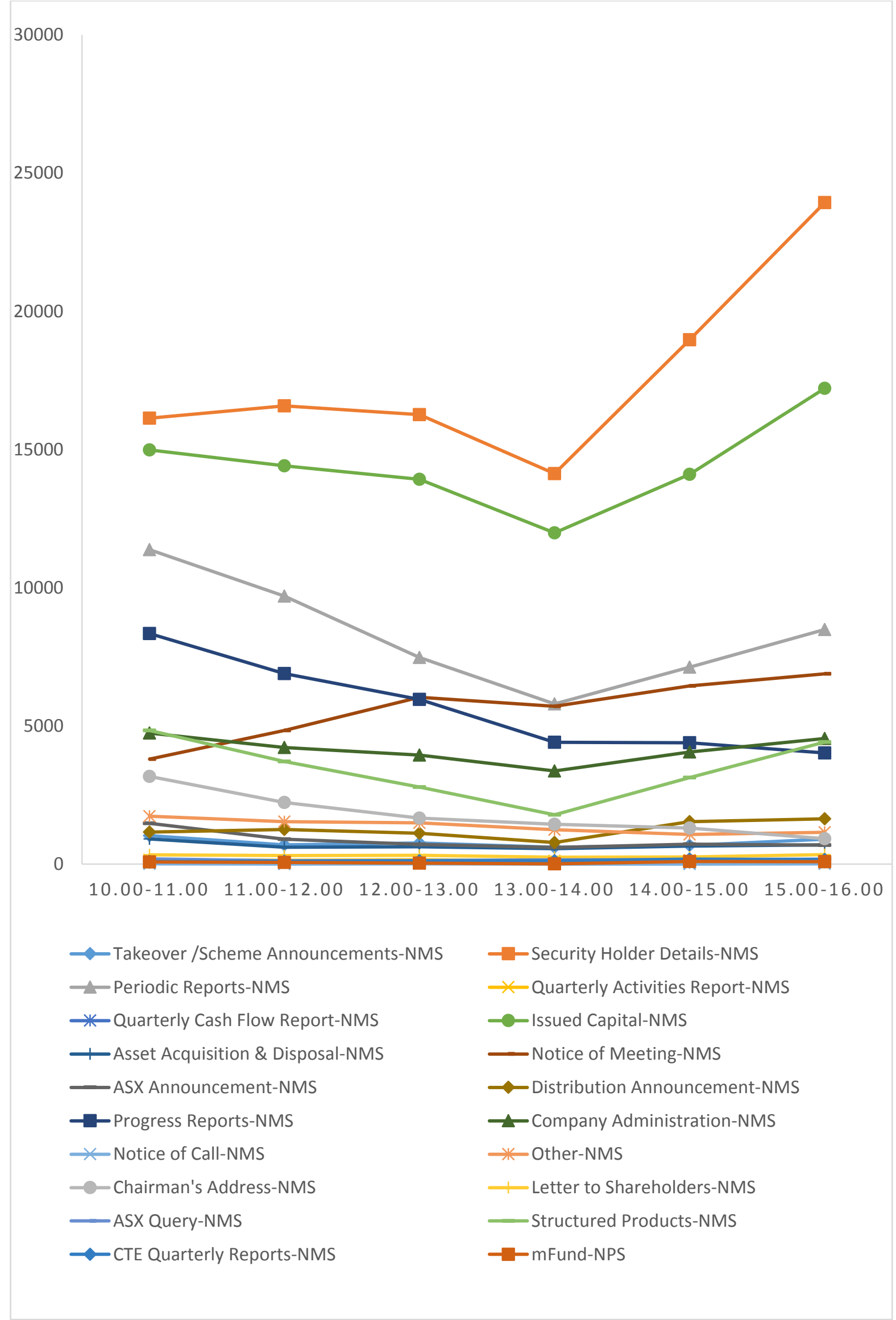

Figure 6-3. Trading Hour Non-market-sensitive Announcements Distribution by Time 
In order to further understand how the corporate disclosure behaviour changes with time, Table 6-4 presents the announcement summary statistics ${ }^{36}$ from 2004 to 2014 year by year (the year 2004 statistics are half a year: 01/07/2004 to 31/12/2004). The announcement distribution follows broader economic cycles. The distribution pattern is a reverse U shape (see Figure 64), with more announcements released around the GFC period. Figure $6-5^{37}$ reveals how release time patterns change over ten years, in terms of pre-trading, trading hour and post-trading announcements. The proportion of trading hour announcements reduced over time, while the percentage of pre-trading announcements increased, especially for market-sensitive announcements after GFC. Before 2008 there was a significant difference (6-7\%) between pretrading and trading hour announcements. The difference has become less over time. In 2014, about the same amount of market-sensitive announcements were released during pre-trading hours as those released during trading hours, which provides further evidence that companies tend to release market-sensitive information as early as possible.

\footnotetext{
${ }^{36} \mathrm{mFund}$ is excluded from the statistical summary because mFund is not a listed entity. Appendix II shows the announcement distribution over time as to each category, which is coded according to the Primary Report Code set by ASX.

37 In Figure 6-5, weekend announcements are included in the Post-trading announcements.
} 


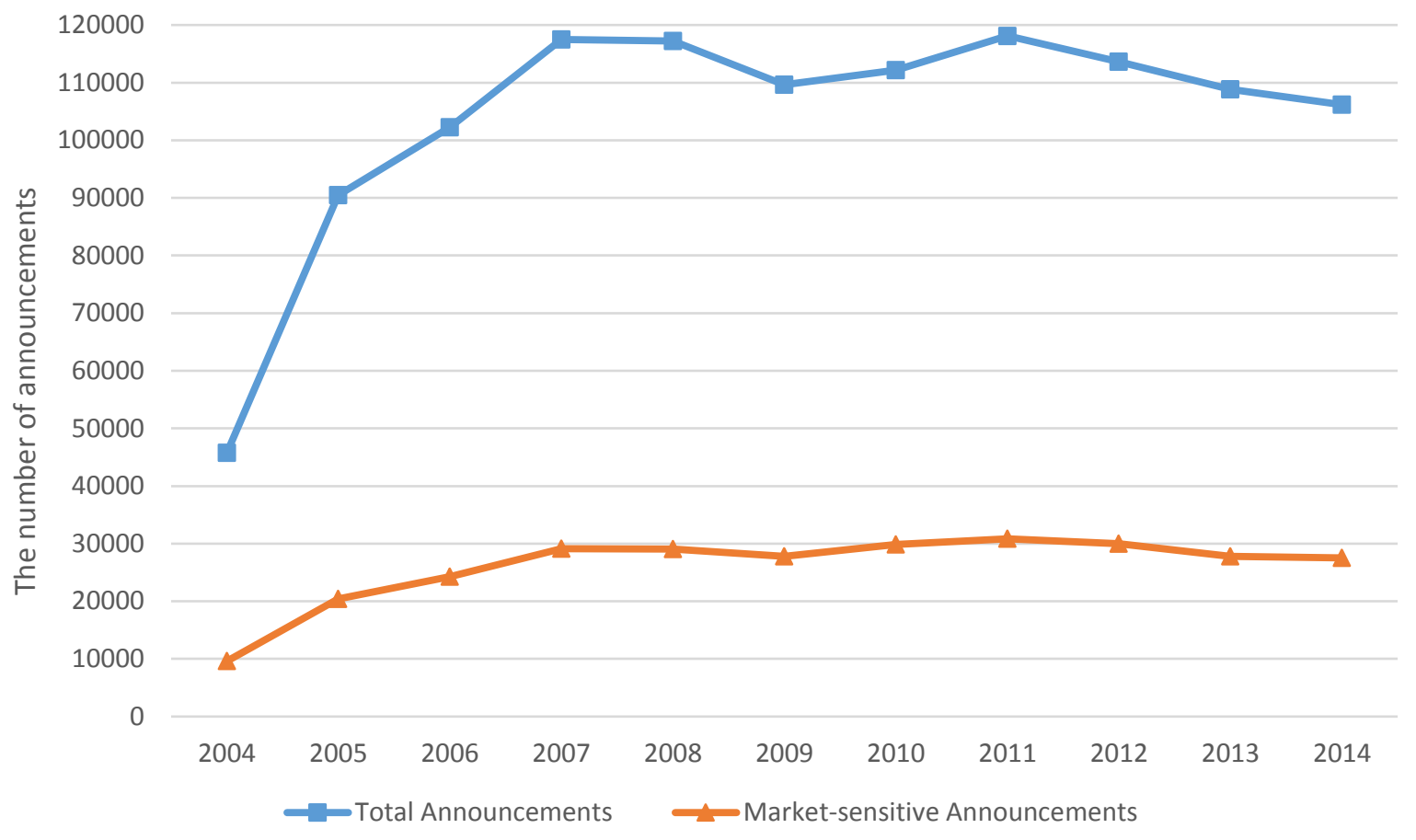

Figure 6-4. Yearly Number of Announcements

$60.00 \%$

$50.00 \%$

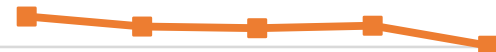

$40.00 \%$

$30.00 \%$

$\stackrel{\frac{1}{2}}{\stackrel{5}{2}}$

它

$20.00 \%$

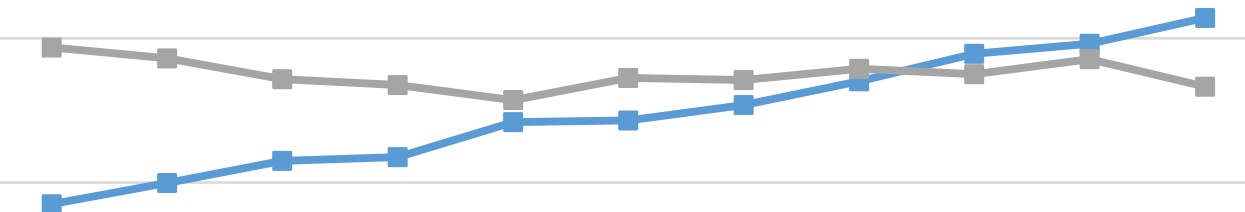

$10.00 \%$

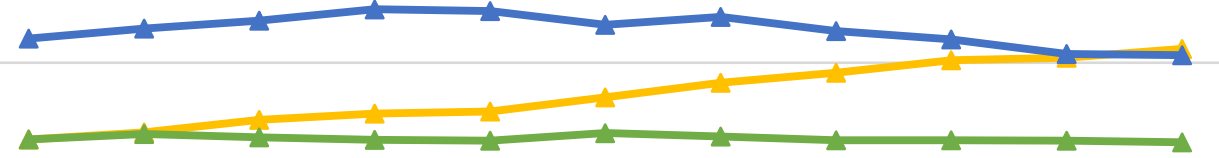

$0.00 \%$ $20042005 \quad 2006$

$200720082009 \quad 2010$

$20112012 \quad 2013 \quad 2014$

- Pre trading

- Pre trading-MS
- Trading hours

—Trading hours-MS
- Post trading

$\longrightarrow$ Post trading-MS

Figure 6-5. Yearly Proportion of Announcements Released Pre, During and Post Trading Hour 
Table 6-4. Announcement Distribution Pattern from 2004 to 2014

\begin{tabular}{|c|c|c|c|c|c|c|c|c|c|c|c|c|}
\hline Announcements & $\mathbf{2 0 0 4}^{\#}$ & 2005 & 2006 & 2007 & 2008 & 2009 & 2010 & 2011 & 2012 & 2013 & 2014 & Total \\
\hline Pre trading & 8,467 & 18,057 & 21,991 & 25,546 & 28,364 & 26,660 & 28,470 & 31,923 & 32,876 & 32,233 & 33,364 & 287,951 \\
\hline $10.00-11.00^{*}$ & 5,007 & 9,812 & 10,897 & 12,890 & 12,796 & 11,025 & 10,954 & 11,160 & 10,568 & 9,474 & 9,477 & 114,060 \\
\hline $11.00-12.00^{*}$ & 4,170 & 7,947 & 9,326 & 10,945 & 10,421 & 9,240 & 8,967 & 8,883 & 8,199 & 7,580 & 7,121 & 92,799 \\
\hline $12.00-13.00^{*}$ & 3,783 & 7,364 & 8,475 & 9,453 & 9,324 & 8,155 & 8,455 & 8,394 & 7,722 & 7,089 & 6,892 & 85,106 \\
\hline $13.00-14.00^{*}$ & 2,941 & 5,762 & 6,668 & 7,968 & 7,503 & 7,041 & 7,317 & 7,053 & 6,628 & 6,218 & 5,821 & 70,920 \\
\hline $14.00-15.00^{*}$ & 3,695 & 7,259 & 7,994 & 8,943 & 8,714 & 8,227 & 8,372 & 8,279 & 7,723 & 7,087 & 7,113 & 83,406 \\
\hline $15.00-16.00$ & 4,259 & 8,397 & 9,110 & 10,299 & 9,971 & 9,406 & 9,232 & 9,469 & 8,641 & 8,070 & 8,082 & 94,936 \\
\hline Post trading & 13,442 & 25,872 & 27,761 & 31,369 & 30,102 & 29,844 & 30,406 & 32,944 & 31,210 & 31,086 & 28,298 & 312,334 \\
\hline Weekends & 0 & 22 & 13 & 68 & 36 & 53 & 7 & 13 & 41 & 1 & 1 & 255 \\
\hline Trading hours & 23,855 & 46,541 & 52,470 & 60,498 & 58,729 & 53,094 & 53,297 & 53,238 & 49,481 & 45,518 & 44,506 & 541,227 \\
\hline \% Each Year & $52.13 \%$ & $51.43 \%$ & $51.32 \%$ & $51.50 \%$ & $50.10 \%$ & $48.42 \%$ & $47.51 \%$ & $45.07 \%$ & $43.55 \%$ & $41.82 \%$ & $41.92 \%$ & $47.40 \%$ \\
\hline Off-trading hours & 21,909 & 43,951 & 49,765 & 56,983 & 58,502 & 56,557 & 58,883 & 64,880 & 64,127 & 63,320 & 61,663 & 600,540 \\
\hline \% Each Year & $47.87 \%$ & $48.57 \%$ & $48.68 \%$ & $48.50 \%$ & $49.90 \%$ & $51.58 \%$ & $52.49 \%$ & $54.93 \%$ & $56.45 \%$ & $58.18 \%$ & $58.08 \%$ & $52.60 \%$ \\
\hline Total & 45,764 & 90,492 & 102,235 & 117,481 & 117,231 & 109,651 & 112,180 & 118,118 & 113,608 & 108,838 & 106,169 & $1,141,767$ \\
\hline$\%$ Total Number & $4.01 \%$ & $7.93 \%$ & $8.95 \%$ & $10.29 \%$ & $10.27 \%$ & $9.60 \%$ & $9.83 \%$ & $10.35 \%$ & $9.95 \%$ & $9.53 \%$ & $9.30 \%$ & $100.00 \%$ \\
\hline $\begin{array}{l}\text { Market-sensitive } \\
\text { Announcements }\end{array}$ & $\mathbf{2 0 0 4}^{\#}$ & 2005 & 2006 & 2007 & 2008 & 2009 & 2010 & 2011 & 2012 & 2013 & 2014 & Total \\
\hline Pre trading & 2,134 & 4,670 & 6,155 & 7,579 & 7,760 & 8,318 & 9,644 & 10,976 & 11,536 & 11,247 & 11,625 & 91,644 \\
\hline $10.00-11.00^{*}$ & 1,354 & 2,798 & 3,475 & 4,693 & 4,622 & 3,844 & 4,208 & 4,136 & 3,919 & 3,326 & 3,213 & 39,588 \\
\hline $11.00-12.00^{*}$ & 1,001 & 1,986 & 2,383 & 3,043 & 2,886 & 2,352 & 2,565 & 2,423 & 2,222 & 1,867 & 1,836 & 24,564 \\
\hline $12.00-13.00^{*}$ & 825 & 1,795 & 2,108 & 2,343 & 2,474 & 2,075 & 2,270 & 2,284 & 2,044 & 1,703 & 1,675 & 21,596 \\
\hline $13.00-14.00^{*}$ & 634 & 1,416 & 1,668 & 1,942 & 1,949 & 1,800 & 1,966 & 1,842 & 1,648 & 1,560 & 1,448 & 17,873 \\
\hline $14.00-15.00^{*}$ & 718 & 1,588 & 1,817 & 2,054 & 1,993 & 1,889 & 1,901 & 1,822 & 1,662 & 1,527 & 1,479 & 18,450 \\
\hline $15.00-16.00$ & 807 & 1,603 & 1,757 & 2,023 & 1,989 & 1,884 & 1,864 & 1,896 & 1,694 & 1,563 & 1,511 & 18,591 \\
\hline Post trading & 2,131 & 4,569 & 4,913 & 5,424 & 5,363 & 5,595 & 5,454 & 5,458 & 5,233 & 4,976 & 4,745 & 53,861 \\
\hline Weekends & 0 & 1 & 0 & 19 & 4 & 12 & 0 & 2 & 20 & 1 & 1 & 60 \\
\hline Trading hours & 5,339 & 11,186 & 13,208 & 16,098 & 15,913 & 13,844 & 14,774 & 14,403 & 13,189 & 11,546 & 11,162 & 140,662 \\
\hline \% Each Year MS & $55.59 \%$ & $54.76 \%$ & $54.41 \%$ & $55.28 \%$ & $54.80 \%$ & $49.85 \%$ & $49.46 \%$ & $46.70 \%$ & $44.00 \%$ & $41.58 \%$ & $40.54 \%$ & $49.14 \%$ \\
\hline Off-trading hours & 4,265 & 9,240 & 11,068 & 13,022 & 13,127 & 13,925 & 15,098 & 16,436 & 16,789 & 16,224 & 16,371 & 145,565 \\
\hline \% Each Year MS & $44.41 \%$ & $45.24 \%$ & $45.59 \%$ & $44.72 \%$ & $45.20 \%$ & $50.15 \%$ & $50.54 \%$ & $53.30 \%$ & $56.00 \%$ & $58.42 \%$ & $59.46 \%$ & $50.86 \%$ \\
\hline Market-sensitive Total & 9,604 & 20,426 & 24,276 & 29,120 & 29,040 & 27,769 & 29,872 & 30,839 & 29,978 & 27,770 & 27,533 & 286,227 \\
\hline \% Total MS & $3.36 \%$ & $7.14 \%$ & $8.48 \%$ & $10.17 \%$ & $10.15 \%$ & $9.70 \%$ & $10.44 \%$ & $10.77 \%$ & $10.47 \%$ & $9.70 \%$ & $9.62 \%$ & $100.00 \%$ \\
\hline \% Each Year Total & $20.99 \%$ & $22.57 \%$ & $23.75 \%$ & $24.79 \%$ & $24.77 \%$ & $25.32 \%$ & $26.63 \%$ & $26.11 \%$ & $26.39 \%$ & $25.51 \%$ & $25.93 \%$ & $25.07 \%$ \\
\hline
\end{tabular}

Note: * excluding the minute in the time window. Pre trading: 00.00-9.59; Post trading: 16.01-23.59. The announcement release time is rounded up to one minute.

\# the number of announcements for 2004 is half year from 01/07/2004 to 31/12/2004. 


\subsection{Immediate Price and Volume Reaction $t$-test Results}

This study focuses on trading hour announcements to explore and compare the immediate market reactions to different types of announcements. This is because there may be more than one category of announcements released during off-trading hours in one day. The overall offtrading hour announcements drive the price change between the closing price and the opening price on the next trading day. Thus, it is difficult to measure the magnitudes of market reactions to each announcement category in off-trading hours. Moreover, this study's three hour time window can only capture the immediate market responses to 'normal' announcements that do not induce longer trading halts, because more important announcements may be accompanied by a longer trading halt. However, 'normal' is not equal to 'not important'. The $t$-tests can show whether the abnormal returns or abnormal trading volumes are significant different from zero $(0)$.

Table 6-5 shows the $t$-test results of CARs based on overall announcements and Table 6-6 provides the results of market-sensitive announcements. Although CARs reveal whether the abnormal returns exist after announcements are released, other factors may impact on share prices and returns. It could be a good or bad trading day due to the macroeconomic environment or an influential event. So, as a robustness test, this study tests the differences between CARs before and after announcements are released over the equivalent time window ( $\triangle \mathrm{CAR}$ ). Table 6-7 indicates the $\triangle$ CAR $t$-test results of the full sample, and Table 6-8 shows the results of the market-sensitive announcement subsamples. In addition to share price reactions to announcements, this study explores how trading volumes change around the release of the announcements. By examining the ATVs, Table 6-9 is the $t$-test results of volume reactions to 
different types of announcements based on the full sample. Table 6-10 shows the ATV $t$-test results ${ }^{38}$ of market-sensitive announcements.

${ }^{38}$ In the $t$-tests, the unit of ATV is thousand ('000), while in regression results, the unit of ATV is million ('000,000) 
Table 6-5. Intraday Cumulative Abnormal Returns of All Announcements

\begin{tabular}{|c|c|c|c|c|c|c|c|c|}
\hline Main Category & CAR10 & CAR15 & CAR30 & CAR60 & CAR90 & CAR120 & CAR150 & CAR180 \\
\hline \multicolumn{9}{|c|}{ Investment/project-related announcements: } \\
\hline $\mathbf{N}$ & 5,498 & 5,394 & 5,099 & 4,483 & 3,957 & 3,506 & 3,062 & 2,677 \\
\hline Takeover/Scheme Announcements & $0.060 \% * * *$ & $0.125 \% * * *$ & $0.159 \% * * *$ & $0.315 \% * * *$ & $0.393 \% * * *$ & $0.441 \% * * *$ & $0.427 \% * * *$ & $0.377 \% * * *$ \\
\hline$t$-value & 4.52 & 6.10 & 5.33 & 6.25 & 6.16 & 6.40 & 5.66 & 4.77 \\
\hline $\mathbf{N}$ & 60,774 & 59,507 & 55,945 & 49,448 & 43,668 & 38,355 & 33,734 & 29,100 \\
\hline Issued Capital & $0.003 \%$ & $0.010 \%$ & $-0.001 \%$ & $-0.001 \%$ & $-0.035 \% * *$ & $-0.031 \%$ & $-0.008 \%$ & $-0.040 \% *$ \\
\hline$t$-value & 0.37 & 1.10 & -0.08 & -0.05 & -2.04 & -1.62 & -0.37 & -1.74 \\
\hline $\mathbf{N}$ & 7,437 & 7,340 & 7,008 & 6,418 & 5,801 & 5,177 & 4,701 & 4,186 \\
\hline Asset Acquisition \& Disposal & $0.513 \% * * *$ & $0.830 \% * * *$ & $1.090 \% * * *$ & $1.160 \% * * *$ & $1.160 \% * * *$ & $1.190 \% * * *$ & $1.190 \% * * *$ & $1.170 \% * * *$ \\
\hline$t$-value & 15.42 & 18.53 & 18.21 & 17.78 & 16.24 & 15.24 & 14.31 & 13.44 \\
\hline $\mathbf{N}$ & 57,892 & 57,372 & 55,729 & 52,255 & 48,498 & 44,491 & 40,602 & 36,369 \\
\hline Progress Report & $0.692 \% * * *$ & $1.010 \% * * *$ & $1.130 \% * * *$ & $1.220 \% * * *$ & $1.250 \% * * *$ & $1.250 \% * * *$ & $1.240 \% * * *$ & $1.250 \% * * *$ \\
\hline$t$-value & 49.20 & 55.80 & 53.37 & 49.10 & 44.94 & 41.36 & 38.24 & 35.54 \\
\hline $\mathbf{N}$ & 6,775 & 6,680 & 6,434 & 5,898 & 5,397 & 4,905 & 4,393 & 3,794 \\
\hline Other & $0.159 \% * * *$ & $0.239 \% * * *$ & $0.303 \% * * *$ & $0.362 \% * * *$ & $0.420 \% * * *$ & $0.447 \% * * *$ & $0.490 \% * * *$ & $0.471 \% * * *$ \\
\hline$t$-value & 7.82 & 9.21 & 9.40 & 8.77 & 8.73 & 8.42 & 8.25 & 7.06 \\
\hline \multicolumn{9}{|l|}{ Earnings-related announcements: } \\
\hline $\mathbf{N}$ & 32,989 & 32,347 & 30,591 & 27,220 & 24,259 & 21,594 & 19,294 & 17,003 \\
\hline Periodic Reports & $0.109 \% * * *$ & $0.159 \% * * *$ & $0.181 \% * * *$ & $0.196 \% * * *$ & $0.211 \% * * *$ & $0.222 \% * * *$ & $0.216 \% * * *$ & $0.221 \% * * *$ \\
\hline$t$-value & 11.47 & 12.18 & 10.48 & 8.94 & 8.36 & 7.81 & 6.93 & 6.55 \\
\hline $\mathbf{N}$ & 4,813 & 4,712 & 4,449 & 3,920 & 3,446 & 3,050 & 2,705 & 2,415 \\
\hline Distribution Announcement & $0.070 \% * * *$ & $0.103 \% * * *$ & $0.157 \% * * *$ & $0.200 \% * * *$ & $0.233 \% * * *$ & $0.266 \% * * *$ & $0.276 \% * * *$ & $0.277 \% * * *$ \\
\hline$t$-value & 5.30 & 6.07 & 6.91 & 6.02 & 6.09 & 5.72 & 5.68 & 6.08 \\
\hline \multicolumn{9}{|l|}{ Quarterly reports: } \\
\hline $\mathbf{N}$ & 10,101 & 9,909 & 9,388 & 8,364 & 7,357 & 6,387 & 5,546 & 4,664 \\
\hline Quarterly Activities Report & $0.026 \% * *$ & $0.050 \% * * *$ & $0.040 \%$ & $0.004 \%$ & $0.034 \%$ & $-0.009 \%$ & $-0.003 \%$ & $-0.078 \%$ \\
\hline$t$-value & 2.21 & 2.70 & 1.49 & 0.11 & 0.79 & -0.17 & -0.05 & -1.16 \\
\hline $\mathbf{N}$ & 3,877 & 3,794 & 3,564 & 3,147 & 2,743 & 2,344 & 1,969 & 1,625 \\
\hline Quarterly Cash Flow Report & $0.019 \%$ & $0.001 \%$ & $-0.052 \%$ & $-0.134 \% * *$ & $-0.152 \% * *$ & $-0.158 \% *$ & $-0.169 \% *$ & $-0.245 \% * * *$ \\
\hline$t$-value & 1.03 & 0.03 & -1.29 & -2.37 & -2.30 & -1.93 & -1.73 & -2.22 \\
\hline $\mathbf{N}$ & 2,965 & 2,897 & 2,673 & 2,289 & 1,930 & 1,619 & 1,393 & 1,139 \\
\hline CTE Quarterly Reports & $0.042 \%$ & $0.070 \%$ & $0.146 \% *$ & $0.251 \% * * *$ & $0.364 \% * * *$ & $0.457 \% * * *$ & $0.489 \% * * *$ & $0.335 \% *$ \\
\hline$t$-value & 0.91 & 1.17 & 1.94 & 2.61 & 2.97 & 3.27 & 2.78 & 1.74 \\
\hline
\end{tabular}




\begin{tabular}{|c|c|c|c|c|c|c|c|c|}
\hline Main Category & CAR10 & CAR15 & CAR30 & CAR60 & CAR90 & CAR120 & CAR150 & CAR180 \\
\hline \multicolumn{9}{|l|}{ ASX-related announcements: } \\
\hline $\mathbf{N}$ & 3,704 & 3,645 & 3,506 & 3,226 & 2,949 & 2,652 & 2,371 & 2,034 \\
\hline ASX Announcement & $0.009 \%$ & $0.004 \%$ & $0.011 \%$ & $-0.088 \%$ & $-0.047 \%$ & $-0.014 \%$ & $0.054 \%$ & $0.091 \%$ \\
\hline$t$-value & 1.11 & 0.24 & 0.25 & -1.60 & -0.67 & -0.18 & 0.68 & 0.96 \\
\hline $\mathbf{N}$ & 2,360 & 2,314 & 2,174 & 1,893 & 1,598 & 1,290 & 1,006 & 738 \\
\hline ASX Query & $-0.382 \% * * *$ & $-0.654 \% * * *$ & $-0.752 \% * * *$ & $-0.954 \% * * *$ & $-1.070 \% * * *$ & $-1.220 \% * * *$ & $-1.050 \% * * *$ & $-0.939 \% * * *$ \\
\hline$t$-value & -6.31 & -8.09 & -7.41 & -7.81 & -6.87 & -6.66 & -5.20 & -3.85 \\
\hline \multicolumn{9}{|c|}{ Administrative announcements: } \\
\hline $\mathbf{N}$ & 72,334 & 70,542 & 65,857 & 56,897 & 48,788 & 42,068 & 36,515 & 31,622 \\
\hline Security Holder Details & $0.022 \% * * *$ & $0.039 \% * * *$ & $0.062 \% * * *$ & $0.108 \% * * *$ & $0.134 \% * * *$ & $0.153 \% * * *$ & $0.147 \% * * *$ & $0.129 \% * * *$ \\
\hline$t$-value & 4.50 & 6.92 & 8.20 & 9.68 & 9.80 & 9.49 & 7.82 & 6.34 \\
\hline $\mathbf{N}$ & 20,638 & 20,239 & 19,011 & 16,631 & 14,328 & 12,210 & 10,254 & 8,392 \\
\hline Notice of Meeting & $0.005 \%$ & $-0.002 \%$ & $0.011 \%$ & $0.004 \%$ & $0.023 \%$ & $0.013 \%$ & $0.023 \%$ & $-0.010 \%$ \\
\hline$t$-value & 0.48 & -0.16 & 0.68 & 0.16 & 0.83 & 0.41 & 0.59 & -0.22 \\
\hline $\mathbf{N}$ & 13,723 & 13,457 & 12,745 & 11,410 & 10,107 & 8,904 & 7,871 & 6,847 \\
\hline Company Administration & $0.033 \% * *$ & $0.027 \%$ & $0.053 \% * *$ & $0.106 \% * * *$ & $0.077 \% * *$ & $0.118 \% * * *$ & $0.096 \% * *$ & $0.090 \% *$ \\
\hline$t$-value & 2.19 & 1.56 & 2.49 & 3.55 & 2.26 & 2.98 & 2.17 & 1.81 \\
\hline $\mathbf{N}$ & 9 & 9 & 8 & 7 & 7 & 7 & 7 & 7 \\
\hline Notice of Call & $0.545 \%$ & $0.544 \%$ & $0.550 \%$ & $0.970 \%$ & $0.838 \%$ & $0.856 \%$ & $0.886 \%$ & $-0.168 \%$ \\
\hline$t$-value & 0.94 & 0.94 & 0.86 & 1.39 & 1.16 & 1.13 & 1.13 & -0.38 \\
\hline $\mathbf{N}$ & 7,705 & 7,640 & 7,445 & 7,063 & 6,553 & 5,967 & 5,368 & 4,799 \\
\hline Chairman's Address & $0.053 \% * * *$ & $0.069 \% * * *$ & $0.063 \% * *$ & $0.088 \% * *$ & $0.116 \% * * *$ & $0.119 \% * * *$ & $0.192 \% * * *$ & $0.199 \% * * *$ \\
\hline$t$-value & 3.66 & 3.70 & 2.52 & 2.57 & 2.88 & 2.65 & 3.84 & 3.54 \\
\hline $\mathbf{N}$ & 1,196 & 1,164 & 1,117 & 966 & 871 & 773 & 682 & 598 \\
\hline Letter to Shareholders & $0.069 \% * *$ & $0.088 \% * *$ & $0.162 \% * *$ & $0.099 \%$ & $0.219 \% * *$ & $0.302 \% * *$ & $0.443 \% *$ & $0.158 \%$ \\
\hline$t$-value & 2.04 & 2.14 & 2.57 & 1.25 & 2.16 & 2.55 & 3.16 & 0.85 \\
\hline $\mathbf{N}$ & 3,218 & 3,123 & 2,874 & 2,493 & 2,124 & 1,870 & 1,632 & 1,491 \\
\hline Structured Products & $-0.001 \%$ & $0.000 \%$ & $-0.007 \%$ & $-0.023 \% * * *$ & $-0.024 \% * *$ & $-0.026 \% * *$ & $-0.028 \% * *$ & $-0.045 \% * * *$ \\
\hline$t$-value & -0.19 & -0.11 & -1.27 & -2.98 & -2.22 & -2.03 & -1.97 & -2.68 \\
\hline
\end{tabular}


Table 6-6. Intraday Cumulative Abnormal Returns of Market-sensitive Announcements

\begin{tabular}{|c|c|c|c|c|c|c|c|c|}
\hline Main Category & CAR10 & CAR15 & CAR30 & CAR60 & CAR90 & CAR120 & CAR150 & CAR180 \\
\hline \multicolumn{9}{|c|}{ Investment/project-related announcements: } \\
\hline $\mathbf{N}$ & 2,415 & 2,371 & 2,244 & 1,973 & 1,739 & 1,528 & 1,316 & 1,139 \\
\hline Takeover/Scheme Announcements & $0.106 \% * * *$ & $0.202 \% * * *$ & $0.247 \% * * *$ & $0.438 \% * * *$ & $0.664 \% * * *$ & $0.779 \% * * *$ & $0.758 \% * * *$ & $0.715 \% * * *$ \\
\hline$t$-value & 4.41 & 5.23 & 5.12 & 4.93 & 6.04 & 6.34 & 5.50 & 4.65 \\
\hline $\mathbf{N}$ & 5,015 & 4,941 & 4,657 & 4,139 & 3,690 & 3,279 & 2,960 & 2,576 \\
\hline Issued Capital & $0.224 \% * * *$ & $0.330 \% * * *$ & $0.398 \% * * *$ & $0.422 \% * * *$ & $0.426 \% * * *$ & $0.479 \% * * *$ & $0.535 \% * * *$ & $0.406 \% * * *$ \\
\hline$t$-value & 5.21 & 4.82 & 4.73 & 4.04 & 3.78 & 4.00 & 4.10 & 2.97 \\
\hline $\mathbf{N}$ & 5,178 & 5,121 & 4,921 & 4,534 & 4,134 & 3,701 & 3,394 & 3,042 \\
\hline Asset Acquisition \& Disposal & $0.648 \% * * *$ & $1.100 \% * * *$ & $1.380 \% * * *$ & $1.470 \% * * *$ & $1.470 \% * * *$ & $1.520 \% * * *$ & $1.500 \% * * *$ & $1.460 \% * * *$ \\
\hline$t$-value & 15.33 & 18.63 & 18.21 & 17.66 & 16.30 & 15.22 & 14.40 & 13.54 \\
\hline $\mathbf{N}$ & 33,799 & 33,508 & 32,658 & 30,828 & 28,807 & 26,600 & 24,369 & 21,922 \\
\hline Progress Report & $1.060 \% * * *$ & $1.590 \% * * *$ & $1.710 \% * * *$ & $1.770 \% * * *$ & $1.770 \% * * *$ & $1.760 \% * * *$ & $1.740 \% * * *$ & $1.710 \% * * *$ \\
\hline$t$-value & 48.35 & 55.19 & 51.91 & 46.74 & 41.88 & 38.58 & 35.88 & 33.11 \\
\hline $\mathbf{N}$ & 1373 & 1358 & 1311 & 1225 & 1132 & 1009 & 924 & 836 \\
\hline Other & $0.343 \% * * *$ & $0.567 \% * * *$ & $0.656 \% * * *$ & $0.789 \% * * *$ & $0.878 \% * * *$ & $0.878 \% * * *$ & $0.853 \% * * *$ & $0.950 \% * * *$ \\
\hline$t$-value & 6.02 & 7.03 & 6.53 & 7.06 & 6.90 & 6.39 & 5.86 & 5.97 \\
\hline \multicolumn{9}{|l|}{ Earnings-related announcements: } \\
\hline $\mathbf{N}$ & 9,200 & 9,028 & 8,520 & 7,645 & 6,837 & 6,128 & 5,475 & 4,815 \\
\hline Periodic Reports & $0.264 \% * * *$ & $0.397 \% * * *$ & $0.462 \% * * *$ & $0.466 \% * * *$ & $0.491 \% * * *$ & $0.499 \% * * *$ & $0.497 \% * * *$ & $0.494 \% * * *$ \\
\hline$t$-value & 9.54 & 10.04 & 8.97 & 7.44 & 6.76 & 6.22 & 5.70 & 5.23 \\
\hline $\mathbf{N}$ & 1,518 & 1,480 & 1,388 & 1,221 & 1,071 & 964 & 854 & 741 \\
\hline Distribution Announcement & $0.195 \% * * *$ & $0.271 \% * * *$ & $0.440 \% * * *$ & $0.551 \% * * *$ & $0.678 \% * * *$ & $0.718 \% * * *$ & $0.771 \% * * *$ & $0.797 \% * * *$ \\
\hline$t$-value & 5.48 & 5.93 & 6.88 & 5.78 & 6.42 & 5.45 & 5.82 & 6.57 \\
\hline \multicolumn{9}{|l|}{ Quarterly reports: } \\
\hline $\mathbf{N}$ & 9,696 & 9,505 & 8,999 & 8,004 & 7,028 & 6,097 & 5,278 & 4,429 \\
\hline Quarterly Activities Report & $0.027 \% * *$ & $0.052 \% * * *$ & $0.042 \%$ & $0.009 \%$ & $0.040 \%$ & $-0.001 \%$ & $0.010 \%$ & $-0.068 \%$ \\
\hline$t$-value & 2.29 & 2.73 & 1.51 & 0.23 & 0.90 & -0.01 & 0.16 & -0.98 \\
\hline $\mathbf{N}$ & 3,792 & 3,715 & 3,490 & 3,084 & 2,690 & 2,299 & 1,929 & 1,596 \\
\hline Quarterly Cash Flow Report & $0.020 \%$ & $0.001 \%$ & $-0.052 \%$ & $-0.143 \% * *$ & $-0.158 \% * *$ & $-0.167 \% * *$ & $-0.169 \% *$ & $-0.256 \% * *$ \\
\hline$t$-value & 1.04 & 0.03 & -1.26 & -2.50 & -2.35 & -2.01 & -1.71 & -2.30 \\
\hline $\mathbf{N}$ & 2,863 & 2,796 & 2,583 & 2,209 & 1,866 & 1,568 & 1,351 & 1,103 \\
\hline CTE Quarterly Reports & $0.027 \%$ & $0.057 \%$ & $0.125 \%$ & $0.226 \% * *$ & $0.311 \% * *$ & $0.442 \% * * *$ & $0.463 \% * *$ & $0.303 \%$ \\
\hline$t$-value & 0.57 & 0.94 & 1.62 & 2.32 & 2.49 & 3.10 & 2.57 & 1.55 \\
\hline
\end{tabular}




\begin{tabular}{|c|c|c|c|c|c|c|c|c|}
\hline Main Category & CAR10 & CAR15 & CAR30 & CAR60 & CAR90 & CAR120 & CAR150 & CAR180 \\
\hline \multicolumn{9}{|l|}{ ASX-related announcements: } \\
\hline $\mathbf{N}$ & 2,726 & 2,689 & 2,596 & 2,394 & 2,201 & 1,988 & 1,783 & 1,540 \\
\hline ASX Announcement & $0.005 \%$ & $0.002 \%$ & $0.026 \%$ & $-0.087 \%$ & $-0.030 \%$ & $-0.004 \%$ & $0.065 \%$ & $0.133 \%$ \\
\hline$t$-value & 0.95 & 0.12 & 0.46 & -1.23 & -0.33 & -0.04 & 0.67 & 1.12 \\
\hline $\mathbf{N}$ & 1,886 & 1,849 & 1,735 & 1,506 & 1,254 & 999 & 764 & 546 \\
\hline ASX Query & $-0.471 \% * * *$ & $-0.813 \% * * *$ & $-0.940 \% * * *$ & $-1.130 \% * * *$ & $-1.310 \% * * *$ & $-1.440 \% * * *$ & $-1.240 \% * * *$ & $-1.110 \% * * *$ \\
\hline$t$-value & -6.35 & -8.21 & -7.54 & -7.54 & -6.76 & -6.39 & -4.97 & -3.61 \\
\hline \multicolumn{9}{|c|}{ Administrative announcements: } \\
\hline $\mathbf{N}$ & 473 & 464 & 444 & 411 & 369 & 337 & 303 & 269 \\
\hline Security Holder Details & $0.442 \% * * *$ & $0.809 \% * * *$ & $1.030 \% * * *$ & $0.993 \% * * *$ & $0.867 \% * * *$ & $1.160 \% * * *$ & $0.784 \% * * *$ & $1.070 \% * * *$ \\
\hline$t$-value & 4.60 & 5.50 & 5.19 & 4.22 & 3.41 & 3.55 & 2.05 & 2.50 \\
\hline $\mathbf{N}$ & 117 & 117 & 111 & 100 & 92 & 82 & 72 & 60 \\
\hline Notice of Meeting & $0.169 \%$ & $0.312 \%$ & $0.405 \%$ & $0.127 \%$ & $0.179 \%$ & $0.055 \%$ & $0.188 \%$ & $0.376 \%$ \\
\hline$t$-value & 0.94 & 1.43 & 1.51 & 0.41 & 0.40 & 0.12 & 0.47 & 0.76 \\
\hline $\mathbf{N}$ & 706 & 692 & 662 & 607 & 559 & 505 & 453 & 392 \\
\hline Company Administration & $0.123 \%$ & $0.103 \%$ & $0.121 \%$ & $0.386 \% * *$ & $0.502 \% * * *$ & $0.679 \% * * *$ & $\mathbf{0 . 5 8 5 \%} \% * *$ & $0.416 \% *$ \\
\hline$t$-value & 1.51 & 0.97 & 0.84 & 2.17 & 2.68 & 3.35 & 2.74 & 1.94 \\
\hline $\mathbf{N}$ & 0 & 0 & 0 & 0 & 0 & 0 & 0 & 0 \\
\hline Notice of Call & - & - & - & - & - & - & - & - \\
\hline$t$-value & - & - & - & - & - & - & - & - \\
\hline $\mathbf{N}$ & 593 & 586 & 577 & 555 & 520 & 473 & 428 & 401 \\
\hline Chairman's Address & $0.035 \%$ & $0.104 \%$ & $0.046 \%$ & $0.156 \%$ & $0.284 \%$ & $0.275 \%$ & $0.284 \%$ & $0.344 \%$ \\
\hline$t$-value & 0.49 & 1.14 & 0.36 & 1.02 & 1.42 & 1.22 & 1.22 & 1.40 \\
\hline $\mathbf{N}$ & 14 & 14 & 12 & 12 & 12 & 10 & 10 & 9 \\
\hline Letter to Shareholders & $0.689 \%$ & $0.666 \%$ & $0.254 \%$ & $-0.773 \%$ & $-0.305 \%$ & $-0.115 \%$ & $-0.287 \%$ & $2.810 \%$ \\
\hline$t$-value & 0.73 & 0.50 & 0.10 & -0.30 & -0.10 & -0.03 & -0.07 & 1.03 \\
\hline $\mathbf{N}$ & 1 & 1 & 0 & 0 & 0 & 0 & 0 & 0 \\
\hline Structured Products & $-0.259 \%$ & $-0.291 \%$ & - & - & - & - & - & - \\
\hline$t$-value & - & - & - & - & - & - & - & - \\
\hline
\end{tabular}


Table 6-7. The Difference between Intraday Cumulative Abnormal Returns before and after the Announcements

\begin{tabular}{|c|c|c|c|c|c|c|c|c|}
\hline Main Category & $\Delta$ CAR10 & $\Delta$ CAR15 & $\Delta$ CAR30 & $\Delta$ CAR60 & ACAR90 & $\Delta$ CAR120 & $\Delta$ CAR150 & $\Delta$ CAR180 \\
\hline \multicolumn{9}{|c|}{ Investment/project-related announcements: } \\
\hline $\mathbf{N}$ & 5,230 & 4,999 & 4,400 & 3,296 & 2,376 & 1,498 & 633 & 1 \\
\hline Takeover/Scheme Announcements & $0.060 \% * * *$ & $0.116 \% * * *$ & $0.131 \% * * *$ & $0.255 \% * * *$ & $0.304 \% * * *$ & $0.383 \% * * *$ & $0.457 \% * *$ & $-6.260 \%$ \\
\hline$t$-value & 3.55 & 4.37 & 3.33 & 4.03 & 3.72 & 3.54 & 2.47 & - \\
\hline $\mathbf{N}$ & 58,306 & 55,949 & 49,382 & 37,453 & 26,437 & 16,038 & 7,102 & 17 \\
\hline Issued Capital & $0.010 \%$ & $0.018 \% *$ & $0.015 \%$ & $0.030 \%$ & $0.049 \% *$ & $0.020 \%$ & $0.110 \% *$ & $0.846 \%$ \\
\hline$t$-value & 1.17 & 1.71 & 0.99 & 1.43 & 1.72 & 0.51 & 1.71 & 0.7 \\
\hline $\mathbf{N}$ & 7,060 & 6,720 & 5,870 & 4,411 & 3,059 & 1,849 & 822 & 2 \\
\hline Asset Acquisition \& Disposal & $0.484 \% * * *$ & $0.792 \% * * *$ & $1.080 \% * * *$ & $1.150 \% * * *$ & $1.020 \% * * *$ & $0.994 \% * * *$ & $0.995 \% * * *$ & $-1.100 \%$ \\
\hline$t$-value & 13.45 & 16.18 & 15.51 & 13.04 & 9.02 & 6.37 & 3.82 & -0.44 \\
\hline $\mathbf{N}$ & 54,795 & 52,596 & 46,616 & 35,714 & 25,084 & 15,339 & 6,744 & 21 \\
\hline Progress Report & $0.699 \% * * *$ & $1.010 \% * * *$ & $1.090 \% * * *$ & $1.170 \% * * *$ & $1.190 \% * * *$ & $1.150 \% * * *$ & $1.080 \% * * *$ & $1.470 \%$ \\
\hline$t$-value & 44.57 & 49.79 & 43.67 & 35.35 & 27.12 & 19.07 & 11.75 & 1.67 \\
\hline $\mathbf{N}$ & 6,396 & 6,179 & 5,523 & 4,250 & 3,130 & 2,004 & 953 & 2 \\
\hline Other & $0.151 \% * * *$ & $0.229 \% * * *$ & $0.302 \% * * *$ & $0.364 \% * * *$ & $0.465 \% * * *$ & $0.481 \% * * *$ & $0.619 \% * * *$ & $-3.310 \%$ \\
\hline$t$-value & 6.42 & 7.74 & 7.64 & 6.47 & 6.09 & 4.72 & 3.98 & -1.04 \\
\hline \multicolumn{9}{|l|}{ Earnings-related announcements: } \\
\hline $\mathbf{N}$ & 31,205 & 29,749 & 25,783 & 18,825 & 12,882 & 7,646 & 3,267 & 8 \\
\hline Periodic Reports & $0.105 \% * * *$ & $0.161 \% * * *$ & $0.180 \% * * *$ & $0.203 \% * * *$ & $0.242 \% * * *$ & $0.235 \% * * *$ & $0.173 \% *$ & $-1.800 \%$ \\
\hline$t$-value & 9.52 & 10.85 & 8.44 & 6.52 & 5.88 & 4.00 & 1.77 & -1.04 \\
\hline $\mathbf{N}$ & 4,623 & 4,449 & 3,961 & 2,977 & 2,086 & 1,244 & 525 & 0 \\
\hline Distribution Announcement & $0.089 \% * * *$ & $0.113 \% * * *$ & $0.176 \% * * *$ & $0.209 \% * * *$ & $0.254 \% * * *$ & $0.309 \% * * *$ & $0.250 \% *$ & - \\
\hline$t$-value & 5.56 & 5.43 & 6.04 & 4.94 & 4.20 & 3.23 & 1.82 & - \\
\hline \multicolumn{9}{|l|}{ Quarterly reports: } \\
\hline $\mathbf{N}$ & 9,785 & 9,395 & 8,391 & 6,435 & 4,495 & 2,734 & 1,193 & 0 \\
\hline Quarterly Activities Report & $0.026 \%$ & $0.048 \% *$ & $0.049 \%$ & $0.025 \%$ & $0.083 \%$ & $-0.058 \%$ & $0.263 \%$ & - \\
\hline$t$-value & 1.49 & 1.94 & 1.36 & 0.47 & 1.18 & -0.56 & 1.63 & - \\
\hline $\mathbf{N}$ & 3,792 & 3,640 & 3,253 & 2,528 & 1,796 & 1,081 & 452 & 0 \\
\hline Quarterly Cash Flow Report & $0.032 \%$ & $0.032 \%$ & $-0.044 \%$ & $-0.109 \%$ & $-0.101 \%$ & $0.110 \%$ & $0.188 \%$ & - \\
\hline$t$-value & 1.35 & 0.95 & -0.81 & -1.26 & -0.81 & 0.57 & 0.58 & - \\
\hline $\mathbf{N}$ & 2,877 & 2,755 & 2,392 & 1,772 & 1,184 & 686 & 295 & 0 \\
\hline CTE Quarterly Reports & $0.013 \%$ & $0.032 \%$ & $0.133 \%$ & $0.305 \% * *$ & $0.517 \% * * *$ & $0.785 \% * * *$ & $1.040 \% * *$ & - \\
\hline$t$-value & 0.25 & 0.44 & 1.58 & 2.23 & 2.73 & 3.28 & 2.35 & - \\
\hline
\end{tabular}




\begin{tabular}{|c|c|c|c|c|c|c|c|c|}
\hline Main Category & $\triangle$ CAR10 & $\Delta$ CAR15 & $\Delta$ CAR30 & $\Delta$ CAR60 & ACAR90 & $\triangle$ CAR120 & $\Delta$ CAR150 & $\Delta$ CAR180 \\
\hline \multicolumn{9}{|l|}{ ASX-related announcements: } \\
\hline $\mathbf{N}$ & 3,643 & 3,526 & 3,165 & 2,466 & 1,762 & 1,113 & 516 & 0 \\
\hline ASX Announcement & $0.007 \%$ & $-0.004 \%$ & $-0.096 \%$ & $-0.564 \% * * *$ & $-0.892 \% * * *$ & $-0.956 \% * * *$ & $-0.959 \% * *$ & - \\
\hline$t$-value & 0.63 & -0.16 & -1.49 & -4.97 & -4.91 & -3.02 & -2.02 & - \\
\hline $\mathbf{N}$ & 2,282 & 2,190 & 1,962 & 1,590 & 1,206 & 815 & 407 & 1 \\
\hline ASX Query & $-0.359 \% * * *$ & $-0.640 \% * * *$ & $-0.554 \% * * *$ & $-0.688 \% * * *$ & $-0.603 \% * *$ & $-1.550 \% * * *$ & $-3.080 \% * * *$ & $-30.050 \%$ \\
\hline$t$-value & -4.93 & -6.41 & -4.01 & -3.79 & -2.39 & -4.08 & -5.15 & - \\
\hline \multicolumn{9}{|c|}{ Administrative announcements: } \\
\hline $\mathbf{N}$ & 69,798 & 66,837 & 59,051 & 44,163 & 30,131 & 17,744 & 7,444 & 19 \\
\hline Security Holder Details & $0.017 \% * *$ & $\mathbf{0 . 0 3 0 \%} * * *$ & $0.067 \% * * *$ & $0.117 \% * * *$ & $0.126 \% * * *$ & $0.117 \% * * *$ & $0.261 \% * * *$ & $2.300 \% * * *$ \\
\hline$t$-value & 2.39 & 3.57 & 5.77 & 6.14 & 4.80 & 2.70 & 4.15 & 3.17 \\
\hline $\mathbf{N}$ & 19,953 & 19,333 & 17,349 & 13,440 & 9,671 & 5,992 & 2,593 & 8 \\
\hline Notice of Meeting & $0.005 \%$ & $-0.001 \%$ & $-0.011 \%$ & $-0.042 \%$ & $-0.052 \%$ & $-0.110 \%$ & $-0.091 \%$ & $3.050 \%$ \\
\hline$t$-value & 0.34 & -0.04 & -0.49 & -1.18 & -1.08 & -1.60 & -0.76 & 1.17 \\
\hline $\mathbf{N}$ & 13,019 & 12,484 & 11,063 & 8,342 & 5,818 & 3,553 & 1,516 & 10 \\
\hline Company Administration & $0.026 \%$ & $0.037 \% *$ & $0.054 \% *$ & $0.089 \% *$ & $0.039 \%$ & $0.081 \%$ & $-0.025 \%$ & $0.746 \%$ \\
\hline$t$-value & 1.34 & 1.66 & 1.77 & 1.95 & 0.64 & 0.89 & -0.15 & 0.51 \\
\hline $\mathbf{N}$ & 9 & 8 & 7 & 6 & 4 & 1 & 0 & 0 \\
\hline Notice of Call & $0.693 \%$ & $0.924 \%$ & $1.550 \%$ & $1.930 \%$ & $0.474 \%$ & $0.406 \%$ & - & - \\
\hline$t$-value & 0.95 & 1.12 & 1.07 & 1.23 & 5.57 & - & - & - \\
\hline $\mathbf{N}$ & 7,194 & 6,938 & 6,188 & 4,540 & 3,163 & 1,983 & 861 & 3 \\
\hline Chairman's Address & $0.058 \% * * *$ & $0.068 \% * * *$ & $0.046 \%$ & $-0.035 \%$ & $-0.027 \%$ & $0.025 \%$ & $0.188 \%$ & $-2.880 \%$ \\
\hline$t$-value & 2.83 & 2.71 & 1.19 & -0.59 & -0.36 & 0.26 & 1.21 & -2.12 \\
\hline $\mathbf{N}$ & 1,143 & 1,086 & 970 & 730 & 522 & 316 & 131 & 1 \\
\hline Letter to Shareholders & $0.106 \% * *$ & $0.139 \% * *$ & $0.176 \% *$ & $0.062 \%$ & $0.322 \% *$ & $0.219 \%$ & $0.696 \%$ & $-1.810 \%$ \\
\hline$t$-value & 2.12 & 2.22 & 1.92 & 0.55 & 1.92 & 0.95 & 1.44 & - \\
\hline $\mathbf{N}$ & 3,113 & 2,977 & 2,580 & 1,907 & 1,294 & 746 & 273 & 0 \\
\hline Structured Products & $-0.005 \%$ & $-0.003 \%$ & $-0.007 \%$ & $-0.013 \%$ & $-0.034 \% * *$ & $-\mathbf{0 . 0 8 8 \%} \% * *$ & $-0.047 \%$ & - \\
\hline$t$-value & -1.03 & -0.56 & -0.94 & -1.21 & -2.08 & -3.59 & -0.90 & - \\
\hline
\end{tabular}


Table 6-8. The Difference between Intraday Cumulative Abnormal Returns before and after the Market-sensitive Announcements

\begin{tabular}{|c|c|c|c|c|c|c|c|c|}
\hline Main Category & $\Delta$ CAR10 & $\Delta$ CAR15 & $\Delta$ CAR30 & $\Delta$ CAR60 & ACAR90 & $\Delta$ CAR120 & $\Delta$ CAR150 & $\Delta$ CAR180 \\
\hline \multicolumn{9}{|c|}{ Investment/project-related announcements: } \\
\hline $\mathbf{N}$ & 2,323 & 2,213 & 1,951 & 1,470 & 1,063 & 669 & 288 & 0 \\
\hline Takeover/Scheme Announcements & $0.097 \% * * *$ & $0.176 \% * * *$ & $0.231 \% * * *$ & $0.324 \% * * *$ & $0.400 \% * * *$ & $0.581 \% * * *$ & $0.575 \% * *$ & - \\
\hline$t$-value & 3.47 & 3.66 & 3.72 & 3.05 & 3 & 3.34 & 1.99 & - \\
\hline $\mathbf{N}$ & 4,783 & 4,553 & 3,924 & 2,876 & 1,975 & 1,174 & 522 & 2 \\
\hline Issued Capital & $0.201 \% * * *$ & $0.344 \% * * *$ & $0.409 \% * * *$ & $0.450 \% * * *$ & $0.434 \% * *$ & $0.455 \% * *$ & $0.923 \% * *$ & $5.950 \%$ \\
\hline$t$-value & 4.1 & 4.63 & 3.92 & 3.14 & 2.47 & 1.98 & 2.55 & 9.86 \\
\hline $\mathbf{N}$ & 4,925 & 4,692 & 4,107 & 3,082 & 2,108 & 1,259 & 564 & 1 \\
\hline Asset Acquisition \& Disposal & $0.635 \% * * *$ & $1.090 \% * * *$ & $1.410 \% * * *$ & $1.530 \% * * *$ & $1.440 \% * * *$ & $1.490 \% * * *$ & $1.540 \% * * *$ & $-3.600 \%$ \\
\hline$t$-value & 13.81 & 16.94 & 15.79 & 13.36 & 9.60 & 7.08 & 4.32 & - \\
\hline $\mathbf{N}$ & 32,072 & 30,682 & 27,005 & 20,592 & 14,386 & 8,796 & 3,874 & 14 \\
\hline Progress Report & $1.070 \% * * *$ & $1.580 \% * * *$ & $1.670 \% * * *$ & $1.720 \% * * *$ & $1.700 \% * * *$ & $1.620 \% * * *$ & $1.460 \% * * *$ & $1.950 \%$ \\
\hline$t$-value & 45.19 & 50.73 & 43.88 & 34.76 & 25.78 & 17.95 & 11.32 & 1.68 \\
\hline $\mathbf{N}$ & 1,304 & 1,256 & 1,095 & 841 & 614 & 366 & 163 & 0 \\
\hline Other & $0.405 \% * * *$ & $0.631 \% * * *$ & $0.732 \% * * *$ & $0.790 \% * * *$ & $0.932 \% * * *$ & $0.692 \% * *$ & $0.759 \%$ & - \\
\hline$t$-value & 6.44 & 7.03 & 6.60 & 5.40 & 4.49 & 2.59 & 1.62 & - \\
\hline \multicolumn{9}{|l|}{ Earnings-related announcements: } \\
\hline $\mathbf{N}$ & 8,735 & 8,322 & 7,154 & 5,218 & 3,572 & 2,170 & 948 & 0 \\
\hline Periodic Reports & $0.248 \% * * *$ & $0.396 \% * * *$ & $0.450 \% * * *$ & $0.441 \% * * *$ & $0.586 \% * * *$ & $0.505 \% * * *$ & $0.246 \%$ & - \\
\hline$t$-value & 8.37 & 9.41 & 7.44 & 5.28 & 5.38 & 3.54 & 1.04 & - \\
\hline $\mathbf{N}$ & 1,465 & 1,407 & 1,234 & 911 & 648 & 396 & 172 & 0 \\
\hline Distribution Announcement & $0.200 \% * * *$ & $0.262 \% * * *$ & $0.459 \% * * *$ & $0.575 \% * * *$ & $0.780 \% * * *$ & $0.890 \% * * *$ & $0.809 \% * *$ & - \\
\hline$t$-value & 5.08 & 5.03 & 6.04 & 5.15 & 4.89 & 3.38 & 2.51 & - \\
\hline \multicolumn{9}{|l|}{ Quarterly reports: } \\
\hline $\mathbf{N}$ & 9,399 & 9,016 & 8,057 & 6,181 & 4,309 & 2,626 & 1,149 & 0 \\
\hline Quarterly Activities Report & $0.031 \% *$ & $0.053 \% * *$ & $0.058 \%$ & $0.041 \%$ & $0.106 \%$ & $-0.037 \%$ & $0.259 \%$ & - \\
\hline$t$-value & 1.79 & 2.08 & 1.56 & 0.75 & 1.47 & -0.35 & 1.58 & - \\
\hline $\mathbf{N}$ & 3,709 & 3,564 & 3,184 & 2,474 & 1,755 & 1,057 & 439 & 0 \\
\hline Quarterly Cash Flow Report & $0.033 \%$ & $0.034 \%$ & $-0.053 \%$ & $-0.128 \%$ & $-0.126 \%$ & $0.061 \%$ & $0.091 \%$ & - \\
\hline$t$-value & 1.4 & 1.02 & -0.97 & -1.45 & -1 & 0.31 & 0.28 & - \\
\hline $\mathbf{N}$ & 2,781 & 2,661 & 2,312 & 1,709 & 1,145 & 662 & 285 & 0 \\
\hline CTE Quarterly Reports & $0.002 \%$ & $0.023 \%$ & $0.121 \%$ & $0.297 \% * *$ & $0.482 \% * *$ & $0.821 \% * * *$ & $1.070 \% * *$ & - \\
\hline$t$-value & 0.03 & 0.31 & 1.41 & 2.12 & 2.49 & 3.31 & 2.33 & - \\
\hline
\end{tabular}




\begin{tabular}{|c|c|c|c|c|c|c|c|c|}
\hline Main Category & $\Delta$ CAR10 & $\Delta$ CAR15 & $\Delta$ CAR30 & $\triangle \mathrm{CAR60}$ & $\Delta$ CAR90 & ACAR120 & $\Delta$ CAR150 & $\Delta$ CAR180 \\
\hline \multicolumn{9}{|l|}{ ASX-related announcements: } \\
\hline $\mathbf{N}$ & 2,683 & 2,602 & 2,328 & 1,791 & 1,289 & 815 & 372 & 0 \\
\hline ASX Announcement & $0.004 \%$ & $-0.011 \%$ & $-0.114 \%$ & $-0.668 \% * * *$ & $-\mathbf{0 . 8 9 0 \%} * * *$ & $-0.970 \% * * *$ & $-1.270 \% * *$ & - \\
\hline$t$-value & 0.39 & -0.40 & -1.37 & -4.58 & -3.91 & -2.61 & -2.23 & - \\
\hline $\mathbf{N}$ & 1,830 & 1,757 & 1,583 & 1,310 & 1,001 & 684 & 347 & 1 \\
\hline ASX Query & $-0.449 \% * * *$ & $-0.795 \% * * *$ & $-0.697 \% * * *$ & $-0.786 \% * * *$ & $-0.717 \% * *$ & $-1.710 \% * * *$ & $-3.400 \% * * *$ & $-30.050 \%$ \\
\hline$t$-value & -5.07 & -6.54 & -4.18 & -3.67 & -2.42 & -3.86 & -4.97 & - \\
\hline \multicolumn{9}{|c|}{ Administrative announcements: } \\
\hline $\mathbf{N}$ & 450 & 421 & 350 & 275 & 191 & 119 & 52 & 0 \\
\hline Security Holder Details & $0.344 \% * * *$ & $0.654 \% * * *$ & $0.744 \% * * *$ & $0.799 \% * *$ & $0.150 \%$ & $0.393 \%$ & $-1.360 \%$ & - \\
\hline$t$-value & 3.21 & 3.63 & 2.7 & 2.23 & 0.34 & 0.65 & -1.34 & - \\
\hline $\mathbf{N}$ & 110 & 106 & 92 & 70 & 53 & 32 & 12 & 0 \\
\hline Notice of Meeting & $0.088 \%$ & $0.235 \%$ & $0.227 \%$ & $-0.107 \%$ & $-0.567 \%$ & $0.073 \%$ & $1.130 \%$ & - \\
\hline$t$-value & 0.44 & 0.89 & 0.53 & -0.22 & -0.76 & 0.08 & 1.27 & - \\
\hline $\mathbf{N}$ & 665 & 633 & 549 & 419 & 310 & 199 & 89 & 2 \\
\hline Company Administration & $0.103 \%$ & $0.107 \%$ & $0.197 \%$ & $0.563 \% *$ & $0.415 \%$ & $0.925 \% *$ & $0.978 \%$ & $-0.559 \%$ \\
\hline$t$-value & 1.12 & 0.90 & 0.92 & 1.84 & 1.28 & 1.89 & 0.99 & -0.50 \\
\hline $\mathbf{N}$ & 0 & 0 & 0 & 0 & 0 & 0 & 0 & 0 \\
\hline Notice of Call & - & - & - & - & - & - & - & - \\
\hline$t$-value & - & - & - & - & - & - & - & - \\
\hline $\mathbf{N}$ & 552 & 532 & 465 & 327 & 202 & 117 & 44 & 0 \\
\hline Chairman's Address & $0.116 \%$ & $0.197 \% *$ & $0.051 \%$ & $0.053 \%$ & $0.253 \%$ & $0.523 \%$ & $0.691 \%$ & - \\
\hline$t$-value & 1.47 & 1.84 & 0.33 & 0.23 & 0.74 & 1.05 & 1.31 & - \\
\hline $\mathbf{N}$ & 13 & 12 & 10 & 7 & 6 & 3 & 2 & 0 \\
\hline Letter to Shareholders & $0.788 \%$ & $1.170 \%$ & $0.143 \%$ & $-4.780 \%$ & $-5.870 \%$ & $-8.930 \%$ & $-14.720 \%$ & - \\
\hline$t$-value & 0.76 & 0.75 & 0.04 & -1.63 & -1.43 & -0.81 & -0.71 & - \\
\hline $\mathbf{N}$ & 1 & 1 & 0 & 0 & 0 & 0 & 0 & 0 \\
\hline Structured Products & $-0.880 \%$ & $-1.160 \%$ & - & - & - & - & - & - \\
\hline$t$-value & - & - & - & - & - & - & - & - \\
\hline
\end{tabular}


Table 6-9. Intraday Abnormal Trading Volumes of All Announcements

\begin{tabular}{|c|c|c|c|c|c|c|c|c|}
\hline Main Category & ATV10 & ATV 15 & ATV 30 & ATV 60 & ATV 90 & ATV 120 & ATV 150 & ATV 180 \\
\hline \multicolumn{9}{|c|}{ Investment/project-related announcements: } \\
\hline $\mathbf{N}$ & 7,388 & 7,042 & 6,161 & 4,744 & 3,518 & 2,368 & 1,171 & 19 \\
\hline Takeover/Scheme Announcements & $21.063 * * *$ & $33.557 *$ & $101.497 * * *$ & $147.169 * * *$ & $269.222 * * *$ & 283.080*** & 195.257 & 304.982 \\
\hline$t$-value & 2.94 & 1.92 & 3.81 & 3.07 & 3.67 & 2.64 & 1.52 & 1.24 \\
\hline $\mathbf{N}$ & 82,565 & 79,292 & 70,771 & 55,613 & 41,109 & 26,700 & 13,126 & 220 \\
\hline Issued Capital & $16.796 * * *$ & $32.089 * * *$ & $63.365 * * *$ & $92.151 * * *$ & $84.502 * * *$ & 32.533* & 10.798 & 41.074 \\
\hline$t$-value & 6.04 & 9.46 & 11.38 & 10.03 & 6.89 & 1.89 & 0.41 & 0.52 \\
\hline $\mathbf{N}$ & 11,074 & 10,561 & 9,302 & 7,232 & 5,236 & 3,413 & 1,714 & 23 \\
\hline Asset Acquisition \& Disposal & $60.764 * * *$ & $141.296 * * *$ & $290.405 * * *$ & $425.923 * * *$ & 417.137*** & $429.768 * * *$ & $474.594 * * *$ & -137.213 \\
\hline$t$-value & 7.45 & 10.07 & 11.86 & 9.83 & 8.51 & 6.02 & 3.93 & -0.85 \\
\hline $\mathbf{N}$ & 76,273 & 73,150 & 65,273 & 51,243 & 37,266 & 24,377 & 11,900 & 196 \\
\hline Progress Report & $108.008 * * *$ & $243.645 * * *$ & $394.535 * * *$ & $541.795 * * *$ & $610.391 * * *$ & $715.668 * * *$ & $708.177 * * *$ & $-1,065.043$ \\
\hline$t$-value & 26.12 & 32.67 & 28.44 & 21.49 & 15.41 & 10.41 & 4.96 & -1.35 \\
\hline $\mathbf{N}$ & 8,628 & 8,331 & 7,506 & 5,944 & 4,481 & 3,026 & 1,547 & 29 \\
\hline Other & $33.780 * * *$ & $66.280 * * *$ & $91.420 * * *$ & $134.591 * * *$ & $114.620 * *$ & $129.273 * *$ & $246.601 * *$ & $4,403.968$ \\
\hline$t$-value & 4.58 & 6.06 & 5.49 & 4.75 & 2.47 & 2.22 & 2.04 & 0.80 \\
\hline \multicolumn{9}{|l|}{ Earnings-related announcements: } \\
\hline $\mathbf{N}$ & 53,219 & 50,837 & 45,016 & 34,797 & 24,713 & 15,794 & 7,583 & 122 \\
\hline Periodic Reports & $15.923 * * *$ & $35.948 * * *$ & $70.179 * * *$ & $101.115 * * *$ & $110.248 * * *$ & $82.880 * * *$ & $90.483 * *$ & 153.086 \\
\hline$t$-value & 9.70 & 13.20 & 13.75 & 10.67 & 7.13 & 3.73 & 2.47 & 1.61 \\
\hline $\mathbf{N}$ & 6,097 & 5,856 & 5,220 & 4,067 & 2,920 & 1,830 & 841 & 7 \\
\hline Distribution Announcement & $7.327 * *$ & $24.742 * * *$ & $41.447 * * *$ & $51.553 * * *$ & 4.045 & -25.025 & -16.597 & -26.175 \\
\hline$t$-value & 2.00 & 3.06 & 3.59 & 3.86 & 0.21 & -1.31 & -0.56 & -0.82 \\
\hline \multicolumn{9}{|l|}{ Quarterly reports: } \\
\hline $\mathbf{N}$ & 15,927 & 15,411 & 14,036 & 11,244 & 8,370 & 5,554 & 2,800 & 48 \\
\hline Quarterly Activities Report & 0.138 & 23.642*** & $45.292 * * *$ & $\mathbf{5 9 . 8 0 0 * * *}$ & $46.579 * * *$ & -15.159 & -34.900 & -156.179 \\
\hline$t$-value & 0.06 & 4.23 & 6.68 & 5.77 & 3.22 & -0.63 & -0.67 & -1.75 \\
\hline $\mathbf{N}$ & 6,593 & 6,403 & 5,844 & 4,780 & 3,668 & 2,420 & 1,202 & 22 \\
\hline Quarterly Cash Flow Report & 2.188 & $17.737 * * *$ & $27.872 * * *$ & $45.962 * * *$ & 24.876 & -21.559 & 7.546 & 19.097 \\
\hline$t$-value & 0.59 & 3.39 & 3.39 & 3.46 & 1.24 & -0.69 & 0.20 & 0.38 \\
\hline $\mathbf{N}$ & 6,133 & 5,934 & 5,339 & 4,213 & 3,108 & 2,060 & 1,063 & 23 \\
\hline CTE Quarterly Reports & $16.444 * * *$ & $41.041 * * *$ & $69.992 * * *$ & $69.996 * * *$ & $63.425 * * *$ & 58.699 & 12.587 & 4.305 \\
\hline$t$-value & 3.45 & 5.53 & 5.08 & 3.11 & 2.97 & 1.47 & 0.46 & 0.10 \\
\hline
\end{tabular}




\begin{tabular}{|c|c|c|c|c|c|c|c|c|}
\hline Main Category & ATV10 & ATV 15 & ATV 30 & ATV 60 & ATV 90 & ATV 120 & ATV 150 & ATV 180 \\
\hline \multicolumn{9}{|l|}{ ASX-related announcements: } \\
\hline $\mathbf{N}$ & 8,903 & 8,154 & 6,695 & 5,016 & 3,672 & 2,393 & 1,283 & 19 \\
\hline ASX Announcement & 1.730 & 2.906 & $339.088 * * *$ & $\mathbf{5 3 1 . 8 4 5 * * *}$ & $602.691 * *$ & 889.327* & 1246.212 & 198.375 \\
\hline$t$-value & 0.20 & 0.27 & 6.63 & 3.66 & 2.34 & 1.91 & 1.17 & 0.50 \\
\hline $\mathbf{N}$ & 3,041 & 2,884 & 2,555 & 2,053 & 1,569 & 1,074 & 582 & 9 \\
\hline ASX Query & $34.577 *$ & $160.164 * * *$ & $266.229 * * *$ & $335.580 * * *$ & -24.697 & $-375.651 * *$ & $-1,423.748 * * *$ & $-2,444.690$ \\
\hline$t$-value & 1.96 & 4.62 & 5.51 & 3.72 & -0.20 & -2.37 & -5.40 & -1.10 \\
\hline \multicolumn{9}{|c|}{ Administrative announcements: } \\
\hline $\mathbf{N}$ & 96,485 & 93,110 & 83,489 & 64,814 & 47,033 & 29,976 & 14,327 & 234 \\
\hline Security Holder Details & $8.143 * * *$ & $6.798 * * *$ & $12.350 * * *$ & 6.274 & $-22.207 * * *$ & $-70.309 * * *$ & $-115.546^{* * * *}$ & -147.270 \\
\hline$t$-value & 3.70 & 2.84 & 3.20 & 1.08 & -2.74 & -5.72 & -5.86 & -1.61 \\
\hline $\mathbf{N}$ & 31,001 & 30,114 & 27,553 & 22,512 & 17,263 & 11,540 & 5,850 & 81 \\
\hline Notice of Meeting & 2.978 & $5.271 *$ & $8.688 * *$ & 8.854 & -0.481 & $-37.974 * * *$ & $-41.118 * *$ & -99.848 \\
\hline$t$-value & 1.20 & 1.84 & 2.08 & 1.27 & -0.05 & -3.16 & -2.25 & -1.48 \\
\hline $\mathbf{N}$ & 20,782 & 19,983 & 17,941 & 14,114 & 10,407 & 6,743 & 3,291 & 68 \\
\hline Company Administration & $8.820 * * *$ & $16.228 * * *$ & $36.774 * * *$ & $53.817 * * *$ & 31.764* & -1.515 & -44.715 & 77.356 \\
\hline$t$-value & 3.07 & 4.52 & 6.09 & 4.59 & 1.89 & -0.07 & -1.22 & 1.29 \\
\hline $\mathbf{N}$ & 15 & 14 & 12 & 10 & 6 & 5 & 1 & 0 \\
\hline Notice of Call & -18.160 & -12.629 & -23.012 & -30.506 & -1.606 & -2.655 & 32.686 & - \\
\hline$t$-value & -1.16 & -1.28 & -1.19 & -1.03 & -0.20 & -0.18 & - & - \\
\hline $\mathbf{N}$ & 10,573 & 10,206 & 9,207 & 7,055 & 4,985 & 3,291 & 1,625 & 29 \\
\hline Chairman's Address & $19.744 * * *$ & $29.578 * * *$ & $50.660 * * *$ & $83.285 * * *$ & $112.125 * * *$ & $67.661 *$ & 24.477 & -445.617 \\
\hline$t$-value & 4.42 & 3.96 & 5.20 & 6.23 & 4.39 & 1.87 & 0.39 & -1.30 \\
\hline $\mathbf{N}$ & 1,615 & 1,559 & 1,397 & 1,091 & 822 & 552 & 267 & 6 \\
\hline Letter to Shareholders & $35.326 * * *$ & $42.900 * * *$ & $47.158 * *$ & 38.913 & 62.648 & 58.529 & 21.888 & 61.373 \\
\hline$t$-value & 4.58 & 3.92 & 2.56 & 1.64 & 1.14 & 1.44 & 0.27 & 1.33 \\
\hline $\mathbf{N}$ & 3,309 & 3,169 & 2,759 & 2,058 & 1,428 & 841 & 321 & 1 \\
\hline Structured Products & 3.389* & $3.887 *$ & 3.375 & $19.566 * *$ & $-54.149 * * *$ & $-134.840 * * *$ & $-271.572 * * *$ & -467.660 \\
\hline$t$-value & 1.86 & 1.83 & 1.02 & 2.58 & -3.48 & -5.83 & -5.63 & - \\
\hline
\end{tabular}


Table 6-10. Intraday Abnormal Trading Volumes of Market-sensitive Announcements

\begin{tabular}{|c|c|c|c|c|c|c|c|c|}
\hline Main Category & ATV10 & ATV 15 & ATV 30 & ATV 60 & ATV 90 & ATV 120 & ATV 150 & ATV 180 \\
\hline \multicolumn{9}{|c|}{ Investment/project-related announcements: } \\
\hline $\mathbf{N}$ & 3,431 & 3,253 & 2,816 & 2,168 & 1,624 & 1,084 & 545 & 9 \\
\hline Takeover/Scheme Announcements & $32.767 * * *$ & $95.470 * * *$ & $238.574 * * *$ & $371.185 * * *$ & $610.745 * * *$ & $755.234 * * *$ & $522.983 * * *$ & 801.336 \\
\hline$t$-value & 2.67 & 4.96 & 5.45 & 4.42 & 4.42 & 3.76 & 3.01 & 1.71 \\
\hline $\mathbf{N}$ & 8,750 & 8,208 & 7,048 & 5,387 & 3,943 & 2,549 & 1,317 & 23 \\
\hline Issued Capital & $80.298 * * *$ & $188.400 * * *$ & $434.295 * * *$ & $673.878 * * *$ & $737.773 * * *$ & $704.455 * * *$ & $779.951 * * *$ & 741.860 \\
\hline$t$-value & 5.61 & 8.93 & 10.69 & 10.04 & 8.81 & 6.75 & 5.16 & 1.70 \\
\hline $\mathbf{N}$ & 7,771 & 7,405 & 6,513 & 5,044 & 3,616 & 2,320 & 1,154 & 16 \\
\hline Asset Acquisition \& Disposal & $75.695 * * *$ & $186.200 * * *$ & $376.810 * * *$ & $547.099 * * *$ & $550.730 * * *$ & $593.225 * * *$ & $694.415 * * *$ & -255.589 \\
\hline$t$-value & 6.72 & 9.50 & 11.23 & 9.08 & 8.10 & 5.87 & 4.03 & -1.15 \\
\hline $\mathbf{N}$ & 45,863 & 43,793 & 38,725 & 30,274 & 21,962 & 14,297 & 7,024 & 118 \\
\hline Progress Report & $153.383 * * *$ & $364.896 * * *$ & $595.787 * * *$ & $827.211 * * *$ & $930.980 * * *$ & $1,067.069 * * *$ & $939.156 * * *$ & $-1,271.655$ \\
\hline$t$-value & 25.19 & 32.29 & 29.17 & 23.34 & 18.65 & 13.10 & 7.61 & -1.01 \\
\hline $\mathbf{N}$ & 1,625 & 1,562 & 1,366 & 1,068 & 781 & 486 & 233 & 4 \\
\hline Other & $59.122 * * *$ & $156.299 * * *$ & $265.720 * * *$ & $360.895 * * *$ & $323.803 * *$ & $467.677 * *$ & 709.530* & 265.549 \\
\hline$t$-value & 3.91 & 4.66 & 4.44 & 3.81 & 2.15 & 2.55 & 1.81 & 1.21 \\
\hline \multicolumn{9}{|l|}{ Earnings-related announcements: } \\
\hline $\mathbf{N}$ & 14,424 & 13,696 & 11,926 & 9,110 & 6,606 & 4,379 & 2,181 & 36 \\
\hline Periodic Reports & $41.957 * * *$ & $97.719 * * *$ & $181.487 * * *$ & $263.511 * * *$ & 309.370*** & $316.629 * * *$ & $368.180 * * *$ & $520.730 *$ \\
\hline$t$-value & 8.91 & 11.21 & 12.43 & 9.10 & 6.57 & 4.29 & 3.08 & 1.85 \\
\hline $\mathbf{N}$ & 2,111 & 2,010 & 1,775 & 1,359 & 987 & 640 & 304 & 3 \\
\hline Distribution Announcement & 6.612 & $47.896 * *$ & $89.640 * * *$ & $115.106 * * *$ & 50.062 & 21.996 & 75.039 & -66.252 \\
\hline$t$-value & 0.98 & 2.23 & 2.84 & 3.29 & 1.12 & 0.63 & 1.45 & -0.92 \\
\hline \multicolumn{9}{|l|}{ Quarterly reports: } \\
\hline $\mathbf{N}$ & 15,233 & 14,743 & 13,432 & 10,769 & 8,014 & 5,323 & 2,682 & 47 \\
\hline Quarterly Activities Report & 0.463 & $25.385 * * *$ & $47.946 * * *$ & $64.228 * * *$ & $49.898 * * *$ & -13.529 & -34.682 & $-159.502 *$ \\
\hline$t$-value & 0.19 & 4.38 & 6.81 & 5.99 & 3.31 & -0.54 & -0.64 & -1.75 \\
\hline $\mathbf{N}$ & 6,379 & 6,201 & 5,666 & 4,638 & 3,565 & 2,356 & 1,165 & 22 \\
\hline Quarterly Cash Flow Report & 2.146 & $19.715 * * *$ & $32.134 * * *$ & $47.921 * * *$ & 25.171 & -21.247 & 11.408 & 19.097 \\
\hline$t$-value & 0.56 & 3.81 & 4.06 & 3.50 & 1.22 & -0.66 & 0.29 & 0.38 \\
\hline $\mathbf{N}$ & 5,688 & 5,502 & 4,955 & 3,896 & 2,893 & 1,919 & 989 & 22 \\
\hline CTE Quarterly Reports & $18.477 * * *$ & $45.216 * * *$ & $75.007 * * *$ & $75.082 * * *$ & $62.485 * * *$ & 59.303 & 8.713 & 2.456 \\
\hline$t$-value & 3.83 & 5.81 & 5.12 & 3.15 & 2.77 & 1.39 & 0.30 & 0.06 \\
\hline
\end{tabular}




\begin{tabular}{|c|c|c|c|c|c|c|c|c|}
\hline Main Category & ATV10 & ATV 15 & ATV 30 & ATV 60 & ATV 90 & ATV 120 & ATV 150 & ATV 180 \\
\hline \multicolumn{9}{|l|}{ ASX-related announcements: } \\
\hline $\mathbf{N}$ & 6,443 & 5,830 & 4,741 & 3,574 & 2,644 & 1,746 & 931 & 11 \\
\hline ASX Announcement & 1.624 & 2.056 & $483.663 * * *$ & $799.186 * * *$ & $948.073 * * *$ & $1,334.545 * * *$ & $1,769.970$ & 614.989 \\
\hline$t$-value & 0.13 & 0.14 & 6.75 & 3.94 & 2.67 & 2.10 & 1.21 & 0.99 \\
\hline $\mathbf{N}$ & 2,246 & 2,120 & 1,871 & 1,528 & 1,165 & 800 & 434 & 6 \\
\hline ASX Query & $41.647 *$ & $209.275 * * *$ & $354.883 * * *$ & $413.828 * * *$ & 19.416 & $-407.288 * *$ & $-1639.534 * * *$ & -3733.586 \\
\hline$t$-value & 1.77 & 4.48 & 5.47 & 3.82 & 0.12 & -2.13 & -5.58 & -1.13 \\
\hline \multicolumn{9}{|c|}{ Administrative announcements: } \\
\hline $\mathbf{N}$ & 709 & 669 & 561 & 431 & 321 & 220 & 108 & 0 \\
\hline Security Holder Details & 227.000 & $301.204 *$ & 338.361 & 410.045 & $688.339 * *$ & 842.727* & $1,092.110$ & - \\
\hline$t$-value & 1.60 & 1.93 & 1.58 & 1.38 & 2.01 & 1.69 & 1.06 & - \\
\hline $\mathbf{N}$ & 230 & 220 & 193 & 154 & 123 & 79 & 42 & 1 \\
\hline Notice of Meeting & 65.372 & 144.397 & $551.316 * *$ & 995.131** & $517.858 * * *$ & 409.893* & 819.097* & -161.011 \\
\hline$t$-value & 1.14 & 1.44 & 2.34 & 2.36 & 2.97 & 1.72 & 1.92 & - \\
\hline $\mathbf{N}$ & 1,128 & 1,071 & 948 & 754 & 585 & 403 & 192 & 2 \\
\hline Company Administration & $28.149 * *$ & $94.634 * * *$ & $156.916 * * *$ & 143.585 & 202.354* & 303.139* & 44.080 & 411.350 \\
\hline$t$-value & 2.12 & 3.89 & 3.27 & 1.49 & 1.92 & 1.91 & 0.12 & 0.98 \\
\hline $\mathbf{N}$ & 1 & 1 & 1 & 1 & 0 & 0 & 0 & 0 \\
\hline Notice of Call & 0 & 0 & 0 & 0 & - & - & - & - \\
\hline$t$-value & - & - & - & - & - & - & - & - \\
\hline $\mathbf{N}$ & 799 & 766 & 672 & 487 & 315 & 193 & 84 & 2 \\
\hline Chairman's Address & $10.329 * *$ & $46.746 * * *$ & $115.327 * * *$ & $167.778 * * *$ & $256.843 * * *$ & $204.996 * * *$ & 88.916* & -561.995 \\
\hline$t$-value & 2.13 & 4.82 & 5.31 & 4.86 & 4.23 & 3.45 & 1.70 & -0.86 \\
\hline $\mathbf{N}$ & 23 & 22 & 18 & 14 & 10 & 7 & 4 & 0 \\
\hline Letter to Shareholders & 329.193 & 726.411 & $1,334.481$ & 519.948 & 876.984 & $1,202.504$ & $2,118.203$ & - \\
\hline$t$-value & 1.54 & 1.44 & 1.34 & 1.35 & 1.69 & 1.57 & 1.68 & - \\
\hline $\mathbf{N}$ & 1 & 1 & 0 & 0 & 0 & 0 & 0 & 0 \\
\hline Structured Products & 22.551 & 43.990 & - & - & - & - & - & - \\
\hline$t$-value & - & - & - & - & - & - & - & - \\
\hline
\end{tabular}


The research sample is reduced when calculating CARs, $\triangle$ CARs and ATVs over time, as the maximum examining window is three hours. For instance, when calculating 60 minute CARs, the announcements released before 3.00 p.m. are covered. When calculating 60 minute $\triangle \mathrm{CARs}$ or ATVs, the announcements released between 11.00a.m. and 3.00p.m. are covered. The 180 minute $\triangle \mathrm{CAR}$ and ATV $t$-test results are not valid statistically due to the small number of observations, because only the announcements released exactly at $1.00 \mathrm{p} . \mathrm{m}$. are covered. ${ }^{39}$ When interpreting the $t$-test results, this study focuses on the announcement categories as it is easier and clearer to explain the results according to each category (19 categories in total) than tables (Table 6-5-6-10). The differences between the $t$-test results of the full sample and market-sensitive subsamples confirm that, to some extent, the ASX gets the 'market-sensitivity tag' right, because the share price reactions are driven mostly by the market-sensitive announcements. There are lower CAR/ $\triangle \mathrm{CAR} / \mathrm{ATV}$ values and fewer CARs/ $\triangle \mathrm{CARs} / \mathrm{ATV}$ s that pass the statistical $t$-test for all categories in the non-market-sensitive announcement subsamples (detailed $t$-test results are shown in Appendix III). When analysing the results, this study divides the 19 categories into five groups according to the announcement category definition, and Section 6.2 focuses on CAR figures to avoid repetition. The five announcement groups are as follows.

Investment/project-related announcements: Takeover/Scheme Announcements, Issued Capital, Asset Acquisition \& Disposal, Progress Reports, and Other. Announcement category Other is classified here because it may have important announcements such as open briefings, a type of market update on issues about which investors are concerned.

Earnings-related announcements: Periodic Reports and Distribution Announcements.

\footnotetext{
${ }^{39}$ The 180 minute $\triangle$ CARs / ATVs require valid trading price / volume data 180 minutes before and after the announcements are released. The normal trading hours are six hours from 10.00a.m. to 4.00p.m..
} 
Quarterly reports: Quarterly Activities Reports, Quarterly Cash Flow Reports and CTE Quarterly Reports.

ASX-related announcements: ASX Announcement and ASX Query.

Administrative announcements: Security Holder Details, Notice of Meeting, Company Administration, Notice of Call, Chairman's Address, Letter to Shareholders, and Structured Products. Administrative announcements are mainly non-market-sensitive, which may not cause significant market responses. Although Structured Products are not administrative information, announcements in Structured Products are unlikely to be material because few such announcements are classified as market-sensitive.

\subsubsection{Investment/project-related Announcements}

There are five announcement categories in the Investment/project-related announcement group: Takeover/Scheme Announcements, Issued Capital, Asset Acquisition \& Disposal, Progress Reports, and Other. The $t$-test results show that all five categories have robust positive market responses. Both $\mathrm{CAR}$ and $\triangle \mathrm{CAR} t$-test show consistent patterns although there are slight differences in the mean values and the peak time.

\section{Takeover/Scheme Announcements}

The intraday CAR increases with time, peaking at $0.441 \%, 120$ minutes after the announcement is released; then there is a slight decrease at 150 and 180 minutes. Restricting the samples to the market-sensitive announcements, the CAR pattern is similar to the full sample over time, but with a higher value (the peak is $0.779 \%$ at 120 minutes). A relevant pattern can be seen when dealing with the trading volume because the trading activities increase until the CARs reach their highest level at 120 minutes: thereafter there are no significant ATVs based on the 
full sample. In the market-sensitive subsample, the ATVs also decrease from 755.234 at 120 minutes to 522.983 at 150 minutes.

\section{Issued Capital}

As to Issued Capital, consistent CAR/ $\triangle \mathrm{CAR}$ results are only observed in the market-sensitive subsample, which means only market-sensitive announcements have robust price reactions. In the market-sensitive subsample, the CAR increases over time, peaking at $0.535 \%, 150$ minutes after the announcement is released; then there is a slight decrease at 180 minutes. Trading activities increase in the market-sensitive subsample where CARs are positive.

\section{Asset Acquisition \& Disposal}

This announcement category has the second-largest share price reaction. The intraday CAR increases with time, peaking at 1.190\%, 120 and 150 minutes after the announcement is released; then there is a slight decrease at 180 minutes. Looking at the market-sensitive subsample, CARs have higher values (the peak is $1.520 \%$ at 120 minutes). Trading activities keep increasing until the CARs reach their highest level at 150 minutes, based on the full sample (474.594) and the market-sensitive subsample (694.415).

\section{Progress Report}

This announcement category has the largest positive share price reaction. The intraday CAR increases with time, peaking at $1.250 \%, 90$ minutes after the announcement is released, and then stabilises at this level. The market-sensitive announcement CAR peak is $1.770 \%$ at 60 minutes, and then stabilises at this level for one hour, before declining slightly to $1.710 \%$ at 180 minutes. Trading activities increase with time, peaking at 120 minutes: 715.668 for the full 
sample and 1,067.069 for the market-sensitive subsample. There is a slight ATV decrease at 150 minutes for the full sample and market-sensitive subsample.

\section{Other}

The intraday CAR increases with time, peaking at $0.490 \%, 150$ minutes after the announcement is released; then there is a slight decrease at 180 minutes. In the market-sensitive announcements, the CAR first peaks $0.878 \%$ at 90 minutes and again with $0.950 \%$ at 180 minutes. Trading volumes increase to peak at 150 minutes: 246.601 for the full sample, and 709.530 for the market-sensitive subsample.

\subsubsection{Earnings-related Announcements}

There are two types of announcements which are directly related to earnings information: Periodic Reports and Distribution Announcements. The $t$-test results also show robust positive price reactions to the two categories. Investment/project and Earnings-related announcements are normally interpreted as important announcements. It is not surprising that these announcements tend to have robust and large market responses based on the $t$-test results.

\section{Periodic Reports}

The intraday CAR increases with time, peaking at $0.222 \%, 120$ minutes after the announcement is released; then there is a slight decrease at 150 and 180 minutes. For market-sensitive announcements, CARs have a higher value (the peak is $0.499 \%$ at 120 minutes). ATVs increase with time, peaking at 110.248 at 90 minutes; then decrease slightly. Trading activities keep increasing as time passes in the market-sensitive subsample. 


\section{Distribution Announcement}

The intraday CAR increases with time, peaking at $0.277 \%, 180$ minutes after the announcement is released. The market-sensitive announcement CARs have higher values (the peak is $0.797 \%$ at 180 minutes). A different pattern can be seen when dealing with the trading volume. For the full sample and the market-sensitive subsample, the trading volumes increase only within 60 minutes, indicating that investors tend to hold on to their shares.

\subsubsection{Quarterly Reports}

There are three types of quarterly reports: Quarterly Activities Report, Quarterly Cash Flow Reports and CTE Quarterly Reports. Quarterly reports meet disclosing requirements for special companies, such as mining companies, oil and gas companies, investment companies and newly-listed companies with cash as their main asset. The $t$-test results with no consistent market reactions to Quarterly Activities/Cash Flow Report may be due to the high risk and unstable nature of mining companies and oil and gas companies. There are some positive market reactions to CTE Quarterly Reports.

\section{Commitments Test Entity (CTE) Quarterly Reports}

The significant CAR shows from 30 minutes after the announcements are released. It increases with time, peaking at $0.489 \%, 150$ minutes after release; then there is a slight decrease at 180 minutes. The market-sensitive announcement CARs have higher values (the peak is $0.463 \%$ at 150 minutes). Significant ATVs exist within 90 minutes while CARs and $\triangle$ CARs last longer. Trading activities increase with time until 60 minutes; then there is a light decrease at 90 minutes in the full sample and the market-sensitive subsample. 


\subsubsection{ASX-related Announcements}

Two types of announcements relate to the stock market ASX: ASX Announcement and ASX Query. Because ASX Announcements are more likely to be related to trading halts, there are few significant market responses, especially price reactions. ASX Query is the only category that induces robust negative market reactions.

\section{ASX Query}

The largest negative CAR is observed at $-1.220 \%, 120$ minutes after the announcement is released; then slightly less negative at 150 and 180 minutes. The ASX Query differs from other announcement categories. Most ASX Queries refer to an ASX Price Query. Most of the time, a company receives an ASX Price Query after its share price changes significantly over a short period. The query is released when the company responds to it. If the company does not provide any new information to explain the share price variation in its response, the market reacts to the price query reversely (Drienko \& Sault, 2013). In the market-sensitive subsample, the CARs have much larger negative values $(-1.440 \%$ at 120 minutes). A relevant pattern can be seen when dealing with the trading volume. Trading activities decrease at 120 minutes when CARs reach their highest negative value, although positive ATVs exist within 60 minutes. This ATV pattern of ASX Queries is consistent with Drienko and Sault (2013): it suggests that the ASX Queries help correct investors' trading activities to some extent, as significant trading price and volume fluctuations are the main reasons for issuing a ASX Query. 


\subsubsection{Administrative Announcements}

Administrative announcements include Security Holder Details, Notice of Meeting, Company Administration, Notice of Call, Chairman's Address, Letter to Shareholders, and Structured Products. These announcement categories are suspected to be non-material, because the majority announcements in each category are classified as non-market-sensitive according to the descriptive analysis in this chapter.

\section{Security Holder Details}

The intraday CAR increases with time, peaking at $0.153 \%, 120$ minutes after the announcement is released; then there is a slight decrease at 150 and 180 minutes. The market-sensitive announcement CARs have higher values (the peak is $1.160 \%$ at 120 minutes). Although the $t$ tests show robust positive price reactions, the ATVs are relatively small in this announcement category and no consistent significant ATVs are observed for the market-sensitive subsample, indicating small market responses. The negative ATVs after 90 minutes in the full sample are caused by non-market-sensitive announcements. A possible explanation is that investors trade more in the morning than afternoon if there is no material information in the market. For longer time windows, such as $[0,120 \mathrm{~min}]$ and $[0,150 \mathrm{~min}]$, the $t$-test samples only cover announcements released between 12:00noon - 2:00p.m. and 12:30pm - 1:30pm.

\section{Notice of Meeting}

There are no consistent market reactions observed although some positive ATVs exist in the market-sensitive announcements from 30 minutes. Negative ATVs are also observed at 120 and 150 minutes, which may confirm that investors trade more in the morning than afternoon when there is no material information. 
The $t$-test results are not robust or significant for other categories: Company Administration, Notice of Call, Chairman's Address, Letter to shareholders and Structured Products.

These $t$-test results provide some insights into the market's immediate reactions to different types of announcements. On average, the market reacts to different announcements differently, depending on the nature and predictability of the information. Investment/project and earningsrelated announcements can cause positive market reactions. An ASX Query is the only category with negative market reactions. The $t$-tests also confirms there are small or even no market reactions to administrative announcements. In addition, there are larger price and volume reactions to unexpected announcements (Progress Report, Asset Acquisition \& Disposal, Takeover/Scheme Announcements, and ASX Query) than expected announcements (Period Reports and Distribution Announcements). This conclusion is further confirmed by the regression results.

\subsection{Immediate Price and Volume Reaction Regressions}

\subsubsection{Descriptive Analysis for the Regression Samples}

Table 6-14 shows the statistical summary of the regression sample for the immediate market reactions to trading hour announcements. The table covers the market-sensitive announcements, and the price/volume change around the release of those announcements. ${ }^{40}$ In order to control

\footnotetext{
${ }^{40}$ This restriction is because of multicollinearity issues which occur when testing market reactions to non-market sensitive announcements. For non-market sensitive announcement regressions, the Variance Inflation Factor (VIF) values of the independent variables (announcement types) are larger than 10, suggesting serious multicollinearity problems exist. In future research, I will try to find other ways to categorise announcements and run the test for separate group of announcements.
} 
the GFC impacts, the regressions are run for three time periods respectively: before the GFC (before 2008), during the GFC (from 2008 to 2010), and after the GFC (since 2010).

In Table 6-11, the means of CARs, $\triangle \mathrm{CARs}$ and ATVs are based on all types of announcements, not for each category (the $t$-tests show each category's mean). Larger market reactions are observed during the GFC, and smaller reactions after it. Average CARs increase with time in general, while average $\triangle \mathrm{CARs}$ start dropping from 90 minutes. This difference could be caused by the reduced samples when calculating $\triangle$ CARs. The normal trading hours are 10:00a.m. 4:00p.m. Taking the time window $[0,120 \mathrm{~min}]$ as an example, the CAR120min sample covers announcements released between 10:00a.m. and 2:00p.m. but $\triangle \mathrm{CAR} 120 \mathrm{~min}$ sample covers announcements released between 12 noon and 2:00p.m. because 120 minute share price information is required before and after the announcement release. This may again support that the companies try to release market-sensitive information as early as possible. ${ }^{41}$

Table 6-11 also shows the proportion of each announcement category in the regression sample, and the percentage of total announcements that belong to the Resource or Financial Sector. Progress Reports contain the largest proportion of announcements, at $42.85 \%, 44.12 \%$, and $41.68 \%$ respectively before, during and since the GFC. Three announcement categories have few observations in the samples: Notice of Call, Letter to Shareholders, and Structured Products (fewer than 10 announcements in each period). More than $60 \%$ of the announcements are released by Resource Sector companies, and fewer than $10 \%$ are released by Financial Sector companies.

\footnotetext{
${ }^{41}$ Appendix IV provides the average CARs/ $\triangle \mathrm{CARs}$ /ATVs of the market-sensitive announcements released at different hours (10-11, 11-12, 12-1p.m. etc.), which also shows that announcements released in the morning have larger market responses then those released in the afternoon.
} 
In the immediate market reaction regression models, the dependent (CARs, $\triangle \mathrm{CARs}$ and ATVs) and independent (announcement types) variables are intraday data, while control variables, such as firm size, are annual data. There could be hundreds of announcements released in one firmyear and all such announcements are controlled by the same firm size. As a result, in the immediate market reaction's research samples, the statistical summary of the control variables cannot reveal these variables' real distribution. The descriptive analysis of the annual financial data will be displayed in the Chapter Seven: Research Analysis on the Timeliness of Price Discovery.

Table 6-11. Statistic Summary of the Regressions for the Market Immediate Reactions Panel A: Before the Global Financial Crisis

\begin{tabular}{|c|c|c|c|c|c|c|}
\hline Variable & $\mathbf{N}$ & Mean & Median & Std Dev & Minimum & Maximum \\
\hline CAR10min & 23,132 & 0.0070 & 0.0001 & 0.0305 & -0.3407 & 0.5390 \\
\hline CAR15min & 22,838 & 0.0091 & 0.0003 & 0.0379 & -0.5103 & 0.5110 \\
\hline CAR30min & 21,911 & 0.0102 & 0.0005 & 0.0438 & -0.5327 & 0.7999 \\
\hline CAR60min & 20,128 & 0.0106 & 0.0008 & 0.0489 & -0.5234 & 1.1777 \\
\hline CAR90min & 18,346 & 0.0110 & 0.0011 & 0.0540 & -0.5793 & 1.4648 \\
\hline CAR120min & 16,500 & 0.0111 & 0.0013 & 0.0552 & -0.6110 & 1.3403 \\
\hline CAR150min & 14,921 & 0.0114 & 0.0017 & 0.0563 & -0.5817 & 1.1740 \\
\hline CAR180min & 13,257 & 0.0115 & 0.0019 & 0.0570 & -0.7544 & 1.2232 \\
\hline$\triangle \mathrm{CAR} 10 \mathrm{~min}$ & 22,133 & 0.0070 & 0.0002 & 0.0322 & -0.6270 & 0.5391 \\
\hline$\Delta$ CAR15min & 21,230 & 0.0090 & 0.0004 & 0.0393 & -0.4786 & 0.5113 \\
\hline$\triangle \mathrm{CAR} 30 \mathrm{~min}$ & 18,680 & 0.0101 & 0.0008 & 0.0469 & -1.0568 & 0.8001 \\
\hline$\triangle \mathrm{CAR} 60 \mathrm{~min}$ & 14,147 & 0.0102 & 0.0012 & 0.0557 & -1.1641 & 1.1786 \\
\hline$\triangle \mathrm{CAR} 90 \mathrm{~min}$ & 9,895 & 0.0105 & 0.0016 & 0.0625 & -0.5381 & 1.4679 \\
\hline$\Delta \mathrm{CAR} 120 \mathrm{~min}$ & 5,965 & 0.0099 & 0.0019 & 0.0649 & -0.5376 & 1.3443 \\
\hline$\Delta \mathrm{CAR} 150 \mathrm{~min}$ & 2,618 & 0.0084 & 0.0023 & 0.0637 & -0.3359 & 0.4313 \\
\hline$\Delta \mathrm{CAR} 180 \mathrm{~min}$ & 6 & 0.0446 & 0.0296 & 0.0547 & 0.0002 & 0.1405 \\
\hline ATV10min & 23,069 & 0.1234 & 0.0000 & 0.9370 & -12.0790 & 59.0309 \\
\hline ATV15min & 22,394 & 0.2556 & 0.0035 & 1.8229 & -51.3314 & 80.3254 \\
\hline ATV30min & 20,051 & 0.4053 & 0.0120 & 2.7749 & -54.8598 & 111.4206 \\
\hline ATV60min & 15,830 & 0.5090 & 0.0190 & 3.5297 & -64.9765 & 121.0162 \\
\hline ATV90min & 11,562 & 0.5873 & 0.0190 & 5.1971 & -72.0151 & 286.8731 \\
\hline ATV120min & 7,493 & 0.6606 & 0.0155 & 7.2676 & -83.2805 & 425.6872 \\
\hline ATV150min & 3,696 & 0.5702 & 0.0201 & 7.0542 & -110.3176 & 290.4954 \\
\hline ATV180min & 61 & 0.2938 & 0.0030 & 2.1164 & -4.6950 & 8.9977 \\
\hline Variable & $\mathbf{N}$ & $\begin{array}{l}\text { The } \mathrm{Nu} \\
\text { Announ }\end{array}$ & $\begin{array}{l}\text { ber of } \\
\text { ements }\end{array}$ & \multicolumn{3}{|c|}{ Percentage of Announcements } \\
\hline Takeover / Scheme Announcements & 23,353 & & 417 & & & 0.0179 \\
\hline Security Holder Details & 23,353 & & 114 & & & $0.49 \%$ \\
\hline Periodic Reports & 23,353 & & 3,074 & & & $13.16 \%$ \\
\hline Quarterly Activities Report & 23,353 & & 2,372 & & & $10.16 \%$ \\
\hline Quarterly Cash Flow Report & 23,353 & & 697 & & & $2.98 \%$ \\
\hline Issued Capital & 23,353 & & 1,514 & & & $6.48 \%$ \\
\hline Asset Acquisition \& Disposal & 23,353 & & 1,831 & & & $7.84 \%$ \\
\hline Notice of Meeting & 23,353 & & 24 & & & $0.10 \%$ \\
\hline ASX Announcement & 23,353 & & 484 & & & $2.07 \%$ \\
\hline Distribution Announcement & 23,353 & & 446 & & & $1.91 \%$ \\
\hline
\end{tabular}




\begin{tabular}{|c|c|c|c|c|c|c|}
\hline Progress Reports & 23,353 & & 10,006 & & & $42.85 \%$ \\
\hline Company Administration & 23,353 & & 47 & & & $0.20 \%$ \\
\hline Notice of Call & 23,353 & & 0 & & & $0.00 \%$ \\
\hline Other & 23,353 & & 722 & & & $3.09 \%$ \\
\hline Chairman's Address & 23,353 & & 231 & & & $0.99 \%$ \\
\hline Letter to Shareholders & 23,353 & & 6 & & & $0.03 \%$ \\
\hline ASX Query & 23,353 & & 394 & & & $1.69 \%$ \\
\hline Structured Products & 23,353 & & 1 & & & $0.00 \%$ \\
\hline CTE Quarterly Reports & 23,353 & & 973 & & & $4.17 \%$ \\
\hline Resource Sector & 23,353 & & 14,144 & & & $60.57 \%$ \\
\hline Financial Sector & 23,353 & & 2,181 & & & $9.34 \%$ \\
\hline Panel B: During the Global Financ & Crisis & & & & & \\
\hline Variable & $\mathbf{N}$ & Mean & Median & Std Dev & Minimum & Maximum \\
\hline CAR10min & 22,638 & 0.0053 & 0.0001 & 0.0331 & -0.4693 & 1.0990 \\
\hline CAR15min & 22,392 & 0.0097 & 0.0004 & 0.0484 & -0.7425 & 1.1632 \\
\hline CAR30min & 21,508 & 0.0106 & 0.0007 & 0.0577 & -0.6938 & 1.4338 \\
\hline CAR60min & 19,749 & 0.0111 & 0.0011 & 0.0646 & -0.7409 & 1.1624 \\
\hline CAR90min & 17,925 & 0.0119 & 0.0016 & 0.0696 & -1.0646 & 1.3247 \\
\hline CAR120min & 16,183 & 0.0124 & 0.0018 & 0.0718 & -0.9306 & 1.4388 \\
\hline CAR150min & 14,506 & 0.0123 & 0.0023 & 0.0740 & -0.9828 & 1.6898 \\
\hline CAR180min & 12,744 & 0.0121 & 0.0026 & 0.0739 & -0.9825 & 1.3851 \\
\hline$\Delta$ CAR10min & 21,754 & 0.0053 & 0.0002 & 0.0355 & -0.4699 & 1.0996 \\
\hline$\Delta$ CAR15min & 20,861 & 0.0096 & 0.0005 & 0.0511 & -0.7436 & 1.1637 \\
\hline$\triangle \mathrm{CAR} 30 \mathrm{~min}$ & 18,373 & 0.0100 & 0.0009 & 0.0625 & -0.8082 & 1.4342 \\
\hline$\triangle$ CAR60min & 13,941 & 0.0102 & 0.0014 & 0.0708 & -0.8102 & 0.8639 \\
\hline$\triangle$ CAR90min & 9,642 & 0.0105 & 0.0021 & 0.0770 & -0.9526 & 0.7998 \\
\hline$\Delta$ CAR120min & 5,976 & 0.0095 & 0.0025 & 0.0798 & -0.9301 & 0.8052 \\
\hline$\Delta \mathrm{CAR} 150 \mathrm{~min}$ & 2,675 & 0.0090 & 0.0032 & 0.0873 & -0.7779 & 0.8118 \\
\hline$\Delta$ CAR180min & 7 & 0.0175 & 0.0161 & 0.0299 & -0.0269 & 0.0634 \\
\hline ATV10min & 22,322 & 0.1011 & 0.0000 & 1.3580 & -28.7568 & 61.6333 \\
\hline ATV15min & 21,595 & 0.3219 & 0.0000 & 2.4288 & -32.0931 & 99.1153 \\
\hline ATV30min & 19,410 & 0.5486 & 0.0077 & 4.0865 & -27.1387 & 182.7609 \\
\hline ATV60min & 15,405 & 0.7716 & 0.0137 & 6.2513 & -69.1940 & 231.9647 \\
\hline ATV90min & 11,299 & 0.7850 & 0.0130 & 6.9136 & -76.4323 & 254.1747 \\
\hline ATV120min & 7,511 & 0.7995 & 0.0103 & 7.6790 & -73.5297 & 179.7324 \\
\hline ATV150min & 3,818 & 0.9063 & 0.0098 & 9.1141 & -151.6879 & 157.7119 \\
\hline ATV180min & 46 & 0.5585 & -0.0109 & 2.3876 & -1.5662 & 13.8794 \\
\hline Variable & $\mathbf{N}$ & $\begin{array}{l}\text { The NuI } \\
\text { Announ }\end{array}$ & $\begin{array}{l}\text { nber of } \\
\text { ements }\end{array}$ & Percen & age of Annor & ncements \\
\hline Takeover / Scheme Announcements & 22,639 & & 460 & & & $2.03 \%$ \\
\hline Security Holder Details & 22,639 & & 165 & & & $0.73 \%$ \\
\hline Periodic Reports & 22,639 & & 2,425 & & & $10.71 \%$ \\
\hline Quarterly Activities Report & 22,639 & & 2,752 & & & $12.16 \%$ \\
\hline Quarterly Cash Flow Report & 22,639 & & 1,100 & & & $4.86 \%$ \\
\hline Issued Capital & 22,639 & & 1,426 & & & $6.30 \%$ \\
\hline Asset Acquisition \& Disposal & 22,639 & & 1,406 & & & $6.21 \%$ \\
\hline Notice of Meeting & 22,639 & & 35 & & & $0.15 \%$ \\
\hline ASX Announcement & 22,639 & & 592 & & & $2.61 \%$ \\
\hline Distribution Announcement & 22,639 & & 333 & & & $1.47 \%$ \\
\hline Progress Reports & 22,639 & & 9,989 & & & $44.12 \%$ \\
\hline Company Administration & 22,639 & & 137 & & & $0.61 \%$ \\
\hline Notice of Call & 22,639 & & 0 & & & $0.00 \%$ \\
\hline Other & 22,639 & & 257 & & & $1.14 \%$ \\
\hline Chairman's Address & 22,639 & & 174 & & & $0.77 \%$ \\
\hline Letter to Shareholders & 22,639 & & 4 & & & $0.02 \%$ \\
\hline ASX Query & 22,639 & & 622 & & & $2.75 \%$ \\
\hline Structured Products & 22,639 & & 0 & & & $0.00 \%$ \\
\hline CTE Quarterly Reports & 22,639 & & 762 & & & $3.37 \%$ \\
\hline Resource Sector & 22,639 & & 15,294 & & & $67.56 \%$ \\
\hline Financial Sector & 22,639 & & 1,905 & & & $8.41 \%$ \\
\hline
\end{tabular}




\begin{tabular}{|c|c|c|c|c|c|c|}
\hline \multicolumn{7}{|c|}{ Panel C: After the Global Financial Crisis } \\
\hline Variable & $\mathbf{N}$ & Mean & Median & Std Dev & Minimum & Maximum \\
\hline CAR10min & 21,625 & 0.0042 & 0.0001 & 0.0326 & -0.6932 & 0.6940 \\
\hline CAR15min & 21,377 & 0.0065 & 0.0002 & 0.0435 & -0.6935 & 0.6943 \\
\hline CAR30min & 20,512 & 0.0075 & 0.0003 & 0.0516 & -0.7316 & 0.8482 \\
\hline CAR60min & 18,776 & 0.0081 & 0.0005 & 0.0599 & -0.8983 & 1.1882 \\
\hline CAR90min & 17,149 & 0.0080 & 0.0006 & 0.0636 & -0.8113 & 1.1368 \\
\hline CAR120min & 15,426 & 0.0079 & 0.0008 & 0.0672 & -0.8106 & 1.1306 \\
\hline CAR150min & 13,803 & 0.0083 & 0.0009 & 0.0691 & -0.7719 & 1.0991 \\
\hline CAR180min & 12,104 & 0.0080 & 0.0011 & 0.0711 & -0.8106 & 1.0995 \\
\hline$\triangle \mathrm{CAR} 10 \mathrm{~min}$ & 20,546 & 0.0041 & 0.0001 & 0.0348 & -0.6942 & 0.6948 \\
\hline$\Delta$ CAR15min & 19,676 & 0.0062 & 0.0002 & 0.0456 & -0.6948 & 0.6949 \\
\hline$\triangle \mathrm{CAR} 30 \mathrm{~min}$ & 17,234 & 0.0072 & 0.0004 & 0.0549 & -0.7321 & 0.8482 \\
\hline$\triangle$ CAR60min & 13,054 & 0.0077 & 0.0007 & 0.0656 & -0.6943 & 1.1909 \\
\hline$\Delta$ CAR90min & 9,259 & 0.0073 & 0.0009 & 0.0734 & -0.7561 & 1.1405 \\
\hline$\Delta \mathrm{CAR} 120 \mathrm{~min}$ & 5,677 & 0.0064 & 0.0013 & 0.0855 & -1.3842 & 1.1799 \\
\hline$\Delta \mathrm{CAR} 150 \mathrm{~min}$ & 2,502 & 0.0051 & 0.0018 & 0.0844 & -0.7352 & 0.6941 \\
\hline$\Delta \mathrm{CAR} 180 \mathrm{~min}$ & 5 & -0.0655 & -0.0169 & 0.1331 & -0.3005 & 0.0192 \\
\hline ATV10min & 21,244 & 0.0979 & 0.0000 & 1.2597 & -31.8227 & 101.7178 \\
\hline ATV15min & 20,537 & 0.2572 & 0.0000 & 2.1741 & -64.1096 & 101.6178 \\
\hline ATV30min & 18,359 & 0.4116 & 0.0013 & 3.5804 & -146.0060 & 187.0985 \\
\hline ATV60min & 14,588 & 0.5875 & 0.0066 & 6.1584 & -121.8175 & 355.0693 \\
\hline ATV90min & 10,959 & 0.5955 & 0.0073 & 6.9306 & -128.8577 & 368.7490 \\
\hline ATV120min & 7,308 & 0.5993 & 0.0050 & 9.4145 & -210.6111 & 461.9228 \\
\hline ATV150min & 3,656 & 0.2813 & 0.0053 & 10.3411 & -435.0011 & 225.3376 \\
\hline ATV180min & 59 & -3.9279 & -0.0030 & 19.0946 & -116.7922 & 3.6700 \\
\hline \multicolumn{7}{|c|}{$\begin{array}{l}\text { The Number of } \\
\text { Announcements }\end{array}$} \\
\hline Takeover / Scheme Announcements & 21,628 & & 353 & & & $1.63 \%$ \\
\hline Security Holder Details & 21,628 & & 114 & & & $0.53 \%$ \\
\hline Periodic Reports & 21,628 & & 2,478 & & & $11.46 \%$ \\
\hline Quarterly Activities Report & 21,628 & & 3,027 & & & $14.00 \%$ \\
\hline Quarterly Cash Flow Report & 21,628 & & 1,335 & & & $6.17 \%$ \\
\hline Issued Capital & 21,628 & & 1,250 & & & $5.78 \%$ \\
\hline Asset Acquisition \& Disposal & 21,628 & & 1,176 & & & $5.44 \%$ \\
\hline Notice of Meeting & 21,628 & & 29 & & & $0.13 \%$ \\
\hline ASX Announcement & 21,628 & & 545 & & & $2.52 \%$ \\
\hline Distribution Announcement & 21,628 & & 383 & & & $1.77 \%$ \\
\hline Progress Reports & 21,628 & & 9,014 & & & $41.68 \%$ \\
\hline Company Administration & 21,628 & & 407 & & & $1.88 \%$ \\
\hline Notice of Call & 21,628 & & 0 & & & $0.00 \%$ \\
\hline Other & 21,628 & & 160 & & & $0.74 \%$ \\
\hline Chairman's Address & 21,628 & & 128 & & & $0.59 \%$ \\
\hline Letter to Shareholders & 21,628 & & 3 & & & $0.01 \%$ \\
\hline ASX Query & 21,628 & & 576 & & & $2.66 \%$ \\
\hline Structured Products & 21,628 & & 0 & & & $0.00 \%$ \\
\hline CTE Quarterly Reports & 21,628 & & 650 & & & $3.01 \%$ \\
\hline Resource Sector & 21,628 & & 14,889 & & & $68.84 \%$ \\
\hline Financial Sector & 21,628 & & 1,564 & & & $7.23 \%$ \\
\hline
\end{tabular}




\subsubsection{Share Price Reactions to Different Announcements}

In regression analysis, this study first examines whether or not there are serious multicollinearity problems indicated by VIF values in the models. The regressions tests exclude the Quarterly Activity Report and Quarterly Cash Flow Report due to multicollinearity problems, because the variables Quarterly Activity/Cash Flow Reports, Progress Reports and Resource Sector have large VIF values $(>10)$. The multicollinearity is possibly caused by mining and exploration (i.e. resource sector) companies which are required to disclose both Quarterly Activity Reports and Quarterly Cash Flow Reports. In many cases, Quarterly Activity Reports and Quarterly Cash Flow Reports are combined in one announcement. It would be difficult to explain the results if only exclude one type of quarterly reports. For example, if excluding Quarterly Activity Reports, the independent variable Quarterly Cash Flow Reports cannot represent the announcement category, because many cash flow reports are not covered by the sample. Multi-categorisation is one limitation in this study. Without the quarterly reports, the VIF values for other variables are less than 3.35, indicating no multicollinearity among independent and control variables.

Tables 6-12 - 6-14 show the regression results of market-sensitive announcements when using CARs as dependent variables without separating good and bad news during the pre-GFC, GFC, and post-GFC periods. The subsequent tables, Tables 6-15 - 6-17, show the corresponding robust test results when using $\triangle \mathrm{CARs}$ as dependent variables. When both CAR tests and $\triangle \mathrm{CAR}$ tests show consistently significant results, it can be argued that the share price movements are caused by announcement releases in the specific category. The $\triangle \mathrm{CAR}$ measures the change of CAR over the same time, which controls the influences from the macroeconomic environment and concurrent or off-hour disclosures to some extent. For example, if it is a bull market day, 
the $\triangle \mathrm{CAR}$ measure can counteract the price effects from the macroeconomic environment. Additionally, if a company's share price increases significantly for the whole day due to a market-sensitive announcement released in the morning, the $\triangle \mathrm{CAR}$ measure can also counteract this impact. This is because it is unlikely that two different types of market-sensitive announcements are released simultaneously. Even so, the research sample contains enough observations to eliminate the rare and random impacts from statistical tests.

In Tables 6-12 -6-17, the significant coefficients indicate the average magnitudes of share price reactions to different types of announcements across different time windows. Without taking good/bad news into account, the market tends to react positively to trading hour announcements, except for ASX Queries which induce negative share price reactions. All other significant results show positive price reactions, regardless of the GFC influence. According to the coefficients, the announcements from the largest to the smallest positive share price reactions are as follows.

Before the GFC: Progress Reports, Asset Acquisition \& Disposal, Distribution Announcements, Other, and Periodic Reports.

During the GFC: Asset Acquisition \& Disposal, Progress Reports, Distribution Announcements, Other, Periodic Reports and Issued Capital.

After the GFC: Progress Reports, Asset Acquisition \& Disposal, Distribution Announcements, and Periodic Reports.

This study divides the 19 announcement categories into five groups: investment/project-related announcements, earnings-related announcements, quarterly reports, administrative announcements, and ASX-related announcements. It is worth noting that the announcements 
with robust positive price reactions are either investment/project-related or earnings-related, and that the ASX-related announcement, ASX Query, is the only category which has negative price reactions. The share price reactions to quarterly reports and administrative announcements are not either significant or robust, based on the regression results of CARs and $\triangle$ CARs. The regression analysis focuses on the figures from CAR test results to avoid repetition from the robust $\triangle \mathrm{CAR}$ tests. 
Table 6-12. The Regression Results of Market-sensitive Announcements - Intraday CARs before the GFC

\begin{tabular}{|c|c|c|c|c|c|c|c|c|}
\hline Variable & CAR10min & CAR15min & CAR30min & CAR60min & CAR90min & CAR120min & CAR150min & CAR180min \\
\hline \multirow[t]{2}{*}{ Intercept } & $0.0066 * * *$ & $0.0117 * * *$ & $\mathbf{0 . 0 1 3 3}^{* * * *}$ & $0.0129 * * *$ & $\mathbf{0 . 0 1 3 8}^{* * *}$ & $0.0156 * * *$ & $0.0147 * * *$ & $0.0143 * * *$ \\
\hline & $(7.39)$ & (10.56) & (10.14) & $(8.37)$ & $(7.60)$ & $(7.85)$ & $(6.78)$ & $(5.97)$ \\
\hline \multirow{2}{*}{$\begin{array}{l}\text { Takeover / Scheme } \\
\text { Announcements }\end{array}$} & $0.0030 *$ & $0.0045 * *$ & $0.0047 *$ & $0.0074 * * *$ & $0.0083 * *$ & $0.0111 * * *$ & $0.0104 * * *$ & $0.0104 * *$ \\
\hline & (1.87) & $(2.21)$ & (1.96) & (2.59) & (2.49) & (3.04) & (2.60) & (2.43) \\
\hline \multirow[t]{2}{*}{ Security Holder Details } & $0.0070 * *$ & $0.0105 * * *$ & $0.0108 * *$ & $0.0149 * * *$ & $0.0137 * *$ & $0.0137 * *$ & $0.0228 * * *$ & $0.0222 * * *$ \\
\hline & $(2.45)$ & (2.92) & $(2.54)$ & $(2.96)$ & $(2.37)$ & $(2.19)$ & $(3.42)$ & $(3.15)$ \\
\hline \multirow[t]{2}{*}{ Periodic Reports } & $0.0047 * * *$ & $0.0063 * * *$ & $0.0072 * * *$ & $0.0080 * * *$ & $0.0074 * * *$ & $0.0074 * * *$ & $0.0084 * * *$ & $0.0079 * * *$ \\
\hline & (5.49) & $(5.84)$ & (5.64) & $(5.30)$ & (4.17) & (3.81) & (4.02) & (3.47) \\
\hline \multirow[t]{2}{*}{ Issued Capital } & $0.0017 *$ & 0.0016 & $0.0036 * *$ & $0.0034 * *$ & 0.0022 & 0.0017 & 0.0038 & $0.0042 *$ \\
\hline & (1.74) & $(1.31)$ & $(2.47)$ & $(1.97)$ & $(1.12)$ & $(0.76)$ & $(1.63)$ & $(1.66)$ \\
\hline \multirow{2}{*}{$\begin{array}{l}\text { Asset Acquisition \& } \\
\text { Disposal }\end{array}$} & 0.0073 & $0.0111 * * *$ & $0.0130 * * *$ & $0.0140 * * *$ & $0.0142 * * *$ & $0.0147 * * *$ & $0.0158 * * *$ & $0.0138 * * * *$ \\
\hline & $(7.80)$ & $(9.45)$ & (9.39) & (8.60) & (7.48) & (7.11) & (7.15) & (5.77) \\
\hline \multirow[t]{2}{*}{ Notice of Meeting } & -0.0012 & -0.0014 & 0.0027 & 0.0033 & 0.0035 & 0.0036 & 0.0036 & 0.0034 \\
\hline & $(-0.19)$ & $(-0.18)$ & $(0.29)$ & $(0.31)$ & $(0.29)$ & $(0.28)$ & $(0.27)$ & $(0.22)$ \\
\hline \multirow[t]{2}{*}{ ASX Announcement } & -0.0004 & -0.0017 & 0.0014 & 0.0027 & 0.0006 & -0.0018 & 0.0018 & 0.0038 \\
\hline & $(-0.26)$ & $(-0.84)$ & $(0.61)$ & $(0.95)$ & $(0.17)$ & $(-0.48)$ & $(0.46)$ & $(0.88)$ \\
\hline \multirow{2}{*}{$\begin{array}{l}\text { Distribution } \\
\text { Announcement }\end{array}$} & $0.0052 * *$ & $0.0075 * * *$ & $0.0096 * * *$ & $0.0110 * * * *$ & $0.0116 * * *$ & $0.0111 * * *$ & $0.0128 * * * *$ & $0.0109 * *$ \\
\hline & (3.17) & (3.70) & (3.95) & (3.79) & (3.36) & (3.00) & (3.16) & $(2.50)$ \\
\hline \multirow[t]{2}{*}{ Progress Reports } & $0.0127 * * *$ & $0.0177 * * *$ & $0.0188 * * *$ & $0.0193 * * *$ & $0.0194 * * *$ & $0.0189 * * *$ & $0.0194 * * *$ & $0.0188 * * *$ \\
\hline & $(20.55)$ & $(22.60)$ & (20.16) & (17.57) & $(15.05)$ & (13.46) & (12.66) & $(11.23)$ \\
\hline \multirow[t]{2}{*}{ Company Administration } & 0.0049 & $0.0118 * *$ & $0.0110 *$ & 0.0122 & 0.0098 & 0.0027 & 0.0049 & 0.0002 \\
\hline & $(1.05)$ & (2.12) & (1.70) & $(1.62)$ & $(1.17)$ & $(0.29)$ & $(0.48)$ & $(0.02)$ \\
\hline Notice of Call & - & - & - & - & - & - & - & - \\
\hline \multirow[t]{2}{*}{ Other } & $0.0059 * * *$ & $0.0100 * * *$ & $0.0107 * * *$ & $0.0119 * * *$ & $0.0124 * * *$ & $0.0117 * * *$ & $0.0123 * * *$ & $0.0133 * * *$ \\
\hline & (4.34) & $(6.20)$ & $(5.63)$ & (5.40) & (4.84) & (4.23) & (4.14) & (4.17) \\
\hline \multirow[t]{2}{*}{ Chairman's Address } & 0.0024 & $0.0046 *$ & 0.0037 & 0.0057 & $0.0067 *$ & 0.0056 & 0.0089* & $0.0095 *$ \\
\hline & $(1.13)$ & $(1.75)$ & $(1.20)$ & $(1.63)$ & (1.66) & $(1.27)$ & (1.89) & $(1.93)$ \\
\hline
\end{tabular}




\begin{tabular}{|c|c|c|c|c|c|c|c|c|}
\hline Variable & CAR10min & CAR15min & CAR30min & CAR60min & CAR90min & CAR120min & CAR150min & CAR180min \\
\hline \multirow{2}{*}{ Letter to Shareholders } & -0.0007 & 0.0169 & $0.0435 * *$ & 0.0265 & 0.0416* & $\mathbf{0 . 1 0 2 1} * * *$ & $\mathbf{0 . 0 8 9 3}^{*} * *$ & $\mathbf{0 . 0 7 5 2} * *$ \\
\hline & $(-0.06)$ & (1.12) & (2.03) & (1.23) & (1.75) & $(3.25)$ & (2.78) & (2.31) \\
\hline \multirow[t]{2}{*}{ ASX Query } & $-0.0085 * * *$ & $-0.0124 * * *$ & $-0.0141 * * *$ & $-0.0154 * * *$ & $-0.0164 * * *$ & $-0.0212 * * *$ & $-0.0192 * * *$ & $-0.0243 * * *$ \\
\hline & $(-5.11)$ & $(-6.14)$ & $(-5.87)$ & $(-5.30)$ & $(-4.71)$ & $(-5.38)$ & $(-4.25)$ & $(-4.79)$ \\
\hline \multirow[t]{2}{*}{ Structured Products } & 0.0020 & 0.0040 & - & - & - & - & - & - \\
\hline & $(0.07)$ & $(0.11)$ & - & - & - & - & - & - \\
\hline \multirow[t]{2}{*}{ CTE Quarterly Reports } & -0.0012 & $-0.0033^{* *}$ & -0.0025 & -0.0008 & -0.0017 & -0.0001 & 0.0007 & -0.0010 \\
\hline & $(-1.06)$ & $(-2.23)$ & $(-1.43)$ & $(-0.39)$ & $(-0.66)$ & $(-0.02)$ & $(0.22)$ & $(-0.28)$ \\
\hline \multirow[t]{2}{*}{ Bid-Ask Spread } & 0.0000 & -0.0026 & -0.0006 & 0.0022 & 0.0055 & 0.0075 & 0.0018 & -0.0025 \\
\hline & $(0.01)$ & $(-0.53)$ & $(-0.11)$ & $(0.32)$ & $(0.62)$ & $(0.80)$ & $(0.20)$ & $(-0.16)$ \\
\hline \multirow[t]{2}{*}{ Size } & $-0.0015 * * *$ & $-0.0023 * * *$ & $-0.0025 * * *$ & $-0.0027 * * *$ & $-0.0029 * * *$ & $-\mathbf{0 . 0 0 3 0} * * *$ & $-0.0031 * * *$ & $-0.0029 * * *$ \\
\hline & $(-12.78)$ & $(-16.98)$ & $(-15.7)$ & $(-14.33)$ & $(-13.18)$ & $(-12.66)$ & $(-11.94)$ & $(-10.44)$ \\
\hline \multirow[t]{2}{*}{ Book-to-Market Ratio } & $-0.0011 * * *$ & $-0.0012 * * *$ & $-0.0013 * * *$ & -0.0006 & -0.0006 & -0.0012 & -0.0007 & -0.0009 \\
\hline & $(-3.35)$ & $(-2.93)$ & $(-2.62)$ & $(-1.05)$ & $(-0.93)$ & $(-1.63)$ & $(-0.83)$ & $(-0.88)$ \\
\hline \multirow[t]{2}{*}{ Leverage } & $0.0001 * * *$ & $0.0001 * * *$ & $0.0001 * * *$ & $0.0001 * * *$ & $0.0001 * * *$ & $0.0001 * * *$ & $0.0001 * * *$ & $0.0001 * * *$ \\
\hline & (4.66) & $(6.20)$ & (6.28) & (4.49) & $(6.46)$ & $(4.82)$ & $(4.25)$ & (4.68) \\
\hline \multirow[t]{2}{*}{ ROA } & -0.0002 & -0.0003 & -0.0005 & $-0.0008 * *$ & $-0.0014 * * *$ & $-0.0013 * * *$ & $-0.0020 * * *$ & $-0.0023 * * *$ \\
\hline & $(-1.00)$ & $(-1.06)$ & $(-1.54)$ & $(-2.09)$ & $(-3.39)$ & $(-3.16)$ & $(-3.51)$ & $(-3.46)$ \\
\hline \multirow[t]{2}{*}{ Resource Sector } & $-0.0013^{* *}$ & $-0.0034 * * *$ & $-0.0045 * * *$ & $-0.0043 * * *$ & $-0.0043 * * *$ & $-0.0056 * * *$ & $-0.0051^{* * *}$ & $-0.0046 * * *$ \\
\hline & $(-2.47)$ & $(-5.36)$ & $(-5.98)$ & $(-4.90)$ & $(-4.22)$ & $(-5.06)$ & $(-4.33)$ & $(-3.64)$ \\
\hline \multirow[t]{2}{*}{ Financial Sector } & $-0.0017 * *$ & $-0.0031 * * *$ & $-0.0040 * * *$ & $-0.0027 *$ & -0.0015 & -0.0021 & -0.0020 & -0.0012 \\
\hline & $(-2.07)$ & $(-3.14)$ & $(-3.41)$ & $(-1.96)$ & $(-0.92)$ & $(-1.24)$ & $(-1.08)$ & $(-0.61)$ \\
\hline Max. VIF & 2.34 & 2.50 & 2.53 & 2.58 & 2.65 & 2.72 & 2.79 & 2.90 \\
\hline F Value & 42.42 & 59.64 & 49.92 & 37.46 & 31.53 & 27.76 & 23.80 & 20.17 \\
\hline R-Square & $4.64 \%$ & $5.71 \%$ & $4.80 \%$ & $3.97 \%$ & $3.68 \%$ & $3.61 \%$ & $3.43 \%$ & $3.28 \%$ \\
\hline Adj R-Sq & $4.53 \%$ & $5.62 \%$ & $4.71 \%$ & $3.86 \%$ & $3.56 \%$ & $3.48 \%$ & $3.29 \%$ & $3.12 \%$ \\
\hline $\mathbf{N}$ & 20,065 & 22,663 & 21,785 & 19,970 & 18,175 & 16,341 & 14,760 & 13,112 \\
\hline
\end{tabular}


Table 6-13. The Regression Results of Market-sensitive Announcements - Intraday CARs during the GFC

\begin{tabular}{|c|c|c|c|c|c|c|c|c|}
\hline Variable & CAR10min & CAR15min & CAR30min & CAR60min & CAR90min & CAR120min & CAR150min & CAR180min \\
\hline \multirow[t]{2}{*}{ Intercept } & $0.0058 * * *$ & $0.0109 * * *$ & $0.0111 * * *$ & $0.0128 * * *$ & $0.0162 * * *$ & $\mathbf{0 . 0 1 7 0} * * *$ & $\mathbf{0 . 0 1 8 3}^{* * *}$ & $\mathbf{0 . 0 1 8 3} * * *$ \\
\hline & $(5.56)$ & $(8.02)$ & $(6.70)$ & $(6.51)$ & $(7.16)$ & $(6.92)$ & $(6.78)$ & $(6.27)$ \\
\hline \multirow{2}{*}{$\begin{array}{l}\text { Takeover / Scheme } \\
\text { Announcements }\end{array}$} & 0.0021 & $0.0055 * *$ & $0.0082 * * *$ & $0.0108 * * *$ & $0.0118 * * *$ & $0.0119 * * *$ & $0.0124 * *$ & $0.0143 * * *$ \\
\hline & $(1.18)$ & $(2.30)$ & $(2.75)$ & (3.02) & $(2.85)$ & (2.59) & $(2.41)$ & (2.59) \\
\hline \multirow[t]{2}{*}{ Security Holder Details } & $0.0052 *$ & $0.0110 * * *$ & $0.0152 * * *$ & $0.0127 * *$ & 0.0085 & 0.0080 & -0.0022 & 0.0016 \\
\hline & $(1.71)$ & $(2.81)$ & $(3.23)$ & $(2.30)$ & $(1.32)$ & $(1.15)$ & $(-0.29)$ & $(0.19)$ \\
\hline \multirow[t]{2}{*}{ Periodic Reports } & $0.0040 * * *$ & $0.0063 * * *$ & $0.0077 * * *$ & $0.0092 * * *$ & $0.0094 * * *$ & $0.0092 * * *$ & $0.0085 * * *$ & $0.0084 * * *$ \\
\hline & $(3.67)$ & $(4.50)$ & $(4.46)$ & $(4.49)$ & $(4.01)$ & $(3.65)$ & $(3.07)$ & $(2.80)$ \\
\hline \multirow[t]{2}{*}{ Issued Capital } & $0.0034 * * *$ & $0.0054 * * *$ & $0.0068 * * *$ & $0.0072 * * *$ & $0.0073 * * *$ & $0.0091 * * *$ & $0.0077 * *$ & 0.0031 \\
\hline & (2.93) & $(3.51)$ & $(3.62)$ & $(3.26)$ & $(2.87)$ & $(3.28)$ & $(2.56)$ & $(0.96)$ \\
\hline \multirow{2}{*}{$\begin{array}{l}\text { Asset Acquisition \& } \\
\text { Disposal }\end{array}$} & $0.0089 * * *$ & $0.0158 * * *$ & $0.0203 * * *$ & $0.0227 * * *$ & $0.0208 * * *$ & $0.0200 * * *$ & $0.0185 * * *$ & $0.0194 * * *$ \\
\hline & (7.45) & (10.17) & (10.78) & (10.24) & (8.26) & (7.34) & (6.26) & (6.18) \\
\hline \multirow[t]{2}{*}{ Notice of Meeting } & 0.0011 & 0.0052 & 0.0049 & -0.0005 & -0.0016 & -0.0010 & 0.0063 & 0.0072 \\
\hline & $(0.17)$ & $(0.64)$ & $(0.50)$ & $(-0.05)$ & $(-0.12)$ & $(-0.07)$ & $(0.40)$ & $(0.42)$ \\
\hline \multirow[t]{2}{*}{ ASX Announcement } & -0.0008 & -0.0019 & -0.0032 & -0.0052 & $-0.0063 *$ & $-0.0071 *$ & $-0.0081 *$ & -0.0058 \\
\hline & $(-0.47)$ & $(-0.87)$ & $(-1.17)$ & $(-1.57)$ & $(-1.68)$ & $(-1.76)$ & $(-1.82)$ & $(-1.18)$ \\
\hline \multirow{2}{*}{$\begin{array}{l}\text { Distribution } \\
\text { Announcement }\end{array}$} & 0.0039* & $0.0077 * * *$ & $0.0117 * * *$ & $0.0123 * * *$ & $0.0124 * *$ & $0.0130 * *$ & $0.0123 * *$ & $0.0181 * * *$ \\
\hline & $(1.71)$ & $(2.63)$ & $(3.22)$ & $(2.85)$ & $(2.51)$ & $(2.43)$ & $(2.14)$ & $(2.90)$ \\
\hline \multirow[t]{2}{*}{ Progress Reports } & $0.0118 * * *$ & $0.0178 * * *$ & $0.0193 * * *$ & $0.0199 * * *$ & $0.0186 * * *$ & $0.0180 * * *$ & $0.0169 * * *$ & $0.0166 * * *$ \\
\hline & (16.80) & (19.25) & $(17.05)$ & $(14.85)$ & (12.10) & $(10.83)$ & $(9.24)$ & $(8.40)$ \\
\hline \multirow[t]{2}{*}{ Company Administration } & 0.0039 & 0.0025 & 0.0033 & 0.0056 & 0.0093 & 0.0097 & 0.0064 & 0.0086 \\
\hline & $(1.18)$ & $(0.59)$ & $(0.64)$ & $(0.93)$ & $(1.39)$ & $(1.36)$ & $(0.83)$ & $(1.04)$ \\
\hline Notice of Call & - & - & - & - & - & - & - & - \\
\hline \multirow[t]{2}{*}{ Other } & 0.0031 & $0.0064 * *$ & $0.0076 * *$ & $0.0115 * * *$ & $0.0126 * * *$ & $0.0136 * *$ & $0.0114 * *$ & 0.0118* \\
\hline & $(1.24)$ & $(2.06)$ & $(1.99)$ & $(2.60)$ & $(2.55)$ & $(2.54)$ & $(1.98)$ & $(1.95)$ \\
\hline \multirow[t]{2}{*}{ Chairman's Address } & 0.0037 & 0.0056 & 0.0043 & 0.0064 & 0.0093 & 0.0078 & 0.0098 & $0.0131 *$ \\
\hline & $(1.23)$ & $(1.47)$ & $(0.94)$ & $(1.22)$ & $(1.61)$ & $(1.27)$ & $(1.45)$ & (1.88) \\
\hline
\end{tabular}




\begin{tabular}{|c|c|c|c|c|c|c|c|c|}
\hline Variable & CAR10min & CAR15min & CAR30min & CAR60min & CAR90min & CAR120min & CAR150min & CAR180min \\
\hline \multirow[t]{2}{*}{ Letter to Shareholders } & 0.0221 & -0.0086 & -0.0401 & $-0.0622 *$ & $-0.0677^{*}$ & -0.0870 ** & $-0.0853^{* *}$ & -0.0068 \\
\hline & $(1.27)$ & $(-0.36)$ & $(-1.41)$ & $(-1.94)$ & $(-1.96)$ & $(-2.46)$ & $(-2.34)$ & $(-0.16)$ \\
\hline \multirow[t]{2}{*}{ ASX Query } & $-0.0051 * * *$ & $-0.0085 * * *$ & $-0.0101 * * *$ & $-0.0099 * * *$ & $-0.0111 * * *$ & $-0.0107 * * *$ & $-\mathbf{0 . 0 1 1 4} * *$ & $-0.0117 * *$ \\
\hline & $(-3.10)$ & $(-4.07)$ & $(-3.93)$ & $(-3.24)$ & $(-3.11)$ & $(-2.68)$ & $(-2.45)$ & $(-2.10)$ \\
\hline Structured Products & - & - & - & - & - & - & - & - \\
\hline \multirow[t]{2}{*}{ CTE Quarterly Reports } & -0.0008 & -0.0022 & -0.0022 & -0.0036 & -0.0008 & -0.0006 & -0.0013 & -0.0016 \\
\hline & $(-0.51)$ & $(-1.06)$ & $(-0.86)$ & $(-1.18)$ & $(-0.21)$ & $(-0.16)$ & $(-0.28)$ & $(-0.34)$ \\
\hline \multirow[t]{2}{*}{ Bid-Ask Spread } & -0.0001 & -0.0002 & 0.0023 & $\mathbf{0 . 0 1 5 3 *}$ & 0.0131 & 0.0206 & 0.0092 & 0.0058 \\
\hline & $(-0.20)$ & $(-0.23)$ & $(0.27)$ & $(1.81)$ & $(1.06)$ & $(1.54)$ & $(0.65)$ & $(0.40)$ \\
\hline \multirow[t]{2}{*}{ Size } & $-0.0013 * * *$ & $-0.0023 * * *$ & $-0.0025 * * *$ & $-0.0028 * * *$ & $-0.0030 * * *$ & $-0.0030 * * *$ & $-0.0030 * * *$ & $-0.0032 * * *$ \\
\hline & $(-9.31)$ & $(-13.87)$ & $(-12.25)$ & $(-11.75)$ & $(-11.04)$ & $(-9.89)$ & $(-9.14)$ & $(-9.28)$ \\
\hline \multirow[t]{2}{*}{ Book-to-Market Ratio } & $-0.0006 * * *$ & $-0.0007 * * *$ & -0.0005 & -0.0004 & -0.0005 & -0.0003 & -0.0006 & -0.0010 \\
\hline & $(-2.90)$ & $(-2.58)$ & $(-1.43)$ & $(-0.90)$ & $(-1.08)$ & $(-0.54)$ & $(-1.04)$ & $(-1.59)$ \\
\hline \multirow[t]{2}{*}{ Leverage } & -0.0001 & -0.0001 & -0.0001 & -0.0001 & $-0.0005 * * *$ & $-0.0008 * * *$ & $-0.0008 * * *$ & -0.0002 \\
\hline & $(-0.79)$ & $(-0.67)$ & $(-0.62)$ & $(-0.21)$ & $(-2.68)$ & $(-4.27)$ & $(-4.25)$ & $(-0.68)$ \\
\hline \multirow[t]{2}{*}{ ROA } & -0.0001 & -0.0001 & -0.0001 & -0.0001 & -0.0001 & -0.0001 & -0.0002 & -0.0002 \\
\hline & $(-0.57)$ & $(-0.16)$ & $(-0.12)$ & $(-0.20)$ & $(-0.83)$ & $(-0.80)$ & $(-1.21)$ & $(-1.16)$ \\
\hline \multirow[t]{2}{*}{ Resource Sector } & -0.0007 & -0.0013 & -0.0016 & $-0.0028 * *$ & $-0.0041 * * *$ & $-0.0050 * * *$ & $-0.0055 * * *$ & $-0.0041 * *$ \\
\hline & $(-0.98)$ & $(-1.52)$ & $(-1.46)$ & $(-2.26)$ & $(-2.91)$ & $(-3.28)$ & $(-3.28)$ & $(-2.30)$ \\
\hline \multirow[t]{2}{*}{ Financial Sector } & -0.0009 & -0.0009 & -0.0019 & -0.0023 & $-0.0043 *$ & $-0.0063 * * *$ & $-0.0065 * *$ & $-0.0065 * *$ \\
\hline & $(-0.82)$ & $(-0.67)$ & $(-1.15)$ & $(-1.17)$ & $(-1.92)$ & $(-2.62)$ & $(-2.47)$ & $(-2.33)$ \\
\hline Max. VIF & 1.97 & 2.07 & 2.09 & 2.13 & 2.19 & 2.23 & 2.27 & 2.35 \\
\hline F Value & 24.31 & 36.57 & 29.72 & 23.91 & 18.3 & 15.96 & 13.2 & 10.81 \\
\hline R-Square & $2.66 \%$ & $3.49 \%$ & $2.97 \%$ & $2.62 \%$ & $2.22 \%$ & $2.14 \%$ & $1.98 \%$ & $1.85 \%$ \\
\hline Adj R-Sq & $2.55 \%$ & $3.40 \%$ & $2.87 \%$ & $2.51 \%$ & $2.10 \%$ & $2.01 \%$ & $1.83 \%$ & $1.68 \%$ \\
\hline $\mathbf{N}$ & 19,566 & 22,240 & 21,395 & 19,609 & 17,781 & 16,040 & 14,368 & 12,631 \\
\hline
\end{tabular}

$*, * *$ and $* * *$ denote significance at the $10 \%, 5 \%$ and $1 \%$ levels respectively (two-tailed). 
Table 6-14. The Regression Results of Market-sensitive Announcements - Intraday CARs after the GFC

\begin{tabular}{|c|c|c|c|c|c|c|c|c|}
\hline Variable & CAR10min & CAR15min & CAR30min & CAR60min & CAR90min & CAR120min & CAR150min & CAR180min \\
\hline \multirow[t]{2}{*}{ Intercept } & $0.0054 * * *$ & $0.0065 * * *$ & $0.0087 * * *$ & $0.0101 * * *$ & $0.0108 * * * *$ & $0.0104 * * *$ & $0.0094 * * *$ & $0.0084 * * *$ \\
\hline & $(5.59)$ & $(5.41)$ & $(5.97)$ & $(5.61)$ & $(5.39)$ & $(4.55)$ & $(3.74)$ & (3.00) \\
\hline \multirow{2}{*}{$\begin{array}{l}\text { Takeover / Scheme } \\
\text { Announcements }\end{array}$} & 0.0030 & $0.0064 * * *$ & $0.0074 * *$ & $0.0095 * *$ & $0.0116 * * *$ & $0.0129 * * *$ & $0.0140 * * *$ & $0.0151 * * *$ \\
\hline & $(1.55)$ & $(2.65)$ & $(2.51)$ & $(2.51)$ & (2.77) & (2.69) & $(2.61)$ & (2.59) \\
\hline \multirow[t]{2}{*}{ Security Holder Details } & 0.0053 & $0.0074 *$ & 0.0070 & 0.0072 & 0.0060 & 0.0095 & 0.0062 & 0.0148* \\
\hline & $(1.59)$ & $(1.79)$ & $(1.41)$ & $(1.20)$ & $(0.89)$ & $(1.30)$ & $(0.79)$ & $(1.72)$ \\
\hline \multirow[t]{2}{*}{ Periodic Reports } & $0.0033 * * *$ & $0.0064 * * *$ & $0.0073 * * *$ & $0.0064 * * *$ & $0.0067 * * *$ & $0.0059 * *$ & $0.0062 * *$ & $0.0089 * * *$ \\
\hline & $(3.29)$ & $(5.14)$ & $(4.82)$ & $(3.40)$ & (3.19) & $(2.48)$ & $(2.39)$ & $(3.10)$ \\
\hline \multirow[t]{2}{*}{ Issued Capital } & $0.0020 *$ & 0.0023 & 0.0022 & 0.0019 & 0.0018 & 0.0022 & 0.0022 & 0.0055 \\
\hline & $(1.71)$ & $(1.60)$ & $(1.29)$ & $(0.89)$ & $(0.72)$ & $(0.80)$ & $(0.73)$ & (1.63) \\
\hline \multirow{2}{*}{$\begin{array}{l}\text { Asset Acquisition \& } \\
\text { Disposal }\end{array}$} & $0.0075 * * *$ & $0.0119 * * *$ & $0.0147 * * *$ & $0.0140 * * *$ & $0.0139 * * *$ & $0.0155 * * *$ & $0.0156 * * *$ & $0.0175 * * *$ \\
\hline & $(6.32)$ & (8.12) & (8.33) & $(6.44)$ & (5.77) & (5.67) & $(5.22)$ & $(5.32)$ \\
\hline \multirow[t]{2}{*}{ Notice of Meeting } & 0.0082 & 0.0097 & 0.0114 & 0.0106 & 0.0143 & 0.0106 & 0.0074 & 0.0122 \\
\hline & $(1.29)$ & $(1.19)$ & $(1.17)$ & $(0.89)$ & $(1.04)$ & $(0.69)$ & $(0.45)$ & $(0.70)$ \\
\hline \multirow[t]{2}{*}{ ASX Announcement } & -0.0006 & -0.0004 & 0.0005 & 0.0006 & -0.0008 & 0.0010 & 0.0013 & 0.0032 \\
\hline & $(-0.37)$ & $(-0.18)$ & $(0.19)$ & $(0.20)$ & $(-0.22)$ & $(0.25)$ & $(0.30)$ & $(0.67)$ \\
\hline \multirow{2}{*}{$\begin{array}{l}\text { Distribution } \\
\text { Announcement }\end{array}$} & $0.0052 * * *$ & $0.0070 * * *$ & $0.0089 * * *$ & $0.0097 * * *$ & $0.0115 * * *$ & $0.0106 * *$ & $0.0119 * *$ & $0.0127 * *$ \\
\hline & $(2.59)$ & $(2.81)$ & $(2.94)$ & $(2.58)$ & $(2.71)$ & $(2.21)$ & $(2.27)$ & (2.19) \\
\hline \multirow[t]{2}{*}{ Progress Reports } & $0.0098 * * *$ & $0.0142 * * *$ & $0.0158 * * *$ & $0.0174 * * *$ & $0.0169 * * *$ & $0.0170 * * *$ & $0.0165 * * *$ & $0.0181 * * *$ \\
\hline & (15.03) & (17.54) & $(16.26)$ & $(14.48)$ & $(12.57)$ & (11.14) & $(9.82)$ & $(9.62)$ \\
\hline \multirow[t]{2}{*}{ Company Administration } & 0.0011 & 0.0021 & 0.0031 & $0.0067 * *$ & $0.0083 * *$ & $0.0101 * *$ & $0.0084 *$ & 0.0069 \\
\hline & $(0.59)$ & $(0.93)$ & $(1.11)$ & $(1.98)$ & $(2.23)$ & $(2.42)$ & $(1.84)$ & $(1.37)$ \\
\hline Notice of Call & - & - & - & - & - & - & - & - \\
\hline \multirow[t]{2}{*}{ Other } & 0.0022 & 0.0045 & 0.0060 & 0.0088* & 0.0084 & 0.0078 & 0.0090 & 0.0101 \\
\hline & $(0.79)$ & $(1.29)$ & $(1.43)$ & $(1.73)$ & $(1.53)$ & $(1.26)$ & $(1.37)$ & $(1.41)$ \\
\hline \multirow[t]{2}{*}{ Chairman's Address } & 0.0035 & 0.0057 & 0.0075 & 0.0064 & 0.0048 & 0.0056 & 0.0002 & -0.0018 \\
\hline & $(1.07)$ & $(1.46)$ & $(1.60)$ & $(1.14)$ & $(0.77)$ & $(0.81)$ & $(0.02)$ & $(-0.23)$ \\
\hline
\end{tabular}




\begin{tabular}{|c|c|c|c|c|c|c|c|c|}
\hline Variable & CAR10min & CAR15min & CAR30min & CAR60min & CAR90min & CAR120min & CAR150min & CAR180min \\
\hline \multirow[t]{2}{*}{ Letter to Shareholders } & 0.0025 & 0.0027 & 0.0016 & 0.0050 & 0.0047 & 0.0050 & 0.0129 & 0.0154 \\
\hline & $(0.13)$ & $(0.11)$ & $(0.05)$ & $(0.12)$ & $(0.11)$ & $(0.10)$ & $(0.26)$ & $(0.31)$ \\
\hline \multirow[t]{2}{*}{ ASX Query } & $-0.0044 * * *$ & $-0.0071 * * *$ & $-0.0083 * * *$ & $-0.0111 * * *$ & $-0.0175 * * *$ & $-0.0213 * * *$ & $-0.0148 * * *$ & -0.0036 \\
\hline & $(-2.77)$ & $(-3.68)$ & $(-3.53)$ & $(-3.69)$ & $(-4.96)$ & $(-5.04)$ & $(-2.88)$ & $(-0.58)$ \\
\hline Structured Products & - & - & - & - & - & - & - & - \\
\hline \multirow[t]{2}{*}{ CTE Quarterly Reports } & $-0.0034 * *$ & -0.0021 & -0.0028 & -0.0040 & -0.0055 & -0.0055 & -0.0058 & -0.0042 \\
\hline & $(-2.22)$ & $(-1.08)$ & $(-1.19)$ & $(-1.33)$ & $(-1.64)$ & $(-1.44)$ & $(-1.36)$ & $(-0.86)$ \\
\hline \multirow[t]{2}{*}{ Bid-Ask Spread } & -0.0034 & -0.0063 & -0.0055 & -0.0005 & -0.0047 & -0.0106 & -0.0106 & -0.0040 \\
\hline & $(-0.92)$ & $(-1.34)$ & $(-0.95)$ & $(-0.07)$ & $(-0.50)$ & $(-1.10)$ & $(-1.02)$ & $(-0.34)$ \\
\hline \multirow[t]{2}{*}{ Size } & $-0.0013 * * *$ & $-0.0018 * * *$ & $-0.0021 * * *$ & $-0.0023 * * *$ & $-0.0024 * * *$ & $-0.0021 * * *$ & $-0.0021 * * *$ & $-0.0020 * * *$ \\
\hline & $(-9.24)$ & $(-10.97)$ & $(-10.8)$ & $(-9.78)$ & $(-9.09)$ & $(-7.16)$ & $(-6.34)$ & $(-5.53)$ \\
\hline \multirow[t]{2}{*}{ Book-to-Market Ratio } & -0.0002 & -0.0003 & -0.0004 & -0.0003 & -0.0002 & 0.0001 & 0.0005 & 0.0007 \\
\hline & $(-1.13)$ & $(-1.34)$ & $(-1.54)$ & $(-0.85)$ & $(-0.57)$ & $(0.16)$ & (1.07) & (1.49) \\
\hline \multirow[t]{2}{*}{ Leverage } & 0.0001 & 0.0001 & 0.0001 & 0.0001 & -0.0001 & -0.0002 & -0.0003 & -0.0003 \\
\hline & $(0.44)$ & $(0.26)$ & $(0.14)$ & $(0.19)$ & $(-0.65)$ & $(-0.86)$ & $(-1.19)$ & $(-1.04)$ \\
\hline \multirow[t]{2}{*}{ ROA } & $-0.0002 * *$ & $-0.0004 * * *$ & $-0.0003 * * *$ & -0.0002 & -0.0002 & -0.0003 & 0.0002 & 0.0002 \\
\hline & $(-1.97)$ & $(-3.94)$ & $(-2.75)$ & $(-1.21)$ & $(-1.36)$ & $(-1.55)$ & $(0.86)$ & (0.89) \\
\hline \multirow[t]{2}{*}{ Resource Sector } & $-0.0012 *$ & -0.0007 & $-0.0018^{*}$ & $-0.0031 * *$ & $-0.0037 * * *$ & $-0.0053 * * *$ & $-0.0044 * * *$ & $-0.0067 * * *$ \\
\hline & $(-1.78)$ & $(-0.82)$ & $(-1.82)$ & $(-2.56)$ & $(-2.80)$ & $(-3.55)$ & $(-2.70)$ & $(-3.78)$ \\
\hline \multirow[t]{2}{*}{ Financial Sector } & -0.0015 & -0.0019 & $-0.0028 *$ & -0.0025 & -0.0029 & -0.0035 & -0.0030 & $-0.0050 *$ \\
\hline & $(-1.41)$ & $(-1.46)$ & $(-1.75)$ & $(-1.26)$ & $(-1.30)$ & $(-1.38)$ & $(-1.08)$ & $(-1.65)$ \\
\hline Max. VIF & 1.80 & 1.84 & 1.86 & 1.89 & 1.93 & 1.98 & 2.03 & 2.10 \\
\hline F Value & 19.77 & 27.59 & 24.13 & 19.31 & 16.55 & 13.33 & 9.61 & 7.97 \\
\hline R-Square & $2.17 \%$ & $2.78 \%$ & $2.54 \%$ & $2.23 \%$ & $2.10 \%$ & $1.89 \%$ & $1.53 \%$ & $1.45 \%$ \\
\hline Adj R-Sq & $2.06 \%$ & $2.68 \%$ & $2.43 \%$ & $2.12 \%$ & $1.97 \%$ & $1.74 \%$ & $1.37 \%$ & $1.26 \%$ \\
\hline $\mathbf{N}$ & 19,672 & 21,228 & 20,402 & 18,637 & 17,012 & 15,285 & 13,657 & 11,968 \\
\hline
\end{tabular}

$*, * *$ and $* * *$ denote significance at the $10 \%, 5 \%$ and $1 \%$ levels respectively (two-tailed). 
Table 6-15. The Regression Results of Market-sensitive Announcements - Intraday $\triangle C A R s$ before the GFC

\begin{tabular}{|c|c|c|c|c|c|c|c|}
\hline Variable & $\triangle \mathrm{CAR} 10 \mathrm{~min}$ & $\triangle \mathrm{CAR} 15 \mathrm{~min}$ & $\Delta$ CAR30min & $\triangle \mathrm{CAR60min}$ & $\triangle$ CAR90min & $\Delta$ CAR120min & $\Delta$ CAR150min \\
\hline \multirow[t]{2}{*}{ Intercept } & $\mathbf{0 . 0 0 7 0} * * *$ & $0.0120 * * *$ & $0.0151 * * *$ & $0.0143 * * *$ & $0.0147 * * *$ & $0.0168 * * *$ & 0.0103* \\
\hline & (7.28) & (10.09) & (9.98) & $(6.93)$ & $(5.32)$ & $(4.57)$ & $(1.79)$ \\
\hline \multirow[t]{2}{*}{ Takeover / Scheme Announcements } & 0.0024 & 0.0033 & 0.0025 & 0.0048 & 0.0069 & 0.0105 & 0.0043 \\
\hline & $(1.43)$ & $(1.54)$ & $(0.91)$ & $(1.29)$ & $(1.43)$ & $(1.64)$ & $(0.43)$ \\
\hline \multirow[t]{2}{*}{ Security Holder Details } & 0.0044 & $0.0089 * *$ & 0.0076 & $0.0175 * *$ & 0.0093 & 0.0115 & 0.0208 \\
\hline & $(1.46)$ & $(2.28)$ & $(1.48)$ & $(2.57)$ & $(1.02)$ & $(0.95)$ & $(1.16)$ \\
\hline \multirow[t]{2}{*}{ Periodic Reports } & $0.0042 * * *$ & $0.0054 * * *$ & $0.0051 * * *$ & $0.0070 * * *$ & $0.0082 * * *$ & $0.0099 * * *$ & 0.0052 \\
\hline & $(4.49)$ & (4.64) & (3.44) & (3.44) & $(3.00)$ & (2.74) & $(0.95)$ \\
\hline \multirow[t]{2}{*}{ Issued Capital } & 0.0012 & 0.0013 & 0.0013 & 0.0009 & 0.0015 & -0.0001 & 0.0036 \\
\hline & $(1.11)$ & $(0.97)$ & $(0.77)$ & $(0.40)$ & $(0.50)$ & $(0.00)$ & $(0.60)$ \\
\hline \multirow[t]{2}{*}{ Asset Acquisition \& Disposal } & $0.0070 * * *$ & $0.0107 * * *$ & $0.0127 * * *$ & $0.0158 * * *$ & $0.0177 * * *$ & $0.0203 * * *$ & $0.0250 * * *$ \\
\hline & $(6.92)$ & $(8.44)$ & $(7.95)$ & (7.18) & $(5.89)$ & $(5.09)$ & (4.23) \\
\hline \multirow[t]{2}{*}{ Notice of Meeting } & -0.0015 & -0.0042 & 0.0015 & 0.0104 & 0.0140 & 0.0197 & 0.0288 \\
\hline & $(-0.23)$ & $(-0.49)$ & $(0.13)$ & $(0.68)$ & $(0.75)$ & $(0.92)$ & $(0.79)$ \\
\hline \multirow[t]{2}{*}{ ASX Announcement } & -0.0004 & -0.0032 & -0.0020 & 0.0012 & -0.0001 & -0.0022 & -0.0044 \\
\hline & $(-0.27)$ & $(-1.51)$ & $(-0.75)$ & $(0.31)$ & $(-0.02)$ & $(-0.33)$ & $(-0.44)$ \\
\hline \multirow[t]{2}{*}{ Distribution Announcement } & $0.0051 * * *$ & $0.0072 * * *$ & $0.0085 * * *$ & $0.0125 * * *$ & $0.0157 * * *$ & $0.0190 * * *$ & $0.0238 * *$ \\
\hline & $(2.91)$ & $(3.31)$ & $(3.06)$ & $(3.29)$ & $(3.05)$ & $(2.72)$ & $(2.30)$ \\
\hline \multirow[t]{2}{*}{ Progress Reports } & $0.0125 * * *$ & $0.0171 * * *$ & $0.0172 * * *$ & $0.0184 * * *$ & $0.0212 * * *$ & $0.0210 * * *$ & $0.0195 * * *$ \\
\hline & (18.67) & (20.31) & (16.19) & (12.75) & (10.99) & $(8.27)$ & (5.11) \\
\hline \multirow[t]{2}{*}{ Company Administration } & 0.0056 & 0.0119* & 0.0076 & $0.0220 * *$ & 0.0061 & -0.0024 & -0.0079 \\
\hline & $(1.09)$ & (1.95) & $(0.96)$ & (2.02) & $(0.43)$ & $(-0.13)$ & $(-0.37)$ \\
\hline Notice of Call & - & - & - & - & - & - & - \\
\hline \multirow[t]{2}{*}{ Other } & $0.0058 * * *$ & $0.0101 * * *$ & $0.0105 * * *$ & $0.0111 * * *$ & $0.0129 * * *$ & $0.0129 * *$ & 0.0117 \\
\hline & $(3.95)$ & $(5.82)$ & $(4.73)$ & $(3.70)$ & $(3.28)$ & $(2.47)$ & $(1.50)$ \\
\hline \multirow[t]{2}{*}{ Chairman's Address } & 0.0030 & $0.0056 * *$ & 0.0045 & 0.0069 & 0.0061 & 0.0064 & 0.0170 \\
\hline & $(1.30)$ & (1.96) & $(1.26)$ & (1.34) & $(0.81)$ & $(0.53)$ & $(0.93)$ \\
\hline
\end{tabular}




\begin{tabular}{|c|c|c|c|c|c|c|c|}
\hline Variable & $\triangle \mathrm{CAR} 10 \mathrm{~min}$ & $\Delta$ CAR15min & $\triangle$ CAR30min & $\triangle \mathrm{CAR60min}$ & $\triangle$ CAR90min & $\Delta$ CAR120min & $\triangle$ CAR150min \\
\hline \multirow[t]{2}{*}{ Letter to Shareholders } & -0.0007 & 0.0167 & 0.0434* & $-0.0642 * *$ & $-0.0708 * *$ & - & - \\
\hline & $(-0.05)$ & $(1.06)$ & (1.88) & $(-2.02)$ & $(-1.99)$ & - & - \\
\hline \multirow[t]{2}{*}{ ASX Query } & $-0.0094 * * *$ & $-0.0131 * * *$ & $-0.0127 * * *$ & $-0.0114 * * *$ & $-0.0091 *$ & $-0.0147 * *$ & -0.0093 \\
\hline & $(-5.24)$ & $(-5.95)$ & $(-4.54)$ & $(-3.07)$ & $(-1.89)$ & $(-2.40)$ & $(-1.02)$ \\
\hline \multirow[t]{2}{*}{ Structured Products } & -0.0047 & -0.0054 & - & - & - & - & - \\
\hline & $(-0.16)$ & $(-0.14)$ & - & - & - & - & - \\
\hline \multirow[t]{2}{*}{ CTE Quarterly Reports } & -0.0013 & $-0.0040 * *$ & $-0.0043 * *$ & -0.0027 & -0.0008 & 0.0064 & 0.0108 \\
\hline & $(-1.05)$ & $(-2.51)$ & $(-2.14)$ & $(-0.95)$ & $(-0.20)$ & $(1.24)$ & $(1.39)$ \\
\hline \multirow[t]{2}{*}{ Bid-Ask Spread } & -0.0001 & -0.0014 & -0.0007 & 0.0029 & 0.0068 & 0.0279 & -0.0009 \\
\hline & $(-0.02)$ & $(-0.27)$ & $(-0.11)$ & $(0.34)$ & $(0.54)$ & $(1.01)$ & $(-0.02)$ \\
\hline \multirow[t]{2}{*}{ Size } & $-0.0015 * * *$ & $-0.0023 * * *$ & $-0.0026 * * *$ & $-0.0028 * * *$ & $-0.0031 * * *$ & $-0.0034 * * *$ & $-0.0023 * * *$ \\
\hline & $(-11.77)$ & $(-15.61)$ & $(-13.78)$ & $(-11.03)$ & $(-9.01)$ & $(-7.41)$ & $(-3.19)$ \\
\hline \multirow[t]{2}{*}{ Book-to-Market Ratio } & $-0.0011 * * *$ & $-0.0012 * * *$ & $-0.0015 * * *$ & -0.0010 & -0.0012 & $-0.0023 *$ & -0.0007 \\
\hline & $(-3.34)$ & $(-2.74)$ & $(-2.81)$ & $(-1.43)$ & $(-1.31)$ & $(-1.82)$ & $(-0.44)$ \\
\hline \multirow[t]{2}{*}{ Leverage } & $0.0001 * * *$ & $0.0001 * * *$ & $0.0001 * * *$ & $0.0001 * * *$ & $0.0001 * * *$ & $0.0001 * *$ & 0.0001 \\
\hline & (3.82) & $(5.40)$ & $(6.65)$ & (3.93) & $(6.84)$ & $(2.05)$ & $(1.00)$ \\
\hline \multirow[t]{2}{*}{ ROA } & -0.0001 & -0.0001 & -0.0001 & -0.0002 & $-0.0010 *$ & -0.0008 & -0.0035 \\
\hline & $(-0.33)$ & $(-0.20)$ & $(-0.31)$ & $(-0.36)$ & $(-1.73)$ & $(-1.34)$ & $(-1.25)$ \\
\hline \multirow[t]{2}{*}{ Resource Sector } & $-0.0013 * *$ & $-0.0033 * * *$ & $-0.0046 * * *$ & $-0.0045 * * *$ & $-0.0060 * * *$ & $-0.0081 * * *$ & $-0.0068 * *$ \\
\hline & $(-2.27)$ & $(-4.75)$ & $(-5.24)$ & $(-3.67)$ & $(-3.6)$ & $(-3.65)$ & $(-2.04)$ \\
\hline \multirow[t]{2}{*}{ Financial Sector } & $-0.0018 * *$ & $-0.0030 * * *$ & $-0.0037 * * *$ & -0.0031 & -0.0015 & -0.0029 & -0.0042 \\
\hline & $(-2.10)$ & $(-2.84)$ & $(-2.70)$ & $(-1.61)$ & $(-0.57)$ & $(-0.86)$ & $(-0.80)$ \\
\hline Max. VIF & 2.33 & 2.47 & 2.43 & 2.39 & 2.37 & 2.32 & 2.34 \\
\hline F Value & 36.11 & 49.59 & 35.59 & 20.19 & 15.38 & 9.03 & 3.58 \\
\hline R-Square & $4.14 \%$ & $5.14 \%$ & $4.05 \%$ & $3.07 \%$ & $3.34 \%$ & $3.13 \%$ & $2.84 \%$ \\
\hline Adj R-Sq & $4.03 \%$ & $5.04 \%$ & $3.94 \%$ & $2.92 \%$ & $3.13 \%$ & $2.78 \%$ & $2.05 \%$ \\
\hline $\mathbf{N}$ & 19,241 & 21,062 & 18,579 & 14,044 & 9,808 & 5,896 & 2,589 \\
\hline
\end{tabular}


Table 6-16. The Regression Results of Market-sensitive Announcements - Intraday $\triangle$ CARs during the GFC

\begin{tabular}{|c|c|c|c|c|c|c|c|}
\hline Variable & $\Delta$ CAR10min & $\Delta$ CAR15min & $\triangle$ CAR30min & $\triangle$ CAR60min & $\triangle$ CAR90min & $\Delta$ CAR120min & $\triangle$ CAR150min \\
\hline \multirow[t]{2}{*}{ Intercept } & $0.0057 * * *$ & $0.0102 * * *$ & $0.0082 * * *$ & $\mathbf{0 . 0 1 0 0} * * *$ & $0.0141 * * *$ & $\mathbf{0 . 0 1 0 0} * *$ & 0.0001 \\
\hline & $(5.03)$ & $(6.87)$ & $(4.25)$ & (3.93) & (3.96) & (2.14) & $(0.00)$ \\
\hline \multirow[t]{2}{*}{ Takeover / Scheme Announcements } & 0.0018 & $0.0044 *$ & $0.0085 * *$ & $0.0098 * *$ & 0.0015 & -0.0012 & 0.0014 \\
\hline & $(0.89)$ & (1.68) & (2.48) & $(2.20)$ & $(0.26)$ & $(-0.15)$ & $(0.11)$ \\
\hline \multirow[t]{2}{*}{ Security Holder Details } & 0.0042 & $0.0101 * *$ & $0.0107 *$ & 0.0053 & -0.0096 & -0.0094 & $-0.0345 *$ \\
\hline & $(1.27)$ & (2.36) & (1.88) & $(0.73)$ & $(-0.99)$ & $(-0.72)$ & $(-1.66)$ \\
\hline \multirow[t]{2}{*}{ Periodic Reports } & $0.0040 * * *$ & $0.0067 * * *$ & $0.0091 * * *$ & $0.0076 * * *$ & $0.0066 *$ & 0.0040 & 0.0054 \\
\hline & (3.37) & (4.37) & $(4.51)$ & $(2.87)$ & (1.89) & $(0.87)$ & $(0.70)$ \\
\hline \multirow[t]{2}{*}{ Issued Capital } & $0.0038 * * *$ & $0.0056 * * *$ & $0.0072 * * *$ & $0.0059 * *$ & 0.0008 & 0.0064 & $0.0167 * *$ \\
\hline & $(3.01)$ & (3.36) & $(3.30)$ & (2.06) & $(0.21)$ & $(1.28)$ & (2.03) \\
\hline \multirow[t]{2}{*}{ Asset Acquisition \& Disposal } & $0.0086^{* * * *}$ & $0.0157 * * *$ & $0.0214 * * *$ & $0.0242 * * *$ & $0.0183^{* * *}$ & $0.0166 * * *$ & $0.0178^{* *}$ \\
\hline & (6.63) & $(9.24)$ & (9.69) & $(8.39)$ & $(4.80)$ & (3.24) & (2.12) \\
\hline \multirow[t]{2}{*}{ Notice of Meeting } & -0.0010 & 0.0041 & 0.0017 & -0.0031 & -0.0161 & -0.0094 & 0.0379 \\
\hline & $(-0.14)$ & $(0.46)$ & $(0.14)$ & $(-0.20)$ & $(-0.81)$ & $(-0.37)$ & $(0.62)$ \\
\hline \multirow[t]{2}{*}{ ASX Announcement } & -0.0009 & -0.0015 & -0.0038 & $-0.0098 * *$ & $-0.0183 * * *$ & $-0.0154 * *$ & -0.0171 \\
\hline & $(-0.54)$ & $(-0.62)$ & $(-1.23)$ & $(-2.39)$ & $(-3.42)$ & $(-2.20)$ & $(-1.50)$ \\
\hline \multirow[t]{2}{*}{ Distribution Announcement } & 0.0040 & $0.0072 * *$ & $0.0114 * * *$ & $0.0112 * *$ & 0.0086 & 0.0111 & -0.0017 \\
\hline & (1.59) & $(2.28)$ & $(2.75)$ & $(2.08)$ & $(1.24)$ & $(1.23)$ & $(-0.11)$ \\
\hline \multirow[t]{2}{*}{ Progress Reports } & $0.0118 * * *$ & $0.0178 * * *$ & $0.0196 * * *$ & $0.0190 * * *$ & $0.0150 * * *$ & $0.0141 * * *$ & $0.0135 * * *$ \\
\hline & $(15.47)$ & (17.65) & (15.04) & (11.25) & (6.69) & $(4.77)$ & $(2.81)$ \\
\hline \multirow[t]{2}{*}{ Company Administration } & 0.0030 & 0.0015 & 0.0067 & 0.0057 & 0.0048 & -0.0002 & 0.0023 \\
\hline & $(0.82)$ & $(0.31)$ & (1.06) & $(0.69)$ & $(0.47)$ & $(-0.01)$ & $(0.11)$ \\
\hline Notice of Call & - & - & - & - & - & - & - \\
\hline \multirow[t]{2}{*}{ Other } & $0.0054 * *$ & $0.0080 * *$ & $0.0104 * *$ & $0.0123 * *$ & $0.0149 * *$ & 0.0134 & 0.0012 \\
\hline & (1.99) & (2.33) & $(2.30)$ & (2.07) & (1.97) & (1.26) & $(0.06)$ \\
\hline \multirow[t]{2}{*}{ Chairman's Address } & 0.0048 & 0.0057 & 0.0029 & -0.0010 & -0.0051 & -0.0086 & -0.0100 \\
\hline & $(1.46)$ & $(1.37)$ & $(0.52)$ & $(-0.13)$ & $(-0.49)$ & $(-0.67)$ & $(-0.42)$ \\
\hline
\end{tabular}




\begin{tabular}{|c|c|c|c|c|c|c|c|}
\hline Variable & $\triangle \mathrm{CAR} 10 \mathrm{~min}$ & $\Delta$ CAR15min & $\triangle$ CAR30min & $\triangle \mathrm{CAR60min}$ & $\triangle$ CAR90min & $\triangle \mathrm{CAR} 120 \mathrm{~min}$ & $\Delta$ CAR150min \\
\hline \multirow[t]{2}{*}{ Letter to Shareholders } & 0.0264 & -0.0010 & $-0.0637^{*}$ & -0.0509 & -0.0544 & $-0.0984 * *$ & $-0.1474 * *$ \\
\hline & $(1.23)$ & $(-0.03)$ & $(-1.78)$ & $(-1.25)$ & $(-1.23)$ & $(-2.14)$ & $(-2.41)$ \\
\hline \multirow[t]{2}{*}{ ASX Query } & $-0.0046 * * *$ & $-0.0073 * * *$ & $-\mathbf{0 . 0 0 7 0} * *$ & $-0.0065 *$ & $-0.0097 * *$ & $-0.0160 * *$ & $-0.0302 * * *$ \\
\hline & $(-2.60)$ & $(-3.20)$ & $(-2.38)$ & $(-1.77)$ & $(-2.15)$ & $(-2.86)$ & $(-3.55)$ \\
\hline Structured Products & - & - & - & - & - & - & - \\
\hline \multirow[t]{2}{*}{ CTE Quarterly Reports } & -0.0009 & -0.0018 & -0.0002 & 0.0010 & 0.0007 & 0.0043 & 0.0104 \\
\hline & $(-0.57)$ & $(-0.8)$ & $(-0.08)$ & $(0.27)$ & $(0.14)$ & $(0.62)$ & $(0.86)$ \\
\hline \multirow[t]{2}{*}{ Bid-Ask Spread } & -0.0001 & -0.0001 & 0.0003 & $0.0220 *$ & 0.0156 & 0.0521 & 0.0087 \\
\hline & $(-0.15)$ & $(-0.18)$ & $(0.03)$ & (1.81) & $(0.59)$ & (1.33) & $(0.13)$ \\
\hline \multirow[t]{2}{*}{ Size } & $-0.0013 * * *$ & $-0.0022 * * *$ & $-0.0023 * * *$ & $-0.0022 * * *$ & $-0.0021 * * *$ & $-0.0010 *$ & 0.0002 \\
\hline & $(-8.73)$ & $(-12.16)$ & $(-9.44)$ & $(-7.08)$ & $(-4.70)$ & $(-1.75)$ & $(0.19)$ \\
\hline \multirow[t]{2}{*}{ Book-to-Market Ratio } & $-0.0008 * * *$ & $-0.0008 * *$ & -0.0002 & 0.0001 & 0.0003 & 0.0005 & 0.0005 \\
\hline & $(-3.23)$ & $(-2.56)$ & $(-0.37)$ & $(0.14)$ & $(0.39)$ & $(0.52)$ & $(0.28)$ \\
\hline \multirow[t]{2}{*}{ Leverage } & -0.0001 & -0.0001 & -0.0001 & -0.0001 & $-0.0006 * * *$ & -0.0008 & -0.0006 \\
\hline & $(-0.77)$ & $(-0.74)$ & $(-1.02)$ & $(-0.69)$ & $(-2.98)$ & $(-1.51)$ & $(-0.72)$ \\
\hline \multirow[t]{2}{*}{ ROA } & -0.0001 & -0.0001 & -0.0001 & -0.0002 & 0.0001 & 0.0010 & -0.0052 \\
\hline & $(-0.54)$ & $(-0.13)$ & $(-0.31)$ & $(-0.84)$ & $(0.07)$ & $(0.44)$ & $(-1.30)$ \\
\hline \multirow[t]{2}{*}{ Resource Sector } & -0.0002 & -0.0009 & -0.0005 & $-0.0029 *$ & $-0.0039 *$ & -0.0047 & 0.0009 \\
\hline & $(-0.25)$ & $(-0.95)$ & $(-0.38)$ & $(-1.75)$ & $(-1.78)$ & $(-1.63)$ & $(0.20)$ \\
\hline \multirow[t]{2}{*}{ Financial Sector } & -0.0006 & -0.0009 & -0.0006 & 0.0000 & -0.0016 & -0.0026 & 0.0028 \\
\hline & $(-0.53)$ & $(-0.60)$ & $(-0.32)$ & $(0.01)$ & $(-0.46)$ & $(-0.58)$ & $(0.36)$ \\
\hline Max. VIF & 1.94 & 2.03 & 2.00 & 1.98 & 2.00 & 2.01 & 2.03 \\
\hline F Value & 20.62 & 29.21 & 20.42 & 12.64 & 7.02 & 3.87 & 2.7 \\
\hline R-Square & $2.35 \%$ & $3.01 \%$ & $2.40 \%$ & $1.97 \%$ & $1.60 \%$ & $1.43 \%$ & $2.22 \%$ \\
\hline Adj R-Sq & $2.24 \%$ & $2.91 \%$ & $2.29 \%$ & $1.82 \%$ & $1.37 \%$ & $1.06 \%$ & $1.40 \%$ \\
\hline $\mathbf{N}$ & 18,871 & 20,721 & 18,271 & 13,844 & 9,551 & 5,906 & 2,637 \\
\hline
\end{tabular}


Table 6-17. The Regression Results of Market-sensitive Announcements - Intraday $\triangle$ CARs after the GFC

\begin{tabular}{|c|c|c|c|c|c|c|c|}
\hline Variable & $\triangle$ CAR10min & $\triangle$ CAR15min & $\Delta$ CAR30min & $\triangle$ CAR60min & $\triangle$ CAR90min & $\triangle$ CAR120min & $\triangle$ CAR150min \\
\hline \multirow[t]{2}{*}{ Intercept } & $\mathbf{0 . 0 0 5 0} * * *$ & $0.0067 * * *$ & $\mathbf{0 . 0 0 7 0} * * *$ & $\mathbf{0 . 0 0 7 0} * * *$ & $0.0065 * *$ & 0.0053 & 0.0059 \\
\hline & $(4.70)$ & $(5.06)$ & $(4.14)$ & (2.99) & (2.09) & $(1.13)$ & $(0.83)$ \\
\hline \multirow[t]{2}{*}{ Takeover / Scheme Announcements } & 0.0029 & $0.0054 * *$ & $0.0070 * *$ & 0.0054 & 0.0068 & 0.0100 & 0.0163 \\
\hline & $(1.36)$ & $(2.04)$ & $(2.06)$ & $(1.08)$ & $(1.06)$ & $(1.03)$ & $(1.06)$ \\
\hline \multirow[t]{2}{*}{ Security Holder Details } & 0.0054 & 0.0042 & 0.0034 & 0.0055 & 0.0038 & -0.0027 & -0.0302 \\
\hline & $(1.43)$ & $(0.90)$ & $(0.54)$ & $(0.64)$ & $(0.34)$ & $(-0.18)$ & $(-1.32)$ \\
\hline \multirow[t]{2}{*}{ Periodic Reports } & $0.0032 * * *$ & $0.0065 * * *$ & $0.0073 * * *$ & $0.0070 * * *$ & $0.0063 *$ & 0.0036 & -0.0012 \\
\hline & $(2.95)$ & $(4.75)$ & $(4.20)$ & $(2.84)$ & $(1.90)$ & $(0.71)$ & $(-0.16)$ \\
\hline \multirow[t]{2}{*}{ Issued Capital } & 0.0015 & 0.0022 & 0.0038* & $0.0061 * *$ & $0.0088 * *$ & 0.0054 & 0.0050 \\
\hline & $(1.22)$ & $(1.44)$ & (1.89) & $(2.16)$ & $(2.27)$ & $(0.93)$ & $(0.57)$ \\
\hline \multirow[t]{2}{*}{ Asset Acquisition \& Disposal } & $0.0073 * * *$ & $0.0119 * * *$ & $0.0155 * * *$ & $0.0141 * * *$ & $0.0114 * * *$ & $0.0123 * *$ & 0.0019 \\
\hline & $(5.61)$ & $(7.42)$ & $(7.48)$ & $(4.97)$ & $(3.03)$ & $(2.17)$ & $(0.22)$ \\
\hline \multirow[t]{2}{*}{ Notice of Meeting } & 0.0074 & 0.0077 & 0.0047 & -0.0033 & -0.0104 & 0.0036 & - \\
\hline & $(1.05)$ & $(0.85)$ & $(0.41)$ & $(-0.20)$ & $(-0.45)$ & $(0.08)$ & - \\
\hline \multirow[t]{2}{*}{ ASX Announcement } & -0.0006 & -0.0009 & -0.0010 & -0.0027 & $-0.0097 *$ & -0.0019 & -0.0094 \\
\hline & $(-0.32)$ & $(-0.42)$ & $(-0.36)$ & $(-0.69)$ & $(-1.82)$ & $(-0.25)$ & $(-0.78)$ \\
\hline \multirow[t]{2}{*}{ Distribution Announcement } & $0.0054 * *$ & $0.0069 * *$ & $0.0096 * * *$ & $0.0082 *$ & 0.0101 & -0.0004 & 0.0018 \\
\hline & $(2.50)$ & $(2.57)$ & $(2.78)$ & $(1.71)$ & $(1.59)$ & $(-0.04)$ & $(0.12)$ \\
\hline \multirow[t]{2}{*}{ Progress Reports } & $0.0102 * * *$ & $0.0141 * * *$ & $0.0155 * * *$ & $0.0170 * * *$ & $0.0160 * * *$ & $0.0146 * * *$ & 0.0073 \\
\hline & (14.48) & (16.03) & (13.72) & (10.94) & (7.74) & (4.73) & $(1.59)$ \\
\hline \multirow[t]{2}{*}{ Company Administration } & 0.0010 & 0.0021 & 0.0023 & $0.0074 *$ & 0.0086 & $0.0166 * *$ & 0.0123 \\
\hline & $(0.52)$ & $(0.82)$ & $(0.73)$ & (1.69) & $(1.52)$ & $(2.01)$ & $(0.96)$ \\
\hline Notice of Call & - & - & - & - & - & - & - \\
\hline \multirow[t]{2}{*}{ Other } & 0.0033 & 0.0047 & 0.0077 & $0.0124 *$ & $0.0150 *$ & 0.0051 & 0.0112 \\
\hline & $(1.05)$ & $(1.21)$ & $(1.54)$ & (1.82) & (1.69) & $(0.38)$ & $(0.58)$ \\
\hline \multirow[t]{2}{*}{ Chairman's Address } & 0.0039 & 0.0067 & 0.0083 & 0.0031 & 0.0116 & 0.0197 & 0.0132 \\
\hline & $(1.08)$ & $(1.55)$ & $(1.48)$ & $(0.38)$ & $(1.05)$ & $(1.30)$ & $(0.51)$ \\
\hline
\end{tabular}




\begin{tabular}{|c|c|c|c|c|c|c|c|}
\hline Variable & $\Delta$ CAR10min & $\Delta$ CAR15min & $\Delta$ CAR30min & $\Delta$ CAR60min & $\triangle \mathrm{CAR} 90 \mathrm{~min}$ & $\Delta$ CAR120min & $\Delta$ CAR150min \\
\hline \multirow[t]{2}{*}{ Letter to Shareholders } & 0.0067 & 0.0068 & 0.0060 & 0.0070 & - & - & - \\
\hline & $(0.32)$ & $(0.26)$ & $(0.19)$ & $(0.11)$ & - & - & - \\
\hline \multirow[t]{2}{*}{ ASX Query } & $-0.0035 * *$ & $-0.0068 * * *$ & $-0.0044 *$ & $-0.0082 * *$ & $-0.0090 * *$ & $-0.0193 * * *$ & $-0.0569 * * *$ \\
\hline & $(-2.09)$ & $(-3.29)$ & $(-1.72)$ & $(-2.37)$ & $(-2.00)$ & $(-3.05)$ & $(-6.24)$ \\
\hline Structured Products & - & - & - & - & - & - & - \\
\hline \multirow[t]{2}{*}{ CTE Quarterly Reports } & $-0.0041 * *$ & -0.0033 & -0.0015 & -0.0014 & 0.0003 & -0.0028 & -0.0003 \\
\hline & $(-2.50)$ & $(-1.56)$ & $(-0.55)$ & $(-0.37)$ & $(0.06)$ & $(-0.36)$ & $(-0.02)$ \\
\hline \multirow[t]{2}{*}{ Bid-Ask Spread } & -0.0030 & -0.0064 & -0.0041 & 0.0031 & -0.0020 & -0.0153 & -0.0246 \\
\hline & $(-0.76)$ & $(-1.29)$ & $(-0.66)$ & $(0.32)$ & $(-0.09)$ & $(-0.73)$ & $(-0.96)$ \\
\hline \multirow[t]{2}{*}{ Size } & $-0.0012 * * *$ & $-0.0017 * * *$ & $-0.0018 * * *$ & $-0.0021 * * *$ & $-0.0020 * * *$ & -0.0010 & -0.0005 \\
\hline & $(-8.08)$ & $(-9.82)$ & $(-8.20)$ & $(-6.67)$ & $(-4.92)$ & $(-1.57)$ & $(-0.57)$ \\
\hline \multirow[t]{2}{*}{ Book-to-Market Ratio } & -0.0002 & $-0.0006 * *$ & $-0.0006 *$ & -0.0003 & -0.0004 & $-0.0023 * *$ & $-0.0023 *$ \\
\hline & $(-1.06)$ & $(-2.46)$ & $(-1.78)$ & $(-0.76)$ & $(-0.71)$ & $(-2.53)$ & $(-1.76)$ \\
\hline \multirow[t]{2}{*}{ Leverage } & 0.0001 & 0.0001 & -0.0001 & -0.0001 & -0.0001 & -0.0001 & 0.0005 \\
\hline & $(0.36)$ & $(0.25)$ & $(-0.15)$ & $(-0.48)$ & $(-0.09)$ & $(-0.25)$ & $(0.58)$ \\
\hline \multirow[t]{2}{*}{ ROA } & $-0.0002 *$ & $-0.0003 * * *$ & $-\mathbf{0 . 0 0 0 2} * *$ & -0.0001 & 0.0000 & -0.0003 & 0.0003 \\
\hline & $(-1.80)$ & $(-3.67)$ & $(-2.17)$ & $(-0.28)$ & $(-0.05)$ & $(-0.75)$ & $(0.25)$ \\
\hline \multirow[t]{2}{*}{ Resource Sector } & -0.0012 & -0.0007 & -0.0006 & 0.0001 & 0.0009 & 0.0004 & 0.0043 \\
\hline & $(-1.64)$ & $(-0.77)$ & $(-0.52)$ & $(0.08)$ & $(0.43)$ & $(0.13)$ & $(0.90)$ \\
\hline \multirow[t]{2}{*}{ Financial Sector } & -0.0013 & -0.0015 & -0.0024 & -0.0013 & -0.0016 & -0.0023 & -0.0044 \\
\hline & $(-1.11)$ & $(-1.07)$ & $(-1.27)$ & $(-0.48)$ & $(-0.44)$ & $(-0.42)$ & $(-0.56)$ \\
\hline Max. VIF & 1.79 & 1.81 & 1.80 & 1.79 & 1.78 & 1.80 & 1.80 \\
\hline F Value & 17.55 & 22.97 & 15.50 & 10.00 & 5.65 & 3.15 & 3.42 \\
\hline R-Square & $2.02 \%$ & $2.52 \%$ & $1.95 \%$ & $1.67 \%$ & $1.28 \%$ & $1.16 \%$ & $2.71 \%$ \\
\hline Adj R-Sq & $1.91 \%$ & $2.41 \%$ & $1.83 \%$ & $1.51 \%$ & $1.05 \%$ & $0.79 \%$ & $1.92 \%$ \\
\hline $\mathbf{N}$ & 18,705 & 19,541 & 17,135 & 12,938 & 9,188 & 5,636 & 2,483 \\
\hline
\end{tabular}


ASX Query: Most ASX Queries concern ASX Price Queries. A company receives an ASX Price Query after its share price changes significantly over a short period. If the company does not provide any new information to explain why the share price increases/decreases in its response, the market reacts to the price query reversely (Drienko \& Sault, 2013). Because most of the cases involve price increases, the coefficients show a significant negative association between CARs and ASX Queries, which is different from other announcement categories. Before the GFC, although there is a small fluctuation from 120 to 150 minutes, the CARs decline with time, reaching the bottom (coefficient -0.0243) at three hours after the ASX Query was released. During the GFC, the largest negative share price reaction happened at three hours after the announcement was released, but from 30 minutes the coefficients fluctuated slightly from -0.01 to -0.0117 . Since the GFC, the CARs of an ASX Query reach the bottom at two hours after the announcements are released (coefficient -0.0213).

Progress Reports: The CAR regression results reveal that share prices react the most to the Progress Reports before and after the GFC. During the GFC, Progress Reports have the secondlargest price reactions. The CARs peaked at 90 minutes after the announcements released before the GFC (coefficient 0.0194). During the GFC, there were higher CARs observed, peaking more quickly at one hour (coefficient 0.0199). Since the GFC, the coefficients are smaller, with the first CAR peaking at 60 minutes (coefficient 0.0174 ) and a second peak at 180 minutes (coefficient 0.0181). Progress Reports induced slightly larger price reactions during the GFC, but the coefficients in the three periods are close to each other, indicating relative small influences from the GFC on the price reactions to Progress Reports. The large positive price reactions also reveal that the market interprets the operation updates on investment/projects as good news on average, and that the share prices adjust to this update information promptly. 
Asset Acquisition \& Disposal: Announcements from Asset Acquisition \& Disposal had the largest share price responses during the GFC, and the second largest price responses before and after the GFC. Similar to Progress Reports, the CARs of Asset Acquisition \& Disposal peak at 150 minutes (coefficient 0.0158 ) after the announcements were released prior to the GFC, and at one hour (coefficient 0.0227) during the GFC. Since then (but with small fluctuations) the CARs keep increasing over the three hours (coefficient 0.0156 at 150 minutes and 0.0175 at 180 minutes). The coefficients over different periods show that the GFC has a relative large impact on the share price reactions to Asset Acquisition \& Disposal. A possible reason is Asset Acquisition \& Disposal announcements signal the market that the company has fewer or no liquidity problems. Acquiring new assets could indicate that the company still has enough cash, while disposing assets could imply liquidity issues so the company has to dispose of some assets for cash; but meanwhile the cash inflows from disposing assets might be a good sign if the liquidity issue could be solved.

Distribution Announcements: The CAR regression results show that Distribution Announcements have medium share price reactions. Distribution Announcements could have induced slightly larger abnormal returns during the GFC, and slightly smaller returns since then. The robust $\triangle \mathrm{CAR}$ tests indicate that the significant price reactions are observed with smaller values over shorter time windows (within 60 minutes) during and after the GFC. There are at least two possible reasons. One is that the dividend information was expected (or known) from other announcements, especially off-hour announcements, so investors were trading based on their knowledge even before the Distribution Announcement was released. Another is that the Distribution Announcements that are released early may cause larger market reactions, such as announcements between 10:00am and 11:30am, which the $\triangle \mathrm{CAR}$ measurement could not capture due to the reduced sample size. The $\triangle \mathrm{CAR} 90 \mathrm{~min}$ sample convers the announcements 
released in the period 11:30a.m. (90 minutes after the market opens) - 2:30p.m. (90 minutes before the market closes), while the CAR 90min sample is taken from the announcements made between 10:00a.m. and 2:30p.m.

Other: The announcement category Other has medium share price reactions similar to Distribution Announcements before and during the GFC (coefficients 0.0133 at 180 minutes, and 0.0136 at 120 minutes respectively). Since the GFC, the CAR regressions do not show significant price responses to Other. This does not mean that the market did not react to this category but only that no significant result is caused by mixing good news with bad news in one sample. Once the good news is separated from the bad, the results show there have been robust positive market reactions to Other after the GFC. This is further discussed in 6.3.3.

Periodic Reports: The share prices react the least to the Periodic Reports over the three hour time window: the CAR coefficients are smaller than 0.01 (the largest coefficient is 0.0094 at 90 minutes during the GFC). Both Periodic Reports and Distribution Announcements are directly related to earnings information. Distribution Announcements have larger market consequences perhaps because dividend information tends to be good news but periodic financial reports can be either good or bad news. The positive market effects caused by good news Periodic Reports can be reduced by the negative market effects of bad news Periodic Reports. Further discussion is in Section 6.3.3.

Issued Capital: Small share price reactions to Issued Capital are observed only during the GFC, with the largest coefficient 0.0091 at 120 minutes after the announcement was released. However, when taking good/bad news into account, clear price reactions are observed in good/bad news subsamples regardless of the GFC influences. The average positive market 
reactions during the GFC could be because the cash inflow from successful capital issues may have helped companies to overcome the difficult times, relieving some financial pressure.

From the above analysis, the market reacts to different announcements differently, as reflected in abnormal share price returns. There are two possible reasons for the different market reaction magnitudes. One is the information materiality (i.e. the attention that the investors paid to the announcements): if one type of announcement signals positive and important information to investors, this may cause large CARs to occur. The other possible reason is information predictability: if the information can be expected because of other relevant information available in the previous days or months, share prices could adjust to the information more smoothly. The information contained in Periodic Reports is predictable, while the announcements regarding Progress Reports, Asset Acquisition \& Disposal and ASX Queries are relatively unexpected.

In terms of the announcement categories with insignificant and/or non-robust results, most of those categories are administrative announcements. The results show that administrative announcements do not induce significant abnormal share price returns, even though they are tagged as 'market-sensitive' by the ASX. Takeover/Scheme Announcements is the only category showing insignificant and/or non-robust results in the investment/project-related announcement group. This finding may need to be further explored in future research, because consistent and significant CARs have been observed after the Takeover Announcement release but $\triangle \mathrm{CAR}$ tests show inconsistent results. A possible reason is that this study did not distinguish between acquiring and target companies in a takeover event. It is also possible that important takeover announcements are released during off-hours, which cause significant CARs once the 
market is open. It would be valuable to examine the differences between trading- and off-hour announcements.

In addition to the impacts of different announcement types on the intraday CARs, firm size is an important factor that influences the intraday market reactions to disclosures. The negative coefficients of firm size show that large companies tend to have smaller market responses after information is released. This is because the stock prices of large companies are more stable, and are less likely to be influenced by a single information release. Leverage had a small positive impact on the intraday CARs only before the GFC. Companies with more debt might have had higher intraday abnormal returns prior to the GFC. Moreover, before the GFC ${ }^{42}$, information released by resource sector companies may have had smaller stock abnormal returns over the three-hour time windows, while information released by the financial sector may have induced smaller returns within one hour.

\subsubsection{Share Price Reactions to Good and Bad News as to Each Announcement Category}

This study compares the magnitude of market reactions to different information. The regression results presented in Section 6.3.2 may not reveal the 'true' magnitude of share price reactions due to the mixed good and bad news sample. There may be either negative or positive information in the same type of announcements (for example, Periodic Reports). The negative market reactions to the bad news could be offset by the positive market reactions to good news. The stock price also could be more sensitive to bad news than to good news, or vice versa. In order to further examine the magnitude of market reactions to different announcement categories, this study separates the market-sensitive announcement sample into bad news sub-

\footnotetext{
${ }^{42}$ During and after the GFC, the regressions do not show robust results in the $\triangle \mathrm{CAR}$ tests.
} 
samples (when CARs are negative) and good news sub-samples (when CARs are positive). The regression results of good/bad subsamples show better R-square results than full samples, providing better explanations for the associations between intraday CARs and announcement categories.

Tables 6-18 - 6-20 indicate the regression results of bad news subsamples using CARs as dependent variables during the pre-GFC, GFC, and post-GFC periods. Tables 6-21 - 6-23 are the corresponding robust test results using $\triangle \mathrm{CARs}$ as dependent variables. The results indicate that the CARs are significantly negatively associated with the release of ASX Query, Issued Capital, Periodic Reports, and Progress Reports no matter whether before, during or after the GFC. Asset Acquisition \& Disposal announcements had small negative market responses only during the GFC.

According to the regression results for the market-sensitive announcements with good news (CAR results are shown in Tables 6-24 - 6-26, and robust $\Delta$ CAR results in Tables 6-27 - 6-29), the market reacts positively to Progress Reports, Asset Acquisition \& Disposal, Periodic Reports, Distribution Announcements, Issued Capital, Other and ASX Query regardless of the GFC impact. The results also indicate that announcements with good news have larger share price reactions than those with bad news. 
Table 6-18. The Regression Results of Market-sensitive Announcements with Bad News - Intraday CARs before the GFC

\begin{tabular}{|c|c|c|c|c|c|c|c|c|}
\hline Variable & CAR10min & CAR15min & CAR30min & CAR60min & CAR90min & CAR120min & CAR150min & CAR180min \\
\hline \multirow[t]{2}{*}{ Intercept } & $\mathbf{- 0 . 0 0 2 7} * * *$ & $\mathbf{- 0 . 0 0 8 7} * * *$ & $-0.0141 * * *$ & $-0.0218 * * *$ & $-\mathbf{0 . 0 2 8 0} * * *$ & $-0.0301 * * *$ & $-\mathbf{0 . 0 3 3 6} * * *$ & $-\mathbf{0 . 0 3 8 3} * * *$ \\
\hline & $(-3.52)$ & $(-7.75)$ & $(-10.68)$ & $(-13.96)$ & $(-15.70)$ & $(-15.11)$ & $(-15.59)$ & $(-16.40)$ \\
\hline \multirow{2}{*}{$\begin{array}{l}\text { Takeover / Scheme } \\
\text { Announcements }\end{array}$} & -0.0021 & -0.0014 & -0.0019 & -0.0024 & -0.0024 & -0.0006 & -0.0002 & 0.0005 \\
\hline & $(-1.52)$ & $(-0.76)$ & $(-0.80)$ & $(-0.85)$ & $(-0.75)$ & $(-0.17)$ & $(-0.05)$ & $(0.13)$ \\
\hline \multirow[t]{2}{*}{ Security Holder Details } & -0.0016 & -0.0016 & -0.0013 & -0.0011 & -0.0011 & -0.0009 & -0.0021 & -0.0033 \\
\hline & $(-0.68)$ & $(-0.45)$ & $(-0.30)$ & $(-0.23)$ & $(-0.20)$ & $(-0.16)$ & $(-0.31)$ & $(-0.47)$ \\
\hline \multirow[t]{2}{*}{ Periodic Reports } & $-0.0043 * * *$ & $-0.0081 * * *$ & $-0.0102 * * *$ & $-0.0137 * * *$ & $-0.0145 * * *$ & $-0.0131 * * *$ & $-0.0129 * * *$ & $-0.0123 * * *$ \\
\hline & $(-5.85)$ & $(-7.60)$ & $(-8.15)$ & $(-9.19)$ & $(-8.43)$ & $(-6.98)$ & $(-6.37)$ & $(-5.66)$ \\
\hline \multirow[t]{2}{*}{ Issued Capital } & $-0.0050 * * *$ & $-0.0101 * * *$ & $-0.0103 * * *$ & $-0.0127 * * *$ & $-0.0120 * * *$ & $-0.0146 * * *$ & $-0.0137 * * *$ & $-0.0095 * * *$ \\
\hline & $(-5.98)$ & $(-8.48)$ & $(-7.40)$ & $(-7.69)$ & $(-6.32)$ & $(-6.93)$ & $(-6.01)$ & $(-3.93)$ \\
\hline \multirow{2}{*}{$\begin{array}{l}\text { Asset Acquisition \& } \\
\text { Disposal }\end{array}$} & -0.0008 & -0.0013 & -0.0011 & -0.0006 & -0.0007 & -0.0009 & -0.0012 & 0.0004 \\
\hline & $(-0.95)$ & $(-1.10)$ & $(-0.73)$ & $(-0.34)$ & $(-0.34)$ & $(-0.41)$ & $(-0.50)$ & $(0.17)$ \\
\hline \multirow[t]{2}{*}{ Notice of Meeting } & $-0.0124 * *$ & $-0.0135 *$ & -0.0127 & $-0.0188 *$ & -0.0122 & -0.0097 & -0.0105 & -0.0084 \\
\hline & $(-2.30)$ & $(-1.70)$ & $(-1.33)$ & $(-1.65)$ & $(-1.09)$ & $(-0.84)$ & $(-0.85)$ & $(-0.64)$ \\
\hline \multirow[t]{2}{*}{ ASX Announcement } & $0.0023 *$ & $0.0043 * *$ & $0.0062 * * *$ & $0.0088 * * *$ & $0.0096 * * *$ & $0.0080 * *$ & $0.0068 *$ & $0.0090 * *$ \\
\hline & $(1.75)$ & $(2.27)$ & $(2.87)$ & $(3.19)$ & $(3.21)$ & $(2.45)$ & $(1.88)$ & $(2.40)$ \\
\hline \multirow{2}{*}{$\begin{array}{l}\text { Distribution } \\
\text { Announcement }\end{array}$} & -0.0012 & -0.0022 & -0.0020 & -0.0027 & -0.0014 & -0.0013 & 0.0001 & 0.0013 \\
\hline & $(-0.84)$ & $(-1.08)$ & $(-0.78)$ & $(-0.90)$ & $(-0.40)$ & $(-0.34)$ & $(0.01)$ & $(0.31)$ \\
\hline \multirow[t]{2}{*}{ Progress Reports } & $-0.0026 * * *$ & $-0.0052 * * *$ & $-0.0064 * * *$ & $-0.0062 * * *$ & $-0.0059 * * *$ & $-0.0057 * * *$ & $-0.0047 * * *$ & $-0.0042 * * *$ \\
\hline & $(-4.82)$ & $(-6.60)$ & $(-6.97)$ & $(-5.80$ & $(-4.80)$ & $(-4.19)$ & $(-3.19)$ & $(-2.65)$ \\
\hline \multirow[t]{2}{*}{ Company Administration } & 0.0003 & -0.0020 & $-0.0149 * *$ & $-0.0287 * * *$ & $-0.0168 * *$ & $-0.0174 *$ & -0.0169 & $-0.0285 * *$ \\
\hline & $(0.07)$ & $(-0.31)$ & $(-2.24)$ & $(-3.29)$ & $(-2.00)$ & $(-1.94)$ & $(-1.64)$ & $(-2.42)$ \\
\hline Notice of Call & - & - & - & - & - & - & - & - \\
\hline \multirow[t]{2}{*}{ Other } & -0.0015 & $-0.0037 * *$ & $-0.0045 * *$ & -0.0027 & $-0.0048 *$ & -0.0043 & -0.0038 & -0.0012 \\
\hline & $(-1.20)$ & $(-2.15)$ & $(-2.23)$ & $(-1.19)$ & $(-1.82)$ & $(-1.54)$ & $(-1.27)$ & $(-0.39)$ \\
\hline \multirow[t]{2}{*}{ Chairman's Address } & $-0.0076 * * *$ & $-0.0065 * *$ & $-0.0098 * * *$ & $-0.0081 * *$ & $-0.0071 *$ & $-0.0105 * *$ & -0.0071 & -0.0045 \\
\hline & $(-4.02)$ & $(-2.48)$ & $(-3.28)$ & $(-2.35)$ & $(-1.80)$ & $(-2.28)$ & $(-1.47)$ & $(-0.92)$ \\
\hline
\end{tabular}




\begin{tabular}{|c|c|c|c|c|c|c|c|c|}
\hline Variable & CAR10min & CAR15min & CAR30min & CAR60min & CAR90min & CAR120min & CAR150min & CAR180min \\
\hline \multirow[t]{2}{*}{ Letter to Shareholders } & -0.0146 & 0.0052 & 0.0007 & -0.0142 & -0.0238 & -0.0137 & -0.0136 & -0.0095 \\
\hline & $(-1.49)$ & $(0.21)$ & $(0.02)$ & $(-0.76)$ & $(-0.96)$ & $(-0.38)$ & $(-0.37)$ & $(-0.26)$ \\
\hline \multirow[t]{2}{*}{ ASX Query } & $-0.0144 * * *$ & $-0.0208 * * *$ & $-0.0227 * * *$ & $-0.0210 * * *$ & $-0.0248 * * *$ & $-0.0252 * * *$ & $-0.0259 * * *$ & $-0.0273 * * *$ \\
\hline & $(-11.14)$ & $(-11.63)$ & $(-10.93)$ & $(-8.59)$ & $(-8.16)$ & $(-7.41)$ & $(-6.54)$ & $(-6.45)$ \\
\hline \multirow[t]{2}{*}{ Structured Products } & 0.0008 & 0.0010 & - & - & - & - & - & - \\
\hline & $(0.05)$ & $(0.04)$ & - & - & - & - & - & - \\
\hline \multirow[t]{2}{*}{ CTE Quarterly Reports } & -0.0002 & -0.0005 & -0.0001 & 0.0014 & 0.0022 & $0.0049 *$ & 0.0044 & 0.0048 \\
\hline & $(-0.15)$ & $(-0.32)$ & $(-0.05)$ & $(0.67)$ & $(0.89)$ & (1.76) & $(1.48)$ & $(1.45)$ \\
\hline \multirow[t]{2}{*}{ Bid-Ask Spread } & 0.0019 & 0.0037 & 0.0029 & 0.0044 & 0.0089 & 0.0094 & 0.0096 & $0.0299 * *$ \\
\hline & $(0.54)$ & $(0.77)$ & $(0.57)$ & $(0.81)$ & $(1.28)$ & $(1.25)$ & $(1.27)$ & (1.97) \\
\hline \multirow[t]{2}{*}{ Size } & 0.0001 & $0.0009 * * *$ & $0.0016 * * *$ & $0.0023 * * *$ & $0.0030 * * *$ & $0.0032 * * *$ & $0.0036 * * *$ & $0.0040 * * *$ \\
\hline & $(1.34)$ & $(6.71)$ & $(9.41)$ & (11.65) & $(13.65)$ & $(13.31)$ & (13.67) & (14.27) \\
\hline \multirow[t]{2}{*}{ Book-to-Market Ratio } & -0.0002 & 0.0004 & 0.0002 & 0.0005 & 0.0005 & 0.0000 & 0.0006 & 0.0003 \\
\hline & $(-0.74)$ & $(1.11)$ & $(0.41)$ & $(0.83)$ & $(0.76)$ & (0.03) & $(0.65)$ & $(0.36)$ \\
\hline \multirow[t]{2}{*}{ Leverage } & 0.0001 & 0.0001 & $-0.0001 *$ & $-0.0001 * * *$ & $-0.0001 * *$ & $-0.0001 *$ & -0.0001 & -0.0001 \\
\hline & $(0.06)$ & $(0.97)$ & $(-1.93)$ & $(-3.00)$ & $(-2.14)$ & $(-1.7)$ & $(-0.73)$ & $(-0.84)$ \\
\hline \multirow[t]{2}{*}{ ROA } & -0.0001 & 0.0001 & 0.0001 & 0.0003 & -0.0002 & -0.0002 & -0.0006 & -0.0006 \\
\hline & $(-0.08)$ & $(0.50)$ & $(0.11)$ & $(0.76)$ & $(-0.59)$ & $(-0.46)$ & $(-1.13)$ & $(-0.96)$ \\
\hline \multirow[t]{2}{*}{ Resource Sector } & $-0.0015 * * *$ & $-0.0014 * *$ & -0.0012 & -0.0002 & 0.0001 & -0.0001 & -0.0003 & 0.0012 \\
\hline & $(-3.06)$ & $(-2.07)$ & $(-1.53)$ & $(-0.25)$ & $(0.14)$ & $(-0.11)$ & $(-0.27)$ & $(0.88)$ \\
\hline \multirow[t]{2}{*}{ Financial Sector } & $0.0027 * * *$ & $0.0036 * * *$ & $0.0047 * * *$ & $0.0080 * * *$ & $0.0076 * * *$ & $0.0072 * * *$ & $0.0079 * * *$ & $0.0074 * * *$ \\
\hline & (3.78) & $(3.53)$ & $(3.95)$ & $(5.58)$ & (4.69) & (4.03) & (4.18) & (3.70) \\
\hline Max. VIF & 2.08 & 2.09 & 2.10 & 2.17 & 2.23 & 2.29 & 2.36 & 2.46 \\
\hline F Value & 10.50 & 15.33 & 18.85 & 21.95 & 21.44 & 19.63 & 18.40 & 18.60 \\
\hline R-Square & $2.68 \%$ & $3.63 \%$ & $4.46 \%$ & $5.55 \%$ & $5.92 \%$ & $6.00 \%$ & $6.23 \%$ & $7.01 \%$ \\
\hline Adj R-Sq & $2.42 \%$ & $3.40 \%$ & $4.22 \%$ & $5.30 \%$ & $5.65 \%$ & $5.70 \%$ & $5.89 \%$ & $6.63 \%$ \\
\hline $\mathbf{N}$ & 8,792 & 9,378 & 8,909 & 8,243 & 7,514 & 6,785 & 6,113 & 5,453 \\
\hline
\end{tabular}


Table 6-19. The Regression Results of Market-sensitive Announcements with Bad News - Intraday CARs during the GFC

\begin{tabular}{|c|c|c|c|c|c|c|c|c|}
\hline Variable & CAR10min & CAR15min & CAR30min & CAR60min & CAR90min & CAR120min & CAR150min & CAR180min \\
\hline \multirow[t]{2}{*}{ Intercept } & $-0.0008 * * *$ & $-0.0075 * * *$ & $-\mathbf{0 . 0 1 7 8} * * *$ & $-0.0274 * * *$ & $-\mathbf{0 . 0 3 1 0} * * *$ & $-\mathbf{0 . 0 3 3 1} * * *$ & $-0.0363 * * *$ & $-0.0380 * * *$ \\
\hline & $(-0.90)$ & $(-5.07)$ & $(-9.39)$ & $(-12.08)$ & $(-12.22)$ & $(-12.34)$ & $(-11.53)$ & $(-11.19)$ \\
\hline \multirow{2}{*}{$\begin{array}{l}\text { Takeover / Scheme } \\
\text { Announcements }\end{array}$} & -0.0011 & -0.0014 & -0.0001 & -0.0008 & -0.0044 & -0.0047 & -0.0043 & -0.0067 \\
\hline & $(-0.70)$ & $(-0.56)$ & $(-0.02)$ & $(-0.21)$ & $(-0.99)$ & $(-0.99)$ & $(-0.75)$ & $(-1.08)$ \\
\hline \multirow[t]{2}{*}{ Security Holder Details } & -0.0025 & -0.0037 & -0.0055 & -0.0091 & -0.0108 & $-0.0171 * *$ & $-0.0279 * * *$ & $-0.0379 * * *$ \\
\hline & $(-0.94)$ & $(-0.91)$ & $(-1.01)$ & $(-1.51)$ & $(-1.58)$ & $(-2.32)$ & $(-3.55)$ & $(-4.23)$ \\
\hline \multirow[t]{2}{*}{ Periodic Reports } & $-0.0046 * * * *$ & $-0.0101 * * *$ & $-0.0129 * * *$ & $-0.0146 * * *$ & $-0.0161 * * *$ & $-0.0191 * * *$ & $-0.0220 * * *$ & $-0.0203^{* * * *}$ \\
\hline & $(-4.93)$ & $(-6.58)$ & $(-6.56)$ & $(-6.17)$ & $(-6.14)$ & $(-6.87)$ & $(-7.14)$ & $(-6.19)$ \\
\hline \multirow[t]{2}{*}{ Issued Capital } & $-0.0068 * * *$ & $-0.0133 * * *$ & $-0.0167 * * *$ & $-0.0183 * * *$ & $-0.0206 * * *$ & $-0.0188 * * *$ & $-0.0199 * * *$ & $-\mathbf{0 . 0 2 3 5} * * *$ \\
\hline & $(-6.78)$ & $(-8.22)$ & $(-7.92)$ & $(-7.24)$ & $(-7.34)$ & $(-6.28)$ & $(-6.09)$ & $(-6.82)$ \\
\hline \multirow{2}{*}{$\begin{array}{l}\text { Asset Acquisition \& } \\
\text { Disposal }\end{array}$} & $-0.0039 * * *$ & $-0.0060 * * *$ & $-0.0064 * * *$ & $-0.0057 * * *$ & $-0.0081 * * *$ & $-0.0088 * * *$ & $-0.0088 * * *$ & $-0.0062 *$ \\
\hline & $(-3.61)$ & $(-3.37)$ & $(-2.82)$ & $(-2.13)$ & $(-2.76)$ & $(-2.87)$ & $(-2.59)$ & $(-1.69)$ \\
\hline \multirow[t]{2}{*}{ Notice of Meeting } & -0.0018 & -0.0020 & -0.0049 & -0.0021 & -0.0220 & -0.0199 & 0.0040 & 0.0149 \\
\hline & $(-0.38)$ & $(-0.26)$ & $(-0.45)$ & $(-0.15)$ & $(-1.28)$ & $(-1.17)$ & $(0.20)$ & $(0.68)$ \\
\hline \multirow[t]{2}{*}{ ASX Announcement } & 0.0014 & $0.0046 * *$ & 0.0020 & 0.0043 & 0.0042 & 0.0034 & 0.0027 & 0.0037 \\
\hline & $(1.07)$ & $(1.96)$ & $(0.67)$ & $(1.20)$ & $(1.06)$ & $(0.80)$ & $(0.58)$ & $(0.72)$ \\
\hline \multirow{2}{*}{$\begin{array}{l}\text { Distribution } \\
\text { Announcement }\end{array}$} & $-0.0037 *$ & -0.0041 & -0.0031 & -0.0054 & -0.0051 & -0.0096 & -0.0059 & 0.0040 \\
\hline & $(-1.84)$ & $(-1.30)$ & $(-0.72)$ & $(-1.06)$ & $(-0.91)$ & $(-1.59)$ & $(-0.92)$ & $(0.58)$ \\
\hline \multirow[t]{2}{*}{ Progress Reports } & $-0.0039 * * *$ & $-0.0090 * * *$ & $-0.0105 * * *$ & $-0.0103 * * *$ & $-0.0128 * * *$ & $-0.0128 * * *$ & $-0.0126 * * *$ & $-0.0116 * * *$ \\
\hline & $(-6.44)$ & $(-8.84)$ & $(-8.14)$ & $(-6.79)$ & $(-7.60)$ & $(-7.16)$ & $(-6.36)$ & $(-5.44)$ \\
\hline \multirow[t]{2}{*}{ Company Administration } & -0.0008 & -0.0073 & -0.0055 & -0.0016 & -0.0025 & -0.0005 & -0.0022 & 0.0002 \\
\hline & $(-0.27)$ & $(-1.61)$ & $(-1.00)$ & $(-0.24)$ & $(-0.32)$ & $(-0.06)$ & $(-0.26)$ & $(0.02)$ \\
\hline Notice of Call & - & - & - & - & - & - & - & - \\
\hline \multirow[t]{2}{*}{ Other } & -0.0025 & -0.0025 & $-0.0076 *$ & -0.0024 & -0.0038 & -0.0002 & -0.0020 & -0.0030 \\
\hline & $(-1.20)$ & $(-0.75)$ & $(-1.73)$ & $(-0.45)$ & $(-0.65)$ & $(-0.04)$ & $(-0.32)$ & $(-0.45)$ \\
\hline \multirow[t]{2}{*}{ Chairman's Address } & -0.0029 & -0.0043 & $-0.0101 * *$ & $-0.0127 * *$ & $-0.0170 * *$ & $-0.0183 * * *$ & -0.0110 & -0.0079 \\
\hline & $(-1.12)$ & $(-1.04)$ & $(-1.97)$ & $(-2.10)$ & $(-2.55)$ & $(-2.66)$ & $(-1.46)$ & $(-1.01)$ \\
\hline
\end{tabular}




\begin{tabular}{|c|c|c|c|c|c|c|c|c|}
\hline Variable & CAR10min & CAR15min & CAR30min & CAR60min & CAR90min & CAR120min & CAR150min & CAR180min \\
\hline \multirow[t]{2}{*}{ Letter to Shareholders } & -0.0073 & $-0.0649 * * *$ & $-\mathbf{0 . 0 8 9 8} * * *$ & $-0.1263 * * *$ & $-0.1504 * * *$ & $-\mathbf{0 . 1 6 8 5} * * *$ & $-0.1618 * * *$ & -0.0648 \\
\hline & $(-0.51)$ & $(-2.66)$ & $(-2.93)$ & $(-3.65)$ & $(-4.15)$ & $(-4.68)$ & $(-4.31)$ & $(-1.22)$ \\
\hline \multirow[t]{2}{*}{ ASX Query } & $-0.0159 * * *$ & $-0.0204 * * *$ & $-0.0256 * * *$ & $-0.0225 * * *$ & $-0.0227 * * *$ & $-0.0216 * * *$ & $-0.0250 * * *$ & $-0.0226 * * *$ \\
\hline & $(-11.53)$ & $(-10.16)$ & $(-9.54)$ & $(-7.23)$ & $(-6.63)$ & $(-5.71)$ & $(-5.51)$ & $(-4.17)$ \\
\hline Structured Products & - & - & - & - & - & - & - & - \\
\hline \multirow[t]{2}{*}{ CTE Quarterly Reports } & $-0.0027 * *$ & -0.0021 & 0.0018 & 0.0029 & 0.0019 & 0.0010 & 0.0018 & 0.0020 \\
\hline & $(-2.15)$ & $(-0.98)$ & $(0.68)$ & $(0.88)$ & $(0.49)$ & $(0.24)$ & $(0.38)$ & $(0.36)$ \\
\hline \multirow[t]{2}{*}{ Bid-Ask Spread } & 0.0001 & 0.0003 & 0.0095 & 0.0191* & 0.0193 & $0.0261 * *$ & $0.0266 * *$ & 0.0214 \\
\hline & $(0.27)$ & $(0.50)$ & (1.09) & (1.74) & $(1.58)$ & (2.03) & (1.99) & $(1.40)$ \\
\hline \multirow[t]{2}{*}{ Size } & -0.0002 & $0.0007 * * *$ & $0.0018 * * *$ & $0.0027 * * *$ & $0.0032 * * *$ & $0.0035 * * *$ & $0.0039 * * *$ & $0.0037 * * *$ \\
\hline & $(-1.28)$ & (3.55) & (7.53) & (9.66) & (10.37) & $(10.75)$ & $(9.90)$ & (8.91) \\
\hline \multirow[t]{2}{*}{ Book-to-Market Ratio } & -0.0001 & 0.0003 & 0.0001 & 0.0006 & 0.0006 & 0.0007 & 0.0002 & -0.0001 \\
\hline & $(-0.15)$ & (1.05) & $(0.18)$ & $(1.21)$ & $(1.24)$ & $(1.24)$ & $(0.36)$ & $(-0.11)$ \\
\hline \multirow[t]{2}{*}{ Leverage } & -0.0001 & -0.0001 & 0.0002 & -0.0001 & 0.0001 & 0.0001 & 0.0002 & 0.0002 \\
\hline & $(-0.26)$ & $(-0.67)$ & $(1.16)$ & $(-0.71)$ & $(0.11)$ & $(0.19)$ & $(0.76)$ & $(0.74)$ \\
\hline \multirow[t]{2}{*}{ ROA } & 0.0001 & -0.0001 & 0.0001 & -0.0001 & -0.0001 & -0.0001 & 0.0012 & 0.0020 \\
\hline & $(0.01)$ & $(-0.08)$ & $(0.06)$ & $(-0.06)$ & $(-0.04)$ & $(-0.19)$ & $(0.80)$ & $(1.25)$ \\
\hline \multirow[t]{2}{*}{ Resource Sector } & $-0.0016 * * *$ & -0.0011 & -0.0004 & -0.0006 & -0.0007 & -0.0016 & -0.0013 & -0.0009 \\
\hline & $(-2.70)$ & $(-1.09)$ & $(-0.31)$ & $(-0.42)$ & $(-0.44)$ & $(-0.91)$ & $(-0.70)$ & $(-0.45)$ \\
\hline \multirow[t]{2}{*}{ Financial Sector } & 0.0006 & 0.0008 & -0.0005 & -0.0011 & -0.0001 & -0.0020 & -0.0028 & -0.0041 \\
\hline & $(0.71)$ & $(0.53)$ & $(-0.24)$ & $(-0.47)$ & $(-0.04)$ & $(-0.75)$ & $(-0.93)$ & $(-1.32)$ \\
\hline Max. VIF & 1.85 & 1.88 & 1.85 & 1.89 & 1.96 & 2.00 & 2.04 & 2.09 \\
\hline F Value & 8.48 & 10.18 & 11.36 & 10.93 & 11.52 & 11.11 & 11.48 & 9.92 \\
\hline R-Square & $2.11 \%$ & $2.39 \%$ & $2.76 \%$ & $2.87 \%$ & $3.32 \%$ & $3.58 \%$ & $4.10 \%$ & $4.01 \%$ \\
\hline Adj R-Sq & $1.86 \%$ & $2.16 \%$ & $2.52 \%$ & $2.61 \%$ & $3.03 \%$ & $3.25 \%$ & $3.74 \%$ & $3.61 \%$ \\
\hline $\mathbf{N}$ & 8,678 & 9,163 & 8,822 & 8,166 & 7,397 & 6,613 & 5,935 & 5,244 \\
\hline
\end{tabular}


Table 6-20. The Regression Results of Market-sensitive Announcements with Bad News - Intraday CARs after the GFC

\begin{tabular}{|c|c|c|c|c|c|c|c|c|}
\hline Variable & CAR10min & CAR15min & CAR30min & CAR60min & CAR90min & CAR120min & CAR150min & CAR180min \\
\hline \multirow[t]{2}{*}{ Intercept } & $-0.0042 * * *$ & $-0.01047 * * *$ & $-0.01681 * * *$ & $-0.02608 * * *$ & $-\mathbf{0 . 0 2 9 7 5} * * *$ & $-\mathbf{0 . 0 3 3 8 1} * * *$ & $\mathbf{- 0 . 0 3 7 7 4} * * *$ & $-0.04411 * * *$ \\
\hline & $(-4.17)$ & $(-7.75)$ & $(-9.85)$ & $(-12.22)$ & $(-12.47)$ & $(-12.22)$ & $(-12.62)$ & $(-13.26)$ \\
\hline \multirow{2}{*}{$\begin{array}{l}\text { Takeover / Scheme } \\
\text { Announcements }\end{array}$} & -0.0003 & -0.0005 & -0.0006 & -0.0012 & -0.0006 & 0.0021 & 0.0028 & 0.0037 \\
\hline & $(-0.13)$ & $(-0.18)$ & $(-0.17)$ & $(-0.28)$ & $(-0.13)$ & $(0.36)$ & $(0.45)$ & $(0.56)$ \\
\hline \multirow[t]{2}{*}{ Security Holder Details } & -0.0031 & -0.0039 & -0.0076 & -0.0046 & -0.0102 & -0.0061 & -0.0062 & 0.0035 \\
\hline & $(-0.83)$ & $(-0.82)$ & $(-1.28)$ & $(-0.68)$ & $(-1.30)$ & $(-0.71)$ & $(-0.72)$ & $(0.34)$ \\
\hline \multirow[t]{2}{*}{ Periodic Reports } & $-0.0053 * * *$ & $-0.0084 * * *$ & $-0.0118 * * *$ & $-0.0149 * * *$ & $-0.0166 * * *$ & $-0.0175 * * *$ & $-0.0181 * * *$ & $-0.0157 * * *$ \\
\hline & $(-5.18)$ & $(-6.11)$ & $(-6.78)$ & $(-6.85)$ & $(-6.69)$ & $(-6.10)$ & $(-5.89)$ & $(-4.64)$ \\
\hline \multirow[t]{2}{*}{ Issued Capital } & $-0.0043 * * *$ & $-0.0115 * * *$ & $-0.0132 * * *$ & $-0.0159 * * *$ & $-0.0127 * * *$ & $-0.0120 * * *$ & $-0.0119 * * *$ & $-0.0079 * *$ \\
\hline & $(-3.76)$ & $(-7.36)$ & $(-6.72)$ & $(-6.50)$ & $(-4.67)$ & $(-3.81)$ & $(-3.51)$ & $(-2.11)$ \\
\hline \multirow{2}{*}{$\begin{array}{l}\text { Asset Acquisition \& } \\
\text { Disposal }\end{array}$} & -0.0010 & $-0.0029 *$ & $-0.0038 *$ & -0.0043 & -0.0035 & -0.0028 & -0.0024 & 0.0006 \\
\hline & $(-0.78)$ & $(-1.71)$ & $(-1.78)$ & $(-1.61)$ & $(-1.17)$ & $(-0.83)$ & $(-0.64)$ & $(0.15)$ \\
\hline \multirow[t]{2}{*}{ Notice of Meeting } & 0.0012 & -0.0018 & -0.0031 & -0.0079 & -0.0083 & -0.0076 & -0.0098 & -0.0047 \\
\hline & $(0.16)$ & $(-0.18)$ & $(-0.22)$ & $(-0.56)$ & $(-0.47)$ & $(-0.37)$ & $(-0.47)$ & $(-0.20)$ \\
\hline \multirow[t]{2}{*}{ ASX Announcement } & 0.0018 & $0.0042 * *$ & 0.0048* & $0.0076 * *$ & $0.0091 * *$ & $0.0139 * * *$ & $0.0145 * * *$ & $0.0187 * * *$ \\
\hline & (1.14) & (1.96) & $(1.75)$ & (2.19) & (2.29) & (3.18) & $(3.00)$ & (3.48) \\
\hline \multirow{2}{*}{$\begin{array}{l}\text { Distribution } \\
\text { Announcement }\end{array}$} & -0.0027 & $-0.0049 *$ & $-0.0062 *$ & -0.0066 & -0.0065 & -0.0078 & -0.0047 & -0.0039 \\
\hline & $(-1.30)$ & $(-1.78)$ & $(-1.77)$ & $(-1.49)$ & $(-1.27)$ & $(-1.36)$ & $(-0.73)$ & $(-0.53)$ \\
\hline \multirow[t]{2}{*}{ Progress Reports } & $-0.0047 * * *$ & $-0.0078 * * *$ & $-0.0100 * * *$ & $-0.0117 * * *$ & $-0.0123 * * *$ & $-0.0114 * * *$ & $-0.0108 * * *$ & $-0.0090 * * *$ \\
\hline & $(-7.15)$ & $(-8.76)$ & $(-8.90)$ & $(-8.38)$ & $(-7.82)$ & $(-6.27)$ & $(-5.53)$ & $(-4.16)$ \\
\hline \multirow[t]{2}{*}{ Company Administration } & -0.0029 & $-0.0043^{*}$ & $-0.0053 *$ & -0.0034 & -0.0026 & -0.0006 & -0.0007 & 0.0006 \\
\hline & $(-1.58)$ & $(-1.76)$ & $(-1.68)$ & $(-0.85)$ & $(-0.58)$ & $(-0.13)$ & $(-0.12)$ & $(0.11)$ \\
\hline Notice of Call & - & - & - & - & - & - & - & - \\
\hline \multirow[t]{2}{*}{ Other } & $-0.0064 * *$ & $-0.0087 * *$ & $-0.0094 *$ & -0.0044 & -0.0028 & -0.0038 & -0.0043 & 0.0003 \\
\hline & $(-2.12)$ & $(-2.29)$ & $(-1.89)$ & $(-0.74)$ & $(-0.44)$ & $(-0.51)$ & $(-0.55)$ & $(0.04)$ \\
\hline \multirow[t]{2}{*}{ Chairman's Address } & -0.0025 & -0.0069 & -0.0083 & $-0.0114 *$ & $-0.0175 * *$ & -0.0133 & $-0.0183 * *$ & $-0.0181 * *$ \\
\hline & $(-0.73)$ & $(-1.54)$ & $(-1.45)$ & $(-1.66)$ & $(-2.28)$ & $(-1.59)$ & $(-2.16)$ & $(-2.00)$ \\
\hline
\end{tabular}




\begin{tabular}{|c|c|c|c|c|c|c|c|c|}
\hline Variable & CAR10min & CAR15min & CAR30min & CAR60min & CAR90min & CAR120min & CAR150min & CAR180min \\
\hline \multirow[t]{2}{*}{ Letter to Shareholders } & 0.0024 & -0.0003 & -0.0011 & -0.0136 & -0.0121 & -0.0117 & -0.0060 & -0.0024 \\
\hline & $(0.10)$ & $(-0.01)$ & $(-0.04)$ & $(-0.29)$ & $(-0.24)$ & $(-0.21)$ & $(-0.11)$ & $(-0.04)$ \\
\hline \multirow[t]{2}{*}{ ASX Query } & $-0.0125 * * *$ & $-0.0179 * * *$ & $-0.0227 * * *$ & $-0.0253 * * *$ & $-0.0278 * * *$ & $-0.0335 * * *$ & $-0.0239 * * *$ & $-0.0210 * * *$ \\
\hline & $(-8.29)$ & $(-9.08)$ & $(-9.18)$ & $(-7.99)$ & $(-7.69)$ & $(-7.52)$ & $(-4.43)$ & $(-2.89)$ \\
\hline Structured Products & - & - & - & - & - & - & - & - \\
\hline \multirow[t]{2}{*}{ CTE Quarterly Reports } & $-0.0036 * *$ & -0.0018 & -0.0029 & -0.0046 & -0.0044 & -0.0067 & $-0.0098 * *$ & -0.0076 \\
\hline & $(-2.41)$ & $(-0.86)$ & $(-1.07)$ & $(-1.34)$ & $(-1.17)$ & $(-1.51)$ & $(-2.04)$ & $(-1.34)$ \\
\hline \multirow[t]{2}{*}{ Bid-Ask Spread } & 0.0023 & 0.0023 & 0.0048 & 0.0125 & 0.0091 & 0.0004 & 0.0004 & 0.0079 \\
\hline & $(0.64)$ & $(0.53)$ & $(0.97)$ & (1.30) & (1.16) & $(0.05)$ & $(0.04)$ & $(0.58)$ \\
\hline \multirow[t]{2}{*}{ Size } & $0.0003 * *$ & $0.0012 * * *$ & $0.0019 * * *$ & $0.0029 * * *$ & $0.0034 * * *$ & $0.0041 * * *$ & $0.0046 * * *$ & $0.0051 * * *$ \\
\hline & $(2.45)$ & $(6.58)$ & (8.36) & (10.50) & (11.09) & (11.59) & (12.22) & (12.16) \\
\hline \multirow[t]{2}{*}{ Book-to-Market Ratio } & -0.0001 & 0.0001 & -0.0002 & -0.0002 & -0.0003 & -0.0003 & -0.0004 & -0.0003 \\
\hline & $(-0.48)$ & $(0.11)$ & $(-0.64)$ & $(-0.39)$ & $(-0.55)$ & $(-0.59)$ & $(-0.58)$ & $(-0.40)$ \\
\hline \multirow[t]{2}{*}{ Leverage } & 0.0001 & 0.0001 & 0.0001 & 0.0001 & -0.0001 & -0.0001 & -0.0001 & -0.0001 \\
\hline & $(0.41)$ & $(0.21)$ & $(0.51)$ & $(0.30)$ & $(-0.60)$ & $(-0.43)$ & $(-0.05)$ & $(-0.14)$ \\
\hline \multirow[t]{2}{*}{ ROA } & 0.0003 & 0.0001 & 0.0006 & 0.0001 & 0.0001 & 0.0001 & 0.0001 & -0.0001 \\
\hline & $(1.25)$ & $(0.29)$ & $(1.47)$ & $(0.57)$ & $(0.43)$ & $(0.18)$ & $(0.02)$ & $(-0.08)$ \\
\hline \multirow[t]{2}{*}{ Resource Sector } & 0.0005 & 0.0011 & 0.001713 & 0.0028* & 0.0013 & -0.0008 & -0.0011 & -0.0006 \\
\hline & $(0.75)$ & (1.14) & (1.47) & (1.90) & $(0.81)$ & $(-0.45)$ & $(-0.54)$ & $(-0.28)$ \\
\hline \multirow[t]{2}{*}{ Financial Sector } & $0.0042 * * *$ & $0.0069 * * *$ & $0.0101 * * *$ & $0.0132 * * *$ & $0.0115 * * *$ & $0.0117 * * *$ & $0.0101 * * *$ & $0.0095 * *$ \\
\hline & (3.77) & $(4.75)$ & $(5.34)$ & $(5.58)$ & (4.24) & (3.70) & (3.03) & $(2.58)$ \\
\hline Max. VIF & 1.76 & 1.79 & 1.76 & 1.78 & 1.77 & 1.83 & 1.86 & 1.89 \\
\hline F Value & 6.97 & 11.86 & 13.98 & 15.07 & 14.15 & 14.23 & 13.02 & 11.67 \\
\hline R-Square & $1.68 \%$ & $2.74 \%$ & $3.34 \%$ & $3.91 \%$ & $4.00 \%$ & $4.44 \%$ & $4.55 \%$ & $4.62 \%$ \\
\hline Adj R-Sq & $1.44 \%$ & $2.51 \%$ & $3.10 \%$ & $3.65 \%$ & $3.72 \%$ & $4.13 \%$ & $4.20 \%$ & $4.22 \%$ \\
\hline $\mathbf{N}$ & 8,976 & 9,286 & 8,915 & 8,163 & 7,488 & 6,753 & 6,036 & 5,329 \\
\hline
\end{tabular}

$*, * *$ and $* * *$ denote significance at the $10 \%, 5 \%$ and $1 \%$ levels respectively (two-tailed). 
Table 6-21. The Regression Results of Market-sensitive Announcements with Bad News - Intraday $\triangle$ CARs before the GFC

\begin{tabular}{|c|c|c|c|c|c|c|c|}
\hline Variable & $\triangle \mathrm{CAR} 10 \mathrm{~min}$ & $\triangle$ CAR15min & $\triangle$ CAR30min & $\triangle$ CAR60min & $\triangle \mathrm{CAR90min}$ & $\Delta$ CAR120min & $\Delta$ CAR150min \\
\hline \multirow[t]{2}{*}{ Intercept } & $-0.0047 * * *$ & $-0.0117 * * *$ & $-0.0195 * * *$ & $-\mathbf{0 . 0 3 3 5} * * *$ & $-0.0404 * * *$ & $-0.0427 * * *$ & $-\mathbf{0 . 0 5 1 6} * * *$ \\
\hline & $(-5.14)$ & $(-9.46)$ & $(-11.9)$ & $(-14.71)$ & $(-15.02)$ & $(-11.41)$ & $(-8.27)$ \\
\hline \multirow[t]{2}{*}{ Takeover / Scheme Announcements } & -0.0018 & -0.0021 & -0.0020 & -0.0020 & -0.0017 & 0.0026 & -0.0025 \\
\hline & $(-1.10)$ & $(-1.00)$ & $(-0.68)$ & $(-0.51)$ & $(-0.36)$ & $(0.45)$ & $(-0.27)$ \\
\hline \multirow[t]{2}{*}{ Security Holder Details } & -0.0022 & -0.0022 & -0.0060 & 0.0018 & 0.0001 & 0.0025 & 0.0073 \\
\hline & $(-0.84)$ & $(-0.56)$ & $(-1.00)$ & $(0.25)$ & $(0.01)$ & $(0.21)$ & $(0.39)$ \\
\hline \multirow[t]{2}{*}{ Periodic Reports } & $-0.0043 * * *$ & $-0.0085 * * *$ & $-0.0106 * * *$ & $-0.0137 * * *$ & $-0.0138 * * *$ & $-0.0091 * * *$ & $-0.0115 * *$ \\
\hline & $(-4.9)$ & $(-7.15)$ & $(-6.75)$ & $(-6.19)$ & $(-5.18)$ & $(-2.59)$ & $(-2.03)$ \\
\hline \multirow[t]{2}{*}{ Issued Capital } & $-0.0050 * * *$ & $-0.0100 * * *$ & $-0.0115 * * *$ & $-0.0150 * * *$ & $-0.0130 * * *$ & $-0.0203 * * *$ & $-0.0199 * * *$ \\
\hline & $(-5.18)$ & $(-7.67)$ & $(-6.64)$ & $(-6.26)$ & $(-4.6)$ & $(-5.25)$ & $(-3.24)$ \\
\hline \multirow[t]{2}{*}{ Asset Acquisition \& Disposal } & -0.0010 & -0.0022 & -0.0016 & 0.0001 & 0.0011 & 0.0025 & 0.0009 \\
\hline & $(-0.97)$ & $(-1.60)$ & $(-0.87)$ & $(0.02)$ & $(0.35)$ & $(0.62)$ & $(0.13)$ \\
\hline \multirow[t]{2}{*}{ Notice of Meeting } & $-0.0141 * *$ & $-0.0251 * *$ & -0.0128 & -0.0043 & -0.0025 & -0.0143 & 0.0035 \\
\hline & $(-2.08)$ & $(-2.30)$ & $(-1.02)$ & $(-0.24)$ & $(-0.12)$ & $(-0.48)$ & $(0.11)$ \\
\hline \multirow[t]{2}{*}{ ASX Announcement } & $0.0034 * *$ & 0.0038* & 0.0043 & 0.0048 & -0.0017 & $-0.0143 * *$ & -0.0186 ** \\
\hline & $(2.21)$ & $(1.82)$ & $(1.58)$ & $(1.22)$ & $(-0.36)$ & $(-2.32)$ & $(-1.96)$ \\
\hline \multirow[t]{2}{*}{ Distribution Announcement } & -0.0015 & -0.0033 & -0.0026 & -0.0037 & -0.0012 & -0.0026 & -0.0097 \\
\hline & $(-0.91)$ & $(-1.45)$ & $(-0.87)$ & $(-0.85)$ & $(-0.23)$ & $(-0.35)$ & $(-0.71)$ \\
\hline \multirow[t]{2}{*}{ Progress Reports } & $-0.0029 * * *$ & $-0.0057 * * *$ & $-0.0076 * * *$ & $-0.0074 * * *$ & $-0.0064 * * *$ & $-0.0074 * * *$ & $-0.0077 * *$ \\
\hline & $(-4.52)$ & $(-6.53)$ & $(-6.60)$ & $(-4.80)$ & $(-3.51)$ & $(-3.10)$ & $(-1.99)$ \\
\hline \multirow[t]{2}{*}{ Company Administration } & 0.0015 & -0.0008 & $-0.0188 * *$ & -0.0150 & -0.0099 & -0.0257 & -0.0240 \\
\hline & $(0.32)$ & $(-0.11)$ & $(-2.09)$ & $(-1.17)$ & $(-0.77)$ & $(-1.61)$ & $(-1.16)$ \\
\hline Notice of Call & - & - & - & - & - & - & - \\
\hline \multirow[t]{2}{*}{ Other } & -0.0012 & $-0.0032 *$ & -0.0036 & $-0.0058 *$ & $-0.0083 * *$ & -0.0077 & -0.0125 \\
\hline & $(-0.82)$ & $(-1.76)$ & $(-1.45)$ & $(-1.71)$ & $(-2.11)$ & $(-1.52)$ & $(-1.57)$ \\
\hline \multirow[t]{2}{*}{ Chairman's Address } & $-0.0050 * *$ & $-0.0067 * *$ & $-0.0097 * *$ & -0.0044 & -0.0081 & -0.0109 & 0.0152 \\
\hline & $(-2.30)$ & $(-2.18)$ & $(-2.47)$ & $(-0.82)$ & $(-1.11)$ & $(-0.80)$ & $(0.66)$ \\
\hline
\end{tabular}




\begin{tabular}{|c|c|c|c|c|c|c|c|}
\hline Variable & $\triangle \mathrm{CAR} 10 \mathrm{~min}$ & $\Delta$ CAR15min & $\Delta$ CAR30min & $\triangle$ CAR60min & $\Delta$ CAR90min & $\Delta$ CAR120min & $\Delta C A R 150 \mathrm{~min}$ \\
\hline \multirow[t]{2}{*}{ Letter to Shareholders } & -0.0211 & - & 0.0084 & $-0.0395 *$ & $-0.0421 *$ & - & - \\
\hline & $(-1.56)$ & - & $(0.25)$ & $(-1.71)$ & $(-1.81)$ & - & - \\
\hline \multirow[t]{2}{*}{ ASX Query } & $-0.0159 * * *$ & $-0.0224 * * *$ & $-0.0207 * * *$ & $-0.0217 * * *$ & $-0.0313 * * *$ & $-0.0380 * * * *$ & $-0.0384 * * *$ \\
\hline & $(-10.62)$ & $(-11.24)$ & $(-7.92)$ & $(-6.17)$ & $(-7.20)$ & $(-7.17)$ & $(-4.19)$ \\
\hline \multirow[t]{2}{*}{ Structured Products } & -0.0044 & -0.0066 & - & - & - & - & - \\
\hline & $(-0.23)$ & $(-0.25)$ & - & - & - & - & - \\
\hline \multirow[t]{2}{*}{ CTE Quarterly Reports } & 0.0000 & -0.0016 & -0.0019 & -0.0025 & 0.0006 & $0.0084 *$ & 0.0124 \\
\hline & $(-0.01)$ & $(-1.05)$ & $(-0.88)$ & $(-0.83)$ & $(0.16)$ & (1.72) & $(1.56)$ \\
\hline \multirow[t]{2}{*}{ Bid-Ask Spread } & 0.0026 & 0.0067 & 0.0034 & 0.0043 & 0.0017 & 0.0046 & -0.0138 \\
\hline & $(0.44)$ & $(1.10)$ & $(0.64)$ & $(0.61)$ & $(0.18)$ & $(0.14)$ & $(-0.29)$ \\
\hline \multirow[t]{2}{*}{ Size } & $0.0003 * *$ & $0.0013 * * *$ & $0.0022 * * *$ & $0.0037 * * *$ & $0.0045 * * *$ & $0.0049 * * *$ & $0.0058 * * *$ \\
\hline & $(2.40)$ & $(8.13)$ & (10.72) & (13.17) & (13.54) & (11.24) & (7.32) \\
\hline \multirow[t]{2}{*}{ Book-to-Market Ratio } & -0.0005 & 0.0007 & 0.0004 & 0.0003 & 0.0003 & -0.0010 & 0.0003 \\
\hline & $(-1.47)$ & $(1.49)$ & $(0.65)$ & $(0.34)$ & $(0.29)$ & $(-0.59)$ & $(0.12)$ \\
\hline \multirow[t]{2}{*}{ Leverage } & -0.0001 & 0.0001 & 0.0001 & -0.0001 & $0.0005 *$ & 0.0001 & -0.0001 \\
\hline & $(-0.17)$ & $(0.05)$ & $(0.13)$ & $(-0.94)$ & (1.67) & $(0.82)$ & $(-0.29)$ \\
\hline \multirow[t]{2}{*}{ ROA } & 0.0002 & 0.0004 & 0.0005 & 0.0008 & 0.0002 & 0.0004 & 0.0018 \\
\hline & $(0.62)$ & (1.29) & $(1.15)$ & $(1.43)$ & $(0.36)$ & $(0.63)$ & $(0.63)$ \\
\hline \multirow[t]{2}{*}{ Resource Sector } & $-0.0015 * * *$ & $-0.0017 * *$ & -0.0012 & 0.0013 & -0.0005 & -0.0029 & 0.0001 \\
\hline & $(-2.71)$ & $(-2.24)$ & $(-1.18)$ & $(0.89)$ & $(-0.28)$ & $(-1.25)$ & $(0.01)$ \\
\hline \multirow[t]{2}{*}{ Financial Sector } & $0.0027 * * *$ & $0.0039 * * *$ & $0.0053 * * *$ & $0.0091 * * *$ & $0.0072 * * *$ & 0.0036 & 0.0028 \\
\hline & (3.27) & (3.54) & (3.56) & $(4.32)$ & (2.82) & $(1.01)$ & $(0.46)$ \\
\hline Max. VIF & 2.11 & 2.12 & 2.12 & 2.09 & 2.06 & 2.00 & 1.98 \\
\hline F Value & 9.53 & 16.14 & 15.58 & 17.31 & 16.63 & 14.22 & 6.24 \\
\hline R-Square & $2.59 \%$ & $3.93 \%$ & $4.29 \%$ & $6.10 \%$ & $8.10 \%$ & $10.54 \%$ & $10.59 \%$ \\
\hline Adj R-Sq & $2.32 \%$ & $3.69 \%$ & $4.01 \%$ & $5.75 \%$ & $7.61 \%$ & $9.80 \%$ & $8.90 \%$ \\
\hline $\mathbf{N}$ & 8,271 & 8,695 & 7,671 & 5,884 & 4,174 & 2,557 & 1,128 \\
\hline
\end{tabular}


Table 6-22. The Regression Results of Market-sensitive Announcements with Bad News - Intraday $\triangle$ CARs during the GFC

\begin{tabular}{|c|c|c|c|c|c|c|c|}
\hline Variable & $\triangle \mathrm{CAR} 10 \mathrm{~min}$ & $\triangle$ CAR15min & $\triangle \mathrm{CAR30min}$ & $\triangle$ CAR60min & $\triangle$ CAR90min & $\Delta$ CAR120min & $\triangle \mathrm{CAR} 150 \mathrm{~min}$ \\
\hline \multirow[t]{2}{*}{ Intercept } & $-\mathbf{0 . 0 0 3 8} * * *$ & $-0.0142 * * *$ & $-\mathbf{0 . 0 2 8 3} * * *$ & $-0.0390 * * *$ & $-0.0445 * * *$ & $-0.0519 * * *$ & $-0.0641 * * *$ \\
\hline & $(-3.62)$ & $(-8.67)$ & $(-11.82)$ & $(-11.96)$ & $(-10.43)$ & $(-9.13)$ & $(-7.03)$ \\
\hline \multirow[t]{2}{*}{ Takeover / Scheme Announcements } & -0.0009 & -0.0018 & 0.0017 & -0.0009 & -0.0040 & 0.0011 & 0.0042 \\
\hline & $(-0.49)$ & $(-0.64)$ & $(0.45)$ & $(-0.17)$ & $(-0.59)$ & $(0.12)$ & $(0.31)$ \\
\hline \multirow[t]{2}{*}{ Security Holder Details } & -0.0034 & -0.0046 & -0.0064 & $-0.0138 *$ & $-0.0166^{*}$ & -0.0180 & $-0.0368 *$ \\
\hline & $(-1.14)$ & $(-1.06)$ & $(-1.00)$ & $(-1.76)$ & $(-1.68)$ & $(-1.28)$ & $(-1.77)$ \\
\hline \multirow[t]{2}{*}{ Periodic Reports } & $-0.0034 * * *$ & $-0.0080 * * *$ & $-0.0099 * * *$ & $-0.0124 * * *$ & $-0.0163 * * *$ & $-0.0228 * * *$ & $-0.0345 * * *$ \\
\hline & $(-3.14)$ & $(-4.75)$ & $(-4.20)$ & $(-3.91)$ & $(-3.95)$ & $(-4.08)$ & $(-3.94)$ \\
\hline \multirow[t]{2}{*}{ Issued Capital } & $-0.0062 * * *$ & $-0.0134 * * *$ & $-0.0152 * * *$ & $-0.0150 * * *$ & $-0.0269 * * *$ & $-0.0213 * * *$ & -0.0101 \\
\hline & $(-5.42)$ & $(-7.43)$ & $(-5.95)$ & $(-4.48)$ & $(-6.06)$ & $(-3.41)$ & $(-0.99)$ \\
\hline \multirow[t]{2}{*}{ Asset Acquisition \& Disposal } & $-0.0051 * * *$ & $-0.0067 * * *$ & $-0.0054 * *$ & $-0.0081 * *$ & $-0.0134 * * *$ & $-0.0142 * *$ & $-0.0263 * *$ \\
\hline & $(-4.09)$ & $(-3.42)$ & $(-1.97)$ & $(-2.23)$ & $(-2.86)$ & $(-2.26)$ & $(-2.57)$ \\
\hline \multirow[t]{2}{*}{ Notice of Meeting } & -0.0049 & -0.0061 & -0.0097 & -0.0095 & $-0.0474 *$ & -0.0354 & - \\
\hline & $(-0.76)$ & $(-0.62)$ & $(-0.71)$ & $(-0.45)$ & $(-1.74)$ & $(-1.09)$ & - \\
\hline \multirow[t]{2}{*}{ ASX Announcement } & $0.0031 * *$ & $0.0062 * *$ & 0.0005 & -0.0062 & $-0.0142 * *$ & $-0.0181 * *$ & $-0.0251 * *$ \\
\hline & $(1.98)$ & $(2.41)$ & $(0.13)$ & $(-1.33)$ & $(-2.41)$ & $(-2.36)$ & $(-2.08)$ \\
\hline \multirow[t]{2}{*}{ Distribution Announcement } & -0.0026 & -0.0044 & -0.0034 & -0.0007 & -0.0036 & -0.0052 & -0.0242 \\
\hline & $(-1.15)$ & $(-1.30)$ & $(-0.70)$ & $(-0.10)$ & $(-0.43)$ & $(-0.45)$ & $(-1.33)$ \\
\hline \multirow[t]{2}{*}{ Progress Reports } & $-0.0038 * * *$ & $-0.0092 * * *$ & $-0.0092 * * *$ & $-0.0083 * * *$ & $-0.0140 * * *$ & $-0.0138 * * *$ & $-0.0161 * * *$ \\
\hline & $(-5.45)$ & $(-8.28)$ & $(-5.95)$ & $(-4.14)$ & $(-5.29)$ & $(-3.91)$ & $(-2.95)$ \\
\hline \multirow[t]{2}{*}{ Company Administration } & -0.0001 & -0.0054 & -0.0005 & -0.0019 & 0.0001 & -0.0010 & -0.0102 \\
\hline & $(-0.02)$ & $(-1.14)$ & $(-0.07)$ & $(-0.19)$ & $(0.01)$ & $(-0.06)$ & $(-0.42)$ \\
\hline Notice of Call & - & - & - & - & - & - & - \\
\hline \multirow[t]{2}{*}{ Other } & -0.0017 & -0.0029 & -0.0067 & -0.0004 & -0.0021 & 0.0010 & -0.0085 \\
\hline & $(-0.66)$ & $(-0.77)$ & $(-1.24)$ & $(-0.05)$ & $(-0.23)$ & $(0.08)$ & $(-0.41)$ \\
\hline \multirow[t]{2}{*}{ Chairman's Address } & -0.0014 & -0.0034 & -0.0056 & -0.0072 & $-0.0207 *$ & -0.0213 & -0.0197 \\
\hline & $(-0.46)$ & $(-0.79)$ & $(-0.96)$ & $(-0.92)$ & $(-1.79)$ & $(-1.50)$ & $(-0.70)$ \\
\hline
\end{tabular}




\begin{tabular}{|c|c|c|c|c|c|c|c|}
\hline Variable & $\triangle \mathrm{CAR10min}$ & $\triangle \mathrm{CAR} 15 \mathrm{~min}$ & $\Delta$ CAR30min & $\triangle \mathrm{CAR60min}$ & $\triangle$ CAR90min & $\triangle \mathrm{CAR} 120 \mathrm{~min}$ & $\triangle \mathrm{CAR} 150 \mathrm{~min}$ \\
\hline \multirow[t]{2}{*}{ Letter to Shareholders } & -0.0201 & $\mathbf{- 0 . 0 9 5 2} * * *$ & $-0.2161 * * *$ & $-0.1640 * * *$ & $-0.1914 * * *$ & $-0.2748 * * *$ & $-0.3169 * * *$ \\
\hline & $(-0.87)$ & $(-2.60)$ & $(-4.47)$ & $(-2.93)$ & $(-3.15)$ & $(-4.27)$ & $(-4.73)$ \\
\hline \multirow[t]{2}{*}{ ASX Query } & $-0.0169 * * *$ & $-0.0240 * * *$ & $-0.0285 * * *$ & $-0.0217 * * *$ & $-0.0276 * * *$ & $-0.0337 * * *$ & $-0.0631 * * *$ \\
\hline & $(-10.86)$ & $(-10.74)$ & $(-8.86)$ & $(-5.53)$ & $(-5.64)$ & $(-5.52)$ & $(-6.92)$ \\
\hline Structured Products & - & - & - & - & - & - & - \\
\hline \multirow[t]{2}{*}{ CTE Quarterly Reports } & $-0.0025 *$ & -0.0009 & $0.0066 * *$ & $0.0088 * *$ & 0.0045 & 0.0071 & 0.0029 \\
\hline & $(-1.69)$ & $(-0.39)$ & (2.02) & (2.04) & (0.79) & $(0.90)$ & $(0.22)$ \\
\hline \multirow[t]{2}{*}{ Bid-Ask Spread } & -0.0001 & $0.0182 *$ & 0.0141 & 0.0336 & $0.0722 * *$ & $0.1522 * *$ & 0.1657 \\
\hline & $(-0.02)$ & $(1.75)$ & $(0.99)$ & (1.43) & (1.97) & $(2.15)$ & (1.63) \\
\hline \multirow[t]{2}{*}{ Size } & -0.0001 & $0.0012 * * *$ & $0.0024 * * *$ & $0.0035 * * *$ & $0.0043 * * *$ & $0.0051 * * *$ & $0.0063 * * *$ \\
\hline & $(-0.58)$ & $(6.01)$ & (7.92) & (8.28) & (8.12) & (7.29) & $(5.68)$ \\
\hline \multirow[t]{2}{*}{ Book-to-Market Ratio } & -0.0001 & $0.0006 *$ & 0.0005 & 0.0010 & $0.0016 *$ & 0.0016 & 0.0024 \\
\hline & $(-0.16)$ & (1.72) & (1.13) & (1.31) & (1.66) & $(1.34)$ & $(0.98)$ \\
\hline \multirow[t]{2}{*}{ Leverage } & -0.0001 & -0.0002 & -0.0001 & -0.0003 & -0.0003 & 0.0003 & $0.0020 * *$ \\
\hline & $(-0.27)$ & $(-0.94)$ & $(-0.03)$ & $(-1.06)$ & $(-0.85)$ & $(0.56)$ & (2.43) \\
\hline \multirow[t]{2}{*}{ ROA } & 0.0001 & 0.0001 & $0.0049 * * *$ & $0.0067 * * *$ & $0.0068 * * *$ & $0.0043 *$ & 0.0027 \\
\hline & $(0.31)$ & $(0.16)$ & (4.17) & (4.27) & (3.26) & (1.68) & $(0.61)$ \\
\hline \multirow[t]{2}{*}{ Resource Sector } & -0.0008 & 0.0004 & $0.0028 *$ & 0.0010 & 0.0022 & 0.0005 & 0.0056 \\
\hline & $(-1.16)$ & $(0.38)$ & (1.82) & $(0.47)$ & $(0.81)$ & $(0.15)$ & $(1.02)$ \\
\hline \multirow[t]{2}{*}{ Financial Sector } & 0.0006 & 0.0006 & $0.0050 * *$ & $0.0066^{* *}$ & 0.0053 & 0.0030 & 0.0104 \\
\hline & $(0.60)$ & $(0.35)$ & $(2.15)$ & (2.08) & $(1.31)$ & $(0.52)$ & $(1.12)$ \\
\hline Max. VIF & 1.88 & 1.88 & 1.86 & 1.83 & 1.87 & 1.87 & 1.86 \\
\hline F Value & 7.38 & 11.08 & 12.97 & 10.70 & 10.09 & 7.39 & 6.89 \\
\hline R-Square & $1.89 \%$ & $2.73 \%$ & $3.57 \%$ & $3.80 \%$ & $5.12 \%$ & $5.96 \%$ & $11.32 \%$ \\
\hline Adj R-Sq & $1.63 \%$ & $2.48 \%$ & $3.29 \%$ & $3.44 \%$ & $4.61 \%$ & $5.15 \%$ & $9.68 \%$ \\
\hline $\mathbf{N}$ & 8,474 & 8,728 & 7,738 & 5,987 & 4,134 & 2,590 & 1,155 \\
\hline
\end{tabular}


Table 6-23. The Regression Results of Market-sensitive Announcements with Bad News - Intraday $\triangle$ CARs after the GFC

\begin{tabular}{|c|c|c|c|c|c|c|c|}
\hline Variable & $\triangle$ CAR10min & $\Delta$ CAR15min & $\triangle$ CAR30min & $\triangle$ CAR60min & $\triangle$ CAR90min & $\triangle$ CAR120min & $\Delta$ CAR150min \\
\hline \multirow[t]{2}{*}{ Intercept } & $-0.0066 * * *$ & $-0.0129 * * *$ & $-0.0238 * * *$ & $-\mathbf{0 . 0 3 8 4} * * *$ & $-0.0469 * * *$ & $-0.0598 * * *$ & $-0.0541 * * *$ \\
\hline & $(-5.75)$ & $(-8.62)$ & $(-11.96)$ & $(-14.12)$ & $(-12.58)$ & $(-10.24)$ & $(-6.05)$ \\
\hline \multirow[t]{2}{*}{ Takeover / Scheme Announcements } & -0.0006 & -0.0023 & -0.0003 & -0.0019 & -0.0027 & 0.0014 & 0.0024 \\
\hline & $(-0.24)$ & $(-0.74)$ & $(-0.08)$ & $(-0.34)$ & $(-0.34)$ & $(0.11)$ & $(0.12)$ \\
\hline \multirow[t]{2}{*}{ Security Holder Details } & -0.0019 & $-0.0119 * *$ & $-0.0195 * *$ & -0.0100 & -0.0084 & -0.0101 & $-0.0455 *$ \\
\hline & $(-0.44)$ & $(-2.09)$ & $(-2.58)$ & $(-0.99)$ & $(-0.70)$ & $(-0.55)$ & $(-1.70)$ \\
\hline \multirow[t]{2}{*}{ Periodic Reports } & $-0.0044 * * *$ & $-0.0078 * * *$ & $-0.0099 * * *$ & $-0.0130 * * *$ & $-0.0125 * * *$ & $-0.0155 * *$ & $-0.0191 * *$ \\
\hline & $(-3.73)$ & $(-5.11)$ & $(-4.88)$ & $(-4.62)$ & $(-3.18)$ & $(-2.50)$ & $(-2.09)$ \\
\hline \multirow[t]{2}{*}{ Issued Capital } & $-0.0048 * * *$ & $-0.0108 * * *$ & $-0.0099 * * *$ & $-0.0128 * * *$ & -0.0055 & -0.0076 & -0.0187 \\
\hline & $(-3.57)$ & $(-6.25)$ & $(-4.32)$ & $(-4.04)$ & $(-1.19)$ & $(-1.04)$ & $(-1.63)$ \\
\hline \multirow[t]{2}{*}{ Asset Acquisition \& Disposal } & -0.0005 & -0.0025 & -0.0018 & -0.0030 & -0.0046 & -0.0101 & $-0.0250 * *$ \\
\hline & $(-0.32)$ & $(-1.33)$ & $(-0.69)$ & $(-0.91)$ & $(-1.01)$ & $(-1.31)$ & $(-2.10)$ \\
\hline \multirow[t]{2}{*}{ Notice of Meeting } & -0.0012 & -0.0043 & $-0.0272 *$ & -0.0241 & -0.0269 & 0.0033 & - \\
\hline & $(-0.15)$ & $(-0.41)$ & $(-1.67)$ & $(-1.34)$ & $(-1.11)$ & $(0.06)$ & - \\
\hline \multirow[t]{2}{*}{ ASX Announcement } & $0.0034 *$ & $0.0048 * *$ & 0.0021 & -0.0035 & -0.0083 & 0.0028 & -0.0100 \\
\hline & (1.91) & $(2.00)$ & $(0.69)$ & $(-0.83)$ & $(-1.39)$ & $(0.30)$ & $(-0.71)$ \\
\hline \multirow[t]{2}{*}{ Distribution Announcement } & -0.0018 & -0.0049 & -0.0044 & -0.0064 & -0.0062 & -0.0150 & -0.0137 \\
\hline & $(-0.78)$ & $(-1.61)$ & $(-1.1)$ & $(-1.21)$ & $(-0.84)$ & $(-1.31)$ & $(-0.73)$ \\
\hline \multirow[t]{2}{*}{ Progress Reports } & $-0.0046 * * *$ & $-0.0084 * * *$ & $-0.0099 * * *$ & $-0.0122 * * *$ & $-0.0106 * * *$ & $-0.0124 * * *$ & $-0.0140 * *$ \\
\hline & $(-5.93)$ & $(-8.47)$ & $(-7.43)$ & $(-6.77)$ & $(-4.22)$ & $(-3.12)$ & $(-2.29)$ \\
\hline \multirow[t]{2}{*}{ Company Administration } & -0.0028 & -0.0040 & -0.0045 & -0.0078 & -0.0038 & -0.0019 & -0.0211 \\
\hline & $(-1.32)$ & $(-1.47)$ & $(-1.23)$ & $(-1.50)$ & $(-0.56)$ & $(-0.18)$ & $(-1.31)$ \\
\hline Notice of Call & - & - & - & - & - & - & - \\
\hline \multirow{2}{*}{ Other } & -0.0057 & $-0.0100 * *$ & -0.0069 & 0.0001 & -0.0004 & 0.0000 & -0.0071 \\
\hline & $(-1.6)$ & $(-2.26)$ & $(-1.09)$ & $(0.01)$ & $(-0.03)$ & $(0.00)$ & $(-0.25)$ \\
\hline \multirow[t]{2}{*}{ Chairman's Address } & -0.0016 & -0.0059 & -0.0065 & -0.0116 & -0.0070 & -0.0045 & -0.0015 \\
\hline & $(-0.41)$ & $(-1.22)$ & $(-1.00)$ & $(-1.23)$ & $(-0.47)$ & $(-0.18)$ & $(-0.04)$ \\
\hline
\end{tabular}




\begin{tabular}{|c|c|c|c|c|c|c|c|}
\hline Variable & $\triangle \mathrm{CAR} 10 \mathrm{~min}$ & $\Delta$ CAR15min & $\triangle \mathrm{CAR30min}$ & $\triangle \mathrm{CAR60min}$ & $\triangle$ CAR90min & $\triangle \mathrm{CAR} 120 \mathrm{~min}$ & $\Delta$ CAR150min \\
\hline \multirow[t]{2}{*}{ Letter to Shareholders } & 0.0047 & 0.0013 & 0.0021 & -0.0077 & - & - & - \\
\hline & $(0.18)$ & $(0.05)$ & $(0.07)$ & $(-0.15)$ & - & - & - \\
\hline \multirow[t]{2}{*}{ ASX Query } & $-0.0156 * * *$ & $-0.0219 * * *$ & $-0.0288 * * *$ & -0.0356 **** & $-0.0384 * * *$ & $-0.0591 * * *$ & $-0.0832 * * *$ \\
\hline & $(-8.91)$ & $(-10.08)$ & $(-10.21)$ & $(-9.59)$ & $(-7.70)$ & $(-7.89)$ & $(-7.80)$ \\
\hline Structured Products & - & - & - & - & - & - & - \\
\hline \multirow[t]{2}{*}{ CTE Quarterly Reports } & $-0.0040 * *$ & -0.0027 & 0.0015 & 0.0002 & 0.0032 & 0.0051 & -0.0064 \\
\hline & $(-2.36)$ & $(-1.17)$ & $(0.50)$ & $(0.04)$ & $(0.52)$ & $(0.53)$ & $(-0.45)$ \\
\hline \multirow[t]{2}{*}{ Bid-Ask Spread } & 0.0028 & 0.0034 & 0.0070 & 0.0228 & 0.0217 & -0.0050 & -0.0189 \\
\hline & $(0.78)$ & $(0.63)$ & $(1.00)$ & $(1.22)$ & (1.06) & $(-0.26)$ & $(-0.80)$ \\
\hline \multirow[t]{2}{*}{ Size } & $0.0005 * * *$ & $0.0014 * * *$ & $0.0025 * * *$ & $0.0043 * * *$ & $0.0052 * * *$ & $0.0077 * * *$ & $0.0084 * * *$ \\
\hline & (3.29) & (7.17) & $(9.36)$ & (11.87) & (10.72) & $(9.95)$ & (7.27) \\
\hline \multirow[t]{2}{*}{ Book-to-Market Ratio } & -0.0001 & -0.0002 & -0.0002 & 0.0008 & -0.0003 & -0.0009 & -0.0008 \\
\hline & $(-0.30)$ & $(-0.73)$ & $(-0.50)$ & $(1.53)$ & $(-0.38)$ & $(-0.83)$ & $(-0.55)$ \\
\hline \multirow[t]{2}{*}{ Leverage } & 0.0001 & 0.0001 & 0.0002 & 0.0001 & -0.0005 & -0.0004 & -0.0013 \\
\hline & $(0.17)$ & $(0.07)$ & $(0.88)$ & $(-0.02)$ & $(-1.55)$ & $(-0.76)$ & $(-0.99)$ \\
\hline \multirow[t]{2}{*}{ ROA } & 0.0001 & 0.0001 & 0.0004 & 0.0001 & 0.0001 & -0.0001 & -0.0005 \\
\hline & $(0.82)$ & $(0.47)$ & $(0.84)$ & $(0.06)$ & $(0.36)$ & $(-0.27)$ & $(-0.34)$ \\
\hline \multirow[t]{2}{*}{ Resource Sector } & 0.0002 & 0.0005 & $0.0023 *$ & $0.0032 *$ & 0.0021 & 0.0019 & -0.0093 \\
\hline & $(0.31)$ & $(0.44)$ & $(1.71)$ & (1.70) & $(0.83)$ & $(0.46)$ & $(-1.51)$ \\
\hline \multirow[t]{2}{*}{ Financial Sector } & $0.0044 * * *$ & $0.0073 * * *$ & $0.0110 * * *$ & $0.0134 * * *$ & $0.0128 * * *$ & $0.0166 * *$ & 0.0046 \\
\hline & (3.48) & $(4.48)$ & $(5.03)$ & $(4.43)$ & (3.01) & (2.43) & $(0.49)$ \\
\hline Max. VIF & 1.79 & 1.76 & 1.74 & 1.75 & 1.75 & 1.75 & 1.86 \\
\hline F Value & 7.12 & 12.51 & 14.19 & 14.43 & 11.03 & 9.84 & 7.44 \\
\hline R-Square & $1.78 \%$ & $3.06 \%$ & $3.98 \%$ & $5.24 \%$ & $5.41 \%$ & $7.59 \%$ & $11.97 \%$ \\
\hline Adj R-Sq & $1.53 \%$ & $2.82 \%$ & $3.70 \%$ & $4.88 \%$ & $4.92 \%$ & $6.82 \%$ & $10.36 \%$ \\
\hline $\mathbf{N}$ & 8,659 & 8,731 & 7,561 & 5,762 & 4,069 & 2,538 & 1,116 \\
\hline
\end{tabular}


Table 6-24. The Regression Results of Market-sensitive Announcements with Good News - Intraday CARs before the GFC

\begin{tabular}{|c|c|c|c|c|c|c|c|c|}
\hline Variable & CAR10min & CAR15min & CAR30min & CAR60min & CAR90min & CAR120min & CAR150min & CAR180min \\
\hline \multirow[t]{2}{*}{ Intercept } & $0.0140 * * *$ & $\mathbf{0 . 0 2 5 8}^{* * *}$ & $0.0341 * * *$ & $0.0404 * * *$ & $0.0452 * * *$ & $0.0486 * * *$ & $0.0499 * * *$ & $0.0522 * * *$ \\
\hline & $(10.14)$ & $(16.72)$ & (19.16) & $(20.01)$ & (18.79) & $(18.95)$ & $(18.14)$ & $(17.01)$ \\
\hline \multirow{2}{*}{$\begin{array}{l}\text { Takeover / Scheme } \\
\text { Announcements }\end{array}$} & $0.0068 * * *$ & $0.0094 * * *$ & $0.0079 * *$ & $0.0123 * * *$ & $0.0122 * * *$ & $0.0173 * * *$ & $0.0150 * * *$ & $0.0166 * * *$ \\
\hline & (2.78) & (3.14) & (2.37) & (3.28) & $(2.80)$ & (3.60) & $(2.85)$ & (2.94) \\
\hline \multirow[t]{2}{*}{ Security Holder Details } & $0.0174 * * *$ & $0.0191 * * *$ & $0.0142 * * *$ & $0.0204 * * *$ & $0.0215 * * *$ & $0.0197 * *$ & $\mathbf{0 . 0 3 3 4} * * *$ & $0.0341 * * *$ \\
\hline & $(3.63)$ & $(3.84)$ & $(2.58)$ & $(3.11)$ & $(2.81)$ & $(2.40)$ & $(4.04)$ & $(3.85)$ \\
\hline \multirow[t]{2}{*}{ Periodic Reports } & $0.0127 * * *$ & $0.0170 * * *$ & $0.0185 * * *$ & $0.0206 * * *$ & $0.0199 * * *$ & $0.0204 * * *$ & $0.0214 * * *$ & $0.0199 * * *$ \\
\hline & $(9.37)$ & (11.19) & $(10.54)$ & $(10.31)$ & $(8.43)$ & $(8.06)$ & $(7.89)$ & $(6.78)$ \\
\hline \multirow[t]{2}{*}{ Issued Capital } & $0.0071 * * *$ & $0.0108 * * *$ & $0.0128 * * *$ & $0.0146 * * *$ & $0.0127 * * *$ & $0.0123 * * *$ & $0.0140 * * *$ & $0.0129 * * *$ \\
\hline & $(4.70)$ & $(6.28)$ & $(6.44)$ & $(6.36)$ & $(4.71)$ & $(4.28)$ & $(4.60)$ & $(3.90)$ \\
\hline \multirow{2}{*}{$\begin{array}{l}\text { Asset Acquisition \& } \\
\text { Disposal }\end{array}$} & $0.0119 * * *$ & $0.0165 * * *$ & $0.0166 * * *$ & $0.0171 * * *$ & $0.0155 * * *$ & $0.0161 * * *$ & $0.0169 * * *$ & $0.0129 * * *$ \\
\hline & (8.40) & (10.36) & (9.04) & $(8.17)$ & $(6.35)$ & $(6.21)$ & $(6.11)$ & $(4.33)$ \\
\hline \multirow[t]{2}{*}{ Notice of Meeting } & 0.0075 & 0.0074 & 0.0161 & 0.0128 & 0.0153 & 0.0180 & 0.0194 & 0.0201 \\
\hline & $(0.78)$ & $(0.73)$ & $(1.30)$ & $(0.99)$ & $(0.94)$ & $(1.04)$ & $(1.05)$ & $(0.94)$ \\
\hline \multirow[t]{2}{*}{ ASX Announcement } & -0.0031 & $-0.0072 * *$ & -0.0034 & -0.0032 & -0.0053 & -0.0074 & -0.0005 & 0.0079 \\
\hline & $(-1.34)$ & $(-2.48)$ & $(-0.98)$ & $(-0.80)$ & $(-1.07)$ & $(-1.44)$ & $(-0.09)$ & $(1.33)$ \\
\hline \multirow{2}{*}{$\begin{array}{l}\text { Distribution } \\
\text { Announcement }\end{array}$} & $0.0096 * * *$ & $0.0127 * * *$ & $0.0131 * * *$ & $0.0158 * * *$ & $0.0146 * * *$ & $0.0140 * * *$ & $0.0176 * * *$ & $0.0144 * * *$ \\
\hline & $(3.76)$ & $(4.56)$ & $(4.17)$ & $(4.26)$ & $(3.35)$ & $(3.06)$ & $(3.46)$ & $(2.64)$ \\
\hline \multirow[t]{2}{*}{ Progress Reports } & $0.0204 * * *$ & $0.0260 * * *$ & $0.0256 * * *$ & $0.0259 * * *$ & $0.0244 * * *$ & $0.0238 * * *$ & $0.0244 * * *$ & $0.0219 * * *$ \\
\hline & $(21.17)$ & $(23.29)$ & (19.66) & $(17.46)$ & (13.98) & $(12.72)$ & $(12.05)$ & $(10.00)$ \\
\hline \multirow[t]{2}{*}{ Company Administration } & 0.0048 & $0.0162 * *$ & $0.0236 * * *$ & $0.0261 * * *$ & 0.0198* & 0.0196 & 0.0204 & 0.0099 \\
\hline & $(0.67)$ & $(2.29)$ & $(2.80)$ & $(2.90)$ & $(1.83)$ & $(1.60)$ & (1.64) & $(0.77)$ \\
\hline Notice of Call & - & - & - & - & - & - & - & - \\
\hline \multirow[t]{2}{*}{ Other } & $0.0098 * * *$ & $0.0156 * * *$ & $0.0147 * * *$ & $0.0160 * * *$ & $0.0157 * * *$ & $0.0170 * * *$ & $0.0165 * * *$ & $0.0159 * * *$ \\
\hline & $(4.86)$ & $(7.27)$ & $(5.96)$ & $(5.71)$ & $(4.82)$ & (4.86) & (4.44) & (3.98) \\
\hline \multirow[t]{2}{*}{ Chairman's Address } & $0.0093 * * *$ & $0.0125 * * *$ & $0.0124 * * *$ & $0.0162 * * *$ & $0.0168 * * *$ & $0.0149 * * *$ & $0.0188 * * *$ & $0.0178 * * *$ \\
\hline & $(2.85)$ & $(3.45)$ & $(3.00)$ & $(3.52)$ & $(3.17)$ & $(2.74)$ & $(3.23)$ & $(2.95)$ \\
\hline
\end{tabular}




\begin{tabular}{|c|c|c|c|c|c|c|c|c|}
\hline Variable & CAR10min & CAR15min & CAR30min & CAR60min & CAR90min & CAR120min & CAR150min & CAR180min \\
\hline \multirow[t]{2}{*}{ Letter to Shareholders } & 0.0129 & 0.0110 & 0.0414* & $0.0899 * * *$ & $0.0745 * *$ & $0.1427 * * *$ & $\mathbf{0 . 1 2 3 0} * * *$ & $0.0978 * * *$ \\
\hline & $(0.71)$ & $(0.65)$ & (1.68) & $(2.75)$ & (2.52) & (3.92) & (3.33) & (2.64) \\
\hline \multirow[t]{2}{*}{ ASX Query } & 0.0031 & 0.0037 & $0.0093 * *$ & $0.0108 * *$ & 0.0078 & 0.0034 & 0.0051 & 0.0075 \\
\hline & $(1.06)$ & $(1.15)$ & $(2.41)$ & $(2.31)$ & $(1.50)$ & $(0.58)$ & $(0.79)$ & $(0.97)$ \\
\hline Structured Products & - & - & - & - & - & - & - & - \\
\hline \multirow[t]{2}{*}{ CTE Quarterly Reports } & -0.0021 & $-0.0043 * *$ & $-0.0044 *$ & -0.0026 & -0.0049 & -0.0047 & -0.0007 & -0.0052 \\
\hline & $(-1.16)$ & $(-2.05)$ & $(-1.81)$ & $(-0.92)$ & $(-1.43)$ & $(-1.27)$ & $(-0.18)$ & $(-1.16)$ \\
\hline \multirow[t]{2}{*}{ Bid-Ask Spread } & 0.0016 & -0.0002 & 0.0032 & 0.0035 & 0.0168 & 0.0066 & -0.0009 & -0.0112 \\
\hline & $(0.28)$ & $(-0.03)$ & $(0.36)$ & $(0.28)$ & (1.06) & $(0.40)$ & $(-0.06)$ & $(-0.58)$ \\
\hline \multirow[t]{2}{*}{ Size } & $-0.0027 * * *$ & $-0.0045 * * *$ & $-0.0053 * * *$ & $-0.0063 * * *$ & $-0.0067 * * *$ & $-0.0070 * * *$ & $-0.0073 * * *$ & $-0.0072 * * *$ \\
\hline & $(-15.33)$ & $(-24.2)$ & $(-24.96)$ & $(-25.76)$ & $(-23.46)$ & $(-23.04)$ & $(-22.68)$ & $(-20.36)$ \\
\hline \multirow[t]{2}{*}{ Book-to-Market Ratio } & $-0.0014 * * *$ & $-0.0017 * * *$ & $-0.0015 * *$ & -0.0009 & $-\mathbf{0 . 0 0 1 8} * *$ & $-0.0019 * *$ & $-0.0017 *$ & -0.0012 \\
\hline & $(-2.79)$ & $(-2.94)$ & $(-2.34)$ & $(-1.30)$ & $(-2.10)$ & $(-2.17)$ & $(-1.83)$ & $(-1.00)$ \\
\hline \multirow[t]{2}{*}{ Leverage } & $0.0001 * * *$ & $0.0001 * * *$ & $0.0001 * * *$ & $0.0001 * * *$ & $0.0001 * * *$ & $0.0001 * * *$ & $0.0001 * * *$ & $0.0001 * * *$ \\
\hline & (4.37) & (5.23) & $(6.56)$ & $(5.85)$ & (6.88) & (7.15) & (6.34) & (6.36) \\
\hline \multirow[t]{2}{*}{ ROA } & $-0.0006 *$ & $-0.0008 * *$ & $-0.0013 * * * *$ & $-0.0015 * * *$ & $-0.0027 * * *$ & $-0.0031 * * * *$ & $-0.0032 * * * *$ & $-0.0044 * * *$ \\
\hline & $(-1.70)$ & $(-2.12)$ & $(-3.05)$ & $(-3.14)$ & $(-3.86)$ & $(-4.36)$ & $(-4.32)$ & $(-4.52)$ \\
\hline \multirow[t]{2}{*}{ Resource Sector } & -0.0005 & $-0.0027 * * *$ & $-0.0044 * * *$ & $-0.0043 * * *$ & $-0.0035 * * *$ & $-0.0043 * * *$ & $-0.0040 * * *$ & $-0.0042 * * *$ \\
\hline & $(-0.66)$ & $(-3.26)$ & $(-4.60)$ & $(-3.96)$ & $(-2.73)$ & $(-3.22)$ & $(-2.79)$ & $(-2.77)$ \\
\hline \multirow[t]{2}{*}{ Financial Sector } & $-0.0048 * * *$ & $-0.0068 * * *$ & $-0.0084 * * *$ & $-0.0089 * * *$ & $-0.0070 * * * *$ & $-0.0078 * * * *$ & $-0.0072 * * *$ & $-0.0080 * * *$ \\
\hline & $(-3.92)$ & $(-5.12)$ & $(-5.48)$ & $(-5.12)$ & $(-3.49)$ & $(-3.70)$ & $(-3.16)$ & $(-3.26)$ \\
\hline Max. VIF & 2.60 & 2.91 & 2.99 & 3.02 & 3.10 & 3.18 & 3.25 & 3.35 \\
\hline F Value & 48.52 & 76.57 & 67.14 & 61.22 & 49.63 & 47.3 & 43.67 & 38.04 \\
\hline R-Square & $8.75 \%$ & $11.33 \%$ & $10.34 \%$ & $10.34 \%$ & $9.32 \%$ & $9.85 \%$ & $10.03 \%$ & $9.89 \%$ \\
\hline Adj R-Sq & $8.57 \%$ & $11.18 \%$ & $10.19 \%$ & $10.17 \%$ & $9.13 \%$ & $9.64 \%$ & $9.80 \%$ & $9.63 \%$ \\
\hline $\mathbf{N}$ & 11,152 & 13,211 & 12,826 & 11,698 & 10,647 & 9,546 & 8,637 & 7,647 \\
\hline
\end{tabular}

$*, * *$ and $* * *$ denote significance at the $10 \%, 5 \%$ and $1 \%$ levels respectively (two-tailed). 
Table 6-25. The Regression Results of Market-sensitive Announcements with Good News - Intraday CARs during the GFC

\begin{tabular}{|c|c|c|c|c|c|c|c|c|}
\hline Variable & CAR10min & CAR15min & CAR30min & CAR60min & CAR90min & CAR120min & CAR150min & CAR180min \\
\hline \multirow[t]{2}{*}{ Intercept } & $\mathbf{0 . 0 1 2 6}^{* * *}$ & $\mathbf{0 . 0 2 6 5}{ }^{* * *}$ & $\mathbf{0 . 0 3 6 1} * * *$ & $\mathbf{0 . 0 4 6 0} * * *$ & $\mathbf{0 . 0 5 3 0} * * *$ & $\mathbf{0 . 0 5 4 7} * * *$ & $\mathbf{0 . 0 5 8 9} * * *$ & $0.0628 * * *$ \\
\hline & (7.73) & (14.18) & (16.52) & (18.44) & (18.48) & (17.63) & (17.59) & (17.69) \\
\hline \multirow{2}{*}{$\begin{array}{l}\text { Takeover / Scheme } \\
\text { Announcements }\end{array}$} & $0.0049 *$ & $0.0107 * * *$ & $0.0137 * * *$ & $0.0192 * * *$ & $0.0241 * * *$ & $0.0262 * * *$ & $0.0209 * * *$ & $0.0234 * * *$ \\
\hline & (1.66) & (3.16) & (3.42) & (4.12) & $(4.50)$ & (4.41) & (3.35) & (3.54) \\
\hline \multirow[t]{2}{*}{ Security Holder Details } & $0.0113 * *$ & $0.0233 * * *$ & $0.0261 * * *$ & $0.0299 * * *$ & $0.0236 * * *$ & $0.0242 * * *$ & $0.0221 * *$ & $0.0212 * *$ \\
\hline & (2.39) & (4.19) & (4.32) & (4.12) & (2.78) & (2.73) & $(2.22)$ & (2.19) \\
\hline \multirow[t]{2}{*}{ Periodic Reports } & $0.0112 * * *$ & $0.0168 * * *$ & $0.0190 * * *$ & $0.0201 * * *$ & $0.0228 * * *$ & $0.0245 * * *$ & $0.0243^{* * *}$ & $0.0207 * * *$ \\
\hline & $(6.58)$ & (8.68) & (8.43) & (7.71) & (7.66) & (7.71) & (7.03) & $(5.74)$ \\
\hline \multirow[t]{2}{*}{ Issued Capital } & $0.0125 * * *$ & $0.0209 * * *$ & $0.0229 * * *$ & $0.0233 * * *$ & $0.0257 * * *$ & $0.0261 * * *$ & $0.0270 * * *$ & $0.0227 * * *$ \\
\hline & (6.76) & (9.69) & $(9.22)$ & (8.18) & (7.89) & (7.47) & (7.13) & (5.62) \\
\hline \multirow{2}{*}{$\begin{array}{l}\text { Asset Acquisition \& } \\
\text { Disposal }\end{array}$} & $0.0181 * * *$ & $0.0268 * * *$ & $0.0309 * * *$ & $0.0323 * * *$ & $0.0306^{* * * *}$ & $0.0305^{* * *}$ & $\mathbf{0 . 0 2 7 0}^{* * *}$ & $\mathbf{0 . 0 2 3 0}$ **** \\
\hline & $(9.79)$ & (12.94) & $(12.93)$ & (11.78) & $(9.81)$ & $(9.08)$ & $(7.54)$ & (6.20) \\
\hline \multirow[t]{2}{*}{ Notice of Meeting } & 0.0080 & 0.0129 & 0.0081 & -0.0033 & -0.0006 & -0.0005 & -0.0040 & -0.0056 \\
\hline & $(0.69)$ & $(1.05)$ & $(0.61)$ & $(-0.22)$ & $(-0.04)$ & $(-0.03)$ & $(-0.23)$ & $(-0.29)$ \\
\hline \multirow[t]{2}{*}{ ASX Announcement } & -0.0031 & $-0.0086 * * *$ & $-0.0098 * * *$ & $-0.0148 * * *$ & $-0.0169 * * *$ & $-0.0160 * * *$ & $-0.0173 * * *$ & $-0.0162 * * *$ \\
\hline & $(-1.15)$ & $(-2.68)$ & $(-2.66)$ & $(-3.41)$ & $(-3.44)$ & $(-3.01)$ & $(-2.97)$ & $(-2.66)$ \\
\hline \multirow{2}{*}{$\begin{array}{l}\text { Distribution } \\
\text { Announcement }\end{array}$} & $0.0097 * * *$ & $0.0142 * * *$ & $0.0173 * * *$ & $0.0189 * * *$ & $0.0198 * * *$ & $0.0204 * * *$ & $0.0189 * * *$ & $0.0168 * *$ \\
\hline & $(2.72)$ & (3.52) & (3.75) & (3.54) & (3.18) & (3.13) & (2.66) & $(2.25)$ \\
\hline \multirow[t]{2}{*}{ Progress Reports } & $0.0216 * * *$ & $0.0294 * * *$ & $0.0303 * * *$ & $0.0290 * * *$ & $0.0278^{* * * *}$ & $0.0275 * * *$ & $0.0259 * * *$ & $0.0229 * * *$ \\
\hline & (19.54) & (22.96) & (20.09) & (16.64) & (13.99) & (12.83) & (11.18) & (9.30) \\
\hline \multirow[t]{2}{*}{ Company Administration } & 0.0072 & 0.0084 & 0.0108 & 0.0100 & 0.0092 & 0.0130 & 0.0048 & 0.0037 \\
\hline & $(1.41)$ & $(1.42)$ & $(1.50)$ & $(1.28)$ & $(1.12)$ & $(1.44)$ & $(0.51)$ & $(0.38)$ \\
\hline Notice of Call & - & - & - & - & - & - & - & - \\
\hline \multirow[t]{2}{*}{ Other } & $0.0088 * *$ & $0.0130^{* * * *}$ & $0.0150 * * *$ & $0.0148 * * *$ & $0.0166^{* * * *}$ & $0.0157 * *$ & $0.0147 * *$ & $0.0143^{* *}$ \\
\hline & (2.17) & (2.94) & (3.04) & (2.70) & (2.74) & (2.41) & (2.08) & (1.97) \\
\hline \multirow[t]{2}{*}{ Chairman's Address } & $0.0091 * *$ & $0.0127 * *$ & $0.0141 * *$ & $0.0153 * *$ & $0.0212 * * *$ & $0.0206 * * *$ & $0.0200 * *$ & $0.0206 * *$ \\
\hline & (1.99) & $(2.41)$ & $(2.32)$ & $(2.33)$ & $(2.95)$ & $(2.73)$ & $(2.43)$ & $(2.49)$ \\
\hline
\end{tabular}




\begin{tabular}{|c|c|c|c|c|c|c|c|c|}
\hline Variable & CAR10min & CAR15min & CAR30min & CAR60min & CAR90min & CAR120min & CAR150min & CAR180min \\
\hline \multirow[t]{2}{*}{ Letter to Shareholders } & 0.0498* & 0.0444 & 0.0065 & -0.0024 & 0.0106 & -0.0067 & -0.0108 & 0.0018 \\
\hline & $(1.77)$ & $(1.28)$ & $(0.17)$ & $(-0.06)$ & $(0.23)$ & $(-0.14)$ & $(-0.23)$ & $(0.04)$ \\
\hline \multirow[t]{2}{*}{ ASX Query } & $0.0055 * *$ & $0.0097 * * *$ & $0.0091 * *$ & $0.0132 * * *$ & $0.0190 * * *$ & $0.0195 * * *$ & $0.0157 * *$ & 0.0116 \\
\hline & $(2.04)$ & $(2.95)$ & $(2.47)$ & $(2.98)$ & $(3.57)$ & $(3.32)$ & $(2.45)$ & $(1.53)$ \\
\hline Structured Products & - & - & - & - & - & - & - & - \\
\hline \multirow[t]{2}{*}{ CTE Quarterly Reports } & 0.0007 & -0.0015 & -0.0044 & $-0.0074 *$ & 0.0002 & -0.0009 & -0.0018 & -0.0076 \\
\hline & $(0.30)$ & $(-0.51)$ & $(-1.25)$ & $(-1.82)$ & $(0.04)$ & $(-0.17)$ & $(-0.31)$ & $(-1.28)$ \\
\hline \multirow[t]{2}{*}{ Bid-Ask Spread } & -0.0154 & $-0.0256 * *$ & -0.0068 & 0.0098 & -0.0054 & 0.0040 & -0.0064 & -0.0077 \\
\hline & $(-1.44)$ & $(-2.17)$ & $(-0.53)$ & $(0.99)$ & $(-0.30)$ & $(0.21)$ & $(-0.31)$ & $(-0.42)$ \\
\hline \multirow[t]{2}{*}{ Size } & $-0.0023 * * *$ & $-0.0044 * * *$ & $-0.0056 * * *$ & $-0.0064 * * *$ & $-0.0070 * * *$ & $-0.0070 * * *$ & $-0.0072 * * *$ & $-0.0077 * * *$ \\
\hline & $(-10.92)$ & $(-19.23)$ & $(-21.20)$ & $(-21.23)$ & $(-20.39)$ & $(-18.73)$ & $(-18.15)$ & $(-18.51)$ \\
\hline \multirow[t]{2}{*}{ Book-to-Market Ratio } & $-0.0010 * * *$ & $-0.0013 * * *$ & $-0.0018 * * *$ & $-0.0013 * *$ & $-0.0018 * * *$ & $-0.0018 * * *$ & $-0.0019 * * *$ & $-0.0017 * *$ \\
\hline & $(-2.70)$ & $(-3.20)$ & $(-3.59)$ & $(-2.54)$ & $(-3.01)$ & $(-2.82)$ & $(-2.77)$ & $(-2.39)$ \\
\hline \multirow[t]{2}{*}{ Leverage } & $-0.0002 * *$ & $-\mathbf{0 . 0 0 0 3} * *$ & -0.0001 & $-0.0004 * *$ & $-0.0004 * *$ & $-0.0008 * * *$ & $-0.0009 * * *$ & -0.0002 \\
\hline & $(-2.40)$ & $(-2.39)$ & $(-0.04)$ & $(-2.09)$ & $(-2.34)$ & $(-3.77)$ & $(-4.36)$ & $(-0.49)$ \\
\hline \multirow[t]{2}{*}{ ROA } & -0.0001 & -0.0001 & 0.0001 & 0.0001 & -0.0001 & -0.0001 & -0.0001 & -0.0001 \\
\hline & $(-0.79)$ & $(-0.12)$ & $(0.09)$ & $(0.08)$ & $(-0.87)$ & $(-0.81)$ & $(-0.73)$ & $(-0.76)$ \\
\hline \multirow[t]{2}{*}{ Resource Sector } & -0.0003 & $-0.0023 * *$ & $-0.0024 *$ & $-0.0045 * * *$ & $-0.0050 * * *$ & $-0.0055 * * *$ & $-0.0058 * * *$ & $-0.0039 *$ \\
\hline & $(-0.28)$ & $(-1.99)$ & $(-1.80)$ & $(-2.94)$ & $(-2.90)$ & $(-2.97)$ & $(-2.91)$ & $(-1.87)$ \\
\hline \multirow[t]{2}{*}{ Financial Sector } & -0.0017 & -0.0016 & -0.0013 & $-\mathbf{0 . 0 0 5 0} * *$ & $-0.0068 * *$ & $-0.0079 * * *$ & $-0.0082 * *$ & -0.0050 \\
\hline & $(-1.04)$ & $(-0.85)$ & $(-0.59)$ & $(-2.05)$ & $(-2.45)$ & $(-2.65)$ & $(-2.57)$ & $(-1.50)$ \\
\hline Max. VIF & 2.07 & 2.23 & 2.31 & 2.37 & 2.41 & 2.44 & 2.49 & 2.60 \\
\hline F Value & 29.28 & 50.65 & 45.85 & 40.2 & 33.24 & 28.99 & 26.28 & 23.34 \\
\hline R-Square & $5.63 \%$ & $7.89 \%$ & $7.46 \%$ & $7.20 \%$ & $6.60 \%$ & $6.36 \%$ & $6.44 \%$ & $6.52 \%$ \\
\hline Adj R-Sq & $5.44 \%$ & $7.73 \%$ & $7.29 \%$ & $7.02 \%$ & $6.40 \%$ & $6.14 \%$ & $6.19 \%$ & $6.24 \%$ \\
\hline $\mathbf{N}$ & 10,815 & 13,032 & 12,541 & 11,426 & 10,375 & 9,421 & 8,426 & 7,384 \\
\hline
\end{tabular}

$*, * *$ and $* * *$ denote significance at the $10 \%, 5 \%$ and $1 \%$ levels respectively (two-tailed). 
Table 6-26. The Regression Results of Market-sensitive Announcements with Good News - Intraday CARs after the GFC

\begin{tabular}{|c|c|c|c|c|c|c|c|c|}
\hline Variable & CAR10min & CAR15min & CAR30min & CAR60min & CAR90min & CAR120min & CAR150min & CAR180min \\
\hline \multirow[t]{2}{*}{ Intercept } & $\mathbf{0 . 0 1 3 6}{ }^{* * *}$ & $\mathbf{0 . 0 2 0 8}^{* * *}$ & $\mathbf{0 . 0 3 0 1} * * *$ & $\mathbf{0 . 0 3 7 2} * * *$ & $\mathbf{0 . 0 4 3 8} * * *$ & $0.0490 * * *$ & $0.0509 * * *$ & $0.0557 * * *$ \\
\hline & (9.10) & (12.14) & (15.32) & (15.82) & (17.11) & (17.24) & (16.07) & (15.90) \\
\hline \multirow{2}{*}{$\begin{array}{l}\text { Takeover / Scheme } \\
\text { Announcements }\end{array}$} & $0.0057 *$ & $0.0122 * * *$ & $0.0113 * * *$ & $0.0171 * * *$ & $0.0208 * * *$ & $0.0179 * * *$ & $0.0190 * * *$ & $0.0209 * * *$ \\
\hline & $(1.92)$ & $(3.58)$ & $(2.82)$ & $(3.37)$ & $(3.83)$ & $(2.97)$ & $(2.77)$ & $(2.79)$ \\
\hline \multirow[t]{2}{*}{ Security Holder Details } & $0.0104 * *$ & $0.0151 * * *$ & $0.0154 * *$ & $0.0179 * *$ & $0.0162 *$ & $0.0209 * *$ & $0.0178 *$ & 0.0165 \\
\hline & $(2.14)$ & $(2.60)$ & $(2.36)$ & $(2.21)$ & $(1.90)$ & $(2.26)$ & $(1.70)$ & $(1.54)$ \\
\hline \multirow[t]{2}{*}{ Periodic Reports } & $0.0109 * * *$ & $0.0192 * * *$ & $0.0213 * * *$ & $0.0221 * * *$ & $0.0229 * * *$ & $0.0223 * * *$ & $0.0213 * * *$ & $0.0226 * * *$ \\
\hline & $(6.96)$ & (10.68) & (10.45) & $(9.01)$ & $(8.58)$ & $(7.55)$ & $(6.61)$ & (6.39) \\
\hline \multirow[t]{2}{*}{ Issued Capital } & $0.0078 * * *$ & $0.0134 * * *$ & $0.0145 * * *$ & $0.0176 * * *$ & $0.0158 * * *$ & $0.0172 * * *$ & $0.0158 * * *$ & $0.0186 * * *$ \\
\hline & $(4.36)$ & $(6.55)$ & $(6.14)$ & $(6.07)$ & $(4.89)$ & $(4.78)$ & $(4.00)$ & $(4.19)$ \\
\hline \multirow{2}{*}{$\begin{array}{l}\text { Asset Acquisition \& } \\
\text { Disposal }\end{array}$} & $0.0138 * * *$ & $0.0213 * * *$ & $0.0236 * * *$ & $\mathbf{0 . 0 2 0 0} * * *$ & $0.0207 * * *$ & $0.0207 * * *$ & $0.0184 * * *$ & $0.0180 * * *$ \\
\hline & (7.70) & (10.49) & $(10.25)$ & (7.33) & (6.95) & $(6.29)$ & $(5.15)$ & $(4.60)$ \\
\hline \multirow[t]{2}{*}{ Notice of Meeting } & 0.0115 & 0.0148 & 0.0148 & 0.0225 & 0.0239 & 0.0162 & 0.0128 & 0.0138 \\
\hline & $(1.30)$ & $(1.40)$ & $(1.29)$ & $(1.47)$ & $(1.46)$ & $(0.92)$ & $(0.67)$ & $(0.71)$ \\
\hline \multirow[t]{2}{*}{ ASX Announcement } & -0.0022 & -0.0049 & -0.0035 & -0.0064 & $-0.0096 * *$ & $-0.0093 *$ & $-0.0112 * *$ & $-0.0118 *$ \\
\hline & $(-0.88)$ & $(-1.58)$ & $(-1.04)$ & $(-1.54)$ & $(-2.13)$ & $(-1.79)$ & $(-1.97)$ & $(-1.89)$ \\
\hline \multirow{2}{*}{$\begin{array}{l}\text { Distribution } \\
\text { Announcement }\end{array}$} & $0.0116 * * *$ & $0.0165 * * *$ & $0.0190 * * *$ & $0.0203 * * *$ & $0.0211 * * *$ & $0.0221 * * *$ & $0.0195 * * *$ & $0.0160 * *$ \\
\hline & (3.83) & (4.67) & (4.67) & (4.20) & (4.02) & (3.72) & (3.04) & (2.35) \\
\hline \multirow[t]{2}{*}{ Progress Reports } & $0.0198 * * *$ & $0.0267 * * *$ & $0.0281 * * *$ & $0.0306 * * *$ & $0.0288 * * *$ & $0.0285 * * *$ & $0.0265 * * *$ & $0.0252 * * *$ \\
\hline & $(19.52)$ & $(22.99)$ & $(21.13)$ & (19.14) & $(16.61)$ & $(14.82)$ & $(12.41)$ & (10.53) \\
\hline \multirow[t]{2}{*}{ Company Administration } & 0.0045 & $0.0075 * *$ & $0.0076 * *$ & $0.0109 * *$ & $0.0117 * *$ & $0.0133 * * *$ & $0.0097^{*}$ & 0.0084 \\
\hline & $(1.57)$ & $(2.26)$ & $(2.05)$ & $(2.48)$ & $(2.50)$ & $(2.59)$ & $(1.68)$ & $(1.31)$ \\
\hline Notice of Call & - & - & - & - & - & - & - & - \\
\hline \multirow[t]{2}{*}{ Other } & $0.0078 *$ & $0.0147 * * *$ & $0.0139 * *$ & $0.0163 * *$ & $0.0129 *$ & $0.0128 *$ & 0.0128 & 0.0100 \\
\hline & $(1.86)$ & $(2.94)$ & $(2.52)$ & $(2.44)$ & $(1.80)$ & $(1.66)$ & $(1.58)$ & $(1.12)$ \\
\hline \multirow[t]{2}{*}{ Chairman's Address } & $0.0084 *$ & $0.0139 * *$ & $0.0162 * * *$ & $0.0159 * *$ & $0.0167 * *$ & $0.0185 * *$ & 0.0169* & 0.0156 \\
\hline & (1.72) & $(2.56)$ & $(2.70)$ & (2.24) & $(2.22)$ & (2.16) & (1.76) & $(1.49)$ \\
\hline
\end{tabular}




\begin{tabular}{|c|c|c|c|c|c|c|c|c|}
\hline Variable & CAR10min & CAR15min & CAR30min & CAR60min & CAR90min & CAR120min & CAR150min & CAR180min \\
\hline \multirow[t]{2}{*}{ Letter to Shareholders } & 0.0075 & 0.0032 & 0.0002 & -0.0012 & -0.0042 & -0.0060 & 0.0040 & 0.0031 \\
\hline & $(0.28)$ & $(0.07)$ & $(0.00)$ & $(-0.02)$ & $(-0.07)$ & $(-0.10)$ & $(0.06)$ & $(0.05)$ \\
\hline \multirow[t]{2}{*}{ ASX Query } & $0.0060 * *$ & $0.0076 * *$ & $0.0145 * * *$ & $0.0139 * * *$ & $0.0131 * *$ & $0.0108 *$ & 0.0118 & 0.0111 \\
\hline & $(2.32)$ & $(2.55)$ & (4.18) & (3.16) & $(2.51)$ & (1.78) & $(1.62)$ & $(1.42)$ \\
\hline Structured Products & - & - & - & - & - & - & - & - \\
\hline \multirow[t]{2}{*}{ CTE Quarterly Reports } & -0.0020 & -0.0011 & -0.0022 & -0.0020 & -0.0039 & -0.0032 & -0.0023 & -0.0050 \\
\hline & $(-0.80)$ & $(-0.38)$ & $(-0.68)$ & $(-0.50)$ & $(-0.87)$ & $(-0.64)$ & $(-0.42)$ & $(-0.84)$ \\
\hline \multirow[t]{2}{*}{ Bid-Ask Spread } & -0.0097 & $-0.0218 * *$ & $-0.0299 * *$ & -0.0056 & $-0.0713 * *$ & $-0.0356 *$ & -0.0329 & -0.0184 \\
\hline & $(-1.52)$ & $(-2.53)$ & $(-2.26)$ & $(-0.55)$ & $(-2.55)$ & $(-1.76)$ & $(-1.21)$ & $(-1.22)$ \\
\hline \multirow[t]{2}{*}{ Size } & $-0.0027 * * *$ & $-0.0043^{* * * *}$ & $-0.0051 * * *$ & $-0.0060 * * *$ & $-0.0067 * * *$ & $-0.0072 * * *$ & $-0.0073^{* * *}$ & $-0.0076 * * *$ \\
\hline & $(-13.26)$ & $(-18.94)$ & $(-19.95)$ & $(-19.63)$ & $(-19.98)$ & $(-19.59)$ & $(-17.69)$ & $(-17.09)$ \\
\hline \multirow[t]{2}{*}{ Book-to-Market Ratio } & -0.0001 & -0.0003 & -0.0005 & 0.0001 & 0.0003 & 0.0004 & 0.0008 & 0.0007 \\
\hline & $(-0.17)$ & $(-1.04)$ & $(-1.30)$ & $(0.14)$ & $(0.60)$ & $(0.77)$ & (1.46) & $(1.25)$ \\
\hline \multirow[t]{2}{*}{ Leverage } & -0.0001 & -0.0001 & -0.0001 & 0.0002 & $0.0001 * * *$ & 0.0001 & -0.0002 & -0.0002 \\
\hline & $(-0.29)$ & $(-0.54)$ & $(-0.76)$ & $(0.98)$ & $(0.10)$ & $(0.15)$ & $(-0.52)$ & $(-0.62)$ \\
\hline \multirow[t]{2}{*}{ ROA } & -0.0001 & $-0.0003 * * *$ & $-0.0002 *$ & $-0.0008 * * *$ & $-0.0009 * * *$ & $-0.0010 * * *$ & $-0.0013 *$ & $-0.0012 *$ \\
\hline & $(-1.15)$ & $(-2.95)$ & $(-1.65)$ & $(-3.71)$ & $(-4.00)$ & $(-4.16)$ & $(-1.88)$ & $(-1.73)$ \\
\hline \multirow[t]{2}{*}{ Resource Sector } & $-0.0018 *$ & -0.0011 & $-0.0035 * * *$ & $-0.0045 * * *$ & $-0.0050 * * *$ & $-0.0067 * * *$ & $-0.0055 * * *$ & $-0.0078 * * *$ \\
\hline & $(-1.79)$ & $(-0.99)$ & $(-2.72)$ & $(-2.99)$ & $(-3.07)$ & $(-3.73)$ & $(-2.83)$ & $(-3.71)$ \\
\hline \multirow[t]{2}{*}{ Financial Sector } & $-0.0057 * * *$ & $-0.0081 * * *$ & $-0.0126 * * *$ & $-0.0142 * * *$ & $-0.0146 * * *$ & $-0.0159 * * *$ & $-0.0142 * * *$ & $-0.0159 * * *$ \\
\hline & $(-3.44)$ & $(-4.30)$ & $(-5.88)$ & $(-5.48)$ & $(-5.20)$ & $(-5.12)$ & $(-4.19)$ & $(-4.29)$ \\
\hline Max. VIF & 1.91 & 1.98 & 2.04 & 2.05 & 2.08 & 2.12 & 2.21 & 2.34 \\
\hline F Value & 30.53 & 47.61 & 45.81 & 41.96 & 39.09 & 34.64 & 27.50 & 24.45 \\
\hline R-Square & $5.98 \%$ & $8.14 \%$ & $8.12 \%$ & $8.14 \%$ & $8.32 \%$ & $8.24 \%$ & $7.38 \%$ & $7.52 \%$ \\
\hline Adj R-Sq & $5.79 \%$ & $7.97 \%$ & $7.95 \%$ & $7.95 \%$ & $8.11 \%$ & $8.00 \%$ & $7.11 \%$ & $7.22 \%$ \\
\hline $\mathbf{N}$ & 10,575 & 11,848 & 11,423 & 10,439 & 9,499 & 8,512 & 7,617 & 6,633 \\
\hline
\end{tabular}


Table 6-27. The Regression Results of Market-sensitive Announcements with Good News - Intraday $\triangle$ CARs before the GFC

\begin{tabular}{|c|c|c|c|c|c|c|c|}
\hline Variable & $\Delta$ CAR10min & $\triangle$ CAR15min & $\Delta$ CAR30min & $\triangle$ CAR60min & $\triangle$ CAR90min & $\triangle$ CAR120min & $\Delta$ CAR150min \\
\hline \multirow[t]{2}{*}{ Intercept } & $0.0160 * * *$ & $\mathbf{0 . 0 2 9 4} * * *$ & $0.0406 * * *$ & $\mathbf{0 . 0 5 2 2} * * *$ & $0.0618 * * *$ & $0.0661 * * *$ & $0.0653^{* * * *}$ \\
\hline & (11.47) & (18.45) & $(21.23)$ & (20.95) & (17.69) & (14.82) & $(10.47)$ \\
\hline \multirow[t]{2}{*}{ Takeover / Scheme Announcements } & $0.0058 * *$ & $0.0072 * *$ & 0.0051 & 0.0052 & 0.0079 & 0.0138* & 0.0024 \\
\hline & $(2.30)$ & $(2.35)$ & $(1.41)$ & $(1.18)$ & $(1.29)$ & $(1.67)$ & $(0.19)$ \\
\hline \multirow[t]{2}{*}{ Security Holder Details } & $0.0151 * * *$ & $0.0173 * * *$ & 0.0095 & $0.0261 * * *$ & 0.0107 & 0.0023 & 0.0104 \\
\hline & $(3.06)$ & (3.20) & $(1.55)$ & (3.03) & $(0.88)$ & $(0.16)$ & $(0.54)$ \\
\hline \multirow[t]{2}{*}{ Periodic Reports } & $0.0113 * * *$ & $0.0142 * * *$ & $0.0155 * * *$ & $0.0155 * * *$ & $0.0136 * * *$ & $0.0131 * * *$ & 0.0088 \\
\hline & $(8.21)$ & $(9.16)$ & $(8.24)$ & $(6.26)$ & $(3.97)$ & $(2.96)$ & $(1.48)$ \\
\hline \multirow[t]{2}{*}{ Issued Capital } & $0.0065 * * *$ & $0.0100 * * *$ & $0.0129 * * *$ & $0.0139 * * *$ & $0.0142 * * *$ & $0.0131 * *$ & $0.0151 * *$ \\
\hline & $(4.21)$ & $(5.65)$ & $(5.97)$ & $(4.80)$ & $(3.55)$ & (2.57) & $(2.25)$ \\
\hline \multirow[t]{2}{*}{ Asset Acquisition \& Disposal } & $0.0113 * * *$ & $0.0149 * * *$ & $0.0159 * * *$ & $0.0150 * * *$ & $0.0152 * * *$ & $0.0192 * * *$ & $0.0172 * * *$ \\
\hline & $(7.82)$ & $(9.07)$ & $(8.09)$ & $(5.78)$ & $(4.18)$ & $(4.04)$ & $(2.81)$ \\
\hline \multirow[t]{2}{*}{ Notice of Meeting } & 0.0058 & 0.0024 & 0.0137 & 0.0141 & 0.0149 & 0.0036 & 0.0408 \\
\hline & $(0.63)$ & $(0.24)$ & $(0.95)$ & $(0.81)$ & $(0.69)$ & $(0.16)$ & $(0.82)$ \\
\hline \multirow[t]{2}{*}{ ASX Announcement } & $-0.0039 *$ & $-0.0088 * * *$ & $-0.0061 *$ & 0.0012 & 0.0046 & 0.0068 & 0.0097 \\
\hline & $(-1.67)$ & $(-2.99)$ & $(-1.70)$ & $(0.25)$ & $(0.67)$ & $(0.78)$ & $(0.83)$ \\
\hline \multirow[t]{2}{*}{ Distribution Announcement } & $0.0091 * * *$ & $0.0115 * * *$ & $0.0129 * * *$ & $0.0148 * * *$ & $0.0140 * *$ & $0.0202 * *$ & $0.0211 * *$ \\
\hline & $(3.61)$ & (3.96) & (3.73) & (3.31) & (2.30) & $(2.55)$ & (2.13) \\
\hline \multirow[t]{2}{*}{ Progress Reports } & $0.0197 * * *$ & $0.0246 * * *$ & $0.0243 * * *$ & $0.0241 * * *$ & $0.0255 * * *$ & $0.0243 * * *$ & $0.0183 * * *$ \\
\hline & $(20.21)$ & $(21.62)$ & $(17.89)$ & $(13.45)$ & $(10.24)$ & $(7.53)$ & $(4.21)$ \\
\hline \multirow[t]{2}{*}{ Company Administration } & 0.0060 & 0.0139* & $0.0225 * *$ & $\mathbf{0 . 0 3 3 9} * * *$ & 0.0225 & $0.0428 *$ & 0.0403 \\
\hline & $(0.76)$ & (1.81) & (2.36) & $(2.72)$ & $(1.18)$ & $(1.67)$ & $(1.60)$ \\
\hline Notice of Call & - & - & - & - & - & - & - \\
\hline \multirow[t]{2}{*}{ Other } & $0.0103 * * *$ & $0.0158 * * *$ & $0.0142 * * *$ & $0.0149 * * *$ & $0.0174 * * *$ & $0.0201 * * *$ & $0.0258 * * *$ \\
\hline & $(4.89)$ & $(6.99)$ & $(5.25)$ & $(4.20)$ & $(3.58)$ & $(3.15)$ & $(3.00)$ \\
\hline \multirow[t]{2}{*}{ Chairman's Address } & $0.0098 * * *$ & $0.0125 * * *$ & $0.0113 * *$ & $0.0136 * *$ & 0.0076 & 0.0022 & 0.0128 \\
\hline & $(2.86)$ & $(3.35)$ & $(2.54)$ & $(2.10)$ & $(0.81)$ & $(0.17)$ & $(0.73)$ \\
\hline
\end{tabular}




\begin{tabular}{|c|c|c|c|c|c|c|c|}
\hline Variable & $\Delta$ CAR10min & $\Delta$ CAR15min & $\triangle \mathrm{CAR30min}$ & $\triangle$ CAR60min & $\triangle$ CAR90min & $\triangle \mathrm{CAR} 120 \mathrm{~min}$ & $\Delta$ CAR150min \\
\hline \multirow[t]{2}{*}{ Letter to Shareholders } & 0.0073 & 0.0047 & 0.0361 & - & - & - & - \\
\hline & $(0.45)$ & $(0.30)$ & $(1.45)$ & - & - & - & - \\
\hline \multirow[t]{2}{*}{ ASX Query } & 0.0039 & $0.0061 *$ & $0.0136 * * *$ & $0.0176 * * *$ & $0.0186 * * *$ & $0.0279 * * *$ & $0.0242 * *$ \\
\hline & $(1.27)$ & (1.79) & (3.24) & (3.34) & (2.89) & (3.31) & (2.37) \\
\hline Structured Products & - & - & - & - & - & - & - \\
\hline \multirow[t]{2}{*}{ CTE Quarterly Reports } & -0.0018 & $-0.0040 *$ & -0.0042 & -0.0016 & -0.0001 & 0.0050 & 0.0089 \\
\hline & $(-0.98)$ & $(-1.82)$ & $(-1.62)$ & $(-0.46)$ & $(-0.03)$ & $(0.78)$ & (1.02) \\
\hline \multirow[t]{2}{*}{ Bid-Ask Spread } & -0.0015 & -0.0013 & 0.0025 & 0.0056 & 0.0275 & 0.0265 & -0.0040 \\
\hline & $(-0.30)$ & $(-0.21)$ & $(0.21)$ & $(0.36)$ & (1.03) & $(0.89)$ & $(-0.12)$ \\
\hline \multirow[t]{2}{*}{ Size } & $-0.0028 * * *$ & $-0.0047 * * *$ & $-0.0058 * * *$ & $-0.0074 * * *$ & $-0.0087 * * *$ & $-0.0095 * * *$ & $-0.0083 * * *$ \\
\hline & $(-15.54)$ & $(-24.12)$ & $(-24.73)$ & $(-23.84)$ & $(-20.10)$ & $(-16.94)$ & $(-10.99)$ \\
\hline \multirow[t]{2}{*}{ Book-to-Market Ratio } & $-0.0014 * * *$ & $-0.0021 * * *$ & $-0.0030 * * *$ & $-0.0026 * * *$ & $-0.0025 *$ & $-0.0027 * *$ & -0.0015 \\
\hline & $(-2.91)$ & $(-3.69)$ & $(-4.12)$ & $(-3.20)$ & $(-2.30)$ & $(-2.12)$ & $(-1.07)$ \\
\hline \multirow[t]{2}{*}{ Leverage } & $0.0001 * * *$ & $0.0001 * * *$ & $0.0001 * * *$ & $0.0001 * * *$ & $0.0001 * * *$ & 0.0001 & $0.0001 * *$ \\
\hline & $(3.82)$ & $(5.22)$ & (7.19) & $(4.80)$ & $(5.80)$ & $(1.64)$ & (2.19) \\
\hline \multirow[t]{2}{*}{ ROA } & -0.0005 & $-0.0007 *$ & $-0.0009 * *$ & -0.0009 & $-0.0020 * * *$ & $-0.0015 * *$ & $-0.0108 * * *$ \\
\hline & $(-1.27)$ & $(-1.81)$ & $(-2.23)$ & $(-1.46)$ & $(-3.08)$ & $(-2.04)$ & $(-3.43)$ \\
\hline \multirow[t]{2}{*}{ Resource Sector } & -0.0002 & $-0.0027 * * *$ & $-0.0042 * * *$ & $-0.0049 * * *$ & $-0.0067 * * *$ & $-0.0047 *$ & -0.0047 \\
\hline & $(-0.30)$ & $(-3.09)$ & $(-3.98)$ & $(-3.48)$ & $(-3.38)$ & $(-1.81)$ & $(-1.36)$ \\
\hline \multirow[t]{2}{*}{ Financial Sector } & $-0.0050 * * *$ & $-0.0064 * * *$ & $-0.0081 * * *$ & $-0.0083^{* * * *}$ & $-0.0058^{*}$ & -0.0055 & -0.0061 \\
\hline & $(-4.00)$ & $(-4.60)$ & $(-4.79)$ & $(-3.67)$ & $(-1.85)$ & $(-1.40)$ & $(-1.14)$ \\
\hline Max. VIF & 2.51 & 2.79 & 2.70 & 2.70 & 2.71 & 2.71 & 2.79 \\
\hline F Value & 45.33 & 69.73 & 59.84 & 45.81 & 31.40 & 19.61 & 11.73 \\
\hline R-Square & $8.35 \%$ & $11.06 \%$ & $10.79 \%$ & $10.57 \%$ & $10.51 \%$ & $11.04 \%$ & $14.62 \%$ \\
\hline Adj R-Sq & $8.17 \%$ & $10.90 \%$ & $10.61 \%$ & $10.34 \%$ & $10.18 \%$ & $10.48 \%$ & $13.37 \%$ \\
\hline $\mathbf{N}$ & 10,969 & 12,366 & 10,908 & 8,160 & 5,634 & 3,339 & 1,461 \\
\hline
\end{tabular}


Table 6-28. The Regression Results of Market-sensitive Announcements with Good News - Intraday $\triangle$ CARs during the GFC

\begin{tabular}{|c|c|c|c|c|c|c|c|}
\hline Variable & $\triangle \mathrm{CAR} 10 \mathrm{~min}$ & $\triangle \mathrm{CAR} 15 \mathrm{~min}$ & $\Delta$ CAR30min & $\triangle$ CAR60min & $\Delta$ CAR90min & $\triangle \mathrm{CAR} 120 \mathrm{~min}$ & $\Delta$ CAR150min \\
\hline \multirow[t]{2}{*}{ Intercept } & $0.0141 * * *$ & $\mathbf{0 . 0 2 8 4} * * *$ & $0.0401 * * *$ & $0.0565 * * *$ & $\mathbf{0 . 0 6 3 9} * * *$ & $0.0599 * * *$ & $0.0759 * * *$ \\
\hline & (8.27) & (14.31) & (16.54) & (19.03) & (15.68) & (11.66) & (8.53) \\
\hline \multirow[t]{2}{*}{ Takeover / Scheme Announcements } & 0.0047 & $0.0088 * *$ & $0.0153 * * *$ & $0.0169 * * *$ & 0.0020 & -0.0007 & -0.0091 \\
\hline & $(1.51)$ & $(2.42)$ & (3.40) & (3.17) & $(0.29)$ & $(-0.08)$ & $(-0.65)$ \\
\hline \multirow[t]{2}{*}{ Security Holder Details } & $0.0118 * *$ & $0.0237 * * *$ & $0.0260 * * *$ & $0.0304 * * *$ & $\mathbf{0 . 0 2 3 7 *}$ & 0.0187 & -0.0168 \\
\hline & (2.27) & (3.86) & $(3.52)$ & (3.29) & (1.78) & $(1.17)$ & $(-0.62)$ \\
\hline \multirow[t]{2}{*}{ Periodic Reports } & $0.0111 * * *$ & $0.0163 * * *$ & $0.0206 * * *$ & $0.0187 * * *$ & $0.0188 * * *$ & $0.0177 * * *$ & $0.0179 * *$ \\
\hline & $(6.10)$ & (7.83) & $(8.19)$ & $(5.95)$ & (4.67) & (3.53) & (2.11) \\
\hline \multirow[t]{2}{*}{ Issued Capital } & $0.0130 * * *$ & $0.0205 * * *$ & $0.0239 * * *$ & $0.0209 * * *$ & $0.0192 * * *$ & $0.0179 * * *$ & $0.0155 *$ \\
\hline & $(6.67)$ & $(8.95)$ & $(8.70)$ & $(6.13)$ & (4.39) & (3.33) & (1.78) \\
\hline \multirow[t]{2}{*}{ Asset Acquisition \& Disposal } & $0.0183 * * *$ & $0.0263 * * *$ & $0.0336 * * *$ & $0.0339 * * *$ & $0.0314 * * *$ & $0.0327 * * *$ & $0.0268 * * *$ \\
\hline & (9.39) & (11.89) & (12.54) & (10.37) & $(7.31)$ & (5.89) & (3.01) \\
\hline \multirow[t]{2}{*}{ Notice of Meeting } & 0.0037 & 0.0079 & 0.0090 & -0.0141 & -0.0155 & 0.0024 & -0.0049 \\
\hline & $(0.34)$ & $(0.66)$ & $(0.60)$ & $(-0.85)$ & $(-0.74)$ & $(0.09)$ & $(-0.10)$ \\
\hline \multirow[t]{2}{*}{ ASX Announcement } & $-0.0046^{*}$ & $-0.0092 * * *$ & -0.0051 & $-0.0122 * *$ & $-0.0174 * * *$ & -0.0005 & -0.0119 \\
\hline & $(-1.67)$ & $(-2.79)$ & $(-1.25)$ & $(-2.42)$ & $(-2.64)$ & $(-0.05)$ & $(-0.87)$ \\
\hline \multirow[t]{2}{*}{ Distribution Announcement } & $0.0098 * *$ & $0.0147 * * *$ & $0.0202 * * *$ & $0.0133 * *$ & 0.0093 & 0.0121 & -0.0102 \\
\hline & (2.57) & $(3.41)$ & (3.86) & (2.10) & $(1.17)$ & $(1.28)$ & $(-0.67)$ \\
\hline \multirow[t]{2}{*}{ Progress Reports } & $0.0217 * * *$ & $0.0300 * * *$ & $0.0313 * * *$ & $0.0278 * * *$ & $0.0245 * * *$ & $\mathbf{0 . 0 2 3 3} * * *$ & $0.0201 * * *$ \\
\hline & (18.81) & (22.04) & (19.12) & (13.81) & $(9.43)$ & (7.07) & $(3.68)$ \\
\hline \multirow[t]{2}{*}{ Company Administration } & 0.0063 & 0.0072 & 0.0098 & 0.0062 & 0.0079 & -0.0086 & -0.0122 \\
\hline & $(1.06)$ & $(1.05)$ & $(1.28)$ & $(0.66)$ & $(0.65)$ & $(-0.61)$ & $(-0.53)$ \\
\hline Notice of Call & - & - & - & - & - & - & - \\
\hline \multirow[t]{2}{*}{ Other } & $0.0111 * * *$ & $0.0146^{* * * *}$ & $0.0198 * * *$ & $0.0176^{* * *}$ & $0.0234 * * *$ & $0.0206 *$ & 0.0052 \\
\hline & $(2.68)$ & (3.13) & $(3.57)$ & $(2.60)$ & (2.73) & (1.79) & $(0.27)$ \\
\hline \multirow[t]{2}{*}{ Chairman's Address } & $0.0104 * *$ & $0.0132 * *$ & $0.0170 * *$ & 0.0161 & 0.0108 & 0.0094 & -0.0172 \\
\hline & $(2.10)$ & $(2.25)$ & $(2.20)$ & $(1.60)$ & $(0.85)$ & $(0.62)$ & $(-0.67)$ \\
\hline
\end{tabular}




\begin{tabular}{|c|c|c|c|c|c|c|c|}
\hline Variable & $\Delta$ CAR10min & $\Delta$ CAR15min & $\Delta$ CAR30min & $\triangle \mathrm{CAR60min}$ & $\triangle \mathrm{CAR} 90 \mathrm{~min}$ & $\triangle$ CAR120min & $\Delta$ CAR150min \\
\hline \multirow[t]{2}{*}{ Letter to Shareholders } & 0.0489* & 0.0435 & 0.0052 & -0.0073 & 0.0005 & -0.0158 & 0.0052 \\
\hline & (1.66) & $(1.21)$ & $(0.13)$ & $(-0.17)$ & $(0.01)$ & $(-0.34)$ & $(0.07)$ \\
\hline \multirow[t]{2}{*}{ ASX Query } & $0.0088 * * *$ & $0.0158 * * *$ & $0.0188 * * *$ & $0.0236 * * *$ & $0.0198 * * *$ & $0.0220 * * *$ & 0.0099 \\
\hline & (3.06) & (4.66) & $(4.78)$ & (4.90) & (3.52) & (3.23) & $(0.98)$ \\
\hline Structured Products & - & - & - & - & - & - & - \\
\hline \multirow{2}{*}{ CTE Quarterly Reports } & 0.0008 & -0.0005 & -0.0038 & -0.0049 & -0.0006 & 0.0053 & 0.0045 \\
\hline & $(0.30)$ & $(-0.16)$ & $(-1.02)$ & $(-1.05)$ & $(-0.09)$ & $(0.65)$ & $(0.32)$ \\
\hline \multirow[t]{2}{*}{ Bid-Ask Spread } & -0.0003 & -0.0005 & -0.0117 & 0.0137 & -0.0213 & -0.0049 & 0.0067 \\
\hline & $(-0.44)$ & $(-0.64)$ & $(-0.86)$ & $(1.17)$ & $(-0.77)$ & $(-0.13)$ & $(0.11)$ \\
\hline \multirow[t]{2}{*}{ Size } & $-0.0024 * * *$ & $-0.0045 * * *$ & $-0.0060 * * *$ & $-0.0073 * * *$ & $-0.0070 * * *$ & $-0.0059 * * *$ & $-0.0066 * * *$ \\
\hline & $(-10.71)$ & $(-18.44)$ & $(-20.28)$ & $(-19.96)$ & $(-13.65)$ & $(-9.09)$ & $(-6.02)$ \\
\hline \multirow[t]{2}{*}{ Book-to-Market Ratio } & $-0.0011 * * *$ & $-0.0012 * * *$ & $-0.0011 * *$ & -0.0008 & -0.0004 & 0.0005 & -0.0007 \\
\hline & $(-3.04)$ & $(-2.96)$ & $(-2.02)$ & $(-1.40)$ & $(-0.55)$ & $(0.53)$ & $(-0.36)$ \\
\hline \multirow[t]{2}{*}{ Leverage } & $-0.0002 * *$ & 0.0001 & 0.0001 & 0.0001 & $-0.0006 * * *$ & -0.0009 & $-0.0040 * * *$ \\
\hline & $(-2.39)$ & $(0.06)$ & $(-0.27)$ & $(0.36)$ & $(-3.05)$ & $(-1.53)$ & $(-3.27)$ \\
\hline \multirow[t]{2}{*}{ ROA } & -0.0001 & -0.0001 & -0.0001 & -0.0001 & $-0.0071 * * *$ & $-0.0105 * * *$ & $-0.0089 *$ \\
\hline & $(-0.74)$ & $(-0.19)$ & $(-0.02)$ & $(-0.70)$ & $(-3.50)$ & $(-3.71)$ & $(-1.93)$ \\
\hline \multirow[t]{2}{*}{ Resource Sector } & 0.0004 & -0.0016 & -0.0019 & $-0.0052 * * *$ & $-0.0092 * * *$ & $-0.0066 * *$ & $-0.0114 * *$ \\
\hline & $(0.38)$ & $(-1.30)$ & $(-1.21)$ & $(-2.73)$ & $(-3.70)$ & $(-2.11)$ & $(-2.16)$ \\
\hline \multirow[t]{2}{*}{ Financial Sector } & -0.0016 & -0.0027 & -0.0032 & -0.0034 & -0.0060 & -0.0032 & 0.0015 \\
\hline & $(-0.90)$ & $(-1.37)$ & $(-1.31)$ & $(-1.10)$ & $(-1.52)$ & $(-0.65)$ & $(0.18)$ \\
\hline Max. VIF & 2.00 & 2.16 & 2.12 & 2.11 & 2.12 & 2.15 & 2.23 \\
\hline F Value & 27.06 & 44.91 & 39.93 & 30.08 & 19.81 & 10.68 & 5.20 \\
\hline R-Square & $5.43 \%$ & $7.62 \%$ & $7.71 \%$ & $7.79 \%$ & $7.48 \%$ & $6.66 \%$ & $7.28 \%$ \\
\hline Adj R-Sq & $5.23 \%$ & $7.45 \%$ & $7.52 \%$ & $7.53 \%$ & $7.10 \%$ & $6.03 \%$ & $5.88 \%$ \\
\hline $\mathbf{N}$ & 10,395 & 11,993 & 10,533 & 7,857 & 5,417 & 3,316 & 1,482 \\
\hline
\end{tabular}


Table 6-29. The Regression Results of Market-sensitive Announcements with Good News - Intraday $\triangle$ CARs after the GFC

\begin{tabular}{|c|c|c|c|c|c|c|c|}
\hline Variable & $\Delta$ CAR10min & $\triangle \mathrm{CAR} 15 \mathrm{~min}$ & $\Delta$ CAR30min & $\triangle$ CAR60min & $\triangle$ CAR90min & $\triangle$ CAR120min & $\triangle$ CAR150min \\
\hline \multirow[t]{2}{*}{ Intercept } & $0.0162 * * *$ & $\mathbf{0 . 0 2 5 1} * * *$ & $\mathbf{0 . 0 3 5 5} * * *$ & $0.0412 * * *$ & $0.0526 * * *$ & $0.0656 * * *$ & $\mathbf{0 . 0 5 7 8}^{* * * *}$ \\
\hline & $(10.32)$ & (13.79) & $(16.18)$ & (13.86) & $(13.88)$ & (11.79) & $(7.51)$ \\
\hline \multirow[t]{2}{*}{ Takeover / Scheme Announcements } & 0.0041 & $0.0088 * *$ & $0.0089 * *$ & 0.0093 & $0.0142 *$ & 0.0159 & $0.0361 * *$ \\
\hline & $(1.35)$ & $(2.47)$ & $(2.10)$ & $(1.48)$ & (1.86) & $(1.49)$ & (2.18) \\
\hline \multirow[t]{2}{*}{ Security Holder Details } & $0.0099 *$ & $0.0132 * *$ & $0.0157 * *$ & 0.0109 & 0.0191 & 0.0076 & -0.0048 \\
\hline & $(1.89)$ & (2.13) & (1.98) & (1.05) & $(1.31)$ & $(0.41)$ & $(-0.18)$ \\
\hline \multirow[t]{2}{*}{ Periodic Reports } & $0.0101 * * *$ & $0.0175 * * *$ & $0.0193 * * *$ & $0.0206 * * *$ & $0.0218 * * *$ & $0.0225 * * *$ & $0.0337 * * *$ \\
\hline & $(6.10)$ & $(9.19)$ & $(8.46)$ & $(6.73)$ & $(5.49)$ & (3.96) & $(4.22)$ \\
\hline \multirow[t]{2}{*}{ Issued Capital } & $0.0065 * * *$ & $0.0127 * * *$ & $0.0150 * * *$ & $0.0214 * * *$ & $0.0191 * * *$ & $0.0208 * * *$ & $0.0272 * * *$ \\
\hline & (3.48) & $(5.81)$ & $(5.67)$ & $(5.95)$ & (4.16) & (3.09) & (2.98) \\
\hline \multirow[t]{2}{*}{ Asset Acquisition \& Disposal } & $0.0132 * * *$ & $0.0198 * * *$ & $0.0223 * * *$ & $0.0212 * * *$ & $0.0217 * * *$ & $0.0202 * * *$ & $0.0200 * *$ \\
\hline & $(6.90)$ & $(9.09)$ & $(8.65)$ & $(6.04)$ & $(4.92)$ & $(3.35)$ & $(2.43)$ \\
\hline \multirow[t]{2}{*}{ Notice of Meeting } & 0.0118 & 0.0137 & 0.0098 & 0.0173 & 0.0281 & 0.0004 & - \\
\hline & (1.19) & $(1.12)$ & $(0.76)$ & $(0.81)$ & $(0.87)$ & $(0.01)$ & - \\
\hline \multirow[t]{2}{*}{ ASX Announcement } & -0.0035 & -0.0069 & 0.0001 & 0.0060 & 0.0008 & 0.0078 & 0.0158 \\
\hline & $(-1.27)$ & $(-2.14)$ & $(0.02)$ & (1.16) & $(0.11)$ & $(0.80)$ & $(1.11)$ \\
\hline \multirow[t]{2}{*}{ Distribution Announcement } & $0.0107 * * *$ & $0.0148 * * *$ & $0.0177 * * *$ & $0.0187 * * *$ & $0.0229 * * *$ & 0.0137 & 0.0144 \\
\hline & (3.35) & (4.00) & (4.01) & (3.04) & (2.94) & (1.18) & $(0.98)$ \\
\hline \multirow[t]{2}{*}{ Progress Reports } & $0.0200 * * *$ & $0.0262 * * *$ & $0.0272 * * *$ & $0.0303 * * *$ & $0.0306 * * *$ & $0.0295 * * *$ & $0.0238 * * *$ \\
\hline & $(19.00)$ & $(21.25)$ & (18.61) & $(15.55)$ & $(12.57)$ & $(8.58)$ & $(5.15)$ \\
\hline \multirow[t]{2}{*}{ Company Administration } & 0.0043 & $0.0064 *$ & 0.0064 & $0.0111 * *$ & $0.0157 * *$ & $0.0229 * *$ & $0.0454 * * *$ \\
\hline & $(1.42)$ & (1.80) & $(1.51)$ & (2.08) & $(2.31)$ & $(2.56)$ & (3.26) \\
\hline Notice of Call & - & - & - & - & - & - & - \\
\hline \multirow[t]{2}{*}{ Other } & $0.0086 *$ & $0.0143 * * *$ & $0.0134 * *$ & $0.0184 * *$ & $0.0227 * *$ & 0.0251 & 0.0182 \\
\hline & (1.94) & $(2.68)$ & $(2.17)$ & $(2.16)$ & $(2.23)$ & $(1.64)$ & $(0.99)$ \\
\hline \multirow[t]{2}{*}{ Chairman's Address } & 0.0085 & $0.0152 * *$ & $0.0154 * *$ & 0.0109 & 0.0148 & 0.0133 & 0.0184 \\
\hline & $(1.51)$ & (2.49) & (2.12) & $(1.10)$ & $(1.23)$ & $(0.90)$ & $(0.78)$ \\
\hline
\end{tabular}




\begin{tabular}{|c|c|c|c|c|c|c|c|}
\hline Variable & $\triangle \mathrm{CAR} 10 \mathrm{~min}$ & $\Delta$ CAR15min & $\triangle \mathrm{CAR30min}$ & $\triangle$ CAR60min & $\triangle$ CAR90min & $\triangle \mathrm{CAR} 120 \mathrm{~min}$ & $\Delta$ CAR150min \\
\hline \multirow[t]{2}{*}{ Letter to Shareholders } & 0.0120 & 0.0136 & 0.0109 & - & - & - & - \\
\hline & $(0.44)$ & $(0.30)$ & $(0.21)$ & - & - & - & - \\
\hline \multirow[t]{2}{*}{ ASX Query } & $0.0099 * * *$ & $0.0122 * * *$ & $0.0270 * * *$ & $0.0273 * * *$ & $0.0380 * * *$ & $0.0418 * * *$ & 0.0203* \\
\hline & (3.79) & (3.99) & $(7.55)$ & $(5.89)$ & $(6.52)$ & $(5.47)$ & (1.88) \\
\hline Structured Products & - & - & - & - & - & - & - \\
\hline \multirow[t]{2}{*}{ CTE Quarterly Reports } & -0.0037 & -0.0034 & -0.0049 & 0.0000 & -0.0066 & -0.0136 & 0.0086 \\
\hline & $(-1.43)$ & $(-1.12)$ & $(-1.33)$ & $(0.01)$ & $(-1.06)$ & $(-1.51)$ & $(0.73)$ \\
\hline \multirow[t]{2}{*}{ Bid-Ask Spread } & $-0.0165 * *$ & $-\mathbf{0 . 0 1 5 8} * *$ & $-0.0140 *$ & -0.0055 & $-0.0683^{*}$ & -0.0332 & -0.0493 \\
\hline & $(-2.08)$ & $(-2.26)$ & $(-1.67)$ & $(-0.55)$ & $(-1.70)$ & $(-0.66)$ & $(-0.72)$ \\
\hline \multirow[t]{2}{*}{ Size } & $-0.0028 * * *$ & $-0.0045 * * *$ & $-0.0056 * * *$ & $-0.0064 * * *$ & $-0.0080 * * *$ & $-0.0094 * * *$ & $-0.0095 * * *$ \\
\hline & $(-12.76)$ & $(-18.65)$ & $(-19.18)$ & $(-16.30)$ & $(-15.79)$ & $(-13.13)$ & $(-9.70)$ \\
\hline \multirow[t]{2}{*}{ Book-to-Market Ratio } & -0.0002 & -0.0005 & $-0.0008 * *$ & 0.0002 & 0.0001 & $-0.0021 *$ & -0.0001 \\
\hline & $(-0.53)$ & $(-1.53)$ & $(-2.02)$ & $(0.46)$ & $(0.09)$ & $(-1.81)$ & $(-0.06)$ \\
\hline \multirow[t]{2}{*}{ Leverage } & -0.0001 & -0.0001 & -0.0001 & -0.0001 & 0.0001 & 0.0003 & -0.0002 \\
\hline & $(-0.60)$ & $(-0.82)$ & $(-0.74)$ & $(-0.07)$ & $(0.12)$ & $(0.74)$ & $(-0.27)$ \\
\hline \multirow[t]{2}{*}{ ROA } & -0.0001 & $-0.0003 * * *$ & -0.0001 & $-0.0026 * * * *$ & $-0.0026^{* * * *}$ & $-0.0058 * * *$ & 0.0002 \\
\hline & $(-1.34)$ & $(-2.61)$ & $(-0.89)$ & $(-4.47)$ & $(-4.14)$ & $(-5.87)$ & $(0.12)$ \\
\hline \multirow[t]{2}{*}{ Resource Sector } & $-0.0029 * * *$ & $-0.0022 *$ & $-0.0032 * *$ & -0.0015 & -0.0027 & -0.0035 & $0.0121 * *$ \\
\hline & $(-2.70)$ & $(-1.83)$ & $(-2.21)$ & $(-0.78)$ & $(-1.06)$ & $(-0.98)$ & (2.43) \\
\hline \multirow[t]{2}{*}{ Financial Sector } & $-0.0058 * * *$ & $-0.0082 * * *$ & $-0.0128 * * * *$ & $-0.0142 * * *$ & $-0.0156 * * *$ & $-0.0159 * *$ & -0.0109 \\
\hline & $(-3.28)$ & $(-4.07)$ & $(-5.33)$ & $(-4.29)$ & $(-3.63)$ & $(-2.54)$ & $(-1.28)$ \\
\hline Max. VIF & 1.85 & 1.96 & 1.92 & 1.86 & 1.80 & 1.84 & 1.76 \\
\hline F Value & 29.20 & 43.05 & 37.52 & 30.79 & 24.42 & 16.99 & 7.70 \\
\hline R-Square & $6.04 \%$ & $8.09 \%$ & $7.96 \%$ & $8.29 \%$ & $9.14 \%$ & $10.39 \%$ & $10.27 \%$ \\
\hline Adj R-Sq & $5.83 \%$ & $7.90 \%$ & $7.75 \%$ & $8.02 \%$ & $8.77 \%$ & $9.78 \%$ & $8.94 \%$ \\
\hline $\mathbf{N}$ & 10,018 & 10,791 & 9,564 & 7,174 & 5,119 & 3,098 & 1,367 \\
\hline
\end{tabular}


ASX Queries: In the bad news subsamples, share prices negatively react the most to the ASX Queries, no matter whether before, during or after the GFC. Large share price reactions are observed within a short time. The possible explanation is that the ASX Queries are unexpected. In the good news subsamples, the CAR regression results show relatively small coefficients for the ASX Query, indicating small share price reactions. However, the $\triangle \mathrm{CAR}$ results indicate much larger share price reactions to the ASX Query. This could be because the ASX Queries tend to cause reverse market responses, especially price queries. When a company experiences an abnormal share price increase (decrease), the release of ASX Query would induce a price decrease (increase) to correct the previous abnormal price movements.

Issued Capital: According to the announcement type description, Issued Capital is a complicated announcement category, including both security offerings and security buy-backs. The bad/good news regression results show that Issued Capital can cause either negative or positive share price reactions. The announcement regarding Issued Capital can signal either good or bad news to the market. A company should be able to operate by its internal retained earnings, cash or other assets, so when it needs external capital from equity offerings, it signals bad news. On the other hand, if a company plans to buy back its shares, it signals that the company's share price is undervalued, leading to positive market reactions. The share price reactions were larger during the GFC than non-GFC periods for both bad and good news subsamples. This indicated more sensitive share price reactions to Issued Capital during the GFC, as Issued Capital could change the company's liquidity, especially its cash status. In the good news subsample, Issued Capital has the smallest price reactions among all categories during the non-GFC periods. 
Periodic Reports: It is easy to understand that Periodic Reports could contain either negative or positive information, because the company could have a profit or loss, experience profit increase or decrease, or perform better or worse than management/analyst expectations. The GFC had relatively small impacts on the price reactions to Periodic Reports. Periodic Reports had slightly larger price reactions during the GFC, indicating relatively small GFC impacts. According to the coefficients, Periodic Reports had similar price reactions to Issued Capital in the bad news subsample before the GFC. During the GFC, share prices reacted a little more negatively to Issued Capital, while since then, share prices react a little more negatively to Periodic Reports. In the good news subsample tests, Issued Capital could cause larger CARs than Periodic Reports during the GFC period, while larger share price reactions to Periodic Reports are observed in non-GFC periods. This also reveals that liquidity information was more important than earnings information during the GFC.

Distribution Announcements: Distribution Announcements are directly related to earnings information, because dividends are paid only when the company is in good financial condition. It is not surprising that only significant positive market reactions to Distribution Announcements are shown in the good news subsamples, and no significant results in the bad news subsamples. This further reveals that the market interprets Distribution Announcements as good news in general although the share price reactions are relatively small. In the good news subsamples, the share prices reacted to Distribution Announcements less than Periodic Reports no matter whether before, during or after the GFC. The results also show that share price reactions to Distribution Announcements were larger after the GFC, and could cause similar share price responses as Periodic Reports. This indicates that Distribution Announcements convey information almost as important as Periodic Reports to investors since the GFC. 
Other: According to the good news test coefficients of the announcement category Other, it had relatively small but stable market reactions cross the three periods. The bad news subsamples show no significant results on Other. Similar to Distribution Announcements, category Other is interpreted as good news by the market as no significant negative price responses were observed in bad news subsamples. Announcements under the category Other are viewed as voluntary disclosures, especially the open briefings which provide the company's operation or market update to investors and increase the communication between the company and investors. The results show that this voluntary disclosures tend to signal good news to the market.

Asset Acquisition \& Disposal: The GFC was a sensitive period, and any asset change at that time could make a difference to the company and its share price. This is reflected in the market reactions to Asset Acquisition \& Disposal. During the non-GFC periods, no significant results were shown in the bad news subsamples, and relatively small price reactions were observed in the good news subsamples. Asset Acquisition \& Disposal induced small but significant negative price responses and the largest positive share price reactions only during the GFC. The results also indicate that Asset Acquisition \& Disposal signals good news to the market according to the fact that there were little negative price reactions to bad news but consistent positive price reactions to good news. This could be because asset acquisition-related announcements may indicate potential investment opportunities and earnings increase in the future, and asset disposal-related announcements may imply future cash inflows.

Progress Reports: Regardless of the GFC impact, share prices react to Progress Reports the most, and large positive market reactions to good news are observed within a short time after the announcement is released. This announcement category also has the least negative price reactions to bad news, so Progress Reports could be a positive sign to the market. Although 
Progress Reports are less likely to be predicted, they may contain less negative information than other types of announcements, because as long as the company is still "progressing", a large price decline is unlikely to happen. Companies tend to release project improvements instead of project difficulties in the Progress Reports; this was indicated in the large positive and small negative market reactions in the good and bad subsamples respectively.

From the above analysis, Progress Reports Asset Acquisition \& Disposal, Distribution Announcements, and Other indicate good signs for the market during trading hours. There are consistent positive price reactions to good news but small or no significant negative price reactions to bad news after these announcements are released. Share prices could react to Periodic Reports and Issued Capital both negatively and positively, indicating either good news or bad news can be contained in these announcements. This could be why small positive share price reactions to Periodic Reports and Issued Capital should not be a time to separate good/bad news subsamples, because the positive price consequences of good news are offset by the negative price effects of bad news. An ASX Query is different from other categories, as it can correct previous abnormal price fluctuations at intraday levels. Positive (negative) price reactions to the ASX Query could be expected if there is an abnormal share price decrease (increase) before the ASX Query is released. To some extent, the results support the Continuous Disclosure Regime, especially after the GFC. Except for the ASX Query, the relatively small coefficients indicate listed companies continuously keep investors informed to avoid surprising information or immediate price drops.

When looking at the control variables, there is a positive association between intraday CARs and firm size in the bad news subsamples, and negative association in the good news subsamples. This further confirms that large companies are more stable, suggesting that large 
companies have smaller negative abnormal returns when bad news is released, and have smaller positive returns when good news is released. The leverage's positive impact on the intraday stock abnormal returns is observed only in the good news subsamples before the GFC. Sometimes ROA is negatively associated with intraday CARs in good news subsamples only, which indicates that more profitable companies may experience smaller share price movements when information is released. A possible explanation is that the share prices of more profitable companies are more stable and less impacted by a single piece of information at intraday levels. In terms of the sector influences, when bad news is released, financial companies tend to have less negative abnormal returns during non-GFC periods; when good news is released, both resource and financial companies tend to have smaller positive abnormal returns during nonGFC periods, although the negative effects on the resource sector lasted for a shorter time (30 minutes) in the robust $\triangle \mathrm{CAR}$ tests.

\subsubsection{Regression Results of Trading Volume Reactions to Different Announcements}

Apart from share price reactions, this study also examines and compares trading volume changes concerning the release of different announcements. Tables 6-30-6-32 show the regression results of the trading volume reactions to different announcement categories for all

market-sensitive announcements. In order to explore the impacts of good/bad news on investors' trading behaviour, this study again separates bad news and good news subsamples in Tables 633 - 6-35 $(\triangle \mathrm{CAR}<0)$, and Tables 6-36 - 6-38 $(\Delta \mathrm{CAR}>0)$ respectively. The trading volume analysis can help understand the announcement effects on investors' trading activities. Significant and consistent trading volume reactions are observed around Progress Reports, Asset Acquisition \& Disposal, Issued Capital, and Periodic Reports before, during and after the 
GFC. Relevant patterns are observed in trading volume reactions when comparing them with share price reactions. 
Table 6-30. The Regression Results of Market-sensitive Announcements - Intraday ATVs before the GFC

\begin{tabular}{|c|c|c|c|c|c|c|c|}
\hline Variable & ATV10min & ATV15min & ATV30min & ATV60min & ATV90min & ATV120min & ATV150min \\
\hline \multirow[t]{2}{*}{ Intercept } & 0.0401 & 0.1857 & 0.3870 & 0.5034 & 0.6840 & 0.7611 & 0.3742 \\
\hline & $(1.42)$ & $(3.39)$ & $(4.42)$ & $(4.04)$ & $(3.18)$ & $(2.04)$ & $(0.70)$ \\
\hline \multirow[t]{2}{*}{ Takeover / Scheme Announcements } & $0.0968 *$ & $0.2139 * *$ & $0.3024 *$ & $0.3981 *$ & $0.6654 *$ & $1.3264 *$ & 0.3266 \\
\hline & (1.91) & $(2.15)$ & $(1.87)$ & $(1.74)$ & $(1.71)$ & (1.94) & $(0.34)$ \\
\hline \multirow[t]{2}{*}{ Security Holder Details } & 0.1285 & 0.1590 & -0.3290 & -0.7479 & 0.4182 & 0.1965 & -0.3597 \\
\hline & $(1.42)$ & $(0.89)$ & $(-1.10)$ & $(-1.73)$ & $(0.57)$ & $(0.15)$ & $(-0.19)$ \\
\hline \multirow[t]{2}{*}{ Periodic Reports } & $0.1159 * * *$ & $0.2323 * * *$ & $0.3490 * * *$ & $0.4362 * * *$ & $0.5190 * * *$ & $0.6669 *$ & 0.6161 \\
\hline & $(4.24)$ & $(4.38)$ & $(4.08)$ & $(3.55)$ & $(2.43)$ & $(1.81)$ & $(1.22)$ \\
\hline \multirow[t]{2}{*}{ Issued Capital } & $0.0945 * * *$ & $0.2896 * * *$ & $0.3310 * * *$ & $0.4313 * * *$ & 0.3743 & 0.5052 & 0.4153 \\
\hline & (3.09) & $(4.82)$ & (3.44) & (3.10) & $(1.56)$ & $(1.21)$ & $(0.74)$ \\
\hline \multirow[t]{2}{*}{ Asset Acquisition \& Disposal } & $0.1224 * * *$ & $0.2629 * * *$ & $0.3604 * * *$ & $0.3423 * *$ & $0.4375 *$ & 0.5863 & 0.5541 \\
\hline & $(4.12)$ & $(4.52)$ & $(3.89)$ & $(2.57)$ & $(1.88)$ & $(1.43)$ & $(0.99)$ \\
\hline \multirow[t]{2}{*}{ Notice of Meeting } & -0.0587 & 0.0412 & 0.1882 & 0.2310 & 0.3812 & 0.5596 & 0.5130 \\
\hline & $(-0.31)$ & $(0.11)$ & $(0.29)$ & $(0.24)$ & $(0.24)$ & $(0.23)$ & $(0.14)$ \\
\hline \multirow[t]{2}{*}{ ASX Announcement } & 0.0031 & -0.0265 & 0.0581 & -0.0448 & -0.1306 & -0.2698 & 0.0779 \\
\hline & $(0.07)$ & $(-0.27)$ & $(0.37)$ & $(-0.19)$ & $(-0.33)$ & $(-0.39)$ & $(0.09)$ \\
\hline \multirow[t]{2}{*}{ Distribution Announcement } & $0.0981 *$ & $0.2187 * *$ & $0.3102 *$ & 0.3230 & 0.3301 & 0.5227 & 0.4990 \\
\hline & $(1.91)$ & $(2.16)$ & $(1.91)$ & $(1.38)$ & $(0.82)$ & $(0.74)$ & $(0.50)$ \\
\hline \multirow[t]{2}{*}{ Progress Reports } & $0.2101 * * *$ & $0.4431 * * *$ & $0.6722 * * *$ & $0.7471 * * *$ & $0.9730 * * *$ & $1.3690 * * *$ & $1.4483 * * *$ \\
\hline & (10.70) & (11.43) & (10.87) & $(8.51)$ & $(6.42)$ & $(5.23)$ & (4.04) \\
\hline \multirow[t]{2}{*}{ Company Administration } & 0.1444 & $0.7217 * * *$ & $1.1360 * *$ & 0.8345 & 1.3029 & 2.2592 & -3.3641 \\
\hline & $(0.98)$ & $(2.69)$ & $(2.51)$ & $(1.33)$ & $(1.21)$ & $(1.22)$ & $(-1.41)$ \\
\hline Notice of Call & - & - & - & - & - & - & - \\
\hline \multirow[t]{2}{*}{ Other } & $0.1000 * *$ & $0.3199 * * *$ & $0.4394 * * *$ & $0.3606 * *$ & 0.3669 & $1.0162 *$ & 0.9077 \\
\hline & $(2.34)$ & $(4.03)$ & $(3.45)$ & $(1.98)$ & $(1.17)$ & $(1.86)$ & $(1.18)$ \\
\hline \multirow[t]{2}{*}{ Chairman's Address } & 0.1078 & 0.2225 & 0.3314 & 0.4009 & 0.5539 & 0.7109 & 0.6333 \\
\hline & $(1.59)$ & $(1.69)$ & $(1.59)$ & $(1.27)$ & $(0.93)$ & $(0.58)$ & $(0.35)$ \\
\hline
\end{tabular}




\begin{tabular}{|c|c|c|c|c|c|c|c|}
\hline Variable & ATV10min & ATV15min & ATV30min & ATV60min & ATV90min & ATV120min & ATV150min \\
\hline \multirow[t]{2}{*}{ Letter to Shareholders } & 0.7179* & $1.8051 * *$ & $3.4150 * * *$ & -0.1799 & -0.1660 & -0.1719 & -0.0948 \\
\hline & (1.96) & (2.43) & $(2.76)$ & $(-0.10)$ & $(-0.06)$ & $(-0.03)$ & $(-0.01)$ \\
\hline \multirow[t]{2}{*}{ ASX Query } & $0.1245 * *$ & 0.1631 & $0.3210 *$ & $0.6737 * * *$ & 0.1495 & -0.0216 & -1.1840 \\
\hline & $(2.36)$ & $(1.60)$ & $(1.95)$ & (2.90) & $(0.38)$ & $(-0.03)$ & $(-1.27)$ \\
\hline \multirow[t]{2}{*}{ Structured Products } & 0.0728 & 0.1620 & - & - & - & - & - \\
\hline & $(0.08)$ & $(0.09)$ & - & - & - & - & - \\
\hline \multirow[t]{2}{*}{ CTE Quarterly Reports } & 0.0403 & 0.0550 & 0.0156 & -0.0786 & -0.0982 & 0.1436 & -0.1324 \\
\hline & $(1.12)$ & $(0.76)$ & $(0.13)$ & $(-0.47)$ & $(-0.34)$ & $(0.28)$ & $(-0.19)$ \\
\hline \multirow[t]{2}{*}{ Bid-Ask Spread } & -0.3085 & -0.8164 & $-1.2388 * * *$ & $-1.5398 * * *$ & $-1.8136 *$ & -1.5417 & -0.5802 \\
\hline & $(-2.57)$ & $(-3.42)$ & $(-3.33)$ & $(-2.96)$ & $(-1.94)$ & $(-1.03)$ & $(-0.38)$ \\
\hline \multirow[t]{2}{*}{ Size } & $-0.0202 * * *$ & $-0.0594 * * *$ & $-0.0979 * * *$ & $-0.1044 * * *$ & $-0.1439 * * *$ & $-0.2088 * * *$ & -0.1105 \\
\hline & $(-5.47)$ & $(-8.74)$ & $(-8.99)$ & $(-6.68)$ & $(-5.32)$ & $(-4.47)$ & $(-1.62)$ \\
\hline \multirow[t]{2}{*}{ Book-to-Market Ratio } & -0.0111 & -0.0324 & $-0.0572 *$ & $-0.0867 *$ & -0.1073 & -0.1608 & -0.1142 \\
\hline & $(-1.09)$ & $(-1.63)$ & $(-1.80)$ & $(-1.92)$ & $(-1.43)$ & $(-1.27)$ & $(-0.73)$ \\
\hline \multirow[t]{2}{*}{ Leverage } & 0.0001 & 0.0001 & $0.0006 * * *$ & $0.0010 * * *$ & $0.0015 * * *$ & 0.0002 & -0.0017 \\
\hline & $(0.96)$ & $(1.40)$ & (3.35) & $(4.53)$ & (3.99) & $(0.25)$ & $(-1.73)$ \\
\hline \multirow[t]{2}{*}{ ROA } & -0.0065 & 0.0050 & 0.0042 & 0.0040 & 0.0272 & 0.0433 & -0.9918 \\
\hline & $(-0.81)$ & $(0.35)$ & $(0.19)$ & $(0.13)$ & $(0.59)$ & $(0.64)$ & $(-3.63)$ \\
\hline \multirow[t]{2}{*}{ Resource Sector } & $0.0455 * * *$ & $0.0860 * * *$ & 0.0670 & 0.0517 & -0.0138 & 0.0292 & -0.2958 \\
\hline & (2.76) & (2.72) & $(1.32)$ & $(0.71)$ & $(-0.11)$ & $(0.13)$ & $(-0.95)$ \\
\hline \multirow[t]{2}{*}{ Financial Sector } & -0.0142 & -0.0072 & -0.0486 & -0.1158 & -0.1794 & -0.1374 & -0.1809 \\
\hline & $(-0.56)$ & $(-0.15)$ & $(-0.62)$ & $(-1.01)$ & $(-0.91)$ & $(-0.40)$ & $(-0.38)$ \\
\hline Max. VIF & 2.35 & 2.49 & 2.46 & 2.41 & 2.39 & 2.34 & 2.30 \\
\hline F Value & 10.10 & 14.10 & 14.50 & 9.72 & 5.49 & 3.03 & 2.81 \\
\hline R-Square & $1.15 \%$ & $1.44 \%$ & $1.58 \%$ & $1.34 \%$ & $1.05 \%$ & $0.90 \%$ & $1.68 \%$ \\
\hline Adj R-Sq & $1.03 \%$ & $1.34 \%$ & $1.47 \%$ & $1.21 \%$ & $0.86 \%$ & $0.60 \%$ & $1.08 \%$ \\
\hline $\mathbf{N}$ & 20,023 & 22,218 & 19,942 & 15,711 & 11,443 & 7,384 & 3,639 \\
\hline
\end{tabular}


Table 6-31. The Regression Results of Market-sensitive Announcements - Intraday ATVs during the GFC

\begin{tabular}{|c|c|c|c|c|c|c|c|}
\hline Variable & ATV10min & ATV15min & ATV30min & ATV60min & ATV90min & ATV120min & ATV150min \\
\hline \multirow[t]{2}{*}{ Intercept } & 0.0496 & 0.1857 & 0.3957 & 0.4570 & 0.3527 & 0.5270 & 1.0960 \\
\hline & $(1.17)$ & $(2.66)$ & $(3.19)$ & $(2.15)$ & $(1.27)$ & $(1.40)$ & $(1.69)$ \\
\hline \multirow[t]{2}{*}{ Takeover / Scheme Announcements } & 0.0981 & 0.1872 & 0.2773 & 0.3548 & 0.3227 & 0.5296 & 0.6337 \\
\hline & $(1.31)$ & $(1.50)$ & $(1.26)$ & $(0.93)$ & $(0.65)$ & $(0.77)$ & $(0.56)$ \\
\hline \multirow[t]{2}{*}{ Security Holder Details } & 0.0287 & 0.1291 & 0.1767 & 0.4333 & 0.2361 & 0.5649 & 0.5181 \\
\hline & $(0.23)$ & $(0.64)$ & $(0.49)$ & $(0.71)$ & $(0.29)$ & $(0.51)$ & $(0.27)$ \\
\hline \multirow[t]{2}{*}{ Periodic Reports } & $0.1332 * * *$ & $0.3113 * * *$ & $0.5207 * * *$ & $0.6759 * * *$ & $0.9926 * * *$ & $1.1137 * * *$ & $1.3566 * *$ \\
\hline & (2.99) & (4.29) & (4.04) & (3.03) & (3.44) & $(2.88)$ & (2.03) \\
\hline \multirow[t]{2}{*}{ Issued Capital } & $0.1655 * * *$ & $0.3525 * * *$ & $0.6383 * * *$ & $1.0136 * * *$ & $1.1454 * * *$ & $1.0138 * *$ & $1.6035 * *$ \\
\hline & (3.45) & (4.47) & (4.54) & (4.18) & (3.68) & $(2.41)$ & (2.29) \\
\hline \multirow[t]{2}{*}{ Asset Acquisition \& Disposal } & $0.2540 * * *$ & $0.4936 * * *$ & $0.8650 * * *$ & $1.3403 * * *$ & $1.3640 * * *$ & $1.7510 * * *$ & $2.0819 * * *$ \\
\hline & $(5.15)$ & $(6.16)$ & $(6.11)$ & (5.53) & (4.33) & (4.03) & (2.83) \\
\hline \multirow[t]{2}{*}{ Notice of Meeting } & 0.0272 & 0.0958 & 0.1334 & 0.2520 & 0.4589 & 0.4874 & 0.3642 \\
\hline & $(0.10)$ & $(0.22)$ & $(0.17)$ & $(0.18)$ & $(0.27)$ & $(0.21)$ & $(0.09)$ \\
\hline \multirow[t]{2}{*}{ ASX Announcement } & 0.0118 & -0.0287 & 0.0644 & 0.0145 & 0.0671 & 0.1524 & -0.1511 \\
\hline & $(0.18)$ & $(-0.25)$ & $(0.32)$ & $(0.04)$ & $(0.15)$ & $(0.25)$ & $(-0.15)$ \\
\hline \multirow[t]{2}{*}{ Distribution Announcement } & 0.1140 & $0.2554^{*}$ & $0.4761 *$ & 0.7606 & 0.8733 & 1.3206 & 1.9780 \\
\hline & $(1.20)$ & $(1.70)$ & $(1.77)$ & $(1.65)$ & $(1.47)$ & $(1.65)$ & $(1.51)$ \\
\hline \multirow[t]{2}{*}{ Progress Reports } & $0.2555 * * *$ & $0.5673 * * *$ & $0.9242 * * *$ & $1.3671 * * *$ & $1.5785 * * *$ & $1.8163 * * *$ & $1.9615 * * *$ \\
\hline & $(8.86)$ & (11.91) & (11.02) & $(9.57)$ & $(8.55)$ & (7.30) & (4.67) \\
\hline \multirow[t]{2}{*}{ Company Administration } & 0.1029 & 0.2916 & 0.5317 & 1.1208 & 1.1328 & 1.7883 & 2.7012 \\
\hline & $(0.75)$ & $(1.31)$ & $(1.30)$ & $(1.65)$ & $(1.36)$ & $(1.63)$ & $(1.47)$ \\
\hline Notice of Call & - & - & - & - & - & - & - \\
\hline \multirow[t]{2}{*}{ Other } & 0.0685 & 0.2132 & 0.3976 & 0.8308 & 0.9636 & 0.8975 & 1.6530 \\
\hline & $(0.67)$ & $(1.31)$ & $(1.36)$ & $(1.66)$ & $(1.49)$ & $(0.98)$ & $(0.98)$ \\
\hline \multirow[t]{2}{*}{ Chairman's Address } & 0.0871 & 0.2051 & 0.3689 & 0.5539 & 0.9360 & 1.0802 & 0.8758 \\
\hline & $(0.71)$ & $(1.04)$ & $(1.06)$ & $(0.91)$ & $(1.08)$ & $(0.96)$ & $(0.40)$ \\
\hline
\end{tabular}




\begin{tabular}{|c|c|c|c|c|c|c|c|}
\hline Variable & ATV10min & ATV15min & ATV30min & ATV60min & ATV90min & ATV120min & ATV150min \\
\hline \multirow[t]{2}{*}{ Letter to Shareholders } & 0.8481 & 1.5997 & 2.0053 & 2.5727 & 2.6795 & 3.5403 & 3.9972 \\
\hline & $(1.20)$ & $(1.14)$ & $(0.85)$ & $(0.71)$ & $(0.67)$ & $(0.80)$ & $(0.62)$ \\
\hline \multirow[t]{2}{*}{ ASX Query } & 0.0525 & $0.1964 *$ & $0.4874 * *$ & $0.5919 *$ & 0.0107 & -0.3262 & -0.9140 \\
\hline & $(0.77)$ & (1.82) & $(2.56)$ & (1.85) & $(0.03)$ & $(-0.63)$ & $(-1.11)$ \\
\hline Structured Products & - & - & - & - & - & - & - \\
\hline \multirow[t]{2}{*}{ CTE Quarterly Reports } & 0.0373 & 0.0915 & 0.0924 & 0.2025 & 0.2218 & 0.0676 & -0.3595 \\
\hline & $(0.61)$ & $(0.88)$ & $(0.50)$ & $(0.64)$ & $(0.55)$ & $(0.12)$ & $(-0.38)$ \\
\hline \multirow[t]{2}{*}{ Bid-Ask Spread } & -0.0067 & -0.0190 & -3.1604 & -2.5686 & -6.5340 & -7.8644 & -11.5321 \\
\hline & $(-0.3)$ & $(-0.50)$ & $(-4.29)$ & $(-2.59)$ & $(-3.07)$ & $(-2.46)$ & $(-2.04)$ \\
\hline \multirow[t]{2}{*}{ Size } & $-0.0249 * * *$ & $-0.0653 * * *$ & $-0.1042 * * *$ & $-0.1531 * * *$ & $-0.1670 * * *$ & $-0.2245 * * *$ & $-0.3113 * * *$ \\
\hline & $(-4.46)$ & $(-7.51)$ & $(-6.72)$ & $(-5.73)$ & $(-4.75)$ & $(-4.68)$ & $(-3.79)$ \\
\hline \multirow[t]{2}{*}{ Book-to-Market Ratio } & -0.0049 & -0.0184 & -0.0369 & -0.0488 & -0.0748 & -0.1075 & -0.1799 \\
\hline & $(-0.53)$ & $(-1.20)$ & $(-1.37)$ & $(-1.09)$ & $(-1.23)$ & $(-1.37)$ & $(-1.18)$ \\
\hline \multirow[t]{2}{*}{ Leverage } & -0.0001 & 0.0004 & 0.0005 & 0.0003 & -0.0179 & -0.0302 & -0.0309 \\
\hline & $(-0.11)$ & (0.19) & $(0.16)$ & $(0.05)$ & $(-0.95)$ & $(-1.19)$ & $(-0.93)$ \\
\hline \multirow[t]{2}{*}{ ROA } & -0.0003 & -0.0009 & -0.0023 & -0.0029 & -0.0101 & -0.0096 & -0.0075 \\
\hline & $(-0.15)$ & $(-0.25)$ & $(-0.30)$ & $(-0.25)$ & $(-0.56)$ & $(-0.49)$ & $(-0.32)$ \\
\hline \multirow[t]{2}{*}{ Resource Sector } & 0.0433 & $0.0878 *$ & 0.0772 & 0.1841 & $0.3654 * *$ & 0.3549 & 0.2255 \\
\hline & (1.54) & (1.94) & $(0.96)$ & $(1.32)$ & (2.01) & (1.44) & $(0.54)$ \\
\hline \multirow[t]{2}{*}{ Financial Sector } & -0.0067 & 0.0120 & 0.0293 & 0.0373 & 0.0953 & -0.0282 & -0.2687 \\
\hline & $(-0.16)$ & $(0.17)$ & $(0.23)$ & $(0.17)$ & $(0.33)$ & $(-0.07)$ & $(-0.40)$ \\
\hline Max. VIF & 1.96 & 2.04 & 2.02 & 1.98 & 1.96 & 1.94 & 1.95 \\
\hline F Value & 5.82 & 11.03 & 10.09 & 7.35 & 6.43 & 5.08 & 2.70 \\
\hline R-Square & $0.66 \%$ & $1.12 \%$ & $1.14 \%$ & $1.05 \%$ & $1.25 \%$ & $1.49 \%$ & $1.57 \%$ \\
\hline Adj R-Sq & $0.55 \%$ & $1.02 \%$ & $1.03 \%$ & $0.91 \%$ & $1.06 \%$ & $1.20 \%$ & $0.99 \%$ \\
\hline $\mathbf{N}$ & 19,326 & 21,450 & 19,301 & 15,293 & 11,181 & 7,410 & 3,757 \\
\hline
\end{tabular}


Table 6-32. The Regression Results of Market-sensitive Announcements - Intraday ATVs after the GFC

\begin{tabular}{|c|c|c|c|c|c|c|c|}
\hline Variable & ATV10min & ATV15min & ATV30min & ATV60min & ATV90min & ATV120min & ATV150min \\
\hline \multirow[t]{2}{*}{ Intercept } & 0.0977 & 0.1763 & 0.2791 & 0.2319 & 0.1428 & 0.0731 & 0.3340 \\
\hline & $(2.56)$ & $(2.85)$ & $(2.58)$ & $(1.12)$ & $(0.53)$ & $(0.16)$ & $(0.48)$ \\
\hline \multirow[t]{2}{*}{ Takeover / Scheme Announcements } & 0.0820 & 0.1444 & 0.2874 & 0.4465 & 0.6808 & 1.1323 & 0.5080 \\
\hline & (1.07) & $(1.16)$ & $(1.31)$ & $(0.99)$ & $(1.20)$ & $(1.21)$ & $(0.33)$ \\
\hline \multirow[t]{2}{*}{ Security Holder Details } & 0.2013 & 0.2895 & 0.2234 & 0.3256 & 0.3645 & 0.6838 & -0.2346 \\
\hline & $(1.51)$ & $(1.34)$ & $(0.57)$ & $(0.43)$ & $(0.37)$ & $(0.44)$ & $(-0.10)$ \\
\hline \multirow[t]{2}{*}{ Periodic Reports } & $0.0992 * *$ & $0.1700 * * *$ & $0.2610 * *$ & $0.4627 * *$ & $0.6231 * *$ & $1.0133 * *$ & 1.2267 \\
\hline & (2.50) & (2.66) & $(2.32)$ & (2.12) & (2.16) & (2.11) & $(1.67)$ \\
\hline \multirow[t]{2}{*}{ Issued Capital } & $0.1700 * * *$ & $0.2402 * * *$ & $0.3629 * * *$ & $0.6305 * *$ & $0.8201 * *$ & 1.1101* & 1.0188 \\
\hline & (3.78) & (3.29) & $(2.84)$ & (2.54) & (2.49) & (1.98) & $(1.19)$ \\
\hline \multirow[t]{2}{*}{ Asset Acquisition \& Disposal } & $0.1235 * * *$ & $0.2447 * * *$ & $0.3692 * * *$ & $0.5223 * *$ & 0.5246 & 0.8058 & 0.7083 \\
\hline & (2.63) & (3.24) & (2.79) & (2.06) & $(1.58)$ & $(1.46)$ & $(0.81)$ \\
\hline \multirow[t]{2}{*}{ Notice of Meeting } & $0.4983 *$ & $0.7712 *$ & $1.9055 * * *$ & $3.1031 * *$ & 0.4612 & -0.5020 & 0.3645 \\
\hline & (1.97) & $(1.85)$ & $(2.65)$ & (2.30) & $(0.25)$ & $(-0.14)$ & $(0.06)$ \\
\hline \multirow[t]{2}{*}{ ASX Announcement } & 0.0183 & -0.0228 & 0.0520 & 0.1600 & 0.0813 & -0.0656 & -0.8543 \\
\hline & $(0.29)$ & $(-0.22)$ & $(0.29)$ & $(0.46)$ & $(0.17)$ & $(-0.09)$ & $(-0.71)$ \\
\hline \multirow[t]{2}{*}{ Distribution Announcement } & 0.0759 & 0.1887 & 0.2505 & 0.4624 & 0.6177 & 0.7685 & 0.5456 \\
\hline & $(0.97)$ & $(1.49)$ & (1.13) & $(1.08)$ & $(1.09)$ & $(0.80)$ & $(0.37)$ \\
\hline \multirow[t]{2}{*}{ Progress Reports } & $0.2354 * * *$ & $0.4350 * * *$ & $0.6731 * * *$ & $1.0300 * * *$ & $1.1286 * * *$ & $1.3479 * * *$ & 0.6895 \\
\hline & (9.21) & $(10.50)$ & (9.33) & $(7.47)$ & (6.34) & $(4.52)$ & $(1.51)$ \\
\hline \multirow[t]{2}{*}{ Company Administration } & 0.0626 & 0.0757 & 0.0335 & -0.0762 & 0.1318 & 0.3801 & -0.2941 \\
\hline & $(0.86)$ & $(0.64)$ & $(0.16)$ & $(-0.19)$ & $(0.27)$ & $(0.46)$ & $(-0.22)$ \\
\hline Notice of Call & - & - & - & - & - & - & - \\
\hline \multirow[t]{2}{*}{ Other } & 0.1220 & 0.2531 & 0.3047 & 0.6475 & 0.9146 & 0.5337 & 0.4832 \\
\hline & $(1.08)$ & $(1.41)$ & $(0.96)$ & $(1.04)$ & $(1.15)$ & $(0.40)$ & $(0.25)$ \\
\hline \multirow[t]{2}{*}{ Chairman's Address } & 0.0465 & 0.1457 & 0.3748 & 0.5360 & 0.9343 & 0.8215 & 0.3385 \\
\hline & $(0.36)$ & $(0.71)$ & $(1.03)$ & $(0.73)$ & $(0.93)$ & $(0.52)$ & $(0.13)$ \\
\hline
\end{tabular}




\begin{tabular}{|c|c|c|c|c|c|c|c|}
\hline Variable & ATV10min & ATV15min & ATV30min & ATV60min & ATV90min & ATV120min & ATV150min \\
\hline \multirow[t]{2}{*}{ Letter to Shareholders } & 0.0527 & 0.0456 & 0.0399 & -0.1208 & - & - & - \\
\hline & $(0.07)$ & $(0.04)$ & $(0.02)$ & $(-0.03)$ & - & - & - \\
\hline \multirow[t]{2}{*}{ ASX Query } & 0.0740 & $0.1777 *$ & 0.2420 & -0.0540 & -0.3374 & -0.6724 & $-2.3133 * *$ \\
\hline & $(1.20)$ & (1.82) & $(1.43)$ & $(-0.17)$ & $(-0.81)$ & $(-1.00)$ & $(-2.21)$ \\
\hline Structured Products & - & - & - & - & - & - & - \\
\hline \multirow[t]{2}{*}{ CTE Quarterly Reports } & 0.0353 & 0.0214 & 0.0015 & -0.0435 & 0.0067 & 0.2318 & 0.0593 \\
\hline & $(0.59)$ & $(0.22)$ & $(0.01)$ & $(-0.13)$ & $(0.02)$ & $(0.32)$ & $(0.06)$ \\
\hline \multirow[t]{2}{*}{ Bid-Ask Spread } & -0.1964 & $-0.4159 *$ & -0.6698 & -1.0496 & -3.0392 & -1.6754 & -0.9723 \\
\hline & $(-1.34)$ & $(-1.75)$ & $(-1.63)$ & $(-1.25)$ & $(-1.54)$ & $(-0.75)$ & $(-0.32)$ \\
\hline \multirow[t]{2}{*}{ Size } & $-0.0182 * * *$ & $-0.0355 * * *$ & $-0.0473 * * *$ & $-0.0540 *$ & -0.0562 & -0.0826 & -0.0869 \\
\hline & $(-3.41)$ & $(-4.30)$ & $(-3.26)$ & $(-1.93)$ & $(-1.53)$ & $(-1.34)$ & $(-0.90)$ \\
\hline \multirow[t]{2}{*}{ Book-to-Market Ratio } & -0.0091 & 0.0247 & 0.0247 & 0.0100 & 0.0228 & -0.0801 & 0.0292 \\
\hline & $(-1.29)$ & $(2.14)$ & $(1.18)$ & $(0.25)$ & $(0.46)$ & $(-0.94)$ & $(0.23)$ \\
\hline \multirow[t]{2}{*}{ Leverage } & -0.0006 & -0.0014 & -0.0024 & -0.0011 & -0.0010 & -0.0003 & 0.0077 \\
\hline & $(-0.22)$ & $(-0.33)$ & $(-0.32)$ & $(-0.06)$ & $(-0.04)$ & $(-0.01)$ & $(0.10)$ \\
\hline \multirow[t]{2}{*}{ ROA } & -0.0045 & $-0.0087 *$ & -0.0076 & -0.0097 & -0.0073 & -0.0194 & -0.0042 \\
\hline & $(-1.46)$ & $(-1.94)$ & $(-1.02)$ & $(-0.67)$ & $(-0.44)$ & $(-0.71)$ & $(-0.03)$ \\
\hline \multirow[t]{2}{*}{ Resource Sector } & -0.0419 & -0.0553 & -0.0881 & 0.0166 & 0.0950 & 0.2527 & -0.1212 \\
\hline & $(-1.60)$ & $(-1.32)$ & $(-1.20)$ & $(0.12)$ & $(0.51)$ & $(0.82)$ & $(-0.25)$ \\
\hline \multirow[t]{2}{*}{ Financial Sector } & $-0.0955 * *$ & $-0.1645 * *$ & $-0.2806 * *$ & -0.3053 & -0.3735 & -0.3583 & -0.7551 \\
\hline & $(-2.26)$ & $(-2.43)$ & $(-2.34)$ & $(-1.30)$ & $(-1.20)$ & $(-0.68)$ & $(-0.95)$ \\
\hline Max. VIF & 1.80 & 1.81 & 1.80 & 1.77 & 1.74 & 1.76 & 1.73 \\
\hline F Value & 5.89 & 8.19 & 6.25 & 3.93 & 3.02 & 1.65 & 0.74 \\
\hline R-Square & $0.67 \%$ & $0.88 \%$ & $0.75 \%$ & $0.60 \%$ & $0.58 \%$ & $0.48 \%$ & - \\
\hline Adj R-Sq & $0.55 \%$ & $0.77 \%$ & $0.63 \%$ & $0.44 \%$ & $0.39 \%$ & $0.19 \%$ & - \\
\hline $\mathbf{N}$ & 19,336 & 20,398 & 18,258 & 14,456 & 10,857 & 7,232 & 3,612 \\
\hline
\end{tabular}

Note: The ATV150min regression model does not pass the significant test (F value $=0.74)$.

$*, * *$ and $* * *$ denote significance at the $10 \%, 5 \%$ and $1 \%$ levels respectively (two-tailed). 
Table 6-33. The Regression Results of Market-sensitive Announcements with Bad News - Intraday ATVs before the GFC

\begin{tabular}{|c|c|c|c|c|c|c|c|}
\hline Variable & ATV10min & ATV15min & ATV30min & ATV60min & ATV90min & ATV120min & ATV150min \\
\hline \multirow[t]{2}{*}{ Intercept } & -0.0173 & 0.0173 & 0.0848 & 0.0939 & 0.2628 & 0.1583 & -0.4245 \\
\hline & $(-0.44)$ & $(0.27)$ & $(0.92)$ & $(0.59)$ & $(1.05)$ & $(0.38)$ & $(-0.46)$ \\
\hline \multirow[t]{2}{*}{ Takeover / Scheme Announcements } & 0.0766 & 0.1998* & 0.2686 & $0.7400 * * *$ & $1.1744 * * *$ & $2.2609 * * *$ & 0.8537 \\
\hline & $(1.13)$ & (1.83) & $(1.62)$ & $(2.64)$ & $(2.74)$ & $(3.50)$ & $(0.62)$ \\
\hline \multirow[t]{2}{*}{ Security Holder Details } & 0.0680 & -0.0058 & $-1.5756 * * *$ & $-1.7161 * * *$ & 0.2543 & 0.4417 & 0.7063 \\
\hline & $(0.61)$ & $(-0.03)$ & $(-4.66)$ & $(-3.48)$ & $(0.33)$ & $(0.33)$ & $(0.25)$ \\
\hline \multirow[t]{2}{*}{ Periodic Reports } & $0.0742 * *$ & $0.1887 * * *$ & $0.2271 * *$ & $0.3413 * *$ & $0.4813^{*}$ & 0.6208 & 1.4415 \\
\hline & $(2.00)$ & (3.08) & $(2.55)$ & $(2.20)$ & (1.93) & $(1.58)$ & $(1.73)$ \\
\hline \multirow[t]{2}{*}{ Issued Capital } & $0.0839 * *$ & $0.3091 * * *$ & $0.5047 * * *$ & $0.6614 * * *$ & $0.4573 *$ & 0.7037 & 0.6104 \\
\hline & (2.03) & (4.57) & (5.16) & (3.93) & (1.74) & $(1.63)$ & $(0.67)$ \\
\hline \multirow[t]{2}{*}{ Asset Acquisition \& Disposal } & 0.0639 & $0.1622 * *$ & $0.1732 *$ & 0.0977 & 0.2548 & 0.5774 & 1.1497 \\
\hline & $(1.53)$ & $(2.30)$ & (1.69) & $(0.55)$ & $(0.89)$ & $(1.29)$ & $(1.13)$ \\
\hline \multirow[t]{2}{*}{ Notice of Meeting } & 0.0567 & 0.1212 & 0.0356 & 0.0665 & 0.2913 & -0.6445 & 1.3125 \\
\hline & $(0.20)$ & $(0.21)$ & $(0.05)$ & $(0.05)$ & $(0.16)$ & $(-0.19)$ & $(0.27)$ \\
\hline \multirow[t]{2}{*}{ ASX Announcement } & 0.0031 & -0.0243 & -0.0199 & -0.0208 & -0.0971 & -0.2724 & 0.6278 \\
\hline & $(0.05)$ & $(-0.23)$ & $(-0.13)$ & $(-0.08)$ & $(-0.23)$ & $(-0.40)$ & $(0.45)$ \\
\hline \multirow[t]{2}{*}{ Distribution Announcement } & 0.0631 & 0.1509 & 0.1434 & 0.1982 & 0.2052 & 0.4608 & 0.7712 \\
\hline & $(0.88)$ & (1.29) & $(0.84)$ & $(0.65)$ & $(0.41)$ & $(0.55)$ & $(0.38)$ \\
\hline \multirow[t]{2}{*}{ Progress Reports } & $0.1164 * * *$ & $0.2344 * * *$ & $0.3452 * * *$ & $0.4088 * * *$ & $0.5838 * * *$ & $0.8714 * * *$ & 1.5373 \\
\hline & (4.30) & $(5.22)$ & $(5.34)$ & (3.77) & $(3.44)$ & $(3.27)$ & $(2.67)$ \\
\hline \multirow[t]{2}{*}{ Company Administration } & 0.1302 & 0.3008 & 0.4003 & 0.7651 & 0.1500 & $3.1603 *$ & 0.8508 \\
\hline & $(0.67)$ & $(0.86)$ & $(0.79)$ & $(0.85)$ & $(0.13)$ & (1.77) & $(0.28)$ \\
\hline Notice of Call & - & - & - & - & - & - & - \\
\hline \multirow[t]{2}{*}{ Other } & 0.0519 & $0.2960 * * *$ & $0.4148 * * *$ & -0.0230 & -0.3739 & 0.4182 & 0.7141 \\
\hline & $(0.86)$ & (3.12) & (2.95) & $(-0.10)$ & $(-1.03)$ & $(0.74)$ & $(0.61)$ \\
\hline \multirow[t]{2}{*}{ Chairman's Address } & 0.0889 & 0.1774 & 0.2590 & 0.4305 & 0.8185 & 1.2600 & 1.0365 \\
\hline & $(0.96)$ & $(1.12)$ & $(1.16)$ & $(1.15)$ & $(1.21)$ & $(0.83)$ & $(0.30)$ \\
\hline
\end{tabular}




\begin{tabular}{|c|c|c|c|c|c|c|c|}
\hline Variable & ATV10min & ATV15min & ATV30min & ATV60min & ATV90min & ATV120min & ATV150min \\
\hline \multirow[t]{2}{*}{ Letter to Shareholders } & 0.0521 & - & -0.0541 & -0.0694 & -0.0604 & - & - \\
\hline & $(0.09)$ & - & $(-0.03)$ & $(-0.04)$ & $(-0.03)$ & - & - \\
\hline \multirow[t]{2}{*}{ ASX Query } & $0.2183 * * *$ & $0.2358 * *$ & $0.7142 * * *$ & $0.9937 * * *$ & 0.0427 & -0.2525 & -1.2665 \\
\hline & $(3.43)$ & (2.28) & $(4.85)$ & $(4.03)$ & $(0.11)$ & $(-0.43)$ & $(-0.94)$ \\
\hline \multirow[t]{2}{*}{ Structured Products } & 0.0244 & 0.0636 & - & - & - & - & - \\
\hline & $(0.03)$ & $(0.05)$ & - & - & - & - & - \\
\hline \multirow[t]{2}{*}{ CTE Quarterly Reports } & 0.0639 & 0.0824 & 0.0470 & 0.0601 & 0.1432 & 0.5148 & 0.5846 \\
\hline & $(1.32)$ & $(1.02)$ & $(0.39)$ & $(0.29)$ & $(0.43)$ & $(0.94)$ & $(0.50)$ \\
\hline \multirow[t]{2}{*}{ Bid-Ask Spread } & -0.4063 & $-0.6287 * *$ & -0.4709 & -0.6513 & -0.8640 & -4.4731 & -4.2613 \\
\hline & $(-1.63)$ & $(-1.99)$ & $(-1.57)$ & $(-1.33)$ & $(-1.02)$ & $(-1.25)$ & $(-0.60)$ \\
\hline \multirow[t]{2}{*}{ Size } & -0.0036 & $-0.0179 * *$ & $-0.0222 *$ & -0.0069 & -0.0304 & -0.0566 & -0.0413 \\
\hline & $(-0.69)$ & $(-2.23)$ & $(-1.92)$ & $(-0.35)$ & $(-0.99)$ & $(-1.16)$ & $(-0.36)$ \\
\hline \multirow[t]{2}{*}{ Book-to-Market Ratio } & -0.0071 & -0.0158 & -0.0234 & -0.0739 & -0.0849 & -0.2140 & -0.3254 \\
\hline & $(-0.50)$ & $(-0.70)$ & $(-0.74)$ & $(-1.20)$ & $(-0.92)$ & $(-1.14)$ & $(-0.94)$ \\
\hline \multirow[t]{2}{*}{ Leverage } & -0.0001 & -0.0001 & 0.0001 & 0.0003 & 0.0049 & -0.0005 & -0.0026 \\
\hline & $(-0.59)$ & $(-0.47)$ & $(0.47)$ & $(0.83)$ & $(0.19)$ & $(-0.53)$ & $(-1.98)$ \\
\hline \multirow[t]{2}{*}{ ROA } & -0.0088 & -0.0212 & -0.0347 & -0.0458 & -0.0418 & -0.0537 & -0.3749 \\
\hline & $(-0.79)$ & $(-1.26)$ & $(-1.30)$ & $(-1.11)$ & $(-0.74)$ & $(-0.87)$ & $(-0.88)$ \\
\hline \multirow[t]{2}{*}{ Resource Sector } & 0.0464 & $0.0903 * *$ & 0.0677 & 0.0851 & -0.0482 & 0.1055 & 0.3561 \\
\hline & $(1.95)$ & (2.30) & $(1.19)$ & $(0.86)$ & $(-0.31)$ & $(0.41)$ & $(0.64)$ \\
\hline \multirow[t]{2}{*}{ Financial Sector } & $-0.0078 *$ & -0.0379 & -0.1050 & -0.1511 & -0.2846 & -0.2343 & -0.0815 \\
\hline & $(-0.22)$ & $(-0.66)$ & $(-1.24)$ & $(-1.02)$ & $(-1.19)$ & $(-0.59)$ & $(-0.09)$ \\
\hline Max. VIF & 2.11 & 2.12 & 2.12 & 2.09 & 2.06 & 2.00 & 1.98 \\
\hline F Value & 1.75 & 3.00 & 5.17 & 3.25 & 1.43 & 1.52 & 0.92 \\
\hline R-Square & $0.49 \%$ & $0.76 \%$ & $1.46 \%$ & $1.20 \%$ & $0.75 \%$ & $1.24 \%$ & $1.71 \%$ \\
\hline Adj R-Sq & $0.21 \%$ & $0.50 \%$ & $1.18 \%$ & $0.83 \%$ & $0.23 \%$ & $0.43 \%$ & $-0.15 \%$ \\
\hline $\mathbf{N}$ & 8,271 & 8,695 & 7,671 & 5,884 & 4,174 & 2,557 & 1,128 \\
\hline
\end{tabular}

Note: The ATV150min regression model does not pass the significant test $(\mathrm{F}$ value $=0.92)$

$*, * *$ and $* * *$ denote significance at the $10 \%, 5 \%$ and $1 \%$ levels respectively (two-tailed). 
Table 6-34. The Regression Results of Market-sensitive Announcements with Bad News - Intraday ATVs during the GFC

\begin{tabular}{|c|c|c|c|c|c|c|c|}
\hline Variable & ATV10min & ATV15min & ATV30min & ATV60min & ATV90min & ATV120min & ATV150min \\
\hline \multirow[t]{2}{*}{ Intercept } & 0.0070 & 0.1142 & 0.0758 & -0.1418 & -0.3288 & -0.1199 & -0.1238 \\
\hline & $(0.15)$ & $(1.29)$ & $(0.39)$ & $(-0.39)$ & $(-0.60)$ & $(-0.17)$ & $(-0.10)$ \\
\hline \multirow[t]{2}{*}{ Takeover / Scheme Announcements } & 0.0210 & 0.0505 & 0.2299 & 0.3454 & 0.3344 & 0.2981 & 0.1891 \\
\hline & $(0.26)$ & $(0.33)$ & $(0.74)$ & $(0.60)$ & $(0.38)$ & $(0.27)$ & $(0.10)$ \\
\hline \multirow[t]{2}{*}{ Security Holder Details } & -0.0389 & 0.0341 & 0.1313 & 0.4496 & 0.1778 & 0.4356 & 0.3989 \\
\hline & $(-0.29)$ & $(0.14)$ & $(0.25)$ & $(0.51)$ & $(0.14)$ & $(0.25)$ & $(0.14)$ \\
\hline \multirow[t]{2}{*}{ Periodic Reports } & 0.0841 & $0.2228 * *$ & $0.4476 * *$ & $0.7741 * *$ & $1.2914 * *$ & $1.4568 * *$ & 1.5328 \\
\hline & $(1.72)$ & $(2.46)$ & (2.33) & (2.19) & $(2.43)$ & (2.09) & $(1.29)$ \\
\hline \multirow[t]{2}{*}{ Issued Capital } & 0.1914 & $0.4501 * * *$ & $0.9662 * * *$ & $1.6708 * * *$ & $1.3187 * *$ & $1.8455 * *$ & 1.6650 \\
\hline & $(3.67)$ & $(4.63)$ & $(4.65)$ & $(4.45)$ & $(2.31)$ & $(2.37)$ & $(1.21)$ \\
\hline \multirow[t]{2}{*}{ Asset Acquisition \& Disposal } & 0.0555 & $0.2041 *$ & $0.5469 * *$ & $0.7517 *$ & $1.3614 * *$ & $1.7595 * *$ & 3.1436 \\
\hline & $(0.99)$ & (1.93) & $(2.47)$ & $(1.85)$ & $(2.26)$ & $(2.25)$ & $(2.27)$ \\
\hline \multirow[t]{2}{*}{ Notice of Meeting } & 0.0163 & 0.0052 & 0.1720 & 0.2217 & 0.8946 & -0.8644 & - \\
\hline & $(0.06)$ & $(0.01)$ & $(0.16)$ & $(0.09)$ & $(0.25)$ & $(-0.21)$ & - \\
\hline \multirow[t]{2}{*}{ ASX Announcement } & -0.0030 & -0.0384 & 0.1088 & 0.0615 & -0.0799 & 0.0766 & -0.4362 \\
\hline & $(-0.04)$ & $(-0.28)$ & $(0.39)$ & $(0.12)$ & $(-0.11)$ & $(0.08)$ & $(-0.27)$ \\
\hline \multirow[t]{2}{*}{ Distribution Announcement } & 0.0424 & 0.1602 & 0.5502 & 0.8185 & 0.7435 & 1.1953 & 1.7365 \\
\hline & $(0.41)$ & $(0.88)$ & $(1.41)$ & $(1.13)$ & $(0.69)$ & $(0.82)$ & $(0.70)$ \\
\hline \multirow[t]{2}{*}{ Progress Reports } & $0.0905 * * *$ & $0.3170 * * *$ & $0.7057 * * *$ & $1.1399 * * *$ & $1.4943 * * *$ & $1.6167 * * *$ & 1.2950 \\
\hline & $(2.83)$ & $(5.31)$ & $(5.64)$ & $(5.10)$ & $(4.39)$ & $(3.67)$ & $(1.76)$ \\
\hline \multirow[t]{2}{*}{ Company Administration } & 0.0721 & 0.2537 & 0.7091 & 1.6156 & 0.5842 & 1.3623 & 0.7136 \\
\hline & $(0.51)$ & $(0.99)$ & $(1.12)$ & $(1.43)$ & $(0.39)$ & $(0.64)$ & $(0.21)$ \\
\hline Notice of Call & - & - & - & - & - & - & - \\
\hline \multirow[t]{2}{*}{ Other } & 0.0300 & 0.0862 & 0.2256 & 0.5009 & 0.7146 & 1.1118 & 0.8046 \\
\hline & $(0.26)$ & $(0.43)$ & $(0.51)$ & $(0.62)$ & $(0.61)$ & $(0.69)$ & $(0.28)$ \\
\hline \multirow[t]{2}{*}{ Chairman's Address } & 0.0352 & 0.1512 & 0.3788 & 0.7763 & 0.9940 & 1.2644 & -0.6422 \\
\hline & $(0.26)$ & $(0.65)$ & $(0.80)$ & $(0.89)$ & $(0.67)$ & $(0.71)$ & $(-0.17)$ \\
\hline
\end{tabular}




\begin{tabular}{|c|c|c|c|c|c|c|c|}
\hline Variable & ATV10min & ATV15min & ATV30min & ATV60min & ATV90min & ATV120min & ATV150min \\
\hline \multirow[t]{2}{*}{ Letter to Shareholders } & 0.5080 & 1.6397 & 2.4461 & 2.5818 & 2.4616 & 3.9580 & 5.6519 \\
\hline & $(0.48)$ & $(0.83)$ & $(0.62)$ & $(0.41)$ & $(0.31)$ & $(0.49)$ & $(0.62)$ \\
\hline \multirow[t]{2}{*}{ ASX Query } & 0.0976 & 0.1837 & $0.5290 * *$ & 0.3339 & -0.3435 & -0.5339 & -1.1656 \\
\hline & $(1.38)$ & $(1.52)$ & (2.03) & $(0.76)$ & $(-0.55)$ & $(-0.70)$ & $(-0.94)$ \\
\hline Structured Products & - & - & - & - & - & - & - \\
\hline \multirow[t]{2}{*}{ CTE Quarterly Reports } & 0.0328 & 0.1367 & 0.1070 & 0.2767 & 0.2974 & 0.1347 & -0.1517 \\
\hline & $(0.49)$ & $(1.10)$ & $(0.41)$ & $(0.57)$ & $(0.4)$ & $(0.14)$ & $(-0.09)$ \\
\hline \multirow[t]{2}{*}{ Bid-Ask Spread } & -0.4268 & $-1.3305 * *$ & $-2.2584^{*}$ & $-4.5525 *$ & -6.4374 & -11.6043 & -17.0022 \\
\hline & $(-1.37)$ & $(-2.37)$ & $(-1.95)$ & $(-1.73)$ & $(-1.36)$ & $(-1.31)$ & $(-1.23)$ \\
\hline \multirow[t]{2}{*}{ Size } & -0.0045 & $-0.0298 * * *$ & $-0.0588 * *$ & $-0.0839 *$ & -0.1118 & $-0.1580 *$ & -0.1292 \\
\hline & $(-0.71)$ & $(-2.68)$ & $(-2.37)$ & $(-1.80)$ & $(-1.64)$ & $(-1.79)$ & $(-0.86)$ \\
\hline \multirow[t]{2}{*}{ Book-to-Market Ratio } & 0.0015 & -0.0111 & -0.0195 & 0.0101 & -0.0239 & 0.0043 & 0.0683 \\
\hline & $(0.14)$ & $(-0.56)$ & $(-0.51)$ & $(0.12)$ & $(-0.19)$ & $(0.03)$ & $(0.21)$ \\
\hline \multirow[t]{2}{*}{ Leverage } & 0.0002 & -0.0027 & -0.0050 & -0.0239 & -0.0046 & -0.0371 & -0.0612 \\
\hline & $(0.19)$ & $(-0.28)$ & $(-0.29)$ & $(-0.76)$ & $(-0.09)$ & $(-0.47)$ & $(-0.54)$ \\
\hline \multirow[t]{2}{*}{ ROA } & 0.0001 & -0.0014 & -0.1311 & $-0.3718 * *$ & $-0.8103 * * *$ & $-1.2252 * * *$ & -1.8161 \\
\hline & $(0.04)$ & $(-0.26)$ & $(-1.36)$ & $(-2.11)$ & $(-3.01)$ & $(-3.82)$ & $(-2.98)$ \\
\hline \multirow[t]{2}{*}{ Resource Sector } & 0.0210 & 0.0476 & 0.1999 & $0.4827 * *$ & $0.7066 * *$ & 0.4239 & 0.5199 \\
\hline & $(0.66)$ & $(0.81)$ & (1.60) & (2.12) & (2.07) & (0.95) & $(0.70)$ \\
\hline \multirow[t]{2}{*}{ Financial Sector } & -0.0264 & -0.0030 & -0.0689 & 0.1640 & 0.3503 & -0.0005 & -0.0177 \\
\hline & $(-0.55)$ & $(-0.03)$ & $(-0.37)$ & $(0.47)$ & $(0.67)$ & $(0.00)$ & $(-0.01)$ \\
\hline Max. VIF & 1.88 & 1.88 & 1.86 & 1.83 & 1.87 & 1.87 & 1.86 \\
\hline F Value & 1.06 & 2.71 & 3.18 & 3.02 & 2.61 & 2.52 & 1.34 \\
\hline R-Square & $0.28 \%$ & $0.68 \%$ & $0.90 \%$ & $1.10 \%$ & $1.38 \%$ & $2.11 \%$ & $2.42 \%$ \\
\hline Adj R-Sq & $0.02 \%$ & $0.43 \%$ & $0.62 \%$ & $0.74 \%$ & $0.85 \%$ & $1.27 \%$ & $0.61 \%$ \\
\hline $\mathbf{N}$ & 8,474 & 8,728 & 7,738 & 5,987 & 4,134 & 2,590 & 1,155 \\
\hline
\end{tabular}

Note: The ATV10min and ATV150min regression models do not pass the significant test (F value=1.06, 1.34).

$*, * *$ and $* * *$ denote significance at the $10 \%, 5 \%$ and $1 \%$ levels respectively (two-tailed). 
Table 6-35. The Regression Results of Market-sensitive Announcements with Bad News - Intraday ATVs after the GFC

\begin{tabular}{|c|c|c|c|c|c|c|c|}
\hline Variable & ATV10min & ATV15min & ATV30min & ATV60min & ATV90min & ATV120min & ATV150min \\
\hline \multirow[t]{2}{*}{ Intercept } & 0.0715 & 0.0896 & 0.1588 & 0.2614 & -0.0598 & -0.3664 & -0.1740 \\
\hline & $(1.20)$ & $(1.15)$ & $(1.18)$ & $(1.25)$ & $(-0.22)$ & $(-0.81)$ & $(-0.30)$ \\
\hline \multirow[t]{2}{*}{ Takeover / Scheme Announcements } & 0.0231 & -0.0013 & 0.2080 & 0.2074 & 0.0252 & 0.5005 & 0.3072 \\
\hline & $(0.19)$ & $(-0.01)$ & $(0.73)$ & $(0.48)$ & $(0.04)$ & $(0.52)$ & $(0.24)$ \\
\hline \multirow[t]{2}{*}{ Security Holder Details } & 0.1330 & 0.1585 & 0.5053 & 0.5150 & 0.6528 & 0.9967 & $3.0906 *$ \\
\hline & $(0.58)$ & $(0.53)$ & $(0.99)$ & $(0.66)$ & $(0.73)$ & $(0.70)$ & $(1.77)$ \\
\hline \multirow[t]{2}{*}{ Periodic Reports } & 0.0549 & $0.1610 * *$ & $0.2337 *$ & 0.3478 & $0.5269 *$ & 0.8413* & 1.1179* \\
\hline & $(0.90)$ & $(2.01)$ & $(1.70)$ & $(1.60)$ & (1.79) & (1.75) & $(1.88)$ \\
\hline \multirow[t]{2}{*}{ Issued Capital } & 0.0810 & $0.2714 * * *$ & $0.3622 * *$ & $0.5283 * *$ & $1.1000 * * *$ & $1.7159 * * *$ & $2.0403 * * *$ \\
\hline & $(1.16)$ & $(2.99)$ & $(2.33)$ & $(2.17)$ & $(3.19)$ & $(3.05)$ & $(2.73)$ \\
\hline \multirow[t]{2}{*}{ Asset Acquisition \& Disposal } & 0.0213 & 0.1204 & 0.1360 & 0.1550 & 0.1284 & 0.6401 & 0.3711 \\
\hline & $(0.29)$ & $(1.23)$ & $(0.79)$ & $(0.60)$ & $(0.38)$ & $(1.07)$ & $(0.48)$ \\
\hline \multirow[t]{2}{*}{ Notice of Meeting } & 0.0153 & 0.0193 & -0.0548 & -0.0801 & 0.1725 & -2.5938 & - \\
\hline & $(0.04)$ & $(0.04)$ & $(-0.05)$ & $(-0.06)$ & $(0.10)$ & $(-0.64)$ & - \\
\hline \multirow[t]{2}{*}{ ASX Announcement } & -0.0103 & -0.0585 & -0.1284 & -0.0602 & -0.1973 & -0.5213 & -0.4829 \\
\hline & $(-0.11)$ & $(-0.47)$ & $(-0.64)$ & $(-0.19)$ & $(-0.44)$ & $(-0.73)$ & $(-0.53)$ \\
\hline \multirow[t]{2}{*}{ Distribution Announcement } & 0.0103 & 0.0850 & 0.0913 & 0.1702 & 0.3530 & 0.8802 & 0.9088 \\
\hline & $(0.08)$ & $(0.54)$ & $(0.33)$ & $(0.42)$ & $(0.64)$ & $(0.99)$ & $(0.74)$ \\
\hline \multirow[t]{2}{*}{ Progress Reports } & $0.1532 * * *$ & $0.3133 * * *$ & $0.5355 * * *$ & $0.6939 * * *$ & $0.7621 * * *$ & $1.1696 * * *$ & $0.9047 * *$ \\
\hline & $(3.85)$ & $(6.02)$ & $(5.96)$ & $(5.02)$ & $(4.08)$ & $(3.81)$ & $(2.27)$ \\
\hline \multirow[t]{2}{*}{ Company Administration } & 0.0379 & 0.1190 & 0.1914 & 0.3018 & 0.0550 & 0.5334 & -0.2439 \\
\hline & $(0.35)$ & $(0.83)$ & $(0.78)$ & $(0.75)$ & $(0.11)$ & $(0.63)$ & $(-0.23)$ \\
\hline Notice of Call & - & - & - & - & - & - & - \\
\hline \multirow[t]{2}{*}{ Other } & 0.1543 & 0.2757 & 0.0253 & 0.0812 & 0.3272 & 0.7898 & 0.3396 \\
\hline & $(0.83)$ & $(1.19)$ & $(0.06)$ & $(0.13)$ & $(0.39)$ & $(0.62)$ & $(0.18)$ \\
\hline \multirow[t]{2}{*}{ Chairman's Address } & 0.0213 & 0.2490 & 0.4279 & 0.5446 & 1.2657 & 0.5059 & 0.8443 \\
\hline & $(0.11)$ & $(0.98)$ & $(0.97)$ & $(0.75)$ & $(1.13)$ & $(0.26)$ & $(0.30)$ \\
\hline
\end{tabular}




\begin{tabular}{|c|c|c|c|c|c|c|c|}
\hline Variable & ATV10min & ATV15min & ATV30min & ATV60min & ATV90min & ATV120min & ATV150min \\
\hline \multirow[t]{2}{*}{ Letter to Shareholders } & -0.0077 & 0.0683 & 0.0755 & 0.2001 & - & - & - \\
\hline & $(-0.01)$ & $(0.05)$ & $(0.04)$ & $(0.05)$ & - & - & - \\
\hline \multirow[t]{2}{*}{ ASX Query } & 0.1187 & $0.3068 * * *$ & $0.4648 * *$ & -0.0614 & 0.1949 & 0.0247 & $-2.0849 * * *$ \\
\hline & $(1.30)$ & $(2.71)$ & $(2.44)$ & $(-0.21)$ & $(0.53)$ & $(0.04)$ & $(-3.00)$ \\
\hline Structured Products & - & - & - & - & - & - & - \\
\hline \multirow[t]{2}{*}{ CTE Quarterly Reports } & 0.0835 & 0.1209 & 0.1500 & -0.0263 & 0.3325 & 0.7023 & 0.8438 \\
\hline & $(0.95)$ & $(1.02)$ & $(0.72)$ & $(-0.08)$ & $(0.72)$ & $(0.94)$ & $(0.91)$ \\
\hline \multirow[t]{2}{*}{ Bid-Ask Spread } & -0.0856 & -0.2919 & -0.4853 & -2.0255 & -1.2918 & -0.4310 & -0.2526 \\
\hline & $(-0.47)$ & $(-1.02)$ & $(-1.03)$ & $(-1.41)$ & $(-0.85)$ & $(-0.29)$ & $(-0.16)$ \\
\hline \multirow[t]{2}{*}{ Size } & -0.0083 & $-0.0212 * *$ & -0.0253 & -0.0297 & -0.0289 & -0.0607 & -0.0836 \\
\hline & $(-0.99)$ & $(-2.02)$ & $(-1.40)$ & $(-1.07)$ & $(-0.80)$ & $(-1.01)$ & $(-1.11)$ \\
\hline \multirow[t]{2}{*}{ Book-to-Market Ratio } & -0.0027 & -0.0159 & -0.0269 & -0.0619 & -0.0308 & -0.0437 & -0.0322 \\
\hline & $(-0.25)$ & $(-1.13)$ & $(-0.98)$ & $(-1.51)$ & $(-0.58)$ & $(-0.55)$ & $(-0.36)$ \\
\hline \multirow[t]{2}{*}{ Leverage } & -0.0005 & 0.0020 & -0.0033 & 0.0011 & 0.0055 & -0.0005 & -0.0145 \\
\hline & $(-0.10)$ & $(0.30)$ & $(-0.28)$ & $(0.07)$ & $(0.22)$ & $(-0.01)$ & $(-0.18)$ \\
\hline \multirow[t]{2}{*}{ ROA } & 0.0029 & 0.0020 & -0.0076 & -0.0038 & -0.0042 & -0.0062 & -0.0023 \\
\hline & $(0.31)$ & $(0.16)$ & $(-0.27)$ & $(-0.33)$ & $(-0.32)$ & $(-0.27)$ & $(-0.02)$ \\
\hline \multirow[t]{2}{*}{ Resource Sector } & -0.0434 & 0.0412 & 0.0509 & 0.0627 & $0.3698 *$ & $0.6615 * *$ & 0.6251 \\
\hline & $(-1.05)$ & $(0.76)$ & $(0.55)$ & $(0.44)$ & (1.93) & (2.11) & $(1.56)$ \\
\hline \multirow[t]{2}{*}{ Financial Sector } & -0.0910 & -0.1141 & -0.1980 & -0.2111 & -0.1206 & -0.1859 & -0.4225 \\
\hline & $(-1.40)$ & $(-1.35)$ & $(-1.34)$ & $(-0.91)$ & $(-0.38)$ & $(-0.35)$ & $(-0.68)$ \\
\hline Max. VIF & 1.79 & 1.76 & 1.74 & 1.75 & 1.75 & 1.75 & 1.86 \\
\hline F Value & 1.10 & 2.69 & 2.61 & 1.99 & 1.71 & 1.50 & 1.83 \\
\hline R-Square & $0.28 \%$ & $0.67 \%$ & $0.76 \%$ & $0.76 \%$ & $0.88 \%$ & $1.24 \%$ & $3.23 \%$ \\
\hline Adj R-Sq & $0.02 \%$ & $0.42 \%$ & $0.47 \%$ & $0.38 \%$ & $0.37 \%$ & $0.41 \%$ & $1.46 \%$ \\
\hline $\mathbf{N}$ & 8,659 & 8,731 & 7,561 & 5,762 & 4,069 & 2,538 & 1,116 \\
\hline
\end{tabular}

Note: The ATV10min regression model does not pass the significant test (F value $=1.10)$.

$*, * *$ and $* * *$ denote significance at the $10 \%, 5 \%$ and $1 \%$ levels respectively (two-tailed). 
Table 6-36. The Regression Results of Market-sensitive Announcements with Good News - Intraday ATVs before the GFC

\begin{tabular}{|c|c|c|c|c|c|c|c|}
\hline Variable & ATV10min & ATV15min & ATV30min & ATV60min & ATV90min & ATV120min & ATV150min \\
\hline \multirow[t]{2}{*}{ Intercept } & $0.0864 * *$ & $0.3329 * * *$ & $\mathbf{0 . 6 3 8 1} * * *$ & $0.9879 * * *$ & $1.3897 * * *$ & $1.6257 * *$ & 0.9089 \\
\hline & $(2.05)$ & (3.77) & (4.74) & $(4.64)$ & (3.44) & (2.13) & $(0.83)$ \\
\hline \multirow[t]{2}{*}{ Takeover / Scheme Announcements } & 0.1187 & 0.2057 & 0.2786 & 0.1989 & 0.3132 & 0.6943 & -0.2491 \\
\hline & $(1.55)$ & $(1.21)$ & $(1.11)$ & $(0.52)$ & $(0.44)$ & $(0.49)$ & $(-0.12)$ \\
\hline \multirow[t]{2}{*}{ Security Holder Details } & 0.1930 & 0.2564 & 0.3542 & 0.1197 & 0.6387 & -0.1124 & -1.7182 \\
\hline & $(1.30)$ & $(0.85)$ & $(0.82)$ & $(0.16)$ & $(0.46)$ & $(-0.05)$ & $(-0.51)$ \\
\hline \multirow[t]{2}{*}{ Periodic Reports } & $0.1478 * * *$ & $0.2542 * * *$ & $0.4053 * * *$ & $0.5670 * * *$ & $0.6802 *$ & 0.8247 & 0.5901 \\
\hline & $(3.56)$ & (2.94) & (3.06) & (2.69) & (1.72) & $(1.09)$ & $(0.57)$ \\
\hline \multirow[t]{2}{*}{ Issued Capital } & $0.1046 * *$ & $0.2321 * *$ & 0.2166 & 0.2502 & 0.3638 & 0.4725 & 0.4258 \\
\hline & (2.24) & $(2.35)$ & $(1.42)$ & $(1.01)$ & $(0.79)$ & $(0.54)$ & $(0.36)$ \\
\hline \multirow[t]{2}{*}{ Asset Acquisition \& Disposal } & $0.1677 * * *$ & $0.3311 * * *$ & $0.4670 * * *$ & $0.5305 * *$ & 0.6577 & 0.7546 & 0.2706 \\
\hline & (3.84) & (3.64) & (3.38) & (2.40) & $(1.56)$ & $(0.92)$ & $(0.25)$ \\
\hline \multirow[t]{2}{*}{ Notice of Meeting } & 0.0863 & 0.1905 & 0.3233 & 0.4294 & 0.5962 & 0.7651 & 0.5990 \\
\hline & $(0.31)$ & $(0.34)$ & $(0.32)$ & $(0.29)$ & $(0.24)$ & $(0.21)$ & $(0.07)$ \\
\hline \multirow[t]{2}{*}{ ASX Announcement } & 0.0010 & -0.0276 & 0.1233 & 0.1082 & -0.1466 & -0.2868 & -0.1605 \\
\hline & $(0.01)$ & $(-0.17)$ & $(0.48)$ & $(0.26)$ & $(-0.19)$ & $(-0.19)$ & $(-0.08)$ \\
\hline \multirow[t]{2}{*}{ Distribution Announcement } & 0.1201 & 0.2514 & 0.3713 & 0.4759 & 0.5322 & 0.6758 & 0.4345 \\
\hline & (1.57) & $(1.56)$ & $(1.53)$ & $(1.25)$ & $(0.76)$ & $(0.50)$ & $(0.25)$ \\
\hline \multirow[t]{2}{*}{ Progress Reports } & $0.2702 * * *$ & $0.5648 * * *$ & $0.8080 * * *$ & $1.0020 * * *$ & $1.3624 * * *$ & $1.9909 * * *$ & $1.6878 * *$ \\
\hline & $(9.17)$ & $(8.92)$ & $(8.45)$ & $(6.54)$ & $(4.73)$ & $(3.60)$ & (2.23) \\
\hline \multirow[t]{2}{*}{ Company Administration } & 0.1085 & $1.0494 * *$ & $1.6626 * *$ & 1.4189 & 2.4417 & 2.6888 & $-8.0210 *$ \\
\hline & $(0.46)$ & (2.46) & (2.48) & $(1.33)$ & $(1.11)$ & $(0.61)$ & $(-1.82)$ \\
\hline Notice of Call & - & - & - & - & - & - & - \\
\hline \multirow[t]{2}{*}{ Other } & $0.1439 * *$ & $0.3335 * * *$ & $0.4089 * *$ & $0.6773 * *$ & $0.9694 *$ & 1.6838 & 1.7530 \\
\hline & $(2.26)$ & $(2.66)$ & $(2.15)$ & $(2.24)$ & $(1.73)$ & $(1.54)$ & $(1.17)$ \\
\hline \multirow[t]{2}{*}{ Chairman's Address } & 0.1220 & 0.2669 & 0.3201 & 0.3882 & 0.3496 & 0.6790 & 1.1116 \\
\hline & $(1.18)$ & $(1.29)$ & $(1.02)$ & $(0.70)$ & $(0.32)$ & $(0.30)$ & $(0.36)$ \\
\hline
\end{tabular}




\begin{tabular}{|c|c|c|c|c|c|c|c|}
\hline Variable & ATV10min & ATV15min & ATV30min & ATV60min & ATV90min & ATV120min & ATV150min \\
\hline \multirow[t]{2}{*}{ Letter to Shareholders } & $1.0469 * *$ & $1.7559 * *$ & $5.5945 * * *$ & - & - & - & - \\
\hline & (2.16) & (2.04) & (3.19) & - & - & - & - \\
\hline \multirow[t]{2}{*}{ ASX Query } & 0.0120 & 0.0669 & 0.1788 & 0.3140 & 0.3014 & -0.3631 & -1.1529 \\
\hline & $(0.13)$ & $(0.35)$ & $(0.61)$ & $(0.7)$ & $(0.41)$ & $(-0.25)$ & $(-0.65)$ \\
\hline Structured Products & - & - & - & - & - & - & - \\
\hline \multirow[t]{2}{*}{ CTE Quarterly Reports } & 0.0223 & 0.0318 & -0.0100 & -0.2224 & -0.4017 & 0.0272 & -0.5603 \\
\hline & $(0.40)$ & $(0.26)$ & $(-0.05)$ & $(-0.75)$ & $(-0.69)$ & $(0.02)$ & $(-0.37)$ \\
\hline \multirow[t]{2}{*}{ Bid-Ask Spread } & $-0.2733 *$ & $-0.8535 * *$ & $-3.3006 * * *$ & $-4.5651 * * *$ & $-8.2332 * * *$ & -6.9553 & -3.7006 \\
\hline & $(-1.84)$ & $(-2.46)$ & $(-3.97)$ & $(-3.42)$ & $(-2.67)$ & $(-1.36)$ & $(-0.65)$ \\
\hline \multirow[t]{2}{*}{ Size } & $-0.0332 * * *$ & $-0.0941 * * *$ & $-0.1405 * * *$ & $-0.2054 * * *$ & $-0.2852 * * *$ & $-0.3858 * * *$ & -0.1478 \\
\hline & $(-6.11)$ & $(-8.77)$ & $(-8.46)$ & $(-7.74)$ & $(-5.70)$ & $(-4.03)$ & $(-1.12)$ \\
\hline \multirow[t]{2}{*}{ Book-to-Market Ratio } & -0.0108 & -0.0408 & $-0.0946 *$ & -0.1083 & -0.1484 & -0.1963 & 0.0328 \\
\hline & $(-0.73)$ & $(-1.26)$ & $(-1.85)$ & $(-1.54)$ & $(-1.16)$ & $(-0.89)$ & $(0.14)$ \\
\hline \multirow[t]{2}{*}{ Leverage } & 0.0001 & 0.0002 & $0.0007 * * *$ & $0.0011^{* * * *}$ & $0.0015 * * *$ & 0.0005 & -0.0008 \\
\hline & $(0.96)$ & (1.54) & (3.33) & (3.27) & (3.09) & $(0.41)$ & $(-0.50)$ \\
\hline \multirow[t]{2}{*}{ ROA } & -0.0050 & 0.0214 & 0.0290 & 0.0443 & 0.0528 & 0.1368 & $-1.8623 * * *$ \\
\hline & $(-0.44)$ & $(0.97)$ & $(0.98)$ & $(0.87)$ & $(0.71)$ & (1.06) & $(-3.38)$ \\
\hline \multirow[t]{2}{*}{ Resource Sector } & $0.0460 *$ & $0.0820 *$ & 0.0368 & 0.0250 & -0.0720 & -0.0958 & -0.7064 \\
\hline & (1.91) & (1.69) & $(0.49)$ & $(0.21)$ & $(-0.31)$ & $(-0.22)$ & $(-1.18)$ \\
\hline \multirow[t]{2}{*}{ Financial Sector } & -0.0224 & 0.0304 & -0.0326 & -0.0836 & -0.0701 & 0.0719 & -0.2231 \\
\hline & $(-0.60)$ & $(0.39)$ & $(-0.27)$ & $(-0.43)$ & $(-0.19)$ & $(0.11)$ & $(-0.24)$ \\
\hline Max. VIF & 2.51 & 2.79 & 2.70 & 2.70 & 2.71 & 2.71 & 2.79 \\
\hline F Value & 8.96 & 11.38 & 11.65 & 8.37 & 4.70 & 2.16 & 1.80 \\
\hline R-Square & $1.77 \%$ & $1.99 \%$ & $2.30 \%$ & $2.12 \%$ & $1.73 \%$ & $1.35 \%$ & $2.56 \%$ \\
\hline Adj R-Sq & $1.57 \%$ & $1.81 \%$ & $2.10 \%$ & $1.86 \%$ & $1.36 \%$ & $0.73 \%$ & $1.14 \%$ \\
\hline $\mathbf{N}$ & 10,969 & 12,366 & 10,908 & 8,160 & 5,634 & 3,339 & 1,461 \\
\hline
\end{tabular}

$*, * *$ and $* * *$ denote significance at the $10 \%, 5 \%$ and $1 \%$ levels respectively (two-tailed). 
Table 6-37. The Regression Results of Market-sensitive Announcements with Good News - Intraday ATVs during the GFC

\begin{tabular}{|c|c|c|c|c|c|c|c|}
\hline Variable & ATV10min & ATV15min & ATV30min & ATV60min & ATV90min & ATV120min & ATV150min \\
\hline \multirow[t]{2}{*}{ Intercept } & 0.0991 & $0.2972 * * *$ & $0.6774 * * *$ & $0.8665 * * *$ & 0.4155 & 0.2677 & 0.2436 \\
\hline & (1.44) & (2.79) & $(3.76)$ & $(2.72)$ & $(1.03)$ & $(0.43)$ & $(0.19)$ \\
\hline \multirow[t]{2}{*}{ Takeover / Scheme Announcements } & 0.1649 & 0.2939 & 0.3657 & 0.3819 & 0.3549 & 0.7604 & 0.7819 \\
\hline & $(1.31)$ & $(1.50)$ & $(1.09)$ & $(0.67)$ & $(0.54)$ & $(0.71)$ & $(0.39)$ \\
\hline \multirow[t]{2}{*}{ Security Holder Details } & 0.0971 & 0.2036 & 0.2532 & 0.5298 & 0.5370 & -0.1299 & -1.4652 \\
\hline & $(0.46)$ & $(0.62)$ & $(0.46)$ & $(0.54)$ & $(0.41)$ & $(-0.07)$ & $(-0.38)$ \\
\hline \multirow[t]{2}{*}{ Periodic Reports } & $0.1753^{* *}$ & $0.3834 * * *$ & $0.5357 * * *$ & $0.6944 * *$ & $0.7879 * *$ & $1.2684 * *$ & 1.5621 \\
\hline & $(2.40)$ & $(3.43)$ & $(2.86)$ & $(2.07)$ & (1.98) & (2.07) & $(1.29)$ \\
\hline \multirow[t]{2}{*}{ Issued Capital } & 0.1538* & $0.3385 * * *$ & 0.3793* & 0.5330 & 0.6209 & 1.0735 & 1.4055 \\
\hline & (1.96) & $(2.75)$ & $(1.85)$ & (1.46) & (1.44) & (1.63) & (1.13) \\
\hline \multirow[t]{2}{*}{ Asset Acquisition \& Disposal } & $0.4104 * * *$ & $0.6737 * * *$ & $1.0985 * * *$ & $1.8619 * * *$ & $1.5102 * * *$ & $1.6951 * *$ & 2.1738* \\
\hline & $(5.23)$ & $(5.66)$ & $(5.51)$ & $(5.32)$ & (3.56) & $(2.49)$ & $(1.71)$ \\
\hline \multirow[t]{2}{*}{ Notice of Meeting } & 0.0555 & 0.1143 & 0.0763 & 0.1197 & -0.1663 & 0.3968 & 0.5018 \\
\hline & $(0.13)$ & $(0.18)$ & $(0.07)$ & $(0.07)$ & $(-0.08)$ & $(0.12)$ & $(0.07)$ \\
\hline \multirow[t]{2}{*}{ ASX Announcement } & -0.0009 & -0.0631 & -0.0541 & -0.1245 & -0.1169 & 0.2632 & -0.3730 \\
\hline & $(-0.01)$ & $(-0.36)$ & $(-0.18)$ & $(-0.23)$ & $(-0.18)$ & $(0.25)$ & $(-0.19)$ \\
\hline \multirow[t]{2}{*}{ Distribution Announcement } & 0.1627 & 0.3094 & 0.4315 & 0.7427 & 0.9576 & 1.5425 & 2.0470 \\
\hline & (1.06) & (1.33) & (1.11) & (1.10) & (1.23) & (1.34) & $(0.94)$ \\
\hline \multirow[t]{2}{*}{ Progress Reports } & $0.3806 * * *$ & $0.7239 * * *$ & $1.0925 * * *$ & $1.5590 * * *$ & $1.6523 * * *$ & $2.0201 * * *$ & $2.2513 * * *$ \\
\hline & (8.18) & (9.91) & (8.98) & (7.22) & (6.44) & (5.02) & (2.89) \\
\hline \multirow[t]{2}{*}{ Company Administration } & 0.1100 & 0.2859 & 0.3975 & 1.0313 & 1.8343 & $3.0266 *$ & $6.2384 *$ \\
\hline & $(0.46)$ & $(0.77)$ & $(0.70)$ & $(1.01)$ & $(1.54)$ & (1.77) & (1.90) \\
\hline Notice of Call & - & - & - & - & - & - & - \\
\hline \multirow[t]{2}{*}{ Other } & 0.1018 & 0.3033 & 0.5267 & $1.2025 *$ & 1.1757 & 1.1469 & 1.1337 \\
\hline & $(0.61)$ & $(1.21)$ & $(1.28)$ & $(1.65)$ & $(1.39)$ & $(0.81)$ & $(0.41)$ \\
\hline \multirow[t]{2}{*}{ Chairman's Address } & 0.1291 & 0.2790 & 0.4405 & 0.4550 & 0.6531 & 1.1265 & 0.9859 \\
\hline & $(0.65)$ & $(0.88)$ & $(0.77)$ & $(0.42)$ & $(0.52)$ & $(0.60)$ & $(0.27)$ \\
\hline
\end{tabular}




\begin{tabular}{|c|c|c|c|c|c|c|c|}
\hline Variable & ATV10min & ATV15min & ATV30min & ATV60min & ATV90min & ATV120min & ATV150min \\
\hline \multirow[t]{2}{*}{ Letter to Shareholders } & 1.4169 & 1.5958 & 1.6960 & 2.4351 & 2.8193 & 3.6926 & 3.6680 \\
\hline & $(1.20)$ & $(0.83)$ & $(0.56)$ & $(0.52)$ & $(0.62)$ & $(0.66)$ & $(0.36)$ \\
\hline \multirow[t]{2}{*}{ ASX Query } & 0.0429 & 0.1939 & 0.4673 & $0.9472 *$ & 0.7421 & -0.1723 & -0.2080 \\
\hline & $(0.37)$ & $(1.07)$ & $(1.60)$ & (1.84) & $(1.34)$ & $(-0.21)$ & $(-0.14)$ \\
\hline Structured Products & - & - & - & - & - & - & - \\
\hline \multirow[t]{2}{*}{ CTE Quarterly Reports } & 0.0342 & 0.0163 & 0.0602 & 0.1365 & -0.1065 & -0.1534 & -0.6886 \\
\hline & $(0.34)$ & $(0.10)$ & $(0.22)$ & $(0.28)$ & $(-0.18)$ & $(-0.15)$ & $(-0.35)$ \\
\hline \multirow[t]{2}{*}{ Bid-Ask Spread } & -0.0059 & -0.0158 & $-3.5979 * * *$ & $-2.3004 *$ & $-6.1774 * *$ & $-7.4531 *$ & -8.3143 \\
\hline & $(-0.23)$ & $(-0.37)$ & $(-3.56)$ & $(-1.83)$ & $(-2.27)$ & $(-1.69)$ & $(-0.93)$ \\
\hline \multirow[t]{2}{*}{ Size } & $-0.0419 * * *$ & $-0.0939 * * *$ & $-0.1373 * * *$ & $-0.1946 * * *$ & $-0.1299 * *$ & $-0.1515^{*}$ & -0.1118 \\
\hline & $(-4.66)$ & $(-7.11)$ & $(-6.19)$ & $(-4.99)$ & $(-2.57)$ & $(-1.90)$ & $(-0.72)$ \\
\hline \multirow[t]{2}{*}{ Book-to-Market Ratio } & -0.0074 & -0.0242 & -0.0517 & -0.0512 & -0.0264 & -0.0084 & -0.0131 \\
\hline & $(-0.50)$ & $(-1.07)$ & $(-1.28)$ & $(-0.84)$ & $(-0.35)$ & $(-0.07)$ & $(-0.05)$ \\
\hline \multirow[t]{2}{*}{ Leverage } & -0.0020 & 0.0006 & 0.0008 & 0.0008 & -0.0224 & $-0.2077 * * *$ & $-0.5953 * * *$ \\
\hline & $(-0.53)$ & $(0.28)$ & $(0.24)$ & $(0.14)$ & $(-1.12)$ & $(-2.76)$ & $(-3.37)$ \\
\hline \multirow[t]{2}{*}{ ROA } & -0.0006 & -0.0011 & -0.0017 & -0.0009 & $-0.6471^{* * *}$ & $-0.6457 *$ & -1.0285 \\
\hline & $(-0.14)$ & $(-0.15)$ & $(-0.15)$ & $(-0.05)$ & $(-3.25)$ & $(-1.87)$ & $(-1.56)$ \\
\hline \multirow[t]{2}{*}{ Resource Sector } & 0.0516 & 0.0846 & -0.0754 & -0.0972 & 0.0189 & 0.3203 & -0.0534 \\
\hline & $(1.15)$ & $(1.25)$ & $(-0.66)$ & $(-0.48)$ & $(0.08)$ & $(0.83)$ & $(-0.07)$ \\
\hline \multirow[t]{2}{*}{ Financial Sector } & 0.0257 & 0.0147 & 0.0560 & -0.0057 & -0.0276 & 0.0196 & -0.0423 \\
\hline & $(0.36)$ & $(0.14)$ & $(0.31)$ & $(-0.02)$ & $(-0.07)$ & $(0.03)$ & $(-0.03)$ \\
\hline Max. VIF & 2.00 & 2.16 & 2.12 & 2.11 & 2.12 & 2.15 & 2.23 \\
\hline F Value & 5.40 & 8.56 & 7.45 & 4.70 & 4.40 & 3.02 & 1.63 \\
\hline R-Square & $1.13 \%$ & $1.55 \%$ & $1.53 \%$ & $1.30 \%$ & $1.76 \%$ & $1.98 \%$ & $2.41 \%$ \\
\hline Adj R-Sq & $0.92 \%$ & $1.37 \%$ & $1.33 \%$ & $1.02 \%$ & $1.36 \%$ & $1.32 \%$ & $0.93 \%$ \\
\hline $\mathbf{N}$ & 10,395 & 11,993 & 10,533 & 7,857 & 5,417 & 3,316 & 1,482 \\
\hline
\end{tabular}


Table 6-38. The Regression Results of Market-sensitive Announcements with Good News - Intraday ATVs after the GFC

\begin{tabular}{|c|c|c|c|c|c|c|c|}
\hline Variable & ATV10min & ATV15min & ATV30min & ATV60min & ATV90min & ATV120min & ATV150min \\
\hline \multirow[t]{2}{*}{ Intercept } & $0.1359 * * *$ & $0.2540 * * *$ & $0.4299 * *$ & 0.2701 & 0.6367 & 0.7519 & 0.6726 \\
\hline & $(2.72)$ & $(2.63)$ & $(2.43)$ & $(0.70)$ & $(1.18)$ & $(0.72)$ & $(0.35)$ \\
\hline \multirow[t]{2}{*}{ Takeover / Scheme Announcements } & 0.1015 & 0.2423 & 0.3515 & 0.6599 & 1.4137 & 2.3760 & 1.2204 \\
\hline & $(1.04)$ & $(1.29)$ & $(1.03)$ & $(0.80)$ & $(1.29)$ & $(1.19)$ & $(0.29)$ \\
\hline \multirow[t]{2}{*}{ Security Holder Details } & 0.2648 & 0.3376 & 0.0699 & 0.1961 & 0.0899 & 0.8360 & -4.1972 \\
\hline & (1.58) & (1.03) & $(0.11)$ & $(0.14)$ & $(0.04)$ & $(0.24)$ & $(-0.63)$ \\
\hline \multirow[t]{2}{*}{ Periodic Reports } & $0.1369 * * *$ & $0.1992 * *$ & 0.3091* & 0.6545 & 0.8991 & 1.8242 & 2.9223 \\
\hline & (2.59) & (1.98) & (1.68) & (1.64) & $(1.59)$ & $(1.71)$ & $(1.46)$ \\
\hline \multirow[t]{2}{*}{ Issued Capital } & $0.2475 * * *$ & $0.2267 * *$ & $0.3877 *$ & $0.8548 *$ & 0.8502 & 1.3450 & 1.7386 \\
\hline & (4.19) & (1.97) & (1.82) & (1.82) & $(1.30)$ & $(1.07)$ & $(0.76)$ \\
\hline \multirow[t]{2}{*}{ Asset Acquisition \& Disposal } & $0.2009 * * *$ & $0.3484 * * *$ & $0.5602 * * *$ & $0.8921 *$ & 0.9273 & 1.2840 & 1.3139 \\
\hline & (3.29) & (3.02) & (2.69) & $(1.95)$ & $(1.47)$ & $(1.14)$ & $(0.64)$ \\
\hline \multirow[t]{2}{*}{ Notice of Meeting } & $0.8673 * * *$ & $1.4867 * *$ & $2.9969 * * *$ & 0.9711 & 2.1366 & 1.0810 & - \\
\hline & (2.73) & (2.30) & (2.88) & $(0.35)$ & $(0.46)$ & $(0.12)$ & - \\
\hline \multirow[t]{2}{*}{ ASX Announcement } & 0.0323 & -0.0403 & 0.2843 & 0.4861 & 0.7034 & 0.8651 & -0.8060 \\
\hline & $(0.37)$ & $(-0.24)$ & $(0.90)$ & $(0.72)$ & $(0.73)$ & $(0.48)$ & $(-0.23)$ \\
\hline \multirow[t]{2}{*}{ Distribution Announcement } & 0.1252 & 0.2814 & 0.4045 & 0.7514 & 0.9586 & 1.1274 & 0.7426 \\
\hline & (1.23) & (1.44) & (1.13) & $(0.93)$ & $(0.86)$ & $(0.52)$ & $(0.20)$ \\
\hline \multirow[t]{2}{*}{ Progress Reports } & $0.2896 * * *$ & $0.5198 * * *$ & $0.8048 * * *$ & $1.3152 * * *$ & $1.5506 * * *$ & 1.8614 & 0.7454 \\
\hline & (8.62) & (7.97) & (6.82) & (5.17) & (4.46) & $(2.90)$ & $(0.64)$ \\
\hline \multirow[t]{2}{*}{ Company Administration } & 0.0722 & 0.0347 & -0.1061 & -0.0959 & 0.1612 & 0.4099 & -0.5474 \\
\hline & $(0.74)$ & $(0.19)$ & $(-0.31)$ & $(-0.14)$ & $(0.17)$ & $(0.24)$ & $(-0.16)$ \\
\hline Notice of Call & - & - & - & - & - & - & - \\
\hline \multirow[t]{2}{*}{ Other } & 0.0883 & 0.2417 & 0.2719 & 0.8946 & 1.5366 & 0.6908 & 0.7554 \\
\hline & $(0.63)$ & $(0.86)$ & $(0.55)$ & $(0.80)$ & $(1.06)$ & $(0.24)$ & $(0.16)$ \\
\hline \multirow[t]{2}{*}{ Chairman's Address } & 0.0647 & 0.0573 & 0.3507 & 0.6233 & 0.8720 & 1.2282 & 0.2239 \\
\hline & $(0.36)$ & $(0.18)$ & $(0.60)$ & $(0.48)$ & $(0.50)$ & $(0.44)$ & $(0.04)$ \\
\hline
\end{tabular}




\begin{tabular}{|c|c|c|c|c|c|c|c|}
\hline Variable & ATV10min & ATV15min & ATV30min & ATV60min & ATV90min & ATV120min & ATV150min \\
\hline \multirow{2}{*}{ Letter to Shareholders } & 0.0980 & 0.0085 & -0.0284 & - & - & - & \\
\hline & $(0.11)$ & $(0.00)$ & $(-0.01)$ & - & - & - & 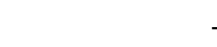 \\
\hline \multirow[t]{2}{*}{ ASX Query } & 0.0068 & 0.0410 & -0.0108 & -0.1104 & -0.5964 & -1.1314 & -2.2672 \\
\hline & $(0.08)$ & $(0.25)$ & $(-0.04)$ & $(-0.18)$ & $(-0.72)$ & $(-0.79)$ & $(-0.84)$ \\
\hline Structured Products & - & - & - & - & - & - & 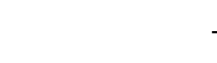 \\
\hline \multirow[t]{2}{*}{ CTE Quarterly Reports } & -0.0245 & -0.0856 & -0.1545 & -0.2517 & -0.5552 & -0.2059 & -0.1144 \\
\hline & $(-0.30)$ & $(-0.53)$ & $(-0.52)$ & $(-0.38)$ & $(-0.62)$ & $(-0.12)$ & $(-0.04)$ \\
\hline \multirow[t]{2}{*}{ Bid-Ask Spread } & $-0.4791 *$ & -0.5485 & -0.8524 & -1.0192 & -9.3187 & -9.5325 & -7.1694 \\
\hline & $(-1.89)$ & $(-1.48)$ & $(-1.26)$ & $(-0.79)$ & $(-1.63)$ & $(-1.00)$ & $(-0.42)$ \\
\hline \multirow[t]{2}{*}{ Size } & $-0.0278 * * *$ & $-0.0527 * * *$ & $-0.0771 * * *$ & $-0.0883 *$ & $-0.1339 *$ & -0.1979 & -0.1675 \\
\hline & $(-4.03)$ & $(-4.12)$ & $(-3.31)$ & $(-1.73)$ & $(-1.86)$ & $(-1.49)$ & $(-0.68)$ \\
\hline \multirow[t]{2}{*}{ Book-to-Market Ratio } & $-0.0159 *$ & $0.0639 * * *$ & $0.0578 *$ & 0.0781 & 0.0716 & -0.1668 & 0.3412 \\
\hline & $(-1.70)$ & (3.53) & $(1.81)$ & $(1.12)$ & $(0.76)$ & $(-0.79)$ & $(0.75)$ \\
\hline \multirow[t]{2}{*}{ Leverage } & -0.0008 & -0.0025 & -0.0018 & -0.0116 & -0.0106 & 0.0060 & 0.0328 \\
\hline & $(-0.27)$ & $(-0.44)$ & $(-0.18)$ & $(-0.26)$ & $(-0.18)$ & $(0.07)$ & $(0.15)$ \\
\hline \multirow[t]{2}{*}{ ROA } & $-0.0053 *$ & $-0.0096 *$ & -0.0069 & -0.0728 & -0.0274 & -0.0169 & -0.0232 \\
\hline & $(-1.73)$ & $(-1.84)$ & $(-0.78)$ & $(-0.97)$ & $(-0.30)$ & $(-0.09)$ & $(-0.07)$ \\
\hline \multirow[t]{2}{*}{ Resource Sector } & -0.0234 & -0.0994 & -0.1748 & 0.0174 & -0.1812 & 0.0764 & -0.5910 \\
\hline & $(-0.69)$ & $(-1.56)$ & $(-1.49)$ & $(0.07)$ & $(-0.50)$ & $(0.11)$ & $(-0.47)$ \\
\hline \multirow[t]{2}{*}{ Financial Sector } & -0.0907 & $-0.1899 *$ & $-0.3353 *$ & -0.3767 & -0.6737 & -0.6565 & -1.4918 \\
\hline & $(-1.62)$ & $(-1.79)$ & $(-1.74)$ & $(-0.88)$ & $(-1.10)$ & $(-0.56)$ & $(-0.70)$ \\
\hline Max. VIF & 1.85 & 1.96 & 1.92 & 1.86 & 1.80 & 1.84 & 1.76 \\
\hline F Value & 6.01 & 6.04 & 4.10 & 2.12 & 1.75 & 0.78 & 0.34 \\
\hline R-Square & $1.31 \%$ & $1.22 \%$ & $0.94 \%$ & $0.62 \%$ & $0.72 \%$ & $0.53 \%$ & $0.51 \%$ \\
\hline Adj R-Sq & $1.09 \%$ & $1.02 \%$ & $0.71 \%$ & $0.33 \%$ & $0.31 \%$ & $-0.15 \%$ & $-0.97 \%$ \\
\hline $\mathbf{N}$ & 10,018 & 10,791 & 9,564 & 7,174 & 5,119 & 3,098 & 1,367 \\
\hline
\end{tabular}

Note: The ATV120min and ATV150min regression models do not pass the significant test ( $\mathrm{F}$ value $=0.78,0.34$ ).

$*, * *$ and $* * *$ denote significance at the $10 \%, 5 \%$ and $1 \%$ levels respectively (two-tailed). 
Progress Reports: Trading volumes react the most to the Progress Reports before, during or after the GFC. Larger coefficients are observed in good news subsamples than in bad news subsamples, indicating that the increased trading volumes are driven by the good news in this category. This is matched with the share price reaction conclusion that Progress Reports tend to be good news. In the bad news subsamples, the relatively larger ATVs could also reveal that investors were trading based on Progress Reports, no matter how the good or bad news was conveyed.

Asset Acquisition \& Disposal: Asset Acquisition \& Disposal had much larger trading volume reactions during the GFC than before or after the GFC. Given the fact that Asset Acquisition \& Disposal could induce the largest share price reactions, it is suggested that Asset Acquisition \& Disposal was more important to investors when making investment decisions during the GFC. Looking at the bad and good news separately, large trading volume reactions existed in both good and bad news subsamples during the GFC, while small trading volume reactions were observed over shorter time windows in good news subsamples during non-GFC periods. It further confirms the importance of Asset Acquisition \& Disposal during the GFC. In addition, the increased trading activities were caused by good news during non-GFC periods, especially the post-GFC period when no significant ATVs observed in bad news subsamples.

Issued Capital: Before separating good and bad news subsamples, the regression results show that Issued Capital had larger trading volume reactions than Asset Acquisition \& Disposal during non-GFC periods. However, after taking good/bad news into account, the trading volume reactions to Issued Capital were larger in the bad news subsamples, and smaller in the good news subsamples no matter whether prior to, during or after the GFC. The share price reaction results indicate that Issued Capital could be either good news or bad news, but the 
trading volume reaction results suggest that investors' trading behaviour was caused by the release of bad news as reflected in the much larger increased trading volumes in the bad news subsamples than the good news subsamples.

Periodic Reports: The trading volumes reacted the least to Periodic Reports in general. Periodic Reports could convey either good news or bad news, which is reflected in the share price reactions. But the ATVs were driven by the good news only before the GFC. Larger trading volume responses were observed in the bad news than good news subsample during the GFC. After the GFC, slightly larger coefficients were observed in the good news subsample only over the short time (30 minutes) while larger trading volume reactions existed in the bad news subsample after 90 minutes. This suggests that investors tend to trade once they receive bad earnings-related news; investors tend to hold their shares when there is good earnings-related news, or at least not to trade immediately after the good news. It is also possible that investors make their buy/sell decisions more promptly once they know from the Periodic Reports that the company is not performing well, while investors take longer to make buy/sell decisions on wellperforming companies.

When compared with share price reaction results, there are three announcement categories with robust share price responses but without significant or consistent trading volume reactions: Distribution Announcements, Other and ASX Query. Similar to Distribution Announcements, announcement category Other is interpreted as good news by the market, because no significant negative price reactions were observed in the bad news subsamples, but there were stable positive stock returns in the good news subsamples. Distribution Announcements are directly related to earnings information, especially good news on dividends. The fact that no significant trading volumes increase after the announcement release in those two categories may further 
support the explanation that investors tend not to trade (at least not to trade immediately) when positive information (especially good earnings news) is released. In terms of ASX Query, ATVs only exist over a short time in the bad news subsamples; 60 minutes before the GFC and 30 minutes after the GFC. Significant trading volume decreases have been observed in the 150 minutes time window since the GFC. The purpose of an ASX Query, especially price query, is to stop abnormal trading activities rather than to induce abnormal trading activities. Therefore, insignificant or negative trading volume reactions may reveal a good sign that an ASX Query helps to correct irrational trading activities to some extent. The ATV results also show that not many announcement categories induce significant trading volume reactions. This could be a good sign for the CDR because if investors are well-informed, they would not make a 'sudden' decision to trade a large number of shares depending on a single piece of information.

In addition to the influences of announcement types on the immediate trading volume reactions to information, firm size is the only control variable that shows significant negative association with ATVs. This suggests that large companies tend to have fewer shares traded immediately after the announcement release, which again confirms the large companies' stability. It is interesting to notice that firm size has no significant impact on ATVs when bad news is released. It suggests that investors tend to trade large companies' shares quickly when there is bad news on the market, but tend to hold their shares for some time when there is good news.

\subsection{Summary}

This chapter has provided the descriptive statistics, $t$-test results and regression results from testing the immediate market reactions to different company announcements. The findings support the proposal that the market reacts to different announcements differently. Both $t$-test 
and regression results show that on average, an ASX Query is the only type of announcement that causes negative market reactions; investment/project- and earnings-related announcements tend to induce positive market reactions in general, while unexpected announcements have larger market responses than expected ones. The GFC significantly affected the immediate market consequences of Issued Capital and Asset Acquisition \& Disposal.

This chapter has also investigated the magnitude of market reactions to announcements after taking good/bad news into account, which also provides insights into the information nature contained in different announcements and the investors' trading activities around the announcement release.

The next chapter presents the research results and analysis of the long-term market effects of different corporate disclosures, as reflected in the timeliness of price discovery, the timeliness with which the value-relevant information is incorporated into share prices before the Preliminary Final Reports are released. 


\section{CHAPTER SEVEN: RESEARCH ANALYSIS ON THE TIMELINESS OF PRICE DISCOVERY}

This study conducts two examinations to evaluate the market effects of different announcements under the CDR. The first is the immediate market reaction tests, shown in Chapter Six; the second is the long-term timeliness of price discovery, presented in this chapter. Based on the Deflated Timeliness Metric equation (5-10) in Chapter Five, this study assumes that when the annual earnings information becomes available to the market, the share price can reflect the 'true' value of the company based on its performance over the fiscal year. It also assumes that investors make their investment decisions based on companies' Preliminary Final Reports, when the earnings information is first released. Given the importance of the Preliminary Final Reports, this chapter reports their descriptive statistics in detail and discusses the market reactions to the reports that are released at different times and on different days. This chapter also presents the empirical results of the tests on the timeliness of price discovery and provides an analysis which attempts to describe and explain the impact of different disclosures on the timeliness of discovery after taking the sector influences and year effects into account.

\subsection{Analysis on Preliminary Final Reports}

A Preliminary Final Report is the officially earliest announcement that informs the market of a company's financial information for the fiscal year (not forecasts), including balance sheet, income statement and cash flow statement. Given the importance of these Reports, this study focuses on this particular type of announcement to investigate in depth the corporate disclosure behaviour and market reactions to earnings information. In addition, the analysis of the Preliminary Final Reports provides insights into the differences between market responses to 
trading-hour and off-hour announcements, which were not covered by the immediate market reaction tests in Chapter Six. By focusing on the Preliminary Final Reports, this study further analyses whether companies tend to release good/bad news at certain times or on certain days.

The release date and time of the preliminary earnings announcements are manually collected by checking the first released financial reports after a fiscal year ends. As a company's first available financial report might be coded as either 'Preliminary Final Report' or 'Annual Report $^{43}$, this study manually works out which announcement first provides the market with financial information. Table 7-1 shows the statistical summary of the month in which the Preliminary Final Reports were released from 01/07/2004 to 31/12/2014, matched with the balance dates. Most companies' fiscal years end in June, and most of the preliminary earnings announcements are released within three months thereafter.

Table 7-1. Preliminary Final Report Distribution by Month

\begin{tabular}{|c|c|c|c|c|c|c|}
\hline \multirow{2}{*}{ Balance Date } & \multicolumn{4}{|c|}{ The Number of Preliminary Financial Reports released within } & \multirow{2}{*}{$\begin{array}{c}>4 \text { months } \\
\left(_{\text {postponed })^{44}}\right.\end{array}$} & \multirow{2}{*}{ Total } \\
\hline & 1 Month & 2 Months & 3 Months & 4 Months & & \\
\hline January & 1 & 15 & 12 & 2 & - & 30 \\
\hline February & 25 & 15 & 3 & - & - & 43 \\
\hline March & 11 & 231 & 55 & 10 & 13 & 320 \\
\hline April & 2 & 19 & 3 & 2 & - & 26 \\
\hline May & - & 9 & - & - & - & 9 \\
\hline June & 172 & 8,994 & 6,955 & 760 & 195 & 17,076 \\
\hline July & 144 & 38 & 3 & 1 & - & 186 \\
\hline August & 2 & 53 & 18 & 1 & - & 74 \\
\hline September & 26 & 178 & 33 & 4 & 1 & 242 \\
\hline October & - & 9 & 9 & - & - & 18 \\
\hline November & - & - & - & - & 2 & 2 \\
\hline December & 24 & 1,272 & 667 & 97 & 36 & 2,096 \\
\hline Total & 407 & 10,833 & 7,758 & 877 & 247 & 20,122 \\
\hline
\end{tabular}

\footnotetext{
${ }^{43}$ This study relies on the SIRCA database regarding company announcements. According to the original data downloaded from the database, some companies do not release Preliminary Final Reports before their Annual Reports. Here, as long as the announcement provides the earnings information for the first time, it is seen as the Preliminary Final Report or preliminary earnings announcement.

${ }^{44}$ Postponed Preliminary Final Reports are excluded from the regression sample, because the periods identified by those report release dates are abnormally too short or too long to calculate the timeliness metric.
} 
The following figures show how these preliminary earnings announcements are distributed over times, days and years. Figures 7-1 and 7-2 show that companies tend to release more preliminary earnings announcements during pre-trading hours. Figure 7-2 also reveals that over the period of study, the proportion of post-trading hour announcements remains relatively stable; from 2004 to 2014, the percentage of trading hour announcements declines gradually, while the percentage of pre-trading hour announcements kept increasing over that time. Figure 7-3 shows that companies release 5\% more earnings announcements on Fridays.

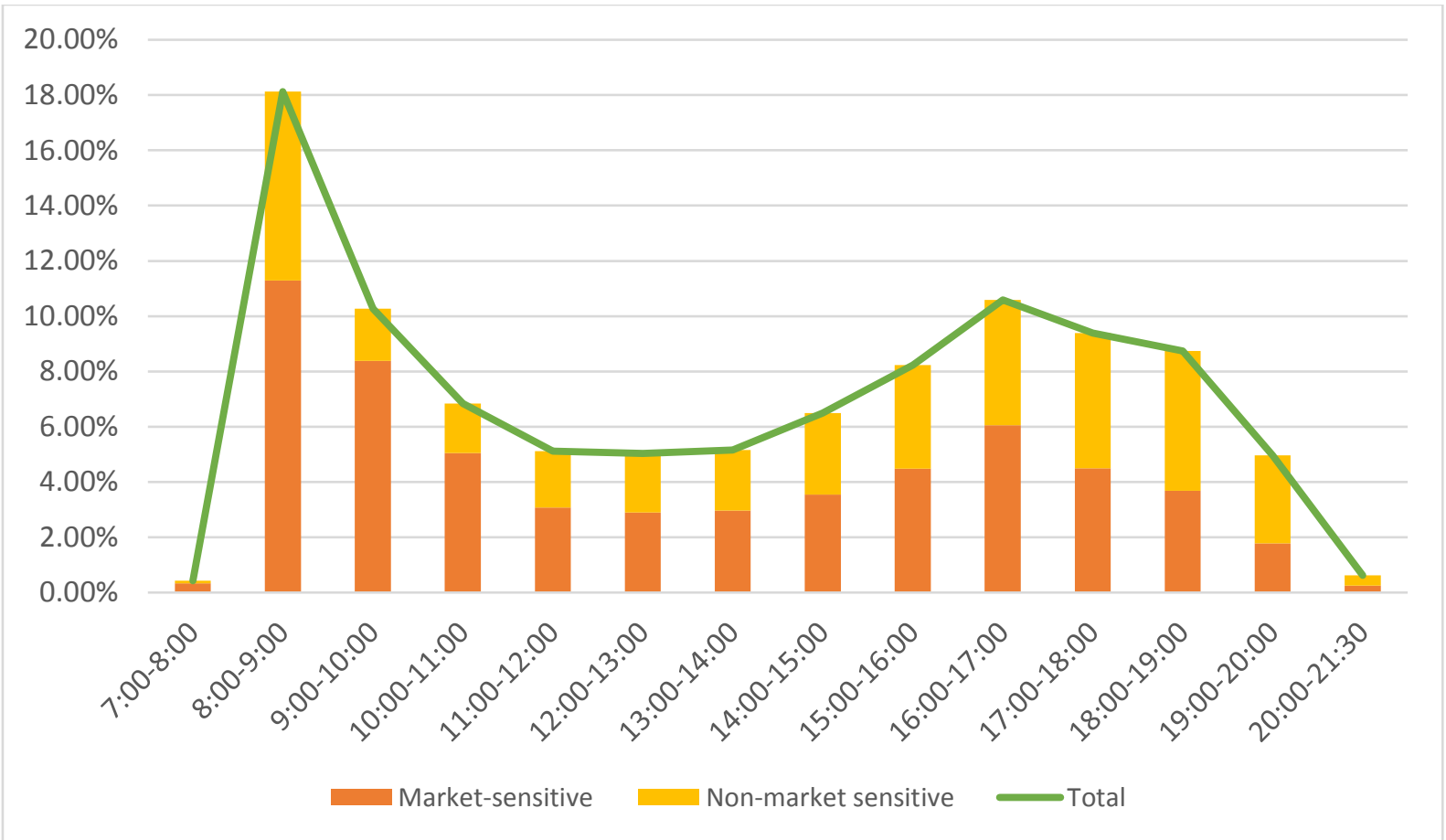

Figure 7-1. Preliminary Final Reports Distribution throughout the Day 


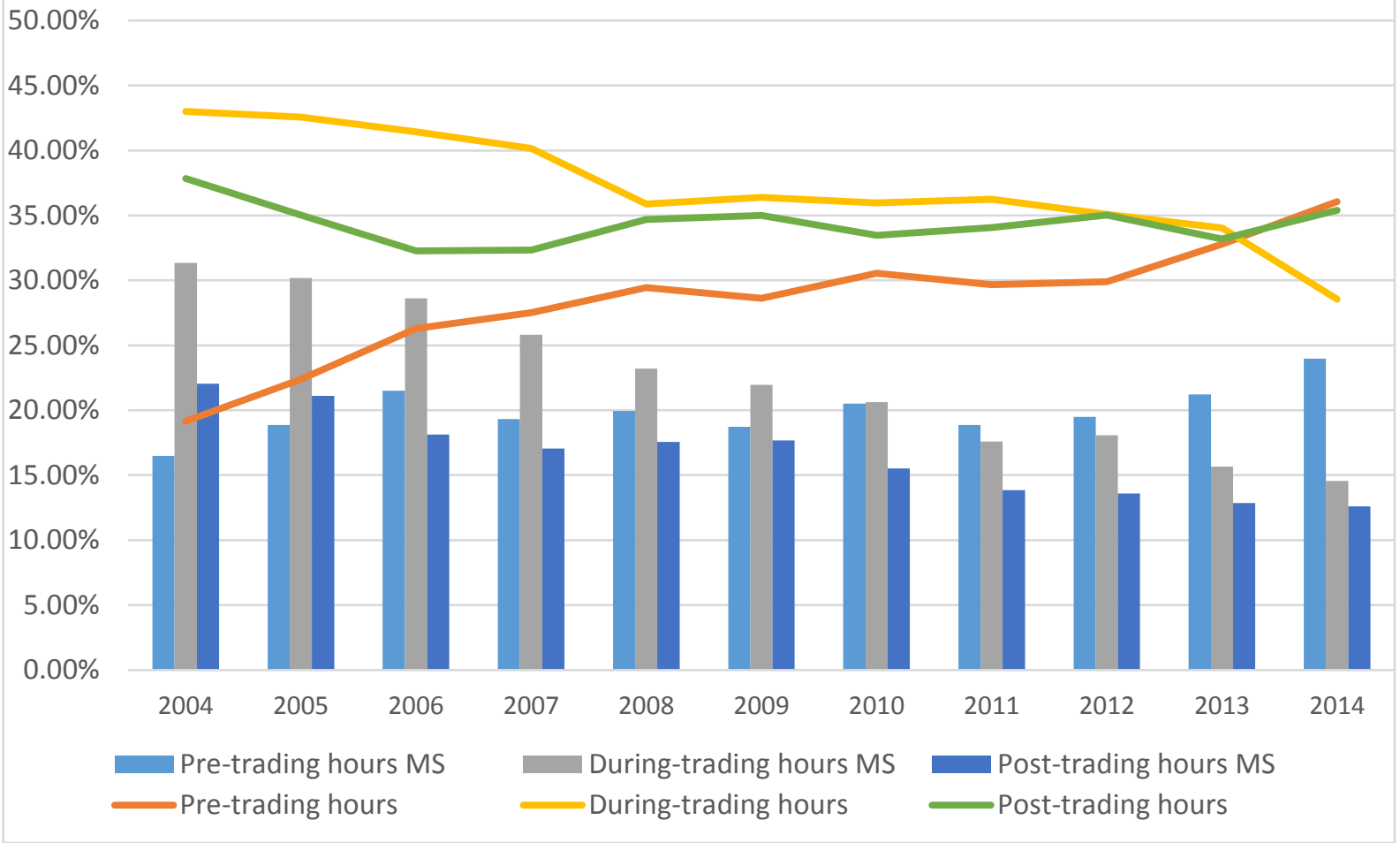

Figure 7-2. The Pre/During/Post Trading Hours Preliminary Final Reports by Year

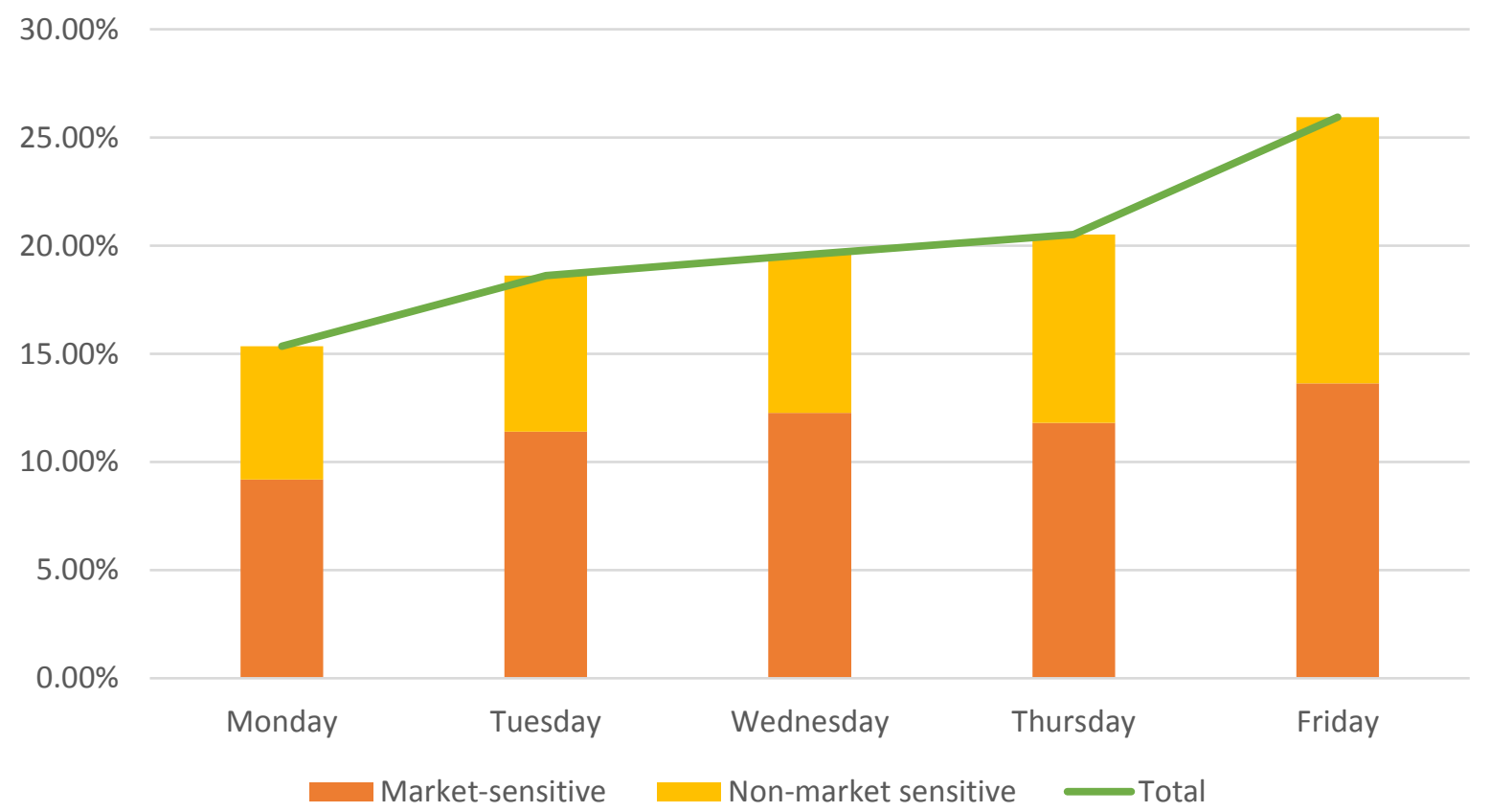

Figure 7-3. Preliminary Final Reports Distribution by Weekdays 
According to the market-adjusted abnormal daily returns when the Preliminary Final Reports become available to the market, the reports are determined as good news if the adjusted return is greater than zero $(>0)$, and as bad news if the adjusted return is smaller than zero $(<0)$. As can be seen in Figure 7-4, more good news earnings announcements are released in the morning, while more bad news earnings announcements are released after the market closes. Figure 7-5 also indicates that more bad news earnings announcements are released on Fridays. To some extent, these patterns reveal that companies tend to release bad news when there could be relatively low investor attention, such as post-trading hours, and/or Fridays.

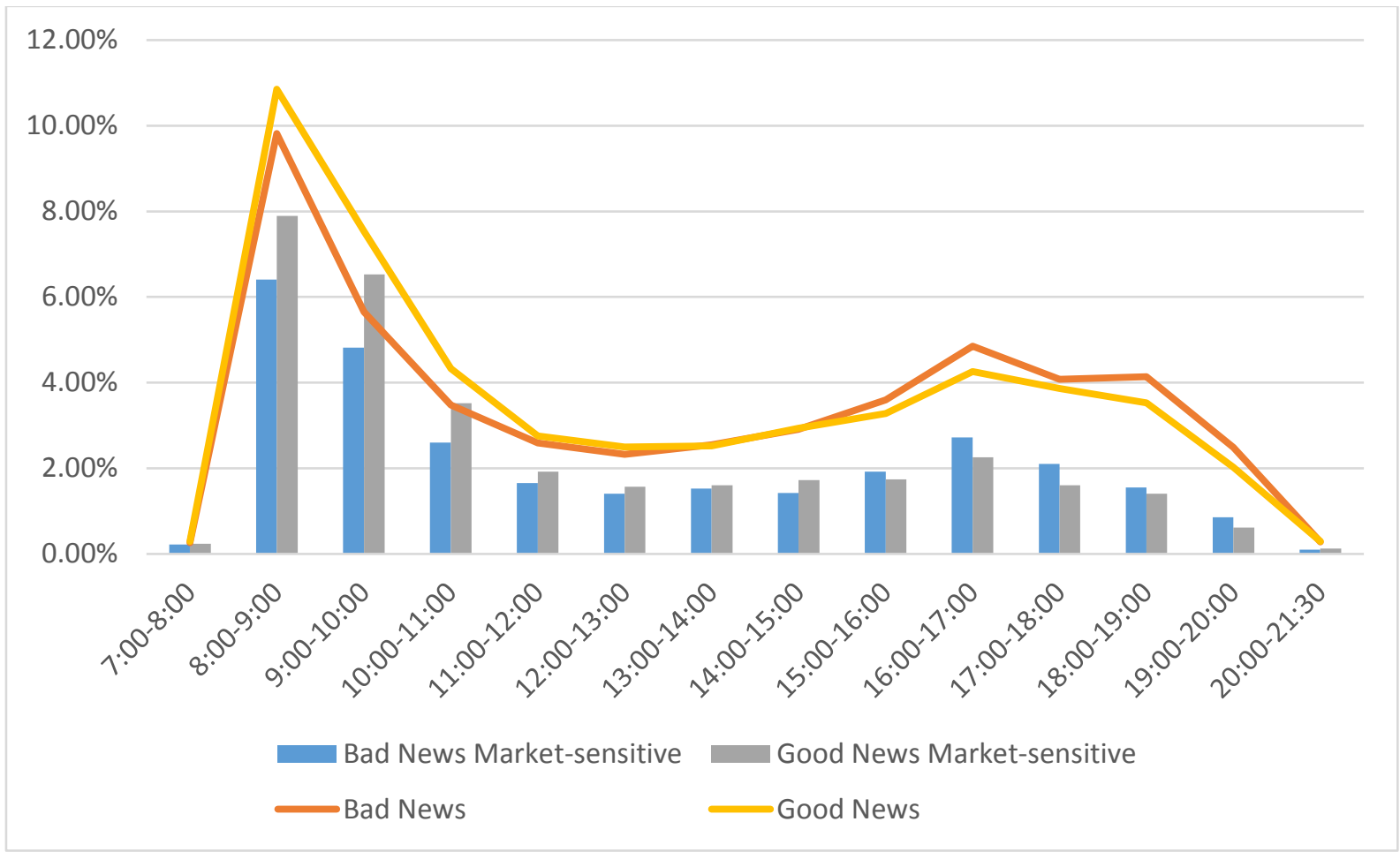

Figure 7-4. Preliminary Final Reports Distribution throughout the Day - Good/Bad News 


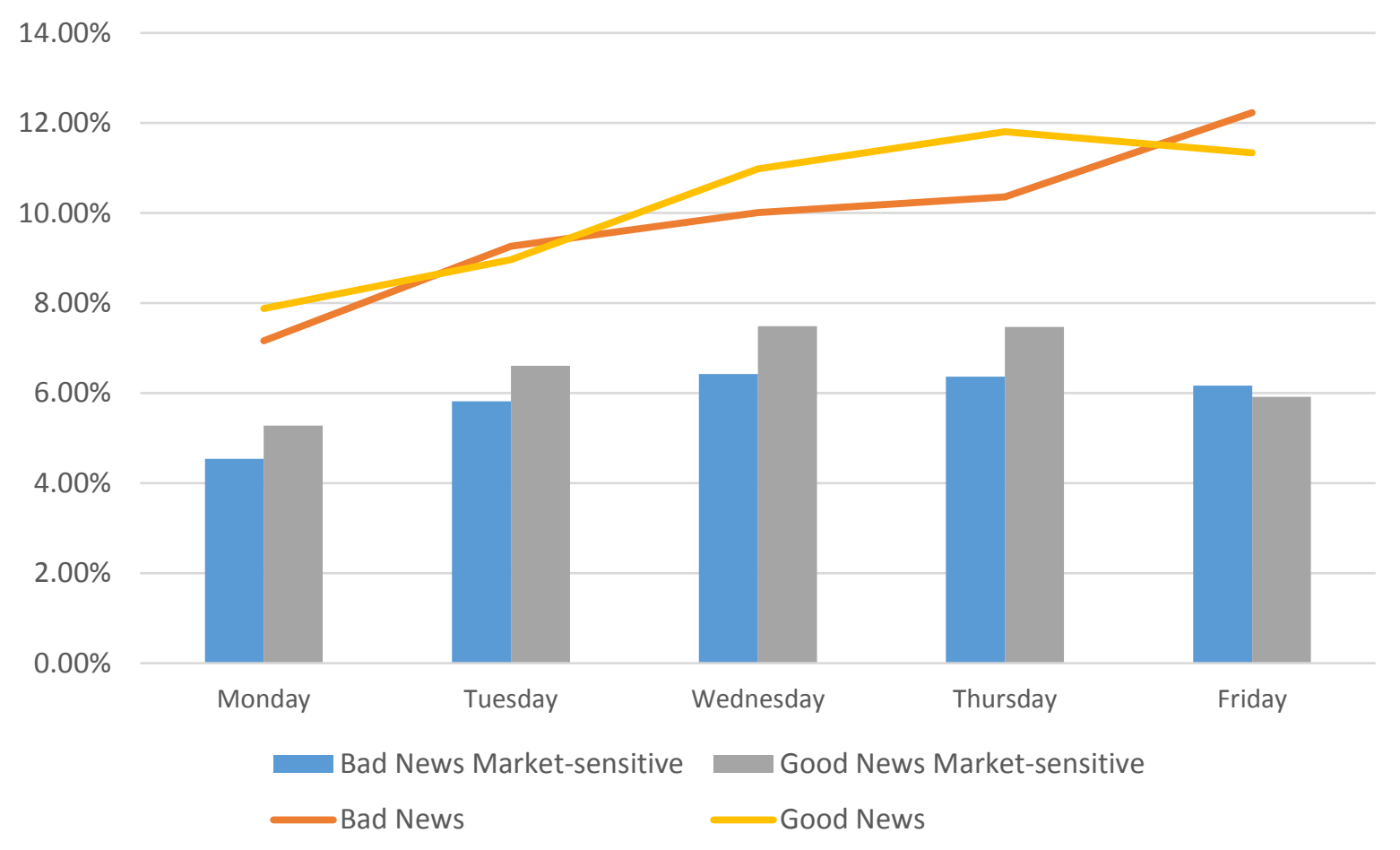

Figure 7-5. Preliminary Final Reports Distribution by Weekdays - Good/Bad News

The analysis of variance (ANOVA), Table 7-2, further confirms that the market-adjusted returns are significantly different for the earnings announcements released at different times. The daily abnormal returns are significantly lower around the Post-trading hour earnings announcements than Pre- and During-trading hour announcements. Table 7-3 shows that the average abnormal return of earnings reports on Fridays are lower, but only significantly lower than on Wednesdays and Thursdays. Comparing the LSD (Least Significant Difference) $t$-test results between Tables 7-2 and 7-3, the time when the announcement is released matters more than the weekday. In other words, companies tend to release good news before or during the market trading hours, and tend to release bad news after the market closes. Compared with other weekdays, companies may release slightly more bad earnings news on Fridays. 
Table 7-2. One-Way ANOVA on the Market-adjusted Returns of Pre/During/Post Trading hours Preliminary Final Reports

Levene's Test for Homogeneity of Abnormal Return Variance. ANOVA of Squared Deviations from Group Means

\begin{tabular}{lccccc} 
Source & DF & Sum of Squares & Mean Square & F Value & Pr $>$ F \\
\hline Pre/During/Post Trade Hours & 2 & 0.0142 & 0.0071 & 1.73 & 0.1778 \\
Error & 12270 & 50.5610 & 0.0041 & & \\
\hline
\end{tabular}

\begin{tabular}{|c|c|c|c|c|c|}
\hline Error & $122 / 0$ & 50.5010 & 0.0041 & & \\
\hline \multicolumn{6}{|c|}{ One-Way Analysis of Variance } \\
\hline Source & DF & Sum of Squares & Mean Square & F Value & $\operatorname{Pr}>\mathrm{F}$ \\
\hline Model & 2 & 0.1637 & 0.0819 & 15.59 & $<.0001$ \\
\hline Error & 12270 & 64.4497 & 0.0053 & & \\
\hline Corrected Total & 12272 & 64.6134 & & & \\
\hline
\end{tabular}

Means of the Preliminary Final Reports' Abnormal Returns

\begin{tabular}{lccc} 
Pre/During/Post-Trade & No. of Observations & Mean & Std Dev \\
\hline Pre-Trade Hours & 4224 & 0.0043 & 0.0672 \\
During Trade Hours & 4389 & 0.0037 & 0.0677 \\
Post-Trade Hours & 3660 & -0.0040 & 0.0831
\end{tabular}

T-Tests (LSD) for the Preliminary Final Reports' Abnormal Returns

Pre/During/Post-Trade Comparison Difference Between Means

Pre-Trade Hours - During Trade Hours

0.0006

$-0.0083$

$95 \%$ Confidence Limits

Post-Trade Hours - Pre-Trade Hours

$-0.0077$

$-0.0024$

Post-Trade Hours - During Trade Hours

0.05

12270

Error Degrees of Freedom

0.0053

Error Mean Square

1.9602

Critical Value of $\mathrm{t}$

$-0.0115-0.0051$

0.0037
-0.0051

$-0.0108 \quad-0.0045$

$* * *$

Note: Comparisons significant at the 0.05 level are indicated by $* * *$. 
Table 7-3. One-Way ANOVA on the Market-adjusted Returns of Preliminary Final Reports on Different Weekdays Levene's Test for Homogeneity of Abnormal Return Variance. ANOVA of Squared Deviations from Group Means

\begin{tabular}{|c|c|c|c|c|c|}
\hline Source & DF & Sum of Squares & Mean Square & F Value & $\operatorname{Pr}>\mathrm{F}$ \\
\hline Week Days & 4 & 0.0177 & 0.0044 & 1.07 & 0.3699 \\
\hline Error & 12268 & 50.8148 & 0.0041 & & \\
\hline \multicolumn{6}{|c|}{ One-Way Analysis of Variance } \\
\hline Source & DF & Sum of Squares & Mean Square & F Value & $\operatorname{Pr}>\mathrm{F}$ \\
\hline Model & 4 & 0.0440 & 0.0110 & 2.09 & 0.0793 \\
\hline Error & 12268 & 64.5694 & 0.0053 & & \\
\hline Corrected Total & 12272 & 64.6134 & & & \\
\hline \multicolumn{6}{|c|}{ Means of the Preliminary Final Reports' Abnormal Returns } \\
\hline Week Days & No. of Observations & Mean & Std Dev & & \\
\hline Mon & 1845 & 0.0021 & 0.0720 & & \\
\hline Tue & 2237 & -0.0004 & 0.0708 & & \\
\hline Wed & 2577 & 0.0035 & 0.0679 & & \\
\hline Thu & 2720 & 0.0036 & 0.0630 & & \\
\hline Fri & 2894 & -0.0007 & 0.0854 & & \\
\hline \multicolumn{6}{|c|}{ T-Tests (LSD) for the Preliminary Final Reports' Abnormal Returns } \\
\hline Week Days Comparison & Difference Between Means & \multicolumn{3}{|c|}{ 95\% Confidence Limits } & \\
\hline Fri - Thu & -0.0043 & -0.0081 & -0.0005 & $* * *$ & \\
\hline Fri - Wed & -0.0041 & -0.0080 & -0.0003 & $* * *$ & \\
\hline Fri - Tue & -0.0003 & -0.0043 & 0.0037 & & \\
\hline Fri - Mon & -0.0027 & -0.0070 & 0.0015 & & \\
\hline Thu - Wed & 0.0002 & -0.0037 & 0.0041 & & \\
\hline Thu - Tue & 0.0040 & -0.0001 & 0.0081 & & \\
\hline Thu - Mon & 0.0015 & -0.0027 & 0.0058 & & \\
\hline Wed - Tue & 0.0038 & -0.0003 & 0.0079 & & \\
\hline Wed - Mon & 0.0014 & -0.0030 & 0.0057 & & \\
\hline Tue - Mon & -0.0025 & -0.0069 & 0.0020 & & \\
\hline Alpha & 0.05 & & & & \\
\hline Error Degrees of Freedom & 12268 & & & & \\
\hline Error Mean Square & 0.0053 & & & & \\
\hline Critical Value of $t$ & 1.9602 & & & & \\
\hline
\end{tabular}

Note: Comparisons significant at the 0.05 level are indicated by $* * *$. 


\subsection{Regressions on the Timeliness of Price Discovery}

In order to test the impacts of announcement frequency on the timeliness of price discovery, this study uses the deflated timeliness metric, the original metric divided by one plus the absolute value of the market-adjusted share price on the Preliminary Final Report release date. To some extent, this deflated timeliness metric can reduce the effects of various security prices. For example, one security could be around 10 dollars per share while another could be 10 cents per share. According to the original metric model, the 10 dollar security is likely to have a larger timeliness metric than the 10 cent security, but it does not necessarily mean a less-timely price discovery for the 10 dollar security. The deflated timeliness measure can control such share price impacts.

With respect to the announcement frequency, this study first examines the association between the timeliness of price discovery and the number of all announcements released over the timeliness metric time window: the period between two adjacent preliminary final report dates. ${ }^{45}$ Then, this study further divides the announcements into two groups, Periodic Reports and Non-periodic Reports. Lastly, this study focuses on the Non-periodic Reports to test whether different types of Non-periodic announcements influence the timeliness of price discovery in different ways. When separating Non-periodic Reports from Periodic Reports, this study include the three types of quarterly reports (Quarterly Activities Reports, Quarterly Cash Flow Reports and CTE Quarterly Reports) in the Non-periodic Reports for two reasons. First, not every company is required to provide quarterly reports; and second, quarterly reports do not

\footnotetext{
45 This timeliness metric time window is important when examining the impacts of announcement frequency on the timeliness of price discovery. As detailed in Table 5-2, the independent variable [announcement frequency (AnnFre)] and two control variables [stock return volatility $(\mathrm{Vol})$ and well-perform company (W/BCompany)] are calculated over the matched time window as the dependent variable [deflated timeliness metric $\left(\right.$ Deflated $\left.M^{c^{\prime}}\right)$ ] calculation. In Chapter Seven, sometimes this study uses the word 'year/firm-year' to explain these variables or the results related to these variables. It means the period between two adjacent preliminary final report dates (not the financial year or calendar year).
} 
provide earnings information (they are either activities reports or cash flow reports). This study explores the impacts of periodic earnings-related announcements on the timeliness of price discovery. Disclosing earnings related periodic reports have a long history, much longer than the CDR, which highlights the importance of the periodic reports. However, it also indicate that non-periodic disclosures represents more the 'continuous disclosures'. The detailed announcement categorisation under the CDR provides an appropriate setting to explore the relationship between the announcement frequency and the timeliness of price discovery. Therefore, this thesis further focuses on the 18 types of non-periodic announcements to examine the impacts of different announcement types on the timeliness of price discovery. Company announcements are categorised by the ASX Primary Report Code. This study explores the effects of both the total announcements and the market-sensitive announcements on the timeliness of price discovery, to examine whether the market-sensitivity could make a difference.

\subsubsection{Descriptive Analysis}

Tables 7-4 and 7-5 are the statistical summaries of the regression samples for the timeliness of price discovery. Table 7-4 presents the mean and percentiles of the dependent variable (deflated timeliness metric) and control variables (financial information such as firm size and book-tomarket ratio). It also provides a basic idea on the number of announcements and marketsensitive announcements released at the firm-year level, including Periodic and Non-periodic Reports. Table 7-5 shows the detailed announcement frequency distribution for each announcement category, focusing on the Non-periodic Reports. The mean of each announcement group/category shows on average how many (market-sensitive) announcements are released in this category at the firm-year level, while the total number of announcements 
shows how many (market-sensitive) announcements are released in this category in the timeliness research sample.

As shown in Table 7-4, at firm-year level, less than one-third of the company announcements are market-sensitive. On average there are 57 total announcements and 16 market-sensitive announcements released by a firm in one year. ${ }^{46}$ Most of the announcements are non-periodic (49 in total, and 13 market-sensitive), and only seven Periodic Reports and two market-sensitive Periodic Reports on average at one firm-year. Table 7-5 reveals that the large announcement categories are Security Holder Details, Issued Capital, and Progress Reports, containing more than eight announcements per firm-year on average. Other announcement categories have three or fewer announcements, on average, released by a company in one year. In addition, on average there are only around five market-sensitive announcements released in the category Progress Report, while fewer than two market-sensitive announcements are released in all other categories. At 95 percentile, apart from Progress Reports, other categories have five or fewer market-sensitive announcements released per firm-year. It shows that there are not many announcements, especially market-sensitive announcements, released as to each category at the firm-year level.

\footnotetext{
${ }^{46}$ Here 'one year' means the period between two adjacent Preliminary Final Reports. The number of announcements is rounded to the nearest whole number.
} 
Table 7-4. Statistic Summary of the Timeliness of Price Discovery Regressions - Total, Periodic and Non-periodic Announcements

\begin{tabular}{|c|c|c|c|c|c|c|c|c|c|}
\hline \multirow{2}{*}{ Variable } & \multirow{2}{*}{$\mathbf{N}$} & \multirow{2}{*}{ Mean } & \multirow{2}{*}{ Median } & \multirow{2}{*}{ Std Dev } & \multirow{2}{*}{$\begin{array}{l}\text { Total Number of } \\
\text { Announcements }\end{array}$} & \multicolumn{4}{|c|}{ Percentiles } \\
\hline & & & & & & 5 & 25 & 75 & 95 \\
\hline Deflated Timeliness Metric & 11,517 & 0.030 & 0.023 & 0.027 & & 0.006 & 0.013 & 0.038 & 0.077 \\
\hline Total Announcements & 11,517 & 56.547 & 50 & 40.506 & 651,247 & 18 & 34 & 69 & 111 \\
\hline Total Announcements - MS & 11,517 & 15.633 & 14 & 10.359 & 180,047 & 4 & 8 & 21 & 35 \\
\hline Periodic Reports & 11,517 & 7.156 & 5 & 11.490 & 82,419 & 2 & 3 & 8 & 17 \\
\hline Periodic Reports - MS & 11,517 & 2.273 & 2 & 2.250 & 26,180 & 0 & 0 & 3 & 6 \\
\hline Non Periodic Reports & 11,517 & 49.390 & 43 & 38.043 & 568,828 & 13 & 28 & 62 & 101 \\
\hline Non Periodic Reports - MS & 11,517 & 13.360 & 11 & 10.905 & 153,867 & 1 & 5 & 19 & 34 \\
\hline Stock Return Volatility & 11,517 & 0.052 & 0.044 & 0.037 & & 0.013 & 0.026 & 0.066 & 0.117 \\
\hline Well-performing Company & 11,517 & 0.489 & 0 & 0.500 & & 0 & 0 & 1 & 1 \\
\hline Size & 11,517 & 4.167 & 3.900 & 2.117 & & 1.179 & 2.614 & 5.445 & 8.134 \\
\hline Book-to-Market Ratio & 11,517 & 0.895 & 0.654 & 1.835 & & 0.090 & 0.332 & 1.163 & 2.703 \\
\hline Leverage & 11,517 & 0.979 & 0.020 & 49.331 & & 0 & 0 & 0.42 & 1.79 \\
\hline ROA & 11,517 & -0.174 & -0.016 & 2.560 & & -0.968 & -0.223 & 0.091 & 0.274 \\
\hline Resource Sector & 11,517 & 0.450 & 0 & 0.497 & & 0 & 0 & 1 & 1 \\
\hline Financial Sector & 11,517 & 0.148 & 0 & 0.355 & & 0 & 0 & 0 & 1 \\
\hline
\end{tabular}

Note: Total Announcements is the number of all announcements released over the firm-year. Periodic Reports is the number of the Type 3 announcements (refer to Table 5-3) released over the firm-year. Non Periodic Reports is the number of the announcements other than Periodic Reports. 'MS' stands for 'market-sensitive' announcements. Stock Return Volatility is the standard deviation of daily market adjusted price return over the year. Well-performing Company equals 1 if the company's stock return is higher than the market index return over the year, otherwise 0. Size is the natural logarithm of the company's market capitalisation. Book-to-Market Ratio is the company's book value on equity divided by its market capitalisation. Leverage is the net gearing ratio, debt divided by equity. ROA is earnings before interests and tax divided by average total asset. Resource Sector equals 1 if the company belongs to energy or materials sector, otherwise 0 . Financial Sector equals 1 if the company belongs to financial sector (including real estate), otherwise 0. 
Table 7-5. Statistic Summary of the Timeliness of Price Discovery Regressions - Different Types of Non-periodic Announcements

\begin{tabular}{|c|c|c|c|c|c|c|c|c|c|}
\hline \multirow{2}{*}{ Variable } & \multirow{2}{*}{$\mathbf{N}$} & \multirow{2}{*}{ Mean } & \multirow{2}{*}{ Median } & \multirow{2}{*}{ Std Dev } & \multirow{2}{*}{$\begin{array}{l}\text { Total Number of } \\
\text { Announcements }\end{array}$} & \multicolumn{4}{|c|}{ Percentiles } \\
\hline & & & & & & 5 & 25 & 75 & 95 \\
\hline Takeover/Scheme Announcements & 11,517 & 0.639 & 0 & 3.489 & 7,362 & 0 & 0 & 0 & 3 \\
\hline Security Holder Details & 11,517 & 12.506 & 10 & 11.217 & 14,4031 & 1 & 5 & 17 & 33 \\
\hline Quarterly Activities Report & 11,517 & 1.767 & 0 & 2.087 & 20,348 & 0 & 0 & 4 & 5 \\
\hline Quarterly Cash Flow Report & 11,517 & 0.708 & 0 & 1.474 & 8,154 & 0 & 0 & 0 & 4 \\
\hline Issued Capital & 11,517 & 12.461 & 8 & 17.070 & 143,515 & 0 & 3 & 16 & 36 \\
\hline Asset Acquisition \& Disposal & 11,517 & 1.388 & 1 & 2.080 & 15,982 & 0 & 0 & 2 & 5 \\
\hline Notice of Meeting & 11,517 & 3.027 & 2 & 1.769 & 34,866 & 1 & 2 & 4 & 6 \\
\hline ASX Announcement & 11,517 & 1.291 & 0 & 2.159 & 14,873 & 0 & 0 & 2 & 6 \\
\hline Distribution Announcement & 11,517 & 0.696 & 0 & 1.901 & 8,021 & 0 & 0 & 0 & 4 \\
\hline Progress Reports & 11,517 & 8.834 & 5 & 11.586 & 101,743 & 0 & 1 & 12 & 29 \\
\hline Company Administration & 11,517 & 2.322 & 2 & 2.331 & 26,747 & 0 & 1 & 3 & 7 \\
\hline Notice of Call & 11,517 & 0.002 & 0 & 0.058 & 21 & 0 & 0 & 0 & 0 \\
\hline Other & 11,517 & 0.933 & 0 & 1.847 & 10,748 & 0 & 0 & 1 & 4 \\
\hline Chairman's Address & 11,517 & 0.998 & 1 & 0.902 & 1,1497 & 0 & 0 & 1 & 3 \\
\hline Letter to Shareholders & 11,517 & 0.192 & 0 & 0.531 & 2,213 & 0 & 0 & 0 & 1 \\
\hline ASX Query & 11,517 & 0.445 & 0 & 0.804 & 5,127 & 0 & 0 & 1 & 2 \\
\hline Structured Products & 11,517 & 0.591 & 0 & 17.412 & 6,801 & 0 & 0 & 0 & 0 \\
\hline CTE Quarterly Reports & 11,517 & 0.589 & 0 & 1.539 & 6,779 & 0 & 0 & 0 & 4 \\
\hline Takeover/Scheme Announcements - MS & 11,517 & 0.300 & 0 & 1.788 & 3,450 & 0 & 0 & 0 & 1 \\
\hline Security Holder Details - MS & 11,517 & 0.101 & 0 & 0.384 & 1,163 & 0 & 0 & 0 & 1 \\
\hline Quarterly Activities Report - MS & 11,517 & 1.687 & 0 & 1.977 & 19,430 & 0 & 0 & 4 & 4 \\
\hline Quarterly Cash Flow Report - MS & 11,517 & 0.688 & 0 & 1.441 & 7,925 & 0 & 0 & 0 & 4 \\
\hline Issued Capital - MS & 11,517 & 1.446 & 1 & 1.725 & 16,657 & 0 & 0 & 2 & 5 \\
\hline Asset Acquisition \& Disposal - MS & 11,517 & 1.011 & 0 & 1.562 & 11,647 & 0 & 0 & 1 & 4 \\
\hline Notice of Meeting - MS & 11,517 & 0.021 & 0 & 0.164 & 242 & 0 & 0 & 0 & 0 \\
\hline ASX Announcement - MS & 11,517 & 1.101 & 0 & 1.846 & 12,675 & 0 & 0 & 2 & 5 \\
\hline Distribution Announcement - MS & 11,517 & 0.238 & 0 & 0.770 & 2,739 & 0 & 0 & 0 & 2 \\
\hline Progress Reports - MS & 11,517 & 5.432 & 3 & 7.115 & 62,566 & 0 & 0 & 8 & 19 \\
\hline Company Administration - MS & 11,517 & 0.163 & 0 & 0.487 & 1,877 & 0 & 0 & 0 & 1 \\
\hline Notice of Call - MS & 11,517 & 0.000 & 0 & 0.023 & 4 & 0 & 0 & 0 & 0 \\
\hline Other - MS & 11,517 & 0.178 & 0 & 0.664 & 2,045 & 0 & 0 & 0 & 1 \\
\hline Chairman's Address - MS & 11,517 & 0.086 & 0 & 0.286 & 989 & 0 & 0 & 0 & 1 \\
\hline Letter to Shareholders - MS & 11,517 & 0.002 & 0 & 0.047 & 25 & 0 & 0 & 0 & 0 \\
\hline ASX Query - MS & 11,517 & 0.348 & 0 & 0.705 & 4,003 & 0 & 0 & 0 & 2 \\
\hline Structured Products - MS & 11,517 & 0.000 & 0 & 0.009 & 1 & 0 & 0 & 0 & 0 \\
\hline CTE Quarterly Reports - MS & 11,517 & 0.558 & 0 & 1.455 & 6,429 & 0 & 0 & 0 & 4 \\
\hline
\end{tabular}




\subsubsection{Correlation Analysis of Variables}

Tables 7-6 to 7-10 show the Pearson correlation among all dependent, independent and control variables. For the regressions on the timeliness of price discovery, the dependent variable is the deflated timeliness metric, and the control variables are daily stock return volatility over the year, well-performing company (the share price performs better than the market index over the year), firm size, book-to-market ratio, leverage, return on total assets, resource sector and financial sector. As to independent variables, this study first uses the total announcement frequency and the total market-sensitive announcement frequency (Table 7-6). Periodic Reports are then separated from Non-periodic Reports (Table 7-7) to test whether they have different impacts on the timeliness of price discovery. Moreover, the market sensitivity is taken into account when testing the Periodic/Non-periodic Report impacts (Table 7-8). Lastly, the Nonperiodic Reports are further divided into detailed announcement categories (Table 7-9), taking market sensitivity into account as well (Table 7-10). Most of the variables do not show large correlation coefficients to suggest a multicollinearity problem. There are significant correlations between firm size and stock return volatility (-0.672), and between resource sector and a few announcement categories, especially Quarterly Activities Reports (0.857). However, there is no serious multicollinearity problem in the regression models according to the Variance Inflation Factor (VIF) value. No VIF value is above 10, indicating no multicollinearity among independent and control variables (Pallant, 2011). The detailed VIF value is shown in Tables 7-10 and 7-11. 
Table 7-6. Correlation Matrix for Deflated Timeliness Metric, Announcement Frequency, Market-sensitive Announcement Frequency and Control Variables

\begin{tabular}{|c|c|c|c|c|c|c|c|c|c|c|c|}
\hline & Deflated $\mathrm{M}^{\mathrm{c}}$ & $\begin{array}{l}\text { All } \\
\text { AnnFre }\end{array}$ & $\begin{array}{l}\text { MS } \\
\text { AnnFre }\end{array}$ & Vol & $\begin{array}{l}\text { W/B } \\
\text { Company }\end{array}$ & Size & BMV & Lev & ROA & $\mathrm{RSec}$ & FSec \\
\hline All Announcements & $0.038 * * *$ & 1 & & & & & & & & & \\
\hline All Announcements - MS & $0.205^{* * *}$ & - & 1 & & & & & & & & \\
\hline Stock Return Volatility & $0.302 * * *$ & $-0.158 * * *$ & $0.119 * * *$ & 1 & & & & & & & \\
\hline Well-performing Company & $-0.043 * * *$ & -0.008 & $-0.020 * *$ & $0.032 * * *$ & 1 & & & & & & \\
\hline Size & $-0.211 * * *$ & $0.342 * * *$ & -0.003 & $-0.672 * * *$ & $0.044 * * *$ & 1 & & & & & \\
\hline Book-to-Market Ratio & $0.021 * *$ & $-0.035 * * *$ & $-0.033 * * *$ & $0.086^{* * *}$ & $-0.073 * * *$ & $-0.126 * * *$ & 1 & & & & \\
\hline Leverage & 0.014 & 0.004 & $0.016^{*}$ & -0.002 & -0.009 & 0.001 & -0.002 & 1 & & & \\
\hline ROA & $-0.060 * * *$ & -0.005 & $-0.044 * * *$ & $-0.121 * * *$ & $0.022 * *$ & $0.092 * * *$ & $0.034 * * *$ & -0.002 & 1 & & \\
\hline Resource Sector & $0.161 * * *$ & $0.082 * * *$ & $0.478 * * *$ & $0.233 * * *$ & 0.006 & $-0.204 * * *$ & $0.027 * * *$ & 0.003 & $-0.021 * *$ & 1 & \\
\hline Financial Sector & $-0.147 * * *$ & $0.104 * * *$ & $-0.228 * * *$ & $-0.212 * * *$ & $-0.017 *$ & $0.204 * * *$ & $0.055^{* * *}$ & 0.002 & $0.034 * * *$ & $-0.377 * * *$ & 1 \\
\hline
\end{tabular}

$*, * *$ and $* * *$ denote significance at the $10 \%, 5 \%$ and $1 \%$ levels respectively (two-tailed).

Table 7-7. Correlation Matrix for Deflated Timeliness Metric, Periodic/Non-periodic Report Frequency and Control Variables

\begin{tabular}{|c|c|c|c|c|c|c|c|c|c|c|c|}
\hline & Deflated $\mathrm{M}^{\mathrm{c}^{\prime}}$ & Type3 & NonType3 & Vol & $\begin{array}{l}\text { W/B } \\
\text { Company }\end{array}$ & Size & BMV & Lev & $\mathrm{ROA}$ & RSec & FSec \\
\hline Periodic Reports & $-0.111 * * *$ & 1 & & & & & & & & & \\
\hline Non-periodic Reports & $0.074 * * *$ & $0.070 * * *$ & 1 & & & & & & & & \\
\hline Stock Return Volatility & $0.302 * * *$ & $-0.210 * * *$ & $-0.105 * * *$ & 1 & & & & & & & \\
\hline Well-performing Company & $-0.043 * * *$ & $-0.024 * * *$ & -0.001 & $0.032 * * *$ & 1 & & & & & & \\
\hline Size & $-0.211 * * *$ & $0.242 * * *$ & $0.291 * * *$ & $-0.672 * * *$ & $0.044 * * *$ & 1 & & & & & \\
\hline Book-to-Market Ratio & $0.021 * *$ & -0.003 & $-0.037 * * *$ & $0.086 * * *$ & $-0.073 * * *$ & $-0.126 * * *$ & 1 & & & & \\
\hline Leverage & 0.014 & 0.001 & 0.004 & -0.002 & -0.009 & 0.001 & -0.002 & 1 & & & \\
\hline ROA & $-0.060 * * *$ & $0.025^{* * *}$ & -0.013 & $-0.121 * * *$ & $0.022 * *$ & $0.092 * * *$ & $0.034 * * *$ & -0.002 & 1 & & \\
\hline Resource Sector & $0.161 * * *$ & $-0.224 * * *$ & $0.155^{* * *}$ & $0.233^{* * *}$ & 0.006 & $-0.204 * * *$ & $0.027 * * *$ & 0.003 & $-0.021 * *$ & 1 & \\
\hline Financial Sector & $-0.147 * * *$ & $0.306^{* * *}$ & $0.018 * *$ & $-0.212 * * *$ & $-0.017 *$ & $0.204 * * *$ & $0.055^{* * *}$ & 0.002 & $0.034 * * *$ & $-0.377 * * *$ & 1 \\
\hline
\end{tabular}

$*, * *$ and $* * *$ denote significance at the $10 \%, 5 \%$ and $1 \%$ levels respectively (two-tailed). 
Table 7-8. Correlation Matrix for Deflated Timeliness Metric, Market-sensitive Periodic/Non-periodic Report Frequency and Control Variables

\begin{tabular}{|c|c|c|c|c|c|c|c|c|c|c|c|}
\hline & Deflated $\mathbf{M}^{\mathrm{c}}$ & Type3MS & $\begin{array}{l}\text { NonType } 3 \\
\text { MS }\end{array}$ & Vol & $\begin{array}{l}\text { W/B } \\
\text { Company }\end{array}$ & Size & BMV & Lev & ROA & $\mathrm{RSec}$ & FSec \\
\hline Periodic Reports - MS & $-0.109 * * *$ & 1 & & & & & & & & & \\
\hline $\begin{array}{l}\text { Non Periodic Announcements - } \\
\text { MS }\end{array}$ & $0.217 * * *$ & $-0.340 * * *$ & 1 & & & & & & & & \\
\hline Stock Return Volatility & $0.302 * * *$ & $-0.287 * * *$ & $0.172 * * *$ & 1 & & & & & & & \\
\hline Well-performing Company & $-0.043 * * *$ & $-0.032 * * *$ & -0.012 & $0.032 * * *$ & 1 & & & & & & \\
\hline Size & $-0.211 * * *$ & $0.408 * * *$ & $-0.087 * * *$ & $-0.672 * * *$ & $0.044 * * *$ & 1 & & & & & \\
\hline Book-to-Market Ratio & $0.021 * *$ & $-0.021 * *$ & $-0.027 * * *$ & $0.086^{* * *}$ & $-0.073 * * *$ & $-0.126^{* * *}$ & 1 & & & & \\
\hline Leverage & 0.014 & 0.004 & 0.014 & -0.002 & -0.009 & 0.001 & -0.002 & 1 & & & \\
\hline ROA & $-0.060 * * *$ & $0.039 * * *$ & $-0.050 * * *$ & $-0.121 * * *$ & $0.022 * *$ & $0.092 * * *$ & $0.034 * * *$ & -0.002 & 1 & & \\
\hline Resource Sector & $0.161 * * *$ & $-0.539 * * *$ & $0.565^{* * *}$ & $0.233 * * *$ & 0.006 & $-0.204 * * *$ & $0.027 * * *$ & 0.003 & $-0.021 * *$ & 1 & \\
\hline Financial Sector & $-0.147 * * *$ & $0.221 * * *$ & $-0.262 * * *$ & $-0.212 * * *$ & $-0.017^{*}$ & $0.204^{* * *}$ & $0.055 * * *$ & 0.002 & $0.034 * * *$ & $-0.377 * * *$ & 1 \\
\hline
\end{tabular}

$*, * *$ and $* * *$ denote significance at the $10 \%, 5 \%$ and $1 \%$ levels respectively (two-tailed). 
Table 7-9. Correlation Matrix for Deflated Timeliness Metric, Announcement Frequency as to Each Category and Control Variables

\begin{tabular}{|c|c|c|c|c|c|c|c|c|c|c|c|c|c|}
\hline & Deflated $\mathrm{M}^{\mathrm{c}^{\prime}}$ & Type 1 & Type2 & Type4 & Type5 & Type6 & Type7 & Type8 & Type9 & Type10 & Type11 & Type12 & Type13 \\
\hline Takeover/Scheme Announcements & 0.015 & 1 & & & & & & & & & & & \\
\hline Security Holder Details & 0.006 & $0.250^{* * *}$ & 1 & & & & & & & & & & \\
\hline Quarterly Activities Report & $0.170^{* * *}$ & $0.024^{* * *}$ & $-0.105^{* * *}$ & 1 & & & & & & & & & \\
\hline Quarterly Cash Flow Report & $0.110^{* * *}$ & $-0.017^{*}$ & $-0.092^{* * *}$ & $0.540^{* * *}$ & 1 & & & & & & & & \\
\hline Issued Capital & 0.008 & $0.029^{* * *}$ & $0.229^{* * * *}$ & 0.014 & 0.006 & 1 & & & & & & & \\
\hline Asset Acquisition \& Disposal & $0.018^{* *}$ & $0.053^{* * *}$ & $0.196^{* * *}$ & $-0.027^{* * *}$ & $-0.042^{* * *}$ & $0.122^{* * *}$ & 1 & & & & & & \\
\hline Notice of Meeting & $0.162^{* * *}$ & $0.079^{* * *}$ & $0.159^{* * *}$ & $0.149^{* * *}$ & $0.117^{* * *}$ & $0.165^{* * *}$ & $0.116^{* * *}$ & 1 & & & & & \\
\hline ASX Announcement & $0.242^{* * *}$ & $0.074^{* * *}$ & $0.083^{* * *}$ & $0.242^{* * *}$ & $0.160^{* * *}$ & $0.141^{* * *}$ & $0.129^{* * *}$ & $0.285^{* * *}$ & 1 & & & & \\
\hline Distribution Announcement & $-0.138^{* * *}$ & $0.019^{* *}$ & $0.224^{* * * *}$ & $-0.251^{* * *}$ & $-0.165^{* * *}$ & $0.143^{* * *}$ & $0.142^{* * *}$ & $-0.052^{* * *}$ & $-0.047^{* * *}$ & 1 & & & \\
\hline Progress Reports & $0.115^{* * *}$ & $0.022^{* *}$ & 0.005 & $0.470^{* * *}$ & $0.222^{* * *}$ & $0.105^{* * *}$ & $0.111^{* * *}$ & $0.143^{* * *}$ & $0.254^{* * *}$ & $-0.120^{* * *}$ & 1 & & \\
\hline Company Administration & $0.067^{* * *}$ & $0.088^{* * *}$ & $0.321^{* * *}$ & $-0.052^{* * *}$ & $-0.026^{* * *}$ & $0.139^{* * *}$ & $0.182^{* * *}$ & $0.236^{* * *}$ & $0.163^{* * *}$ & $0.168^{* * *}$ & $0.071^{* * *}$ & 1 & \\
\hline Notice of Call & 0.012 & -0.006 & 0.005 & 0.006 & 0.005 & 0.007 & -0.007 & 0.011 & $0.022^{* *}$ & -0.008 & 0.018 & 0.012 & 1 \\
\hline Other & $-0.023^{* *}$ & $0.054^{* * *}$ & $0.119^{* * *}$ & $-0.023^{* *}$ & $-0.063^{* * *}$ & $0.133^{* * *}$ & $0.133^{* * *}$ & $0.071^{* * * *}$ & $0.039^{* * *}$ & $0.067^{* * *}$ & $0.144^{* * *}$ & $0.164^{* * *}$ & $0.016^{*}$ \\
\hline Chairman's Address & $-0.066^{* * *}$ & $0.072^{* * *}$ & $0.213^{* * *}$ & $-0.163^{* * *}$ & $-0.129^{* * *}$ & $0.099^{* * *}$ & $0.072^{* * *}$ & $0.075^{\text {*** }}$ & $-0.062^{* * *}$ & $0.106^{* * *}$ & $0.029^{* * *}$ & $0.114^{* * *}$ & -0.005 \\
\hline Letter to Shareholders & -0.011 & $0.131^{* * *}$ & $0.120^{* * *}$ & $-0.056^{* * *}$ & $-0.043^{* * *}$ & $0.096^{* * *}$ & $0.063^{* * *}$ & $0.093^{* * *}$ & $0.055^{* * *}$ & $0.125^{* * *}$ & $0.039^{* * *}$ & $0.129^{* * *}$ & 0.006 \\
\hline ASX Query & $0.185^{* * *}$ & -0.006 & $0.021^{* *}$ & $0.166^{* * *}$ & $0.129^{* * *}$ & $0.043^{* * *}$ & -0.007 & $0.116^{* * *}$ & $0.230^{* * *}$ & $-0.108^{* * *}$ & $0.128^{* * *}$ & $0.075^{* * *}$ & $0.033^{* * *}$ \\
\hline Structured Products & -0.013 & 0.003 & $0.136^{* * *}$ & $-0.029^{* * *}$ & $-0.016^{*}$ & $0.198^{* * *}$ & $0.019^{* *}$ & $0.056^{* * *}$ & $0.022^{* *}$ & $0.195^{* * *}$ & -0.010 & $0.068^{* * *}$ & -0.001 \\
\hline CTE Quarterly Reports & $0.082^{* * *}$ & $-0.041^{* * *}$ & $-0.092^{* * *}$ & $-0.304^{* * *}$ & $-0.173^{* * *}$ & $-0.021^{* *}$ & $-0.036^{* * *}$ & $0.065^{* * *}$ & $0.051^{* * *}$ & $-0.117^{* * *}$ & $-0.045^{* * *}$ & $0.047^{* * *}$ & $0.027^{* * *}$ \\
\hline Stock Return Volatility & $0.302^{* * * *}$ & $-0.058^{* * * *}$ & $-0.224^{* * *}$ & $0.253^{* * *}$ & $0.237^{* * *}$ & $-0.101^{* * * *}$ & $-0.075^{* * *}$ & $0.130^{* * *}$ & $0.140^{* * * *}$ & $-0.273^{* * *}$ & $0.039^{* * *}$ & $-0.050^{* * *}$ & 0.015 \\
\hline Well-performing Company & $-0.043^{* * * *}$ & $0.025^{* * *}$ & -0.009 & 0.007 & $0.018^{*}$ & 0.015 & -0.003 & $0.019^{* *}$ & -0.002 & $-0.029^{* * *}$ & $-0.019^{* *}$ & $-0.065^{* * *}$ & 0.008 \\
\hline Size & $-0.211^{* * *}$ & $0.106^{* * *}$ & $0.425^{* * *}$ & $-0.231^{* * *}$ & $-0.262^{* * *}$ & $0.205^{* * *}$ & $0.194^{* * *}$ & $-0.076^{* * *}$ & $-0.076^{* * *}$ & $0.401^{* * *}$ & 0.005 & $0.188^{* * *}$ & -0.008 \\
\hline Book-to-Market Ratio & $0.021^{* *}$ & -0.011 & -0.006 & $0.036^{* * *}$ & $0.035^{* * *}$ & $-0.034^{* * *}$ & -0.014 & $-0.022^{* *}$ & $-0.025^{* * *}$ & 0.014 & $-0.033^{* * *}$ & -0.008 & -0.015 \\
\hline Leverage & 0.014 & 0.001 & -0.004 & -0.015 & -0.009 & -0.002 & 0.004 & 0.005 & -0.007 & 0.001 & 0.011 & 0.008 & -0.001 \\
\hline $\mathrm{ROA}$ & $-0.060^{* * *}$ & 0.007 & $0.022^{* *}$ & $-0.024^{* * *}$ & $-0.023^{* *}$ & -0.011 & 0.015 & $-0.050^{* * *}$ & $-0.043^{* * *}$ & $0.034^{* * *}$ & $-0.026^{* * *}$ & $-0.025^{* * *}$ & -0.001 \\
\hline Resource Sector & $0.161^{* * *}$ & $0.023^{* *}$ & $-0.095^{* * *}$ & $0.857^{* * *}$ & $0.486^{* * *}$ & 0.012 & $-0.021^{* *}$ & $0.149^{* * *}$ & $0.229^{* * *}$ & $-0.251^{* * *}$ & $0.431^{* * *}$ & $-0.049^{* * *}$ & 0.005 \\
\hline Financial Sector & $-0.147^{* * * *}$ & -0.003 & $0.068^{* * *}$ & $-0.341^{* * *}$ & $-0.197^{* * *}$ & $0.097^{* * *}$ & $0.085^{* * *}$ & $-0.102^{* * *}$ & $-0.114^{* * *}$ & $0.417^{* * *}$ & $-0.228^{* * *}$ & $0.051^{* * * *}$ & -0.009 \\
\hline
\end{tabular}

$*, * *$ and $* * *$ denote significance at the $10 \%, 5 \%$ and $1 \%$ levels respectively (two-tailed). 
Table 7-9 Correlation Matrix for Deflated Timeliness Metric, Announcement Frequency as to Each Category and Control Variables (cont.)

\begin{tabular}{|c|c|c|c|c|c|c|c|c|c|c|c|c|c|c|}
\hline & Type14 & Type15 & Type16 & Type17 & Type 18 & Type18 & Vol & $\begin{array}{r}\mathrm{W} / \mathrm{B} \\
\text { Company } \\
\end{array}$ & Size & BMV & Lev & ROA & $\mathrm{RSec}$ & FSec \\
\hline \multicolumn{15}{|c|}{ Takeover/Scheme Announcements } \\
\hline \multicolumn{15}{|c|}{ Security Holder Details } \\
\hline \multicolumn{15}{|l|}{ Quarterly Activities Report } \\
\hline \multicolumn{15}{|l|}{ Quarterly Cash Flow Report } \\
\hline \multicolumn{15}{|l|}{ Issued Capital } \\
\hline \multicolumn{15}{|l|}{ Asset Acquisition \& Disposal } \\
\hline \multicolumn{15}{|l|}{ Notice of Meeting } \\
\hline \multicolumn{15}{|l|}{ ASX Announcement } \\
\hline \multicolumn{15}{|l|}{ Distribution Announcement } \\
\hline \multicolumn{15}{|l|}{ Progress Reports } \\
\hline \multicolumn{15}{|l|}{ Company Administration } \\
\hline \multicolumn{15}{|l|}{ Notice of Call } \\
\hline Other & 1 & & & & & & & & & & & & & \\
\hline Chairman's Address & $0.097^{* * *}$ & 1 & & & & & & & & & & & & \\
\hline Letter to Shareholders & $0.174^{* * *}$ & $0.093^{* * *}$ & 1 & & & & & & & & & & & \\
\hline ASX Query & $-0.026^{* * *}$ & $-0.048^{* * *}$ & -0.010 & 1 & & & & & & & & & & \\
\hline Structured Products & $0.040^{* * * *}$ & $0.066^{* * * *}$ & $0.032^{* * *}$ & -0.013 & 1 & & & & & & & & & \\
\hline CTE Quarterly Reports & $0.019^{* *}$ & $-0.053^{* * *}$ & $0.025^{* * *}$ & $0.088^{* * *}$ & -0.013 & 1 & & & & & & & & \\
\hline Stock Return Volatility & $-0.167^{* * *}$ & $-0.222^{* * *}$ & $-0.077^{* * *}$ & $0.183^{* * *}$ & $-0.034^{* * *}$ & $0.251^{* * *}$ & 1 & & & & & & & \\
\hline Well-performing Company & -0.008 & $-0.022^{* *}$ & $-0.037^{* * *}$ & $0.044^{* * *}$ & 0.007 & -0.009 & $0.032^{* * *}$ & 1 & & & & & & \\
\hline Size & $0.227^{* * *}$ & $0.297^{* * *}$ & $0.109^{* * *}$ & $-0.075^{* * *}$ & $0.094^{* * *}$ & $-0.255^{* * *}$ & $-0.672^{* * *}$ & $0.044^{* * *}$ & 1 & & & & & \\
\hline Book-to-Market Ratio & $-0.072^{* * * *}$ & $-0.023^{* *}$ & $-0.029^{* * *}$ & $-0.022^{* *}$ & -0.003 & $-0.056^{* * *}$ & $0.086^{* * *}$ & $-0.073^{* * * *}$ & $-0.126^{* * *}$ & 1 & & & & \\
\hline Leverage & 0.001 & $0.032^{* * *}$ & 0.000 & -0.007 & 0.003 & $0.018^{* *}$ & -0.002 & -0.009 & 0.001 & -0.002 & 1 & & & \\
\hline ROA & 0.007 & $0.041^{* * * *}$ & 0.004 & $-0.040^{* * *}$ & 0.003 & $-0.064^{* * *}$ & $-0.121^{* * *}$ & $0.022^{* *}$ & $0.092^{* * *}$ & $0.034^{* * *}$ & -0.002 & 1 & & \\
\hline Resource Sector & -0.010 & $-0.158^{* * *}$ & $-0.065^{* * *}$ & $0.159^{* * *}$ & $-0.030^{* * *}$ & $-0.234^{* * *}$ & $0.233^{* * *}$ & 0.006 & $-0.204^{* * *}$ & $0.027^{* *}$ & 0.003 & $-0.021^{* *}$ & 1 & \\
\hline Financial Sector & 0.004 & $0.038^{* * *}$ & $0.114^{* * *}$ & $-0.131^{* * *}$ & $0.081^{* * *}$ & $-0.088^{* * *}$ & $-0.212^{* * *}$ & $-0.017^{*}$ & $0.204^{* * *}$ & $0.055^{* * *}$ & 0.002 & $0.034^{* * *}$ & $-0.377^{* * * *}$ & 1 \\
\hline
\end{tabular}


Table 7-10. Correlation Matrix for Deflated Timeliness Metric, Market-sensitive Announcement Frequency as to Each Category and Control Variables

\begin{tabular}{|c|c|c|c|c|c|c|c|c|c|c|c|c|c|}
\hline & $\begin{array}{r}\text { Deflated } \\
\mathbf{M}^{\mathbf{c}^{\prime}}\end{array}$ & $\begin{array}{r}\text { Type1 } \\
\text { MS } \\
\end{array}$ & $\begin{array}{r}\text { Type2 } \\
\text { MS }\end{array}$ & $\begin{array}{r}\text { Type4 } \\
\text { MS } \\
\end{array}$ & $\begin{array}{r}\text { Type5 } \\
\text { MS } \\
\end{array}$ & $\begin{array}{r}\text { Type6 } \\
\text { MS } \\
\end{array}$ & $\begin{array}{r}\text { Type7 } \\
\text { MS } \\
\end{array}$ & $\begin{array}{r}\text { Type8 } \\
\text { MS } \\
\end{array}$ & $\begin{array}{r}\text { Type9 } \\
\text { MS } \\
\end{array}$ & $\begin{array}{r}\text { Type } 10 \\
\text { MS } \\
\end{array}$ & $\begin{array}{r}\text { Type11 } \\
\text { MS }\end{array}$ & $\begin{array}{r}\text { Type } 12 \\
\text { MS } \\
\end{array}$ & $\begin{array}{r}\text { Type13 } \\
\text { MS } \\
\end{array}$ \\
\hline Takeover/Scheme Announcements MS & $0.015^{*}$ & 1 & & & & & & & & & & & \\
\hline Security Holder Details MS & $0.025^{* * *}$ & $0.075^{* * *}$ & 1 & & & & & & & & & & \\
\hline Quarterly Activities Report MS & $0.169^{* * *}$ & 0.015 & $0.060^{* * *}$ & 1 & & & & & & & & & \\
\hline Quarterly Cash Flow Report MS & $0.107^{* * *}$ & -0.010 & $0.022^{* *}$ & $0.551^{* * *}$ & 1 & & & & & & & & \\
\hline Issued Capital MS & $0.148^{* * *}$ & 0.002 & $0.087^{* * *}$ & $0.207^{* * *}$ & $0.142^{* * *}$ & 1 & & & & & & & \\
\hline Asset Acquisition \& Disposal MS & $0.019^{* *}$ & $0.032^{* * *}$ & $0.080^{* * *}$ & -0.003 & $-0.020^{* *}$ & $0.162^{* * *}$ & 1 & & & & & & \\
\hline Notice of Meeting MS & $0.044^{* * *}$ & $0.042^{* * *}$ & $0.037^{* * * *}$ & $-0.019^{* *}$ & -0.014 & $0.062^{* * *}$ & $0.033^{* * *}$ & 1 & & & & & \\
\hline ASX Announcement MS & $0.240^{* * *}$ & $0.066^{* * *}$ & $0.071^{* * *}$ & $0.253^{* * *}$ & $0.166^{* * *}$ & $0.367^{* * *}$ & $0.118^{* * *}$ & $0.074^{* * *}$ & 1 & & & & \\
\hline Distribution Announcement MS & $-0.114^{* * *}$ & -0.002 & -0.003 & $-0.234^{* * *}$ & $-0.141^{* * *}$ & -0.007 & $0.131^{* * *}$ & $0.027^{* * *}$ & $-0.074^{* * *}$ & 1 & & & \\
\hline Progress Reports MS & $0.135^{* * *}$ & 0.012 & $0.071^{* * *}$ & $0.519^{* * *}$ & $0.266^{* * *}$ & $0.223^{* * *}$ & $0.117^{* * *}$ & 0.007 & $0.275^{* * *}$ & $-0.154^{* * *}$ & 1 & & \\
\hline Company Administration MS & $0.019^{* *}$ & $0.032^{* * *}$ & $0.023^{* *}$ & $-0.019^{* *}$ & $-0.019^{* *}$ & $0.055^{* * *}$ & $0.047^{* * *}$ & $0.061^{* * *}$ & $0.056^{* * *}$ & 0.007 & $0.025^{* * *}$ & 1 & \\
\hline Notice of Call MS & 0.012 & -0.003 & $0.016^{* *}$ & 0.002 & -0.007 & 0.014 & -0.003 & -0.002 & 0.007 & -0.005 & 0.018 & 0.011 & 1 \\
\hline Other MS & -0.013 & $0.033^{* * *}$ & 0.014 & $-0.043^{* * *}$ & $-0.047^{* * *}$ & $0.029^{* * *}$ & $0.056^{* * *}$ & -0.005 & 0.000 & $0.019^{* *}$ & $0.076^{* * *}$ & $0.033^{* * *}$ & -0.004 \\
\hline Chairman's Address MS & $-0.048^{* * *}$ & $0.028^{* * *}$ & -0.009 & $-0.199^{* * *}$ & $-0.125^{* * *}$ & $-0.060^{* * *}$ & $0.017^{*}$ & -0.013 & $-0.081^{* * *}$ & $0.020^{* *}$ & $-0.108^{* * *}$ & $0.022^{* *}$ & -0.005 \\
\hline Letter to Shareholders MS & 0.012 & $0.030^{* * *}$ & 0.002 & -0.002 & -0.008 & -0.001 & 0.015 & 0.005 & -0.003 & -0.005 & 0.008 & -0.008 & -0.001 \\
\hline ASX Query MS & $0.184^{* * *}$ & -0.001 & $0.039^{* * *}$ & $0.167^{* * *}$ & $0.120^{* * *}$ & $0.132^{* * *}$ & 0.007 & -0.008 & $0.220^{* * *}$ & $-0.085^{* * *}$ & $0.145^{* * *}$ & 0.015 & 0.009 \\
\hline Structured Products MS & -0.006 & 0.004 & -0.002 & 0.011 & -0.004 & 0.003 & 0.012 & -0.001 & -0.001 & -0.003 & $0.037^{* * * *}$ & -0.003 & 0.000 \\
\hline CTE Quarterly Reports MS & $0.079^{* * *}$ & $-0.037^{* * *}$ & -0.009 & $-0.310^{* * *}$ & $-0.174^{* * *}$ & $0.045^{* * *}$ & $-0.036^{* * *}$ & -0.001 & $0.041^{* * *}$ & $-0.097^{* * *}$ & $-0.061^{* * *}$ & $-0.022^{* *}$ & 0.015 \\
\hline Stock Return Volatility & $0.302^{* * *}$ & $-0.046^{* * *}$ & -0.006 & $0.260^{* * *}$ & $0.234^{* * *}$ & $0.147^{* * *}$ & $-0.055^{* * *}$ & 0.000 & $0.148^{* * *}$ & $-0.218^{* * *}$ & $0.070^{* * * *}$ & $-0.053^{* * *}$ & 0.004 \\
\hline Well-performing Company & $-0.043^{* * *}$ & $0.024^{* *}$ & -0.008 & 0.011 & $0.017^{*}$ & -0.004 & -0.010 & $-0.016^{*}$ & -0.005 & $-0.046^{* * *}$ & $-0.017^{*}$ & $-0.063^{* * * *}$ & 0.000 \\
\hline Size & $-0.211^{* * *}$ & $0.088^{* * *}$ & $0.059^{* * *}$ & $-0.249^{* * *}$ & $-0.260^{* * *}$ & $-0.038^{* * *}$ & $0.165^{* * *}$ & $0.020^{* *}$ & $-0.096^{* * *}$ & $0.226^{* * *}$ & $-0.036^{* * *}$ & $0.112^{* * *}$ & $0.001^{* * *}$ \\
\hline Book-to-Market Ratio & $0.021^{* *}$ & -0.011 & -0.007 & $0.037^{* * *}$ & $0.034^{* * *}$ & $-0.017^{*}$ & -0.011 & 0.002 & $-0.030^{* * * *}$ & $0.027^{* * *}$ & $-0.028^{* * *}$ & $0.031^{* * *}$ & -0.001 \\
\hline Leverage & 0.014 & 0.004 & -0.003 & -0.015 & -0.009 & 0.007 & 0.001 & -0.001 & -0.007 & -0.001 & $0.022^{* *}$ & 0.006 & 0.000 \\
\hline ROA & $-0.060^{* * *}$ & 0.006 & 0.002 & $-0.025^{* * *}$ & $-0.022^{* *}$ & $-0.040^{* * *}$ & 0.012 & 0.001 & $-0.047^{* * * *}$ & $0.030^{* * *}$ & $-0.036^{* * *}$ & 0.004 & 0.000 \\
\hline Resource Sector & $0.161^{* * *}$ & $0.020^{* *}$ & $0.055^{* * *}$ & $0.866^{* * *}$ & $0.483^{* * *}$ & $0.198^{* * *}$ & 0.002 & -0.013 & $0.245^{* * *}$ & $-0.240^{* * * *}$ & $0.478^{* * *}$ & $-0.020^{* *}$ & 0.002 \\
\hline Financial Sector & $-0.147^{* * *}$ & -0.010 & $-0.030^{* * *}$ & $-0.345^{* * *}$ & $-0.195^{* * *}$ & $-0.026^{* * *}$ & $0.076^{* * *}$ & $0.033^{* * *}$ & $-0.132^{* * *}$ & $0.422^{* * *}$ & $-0.254^{* * *}$ & 0.005 & -0.006 \\
\hline
\end{tabular}


Table 7-10 Correlation Matrix for Deflated Timeliness Metric, Market-sensitive Announcement Frequency as to Each Category and Control Variables (cont.)

\begin{tabular}{|c|c|c|c|c|c|c|c|c|c|c|c|c|c|c|}
\hline 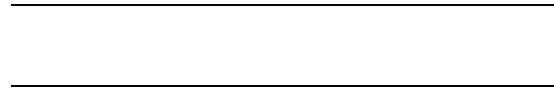 & $\begin{array}{r}\text { Type14 } \\
\text { MS } \\
\end{array}$ & $\begin{array}{r}\text { Type15 } \\
\text { MS }\end{array}$ & $\begin{array}{r}\text { Type16 } \\
\text { MS }\end{array}$ & $\begin{array}{r}\text { Type17 } \\
\text { MS }\end{array}$ & $\begin{array}{r}\text { Type18 } \\
\text { MS }\end{array}$ & $\begin{array}{r}\text { Type18 } \\
\text { MS }\end{array}$ & Vol & $\begin{array}{r}\text { W/B } \\
\text { Company }\end{array}$ & Size & BMV & Lev & $\mathrm{ROA}$ & $\mathrm{RSec}$ & FSec \\
\hline
\end{tabular}

Takeover/Scheme Announcements MS

Security Holder Details MS

Quarterly Activities Report MS

Quarterly Cash Flow Report MS

Issued Capital MS

Asset Acquisition \& Disposal MS

Notice of Meeting MS

ASX Announcement MS

Distribution Announcement MS

Progress Reports MS

Company Administration MS

Notice of Call MS

Other MS

Chairman's Address MS

Letter to Shareholders MS

ASX Query MS

Structured Products MS

CTE Quarterly Reports MS

Stock Return Volatility

Well-performing Company

Size

Book-to-Market Ratio

Leverage

ROA

$\begin{array}{rr}1 & \\ 0.031^{* * *} & 1 \\ 0.004 & -0.001 \\ -0.006 & -0.051^{\text {**** }}\end{array}$

1

$\begin{array}{llll}-0.006 & -0.051^{* * * *} & 0.009 & 1\end{array}$

$\begin{array}{llll}0.026^{* * * *} & -0.003 & 0.000 & -0.005\end{array}$

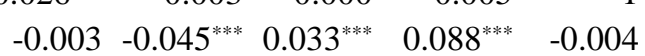

$-0.108^{* * *}-0.132^{* * * *} \quad 0.005 \quad 0.156^{* * * *}-0.005 \quad 0.250 *$

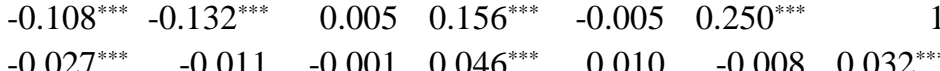

$0.169^{* * * *} \quad 0.163^{* * *} \quad-0.002-0.056^{* * *} \quad 0.012-0.254^{* * *}-0.672^{\text {**** }}$

$\begin{array}{lllllll}-0.031^{* * *} & 0.000 & 0.004 & -0.037^{\text {**** }} & -0.002 & -0.055^{* * *} & 0.086^{\text {**** }}\end{array}$

$-0.002-0.055^{* * *} \quad 0.086^{* * *} \quad-0.073^{* * *}-0.126^{* * * *}$

$\begin{array}{lllllll}0.012 & 0.007 & -0.001 & -0.006 & 0.000 & 0.020^{* * *} & -0.002\end{array}$

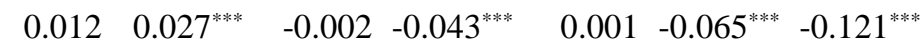

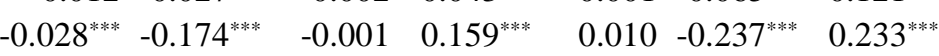

$\begin{array}{lll}-0.009 & 0.001 & -0.002\end{array}$

Resource Sector

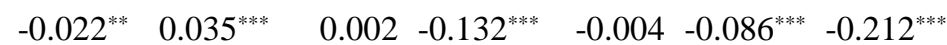

$0.022^{* *} \quad 0.092^{* * *} \quad 0.034^{* * *}-0.002$

$0.006-0.204^{* * *} 0.027^{* * *} 0.003-0.021^{* * *}$

Financial Sector

$0.002-0.132^{* * *}-0.004$

$*, * *$ and $* * *$ denote significance at the $10 \%, 5 \%$ and $1 \%$ levels respectively (two-tailed). 


\subsubsection{The Regression Results on the Timeliness of Price Discovery}

According to the timeliness of price discovery definition, on the day when the annual earnings information becomes available to the market, the share price can reflect the 'true' firm value. The deflated timeliness metric reveals how close, on average, the market-adjusted price is to the 'true' firm value over one year before the current year's earnings information is released. The closer (i.e. smaller) the better, because it means the share price does anticipate the earnings information before the earnings announcement is released. In other words, the earnings information is incorporated into the share price in a timelier manner before the annual earnings information is released.

Tables 7-11 and 7-12 show the regression results for the timeliness of price discovery. Table 711 reveals the impacts of total announcement frequency (Panel A) and Periodic/Non-periodic report frequency (Panel B) on the deflated timeliness metric; it also provides evidence on whether or not announcement sensitivity makes a difference. Table 7-12 focuses on the impacts of the 18 types of Non-periodic Reports on the timeliness of price discovery (Panel A) as well as the announcement market sensitivity influences (Panel B). According to the equation 5-10, the smaller is the timeliness metric, the faster is the timeliness of price discovery. Therefore, the negative coefficients of independent variables indicate that the larger number of announcements (i.e. larger AnnFre) are released (in the announcement category or group) the greater the acceleration of the timeliness of price discovery (i.e. smaller Deflated $M^{c^{\prime}}$ ), meaning the share price gets closer to the 'true' price. The positive coefficients mean that the more announcements (i.e. smaller AnnFre) (in the announcement category or group) are released, the less timely the earnings information is incorporated into share prices (i.e. larger Deflated $M^{c^{\prime}}$ ). Based on the regression results, most of the coefficients are positive, which may indicate that 
more announcements on the market may cause noise information, making the share price move away from the 'true' price. 
Table 7-11. The Impact of Announcement and Periodic/Non-periodic Report Frequency on the Timeliness of Price Discovery

\begin{tabular}{|c|c|c|c|c|c|c|c|c|c|c|c|c|}
\hline \multirow{2}{*}{ Variable } & \multicolumn{6}{|c|}{ Panel A: Total Announcements } & \multicolumn{6}{|c|}{ Panel B: Periodic/Non-Periodic Reports } \\
\hline & Coeff & $t$-stat & VIF & Coeff & $t$-stat & VIF & Coeff & $t$-stat & VIF & Coeff & $t$-stat & VIF \\
\hline Intercept & $0.015470 * * *$ & 11.27 & 0.00 & $0.013380 * * *$ & 9.76 & 0.00 & $0.016160 * * *$ & 11.74 & 0.00 & $0.013110 * * *$ & 9.45 & 0.00 \\
\hline All Announcements & $0.000061 * * *$ & 9.55 & 1.19 & & & & & & & & & \\
\hline All Announcements - MS & & & & $0.000418 * * *$ & 15.78 & 1.35 & & & & & & \\
\hline Periodic Reports & & & & & & & $-0.000062 * * *$ & -2.79 & 1.16 & & & \\
\hline Non-Periodic Reports & & & & & & & $0.000074 * * *$ & 10.92 & 1.18 & & & \\
\hline Periodic Reports - MS & & & & & & & & & & $0.000581 * * *$ & 4.32 & 1.65 \\
\hline Non-Periodic Reports - MS & & & & & & & & & & $0.000413 * * *$ & 15.51 & 1.52 \\
\hline Stock Return Volatility & $0.187470 * * *$ & 20.34 & 2.11 & $0.179530 * * *$ & 19.57 & 2.11 & $0.183650 * * *$ & 19.90 & 2.12 & $0.179140 * * *$ & 19.51 & 2.12 \\
\hline Well-performing Company & $-0.002330 * * *$ & -4.84 & 1.03 & $-0.002160 * * *$ & -4.53 & 1.03 & $-0.002380 * * *$ & -4.96 & 1.03 & $-0.002130 * * *$ & -4.45 & 1.03 \\
\hline Size & $-0.000507 * * *$ & -3.07 & 2.17 & $-0.000382 * *$ & -2.43 & 1.99 & $-0.000506^{* * *}$ & -3.07 & 2.17 & $-0.000442 * * *$ & -2.69 & 2.18 \\
\hline Book-to-Market Ratio & -0.000036 & -0.27 & 1.05 & 0.000034 & 0.26 & 1.05 & -0.000033 & -0.25 & 1.05 & 0.000028 & 0.21 & 1.05 \\
\hline Leverage & 0.000008 & 1.57 & 1.00 & 0.000006 & 1.34 & 1.00 & 0.000008 & 1.57 & 1.00 & 0.000006 & 1.34 & 1.00 \\
\hline ROA & $-0.000208 * *$ & -2.22 & 1.02 & $-0.000184 * *$ & -1.97 & 1.02 & $-0.000204 * *$ & -2.18 & 1.02 & $-0.000185^{* *}$ & -1.99 & 1.02 \\
\hline Resource Sector & $0.003070 * * *$ & 5.76 & 1.25 & -0.000004 & -0.01 & 1.49 & $0.002580 * * *$ & 4.78 & 1.28 & 0.000406 & 0.61 & 1.98 \\
\hline Financial Sector & $-0.005510 * * *$ & -7.47 & 1.22 & $-0.003990 * * *$ & -5.47 & 1.21 & $-0.004660 * * *$ & -6.21 & 1.27 & $-0.003970 * * *$ & -5.44 & 1.21 \\
\hline Year Control & $\mathrm{Y}$ & & & $\mathrm{Y}$ & & & $\mathrm{Y}$ & & & $\mathrm{Y}$ & & \\
\hline F Value & 87.74 & & & 97.64 & & & 85.12 & & & 92.59 & & \\
\hline R-Square & $12.08 \%$ & & & $13.26 \%$ & & & $12.33 \%$ & & & $13.27 \%$ & & \\
\hline Adj R-Sq & $11.94 \%$ & & & $13.12 \%$ & & & $12.19 \%$ & & & $13.13 \%$ & & \\
\hline
\end{tabular}

Note: Dependent variable is the modified deflated timeliness metric (DeflatedMc'). Independent variable is the announcement frequency (AnnFre), which is tested in different announcement groups: 'All Announcements' is the total number of announcements released per firm-year, 'All Announcements - MS' is the number of the market-sensitive announcements released per firm-year; 'Periodic Reports' is the frequency of the specific type of announcement - Periodic Reports - released by a firm during a year, based on the 19 main announcement categories (refer to equation 5-11 and Tables 5-2, 5-3); and 'Non-Periodic Reports' is the number of announcements released in other 18 categories per firm-year.

$*, * *$ and $* * *$ denote significance at the $10 \%, 5 \%$ and $1 \%$ levels respectively (two-tailed). 
Table 7-12. The Impact of Different Non-periodic Announcement Frequency on the Timeliness of Price Discovery

\begin{tabular}{|c|c|c|c|c|c|c|}
\hline \multirow{2}{*}{ Variable } & \multicolumn{3}{|c|}{ Panel A: Announcement Frequency of Each Category } & \multicolumn{3}{|c|}{ Panel B: Market-sensitive Announcement Frequency of Each Category } \\
\hline & Coeff & $t$-stat & VIF & Coeff & $t$-stat & VIF \\
\hline Intercept & $0.012450 * * *$ & 8.71 & 0.00 & $0.014470 * * *$ & 10.48 & 0.00 \\
\hline Takeover/Scheme Announcements & 0.000012 & 0.18 & 1.09 & 0.000213 & 1.62 & 1.03 \\
\hline Security Holder Details & $0.000120 * * *$ & 4.79 & 1.48 & -0.000322 & -0.52 & 1.04 \\
\hline Quarterly Activities Report & $0.000419 *$ & 1.73 & 4.76 & 0.000163 & 0.60 & 5.31 \\
\hline Quarterly Cash Flow Report & $-0.000450 * *$ & -2.35 & 1.49 & $-0.000413 * *$ & -2.10 & 1.49 \\
\hline Issued Capital & -0.000008 & -0.55 & 1.17 & $0.000528 * * *$ & 3.52 & 1.25 \\
\hline Asset Acquisition \& Disposal & 0.000055 & 0.46 & 1.13 & 0.000078 & 0.50 & 1.12 \\
\hline Notice of Meeting & $0.000822 * * *$ & 5.66 & 1.23 & $0.005050 * * *$ & 3.55 & 1.02 \\
\hline ASX Announcement & $0.001890 * * *$ & 15.65 & 1.27 & $0.002230 * * *$ & 15.59 & 1.30 \\
\hline Distribution Announcement & $-0.000506^{* * *}$ & -3.41 & 1.49 & $-0.000659 *$ & -1.93 & 1.29 \\
\hline Progress Reports & $0.000050 * *$ & 2.08 & 1.47 & $0.000121 * * *$ & 2.99 & 1.54 \\
\hline Company Administration & $0.000375 * * *$ & 3.37 & 1.25 & $0.001470 * * *$ & 2.93 & 1.12 \\
\hline Notice of Call & -0.000471 & -0.12 & 1.00 & 0.007170 & 0.70 & 1.00 \\
\hline Other & 0.000023 & 0.16 & 1.44 & 0.000253 & 0.68 & 1.12 \\
\hline Chairman's Address & -0.000085 & -0.30 & 1.18 & 0.000585 & 0.70 & 1.08 \\
\hline Letter to Shareholders & -0.000469 & -1.02 & 1.11 & 0.005970 & 1.20 & 1.00 \\
\hline ASX Query & $0.003070 * * *$ & 10.00 & 1.14 & $0.003980 * * *$ & 11.48 & 1.11 \\
\hline Structured Products & -0.000006 & -0.41 & 1.08 & -0.020260 & -0.81 & 1.00 \\
\hline CTE Quarterly Reports & -0.000049 & -0.27 & 1.45 & -0.000106 & -0.55 & 1.47 \\
\hline Stock Return Volatility & $0.158360 * * *$ & 17.05 & 2.25 & $0.162390 * * *$ & 17.48 & 2.24 \\
\hline Well-performing Company & $-0.002330 * * *$ & -4.94 & 1.04 & $-0.002300 * * *$ & -4.86 & 1.04 \\
\hline Size & $-0.000539 * * *$ & -2.97 & 2.75 & $-0.000392 * *$ & -2.38 & 2.26 \\
\hline Book-to-Market Ratio & 0.000080 & 0.62 & 1.06 & 0.000128 & 0.99 & 1.06 \\
\hline Leverage & $0.000008^{*}$ & 1.81 & 1.00 & $0.000008^{*}$ & 1.70 & 1.00 \\
\hline ROA & -0.000139 & -1.52 & 1.02 & -0.000149 & -1.62 & 1.02 \\
\hline Resource Sector & 0.000063 & 0.07 & 3.95 & 0.000346 & 0.36 & 4.16 \\
\hline Financial Sector & $-0.002740 * * *$ & -3.53 & 1.42 & $-0.003340 * * *$ & -4.29 & 1.42 \\
\hline Year Control & $\mathrm{Y}$ & & & $\mathrm{Y}$ & & \\
\hline F Value & 65.41 & & & 63.34 & & \\
\hline R-Square & $16.63 \%$ & & & $16.18 \%$ & & \\
\hline Adj R-Sq & $16.37 \%$ & & & $15.93 \%$ & & \\
\hline
\end{tabular}


Table 7-11 Panel A shows that both the announcement frequency and the market-sensitive announcement frequency are positively associated with the deflated timeliness metric, indicating that an increased number of announcements and market-sensitive announcements slow the timeliness of price discovery. Panel B reveals that only the Periodic Report frequency can accelerate the timeliness of price discovery to some extent. This is because Periodic Reports can reveal the company's detailed financial status and operation performance through, for example, half-yearly reports, preliminary final reports and annual reports. The market-sensitive Periodic Reports show a significant positive coefficient, suggesting an opposite influence on the timeliness of price discovery. It may be caused by the limited number of market-sensitive Periodic Reports per firm-year. According to the statistical summary in Table 7-4, a quarter (25\%) of the firm-year observations have no market-sensitive Periodic Reports, and threequarters $(75 \%)$ have fewer than three market-sensitive Periodic Reports.

Table 7-12 Panel A reveals that the more Quarterly Cash Flow Reports and Distribution Announcements are released, the faster is the price discovery process. The Quarterly Cash Flow Reports provide investors with more frequent cash flow information, which may help them make accurate judgements on the company's performance. Distribution Announcements can imply a company's earnings information, as only the companies with good profits are able to pay investors dividends. Distribution Announcements could contain value-relevant information to help investors better understand the company's performance. As shown in Panel B, when looking at the market-sensitive Quarterly Cash Flow Reports and Distribution Announcements, the results show that the market-sensitive announcement frequency in these two categories also can promote the timeliness of price discovery. In addition, less timely price discovery is associated with Quarterly Activities Reports, Issued Capital, Progress Reports, some administrative announcements (Security Holder Details, Notice of Meeting, and Company 
Administration) and Announcements from ASX (i.e. ASX Announcements and ASX Queries), because significantly positive coefficients are observed.

Progress Reports and Issued Capital have negative impacts on the timeliness of price discovery, though the impact of Issued Capital is significant only under the market-sensitive announcement subsample. A probable explanation is that companies with these two types of announcements have share prices that tend to fluctuate more. This is supported by the significant immediate market reactions described in Chapter Six. Based on the equation of the timeliness metric, more price fluctuation is likely to influence the timeliness metric value, making it a larger number. This is also supported by the positive coefficient of stock return volatility, suggesting that companies with more volatile stock prices have slower price discovery. The earnings information of these companies is more difficult to be anticipated by share prices. To some extent, the results indicate that Progress Reports and Issued Capital fail to signal the market on the 'true' firm value in the long-term. It is possible that investors' expectations (either optimistic or pessimistic) on firm value is influenced significantly by the information conveyed in Progress Reports and Issued Capital, and when the earnings announcements are available, investors may find the earnings information different from their expectations.

The positive coefficient results could also be caused by the nature of the companies. Companies with quarterly reports are in a special sector or have special characteristics. Companies in the resource sector are required to release Quarterly Activity Reports as that sector is a special sector with higher risk. The positive coefficient of Quarterly Activities Reports may indicate that the timeliness of price discovery is slow for the companies in this sector. Before dividing the Non-periodic announcements into detailed categories, Table 7-11 also shows positive 
coefficient for the resource sector variable, indicating that the timeliness of price discovery is relatively slow in this sector. Companies that receive ASX Announcements or ASX Queries are probably the companies that are likely to have something abnormal happening and/or have disclosure problems. As a result, the ASX may apply a trading halt (or suspension) or send more query letters to the company.

With respect to administrative announcements, Security Holder Details, Notice of Meeting, and Company Administration, the results show that these announcements have negative impacts on the timeliness of price discovery, slowing the process by which value-relevant information is incorporated into share prices. These three types of announcements may show that the companies have an event happening, which could affect investors' investment decisions. For example, more announcements regarding Security Holder Details may show frequent ownership changes, which may influence investors' judgements on share prices. But because the coefficient of Security Holder Details is smaller than other significant coefficients, and the market-sensitive announcement frequency of Security Holder Details is insignificant, the impact of Security Holder Details on the timeliness of price discovery is minor. The Notice of Meeting may be issued when companies need to engage the shareholders over a matter, which necessitates the company calling an extraordinary general meetings to discuss it further. The announcements on Company Administration may change investors' impressions of the company, such as the announcements related to director appointment or resignation (especially directors with good/bad reputations). More administrative activities may imply that the company is making changes, which may influence investors' judgements as to its firm value and make earnings information difficult to anticipate. 
In terms of the announcement categories that show insignificant results, Table7-12 indicates that the following types of announcements have no impact on the timeliness of price discovery: Takeover/Scheme Announcements, Asset Acquisition \& Disposal, Notice of Call, Other, Chairman's Address, Letter to Shareholders, Structured Products, and CTE Quarterly Reports. They have relatively low announcements frequency of occurrence on average. Except for Asset Acquisition \& Disposal with a mean above one (from Table 7-5: 1.388 total number, 1.011 market-sensitive), other categories have less than one announcement released on average per firm-year, which could be a reason for their insignificant effects on the timeliness of price discovery. It is not surprising that administrative announcements (Notice of Call, Chairman's Address, Letter to Shareholders and Structured Products) have no impact on the timeliness because they convey little value-relevant information. The Chairman's Address and Letter to Shareholders often provide a summary of the company's performance and/or operations which are already available to the market. The investment/project-related announcements ${ }^{47}$ may need further exploration in future research. According to the correlation matrix in Tables 7-8 and 79, these announcements are slightly correlated with the deflated timeliness metric, especially Other shows a significantly negative correlation (-0.023) between the number of its total announcement frequency and the timeliness of price discovery. Future analysis could investigate the content of an announcement to measure the informativeness. ${ }^{48}$

Opposite to the unfavourable coefficients from announcement frequency for different announcement groups or categories, some control variables show favourable effects on the timeliness of price discovery. This is consistent with prior literature (Beekes \& Brown, 2006; Beekes et al., 2015; Coulton et al., 2016). ${ }^{49}$ The price discovery process is faster for companies

\footnotetext{
47 The investment/project-related announcements are Takeover/Scheme Announcements, Asset Acquisition \& Disposal and Other.

48 This study did not look at the announcement content due to the big sample size and limited time.

49 Prior literature did not control financial sector.
} 
with good stock price performance, which is when stock prices perform better than the market index over the year. For larger and more profitable companies, value-relevant information can be incorporated into share prices in a more timely fashion. Regarding the sector impact on the timeliness of price discovery, the results suggest that financial companies have timelier price discoveries, while resource companies have less timely price discoveries. This sector impact is clearly shown in Figure 7-6 which presents the average deflated timeliness metric for the financial sector, resource sector and non-resource/financial sector from 2005 to 2014. The financial sector is the top line while the resource sector is the bottom line. Figure 7-6 also reveals that the GFC slowed the timeliness of price discovery, especially for the financial sector.

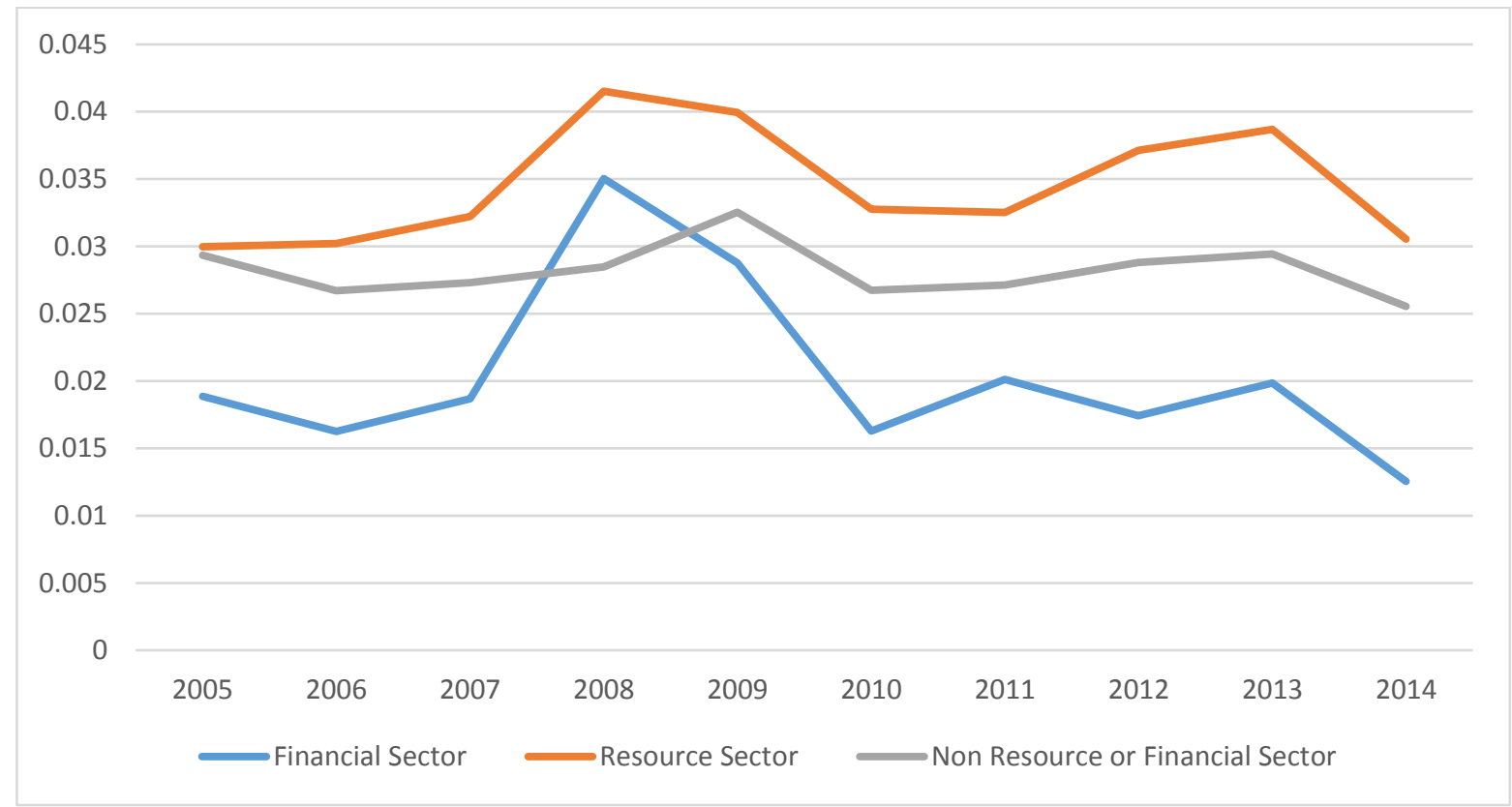

Figure 7-6. The Average Deflated Timeliness Metric for Different Sectors by Year

\subsection{Robustness Tests on the Timeliness of Price Discovery}

This study uses the original Beekes and Brown's (2006) deflated timeliness metric as the dependent variable to re-test the regression models. The robustness tests allow a 10 trading day 'settle down' period for the price discovery process. The results from the robustness tests are 
consistent with the main findings: the details of the results are provided in the Appendix V to this thesis. Comparing the $\mathrm{R}$ squares in the robustness tests with those in the main results, the models with modified timeliness metric are more advantageous with larger $\mathrm{R}$ squares, indicating higher explanatory power. This study also re-tests the models in different industry sectors based on the GICS (Global Industry Classification Standard, 11 sectors), and with different size levels (4 levels according to the Quartiles of firm size) ${ }^{50}$ The results are slightly different between subsamples, but not significantly different to the main findings.

\subsection{Summary}

This chapter has further explored the market effects of Preliminary Final Reports, indicating that bad news tend to be released after the market closes and/or on Fridays. It then presented the descriptive statistics, correlation matrixes and regression results from testing the impacts of announcement frequency on the timeliness of price discovery. Unfortunately, the findings do not confirm that more announcements released on the market could promote the timeliness with which the value-relevant (i.e. earning/income) information is incorporated into share prices before the Preliminary Final Reports are released. Following the step 'All announcements Periodic/Non-periodic announcements - Detailed categories of Non-periodic announcements', the findings reveal that only earnings-related announcements, Periodic Reports and Distribution Announcements show positive impacts on the timeliness of price discovery. The next chapter concludes this study: it provides a brief summary and discussions on the key findings and outlines this study's contributions, limitations and future research directions.

\footnotetext{
${ }^{50}$ As there are too many tables, the results for different industries and size levels will be provided on request but are not included in the Appendix to this thesis.
} 


\section{CHAPTER EIGHT: CONCLUSION}

This last chapter provides a summary of the research described in this thesis. In Section 8.1 it presents a brief summary of the findings, outlines the contributions in Section 8.2, and discusses the limitations and future research directions in Section 8.3.

\subsection{Summary of the Thesis}

This study was motivated to enable a better understanding of the contradiction between an efficient market and a company's opportunistic disclosure behaviour. However, the Australian CDR should constrain such behaviour. Therefore, under the CDR, it is possible to evaluate in depth the market effects of corporate disclosures in the short and the long term. Specifically, this study aims to answer the following Research Questions. (1) How market (price/volume) sensitive is the information contained in each disclosure category in a continuous disclosure environment? (2) How quickly is the value-relevant information incorporated into share prices before the annual earnings announcement is released, and how can company announcements promote this timeliness of price discovery? Two sets of hypotheses were developed accordingly to address the Research Questions. The first hypothesis is tested by exploring the immediate market reactions to different types of announcements, and the second hypothesis is tested by exploring the impacts of announcement frequency as to different announcement categories on the timeliness of price discovery. The market reacts differently to different types of information, supporting Hypothesis 1. But in terms of Hypothesis 2, positive coefficients are observed for most announcement categories, indicating that announcement frequency is associated with the less timely price discovery, which does not confirm that more announcements on the market could promote the timeliness of price discovery. 


\subsubsection{Immediate Market Reaction to Different Announcements}

The results obtained from the immediate market reaction tests identify seven announcement categories that can induce significant share price responses: ASX Query, Progress Reports, Asset Acquisition \& Disposal, Distribution Announcements, Other, Periodic Reports and Issued Capital. According to the regression results for the market-sensitive announcements, ASX Query is the only announcement category that causes immediate negative market responses. There is positive association between intraday CARs and the release of the other six types of announcements. Share prices react the most to Progress Reports and Asset Acquisition \& Disposal, and the least to Periodic Reports and Issued Capital. Distribution Announcements and Other are in the middle. The GFC had significant impact on Asset Acquisition \& Disposal and Issued Capital. Asset Acquisition \& Disposal had the largest positive price responses during the GFC and Issued Capital announcements caused significant positive price responses during that time. A possible reason is Asset Acquisition \& Disposal and Issued Capital announcements might imply fewer or no liquidity problems in companies and there might be potential cash inflows to the company by disposing assets or issuing new capital. This conclusion is based on the fact that there are two possible reasons for the different market reaction magnitudes: information materiality and information predictability. Share prices are sensitive to information: the more important information could cause larger share price responses. Share prices may react more to unexpected 'one-off' announcements than to expected 'routine' announcements.

After taking good and bad news into account, this study further explores and compares the magnitude of positive/negative share price reactions to different announcement categories. In the bad news subsamples, the CARs are significantly negatively associated with the release of 
ASX Query, Issued Capital, Periodic Reports and Progress Reports regardless of the GFC impact. Acquisition \& Disposal during the GFC caused small negative market responses. In the good news subsamples, the share prices react positively to Progress Reports, Asset Acquisition \& Disposal, Periodic Reports, Distribution Announcements, Issued Capital, Other and ASX Query regardless of the GFC impact. The announcements are ranked according to the magnitude of price responses. Combining the results of good and bad news, the immediate market reactions show the nature of the information which is conveyed in different types of announcements. For example, Progress Reports Asset Acquisition \& Disposal, Distribution Announcements, and Other indicate good signs for the market during trading hours. There are consistent positive price reactions to good news but small or no significant negative price reactions to bad news after these announcements are released. Share prices could react to Periodic Reports and Issued Capital both negatively and positively, indicating that either good news or bad news could be contained in these announcements. Finally ASX Query is part of ASX's enforcement practices regarding CDR compliance. When a company experiences abnormal share price increase (decrease), an ASX Query announcement may induce price decrease (increase) to correct the previous abnormal price movements.

In terms of the trading volume reactions, significant ATVs are observed after the release of four types of announcements: Progress Reports, Asset Acquisition \& Disposal, Issued Capital and Periodic Reports, ranking from the largest to the smallest trading volume reactions. After separating good and bad news subsamples, the results show that Progress Reports tend to indicate good signs to the market and cause large trading volumes in general. Asset Acquisition \& Disposal caused significant trading activity increase only during the GFC. Periodic Reports and Issued Capital could contain either good or bad news, but the trading volumes are driven 
by bad news. This could be because investors tend not to trade (at least not to trade immediately) when positive information (especially good earnings news) is released.

The results also reveal that company size is an important factor that influences the intraday market reactions to disclosures. Larger companies tend to have smaller market responses after the information is released, which confirms the likely stability of larger companies. But it is interesting to notice that size has no significant impact on ATVs when bad news is released, which may indicate that investors trade on bad news without taking size into account.

\subsubsection{The Impacts of Announcement Frequency on the Timeliness of Price Discovery}

This study assumes that when the annual earnings information becomes available to the market, the share price can reflect the 'true' value of the company based on its performance over the fiscal year, which is the basis of calculating the timeliness of price discovery. Given the importance of the Preliminary Final Reports and the fact that the immediate market reaction tests only cover announcements released during trading hours, this study investigates in depth the Preliminary Final Reports to further explore the association between corporate disclosure behaviour and its market consequences. Descriptive analysis shows that more good news earnings announcements are released in the morning, while bad news earnings announcements are more often released after the market closes. The results from the ANOVA tests further confirm that the market-adjusted returns around Preliminary Final Reports released post trading hours are significantly less than those released pre- and during trading hours. Regarding release days, bad news earnings announcements are more often released on Fridays, but ANOVA tests find evidence that abnormal returns of earnings announcements released on Fridays are only 
significantly lower than those released on Wednesdays and Thursdays. Therefore, the time when the announcement is released matters more than the release day.

In terms of the timeliness of price discovery test, the results do not confirm that more announcements on the market could promote the timeliness with which the value-relevant (i.e. earning/income) information is incorporated into share prices. Only earnings-related and cash flow announcements show significant positive impacts on the timeliness of price discovery. In other words, Periodic Reports, Distribution Announcements and Quarterly Cash Flow Reports can help stock prices anticipate companies' annual income information before the Preliminary Final Reports are available to the market. However, Issued Capital and Progress Report announcements, which related to investments or projects may slow the price discovery process. These announcements may influence investors' expectations (either optimistic or pessimistic) on company market value, and when the earnings announcements are available, investors may find the earnings information different from their expectations. ASX Announcements and ASX Queries have negative impacts on the timeliness of price discovery, which also reveals that there are disclosure problems in queried companies. In addition to announcement frequency influences, the price discovery process is faster for companies with better share performance, are larger or have greater profitability. Financial companies have timelier price discovery, while resource companies have less timely price discovery. The GFC had a negative impact on the timeliness of price discovery, especially for financial companies. 


\subsection{Contributions}

This study makes several contributions to the literature on the market effects of corporate disclosures. This is the first study that tests the market sensitivity of company announcements under the Australian CDR, and compares the immediate market reactions to different types of information. The intraday market reaction examination reveals the extent to which share prices react to different corporate disclosures within three hours. This study also contributes to the market literature by covering all categories of company announcements, and comparing the market consequences for each type of announcement.

Further, the findings provide evidence on how different information affects share prices and investors' trading behaviour after taking good and bad news into account. This study has revealed that unexpected announcements, such as Progress Reports and ASX Queries, are likely to induce larger share price responses than the expected Periodic Reports. Investors tend to trade immediately when bad news is released through Issued Capital or Periodic Reports, but tend to hold their shares (at least not to trade immediately) when good news is released. This study contributes to literature on both market efficiency and market microstructure by exploring the role of public information in price formation and trading behaviour changes over a short time (measured in minutes and hours) around the information release.

Moreover, the timeliness of price discovery test suggests which type of announcement promotes the timeliness of price discovery, and which type does not. The findings do not confirm the expected result that more announcements on the market could promote the share price to anticipate income information in a timelier manner (i.e. earlier) before the earnings announcements are available to the market. However, this study provides a new perspective on 
the relationship between corporate disclosures and the timeliness of price discovery, which is whether the disclosures, especially non-periodic announcements, could reveal the companies' operational performances, or 'mislead' investors' expectations away from their 'real' performance. It contributes to the timeliness of price discovery literature by linking the announcement frequency of each disclosure category to the timeliness with which annual earnings information is incorporated into share prices.

This study also shows how well the ASX 'market sensitivity tag' operates, building upon the empirical literature on continuous disclosure. There is also a paper by Brown et al. (2006) on how correctly the ASX gets it right on the 'market sensitivity tag', which found greater absolute price changes around market-sensitive announcements than non-market-sensitive ones. This study further examines the importance of announcement types and takes good/bad news into account instead of using the absolute value of price changes. The results from the immediate market reaction tests show that the market reacts strongly to market-sensitive announcements in project- or earnings-related announcement categories but not the administrative announcement categories. This suggests that the CDR applies the market-sensitive label conservatively; that is, the ASX is more likely to label a non-market-sensitive disclosure as being market-sensitive than to label a market-sensitive document as non-market-sensitive. The findings from the timeliness of price discovery tests provide a new perspective on whether it is a good sign to the market if a company releases more market-sensitive announcements. For instance, more market-sensitive announcements may imply that the company has more important projects in process, or, on the other hand, that it faces more problems or difficulties. Investors may either over- or under-react to the information conveyed by those market-sensitive announcements. As a result, investors' expectations on firm value might be 'distorted' by the market-sensitive information, slowing the timeliness of price discovery. 
Lastly, the findings of this study provide some implications for market regulators, investors and internal managers. The findings can help the Australian market regulators review their 'marketsensitive classification' and 'announcement categorisation' systems, to promote company compliance with the CDR. For example, a Progress Report is the announcement category that could induce the largest immediate market reactions, but it has a vague definition and nonspecific subcategories (as shown in Table 4-1). From this study, investors could understand the importance of different announcements, and predict the immediate share price movements according to the announcement types. In addition, this study finds some evidence of companies' strategic timing of good/bad news releases: good news tends to be released more often in the early morning. All this should help them make decisions. Internal managers could gain a better understanding of investors' trading behaviour concerning different information release types, which may help them better comply with the CDR.

\subsection{Limitations and Future Research Suggestions}

There are several limitations in this study, which could provide directions for future research.

First, while many company announcements on the ASX are categorised into more than one announcement category, this study codes the announcements by the first announcement category. Company announcements can be categorised into up to ten subcategories. If an announcement is classified into a few subcategories that lie within the same primary category, it should not have an impact on this study. Sometimes, an announcement is classified into two or more primary categories: this study does not capture the impacts of this multi-categorisation. For future research, categorisation could provide a better and more specific understanding of 
the announcement effects if the research focuses on one type of announcement and the content of the announcement, instead of simply classifying announcements by report type code. For example, separating the Takeover/Scheme announcements for bidders and targets, it could further investigate the information asymmetry or leakage before the announcement.

Secondly, this study does not control for the influences of trading halts. If a stock experiences a trading halt lasting for one hour during trading hours, the stock price is missing or does not change during that hour. This study does not distinguish between this stock and others due to the large sample size. Furthermore, more important announcements are potentially accompanied by a longer trading halt, but this study only captures the immediate market reactions to the announcements that do not induce longer trading halts, so the results may not be generalised for all announcements. Future research could focus on the impact of longer trading halts on market reactions to disclosures. This impact might be either favourable or unfavourable because trading halts can cause low liquidity and high price volatility (Frino et al., 2011). Similar to trading halt effects, it is difficult to measure the impacts of off-hour announcements on share prices, especially when more than one type of announcements are released during off hours, so the research sample only includes trading-hour announcements. This study focuses on the announcement category impact to evaluate the immediate market reactions; therefore, it does not control the influences of overlapping periods on abnormal returns. Future research could further examine whether or not release time can make a difference, not only focusing on the difference between trading- and off- hour announcements, but also more specifically, for example, dividing announcements by (non-overlapping) hours.

A third restriction on this research is regarding the measurements of announcement informativeness and the timeliness of price discovery. When testing the association between 
the timeliness of price discovery and company announcements, the number of announcements may not be a good proxy to represent informativeness. A greater number of announcements does not necessarily mean the information is more useful. In future research, it would be of importance to refine the measurement for announcement informativeness or usefulness: for example, reading the content of announcements, or applying the textual analysis to measure the informativeness of different announcements. As to the timeliness metric, the existing measurements in literature are based on the adjust share prices or the analyst forecast accuracy. However, for future research, it would be essential to develop a duration metric to measure the timeliness of price discovery, which could provide a more straightforward way to estimate the length of price discovery process. 


\section{REFERENCES}

Adams, R. B., Gray, S., \& Nowland, J. (2011). Does gender matter in the boardroom? Evidence from the market reaction to mandatory new director announcements. Available at https://papers.ssrn.com/sol3/papers.cfm?abstract_id=1953152.

Aitken, M., \& Comerton-Forde, C. (2005). Do reductions in tick sizes influence liquidity? Accounting \& Finance, 45(2), 171-184.

Aitken, M., Comerton-Forde, C., \& Frino, A. (2005). Closing call auctions and liquidity. Accounting \& Finance, 45(4), 501-518.

Akhtar, S. (2015). Dividend payout determinants for Australian multinational and domestic corporations. Accounting \& Finance, available at http://onlinelibrary.wiley.com/doi/10.1111/acfi.12137/epdf.

Akyol, A. C., \& Foo, C. C. (2013). Share repurchase reasons and the market reaction to actual share repurchases: Evidence from Australia. International Review of Finance, 13(1), 137.

Alford, A., Jones, J., Leftwich, R., \& Zmijewski, M. (1993). The relative informativeness of accounting disclosures in different countries. Journal of accounting research, 183-223.

Amihud, Y. (2002). Illiquidity and stock returns: cross-section and time-series effects. Journal of financial markets, 5(1), 31-56.

Amihud, Y., \& Mendelson, H. (1986). Asset pricing and the bid-ask spread. Journal of financial Economics, 17(2), 223-249.

Amihud, Y., Mendelson, H., \& Lauterbach, B. (1997). Market microstructure and securities values: Evidence from the Tel Aviv Stock Exchange. Journal of financial Economics, 45(3), 365-390.

ASIC. (2004-2014 ). ASIC Media Release. Retrieved from http://asic.gov.au/aboutasic/media-centre/find-a-media-release/?filter=2014\&financialmarkets=on

ASX. (2014). ASX ComNews ${ }^{\mathrm{TM}}$ Manual - Real-time company announcements, direct from the source. from SIRCA

ASX Listing Rules, (2016).

ASX Listing Rules Guidance Note 8 - Continuous Disclosure: Listing Rule 3.1 - 3.1B.

ASX Listing Rules Guidance Note 14 - ASX Market Announcements Platform.

ASX Listing Rules Guidance Note 23 - Quarterly Cash FLow Reports.

Atiase, R. K., Li, H., Supattarakul, S., \& Tse, S. (2005). Market reaction to multiple contemporaneous earnings signals: Earnings announcements and future earnings guidance. Review of Accounting Studies, 10(4), 497-525. 
Au Yong, H. H., Brown, C., \& Ho, C. C. Y. (2014). Off-Market Buybacks in Australia: Evidence of Abnormal Trading around Key Dates. International Review of Finance, 14(4), 551-585.

Australian Securities and Investments Commission Act 2001, https://www.legislation.gov.au/Details/C2016C01095.

Ayres, I., \& Braithwaite, J. (1992). Responsive Regulation-Transcending the Deregulation Debate. New York, United States: Oxford University Press.

Ball, R., \& Brown, P. (1968). An empirical evaluation of accounting income numbers. Journal of accounting research, 159-178.

Ball, R., \& Shivakumar, L. (2008). How much new information is there in earnings? Journal of accounting research, 46(5), 975-1016.

Barclay, M. J., Holderness, C. G., \& Sheehan, D. P. (2007). Private placements and managerial entrenchment. Journal of Corporate Finance, 13(4), 461-484.

Battalio, R. H., Lerman, A., Livnat, J., \& Mendenhall, R. R. (2012). Who, if anyone, reacts to accrual information? Journal of accounting and economics, 53(1), 205-224.

Beaver, W. H. (1968). The information content of annual earnings announcements. Journal of accounting research, 67-92.

Beaver, W. H., Clarke, R., \& Wright, W. F. (1979). The association between unsystematic security returns and the magnitude of earnings forecast errors. Journal of accounting research, 316-340.

Beekes, W., \& Brown, P. (2006). Do Better-Governed Australian Firms Make More Informative Disclosures? Journal of Business Finance \& Accounting, 33(3-4), 422-450.

Beekes, W., Brown, P., \& Zhang, Q. (2015). Corporate governance and the informativeness of disclosures in Australia: a re-examination. Accounting \& Finance, 55(4), 931-963.

Bhattacharya, S. (1979). Imperfect information, dividend policy, and "the bird in the hand" fallacy. Bell journal of economics, 10(1), 259-270.

Bloch, M., Weatherhead, J., \& Webster, J. (2011). The Development and Enforcement of Australia's Continuous Disclosure Regime'(2011). Company and Securities Law Journal, 29, 253.

Boros, E. (2009). Public and private enforcement of disclosure breaches in Australia. Journal of Corporate Law Studies, 9(2), 409-438.

Brennan, M. J., Chordia, T., Subrahmanyam, A., \& Tong, Q. (2012). Sell-order liquidity and the cross-section of expected stock returns. Journal of financial Economics, 105(3), 523-541.

Brennan, M. J., \& Subrahmanyam, A. (1996). Market microstructure and asset pricing: On the compensation for illiquidity in stock returns. Journal of financial Economics, 41(3), 441-464. 
Brown, C. (2007). The announcement effects of off-market share repurchases in Australia. Australian Journal of Management, 32(2), 369-385.

Brown, C., \& Davis, K. (2012). Taxes, tenders and the design of Australian off-market share repurchases. Accounting \& Finance, 52(s1), 109-135.

Brown, C., \& Norman, D. (2010). Management choice of buyback method: Australian evidence. Accounting \& Finance, 50(4), 767-782.

Brown, P., Finn, F. J., \& Hancock, P. (1977). Dividend changes, earnings reports, and share prices: Some australian findings. Australian Journal of Management, 2(2), 127-147.

Brown, P., Howitt, B., \& Wee, M. (2005). Order Flow and Price Effects Surrounding an ASX Announcement. Paper presented at the Accounting and Finance Association of Australia and New Zealand Conference.

Brown, P., Kwan, R., \& Wee, M. (2006). Investors' order placement strategies around the time of a market sensitive announcement. Working paper. University of Western Australia, Perth, Western Australia. Available at SSRN: https://ssrn.com/abstract=2188744.

Brown, P., Ng, C. K., \& Wee, M. (2003). An Examination of the ASX Announcement Process and Information Pricing Policy. Working paper. University of Western Australia.

Brown, P., Taylor, S. L., \& Walter, T. S. (1999). The impact of statutory sanctions on the level and information content of voluntary corporate disclosure. Abacus, 35(2), 138-162.

Bruner, R. F. (2002). Does M\&A pay? A survey of evidence for the decision-maker. Journal of Applied Finance, 12(1), 48-68.

Burkart, M., \& Panunzi, F. (2006). Takeovers. ECGI - Finance Working Paper No. 118/2006. Available at SSRN: https://papers.ssrn.com/sol3/papers.cfm?abstract_id=884080.

Bushman, R. M., Smith, A. J., \& Wittenberg-Moerman, R. (2010). Price discovery and dissemination of private information by loan syndicate participants. Journal of accounting research, 48(5), 921-972.

Butler, M., Kraft, A., \& Weiss, I. S. (2007). The effect of reporting frequency on the timeliness of earnings: The cases of voluntary and mandatory interim reports. Journal of accounting and economics, 43(2), 181-217.

Campa, J. M., \& Hernando, I. (2004). Shareholder value creation in European M\&As. European financial management, 10(1), 47-81.

Chan, H., Faff, R., Ho, Y. K., \& Ramsay, A. (2007). Management earnings forecasts in a continuous disclosure environment. Pacific Accounting Review, 19(1), 5-30.

Chan, R. S. Y., \& Ho, K. S. J. (2011). Price Sensitive Information Disclosure by Listed Corporations in Hong Kong: Lessons and Experiences from Australia. Australian Journal of Corporate Law, 26(1), 86-111. 
Chang, M., Lim, F., \& Wee, M. (2015). Effects of the Informativeness of Corporate Disclosures on Price Discovery. Paper presented at the The 2015 AFAANZ Conference, Hobart, Tasmania, Australia.

Chapple, L., \& Truong, T. P. (2015). Continuous disclosure compliance: does corporate governance matter? Accounting \& Finance, 55(4), 965-988.

Chen, S.-S., Chou, R. K., \& Lee, Y.-C. (2011). Bidders' strategic timing of acquisition announcements and the effects of payment method on target returns and competing bids. Journal of Banking \& Finance, 35(9), 2231-2244.

Chordia, T., Huh, S.-W., \& Subrahmanyam, A. (2009). Theory-based illiquidity and asset pricing. Review of Financial Studies, 22(9), 3629-3668.

Chordia, T., Roll, R., \& Subrahmanyam, A. (2002). Order imbalance, liquidity, and market returns. Journal of financial Economics, 65(1), 111-130.

Chordia, T., Roll, R., \& Subrahmanyam, A. (2008). Liquidity and market efficiency. Journal of financial Economics, 87(2), 249-268.

Chordia, T., \& Subrahmanyam, A. (2004). Order imbalance and individual stock returns: Theory and evidence. Journal of financial Economics, 72(3), 485-518.

Chordia, T., Subrahmanyam, A., \& Anshuman, V. R. (2001). Trading activity and expected stock returns. Journal of financial Economics, 59(1), 3-32.

Chung, D., \& Hrazdil, K. (2010). Liquidity and market efficiency: A large sample study. Journal of Banking \& Finance, 34(10), 2346-2357.

Comment, R., \& Jarrell, G. A. (1991). The relative signalling power of Dutch-auction and fixedprice self-tender offers and open-market share repurchases. The Journal of finance, 46(4), 1243-1271.

Competition and Consumer Amendment Bill (No. 1) 2011, http://parlinfo.aph.gov.au/parlInfo/download/legislation/bills/r4548_aspassed/toc_pdf/ 11053b01.pdf;fileType=application\%2Fpdf.

Corporate Law Economic Reform Program (Audit Reform and Corporate Disclosure) Act 2004, https://www.legislation.gov.au/Details/C2004A01334.

Corporations Act 2001, https://www.legislation.gov.au/Series/C2004A00818.

Corwin, S. A. (2003). The determinants of underpricing for seasoned equity offers. The Journal of finance, 58(5), 2249-2279.

Coulton, J. J., Dinh, T., \& Jackson, A. B. (2016). The impact of sentiment on price discovery. Accounting \& Finance, 56, 669-694.

D'mello, R., \& Shroff, P. K. (2000). Equity undervaluation and decisions related to repurchase tender offers: An empirical investigation. The Journal of finance, 55(5), 2399-2424. 
da Silva Rosa, R., Limmack, R., Supriadi, \& Woodliff, D. (2004). The equity wealth effects of method of payment in takeover bids for privately held firms. Australian Journal of Management, 29(1_suppl), 93-110.

da Silva Rosa, R., Nguyen, T., \& Walter, T. (2004). Market returns to acquirers of substantial assets. Australian Journal of Management, 29(1 suppl), 111-133.

Dann, L. Y. (1981). Common stock repurchases: An analysis of returns to bondholders and stockholders. Journal of financial Economics, 9(2), 113-138.

Dedman, E., Mouselli, S., Shen, Y., \& Stark, A. W. (2009). Accounting, intangible assets, stock market activity, and measurement and disclosure policy-views from the UK. Abacus, 45(3), 312-341.

deHaan, E., Shevlin, T., \& Thornock, J. (2015). Market (in) attention and the strategic scheduling and timing of earnings announcements. Journal of accounting and economics, 60(1), 36-55.

Donelson, D. C., McInnis, J. M., Mergenthaler, R. D., \& Yu, Y. (2012). The timeliness of bad earnings news and litigation risk. The Accounting Review, 87(6), 1967-1991.

Doyle, J. T., \& Magilke, M. J. (2009). The timing of earnings announcements: An examination of the strategic disclosure hypothesis. The Accounting Review, 84(1), 157-182.

Drienko, J., \& Sault, S. J. (2013). The intraday impact of company responses to exchange queries. Journal of Banking \& Finance, 37(12), 4810-4819.

Drienko, J., Sault, S. J., \& von Reibnitz, A. H. (2016). Company responses to exchange queries in real time. Pacific-Basin Finance Journal, http://dx.doi.org/10.1016/j.pacfin.2016.08.003.

Duffy, M. J. (2012). Testing good securities disclosure: tales of the reasonable investor. Monash University Law Review, 38(2), 25-54.

Dunstan, K., Gallery, G., \& Truong, T. P. (2011). Public regulatory reform and management earnings forecasts in a low private litigation environment. Accounting \& Finance, 51(2), 437-465.

Eckbo, B. E., Masulis, R. W., \& Norli, O. (2007). Security offerings. In B. E. Eckbo (Ed.), Handbook of corporate finance: Empirical corporate finance (Vol. 1, pp. 233-373). Amsterdam, The Netherlands: Elsevier, North-Holland.

Eleswarapu, V. R. (1997). Cost of transacting and expected returns in the Nasdaq market. The Journal of finance, 52(5), 2113-2127.

Fama, E. F. (1970). Efficient capital markets: A review of theory and empirical work. The Journal of finance, 25(2), 383-417.

Fama, E. F., \& French, K. R. (1993). Common risk factors in the returns on stocks and bonds. Journal of financial Economics, 33(1), 3-56. 
Ferguson, A., \& Scott, T. (2016). The determinants and market reaction to Open Briefings: an investor relations option and evidence on the effectiveness of disclosure. Accounting \& Finance, 56(3), 803-843.

Fernández, L. (2012). Price discovery, investor distraction and analyst recommendations under continuous disclosure requirements in Australia. University of Technology, Sydney.

Financial Services Reform Act 2001, https://www.legislation.gov.au/Details/C2005C00498.

Fisse, B., \& Beaton-Wells, C. (2011). The continualregulationofcontinuous disclosure: Informationdisclosureunder the CompetitionandConsumer Amendment Bill (No1) 2011. Competition \& Consumer Law Journal, 19, 127-151.

Florackis, C., Gregoriou, A., \& Kostakis, A. (2011). Trading frequency and asset pricing on the London Stock Exchange: Evidence from a new price impact ratio. Journal of Banking \& Finance, 35(12), 3335-3350.

Foster III, T. W., \& Vickrey, D. (1978). The information content of stock dividend announcements. Accounting Review, 360-370.

Francis, J., Schipper, K., \& Vincent, L. (2002). Expanded disclosures and the increased usefulness of earnings announcements. The Accounting Review, 77(3), 515-546.

Frijns, B., Gilbert, A., \& Tourani-Rad, A. (2008). Insider Trading, Regulation, and The Components of The Bid-Ask spread. Journal of Financial Research, 31(3), 225-246.

Frino, A., Lecce, S., \& Segara, R. (2011). The impact of trading halts on liquidity and price volatility: Evidence from the Australian Stock Exchange. Pacific-Basin Finance Journal, 19(3), 298-307.

Frino, A., Segara, R., \& Zheng, H. (2009). The Impact of Trade Characteristics on Stock Return Volatility: Evidence from the Australian Stock Exchange. Asia-Pacific Journal of Financial Studies, 38(2), 163-186.

Hammersley, J. S., Myers, L. A., \& Shakespeare, C. (2008). Market reactions to the disclosure of internal control weaknesses and to the characteristics of those weaknesses under section 302 of the Sarbanes Oxley Act of 2002. Review of Accounting Studies, 13(1), 141-165.

Hertzel, M., \& Smith, R. L. (1993). Market discounts and shareholder gains for placing equity privately. The Journal of finance, 48(2), 459-485.

Hirshleifer, D., Lim, S. S., \& Teoh, S. H. (2009). Driven to distraction: Extraneous events and underreaction to earnings news. The Journal of finance, 64(5), 2289-2325.

Hirshleifer, D. A., Lim, S. S., \& Teoh, S. H. (2004). Disclosure to an audience with limited attention. Available at SSRN: https://papers.ssrn.com/sol3/papers.cfm?abstract_id=604142.

Hoskin, R. E., Hughes, J. S., \& Ricks, W. E. (1986). Evidence on the incremental information content of additional firm disclosures made concurrently with earnings. Journal of accounting research, 1-32. 
Hsu, G. C. M. (2009). Impact of earnings performance on price-sensitive disclosures under the Australian continuous disclosure regime. Accounting \& Finance, 49(2), 317-339.

Huang, M., Marsden, A., \& Poskitt, R. (2009). The impact of disclosure reform on the NZX's financial information environment. Pacific-Basin Finance Journal, 17(4), 460-478.

Impson, C. M., \& Karafiath, I. (1992). A note on the stock market reaction to dividend announcements. Financial Review, 27(2), 259-271.

John, K., \& Williams, J. (1985). Dividends, dilution, and taxes: A signalling equilibrium. The Journal of finance, 40(4), 1053-1070.

Kim, Y., Li, S., Pan, C., \& Zuo, L. (2013). The role of accounting conservatism in the equity market: Evidence from seasoned equity offerings. The Accounting Review, 88(4), 13271356.

Kohlbeck, M. J., \& Magilke, M. J. (2002). The impact of concurrent conference calls on the information content of earnings announcements. Working paper. The University of Wisconsin. Available at https://papers.ssrn.com/sol3/papers.cfm?abstract_id=302230.

Kothari, S. (2001). Capital markets research in accounting. Journal of accounting and economics, 31(1), 105-231.

KWM. (2016). The review - class actions in Australia 2015/2016. Retrieved from King \& Wood Mallesons (KWM): http://www.kwm.com/en/knowledge/downloads/classactions-2015-2016-year-review-increased-threat-new-normal-20160826

Lam, K. S., \& Tam, L. H. (2011). Liquidity and asset pricing: Evidence from the Hong Kong stock market. Journal of Banking \& Finance, 35(9), 2217-2230.

Lamba, A. S., \& Ramsay, I. (2005). Comparing share buybacks in highly regulated and less regulated market environments. Australian Journal of Corporate Law, 17, 261-280.

Le, H. T., \& Schultz, E. (2007). Toeholds and the bidder shareholder wealth effects of takeover announcements. Australian Journal of Management, 32(2), 315-344.

Lee, B.-S. (1995). The response of stock prices to permanent and temporary shocks to dividends. Journal of Financial and Quantitative analysis, 30(01), 1-22.

Lee, G., \& Masulis, R. W. (2009). Seasoned equity offerings: Quality of accounting information and expected flotation costs. Journal of financial Economics, 92(3), 443-469.

Lerman, A., \& Livnat, J. (2010). The new Form 8-K disclosures. Review of Accounting Studies, 15(4), $752-778$.

Lev, B., \& Ohlson, J. A. (1982). Market-based empirical research in accounting: A review, interpretation, and extension. Journal of accounting research, 249-322.

Low, R., Burdon, M., \& von Nessen, P. (2010). Notification of data breaches under the continuous disclosure regime. Australian Journal of Corporate Law, 25(2), 70-100. 
Matolcsy, Z., Tyler, J., \& Wells, P. (2012). Is continuous disclosure associated with board independence? Australian Journal of Management, 37(1), 99-124.

Mayanja, J. (2010). Enhancing private enforcement of Australia's corporate continuous disclosure regime: why unshackling litigation funders makes eminent sense. Australian Journal of Corporate Law, 25(1), 48-69.

McMullin, J. L., Miller, B. P., \& Twedt, B. J. (2015). Increased Mandated Disclosure Frequency and Price Formation: Evidence from the 8-K Expansion Regulation. Indiana University. Working paper.

Menon, K., \& Williams, D. D. (2010). Investor Reaction to Going Concern Audit Reports. The Accounting Review, 6.

Miller, M. H., \& Rock, K. (1985). Dividend policy under asymmetric information. The Journal of finance, 40(4), 1031-1051.

Mitchell, J., Izan, H., \& Lim, R. (2006). Australian on-market buy-backs: an examination of valuation issues. Multinational Finance Journal, 10(1/2), 43-79.

Mitchell, J. D., \& Dharmawan, G. V. (2007). Incentives for on-market buy-backs: Evidence from a transparent buy-back regime. Journal of Corporate Finance, 13(1), 146-169.

Mucklow, B. (1994). Market microstructure: An examination of the effects on intraday event studies. Contemporary Accounting Research, 10(2), 355-382.

Myers, S. C., \& Majluf, N. S. (1984). Corporate financing and investment decisions when firms have information that investors do not have. Journal of financial Economics, 13(2), 187221.

Nichols, D. C., \& Wahlen, J. M. (2004). How do earnings numbers relate to stock returns? A review of classic accounting research with updated evidence. Accounting Horizons, 18(4), 263-286.

Nofsinger, J. R. (2001). The impact of public information on investors. Journal of Banking \& Finance, 25(7), 1339-1366.

North, G. (2013a). Company Disclosure in Australia. Australia: Thomson Reuters.

North, G. (2013b). The Continuous Disclosure Obligations in Australia: Do They Still Have Substantive Content and Can Those Relying on Public Disclosures Make Confident Informed Decisions? Australian Journal of Corporate Law, 28, 233-252.

O'Hara, M. (1995). Market microstructure theory (Vol. 108): Blackwell Cambridge, MA.

Pallant, J. (2011). SPSS SURVIVAL MANUAL A step by step guide to data analysis using SPSS Retrieved

from http://hbanaszak.mjr.uw.edu.pl/TempTxt/ebooksclub.org_SPSS_Survival_Manual A_Step_by_Step_Guide_to_Data_Analysis_Using the_SPSS_Program_4th_Edition. $\underline{\mathrm{pdf}}$ 
Pattenden, K., \& Twite, G. (2008). Taxes and dividend policy under alternative tax regimes. Journal of Corporate Finance, 14(1), 1-16.

Poskitt, R. (2005). Disclosure regulation and information risk. Accounting \& Finance, 45(3), 457-477.

Ranaldo, A. (2006). Intraday market dynamics around public information arrivals. Working Paper. Available at https://papers.ssrn.com/sol3/papers.cfm?abstract_id=301823.

Reddy Yarram, S. (2014). Factors influencing on-market share repurchase decisions in Australia. Studies in Economics and Finance, 31(3), 255-271.

Rezaee, Z., Espahbodi, R., Espahbodi, P., \& Espahbodi, H. (2012). Firm characteristics and stock price reaction to SOX 404 compliance. Abacus, 48(4), 473-501.

Ritter, A., \& Wells, P. (2006). Identifiable intangible asset disclosures, stock prices and future earnings. Accounting \& Finance, 46(5), 843-863.

Seamer, M. (2014). Does Effective Corporate Governance Facilitate Continuous Market Disclosure? Australian Accounting Review, 24(2), 111-126.

Shivakumar, L. (2000). Do firms mislead investors by overstating earnings before seasoned equity offerings? Journal of accounting and economics, 29(3), 339-371.

Starks, L. T. (1994). Discussion of "Market Microstructure: An Examination of the Effects on Intraday Event Studies". Contemporary Accounting Research, 10(2), 383-386.

Truong, C. (2010). Strategic timing of earnings announcements? Accounting \& Finance, 50(3), 719-738.

Vermaelen, T. (1981). Common stock repurchases and market signalling: An empirical study. Journal of financial Economics, 9(2), 139-183.

Welsh, M. A. (2009). Continuous Disclosure: Testing the Correspondence Between State Enforcement and Compliance. Australian Journal of Corporate Law, 23, 206-232.

Wruck, K. H. (1989). Equity ownership concentration and firm value: Evidence from private equity financings. Journal of financial Economics, 23(1), 3-28. 
APPENDIX I: SAMPLE DETAILS

\begin{tabular}{|c|c|c|c|}
\hline ASX Code & Company Name & Listing Date & Delisting Date \\
\hline $1 \mathrm{AG}$ & Alterra Limited & $16 / 05 / 2008$ & \\
\hline $1 \mathrm{PG}$ & 1-Page Limited & $20 / 04 / 2006$ & \\
\hline $3 \mathrm{DP}$ & Pointerra Limited & $27 / 03 / 2008$ & \\
\hline $3 \mathrm{PL}$ & 3P Learning Limited & $9 / 07 / 2014$ & \\
\hline 4DS & 4DS Memory Limited & $9 / 12 / 2010$ & \\
\hline $88 \mathrm{E}$ & 88 Energy Limited & $20 / 01 / 2000$ & \\
\hline $8 \mathrm{CO}$ & 8Common Ltd & $27 / 08 / 2014$ & \\
\hline $8 \mathrm{IH}$ & 8I Holdings Ltd & $17 / 12 / 2014$ & \\
\hline AAA & BetaShares Australian High Interest Cash ETF & $7 / 03 / 2012$ & \\
\hline $\mathrm{AAB}$ & Absolute Return Fund (The) & $18 / 09 / 2002$ & $15 / 10 / 2004$ \\
\hline AAC & Australian Agricultural Company Limited & $10 / 08 / 2001$ & \\
\hline AAD & Ardent Leisure Group & $2 / 07 / 1998$ & \\
\hline AAG & Aragon Resources Limited & $10 / 08 / 2007$ & $6 / 05 / 2011$ \\
\hline AAH & Arana Therapeutics Limited & 23/01/1986 & $13 / 08 / 2009$ \\
\hline AAI & Alcoa Inc. & $16 / 08 / 2000$ & \\
\hline AAJ & Aruma Resources Limited & $23 / 07 / 2010$ & \\
\hline AAK & Australia China Holdings Limited & $9 / 06 / 1995$ & \\
\hline AAL & APAC Coal Limited & $10 / 07 / 2008$ & \\
\hline AAN & Alinta Limited & $12 / 10 / 2006$ & $6 / 09 / 2007$ \\
\hline AAP & Australian Agricultural Projects Limited & $7 / 01 / 2004$ & \\
\hline AAR & Anglo Australian Resources NL & $13 / 11 / 1986$ & \\
\hline AAS & Asian Pacific Limited & $10 / 01 / 2001$ & $30 / 08 / 2012$ \\
\hline AAT & AAT Corporation Limited & $30 / 01 / 1986$ & $4 / 01 / 2016$ \\
\hline AAU & Adcorp Australia Limited & $4 / 11 / 1999$ & \\
\hline AAX & Ausenco Limited & $15 / 06 / 2006$ & 19/09/2016 \\
\hline AB1 & Animoca Brands Corporation Limited & $30 / 04 / 2007$ & \\
\hline $\mathrm{ABA}$ & Auswide Bank Ltd & $19 / 09 / 1994$ & \\
\hline $\mathrm{ABB}$ & ABB Grain Limited & $22 / 07 / 2002$ & $25 / 09 / 2009$ \\
\hline $\mathrm{ABC}$ & Adelaide Brighton Limited & $5 / 07 / 1960$ & \\
\hline $\mathrm{ABF}$ & Ausmani Limited & $20 / 01 / 1992$ & $4 / 01 / 2016$ \\
\hline $\mathrm{ABL}$ & Abilene Oil and Gas Limited & $9 / 04 / 1970$ & \\
\hline $\mathrm{ABP}$ & Abacus Property Group & $14 / 11 / 2002$ & \\
\hline ABS & A.B.C. Learning Centres Limited & $21 / 03 / 2001$ & $31 / 08 / 2009$ \\
\hline $\mathrm{ABU}$ & ABM Resources NL & $21 / 08 / 1986$ & \\
\hline ABV & Advanced Braking Technology Ltd & $28 / 05 / 2002$ & \\
\hline $\mathrm{ABW}$ & Aurora Absolute Return Fund & $10 / 07 / 2006$ & \\
\hline $\mathrm{ABX}$ & Australian Bauxite Limited & $24 / 12 / 2009$ & \\
\hline ABY & Aditya Birla Minerals Limited & $12 / 05 / 2006$ & $4 / 08 / 2016$ \\
\hline $\mathrm{ACB}$ & A-Cap Resources Limited & $19 / 05 / 2006$ & \\
\hline ACG & AtCor Medical Holdings Limited & $9 / 11 / 2005$ & \\
\hline ACK & Austock Group Limited & $11 / 12 / 2007$ & \\
\hline ACL & Alchemia Limited & $23 / 12 / 2003$ & \\
\hline $\mathrm{ACN}$ & Acer Energy Limited & $6 / 11 / 2003$ & $15 / 01 / 2013$ \\
\hline $\mathrm{ACO}$ & Australian Careers Network Limited & $15 / 12 / 2014$ & \\
\hline ACP & Audalia Resources Limited & $6 / 07 / 2011$ & \\
\hline ACQ & Acorn Capital Investment Fund Limited & $1 / 05 / 2014$ & \\
\hline ACR & Acrux Limited & $29 / 09 / 2004$ & \\
\hline ACS & Accent Resources N.L. & $26 / 08 / 2005$ & \\
\hline $\mathrm{ACU}$ & Activistic Limited & $25 / 08 / 1971$ & \\
\hline $\mathrm{ACW}$ & Actinogen Medical Limited & $16 / 10 / 2007$ & \\
\hline $\mathrm{ACX}$ & Aconex Limited & $9 / 12 / 2014$ & \\
\hline ADA & Adacel Technologies Limited & $27 / 02 / 1998$ & \\
\hline $\mathrm{ADB}$ & Adelaide Bank Limited & $28 / 05 / 1992$ & $29 / 09 / 2008$ \\
\hline ADD & Adavale Resources Limited & $6 / 02 / 1992$ & \\
\hline ADE & Adelaide Energy Limited & $18 / 06 / 2007$ & $17 / 01 / 2012$ \\
\hline
\end{tabular}




\begin{tabular}{|c|c|c|c|}
\hline ASX Code & Company Name & Listing Date & Delisting Date \\
\hline ADG & Adtrans Group Limited & $13 / 08 / 1987$ & $20 / 12 / 2010$ \\
\hline ADI & Adelphi Energy Limited & $3 / 07 / 1980$ & $8 / 09 / 2010$ \\
\hline ADJ & Adslot Ltd & 17/12/1987 & \\
\hline ADN & Adelaide Resources Limited & $17 / 09 / 1996$ & \\
\hline ADO & Anteo Diagnostics Limited & $7 / 04 / 2000$ & \\
\hline ADQ & ADG Global Supply Limited & $6 / 08 / 1998$ & \\
\hline ADT & Advent Limited & 9/05/1991 & $8 / 02 / 2008$ \\
\hline $\mathrm{ADU}$ & Adamus Resources Limited & $7 / 06 / 2001$ & $21 / 12 / 2011$ \\
\hline ADV & Ardiden Limited & $25 / 10 / 2005$ & \\
\hline ADX & ADX Energy Ltd & 29/01/1987 & \\
\hline ADY & Admiralty Resources NL & $1 / 12 / 1983$ & \\
\hline ADZ & Adsteam Marine Limited & $12 / 06 / 1997$ & $17 / 05 / 2007$ \\
\hline AEB & Algae.Tec Limited & $13 / 01 / 2011$ & \\
\hline $\mathrm{AEC}$ & Ammtec Limited & 28/04/1994 & $25 / 11 / 2010$ \\
\hline AED & AED Oil Limited & $3 / 05 / 2005$ & $4 / 01 / 2016$ \\
\hline AEE & Aura Energy Limited & $30 / 05 / 2006$ & \\
\hline $\mathrm{AEF}$ & Australian Ethical Investment Limited & $17 / 12 / 2002$ & \\
\hline AEH & Aveo Healthcare Limited & 18/01/1994 & $23 / 04 / 2014$ \\
\hline AEI & Aeris Environmental Ltd & $12 / 09 / 2000$ & \\
\hline AEJ & Redbank Energy Limited & $11 / 12 / 2006$ & $30 / 08 / 2016$ \\
\hline AEK & Anatolia Energy Limited & $20 / 12 / 2000$ & $12 / 11 / 2015$ \\
\hline AEN & Australian Energy Limited & - & $21 / 04 / 2006$ \\
\hline $\mathrm{AEO}$ & Austereo Group Limited & $5 / 03 / 2001$ & $16 / 05 / 2011$ \\
\hline AES & Advanced Energy Systems Limited & $13 / 10 / 2000$ & $4 / 01 / 2016$ \\
\hline AET & Ausmelt Limited & 24/01/1994 & $23 / 02 / 2010$ \\
\hline AEV & Avenira Limited & $10 / 10 / 2006$ & \\
\hline AEZ & APN European Retail Property Group & $28 / 07 / 2005$ & $4 / 09 / 2012$ \\
\hline AFA & ASF Group Limited & $2 / 01 / 1992$ & \\
\hline AFF & Australian Food \& Fibre Limited & 18/08/1997 & 19/07/2004 \\
\hline$\overline{\mathrm{AFG}}$ & Allco Finance Group Limited & $28 / 02 / 2001$ & $1 / 09 / 2009$ \\
\hline AFI & Australian Foundation Investment Company Limited & $1 / 01 / 1936$ & \\
\hline AFJ & Affinity Education Group Limited & 9/12/2013 & $16 / 12 / 2015$ \\
\hline $\mathrm{AFQ}$ & African Petroleum Corporation Limited & $18 / 10 / 2007$ & $1 / 09 / 2010$ \\
\hline AFR & African Energy Resources Limited & $18 / 04 / 2007$ & \\
\hline AFT & AFT Corporation Limited & 16/01/1992 & \\
\hline AGB & Atlantic Gold Corporation & $8 / 08 / 2014$ & $9 / 03 / 2015$ \\
\hline AGC & Agincourt Resources Limited & $7 / 01 / 2000$ & $20 / 04 / 2007$ \\
\hline AGD & Austral Gold Limited & $18 / 04 / 1997$ & \\
\hline AGE & Alligator Energy Limited & $3 / 02 / 2011$ & \\
\hline AGF & AMP Capital China Growth Fund & $22 / 12 / 2006$ & \\
\hline AGG & AngloGold Ashanti Limited & $16 / 11 / 1999$ & \\
\hline AGI & Ainsworth Game Technology Limited & $17 / 12 / 2001$ & \\
\hline AGJ & Agricultural Land Trust & $10 / 04 / 2003$ & \\
\hline AGL & AGL Energy Limited & $12 / 10 / 2006$ & \\
\hline AGL & Australian Gas Light Company (The) & - & $26 / 10 / 2006$ \\
\hline AGM & Allegiance Mining NL & $29 / 10 / 1993$ & $21 / 07 / 2008$ \\
\hline AGO & Atlas Iron Limited & $17 / 12 / 2004$ & \\
\hline AGP & Anglo Pacific Group Plc & $19 / 05 / 1988$ & 18/06/2010 \\
\hline AGR & Aguia Resources Limited & $13 / 02 / 2008$ & \\
\hline AGS & Alliance Resources Limited & 19/10/1994 & \\
\hline AGU & Aurium Resources Limited & $7 / 08 / 2007$ & $30 / 10 / 2012$ \\
\hline AGX & Agenix Limited & $2 / 01 / 1992$ & \\
\hline AGY & Argosy Minerals Limited & 6/06/1997 & \\
\hline AGZ & A.G.D. Mining Limited & 23/08/1993 & $29 / 08 / 2006$ \\
\hline AHC & AHC Limited & $24 / 03 / 1988$ & $23 / 12 / 2013$ \\
\hline AHF & Australian Dairy Farms Group & $30 / 06 / 2006$ & \\
\hline AHG & Automotive Holdings Group Limited & $3 / 11 / 2005$ & \\
\hline
\end{tabular}




\begin{tabular}{|c|c|c|c|}
\hline ASX Code & Company Name & Listing Date & Delisting Date \\
\hline AHG & Advance Healthcare Group Limited & $7 / 11 / 1991$ & $1 / 09 / 2009$ \\
\hline AHI & Allco HIT Limited & $16 / 02 / 2007$ & $1 / 09 / 2009$ \\
\hline AHJ & Air Change International Limited & $23 / 08 / 2000$ & $4 / 01 / 2016$ \\
\hline AHK & Ark Mines Limited & $9 / 05 / 2011$ & \\
\hline AHL & AHAlife Holdings Limited & 24/03/1997 & \\
\hline AHN & Athena Resources Limited & $22 / 11 / 2006$ & \\
\hline $\mathrm{AHO}$ & Australian Hotel Fund & $2 / 10 / 1996$ & $14 / 11 / 2007$ \\
\hline AHQ & Allegiance Coal Limited & $29 / 05 / 2012$ & \\
\hline AHR & Anchor Resources Limited & $5 / 07 / 2007$ & \\
\hline AHU & Allco Hybrid Investment Trust & $20 / 08 / 2004$ & $14 / 01 / 2009$ \\
\hline AHY & Asaleo Care Limited & $27 / 06 / 2014$ & \\
\hline AHZ & Admedus Ltd & $24 / 03 / 2004$ & \\
\hline AIA & Auckland International Airport Limited & $22 / 02 / 1999$ & \\
\hline AIB & Aurora Global Income Trust & $24 / 12 / 2007$ & \\
\hline AIE & Autodom Limited & $30 / 04 / 1992$ & $30 / 08 / 2013$ \\
\hline AIG & Aircruising Australia Limited & $3 / 07 / 1986$ & $2 / 01 / 2013$ \\
\hline AIH & Alinta Infrastructure Holdings Limited & $4 / 10 / 2005$ & $28 / 02 / 2007$ \\
\hline AII & Abra Mining Limited & $8 / 04 / 2005$ & $5 / 08 / 2011$ \\
\hline AIK & Armidale Investment Corporation Limited & $28 / 01 / 2003$ & \\
\hline $\mathrm{AIO}$ & Asciano Limited & $6 / 06 / 2007$ & 26/08/2016 \\
\hline AIQ & Alternative Investment Trust & $8 / 04 / 2005$ & \\
\hline AIR & Astivita Limited & $16 / 12 / 2009$ & \\
\hline AIS & Aeris Resources Limited & $2 / 02 / 2011$ & \\
\hline AIV & Activex Limited & $11 / 04 / 2006$ & \\
\hline AIY & Authorised Investment Fund Limited & $22 / 04 / 1999$ & \\
\hline AIZ & Air New Zealand Limited & $1 / 10 / 1997$ & \\
\hline AJA & Astro Japan Property Group & $4 / 04 / 2005$ & \\
\hline AJC & Acacia Coal Limited & $3 / 07 / 1986$ & \\
\hline AJD & Asia Pacific Data Centre Group & 9/01/2013 & \\
\hline AJJ & Asian American Medical Group Limited & $19 / 03 / 2007$ & \\
\hline AJL & AJ Lucas Group Limited & $10 / 08 / 1999$ & \\
\hline AJM & Altura Mining Limited & $8 / 01 / 2001$ & \\
\hline AJQ & Armour Energy Limited & $26 / 04 / 2012$ & \\
\hline AJX & Alexium International Group Limited & $22 / 12 / 1999$ & \\
\hline AJY & Asaplus Resources Limited & $16 / 11 / 2012$ & \\
\hline $\mathrm{AKF}$ & Ask Funding Limited & $9 / 08 / 2001$ & \\
\hline AKG & Academies Australasia Group Limited & $1 / 06 / 1977$ & \\
\hline AKI & African Iron Limited & $28 / 05 / 2007$ & $21 / 03 / 2012$ \\
\hline AKK & Austin Exploration Limited & $20 / 07 / 2006$ & \\
\hline AKM & Aspire Mining Limited & $6 / 02 / 2007$ & \\
\hline AKP & Audio Pixel Holdings Limited & $24 / 12 / 2004$ & \\
\hline AKR & Astra Capital Limited & $13 / 11 / 1969$ & $21 / 02 / 2011$ \\
\hline AKS & Australian Masters Corporate Bond Fund No1 Limited & $10 / 06 / 2010$ & $14 / 07 / 2011$ \\
\hline AKT & Australian Masters Corporate Bond Fund No2 Limited & $10 / 06 / 2010$ & $15 / 07 / 2011$ \\
\hline AKU & Australian Masters Corporate Bond Fund No3 Limited & $10 / 06 / 2010$ & $21 / 12 / 2011$ \\
\hline AKW & Arturus Capital Limited & $8 / 05 / 1986$ & $30 / 08 / 2013$ \\
\hline AKX & Australian Masters Corporate Bond Fund No4 Limited & $10 / 05 / 2011$ & $15 / 01 / 2013$ \\
\hline AKY & Australian Masters Corporate Bond Fund No5 Limited & $10 / 05 / 2011$ & $29 / 08 / 2016$ \\
\hline ALB & Albidon Limited & $26 / 03 / 2004$ & $17 / 06 / 2013$ \\
\hline ALC & Alcidion Group Limited & $24 / 06 / 2011$ & \\
\hline ALD & Allied Gold Mining PLC & $8 / 12 / 2003$ & $11 / 09 / 2012$ \\
\hline ALF & Australian Leaders Fund Limited & $10 / 02 / 2004$ & \\
\hline ALH & Australian Leisure and Hospitality Group & - & $18 / 05 / 2005$ \\
\hline ALK & Alkane Resources Ltd & $1 / 05 / 1969$ & \\
\hline ALL & Aristocrat Leisure Limited & $9 / 07 / 1996$ & \\
\hline ALM & Aluminex Resources Limited & $30 / 09 / 2008$ & $31 / 07 / 2009$ \\
\hline ALN & Alinta Limited & $17 / 10 / 2000$ & $26 / 10 / 2006$ \\
\hline
\end{tabular}




\begin{tabular}{|c|c|c|c|}
\hline ASX Code & Company Name & Listing Date & Delisting Date \\
\hline ALO & Auto Group Limited & $9 / 02 / 1994$ & $29 / 08 / 2007$ \\
\hline ALQ & ALS Limited & $1 / 01 / 1952$ & \\
\hline ALR & Aberdeen Leaders Limited & $25 / 08 / 1987$ & \\
\hline ALS & Alesco Corporation Limited & $30 / 06 / 1979$ & $30 / 01 / 2013$ \\
\hline ALT & Analytica Limited & $25 / 10 / 2000$ & \\
\hline ALU & Altium Limited & $4 / 08 / 1999$ & \\
\hline ALW & Alphawest Limited & - & $22 / 11 / 2005$ \\
\hline ALY & Alchemy Resources Limited & $26 / 11 / 2007$ & \\
\hline ALZ & Australand Property Group & 16/06/1997 & $3 / 11 / 2014$ \\
\hline AMA & Ama Group Limited & $31 / 08 / 2006$ & \\
\hline AMB & Ambition Group Limited & 23/11/1999 & \\
\hline AMC & Amcor Limited & $28 / 08 / 1969$ & \\
\hline AME & Alto Metals Limited & $20 / 12 / 2012$ & \\
\hline AMH & AMCIL Limited & $10 / 02 / 2000$ & \\
\hline AMI & Aurelia Metals Limited & $8 / 05 / 2007$ & \\
\hline AML & Aeon Metals Limited & $14 / 06 / 2007$ & \\
\hline AMM & Amcom Telecommunications Limited & 14/07/1994 & $10 / 07 / 2015$ \\
\hline AMN & Agrimin Limited & $9 / 10 / 2007$ & \\
\hline AMO & Ambertech Limited & $10 / 12 / 2004$ & \\
\hline AMP & AMP Limited & $15 / 06 / 1998$ & \\
\hline AMT & Allegra Orthopaedics Limited & $5 / 12 / 2007$ & \\
\hline AMV & Alamain Investments Limited & - & $30 / 08 / 2004$ \\
\hline AMX & Ampella Mining Limited & $13 / 08 / 2007$ & $14 / 04 / 2014$ \\
\hline ANB & Australia New Agribusiness \& Chemical Group Ltd & $7 / 01 / 2011$ & $30 / 08 / 2016$ \\
\hline ANC & Angus \& Coote (Holdings) Limited & $31 / 10 / 1962$ & $3 / 04 / 2007$ \\
\hline AND & Andean Resources Limited & $18 / 12 / 1995$ & $5 / 01 / 2011$ \\
\hline ANE & Auspine Limited & $1 / 01 / 1970$ & $25 / 02 / 2008$ \\
\hline ANG & Austin Engineering Limited & $9 / 03 / 2004$ & \\
\hline ANI & Australian Industrial REIT & $21 / 10 / 2013$ & $3 / 12 / 2015$ \\
\hline ANN & Ansell Limited & $22 / 08 / 1985$ & \\
\hline ANO & Advanced Nano Technologies Limited & $24 / 02 / 2005$ & \\
\hline ANP & Antisense Therapeutics Limited & $20 / 12 / 2001$ & \\
\hline ANQ & AnaeCo Limited & $13 / 02 / 2008$ & \\
\hline ANR & Anatara Lifesciences Ltd & $16 / 10 / 2014$ & \\
\hline ANW & Aus Tin Mining Ltd & $21 / 10 / 2010$ & \\
\hline ANZ & Australia \& New Zealand Banking Group Ltd & $31 / 07 / 1969$ & \\
\hline $\mathrm{AOA}$ & Ausmon Resources Limited & $31 / 03 / 2009$ & \\
\hline AOD & Aurora Dividend Income Trust (Managed Fund) & $17 / 11 / 2005$ & \\
\hline $\mathrm{AOE}$ & Arrow Energy Limited & $17 / 08 / 2000$ & $25 / 08 / 2010$ \\
\hline AOG & Aveo Group & $13 / 12 / 1993$ & \\
\hline $\mathrm{AOH}$ & Altona Mining Limited & $3 / 09 / 2002$ & \\
\hline AOK & Austex Oil Limited & $8 / 01 / 2008$ & \\
\hline $\mathrm{AON}$ & Apollo Minerals Limited & $31 / 10 / 2007$ & \\
\hline AOP & Apollo Consolidated Limited & $29 / 06 / 2005$ & \\
\hline AOU & Auroch Minerals Ltd & $1 / 09 / 2011$ & \\
\hline AOW & American Patriot Oil \& Gas Limited & $9 / 07 / 2014$ & \\
\hline APA & APA Group & $13 / 06 / 2000$ & \\
\hline APB & Arafura Pearls Holdings Limited & $21 / 12 / 2006$ & $30 / 08 / 2013$ \\
\hline APD & APN Property Group Limited & $23 / 06 / 2005$ & \\
\hline APE & AP Eagers Limited & $1 / 01 / 1957$ & \\
\hline$\overline{\mathrm{APG}}$ & Austpac Resources NL & $24 / 07 / 1986$ & \\
\hline$\overline{\mathrm{APH}}$ & Ascent Pharmahealth Ltd & $10 / 06 / 2004$ & $3 / 06 / 2011$ \\
\hline API & Australian Pharmaceutical Industries Limited & $16 / 06 / 1997$ & \\
\hline APK & Australian Power and Gas Company Limited & $15 / 12 / 1999$ & $30 / 10 / 2013$ \\
\hline APL & Australian Plantation Timber Limited & - & $24 / 06 / 2005$ \\
\hline APN & APN News and Media Limited & $21 / 05 / 1992$ & \\
\hline $\mathrm{APO}$ & APN Outdoor Group Limited & $11 / 11 / 2014$ & \\
\hline
\end{tabular}




\begin{tabular}{|c|c|c|c|}
\hline ASX Code & Company Name & Listing Date & Delisting Date \\
\hline APR & API Fund & $22 / 04 / 2005$ & $14 / 11 / 2008$ \\
\hline APW & Aims Property Securities Fund & $17 / 12 / 2004$ & \\
\hline APY & Azonto Petroleum Ltd & $28 / 07 / 2006$ & \\
\hline APZ & Aspen Group & $11 / 04 / 1991$ & \\
\hline AQA & Aquila Resources Limited & $29 / 06 / 2000$ & $30 / 07 / 2014$ \\
\hline $\mathrm{AQC}$ & Australian Pacific Coal Limited & $17 / 12 / 1999$ & \\
\hline AQD & Ausquest Limited & $25 / 11 / 2003$ & \\
\hline $\mathrm{AQE}$ & Aequs Capital Limited & $21 / 08 / 2000$ & $27 / 01 / 2009$ \\
\hline $\mathrm{AQF}$ & Australian Governance Masters Index Fund Limited & $10 / 02 / 2010$ & \\
\hline AQG & Alacer Gold Corp & $7 / 02 / 2011$ & \\
\hline AQI & Alicanto Minerals Limited & $19 / 09 / 2012$ & \\
\hline AQJ & Aus Asia Minerals Limited & $12 / 04 / 2007$ & \\
\hline AQO & Ambassador Oil and Gas Limited & $22 / 12 / 2011$ & $30 / 10 / 2014$ \\
\hline $\mathrm{AQP}$ & Aquarius Platinum Limited & 29/09/1999 & $14 / 04 / 2016$ \\
\hline AQQ & Aphrodite Gold Limited & $7 / 07 / 2010$ & \\
\hline $\mathrm{AQS}$ & Aquis Entertainment Limited & $2 / 12 / 2011$ & \\
\hline AQU & Aquaint Capital Holdings Limited & $11 / 11 / 2013$ & \\
\hline AQX & Alice Queen Limited & $9 / 09 / 2004$ & \\
\hline AQZ & Alliance Aviation Services Limited & $20 / 12 / 2011$ & \\
\hline AR1 & Arrowhead Resources Limited & 9/07/1971 & \\
\hline ARA & Ariadne Australia Limited & $30 / 06 / 1977$ & \\
\hline ARB & ARB Corporation Limited & $14 / 12 / 1989$ & \\
\hline ARC & Australian Rural Capital Limited & $14 / 04 / 2000$ & \\
\hline ARD & Argent Minerals Limited & $3 / 04 / 2008$ & \\
\hline ARE & Argonaut Resources NL & $23 / 01 / 1986$ & \\
\hline ARF & Arena REIT & $13 / 06 / 2013$ & \\
\hline ARG & Argo Investments Limited & $1 / 01 / 1947$ & \\
\hline ARH & Australasian Resources Limited & $3 / 12 / 1981$ & \\
\hline ARI & Arrium Limited & $23 / 10 / 2000$ & \\
\hline ARJ & The Ark Fund Limited & $17 / 09 / 1987$ & $1 / 09 / 2015$ \\
\hline ARK & Ausroc Metals Ltd & $15 / 09 / 2005$ & \\
\hline ARM & Aurora Minerals Limited & $15 / 06 / 2004$ & \\
\hline ARO & Astro Resources NL & $14 / 08 / 1986$ & \\
\hline ARQ & Arc Energy Limited & $21 / 05 / 1987$ & $29 / 08 / 2008$ \\
\hline ARU & Arafura Resources Limited & $5 / 11 / 2003$ & \\
\hline ARV & Artemis Resources Limited & $16 / 03 / 2007$ & \\
\hline ARW & Australian Renewable Fuels Limited & $10 / 05 / 2005$ & \\
\hline ARX & Arc Exploration Limited & $26 / 04 / 1985$ & \\
\hline ASB & Austal Limited & $8 / 12 / 1998$ & \\
\hline ASH & Ashley Services Group Limited & $21 / 08 / 2014$ & \\
\hline ASL & Ausdrill Limited & 6/01/1994 & \\
\hline ASN & Anson Resources Limited & $8 / 07 / 2010$ & \\
\hline ASP & Aspermont Limited & $27 / 04 / 2000$ & \\
\hline ASR & Australian Rural Group Limited & $26 / 08 / 1988$ & 20/10/2011 \\
\hline AST & AusNet Services & $14 / 12 / 2005$ & \\
\hline ASU & Alpha Technologies Corporation Limited & $24 / 09 / 1987$ & 9/05/2011 \\
\hline ASW & Advanced Share Registry Ltd & $10 / 06 / 2008$ & \\
\hline ASX & ASX Limited & $14 / 10 / 1998$ & \\
\hline ASY & AssembleBay Limited & $17 / 02 / 1994$ & \\
\hline ASZ & ASG Group Limited & $10 / 01 / 2000$ & \\
\hline ATA & ATC Alloys Ltd & $3 / 10 / 2006$ & \\
\hline ATC & Altech Chemicals Ltd & $27 / 01 / 2010$ & \\
\hline ATE & Atherton Resources Ltd & $10 / 06 / 2010$ & $31 / 12 / 2015$ \\
\hline ATI & Atlantic Limited & $11 / 06 / 1987$ & $27 / 09 / 2016$ \\
\hline ATM & Aneka Tambang (Persero) Tbk (Pt) & 9/08/1999 & \\
\hline ATP & Atlas Pearls and Perfumes Ltd & $31 / 12 / 1993$ & \\
\hline ATR & Astron Corporation Limited & $11 / 11 / 1986$ & \\
\hline
\end{tabular}




\begin{tabular}{|c|c|c|c|}
\hline ASX Code & Company Name & Listing Date & Delisting Date \\
\hline ATU & Atrum Coal NL & $24 / 07 / 2012$ & \\
\hline ATV & Atlantic Gold NL & 29/03/1994 & $27 / 08 / 2014$ \\
\hline AUB & AUB Group Limited & $16 / 11 / 2005$ & \\
\hline AUC & AusGold Limited & $16 / 12 / 2009$ & \\
\hline AUF & Asian Masters Fund Limited & $4 / 12 / 2007$ & \\
\hline AUG & Augend Ltd & $7 / 12 / 2011$ & \\
\hline AUI & Australian United Investment Company Limited & $1 / 01 / 1974$ & \\
\hline AUK & Augur Resources Limited & $22 / 10 / 2007$ & \\
\hline AUN & Austar United Communications Limited & $20 / 07 / 1999$ & $27 / 04 / 2012$ \\
\hline AUO & Austral Coal Limited & $26 / 05 / 1997$ & $21 / 02 / 2007$ \\
\hline AUP & Aurora Property Buy-Write Income Trust & 26/07/2007 & \\
\hline AUQ & Alara Resources Limited & $24 / 05 / 2007$ & \\
\hline AUS & Auselect Limited & $3 / 05 / 2004$ & $7 / 05 / 2007$ \\
\hline AUT & Aurora Oil \& Gas Limited & 4/09/1986 & $13 / 06 / 2014$ \\
\hline AUW & Australian Wealth Management Limited & $15 / 02 / 2005$ & $15 / 05 / 2009$ \\
\hline AUZ & Australian Mines Limited & $28 / 09 / 2001$ & \\
\hline AVB & Avanco Resources Limited & $13 / 12 / 2007$ & \\
\hline AVD & Antilles Oil and Gas NL & $2 / 06 / 2006$ & \\
\hline AVE & Aevum Limited & $18 / 11 / 2004$ & $29 / 12 / 2010$ \\
\hline AVF & Australian Value Funds Management Limited & - & $30 / 08 / 2004$ \\
\hline AVG & Australian Vintage Ltd & $26 / 03 / 1992$ & \\
\hline AVH & Avita Medical Ltd & $11 / 08 / 1993$ & \\
\hline AVI & Avalon Minerals Limited & $22 / 03 / 2007$ & \\
\hline AVJ & AVJennings Limited & $27 / 01 / 1994$ & \\
\hline AVL & Australian Vanadium Limited & $21 / 02 / 2007$ & \\
\hline AVM & Anvil Mining Limited & $3 / 06 / 2004$ & 20/03/2012 \\
\hline AVO & Avoca Resources Limited & $15 / 04 / 2002$ & $21 / 02 / 2011$ \\
\hline AVQ & Axiom Mining Limited & $10 / 11 / 2006$ & \\
\hline AVR & Avatar Industries Limited & 25/03/1971 & $26 / 11 / 2007$ \\
\hline AVS & Avastra Sleep Centres Limited & $24 / 06 / 2004$ & $14 / 07 / 2011$ \\
\hline AVT & AVT Plasma Limited & 18/10/1994 & $29 / 08 / 2007$ \\
\hline AVX & Avexa Limited & $23 / 09 / 2004$ & \\
\hline AVZ & AVZ Minerals Limited & $14 / 09 / 2007$ & \\
\hline AWB & AWB Limited & $22 / 08 / 2001$ & $10 / 12 / 2010$ \\
\hline AWC & Alumina Limited & $31 / 01 / 1962$ & \\
\hline AWE & AWE Limited & $2 / 07 / 1997$ & \\
\hline AWN & Arowana International Limited & $4 / 06 / 2003$ & \\
\hline AWV & Anova Metals Limited & $18 / 05 / 2011$ & \\
\hline AWY & Australian Whiskey Holdings Limited & $12 / 11 / 2003$ & \\
\hline AXA & AXA Asia Pacific Holdings Limited & $1 / 10 / 1996$ & $31 / 03 / 2011$ \\
\hline AXE & Archer Exploration Limited & $14 / 08 / 2007$ & \\
\hline AXG & Altria Group Inc. & $5 / 11 / 1992$ & $11 / 01 / 2008$ \\
\hline AXI & Axiom Properties Limited & $31 / 01 / 1997$ & \\
\hline AXM & Apex Minerals NL & $5 / 06 / 2002$ & $1 / 09 / 2014$ \\
\hline AXN & Axon Instruments Inc. & - & $9 / 07 / 2004$ \\
\hline AXO & Aurox Resources Limited & $22 / 10 / 2004$ & $1 / 09 / 2010$ \\
\hline AXT & Argo Exploration Limited & $11 / 12 / 2006$ & \\
\hline AXZ & Amex Resources Limited & $11 / 09 / 2007$ & \\
\hline AYA & Attila Resources Limited & $8 / 12 / 2010$ & \\
\hline AYB & Australian Natural Proteins Limited & $28 / 08 / 2001$ & \\
\hline AYC & A1 Consolidated Gold Limited & $21 / 06 / 2012$ & \\
\hline AYD & Australian Masters Yield Fund No 1 Limited & $29 / 11 / 2012$ & \\
\hline AYF & Australian Enhanced Income Fund & $17 / 10 / 2006$ & \\
\hline AYG & Axxis Technology Group Ltd & $13 / 03 / 1986$ & \\
\hline AYH & Australian Masters Yield Fund No 2 Limited & $29 / 11 / 2012$ & \\
\hline AYI & A1 Investments \& Resources Ltd & $2 / 10 / 2007$ & \\
\hline AYJ & Australian Masters Yield Fund No 3 Limited & $31 / 10 / 2013$ & \\
\hline
\end{tabular}




\begin{tabular}{|c|c|c|c|}
\hline ASX Code & Company Name & Listing Date & Delisting Date \\
\hline AYK & Australian Masters Yield Fund No 4 Limited & $31 / 10 / 2013$ & \\
\hline AYM & Australia United Mining Limited & 19/09/2011 & \\
\hline AYN & Alcyone Resources Limited & $16 / 12 / 1993$ & $1 / 09 / 2015$ \\
\hline AYR & Alloy Resources Limited & $6 / 04 / 2006$ & \\
\hline AYT & Adelaide Managed Funds Asset Backed Yield Trust & $31 / 08 / 2006$ & $16 / 11 / 2011$ \\
\hline AYX & Austofix Group Limited & $27 / 02 / 2008$ & $22 / 06 / 2012$ \\
\hline AZA & Anzon Australia Limited & $15 / 12 / 2004$ & $26 / 11 / 2008$ \\
\hline AZC & Australian Zircon NL & $22 / 12 / 1994$ & $4 / 01 / 2016$ \\
\hline AZD & Amazing Loans Limited & $28 / 04 / 2006$ & $8 / 07 / 2009$ \\
\hline AZG & Allmine Group Limited & $28 / 02 / 2011$ & $1 / 09 / 2015$ \\
\hline AZH & Azimuth Resources Limited & $13 / 12 / 2006$ & $24 / 07 / 2013$ \\
\hline AZJ & Aurizon Holdings Limited & $22 / 11 / 2010$ & \\
\hline AZM & Azumah Resources Limited & $9 / 01 / 2006$ & \\
\hline AZO & Apollo Gas Limited & $15 / 12 / 2009$ & 9/02/2011 \\
\hline AZQ & Ascot Resources Limited & $14 / 02 / 2011$ & $29 / 12 / 2015$ \\
\hline AZR & Aztec Resources Limited & $6 / 11 / 1997$ & $22 / 01 / 2007$ \\
\hline AZS & Azure Minerals Limited & $16 / 12 / 2003$ & \\
\hline AZT & Aston Resources Limited & $17 / 08 / 2010$ & $3 / 05 / 2012$ \\
\hline AZU & Azurn International Limited & $19 / 08 / 2009$ & $5 / 08 / 2011$ \\
\hline AZV & Azure Healthcare Limited & $24 / 09 / 2004$ & \\
\hline AZX & Auzex Resources Limited & $4 / 10 / 2005$ & $7 / 05 / 2012$ \\
\hline AZY & Antipa Minerals Limited & $20 / 04 / 2011$ & \\
\hline AZZ & Antares Energy Limited & 24/11/1994 & \\
\hline $\mathrm{BAB}$ & Bullabulling Gold Limited & $23 / 03 / 2012$ & $9 / 10 / 2014$ \\
\hline BAF & Blue Sky Alternatives Access Fund Limited & $16 / 06 / 2014$ & \\
\hline BAL & Bellamy's Australia Limited & $5 / 08 / 2014$ & \\
\hline $\mathrm{BAO}$ & Brookfield Australian Opportunities Fund & $8 / 07 / 2003$ & $30 / 10 / 2012$ \\
\hline BAP & Bapcor Limited & $24 / 04 / 2014$ & \\
\hline BAR & Barra Resources Limited & $12 / 12 / 2000$ & \\
\hline BAS & Bass Strait Oil Company Limited & $30 / 09 / 2004$ & \\
\hline BAU & Bauxite Resources Limited & $22 / 10 / 2007$ & \\
\hline BAX & Baxter Group Limited & $5 / 08 / 2002$ & $20 / 02 / 2007$ \\
\hline BBB & B Digital Limited & $13 / 06 / 2000$ & $8 / 01 / 2007$ \\
\hline BBG & Billabong International Limited & $11 / 08 / 2000$ & \\
\hline BBI & Babcock \& Brown Infrastructure Group & $24 / 06 / 2002$ & 23/11/2009 \\
\hline BBL & Brisbane Broncos Limited & $12 / 05 / 1989$ & \\
\hline BBR & Bora Bora Resources Limited & $11 / 05 / 2012$ & \\
\hline BBX & BBX Minerals Limited & $12 / 08 / 2005$ & \\
\hline $\mathrm{BCC}$ & Buccaneer Energy Limited & $19 / 11 / 2007$ & $1 / 09 / 2015$ \\
\hline BCD & BCD Resources NL & $11 / 03 / 1983$ & \\
\hline BCI & BC Iron Limited & $15 / 12 / 2006$ & \\
\hline $\mathrm{BCK}$ & Brockman Mining Limited & $11 / 01 / 2011$ & \\
\hline BCL & Betcorp Limited & $5 / 05 / 1999$ & $31 / 10 / 2006$ \\
\hline $\mathrm{BCN}$ & Beacon Minerals Limited & 20/10/2006 & \\
\hline $\mathrm{BCO}$ & BCD Resources (Operations) NL & $18 / 06 / 2009$ & $30 / 08 / 2011$ \\
\hline $\mathrm{BCS}$ & BrisConnections Unit Trusts & $31 / 07 / 2008$ & $4 / 01 / 2016$ \\
\hline BCT & Bluechiip Limited & $9 / 06 / 2011$ & \\
\hline BD1 & BARD1 Life Sciences Limited & $18 / 04 / 1991$ & \\
\hline BDI & Blina Minerals NL & $17 / 08 / 2004$ & \\
\hline BDL & Brandrill Limited & $2 / 12 / 1993$ & $16 / 12 / 2009$ \\
\hline $\mathrm{BDM}$ & BioDiem Ltd & $28 / 01 / 2004$ & $18 / 11 / 2013$ \\
\hline BDR & Beadell Resources Limited & $26 / 09 / 2007$ & \\
\hline BDS & Bridgestone Australia Limited & $14 / 04 / 1982$ & $30 / 05 / 2007$ \\
\hline BEC & Becton Property Group & $8 / 07 / 2005$ & $30 / 08 / 2013$ \\
\hline BEG & Bluenergy Group Limited & $3 / 09 / 1970$ & $30 / 08 / 2016$ \\
\hline BEI & Babcock \& Brown Environmental Investments Limited & $18 / 02 / 1998$ & $25 / 06 / 2008$ \\
\hline BEL & Bentley Capital Limited & $7 / 11 / 1986$ & \\
\hline
\end{tabular}




\begin{tabular}{|c|c|c|c|}
\hline ASX Code & Company Name & Listing Date & Delisting Date \\
\hline BEN & Bendigo and Adelaide Bank Limited & $4 / 02 / 1993$ & \\
\hline BEZ & Besra Gold Inc & $5 / 02 / 2010$ & $1 / 09 / 2015$ \\
\hline$\overline{\mathrm{BFG}}$ & Bell Financial Group Limited & $12 / 12 / 2007$ & \\
\hline BGA & Bega Cheese Limited & 19/08/2011 & \\
\hline BGD & BGD Corporation Ltd & $4 / 09 / 1986$ & \\
\hline BGF & Ballarat Goldfields NL & $4 / 07 / 1985$ & $16 / 03 / 2007$ \\
\hline BGG & Blackgold International Holdings Limited & $22 / 02 / 2011$ & \\
\hline BGH & Bligh Resources Limited & $1 / 12 / 2011$ & \\
\hline BGL & Bigair Group Limited & $7 / 04 / 2006$ & \\
\hline BGN & BresaGen Limited & $21 / 09 / 1999$ & $8 / 01 / 2007$ \\
\hline BGR & Bronson Group Limited & 29/05/1987 & \\
\hline BGS & Birimian Limited & $6 / 12 / 2006$ & \\
\hline BHG & Beauty Health Group Limited & 17/08/1994 & $4 / 01 / 2016$ \\
\hline BHL & Bidvest Plc & - & $18 / 08 / 2004$ \\
\hline BHP & BHP Billiton Limited & $13 / 08 / 1885$ & \\
\hline BHU & Beacon Hill Resources Plc & $5 / 04 / 2012$ & $24 / 01 / 2014$ \\
\hline BID & BidEnergy Limited & 27/01/2011 & \\
\hline BIG & Big Un Limited & $16 / 01 / 2004$ & \\
\hline BIR & Burswood Limited & - & $3 / 09 / 2004$ \\
\hline BIS & Bisalloy Steel Group Limited & $23 / 06 / 2003$ & \\
\hline BIT & Biotron Limited & $24 / 01 / 2001$ & \\
\hline BIV & Blue Capital Limited & $18 / 12 / 2003$ & $16 / 05 / 2011$ \\
\hline BKA & Buka Minerals Limited & - & $30 / 06 / 2006$ \\
\hline BKI & BKI Investment Company Limited & $12 / 12 / 2003$ & \\
\hline BKL & Blackmores Limited & $2 / 05 / 1985$ & \\
\hline BKM & BKM Management Limited & $18 / 09 / 1986$ & \\
\hline BKN & Bradken Limited & $18 / 08 / 2004$ & \\
\hline BKP & Baraka Energy \& Resources Limited & $25 / 05 / 2005$ & \\
\hline BKR & Broadcast Production Services Limited & 14/02/1996 & $7 / 10 / 2009$ \\
\hline BKT & Black Rock Mining Limited & $10 / 12 / 2003$ & \\
\hline BKW & Brickworks Limited & $30 / 06 / 1962$ & \\
\hline BKY & Berkeley Energia Limited & $16 / 09 / 2003$ & \\
\hline BLA & Blue Sky Alternative Investments Limited & $24 / 01 / 2012$ & \\
\hline BLD & Boral Limited & $21 / 02 / 2000$ & \\
\hline BLE & Blue Ensign Technologies Limited & 17/12/1999 & $4 / 06 / 2010$ \\
\hline BLG & Bluglass Limited & $22 / 09 / 2006$ & \\
\hline BLK & Blackham Resources Limited & $28 / 09 / 2006$ & \\
\hline BLR & Black Range Minerals Limited & $18 / 12 / 1986$ & $21 / 09 / 2015$ \\
\hline BLT & Benitec Biopharma Limited & $17 / 02 / 1997$ & \\
\hline BLU & BlueFreeway Limited & $21 / 12 / 2006$ & $8 / 07 / 2009$ \\
\hline BLX & Beacon Lighting Group Limited & $15 / 04 / 2014$ & \\
\hline BLY & Boart Longyear Limited & $5 / 04 / 2007$ & \\
\hline BLZ & Blaze International Limited & $18 / 09 / 1996$ & \\
\hline BMB & Balamara Resources Limited & 19/04/1996 & $7 / 05 / 2015$ \\
\hline BMG & BMG Resources Limited & $12 / 05 / 2004$ & \\
\hline BML & Botswana Metals Limited & $18 / 01 / 2008$ & \\
\hline BMM & Bellamel Mining Limited & $2 / 11 / 2007$ & $31 / 12 / 2008$ \\
\hline BMN & Bannerman Resources Limited & $28 / 04 / 2005$ & \\
\hline BMP & BuyMyPlace.com.au Limited & $1 / 12 / 2009$ & \\
\hline BMX & Bemax Resources Limited & $1 / 11 / 1990$ & $29 / 08 / 2008$ \\
\hline BMZ & Black Mountain Resources Limited & 17/02/2011 & \\
\hline BNB & Babcock \& Brown Limited & $6 / 10 / 2004$ & $18 / 06 / 2009$ \\
\hline $\mathrm{BNC}$ & Bioniche Life Sciences Inc & $27 / 01 / 2011$ & $4 / 02 / 2014$ \\
\hline BND & Bandanna Energy Limited & $14 / 05 / 2004$ & $30 / 08 / 2016$ \\
\hline $\mathrm{BNO}$ & Bionomics Limited & $21 / 12 / 1999$ & \\
\hline BNR & Bulletin Resources Limited & $20 / 12 / 2010$ & \\
\hline BNT & Bounty Mining Ltd & $30 / 04 / 2004$ & $4 / 01 / 2016$ \\
\hline
\end{tabular}




\begin{tabular}{|c|c|c|c|}
\hline ASX Code & Company Name & Listing Date & Delisting Date \\
\hline $\mathrm{BNV}$ & Brand New Vintage Limited & $4 / 04 / 2001$ & \\
\hline BOA & Boadicea Resources Limited & $11 / 10 / 2012$ & \\
\hline BOC & Bougainville Copper Limited & $5 / 07 / 1971$ & \\
\hline $\mathrm{BOE}$ & Boss Resources Limited & $25 / 07 / 2007$ & \\
\hline BOK & Black Oak Minerals Limited & $20 / 03 / 2008$ & $30 / 08 / 2016$ \\
\hline $\mathrm{BOL}$ & Boom Logistics Limited & $14 / 10 / 2003$ & \\
\hline BON & Bonaparte Diamond Mines NL & $15 / 10 / 2004$ & $5 / 08 / 2009$ \\
\hline BOP & Birch And Prestige Investment Group Limited & $19 / 08 / 2013$ & \\
\hline BOQ & Bank of Queensland Limited & $31 / 08 / 1975$ & \\
\hline BOT & Botanix Pharmaceuticals Ltd & $24 / 01 / 1985$ & \\
\hline BOW & Bow Energy Limited & $20 / 05 / 2005$ & $23 / 01 / 2012$ \\
\hline BPA & Brookfield Prime Property Fund & $15 / 09 / 2006$ & \\
\hline $\mathrm{BPC}$ & Burns, Philp and Company Limited & $1 / 01 / 1970$ & $20 / 12 / 2006$ \\
\hline BPF & Bulletproof Group Limited & $29 / 03 / 2012$ & \\
\hline BPG & Byte Power Group Limited & $2 / 01 / 1992$ & \\
\hline $\mathrm{BPH}$ & BPH Energy Ltd & $6 / 08 / 2004$ & \\
\hline BPK & Bremer Park Limited & 23/12/1998 & 9/11/2012 \\
\hline BPL & Broken Hill Prospecting Limited & $17 / 02 / 2011$ & \\
\hline BPS & BPS Technology Limited & $9 / 09 / 2014$ & \\
\hline BPT & Beach Energy Limited & $2 / 03 / 1962$ & \\
\hline $\mathrm{BQF}$ & Bakehouse Quarter Fund & $30 / 10 / 2002$ & $15 / 06 / 2007$ \\
\hline BRB & Breaker Resources NL & $20 / 04 / 2012$ & \\
\hline $\mathrm{BRC}$ & Brain Resource Limited & $28 / 08 / 2001$ & \\
\hline BRD & Black Ridge Mining NL & $11 / 06 / 1999$ & \\
\hline BRG & Breville Group Limited & $25 / 05 / 1999$ & \\
\hline BRK & Brookside Energy Limited & $31 / 10 / 2005$ & \\
\hline BRK & BreakFree Limited & - & $23 / 05 / 2005$ \\
\hline BRL & Bathurst Resources Limited & $19 / 12 / 2007$ & \\
\hline BRM & Brockman Resources Limited & $21 / 05 / 1999$ & $2 / 07 / 2012$ \\
\hline BRN & Brainchip Holdings Ltd & $9 / 11 / 2011$ & \\
\hline BRO & Broad Investments Limited & $9 / 11 / 1999$ & \\
\hline BRS & Broadspectrum Limited & $3 / 05 / 2001$ & $28 / 06 / 2016$ \\
\hline BRU & Buru Energy Limited & $1 / 09 / 2008$ & \\
\hline BRW & Breakaway Resources Limited & $22 / 12 / 1993$ & $7 / 11 / 2013$ \\
\hline BRZ & Brazin Limited & $3 / 12 / 1997$ & $25 / 01 / 2007$ \\
\hline$\overline{\mathrm{BSA}}$ & BSA Limited & $15 / 11 / 1999$ & \\
\hline BSE & Base Resources Limited & $2 / 10 / 2008$ & \\
\hline BSG & Bolnisi Gold NL & $19 / 07 / 1984$ & $17 / 01 / 2008$ \\
\hline BSL & BlueScope Steel Limited & $15 / 07 / 2002$ & \\
\hline BSM & Bass Metals Ltd & $20 / 10 / 2005$ & \\
\hline $\mathrm{BSN}$ & Bisan Limited & $29 / 01 / 1987$ & \\
\hline $\mathrm{BSO}$ & Bass Strait Oil Trust & $7 / 07 / 1997$ & $20 / 07 / 2007$ \\
\hline BSP & Black Star Petroleum Limited & $5 / 07 / 2011$ & \\
\hline BSR & Bassari Resources Limited & $7 / 01 / 2008$ & \\
\hline BST & Barrack St Investments Limited & $14 / 08 / 2014$ & \\
\hline BTA & Biota Holdings Limited & $20 / 12 / 1985$ & $13 / 11 / 2012$ \\
\hline BTC & BioTech Capital Limited & $29 / 08 / 2000$ & \\
\hline BTI & Bailador Technology Investments Limited & $21 / 11 / 2014$ & \\
\hline$\overline{\text { BTN }}$ & Brighton Mining Group Limited & $3 / 11 / 2010$ & \\
\hline BTR & Blackthorn Resources Limited & $12 / 11 / 1987$ & $12 / 12 / 2014$ \\
\hline BTT & BT Investment Management Limited & $10 / 12 / 2007$ & \\
\hline BUA & Burrabulla Corporation Limited & $10 / 09 / 2008$ & \\
\hline BUD & Buddy Platform Limited & $18 / 12 / 2006$ & \\
\hline BUE & Bluestone Global Limited & $10 / 01 / 2008$ & $1 / 09 / 2015$ \\
\hline BUG & Buderim Group Limited & $14 / 12 / 1989$ & \\
\hline BUL & Blue Energy Limited & $13 / 10 / 1994$ & \\
\hline BUX & Buxton Resources Limited & $23 / 10 / 2007$ & \\
\hline
\end{tabular}




\begin{tabular}{|c|c|c|c|}
\hline ASX Code & Company Name & Listing Date & Delisting Date \\
\hline BUY & Bounty Oil \& Gas NL & $5 / 02 / 2002$ & \\
\hline BVA & Bravura Solutions Limited & $28 / 06 / 2006$ & $14 / 10 / 2013$ \\
\hline BWD & Blackwood Corporation Limited & $15 / 09 / 2004$ & $24 / 04 / 2014$ \\
\hline BWF & Blackwall Limited & $21 / 10 / 2011$ & \\
\hline BWN & Bowen Energy Limited & $15 / 02 / 2007$ & $6 / 02 / 2013$ \\
\hline BWP & BWP Trust & $16 / 09 / 1998$ & \\
\hline BWR & Blackwall Property Trust & $28 / 10 / 2011$ & \\
\hline BXB & Brambles Limited & $1 / 01 / 1954$ & \\
\hline $\mathrm{BXN}$ & Bioxyne Limited & $14 / 12 / 2000$ & \\
\hline BXP & Bill Express Limited & $21 / 10 / 2004$ & $1 / 09 / 2009$ \\
\hline BYE & Byron Energy Limited & $3 / 06 / 2005$ & \\
\hline BYI & Beyond International Limited & $20 / 04 / 1990$ & \\
\hline BYL & Brierty Limited & $7 / 12 / 2007$ & \\
\hline BYR & Burey Gold Limited & $14 / 12 / 2006$ & \\
\hline BZL & Braziron Limited & 11/11/2010 & $1 / 07 / 2016$ \\
\hline CAA & Capral Limited & $20 / 11 / 1986$ & \\
\hline $\mathrm{CAB}$ & Cabcharge Australia Limited & 14/12/1999 & \\
\hline CAD & Caeneus Minerals Ltd & $11 / 07 / 2000$ & \\
\hline $\mathrm{CAE}$ & Cannindah Resources Limited & $20 / 05 / 2005$ & \\
\hline CAF & Centrepoint Alliance Limited & $25 / 06 / 2002$ & \\
\hline CAG & Cape Range Limited & 9/07/1981 & $24 / 03 / 2016$ \\
\hline CAI & Casinos Austria International Limited & - & $27 / 08 / 2004$ \\
\hline CAJ & Capitol Health Limited & 9/06/2006 & \\
\hline CAL & CITIC Australia Trading Limited & $18 / 06 / 2002$ & $20 / 01 / 2009$ \\
\hline CAM & Clime Capital Limited & $3 / 02 / 2004$ & \\
\hline CAP & Carpentaria Exploration Limited & $14 / 11 / 2007$ & \\
\hline CAQ & CAQ Holdings Limited & $25 / 07 / 2005$ & \\
\hline CAR & Carsales.com Limited & $10 / 09 / 2009$ & \\
\hline CAS & Crusader Resources Limited & $6 / 02 / 2004$ & \\
\hline CAT & Catapult Group International Limited & $19 / 12 / 2014$ & \\
\hline CAT & Catuity Inc. & $23 / 11 / 1999$ & $29 / 08 / 2007$ \\
\hline CAV & Carnavale Resources Limited & $15 / 03 / 2007$ & \\
\hline CAY & Canyon Resources Ltd & $15 / 06 / 2010$ & \\
\hline CAZ & Cazaly Resources Limited & $31 / 10 / 2003$ & \\
\hline CBA & Commonwealth Bank of Australia & $12 / 09 / 1991$ & \\
\hline CBC & CBG Capital Limited & $19 / 12 / 2014$ & \\
\hline CBD & CBD Energy Limited & $29 / 06 / 1988$ & $3 / 02 / 2014$ \\
\hline $\mathrm{CBH}$ & CBH Resources Limited & $6 / 08 / 1992$ & $30 / 09 / 2010$ \\
\hline CBK & CB Australia Limited & $11 / 10 / 1984$ & $4 / 01 / 2016$ \\
\hline CBM & Cambrian Mining Plc & $7 / 09 / 2006$ & $7 / 09 / 2007$ \\
\hline CBQ & Coalbank Limited & $17 / 03 / 2003$ & \\
\hline CBR & Constellation Brands Inc. & $10 / 04 / 2003$ & $24 / 11 / 2010$ \\
\hline CBS & Cabral Resources Limited & $22 / 06 / 1995$ & \\
\hline CBT & Canbet Limited & - & $29 / 12 / 2004$ \\
\hline CBX & Cape Alumina Limited & $29 / 01 / 2009$ & $26 / 11 / 2014$ \\
\hline $\mathrm{CCC}$ & Continental Coal Limited & $10 / 07 / 1985$ & $30 / 08 / 2016$ \\
\hline $\mathrm{CCD}$ & Caledon Resources PLC & $4 / 06 / 2008$ & $30 / 08 / 2011$ \\
\hline CCJ & County International Limited & $15 / 11 / 2011$ & \\
\hline CCK & CCK Financial Solutions Limited & $25 / 11 / 1999$ & $31 / 12 / 2012$ \\
\hline $\mathrm{CCL}$ & Coca-Cola Amatil Limited & $1 / 01 / 1970$ & \\
\hline $\mathrm{CCP}$ & Credit Corp Group Limited & $4 / 09 / 2000$ & \\
\hline CCQ & Contango Capital Partners Limited & $30 / 05 / 2007$ & $25 / 06 / 2012$ \\
\hline $\mathrm{CCU}$ & Cobar Consolidated Resources Limited & $11 / 07 / 2006$ & $1 / 09 / 2014$ \\
\hline $\mathrm{CCV}$ & Cash Converters International & $12 / 02 / 1997$ & \\
\hline $\mathrm{CCZ}$ & Castillo Copper Limited & $14 / 12 / 2010$ & \\
\hline CDA & Codan Limited & $27 / 11 / 2003$ & \\
\hline CDB & Condor Blanco Mines Limited & $25 / 02 / 2011$ & \\
\hline
\end{tabular}




\begin{tabular}{|c|c|c|c|}
\hline ASX Code & Company Name & Listing Date & Delisting Date \\
\hline $\mathrm{CDC}$ & Child Care Centres Australia Limited & - & $31 / 12 / 2004$ \\
\hline CDD & Cardno Limited & $20 / 05 / 2004$ & \\
\hline CDF & Commonwealth Diversified Share Fund & $5 / 06 / 1998$ & $26 / 06 / 2009$ \\
\hline CDG & Cleveland Mining Company Limited & $30 / 07 / 2007$ & \\
\hline $\mathrm{CDH}$ & Chongherr Investments Ltd & $10 / 08 / 1993$ & \\
\hline CDI & Challenger Diversified Property Group & $23 / 10 / 2006$ & $9 / 07 / 2014$ \\
\hline $\mathrm{CDL}$ & Canada Land Limited & $28 / 07 / 1994$ & $23 / 04 / 2013$ \\
\hline CDM & Cadence Capital Limited & $5 / 12 / 2006$ & \\
\hline $\mathrm{CDO}$ & Colorado Group Limited & 9/12/1999 & $15 / 08 / 2007$ \\
\hline CDP & Carindale Property Trust & $17 / 06 / 1999$ & \\
\hline CDR & Commander Communications Limited & $1 / 12 / 2000$ & $1 / 09 / 2009$ \\
\hline CDT & Castle Minerals Limited & $2 / 05 / 2006$ & \\
\hline $\mathrm{CDU}$ & CuDeco Limited & $8 / 04 / 1983$ & \\
\hline CDV & Cardinal Resources Limited & $17 / 08 / 2011$ & \\
\hline CDX & CDS Technologies Limited & $24 / 02 / 1997$ & $19 / 10 / 2007$ \\
\hline CDY & Cellmid Limited & $9 / 12 / 2005$ & \\
\hline CEG & CEC Group Limited & $7 / 06 / 2004$ & $30 / 08 / 2011$ \\
\hline CEH & China Education Limited & $12 / 09 / 1989$ & $21 / 05 / 2008$ \\
\hline CEL & Challenger Energy Limited & $7 / 11 / 2007$ & \\
\hline CEN & Central Australian Phosphate Limited & $16 / 03 / 2007$ & $22 / 01 / 2014$ \\
\hline CEP & Centro Properties Group & - & $18 / 03 / 2005$ \\
\hline CEQ & Central Equity Limited & - & $5 / 07 / 2006$ \\
\hline CER & Centro Retail Group & $17 / 08 / 2005$ & \\
\hline CEU & ConnectEast Group & $23 / 11 / 2004$ & $2 / 11 / 2011$ \\
\hline CEY & Centennial Coal Company Limited & $4 / 08 / 1994$ & $30 / 11 / 2010$ \\
\hline CFE & Cape Lambert Resources Limited & $7 / 06 / 2001$ & \\
\hline CFI & Colonial First Private Capital Limited & $31 / 07 / 1987$ & $21 / 12 / 2007$ \\
\hline CFK & CFK Childcare Centres Limited & $4 / 04 / 2005$ & $1 / 09 / 2009$ \\
\hline $\mathrm{CFU}$ & Ceramic Fuel Cells Limited & $5 / 07 / 2004$ & \\
\hline CGA & Contango Asset Management Limited & $30 / 09 / 2004$ & \\
\hline CGF & Challenger Limited & $23 / 12 / 2003$ & \\
\hline CGG & Citadel Resource Group Limited & $29 / 01 / 1988$ & $18 / 01 / 2011$ \\
\hline CGH & Calibre Group Limited & $2 / 08 / 2012$ & $4 / 01 / 2016$ \\
\hline CGI & Consolidated Global Investments Limited & $24 / 09 / 1987$ & $1 / 09 / 2015$ \\
\hline CGJ & Coles Group Limited & $24 / 09 / 1929$ & $5 / 12 / 2007$ \\
\hline CGL & The Citadel Group Limited & $13 / 11 / 2014$ & \\
\hline CGM & Cougar Metals NL & $16 / 12 / 2003$ & \\
\hline CGN & Crater Gold Mining Limited & $1 / 11 / 2002$ & \\
\hline CGO & CPT Global Limited & $8 / 09 / 2000$ & \\
\hline CGR & CML Group Limited & $23 / 02 / 2010$ & \\
\hline CGS & Cogstate Limited & $13 / 02 / 2004$ & \\
\hline CGT & Castlemaine Goldfields Limited & $3 / 03 / 2005$ & $28 / 09 / 2012$ \\
\hline CGW & Coats Group PLC & $24 / 08 / 1993$ & $27 / 06 / 2016$ \\
\hline CGX & CGA Mining Limited & $11 / 04 / 1991$ & $6 / 02 / 2013$ \\
\hline CHB & Coca-Cola Hellenic Bottling Company S.A. & $1 / 09 / 2000$ & $2 / 09 / 2009$ \\
\hline $\mathrm{CHC}$ & Charter Hall Group & $10 / 06 / 2005$ & \\
\hline CHF & Charter Pacific Corporation Limited & $24 / 02 / 1989$ & \\
\hline CHK & Cohiba Minerals Limited & $1 / 02 / 2012$ & \\
\hline CHL & CCI Holdings Limited & $30 / 01 / 1986$ & $29 / 06 / 2007$ \\
\hline CHN & Chalice Gold Mines Limited & $24 / 03 / 2006$ & \\
\hline $\mathrm{CHO}$ & Choiseul Investments Limited & $28 / 07 / 1971$ & $22 / 12 / 2010$ \\
\hline CHP & Chapmans Limited & $1 / 01 / 1950$ & \\
\hline CHQ & Costaexchange Ltd & $21 / 12 / 1995$ & $21 / 12 / 2009$ \\
\hline CHR & Chalmers Limited & $10 / 06 / 1960$ & \\
\hline CHX & CH4 Gas Limited & $7 / 04 / 2004$ & $5 / 09 / 2006$ \\
\hline CHY & Carter Holt Harvey Limited & - & $5 / 04 / 2006$ \\
\hline $\mathrm{CHZ}$ & Chesser Resources Limited & $29 / 05 / 2007$ & \\
\hline
\end{tabular}




\begin{tabular}{|c|c|c|c|}
\hline ASX Code & Company Name & Listing Date & Delisting Date \\
\hline CIA & Champion Iron Limited & $8 / 06 / 2007$ & \\
\hline CIF & Challenger Infrastructure Fund & $19 / 08 / 2005$ & $2 / 01 / 2013$ \\
\hline $\mathrm{CIH}$ & China Construction Holdings Limited & 24/11/1995 & $27 / 03 / 2009$ \\
\hline CII & CI Resources Limited & $2 / 01 / 1992$ & \\
\hline CIK & China Integrated Media Corporation Limited & $25 / 02 / 2013$ & \\
\hline CIL & Centrebet International Limited & $12 / 07 / 2006$ & $1 / 09 / 2011$ \\
\hline CIM & CIMIC Group Limited & $30 / 04 / 1962$ & \\
\hline CIN & Carlton Investments Limited & 28/02/1971 & \\
\hline CIW & Clime Investment Management Ltd & $15 / 12 / 2000$ & \\
\hline CIX & Calliden Group Limited & $20 / 12 / 1993$ & $29 / 12 / 2014$ \\
\hline CIY & City Pacific Limited & $2 / 07 / 2001$ & $30 / 08 / 2010$ \\
\hline CIZ & Corizon Limited & $26 / 10 / 2010$ & \\
\hline CJC & The Carajas Copper Company Limited & $29 / 08 / 1997$ & \\
\hline CJG & China JXY Group Limited & 24/12/1970 & $30 / 04 / 2013$ \\
\hline $\mathrm{CJO}$ & Cerro Resources NL & $2 / 06 / 1988$ & $1 / 06 / 2013$ \\
\hline CJT & CVC Property Fund & $20 / 02 / 1986$ & $23 / 04 / 2015$ \\
\hline CKA & Cokal Limited & $17 / 04 / 2000$ & \\
\hline CKF & Collins Foods Limited & $4 / 08 / 2011$ & \\
\hline CKL & Colorpak Limited & $15 / 04 / 2004$ & $4 / 05 / 2016$ \\
\hline CKP & Cheviot Kirribilly Vineyard Property Limited & $18 / 05 / 2006$ & $19 / 06 / 2013$ \\
\hline CKT & Challenger Kenedix Japanese Trust & $30 / 04 / 2007$ & $9 / 02 / 2010$ \\
\hline CL8 & Collaborate Corporation Limited & $25 / 09 / 1996$ & \\
\hline CLA & Celsius Coal Limited & 24/03/1988 & \\
\hline CLH & Collection House Limited & $4 / 10 / 2000$ & \\
\hline CLK & Cypress Lakes Group Limited & $15 / 01 / 1998$ & $9 / 12 / 2009$ \\
\hline CLL & P Cleland Enterprises Limited & $8 / 01 / 1987$ & $2 / 05 / 2007$ \\
\hline CLO & Clough Limited & $12 / 03 / 1998$ & $13 / 12 / 2013$ \\
\hline CLQ & Clean TeQ Holdings Limited & $9 / 11 / 2007$ & \\
\hline CLR & Carabella Resources Limited & $17 / 12 / 2010$ & $20 / 02 / 2014$ \\
\hline CLS & CL Asset Holdings Limited & $19 / 11 / 2004$ & $11 / 03 / 2013$ \\
\hline CLT & Cellnet Group Limited & 17/11/1999 & \\
\hline CLV & Clover Corporation Limited & $30 / 11 / 1999$ & \\
\hline CLX & CTI Logistics Limited & $22 / 12 / 1988$ & \\
\hline CLY & Clancy Exploration Limited & $11 / 07 / 2007$ & \\
\hline CLZ & Classic Minerals Limited & $24 / 05 / 2013$ & \\
\hline CM8 & Crowd Mobile Limited & $16 / 12 / 1999$ & \\
\hline CMA & Centuria Metropolitan Reit & $10 / 12 / 2014$ & \\
\hline CMB & Comlabs Limited & - & $2 / 05 / 2012$ \\
\hline $\mathrm{CMC}$ & China Magnesium Corporation Limited & $9 / 11 / 2010$ & \\
\hline CMG & Chandler Macleod Group Limited & $2 / 03 / 2000$ & $20 / 04 / 2015$ \\
\hline CMI & CMI Limited & $22 / 04 / 1993$ & \\
\hline CMJ & Consolidated Media Holdings Limited & $4 / 06 / 1987$ & $21 / 11 / 2012$ \\
\hline CMK & Cumnock Coal Limited & $16 / 12 / 1993$ & $8 / 10 / 2007$ \\
\hline CMM & Capricorn Metals Ltd & $7 / 07 / 2008$ & \\
\hline CMN & Cumminscorp Limited & $16 / 03 / 2006$ & $1 / 09 / 2009$ \\
\hline CMP & Compumedics Limited & $21 / 12 / 2000$ & \\
\hline CMQ & Chemeq Limited & $25 / 08 / 1999$ & $30 / 08 / 2011$ \\
\hline CMR & Compass Resources Limited & $5 / 11 / 1987$ & $1 / 09 / 2015$ \\
\hline CMT & Cott Oil and Gas Limited & $4 / 01 / 2013$ & \\
\hline CMV & CMA Corporation Limited & $12 / 07 / 2005$ & $1 / 09 / 2014$ \\
\hline CMW & Cromwell Property Group & $22 / 02 / 1973$ & \\
\hline CMX & Climax Mining Limited & $28 / 07 / 1971$ & 21/11/2006 \\
\hline CMY & Capital Mining Limited & $22 / 03 / 2007$ & \\
\hline CNA & Coal \& Allied Industries Limited & $30 / 06 / 1962$ & $19 / 12 / 2011$ \\
\hline CNB & CIC Australia Limited & $23 / 07 / 1987$ & $28 / 05 / 2015$ \\
\hline $\mathrm{CNC}$ & Consolidated Capital Investments Limited & $5 / 10 / 1993$ & $30 / 08 / 2012$ \\
\hline CND & Clarius Group Limited & $16 / 01 / 1997$ & \\
\hline
\end{tabular}




\begin{tabular}{|c|c|c|c|}
\hline ASX Code & Company Name & Listing Date & Delisting Date \\
\hline $\mathrm{CNE}$ & Canning Energy Limited & - & $9 / 09 / 2005$ \\
\hline $\mathrm{CNH}$ & China Steel Australia Limited & $29 / 02 / 2008$ & $30 / 08 / 2012$ \\
\hline CNI & Centuria Capital Limited & $26 / 03 / 2002$ & \\
\hline CNJ & Conico Ltd & $18 / 06 / 2007$ & \\
\hline $\mathrm{CNL}$ & Celamin Holdings NL & $17 / 12 / 2009$ & \\
\hline $\mathrm{CNP}$ & CNPR Group & $14 / 08 / 1997$ & $1 / 02 / 2013$ \\
\hline CNQ & Carbine Tungsten Limited & $6 / 06 / 2006$ & \\
\hline CNR & Coonawarra Australia Property Trust & $9 / 05 / 2003$ & $30 / 08 / 2013$ \\
\hline CNT & Centamin Egypt Limited & $8 / 10 / 1970$ & $29 / 01 / 2010$ \\
\hline $\mathrm{CNU}$ & Chorus Limited & $21 / 11 / 2011$ & \\
\hline CNW & Cirrus Networks Holdings Limited & $10 / 02 / 2004$ & \\
\hline $\mathrm{CNX}$ & Carbon Energy Limited & $22 / 12 / 1993$ & \\
\hline $\mathrm{COA}$ & Coates Hire Limited & $6 / 08 / 1996$ & $16 / 01 / 2008$ \\
\hline COE & Cooper Energy Limited & $13 / 03 / 2002$ & \\
\hline $\mathrm{COF}$ & Coffey International Limited & $18 / 01 / 1990$ & $29 / 02 / 2016$ \\
\hline $\mathrm{COH}$ & Cochlear Limited & $4 / 12 / 1995$ & \\
\hline COI & Comet Ridge Limited & $19 / 04 / 2004$ & \\
\hline COJ & Commstrat Limited & $31 / 07 / 1986$ & $1 / 09 / 2015$ \\
\hline $\mathrm{COK}$ & Cockatoo Coal Limited & $19 / 12 / 2005$ & \\
\hline COM & Comops Limited & $7 / 12 / 1999$ & \\
\hline $\mathrm{COO}$ & Corum Group Limited & 9/04/1997 & \\
\hline $\mathrm{COU}$ & Count Financial Limited & $12 / 12 / 2000$ & $12 / 12 / 2011$ \\
\hline $\mathrm{COV}$ & Corvette Resources Limited & $25 / 05 / 2007$ & $24 / 02 / 2010$ \\
\hline $\mathrm{COY}$ & Coppermoly Limited & $31 / 01 / 2008$ & \\
\hline CPA & Commonwealth Property Office Fund & $29 / 04 / 1999$ & $23 / 04 / 2014$ \\
\hline CPI & CPI Group Limited & $1 / 10 / 1992$ & $17 / 05 / 2011$ \\
\hline CPK & CP1 Limited & $20 / 01 / 2003$ & $30 / 08 / 2012$ \\
\hline CPL & Coalspur Mines Limited & $27 / 08 / 1987$ & $29 / 06 / 2015$ \\
\hline CPN & Carpathian Resources Limited & $15 / 06 / 2001$ & $30 / 08 / 2013$ \\
\hline CPR & CLIVPEE Limited & - & $30 / 08 / 2010$ \\
\hline CPS & Computronics Holdings Limited & $17 / 01 / 2003$ & $4 / 01 / 2016$ \\
\hline CPU & Computershare Limited & $27 / 05 / 1994$ & \\
\hline CQC & Cuesta Coal Limited & $4 / 05 / 2012$ & \\
\hline CQO & Charter Hall Office REIT & $9 / 12 / 1993$ & $1 / 05 / 2012$ \\
\hline CQR & Charter Hall Retail REIT & $15 / 11 / 1996$ & \\
\hline CQT & Conquest Mining Limited & $16 / 09 / 1987$ & $3 / 11 / 2011$ \\
\hline CR8 & Cre8tek Limited & $3 / 07 / 1986$ & \\
\hline $\mathrm{CRB}$ & Carbine Resources Limited & $16 / 03 / 2007$ & \\
\hline CRC & Cortona Resources Limited & $21 / 03 / 2006$ & $8 / 02 / 2013$ \\
\hline CRD & Crown Diamonds NL & - & $29 / 06 / 2005$ \\
\hline CRE & Crescent Gold Limited & $29 / 07 / 1999$ & $19 / 05 / 2012$ \\
\hline CRG & Crane Group Limited & $19 / 02 / 1962$ & $6 / 05 / 2011$ \\
\hline CRH & Crowe Horwath Australasia Ltd & $17 / 07 / 1987$ & $8 / 01 / 2015$ \\
\hline CRL & Comet Resources Limited & $2 / 03 / 1994$ & \\
\hline CRM & Carbon Minerals Limited & $31 / 12 / 1980$ & \\
\hline CRT & Consolidated Rutile Limited & $30 / 06 / 1964$ & $2 / 07 / 2009$ \\
\hline CRU & Catalyst Recruitment Systems Limited & $25 / 06 / 1999$ & $2 / 11 / 2006$ \\
\hline CSD & Consolidated Tin Mines Limited & $26 / 02 / 2008$ & \\
\hline CSE & Copper Strike Ltd & $24 / 11 / 2004$ & \\
\hline CSF & Centro Shopping America Trust & $24 / 10 / 2003$ & $31 / 10 / 2007$ \\
\hline $\mathrm{CSH}$ & Concept Hire Limited & $20 / 03 / 1998$ & $16 / 11 / 2007$ \\
\hline CSJ & Credit Suisse GP100 - Australia Trust & $30 / 06 / 2008$ & $1 / 07 / 2013$ \\
\hline CSL & CSL Limited & $8 / 06 / 1994$ & \\
\hline CSM & Consolidated Minerals Limited & $10 / 11 / 1969$ & $25 / 01 / 2008$ \\
\hline CSR & CSR Limited & $31 / 03 / 1962$ & \\
\hline CSS & Clean Seas Tuna Limited & $12 / 12 / 2005$ & \\
\hline CST & Cellestis Limited & $24 / 04 / 2001$ & $31 / 08 / 2011$ \\
\hline
\end{tabular}




\begin{tabular}{|c|c|c|c|}
\hline ASX Code & Company Name & Listing Date & Delisting Date \\
\hline CSU & $\begin{array}{l}\text { Credit Suisse PL100 Emerging Markets Infrastructure } \\
\text { Development Trust }\end{array}$ & 19/12/2007 & $19 / 12 / 2012$ \\
\hline CSV & CSG Limited & $19 / 04 / 2007$ & \\
\hline CSW & Credit Suisse PL100 World Water Trust & $1 / 06 / 2007$ & $25 / 05 / 2012$ \\
\hline CTD & Corporate Travel Management Limited & $15 / 12 / 2010$ & \\
\hline CTE & Cryosite Limited & $9 / 05 / 2002$ & \\
\hline CTI & Chariot Limited & $23 / 12 / 1999$ & $15 / 08 / 2008$ \\
\hline CTL & Citect Corporation Limited & - & $4 / 04 / 2006$ \\
\hline CTM & Centaurus Metals Limited & $12 / 01 / 1989$ & \\
\hline CTN & Contango Microcap Limited & $25 / 03 / 2004$ & \\
\hline CTO & Citigold Corporation Limited & $23 / 12 / 1993$ & \\
\hline CTP & Central Petroleum Limited & $7 / 03 / 2006$ & \\
\hline CTR & Citation Resources Ltd & $2 / 11 / 2006$ & \\
\hline CTX & Caltex Australia Limited & $30 / 06 / 1981$ & \\
\hline CTY & Country Road Limited & $2 / 07 / 1987$ & $5 / 09 / 2014$ \\
\hline CUE & Cue Energy Resources Limited & $11 / 12 / 1995$ & \\
\hline CUL & Cullen Resources Limited & $6 / 04 / 1983$ & \\
\hline CUO & Copperco Limited & $20 / 08 / 1970$ & $1 / 09 / 2009$ \\
\hline CUP & Countplus Limited & $22 / 12 / 2010$ & \\
\hline CUR & Centaurus Resources Limited & $10 / 08 / 2007$ & $1 / 03 / 2010$ \\
\hline CUS & Customers Limited & $22 / 12 / 1989$ & $9 / 07 / 2012$ \\
\hline CUV & Clinuvel Pharmaceuticals Limited & $13 / 02 / 2001$ & \\
\hline CUX & Crossland Strategic Metals Ltd & $13 / 04 / 2007$ & \\
\hline CUY & Curnamona Energy Limited & $19 / 04 / 2005$ & $30 / 07 / 2012$ \\
\hline CVA & Clever Communications Australia Limited & $3 / 11 / 2004$ & $9 / 02 / 2011$ \\
\hline CVB & Cheviot Bridge Limited & $17 / 12 / 1999$ & $25 / 03 / 2010$ \\
\hline $\mathrm{CVC}$ & CVC Limited & $27 / 06 / 1985$ & \\
\hline CVG & Convergent Minerals Limited & $22 / 12 / 2006$ & \\
\hline CVI & CVI Energy Corporation Limited & 28/10/1987 & $5 / 12 / 2011$ \\
\hline $\mathrm{CVN}$ & Carnarvon Petroleum Limited & $16 / 08 / 1984$ & \\
\hline $\mathrm{CVO}$ & Cover-More Group Limited & $19 / 12 / 2013$ & \\
\hline CVR & Central Asia Resources Limited & $13 / 07 / 2007$ & \\
\hline CVS & Cervantes Corporation Limited & $6 / 11 / 2002$ & \\
\hline CVT & Covata Limited & $3 / 01 / 2007$ & \\
\hline CVV & Caravel Minerals Limited & $3 / 11 / 2006$ & \\
\hline CVW & Clearview Wealth Limited & $11 / 12 / 2003$ & \\
\hline CVY & Coventry Resources Limited & $2 / 05 / 2000$ & $9 / 01 / 2013$ \\
\hline CWC & China Waste Corporation Limited & $11 / 06 / 1987$ & \\
\hline CWE & Carnegie Wave Energy Limited & $26 / 10 / 1993$ & \\
\hline CWH & CWH Resources Ltd & $15 / 12 / 1988$ & \\
\hline CWK & Coalworks Limited & $26 / 06 / 2008$ & $23 / 08 / 2012$ \\
\hline CWN & Crown Resorts Limited & $3 / 12 / 2007$ & \\
\hline CWP & Cedar Woods Properties Limited & $31 / 08 / 1994$ & \\
\hline CWT & Challenger Wine Trust & $2 / 07 / 1999$ & $14 / 02 / 2011$ \\
\hline CWY & Cleanaway Waste Management Limited & $3 / 05 / 2005$ & \\
\hline $\mathrm{CXC}$ & Coeur D'Alene Mines Corporation & $12 / 12 / 2007$ & $14 / 12 / 2010$ \\
\hline CXD & CathRx Ltd & $26 / 10 / 2005$ & $2 / 01 / 2013$ \\
\hline $\mathrm{CXH}$ & Compass Hotel Group & $3 / 01 / 2008$ & $30 / 08 / 2011$ \\
\hline CXM & Centrex Metals Limited & $19 / 07 / 2006$ & \\
\hline $\mathrm{CXO}$ & Core Exploration Limited & $11 / 02 / 2011$ & \\
\hline CXP & Corporate Express Australia Limited & $11 / 01 / 1995$ & $8 / 09 / 2010$ \\
\hline CXS & ChemGenex Pharmaceuticals Ltd & $10 / 07 / 1986$ & $22 / 07 / 2011$ \\
\hline $\mathrm{CXU}$ & Cauldron Energy Limited & $28 / 01 / 2005$ & \\
\hline CXX & Cradle Resources Limited & $16 / 09 / 2011$ & \\
\hline CXZ & Connexion Media Limited & $1 / 01 / 1974$ & \\
\hline CYA & Century Australia Investments Limited & $8 / 04 / 2004$ & \\
\hline CYC & Cyclopharm Limited & $18 / 01 / 2007$ & \\
\hline
\end{tabular}




\begin{tabular}{|c|c|c|c|}
\hline ASX Code & Company Name & Listing Date & Delisting Date \\
\hline CYG & Coventry Group Limited & $1 / 11 / 1965$ & \\
\hline CYL & Catalyst Metals Limited & $26 / 07 / 2006$ & \\
\hline $\mathrm{CYO}$ & Clarity Oss Limited & $1 / 09 / 1999$ & \\
\hline CYP & Cynata Therapeutics Limited & $20 / 12 / 2007$ & \\
\hline CYT & Cytopia Limited & $31 / 03 / 1999$ & $5 / 02 / 2010$ \\
\hline CYU & Chinalco Yunnan Copper Resources Ltd & $29 / 10 / 2007$ & \\
\hline CYY & Coventry Resources Inc & $21 / 12 / 2012$ & \\
\hline CZA & Coal Of Africa Limited & $8 / 04 / 1983$ & \\
\hline CZI & Cassini Resources Limited & $9 / 01 / 2012$ & \\
\hline CZL & Consolidated Zinc Limited & $19 / 06 / 2006$ & \\
\hline CZN & Corazon Mining Limited & $30 / 06 / 2005$ & \\
\hline CZR & Coziron Resources Limited & $29 / 08 / 2006$ & \\
\hline CZZ & Capilano Honey Limited & $9 / 07 / 2012$ & \\
\hline D13 & Department 13 International Ltd & $4 / 07 / 2012$ & \\
\hline DAF & Discovery Africa Limited & $22 / 09 / 2011$ & \\
\hline DAU & Dampier Gold Limited & $23 / 08 / 2010$ & \\
\hline DBS & Dark Blue Sea Limited & $10 / 01 / 2001$ & $22 / 01 / 2010$ \\
\hline DCC & DigitalX Limited & $6 / 02 / 1989$ & \\
\hline$\overline{\mathrm{DCG}}$ & Decmil Group Limited & $22 / 04 / 2005$ & \\
\hline DCN & Dacian Gold Limited & $14 / 11 / 2012$ & \\
\hline DDD & 3D Resources Limited & $21 / 03 / 2007$ & \\
\hline DDR & Dicker Data Limited & $24 / 01 / 2011$ & \\
\hline DDT & DataDot Technology Limited & $12 / 01 / 2005$ & \\
\hline DEG & De Grey Mining Limited & $3 / 07 / 2002$ & \\
\hline DES & Destra Corporation Limited & $28 / 02 / 1985$ & $1 / 09 / 2009$ \\
\hline DEX & Dexion Limited & $29 / 04 / 2005$ & $3 / 09 / 2010$ \\
\hline DGA & Digga Australian Mining Fund & $30 / 01 / 2012$ & $30 / 03 / 2015$ \\
\hline DGH & Desane Group Holdings Limited & $15 / 12 / 1988$ & \\
\hline DGM & Deepgreen Minerals Corporation Limited & - & $29 / 12 / 2004$ \\
\hline DGO & DGO Gold Limited & $21 / 12 / 2007$ & \\
\hline DGR & DGR Global Limited & $21 / 08 / 2003$ & \\
\hline DGX & Diploma Group Limited & $5 / 12 / 2007$ & \\
\hline DHR & Dark Horse Resources Limited & $11 / 04 / 2011$ & \\
\hline DID & Didasko Limited & $14 / 09 / 1994$ & 29/08/2006 \\
\hline DIG & Asia Pacific Digital Limited & $1 / 11 / 1985$ & \\
\hline DIO & Dioro Exploration NL & $16 / 07 / 1981$ & $21 / 04 / 2010$ \\
\hline DIR & Direct Nickel Limited & $5 / 11 / 1987$ & $1 / 09 / 2015$ \\
\hline DIT & Deutsche Industrial Trust & 23/09/1997 & \\
\hline DIV & UBS IQ Morningstar Australia Dividend Yield ETF & $15 / 01 / 2014$ & \\
\hline DJS & David Jones Limited & $27 / 11 / 1995$ & $5 / 08 / 2014$ \\
\hline DJW & Djerriwarrh Investments Limited & $28 / 06 / 1995$ & \\
\hline DKM & Duketon Mining Limited & $4 / 08 / 2014$ & \\
\hline DKN & DKN Financial Group Limited & 13/04/1989 & $21 / 10 / 2011$ \\
\hline DKO & Dakota Minerals Limited & $6 / 11 / 1986$ & \\
\hline DKS & Danks Holdings Limited & $1 / 01 / 1974$ & $23 / 12 / 2009$ \\
\hline DLC & Delecta Limited & 15/01/1987 & \\
\hline DLE & Dragon Energy Limited & $18 / 02 / 2009$ & \\
\hline DLS & Drillsearch Energy Limited & $2 / 07 / 1987$ & $2 / 03 / 2016$ \\
\hline DLX & DuluxGroup Limited & $12 / 07 / 2010$ & \\
\hline DM1 & DirectMoney Limited & $16 / 03 / 1989$ & \\
\hline DMA & Dynasty Resources Limited & $6 / 12 / 2005$ & \\
\hline DME & Dome Gold Mines Limited & $22 / 10 / 2013$ & \\
\hline DMG & Dragon Mountain Gold Limited & $31 / 07 / 2007$ & \\
\hline DMI & Dempsey Minerals Limited & $8 / 06 / 2011$ & \\
\hline DML & Discovery Metals Limited & $17 / 12 / 2003$ & $1 / 09 / 2015$ \\
\hline DMM & DMC Mining Limited & $18 / 07 / 2007$ & $1 / 09 / 2010$ \\
\hline DMP & Domino's Pizza Enterprises Limited & $16 / 05 / 2005$ & \\
\hline
\end{tabular}




\begin{tabular}{|c|c|c|c|}
\hline ASX Code & Company Name & Listing Date & Delisting Date \\
\hline DMX & DMX Corporation Limited & $26 / 05 / 2000$ & $10 / 12 / 2013$ \\
\hline DNA & Donaco International Limited & $23 / 12 / 2004$ & \\
\hline DNK & Danakali Limited & $17 / 10 / 2003$ & \\
\hline DNS & Danae Resources NL & - & $30 / 06 / 2006$ \\
\hline DNX & DenX Limited & $12 / 11 / 1980$ & $29 / 08 / 2006$ \\
\hline DOM & Dominion Mining Limited & $30 / 06 / 1981$ & $25 / 02 / 2011$ \\
\hline DOT & Deutsche Office Trust & $13 / 06 / 2000$ & \\
\hline DOW & Downer EDI Limited & $20 / 12 / 1990$ & \\
\hline DRA & Dragon Mining Limited & 19/09/1990 & \\
\hline DRD & DRDGold Limited & 27/05/1999 & $28 / 07 / 2006$ \\
\hline DRG & Draig Resources Limited & $20 / 12 / 2005$ & \\
\hline DRK & Drake Resources Limited & $31 / 03 / 2005$ & \\
\hline DRM & Doray Minerals Limited & $8 / 02 / 2010$ & \\
\hline DRX & Diatreme Resources Limited & $22 / 06 / 2005$ & \\
\hline DSB & Delta SBD Limited & $20 / 12 / 2010$ & \\
\hline DSH & DSHE Holdings Limited & $4 / 12 / 2013$ & $30 / 08 / 2016$ \\
\hline DSQ & DSQ Holdings Limited & $28 / 09 / 2007$ & $30 / 10 / 2012$ \\
\hline DSX & Decimal Software Limited & $21 / 04 / 1994$ & \\
\hline DTE & Dart Energy Limited & $22 / 07 / 2010$ & $22 / 10 / 2014$ \\
\hline DTI & DTI Group Limited & $9 / 12 / 2014$ & \\
\hline DTL & Data3 Limited & $23 / 12 / 1997$ & \\
\hline DTM & Dart Mining NL & $10 / 05 / 2007$ & \\
\hline DTO & D2 Marketing Ltd & $15 / 11 / 2007$ & $14 / 07 / 2010$ \\
\hline DTR & Dateline Resources Limited & $23 / 06 / 2011$ & \\
\hline DTX & Datetix Group Ltd & $5 / 12 / 2014$ & \\
\hline DUB & Dubber Corporation Limited & $30 / 06 / 2000$ & \\
\hline DUE & DUET Group & $13 / 08 / 2004$ & \\
\hline DUI & Diversified United Investment Limited & 19/12/1991 & \\
\hline DVA & Diversa Limited & $16 / 06 / 2000$ & \\
\hline DVC & DCA Group Limited & $17 / 12 / 1987$ & $21 / 12 / 2006$ \\
\hline DVI & Disruptive Investment Group Limited & $22 / 11 / 2004$ & \\
\hline DVL & Dorsavi Ltd & $11 / 12 / 2013$ & \\
\hline DVN & Devine Limited & 25/11/1993 & \\
\hline DWS & DWS Limited & $15 / 06 / 2006$ & \\
\hline DXB & Dimerix Limited & $4 / 02 / 1993$ & \\
\hline DXL & Dyno Nobel Limited & $7 / 04 / 2006$ & $17 / 06 / 2008$ \\
\hline DXS & Dexus Property Group & $6 / 10 / 2004$ & \\
\hline DYE & Dyesol Limited & $31 / 08 / 2005$ & \\
\hline DYL & Deep Yellow Limited & $19 / 12 / 1985$ & \\
\hline E88 & Ensogo Limited & $20 / 12 / 2013$ & \\
\hline EAC & East African Coffee Plantations Limited & - & $19 / 05 / 2005$ \\
\hline EAL & E\&A Limited & $17 / 12 / 2007$ & \\
\hline EAR & Echo Resources Limited & $9 / 05 / 2006$ & \\
\hline EAS & Easton Investments Limited & $2 / 08 / 2005$ & \\
\hline EAU & Eldorado Gold Corporation & $7 / 12 / 2009$ & $3 / 09 / 2012$ \\
\hline EAX & Energy Action Limited & 13/10/2011 & \\
\hline EBG & Eumundi Group Limited & $7 / 12 / 1989$ & \\
\hline EBO & EBOS Group Limited & $6 / 12 / 2013$ & \\
\hline ECG & Ecargo Holdings Limited & 28/11/2014 & \\
\hline ECI & EC-Asia International Limited & $23 / 07 / 2004$ & $29 / 08 / 2007$ \\
\hline ECO & Echoice Limited & 4/09/1970 & $17 / 08 / 2016$ \\
\hline ECV & Ecosave Holdings Limited & $15 / 01 / 2013$ & $17 / 09 / 2015$ \\
\hline EDE & Eden Energy Limited & $6 / 06 / 2006$ & \\
\hline EDN & Elkedra Diamonds NL & $8 / 01 / 2002$ & $28 / 11 / 2007$ \\
\hline EDT & EDT Retail Trust & $26 / 11 / 2003$ & 6/09/2011 \\
\hline EEG & Empire Energy Group Limited & $30 / 08 / 1984$ & \\
\hline EER & East Energy Resources Limited & $19 / 12 / 2007$ & \\
\hline
\end{tabular}




\begin{tabular}{|c|c|c|c|}
\hline ASX Code & Company Name & Listing Date & Delisting Date \\
\hline EEU & BetaShares Euro ETF & $11 / 07 / 2011$ & \\
\hline EFE & Eastern Iron Limited & $16 / 05 / 2008$ & \\
\hline EFG & Everest Financial Group Limited & $8 / 04 / 2005$ & $18 / 07 / 2011$ \\
\hline EFT & Eftel Limited & $21 / 11 / 1996$ & $17 / 06 / 2013$ \\
\hline EGF & Ellerston Gems Fund & $3 / 07 / 2007$ & $11 / 11 / 2008$ \\
\hline EGG & Enero Group Limited & $30 / 04 / 2004$ & \\
\hline EGH & Eureka Group Holdings Limited & $1 / 07 / 2004$ & \\
\hline EGI & Ellerston Global Investments Limited & $20 / 10 / 2014$ & \\
\hline EGL & The Environmental Group Limited & $30 / 06 / 1977$ & \\
\hline EGN & Engenco Limited & $14 / 12 / 2006$ & \\
\hline EGO & Empire Oil \& Gas NL & $15 / 01 / 1998$ & \\
\hline EGS & Eastern Goldfields Limited & $29 / 10 / 2002$ & \\
\hline EGX & EvoGenix Limited & $11 / 08 / 2005$ & $23 / 07 / 2008$ \\
\hline EGY & Energy Technologies Limited & $16 / 08 / 1984$ & \\
\hline EHE & Estia Health Limited & $5 / 12 / 2014$ & \\
\hline EHL & Emeco Holdings Limited & $28 / 07 / 2006$ & \\
\hline EIG & European Investors Global Property Trust & $5 / 04 / 2006$ & $26 / 08 / 2008$ \\
\hline EIM & El Corporation Limited & $22 / 11 / 1984$ & $1 / 09 / 2014$ \\
\hline EKA & Eureka Energy Limited & $21 / 12 / 2005$ & $24 / 08 / 2012$ \\
\hline ELD & Elders Limited & $8 / 04 / 1983$ & \\
\hline ELI & Emerging Leaders Investment Limited & $23 / 03 / 2005$ & $19 / 06 / 2014$ \\
\hline ELK & ELK Petroleum Limited & $17 / 06 / 2005$ & \\
\hline ELL & Ellendale Resources NL & $16 / 02 / 2001$ & $20 / 06 / 2008$ \\
\hline ELM & Elemental Minerals Limited & $21 / 09 / 2005$ & \\
\hline ELR & Elsmore Resources Ltd & $23 / 12 / 2013$ & $16 / 08 / 2016$ \\
\hline ELS & Equity Long/Short Strategies Fund (The) & - & $20 / 07 / 2004$ \\
\hline ELT & Elementos Limited & $23 / 12 / 2009$ & \\
\hline ELX & Ellex Medical Lasers Limited & $12 / 09 / 1994$ & \\
\hline EMB & Embelton Limited & $1 / 01 / 1958$ & \\
\hline EMC & Emefcy Group Limited & $18 / 12 / 2007$ & \\
\hline EME & Energy Metals Limited & $9 / 09 / 2005$ & \\
\hline EMF & Emerging Markets Masters Fund & $11 / 10 / 2012$ & \\
\hline EMG & Emergent Resources Limited & $4 / 08 / 2008$ & \\
\hline EMH & European Metals Holdings Limited & $19 / 07 / 2012$ & \\
\hline EML & Emerchants Limited & $18 / 01 / 2006$ & \\
\hline EMM & Electrometals Technologies Limited & $20 / 03 / 1989$ & $5 / 12 / 2014$ \\
\hline EMP & Emperor Mines Limited & $1 / 01 / 1972$ & $26 / 03 / 2008$ \\
\hline EMR & Emerald Resources NL & $22 / 07 / 1994$ & \\
\hline EMU & Emu NL & $27 / 02 / 2008$ & \\
\hline EMX & Energia Minerals Limited & $24 / 12 / 2009$ & \\
\hline ENA & Ensurance Ltd & $23 / 09 / 2011$ & \\
\hline ENB & Eneabba Gas Limited & $28 / 04 / 2006$ & \\
\hline ENE & Energy Developments Limited & $10 / 11 / 1993$ & $26 / 10 / 2015$ \\
\hline ENG & Engin Limited & $25 / 06 / 1999$ & $9 / 08 / 2011$ \\
\hline ENK & ENK PLC & $29 / 06 / 2010$ & $23 / 10 / 2012$ \\
\hline ENL & Eagle Nickel Limited & $14 / 03 / 2008$ & \\
\hline ENN & Elanor Investor Group & $11 / 07 / 2014$ & \\
\hline ENR & Encounter Resources Limited & $24 / 03 / 2006$ & \\
\hline ENT & Enterprise Metals Limited & $20 / 06 / 2007$ & \\
\hline ENV & Envestra Limited & 29/08/1997 & $20 / 10 / 2014$ \\
\hline ENX & Enegex Limited & $8 / 10 / 2013$ & \\
\hline ENY & Aii S\&P/ASX 200 Energy & $6 / 04 / 2010$ & $13 / 06 / 2013$ \\
\hline ENZ & Enzumo Limited & $23 / 10 / 2007$ & \\
\hline EOC & Endocoal Limited & $30 / 04 / 2010$ & $29 / 07 / 2013$ \\
\hline$\overline{\mathrm{EOL}}$ & Energy One Limited & $15 / 01 / 2007$ & \\
\hline EON & Espreon Limited & $1 / 06 / 2000$ & 29/09/2009 \\
\hline EOR & Enhanced Oil \& Gas Recovery Limited & $10 / 12 / 2004$ & \\
\hline
\end{tabular}




\begin{tabular}{|c|c|c|c|}
\hline ASX Code & Company Name & Listing Date & Delisting Date \\
\hline EOS & Electro Optic Systems Holdings Limited & $23 / 10 / 2000$ & \\
\hline EPA & Ephraim Resources Limited & $1 / 09 / 1949$ & \\
\hline EPD & Empired Ltd & $19 / 10 / 2007$ & \\
\hline EPF & Esplanade Property Fund & $11 / 08 / 1993$ & $19 / 02 / 2009$ \\
\hline EPL & Eyecare Partners Limited & 21/08/1987 & $2 / 07 / 2012$ \\
\hline EPM & Eclipse Metals Ltd & $17 / 02 / 2011$ & \\
\hline EPW & ERM Power Limited & $10 / 12 / 2010$ & \\
\hline EPX & Ethane Pipeline Income Fund & $12 / 09 / 2006$ & $17 / 06 / 2016$ \\
\hline EPY & E-pay Asia Limited & $15 / 11 / 2000$ & $3 / 03 / 2014$ \\
\hline$\overline{\mathrm{EQE}}$ & Equus Mining Limited & $15 / 12 / 1995$ & \\
\hline EQI & Equigold NL & 26/06/1996 & $23 / 06 / 2008$ \\
\hline EQM & Equatorial Mining Limited & $25 / 06 / 1987$ & $26 / 09 / 2006$ \\
\hline EQN & Equinox Minerals Limited & $21 / 06 / 2004$ & $22 / 07 / 2011$ \\
\hline EQT & Equity Trustees Limited & $6 / 08 / 1985$ & \\
\hline EQU & Equator Resources Ltd & $1 / 02 / 2010$ & \\
\hline EQX & Equatorial Resources Limited & $14 / 05 / 1987$ & \\
\hline ERA & Energy Resources of Australia Limited & 19/11/1980 & \\
\hline ERC & eircom Holdings Limited & $14 / 02 / 2005$ & $5 / 01 / 2010$ \\
\hline ERJ & Enerji Ltd & $18 / 04 / 1991$ & \\
\hline ERL & Empire Resources Limited & $1 / 02 / 2007$ & \\
\hline ERM & Emmerson Resources Limited & $17 / 12 / 2007$ & \\
\hline ERX & Emperor Range Group Limited & $12 / 11 / 2014$ & \\
\hline ESG & Eastern Star Gas Limited & $8 / 02 / 2001$ & $21 / 11 / 2011$ \\
\hline ESI & Environmental Clean Technologies Limited & $5 / 02 / 1986$ & \\
\hline ESK & Etherstack PLC & $7 / 09 / 2012$ & \\
\hline ESL & Earth Sanctuaries Limited & - & $21 / 03 / 2005$ \\
\hline ESM & Esperance Minerals Limited & $26 / 09 / 1970$ & \\
\hline ESR & Estrella Resources Limited & $9 / 05 / 2012$ & \\
\hline ESS & Essa Australia Limited & $27 / 10 / 2004$ & $7 / 04 / 2011$ \\
\hline ESV & Eservglobal Limited & $8 / 09 / 2000$ & \\
\hline ETE & Entek Energy Ltd & $16 / 08 / 2004$ & \\
\hline ETF & UBS IQ Morningstar Australia Quality ETF & $18 / 10 / 2012$ & \\
\hline ETR & Etrade Australia Limited & $23 / 10 / 1986$ & $2 / 07 / 2007$ \\
\hline EUM & Eumeralla Resources Ltd & $3 / 05 / 2012$ & \\
\hline EUR & European Lithium Limited & $2 / 12 / 2010$ & \\
\hline EVE & EVE Investments Limited & $8 / 04 / 2004$ & \\
\hline EVM & EnviroMission Limited & $6 / 08 / 2001$ & \\
\hline EVN & Evolution Mining Limited & $20 / 08 / 2002$ & \\
\hline EVO & Evolve Education Group Limited & $5 / 12 / 2014$ & \\
\hline EVR & Endeavour Mining Corporation & $6 / 12 / 2011$ & $12 / 01 / 2016$ \\
\hline EVT & Event Hospitality and Entertainment Ltd & $31 / 01 / 1962$ & \\
\hline EVZ & EVZ Limited & $26 / 04 / 1985$ & \\
\hline EWC & Energy World Corporation Ltd & $8 / 12 / 1988$ & \\
\hline EWN & Erawan Company Limited & $14 / 02 / 1996$ & $15 / 04 / 2009$ \\
\hline EXC & Exterra Resources Limited & $26 / 05 / 2011$ & \\
\hline EXG & Excelsior Gold Limited & $26 / 06 / 2007$ & \\
\hline EXL & Excel Coal Limited & $3 / 05 / 2004$ & $10 / 11 / 2006$ \\
\hline EXM & Excalibur Mining Corporation Limited & $12 / 12 / 1985$ & \\
\hline EXO & Ennox Group Ltd & $18 / 05 / 2007$ & \\
\hline EXR & Elixir Petroleum Limited & $19 / 07 / 2004$ & \\
\hline EXS & Exco Resources Limited & $12 / 11 / 1998$ & $16 / 01 / 2013$ \\
\hline EXT & Extract Resources Ltd & $4 / 08 / 2000$ & $17 / 04 / 2012$ \\
\hline EXU & Explaurum Limited & $15 / 07 / 2005$ & \\
\hline EYM & Elysium Resources Limited & $18 / 10 / 2006$ & \\
\hline EZA & EZA Corporation Ltd & $7 / 10 / 2011$ & \\
\hline EZL & Euroz Limited & $30 / 06 / 1977$ & \\
\hline FAC & Facilitate Digital Holdings Limited & $14 / 09 / 2005$ & $27 / 12 / 2013$ \\
\hline
\end{tabular}




\begin{tabular}{|c|c|c|c|}
\hline ASX Code & Company Name & Listing Date & Delisting Date \\
\hline FAN & Fantastic Holdings Limited & $22 / 09 / 1999$ & \\
\hline FAR & Far Limited & 23/01/1986 & \\
\hline FAS & Fairstar Resources Limited & $30 / 10 / 2006$ & \\
\hline FBR & Fastbrick Robotics Ltd & $8 / 02 / 2001$ & \\
\hline FBU & Fletcher Building Limited & $26 / 03 / 2001$ & \\
\hline FCG & Frontier Capital Group Limited & $6 / 12 / 2011$ & \\
\hline FCN & Falcon Minerals Limited & $20 / 10 / 1988$ & \\
\hline FCO & Farsands Solutions Limited & $30 / 10 / 2003$ & $6 / 12 / 2005$ \\
\hline$\overline{\text { FCR }}$ & Ferrum Crescent Limited & $17 / 11 / 2005$ & \\
\hline FCT & Firstwave Cloud Technology Limited & $11 / 05 / 2011$ & \\
\hline FEA & Forest Enterprises Australia Limited & $6 / 06 / 2000$ & $30 / 08 / 2010$ \\
\hline FEL & Fe Limited & $14 / 10 / 2005$ & \\
\hline FET & Folkestone Education Trust & $22 / 05 / 2003$ & \\
\hline FFG & Fatfish Internet Group Ltd & $8 / 12 / 1995$ & \\
\hline FFI & FFI Holdings Limited & $15 / 01 / 1987$ & \\
\hline FGE & Forge Group Limited & $28 / 06 / 2007$ & $1 / 09 / 2014$ \\
\hline FGF & First Growth Funds Limited & 19/12/1986 & \\
\hline FGI & Flat Glass Industries Limited & $3 / 11 / 2006$ & $3 / 06 / 2016$ \\
\hline FGL & Foster's Group Limited & $4 / 02 / 1982$ & $21 / 12 / 2011$ \\
\hline FGR & First Graphite Limited & $18 / 01 / 1995$ & \\
\hline FGX & Future Generation Investment Company Limited & $6 / 03 / 1997$ & \\
\hline FHM & Freehill Mining Limited & $9 / 04 / 2002$ & $23 / 03 / 2016$ \\
\hline FID & Fiducian Group Limited & $12 / 09 / 2000$ & \\
\hline FIN & Aii S\&P/ASX 200 Financials & $31 / 03 / 2010$ & $13 / 06 / 2013$ \\
\hline FIT & Fitgenes Limited & $10 / 10 / 2003$ & $4 / 01 / 2016$ \\
\hline FIX & Aii S\&P Financials $\mathrm{x}$-A-REIT & $14 / 04 / 2010$ & $13 / 06 / 2013$ \\
\hline FLK & Folkestone Limited & $14 / 06 / 2000$ & \\
\hline FLN & Freelancer Limited & $15 / 11 / 2013$ & \\
\hline FLR & Frankland River Olive Company Limited & $14 / 07 / 2006$ & 21/07/2016 \\
\hline FLT & Flight Centre Travel Group Limited & $1 / 12 / 1995$ & \\
\hline FLX & Felix Resources Limited & $1 / 08 / 1970$ & $31 / 12 / 2009$ \\
\hline FMG & Fortescue Metals Group Ltd & 19/03/1987 & \\
\hline FML & Focus Minerals Limited & $13 / 09 / 2000$ & \\
\hline FMS & Flinders Mines Limited & $20 / 02 / 2002$ & \\
\hline FND & Finders Resources Limited & $8 / 06 / 2007$ & \\
\hline FNP & Freedom Foods Group Limited & $7 / 11 / 1985$ & \\
\hline FNT & Frontier Resources Limited & $9 / 04 / 2003$ & \\
\hline FOA & Foodland Associated Limited & - & $2 / 12 / 2005$ \\
\hline FOD & The Food Revolution Group Limited & $29 / 06 / 2012$ & \\
\hline FOX & Twenty-First Century Fox, Inc. & $4 / 11 / 2004$ & $9 / 05 / 2014$ \\
\hline FOY & Foy Group Limited & $23 / 11 / 1989$ & \\
\hline FPA & Fisher \& Paykel Appliances Holdings Limited & $21 / 11 / 2001$ & $28 / 11 / 2012$ \\
\hline FPH & Fisher \& Paykel Healthcare Corporation Limited & $21 / 11 / 2001$ & \\
\hline FRC & Forte Consolidated Limited & $14 / 04 / 2011$ & \\
\hline FRE & Freshtel Holdings Limited & $26 / 04 / 2005$ & \\
\hline FRI & Finbar Group Limited & 27/01/1995 & \\
\hline FRM & Farm Pride Foods Limited & 19/12/1997 & \\
\hline FRN & Fraser Range Metals Group Ltd & $4 / 08 / 2004$ & \\
\hline FRS & FerrAus Limited & $31 / 12 / 2003$ & $27 / 10 / 2011$ \\
\hline FRT & Farnell \& Thomas Limited & - & $23 / 08 / 2005$ \\
\hline FSA & FSA Group Limited & $11 / 01 / 2001$ & \\
\hline FSE & Firestone Energy Limited & $24 / 06 / 1993$ & $30 / 08 / 2016$ \\
\hline FSF & Fonterra Shareholders' Fund & $30 / 11 / 2012$ & \\
\hline FSI & Flagship Investments Limited & $20 / 12 / 2000$ & \\
\hline FSN & Fusion Resources Limited & $3 / 05 / 2005$ & $8 / 04 / 2009$ \\
\hline FST & Folkestone Social Infrastructure Fund & $25 / 02 / 2011$ & $7 / 01 / 2015$ \\
\hline FTE & Forte Energy NL & $28 / 10 / 1994$ & $20 / 04 / 2015$ \\
\hline
\end{tabular}




\begin{tabular}{|c|c|c|c|}
\hline ASX Code & Company Name & Listing Date & Delisting Date \\
\hline FTH & Fifth Element Resources Limited & $21 / 05 / 2014$ & $27 / 04 / 2015$ \\
\hline FTT & Factor Therapeutics Limited & $19 / 03 / 2004$ & \\
\hline FTZ & Fertoz Limited & $2 / 09 / 2013$ & \\
\hline FUL & Fulcrum Equity Limited & $29 / 09 / 1993$ & $30 / 08 / 2010$ \\
\hline FUN & Funtastic Limited & $14 / 09 / 2000$ & \\
\hline FWD & Fleetwood Corporation Limited & $15 / 04 / 1987$ & \\
\hline FXJ & Fairfax Media Limited & $8 / 05 / 1992$ & \\
\hline FXL & FlexiGroup Limited & $12 / 12 / 2006$ & \\
\hline FXR & Fox Resources Limited & $26 / 04 / 2002$ & $30 / 08 / 2016$ \\
\hline FYI & FYI Resources Limited & $20 / 01 / 1994$ & \\
\hline FZR & Fitzroy River Corporation Ltd & $4 / 03 / 1998$ & \\
\hline G8C & G8 Communications Limited & $16 / 08 / 1984$ & \\
\hline GAP & Gale Pacific Limited & $14 / 12 / 2000$ & \\
\hline GAS & GasNet Australia Group & $17 / 12 / 2001$ & $17 / 11 / 2006$ \\
\hline GAU & Great Australian Resources Limited & $11 / 03 / 2004$ & $17 / 12 / 2009$ \\
\hline GBA & Grandbridge Limited & $12 / 09 / 2000$ & \\
\hline GBE & Globe Metals \& Mining Limited & $9 / 12 / 2005$ & \\
\hline$\overline{\text { GBG }}$ & Gindalbie Metals Ltd & 12/04/1994 & \\
\hline GBI & Genera Biosystems Limited & $11 / 06 / 2008$ & \\
\hline GBM & GBM Gold Ltd & $5 / 03 / 2007$ & \\
\hline GBP & Global Petroleum Limited & $24 / 06 / 1994$ & \\
\hline GBT & GBST Holdings Limited & $28 / 06 / 2005$ & \\
\hline GBX & GB Energy Limited & $7 / 06 / 2007$ & \\
\hline GBZ & GBM Resources Limited & $24 / 10 / 2007$ & \\
\hline GCA & GEC Asian Value Fund & $19 / 07 / 2001$ & $6 / 12 / 2006$ \\
\hline GCE & GEC Eurogrowth Fund & - & $30 / 12 / 2005$ \\
\hline GCG & Greencap Limited & 15/01/1987 & $2 / 12 / 2013$ \\
\hline GCH & GEC Australian Healthcare Fund & 19/07/2001 & $6 / 12 / 2006$ \\
\hline GCL & Gloucester Coal Ltd & 27/06/1985 & $11 / 07 / 2012$ \\
\hline GCN & GoConnect Limited & $22 / 11 / 2000$ & \\
\hline GCR & Golden Cross Resources Limited & $27 / 03 / 1996$ & \\
\hline GCS & Global Construction Services Limited & 17/08/2007 & \\
\hline GCX & Golden China Resources Corporation & $15 / 12 / 2006$ & $10 / 01 / 2008$ \\
\hline GCY & Gascoyne Resources Limited & $11 / 12 / 2009$ & \\
\hline GDA & Gondwana Resources Limited & $2 / 04 / 1987$ & \\
\hline GDI & GDI Property Group & $17 / 12 / 2013$ & \\
\hline GDO & Gold One International Limited & $13 / 02 / 2001$ & $31 / 01 / 2014$ \\
\hline GDY & Geodynamics Limited & $12 / 09 / 2002$ & \\
\hline GED & Golden Deeps Limited & $20 / 08 / 1992$ & \\
\hline GEG & Grays eCommerce Group Limited & $2 / 08 / 2007$ & \\
\hline GEM & G8 Education Limited & $5 / 12 / 2007$ & \\
\hline GEN & Genesis Research \& Development Corporation Limited & $22 / 09 / 2000$ & $30 / 08 / 2013$ \\
\hline GER & Greenearth Energy Limited & $4 / 02 / 2008$ & \\
\hline GES & Genesis Resources Limited & $27 / 10 / 2009$ & \\
\hline GFD & Green's Foods Limited & $10 / 06 / 1993$ & $12 / 03 / 2007$ \\
\hline GFF & Goodman Fielder Limited & $19 / 12 / 2005$ & $20 / 03 / 2015$ \\
\hline GFL & Global Masters Fund Limited & $16 / 05 / 2006$ & \\
\hline GFY & Godfreys Group Limited & $10 / 12 / 2014$ & \\
\hline GGB & GGG Resources Plc & $19 / 05 / 2011$ & $19 / 03 / 2012$ \\
\hline GGE & Grand Gulf Energy Limited & $20 / 03 / 1998$ & \\
\hline GGG & Greenland Minerals and Energy Limited & $20 / 06 / 2006$ & \\
\hline GGH & Global Gold Holdings Limited & $20 / 11 / 2007$ & \\
\hline GGL & The Gruden Group Ltd & $15 / 01 / 2008$ & \\
\hline GGL & Gribbles Group Limited (The) & - & $7 / 01 / 2005$ \\
\hline GGN & Gallery Gold Limited & - & $7 / 04 / 2006$ \\
\hline GGU & GEC Global Fund & 19/07/2001 & $16 / 08 / 2006$ \\
\hline GGX & Gas2Grid Limited & $13 / 05 / 2005$ & \\
\hline
\end{tabular}




\begin{tabular}{|c|c|c|c|}
\hline ASX Code & Company Name & Listing Date & Delisting Date \\
\hline GHC & Generation Healthcare REIT & $15 / 05 / 2006$ & \\
\hline GHG & Grand Hotel Group & $31 / 07 / 1996$ & 9/05/2007 \\
\hline GHT & Geothermal Resources Limited & $21 / 03 / 2006$ & $16 / 01 / 2012$ \\
\hline GIA & Giaconda Limited & $28 / 09 / 2005$ & $30 / 08 / 2013$ \\
\hline GID & GI Dynamics, Inc & $7 / 09 / 2011$ & \\
\hline GIR & Giralia Resources NL & $30 / 07 / 1987$ & $7 / 04 / 2011$ \\
\hline GJT & Galileo Japan Trust & $18 / 12 / 2006$ & \\
\hline GLA & Gladiator Resources Limited & $27 / 06 / 2006$ & \\
\hline GLB & Globe International Limited & $24 / 05 / 2001$ & \\
\hline GLE & GLG Corp Limited & $14 / 12 / 2005$ & \\
\hline GLF & Gulf Industrials Limited & $10 / 08 / 2006$ & \\
\hline GLG & Gerard Lighting Group Limited & - & $26 / 10 / 2012$ \\
\hline GLH & Global Health Limited & $4 / 04 / 2000$ & \\
\hline GLL & Galilee Energy Limited & $15 / 02 / 1996$ & \\
\hline GLN & Gleneagle Gold Limited & $28 / 07 / 2003$ & \\
\hline GLS & Gullivers Travel Group & $15 / 12 / 2004$ & $27 / 09 / 2006$ \\
\hline GLY & Glory Resources Limited & $21 / 01 / 2011$ & $18 / 03 / 2014$ \\
\hline GMA & Genworth Mortgage Insurance Australia Limited & $20 / 05 / 2014$ & \\
\hline GMC & Gulf Manganese Corporation Limited & $3 / 08 / 2007$ & \\
\hline GMD & Genesis Minerals Limited & $2 / 08 / 2007$ & \\
\hline GME & GME Resources Limited & $21 / 08 / 1980$ & \\
\hline GMF & GPT Metro Office Fund & $24 / 10 / 2014$ & \\
\hline GMG & Goodman Group & $2 / 02 / 2005$ & \\
\hline GMI & Global Mining Investments Limited & $8 / 04 / 2004$ & $29 / 11 / 2012$ \\
\hline GML & Gateway Mining Limited & $2 / 08 / 1971$ & \\
\hline GMM & General Mining Corporation Limited & $21 / 12 / 2009$ & $5 / 09 / 2016$ \\
\hline GMN & Gold Mountain Limited & $2 / 09 / 2011$ & \\
\hline GMR & Golden Rim Resources Limited & 13/08/1987 & \\
\hline GMY & Goldfields Money Limited & $22 / 05 / 2012$ & \\
\hline GNB & Greenbox Group Limited & $12 / 11 / 1987$ & $30 / 08 / 2011$ \\
\hline GNC & Graincorp Limited & $30 / 03 / 1998$ & \\
\hline GNE & Genesis Energy Limited & $17 / 04 / 2014$ & \\
\hline GNG & GR Engineering Services Limited & $19 / 04 / 2011$ & \\
\hline GNS & Gunns Limited & $10 / 02 / 1983$ & $30 / 08 / 2013$ \\
\hline GNV & Green Invest Limited & $20 / 02 / 2008$ & $30 / 08 / 2016$ \\
\hline GOE & GO energy Group Limited & $15 / 12 / 2000$ & \\
\hline GOG & Great Artesian Oil \& Gas Limited & $5 / 08 / 2003$ & $12 / 08 / 2008$ \\
\hline GOP & Gippsland Offshore Petroleum Limited & $10 / 01 / 2005$ & $24 / 06 / 2009$ \\
\hline GOR & Gold Road Resources Limited & $4 / 07 / 2006$ & \\
\hline GOW & Gowing Brothers Ltd & $20 / 06 / 1944$ & \\
\hline $\mathrm{GOZ}$ & Growthpoint Properties Australia & $4 / 07 / 2007$ & \\
\hline GPE & Green Pacific Energy Limited & $1 / 01 / 1974$ & $20 / 08 / 2010$ \\
\hline GPH & Goldphyre Resources Limited & $20 / 12 / 2011$ & \\
\hline GPP & Greenpower Energy Limited & $5 / 03 / 2008$ & \\
\hline GPR & Geopacific Resources Ltd & $9 / 05 / 2006$ & \\
\hline GPS & GPS Alliance Holdings Limited & $2 / 07 / 2013$ & \\
\hline GPT & GPT Group & 29/04/1971 & \\
\hline GQH & Gosford Quarry Holdings Limited & $28 / 07 / 2006$ & $16 / 06 / 2008$ \\
\hline GRB & Gage Roads Brewing Co Limited & $13 / 12 / 2006$ & \\
\hline GRD & GRD Limited & $12 / 02 / 1987$ & $4 / 12 / 2009$ \\
\hline GRF & Global Resource Masters Fund Limited & $3 / 02 / 2009$ & $14 / 03 / 2016$ \\
\hline GRG & GRG International LTD & $14 / 10 / 2010$ & $4 / 01 / 2016$ \\
\hline GRL & Gawler Resources Limited & $12 / 09 / 2006$ & $26 / 11 / 2007$ \\
\hline GRN & Gravity Diamonds Limited & $31 / 07 / 1987$ & $31 / 05 / 2007$ \\
\hline GRO & GroPep Limited & $31 / 08 / 2000$ & $4 / 12 / 2006$ \\
\hline GRR & Grange Resources Limited & $27 / 03 / 1986$ & \\
\hline GRV & Greenvale Energy Limited & $28 / 07 / 1971$ & \\
\hline
\end{tabular}




\begin{tabular}{|c|c|c|c|}
\hline ASX Code & Company Name & Listing Date & Delisting Date \\
\hline GRY & Gryphon Minerals Limited & $22 / 04 / 2004$ & \\
\hline GSC & Global Geoscience Limited & $19 / 12 / 2007$ & \\
\hline GSE & Goldsearch Limited & $21 / 07 / 1988$ & \\
\hline GSZ & Global Strategic Metals N.L. & $9 / 04 / 1970$ & $18 / 08 / 2014$ \\
\hline GTE & Great Western Exploration Limited & $5 / 06 / 2007$ & \\
\hline GTG & Genetic Technologies Limited & $30 / 07 / 1987$ & \\
\hline GTK & Gentrack Group Limited & $25 / 06 / 2014$ & \\
\hline GTP & Great Southern Limited & $5 / 07 / 1999$ & $30 / 08 / 2010$ \\
\hline GTR & GTI Resources Limited & $10 / 08 / 2007$ & \\
\hline GTV & Global Television Limited & $1 / 10 / 1987$ & $15 / 01 / 2007$ \\
\hline GUD & GUD Holdings Limited & $1 / 01 / 1959$ & \\
\hline GUI & Guilford Investments Limited & $7 / 09 / 1999$ & $30 / 08 / 2010$ \\
\hline GUJ & Gujarat Nre Resources NL & $25 / 08 / 2005$ & $28 / 03 / 2008$ \\
\hline GUL & Gullewa Limited & $1 / 09 / 1977$ & \\
\hline GVF & Global Value Fund Limited & $21 / 07 / 2014$ & \\
\hline GWA & GWA Group Limited & $20 / 05 / 1993$ & \\
\hline GWR & GWR Group Limited & $24 / 12 / 2004$ & \\
\hline GXL & Greencross Limited & $15 / 06 / 2007$ & \\
\hline GXY & Galaxy Resources Limited & $6 / 02 / 2007$ & \\
\hline GZL & Gazal Corporation Limited & $30 / 04 / 1965$ & \\
\hline HAN & Hanson PLC & $17 / 10 / 2003$ & $30 / 08 / 2007$ \\
\hline $\mathrm{HAO}$ & Haoma Mining NL & $6 / 08 / 1970$ & \\
\hline HAR & Haranga Resources Limited & $9 / 12 / 2010$ & \\
\hline HAS & Hastings Technology Metals Limited & $20 / 02 / 2008$ & \\
\hline HAV & Havilah Resources Limited & $21 / 03 / 2002$ & \\
\hline HAW & Hawthorn Resources Limited & $3 / 07 / 1986$ & \\
\hline $\mathrm{HCC}$ & Hutchisons Child Care Services Ltd & $22 / 04 / 2004$ & $3 / 11 / 2006$ \\
\hline HCD & Huadu City Developments Limited & - & $29 / 08 / 2005$ \\
\hline $\mathrm{HCH}$ & Hot Chili Limited & $3 / 05 / 2010$ & \\
\hline $\mathrm{HCN}$ & Health Communication Network Limited & - & $24 / 02 / 2005$ \\
\hline HCT & Holista Colltech Limited & $26 / 02 / 2004$ & \\
\hline HDF & APA Sub Group & $13 / 12 / 2004$ & $10 / 01 / 2013$ \\
\hline HDG & Hodges Resources Limited & $20 / 10 / 2005$ & $30 / 08 / 2016$ \\
\hline HDI & Handini Resources Limited & $23 / 10 / 2008$ & $30 / 08 / 2011$ \\
\hline HDR & Hardman Resources Limited & 6/08/1987 & 19/01/2007 \\
\hline HDX & Hughes Drilling Limited & $29 / 06 / 2007$ & \\
\hline HEG & Hill End Gold Limited & $17 / 07 / 2003$ & \\
\hline HER & Herald Resources Limited & $16 / 07 / 1981$ & $16 / 12 / 2009$ \\
\hline HFA & HFA Holdings Limited & $28 / 04 / 2006$ & \\
\hline HFR & Highfield Resources Limited & $7 / 02 / 2012$ & \\
\hline HGG & Henderson Group PLC & $23 / 12 / 2003$ & \\
\hline HGL & Hudson Investment Group Limited & $9 / 06 / 1994$ & \\
\hline $\mathrm{HGO}$ & Hillgrove Resources Limited & $22 / 07 / 1994$ & \\
\hline HHL & Hunter Hall International Limited & $28 / 02 / 2001$ & \\
\hline HHM & Hampton Hill Mining NL & $10 / 05 / 1994$ & \\
\hline HHV & Hunter Hall Global Value Limited & $19 / 03 / 2004$ & \\
\hline HHY & Hastings High Yield Fund & $11 / 04 / 2005$ & \\
\hline HIC & Huntley Investment Company Limited & $31 / 01 / 1994$ & $30 / 01 / 2009$ \\
\hline HIG & Highlands Pacific Limited & $12 / 06 / 1997$ & \\
\hline HII & Hire Intelligence International Limited & $18 / 01 / 2002$ & $27 / 02 / 2013$ \\
\hline HIL & Hills Limited & $31 / 01 / 1962$ & \\
\hline HIN & HeartWare International, Inc. & $3 / 11 / 2008$ & $18 / 09 / 2013$ \\
\hline HIT & HiTech Group Australia Limited & $17 / 04 / 2000$ & \\
\hline HJB & HJB Corporation Limited & $12 / 09 / 2000$ & \\
\hline HLO & Helloworld Limited & $13 / 09 / 2000$ & \\
\hline HLS & Hillcrest Litigation Services Limited & $14 / 10 / 1993$ & \\
\hline HLX & Helix Resources Limited & $22 / 05 / 1986$ & \\
\hline
\end{tabular}




\begin{tabular}{|c|c|c|c|}
\hline ASX Code & Company Name & Listing Date & Delisting Date \\
\hline HLY & Hills Motorway Group & - & $22 / 06 / 2005$ \\
\hline HMC & Hydromet Corporation Limited & $1 / 11 / 1984$ & $18 / 07 / 2012$ \\
\hline HME & Home Building Society Limited & $1 / 03 / 2002$ & $24 / 12 / 2007$ \\
\hline HMI & Harvest Minerals Ltd & $15 / 09 / 2010$ & $25 / 07 / 2016$ \\
\hline HMR & Hindmarsh Resources Limited & $11 / 07 / 2005$ & $14 / 07 / 2006$ \\
\hline HMX & Hammer Metals Limited & $25 / 02 / 2003$ & \\
\hline $\mathrm{HNG}$ & HGL Limited & $24 / 08 / 1971$ & \\
\hline HNR & Hannans Ltd & $5 / 12 / 2003$ & \\
\hline HOG & Hawkley Oil and Gas Limited & $5 / 05 / 2006$ & \\
\hline HOM & Homeloans Limited & $19 / 03 / 2001$ & \\
\hline HOR & Horseshoe Metals Limited & $29 / 07 / 2010$ & \\
\hline HPI & Hotel Property Investments & $10 / 12 / 2013$ & \\
\hline HPR & High Peak Royalties Limited & $30 / 03 / 2007$ & \\
\hline HPX & HPAL Limited & $8 / 12 / 2000$ & $15 / 11 / 2007$ \\
\hline HRL & HRL Holdings Ltd & $14 / 11 / 2007$ & \\
\hline HRR & Heron Resources Limited & $7 / 08 / 1996$ & \\
\hline HRS & Hudson Resources Limited & $26 / 11 / 1979$ & $9 / 07 / 2014$ \\
\hline HSK & Heemskirk Consolidated Limited & $28 / 10 / 2004$ & \\
\hline HSN & Hansen Technologies Limited & $1 / 06 / 2000$ & \\
\hline HSO & Healthscope Limited & $28 / 07 / 2014$ & \\
\hline HSP & Healthscope Ltd & $4 / 05 / 1994$ & $14 / 10 / 2010$ \\
\hline HST & Hastie Group Limited & $29 / 03 / 2005$ & $30 / 08 / 2013$ \\
\hline HT8 & Harris Technology Group Limited & 24/06/1999 & \\
\hline HTA & Hutchison Telecommunications (Australia) Limited & 17/08/1999 & \\
\hline HUB & HUB24 Limited & $5 / 07 / 2007$ & \\
\hline HUN & Hunnu Coal Limited & $12 / 02 / 2010$ & $23 / 01 / 2012$ \\
\hline HUO & Huon Aquaculture Group Limited & $23 / 10 / 2014$ & \\
\hline HVN & Harvey Norman Holdings Ltd & $3 / 09 / 1987$ & \\
\hline HWE & Henry Walker Eltin Group Limited & $1 / 06 / 1980$ & $30 / 08 / 2013$ \\
\hline HWG & Hostworks Group Limited & $14 / 12 / 1987$ & $18 / 03 / 2008$ \\
\hline HWT & Harvey World Travel Group Limited & - & $3 / 01 / 2006$ \\
\hline HWW & HWW Limited & - & $20 / 04 / 2006$ \\
\hline HXG & Hexagon Resources Limited & $30 / 10 / 2008$ & \\
\hline HXL & Hexima Limited & $27 / 08 / 2007$ & $17 / 06 / 2011$ \\
\hline HZL & HZ Lim Limited & 9/11/2006 & $30 / 08 / 2012$ \\
\hline HZN & Horizon Oil Limited & $30 / 09 / 1981$ & \\
\hline IAA & iShares Asia 50 ETF & $10 / 09 / 2008$ & \\
\hline $\mathrm{IAB}$ & Inabox Group Limited & $12 / 07 / 2013$ & \\
\hline IAF & iShares Core Composite Bond ETF & $14 / 03 / 2012$ & \\
\hline IAG & Insurance Australia Group Limited & $8 / 08 / 2000$ & \\
\hline IAL & Investment Austasia Limited & - & $26 / 08 / 2004$ \\
\hline IAM & Intiger Group Limited & $18 / 12 / 2001$ & \\
\hline IAS & International All Sports Limited & $6 / 08 / 1999$ & $16 / 10 / 2009$ \\
\hline IAU & Intrepid Mines Limited & $9 / 11 / 1993$ & \\
\hline IBC & Ironbark Capital Limited & $2 / 04 / 1987$ & \\
\hline IBG & Ironbark Zinc Ltd & $16 / 08 / 2006$ & \\
\hline IBK & iShares MSCI BRIC ETF & $10 / 09 / 2008$ & \\
\hline IBR & Iberian Resources Limited & $18 / 11 / 2004$ & $30 / 11 / 2007$ \\
\hline ICG & Inca Minerals Limited & $19 / 06 / 2008$ & \\
\hline $\mathrm{ICH}$ & Infochoice Limited & $21 / 12 / 1999$ & 17/11/2011 \\
\hline $\mathrm{ICL}$ & International Coal Holdings Limited & $7 / 07 / 1994$ & $18 / 03 / 2011$ \\
\hline ICN & Icon Energy Limited & $22 / 09 / 1997$ & \\
\hline ICQ & Icar Asia Limited & $11 / 09 / 2012$ & \\
\hline ICS & ICSGlobal Limited & 23/12/1999 & \\
\hline ICT & iCollege Limited & $24 / 08 / 2009$ & \\
\hline $\mathrm{ICU}$ & Isentric Limited & $12 / 10 / 2000$ & \\
\hline ICZ & Indus Coal Limited & $13 / 03 / 2012$ & \\
\hline
\end{tabular}




\begin{tabular}{|c|c|c|c|}
\hline ASX Code & Company Name & Listing Date & Delisting Date \\
\hline IDA & Indiana Resources Limited & $17 / 08 / 1994$ & \\
\hline IDC & Indochine Mining Limited & $9 / 12 / 2010$ & \\
\hline IDD & Aii S\&P/ASX 200 Industrials & $8 / 04 / 2010$ & $13 / 06 / 2013$ \\
\hline IDE & IDEAS International Limited & $27 / 03 / 2001$ & $26 / 07 / 2012$ \\
\hline IDG & Indago Resources Ltd & 13/11/1986 & $30 / 08 / 2011$ \\
\hline IDL & Industrea Limited & $7 / 06 / 1999$ & $4 / 12 / 2012$ \\
\hline IDM & IDM International Ltd & $8 / 11 / 2006$ & $4 / 01 / 2016$ \\
\hline IDO & Indo Mines Limited & 9/12/1993 & \\
\hline IDP & Indopac Holdings Limited & $4 / 06 / 2007$ & $4 / 01 / 2016$ \\
\hline IDR & Industria REIT & $3 / 12 / 2013$ & \\
\hline IDT & IDT Australia Limited & 24/09/1993 & \\
\hline IDZ & Indoor Skydive Australia Group Limited & $18 / 01 / 2013$ & \\
\hline IEC & Intra Energy Corporation Limited & $4 / 07 / 2007$ & \\
\hline IEM & iShares MSCI Emerging Markets ETF & $10 / 10 / 2007$ & \\
\hline IEQ & International Equities Corporation Limited & 11/03/1993 & \\
\hline IEU & iShares Europe ETF & $10 / 10 / 2007$ & \\
\hline IFL & IOOF Holdings Limited & $5 / 12 / 2003$ & \\
\hline IFM & Infomedia Limited & $16 / 08 / 2000$ & \\
\hline IFN & Infigen Energy & $28 / 10 / 2005$ & \\
\hline IFS & IFS Construction Services Ltd & $16 / 06 / 2004$ & $1 / 09 / 2015$ \\
\hline IFT & Infratil Limited & $30 / 08 / 2010$ & \\
\hline IGB & Ishares Treasury ETF & $14 / 03 / 2012$ & \\
\hline IGD & IAMGold Corporation & $15 / 03 / 2006$ & $30 / 03 / 2007$ \\
\hline IGG & Ingena Group Limited & $18 / 12 / 2007$ & $14 / 01 / 2009$ \\
\hline IGO & Independence Group NL & $17 / 01 / 2002$ & \\
\hline IGR & Integra Mining Limited & $16 / 08 / 2001$ & $21 / 01 / 2013$ \\
\hline IGS & International Goldfields Limited & $8 / 05 / 2002$ & \\
\hline IGX & IGDX Holdings Limited & $9 / 02 / 2007$ & $8 / 07 / 2011$ \\
\hline IHD & iShares S\&P/ASX High Dividend Opportunities ETF & 9/12/2010 & \\
\hline IHK & iShares MSCI Hong Kong ETF & $15 / 11 / 2007$ & \\
\hline IHS & Interpose Holdings Limited & 19/01/2012 & \\
\hline IIF & ING Industrial Fund & 28/11/1991 & $31 / 03 / 2011$ \\
\hline IIL & Innate Immunotherapeutics Limited & $23 / 12 / 2013$ & \\
\hline IIN & iiNET Limited & $20 / 09 / 1999$ & $14 / 09 / 2015$ \\
\hline $\mathrm{IJH}$ & iShares S\&P Mid-Cap ETF & $10 / 10 / 2007$ & \\
\hline IJP & iShares MSCI Japan ETF & $10 / 10 / 2007$ & \\
\hline IJR & iShares S\&P Small-Cap ETF & $10 / 10 / 2007$ & \\
\hline IKO & iShares MSCI South Korea Capped ETF & $15 / 11 / 2007$ & \\
\hline IKW & Ikwezi Minings Limited & $19 / 07 / 2011$ & \\
\hline ILB & Ishares Government Inflation ETF & $14 / 03 / 2012$ & \\
\hline ILC & iShares S\&P/ASX 20 ETF & $9 / 12 / 2010$ & \\
\hline ILU & Iluka Resources Limited & $30 / 06 / 1962$ & \\
\hline IMA & Image Resources NL & $4 / 07 / 2002$ & \\
\hline IMC & Immuron Limited & $30 / 04 / 1999$ & \\
\hline IMD & Imdex Limited & $24 / 09 / 1987$ & \\
\hline IMF & IMF Bentham Limited & $28 / 01 / 2000$ & \\
\hline IMI & IM Medical Ltd & 6/02/1997 & \\
\hline IMU & Imugene Limited & $2 / 12 / 1993$ & \\
\hline INA & Ingenia Communities Group & $1 / 07 / 2004$ & \\
\hline IND & Indus Energy NL & $12 / 02 / 1987$ & \\
\hline INI & Intercoal Limited & $9 / 12 / 1996$ & $13 / 10 / 2006$ \\
\hline INK & Indago Energy Limited & $21 / 04 / 2006$ & \\
\hline INL & Intec Ltd & $2 / 05 / 2002$ & \\
\hline INO & Innovonics Limited & - & $25 / 11 / 2005$ \\
\hline INV & InvestSMART Group Limited & $21 / 04 / 2005$ & \\
\hline INV & InvestorInfo Limited & - & $23 / 12 / 2005$ \\
\hline $\mathrm{IOC}$ & InterOil Corporation & - & $15 / 06 / 2005$ \\
\hline
\end{tabular}




\begin{tabular}{|c|c|c|c|}
\hline ASX Code & Company Name & Listing Date & Delisting Date \\
\hline IOD & IODM Limited & $18 / 11 / 2003$ & \\
\hline IOF & Investa Office Fund & $2 / 04 / 1992$ & \\
\hline IOG & Incremental Oil and Gas Limited & $21 / 01 / 2011$ & \\
\hline $\mathrm{IOH}$ & Iron Ore Holdings Limited & $10 / 05 / 2005$ & $28 / 10 / 2014$ \\
\hline ION & ION Limited & $5 / 12 / 1985$ & $30 / 08 / 2013$ \\
\hline $\mathrm{IOO}$ & iShares Global 100 ETF & $10 / 10 / 2007$ & \\
\hline IOR & IOR Group Limited & $30 / 12 / 2009$ & $5 / 02 / 2010$ \\
\hline IOT & IOT Group Limited & $28 / 01 / 2011$ & \\
\hline IOZ & iShares Core S\&P/ASX 200 ETF & $9 / 12 / 2010$ & \\
\hline IPA & Indigo Properties Australia Limited & $18 / 05 / 2004$ & $4 / 01 / 2016$ \\
\hline IPB & IPB Petroleum Limited & $30 / 04 / 2013$ & \\
\hline IPC & Imperial Pacific Limited & $28 / 07 / 1971$ & \\
\hline IPD & ImpediMed Limited & $24 / 10 / 2007$ & \\
\hline IPE & IPE Limited & $25 / 11 / 2004$ & \\
\hline IPG & Investa Property Group & $1 / 12 / 2000$ & $14 / 09 / 2007$ \\
\hline IPH & IPH Limited & $19 / 11 / 2014$ & \\
\hline IPL & Incitec Pivot Limited & $28 / 07 / 2003$ & \\
\hline IPM & Incremental Petroleum Limited & $20 / 10 / 2005$ & $26 / 03 / 2009$ \\
\hline IPN & Independent Practitioner Network Ltd & $6 / 12 / 1999$ & $14 / 10 / 2008$ \\
\hline IPO & International Petroleum Limited & $2 / 05 / 2006$ & $3 / 09 / 2010$ \\
\hline IPP & iProperty Group Limited & $11 / 09 / 2007$ & $18 / 02 / 2016$ \\
\hline IPT & Impact Minerals Limited & $29 / 11 / 2006$ & \\
\hline IPX & Intrapower Limited & $11 / 07 / 2007$ & $12 / 09 / 2011$ \\
\hline IQE & Intueri Education Group Limited & $23 / 05 / 2014$ & \\
\hline IRC & Intermin Resources Limited & $6 / 02 / 1992$ & \\
\hline IRD & Iron Road Limited & $12 / 06 / 2008$ & \\
\hline IRE & IRESS Limited & $3 / 11 / 2000$ & \\
\hline IRI & Integrated Research Limited & $13 / 12 / 2000$ & \\
\hline IRL & India Resources Limited & $6 / 06 / 2007$ & \\
\hline IRM & Iron Mountain Mining Limited & $25 / 05 / 2007$ & \\
\hline IRN & Indophil Resources NL & $3 / 05 / 2002$ & $2 / 02 / 2015$ \\
\hline IRU & iShares Russell 2000 ETF & $15 / 11 / 2007$ & \\
\hline ISD & iSentia Group Limited & $5 / 06 / 2014$ & \\
\hline ISF & iSOFT Group Limited & $31 / 03 / 2000$ & $3 / 08 / 2011$ \\
\hline ISG & iShares MSCI Singapore ETF & $15 / 11 / 2007$ & \\
\hline ISH & Ishine International Resources Limited & $18 / 12 / 2009$ & \\
\hline ISO & iShares S\&P/ASX Small Ordinaries ETF Index Fund & $9 / 12 / 2010$ & \\
\hline ISS & ISS Group Limited & $30 / 09 / 2004$ & $12 / 08 / 2013$ \\
\hline ISU & iSelect Limited & $24 / 06 / 2013$ & \\
\hline ISX & iSIGNTHIS LTD & $7 / 12 / 1998$ & \\
\hline ITC & Impress Energy Limited & $6 / 10 / 1993$ & $20 / 04 / 2011$ \\
\hline ITD & ITL Limited & $29 / 10 / 2003$ & \\
\hline ITF & Integrated Tree Cropping Limited & $31 / 03 / 2004$ & $26 / 07 / 2006$ \\
\hline ITO & Intoll Group & $16 / 12 / 1996$ & $17 / 12 / 2010$ \\
\hline ITQ & Intecq Limited & $18 / 08 / 1999$ & \\
\hline ITR & Interra Resources Limited & $19 / 06 / 2008$ & $22 / 12 / 2011$ \\
\hline ITW & iShares MSCI Taiwan ETF & $15 / 11 / 2007$ & \\
\hline ITX & itX Group Limited & $12 / 04 / 2007$ & $5 / 01 / 2011$ \\
\hline IUL & Imagine Un Limited & 9/04/1987 & $1 / 09 / 2014$ \\
\hline IVA & Inova Resources Limited & $6 / 08 / 2008$ & 28/11/2013 \\
\hline IVC & InvoCare Limited & $4 / 12 / 2003$ & \\
\hline IVE & iShares MSCI EAFE ETF & $10 / 10 / 2007$ & \\
\hline IVG & Invictus Gold Limited & $27 / 01 / 2011$ & $13 / 01 / 2014$ \\
\hline IVN & Ivanhoe Mines Ltd & - & $13 / 04 / 2005$ \\
\hline IVO & Invigor Group Limited & $3 / 12 / 1999$ & \\
\hline IVQ & Invitrocue Limited & $14 / 12 / 1994$ & \\
\hline IVR & Investigator Resources Ltd & $3 / 04 / 2007$ & \\
\hline
\end{tabular}




\begin{tabular}{|c|c|c|c|}
\hline ASX Code & Company Name & Listing Date & Delisting Date \\
\hline IVS & IVS Holdings Ltd & $8 / 12 / 2003$ & $15 / 03 / 2016$ \\
\hline IVT & Inventis Limited & 14/04/1999 & \\
\hline IVV & iShares S\&P 500 ETF & $10 / 10 / 2007$ & \\
\hline IVX & Invion Limited & $15 / 02 / 2010$ & \\
\hline IWF & Integrated Group Limited & $12 / 10 / 1999$ & $15 / 06 / 2007$ \\
\hline IWG & Iwebgate Limited & $13 / 01 / 2011$ & \\
\hline IWI & International Wine Investment Fund (The) & $22 / 11 / 1996$ & $21 / 07 / 2009$ \\
\hline IWL & IWL Limited & 23/09/1999 & $30 / 11 / 2007$ \\
\hline IXI & iShares Global Consumer Staples ETF & $11 / 03 / 2009$ & \\
\hline IXJ & iShares Global Healthcare ETF & $11 / 03 / 2009$ & \\
\hline IXL & IXLA Limited & 10/09/1986 & $29 / 08 / 2008$ \\
\hline IXP & iShares Global Telecom ETF & $11 / 03 / 2009$ & \\
\hline IYS & IYS Instalment Receipt Limited & - & $17 / 10 / 2006$ \\
\hline IZZ & iShares China Large-Cap ETF & $15 / 11 / 2007$ & \\
\hline JAK & Jackson Minerals Limited & $11 / 06 / 2002$ & $12 / 06 / 2009$ \\
\hline JAL & Jameson Resources Limited & $18 / 12 / 2007$ & \\
\hline JAT & Jatenergy Limited & $30 / 01 / 2008$ & \\
\hline JBH & JB Hi-Fi Limited & $23 / 10 / 2003$ & \\
\hline JBM & Jubilee Mines NL & $5 / 11 / 1987$ & $24 / 04 / 2008$ \\
\hline JCS & Jcurve Solutions Ltd & $8 / 12 / 2000$ & \\
\hline JDV & JDV Limited & - & $19 / 10 / 2005$ \\
\hline JEL & Jacobsen Entertainment Limited & - & $20 / 07 / 2004$ \\
\hline JFG & James Fielding Group & - & $17 / 02 / 2005$ \\
\hline JHC & Japara Healthcare Limited & $17 / 04 / 2014$ & \\
\hline JHX & James Hardie Industries Plc & $1 / 01 / 1951$ & \\
\hline JIN & Jumbo Interactive Limited & $14 / 06 / 1972$ & \\
\hline JKA & Jacka Resources Limited & $9 / 07 / 2010$ & \\
\hline JKL & Just Kapital Litigation Partners Limited & $12 / 05 / 2000$ & \\
\hline JML & Jabiru Metals Limited & $10 / 01 / 2000$ & $22 / 06 / 2011$ \\
\hline JMS & Jupiter Mines Limited & $23 / 12 / 2004$ & $13 / 01 / 2014$ \\
\hline JPR & Jupiter Energy Limited & $12 / 11 / 2003$ & \\
\hline JRL & Jindalee Resources Limited & $11 / 07 / 2002$ & \\
\hline JRV & Jervois Mining Limited & $1 / 11 / 1980$ & \\
\hline JST & Just Group Limited & $7 / 05 / 2004$ & $22 / 09 / 2008$ \\
\hline JVG & JV Global Limited & $5 / 03 / 1986$ & \\
\hline JYC & Joyce Corporation Limited & 17/12/1986 & \\
\hline KAB & Kaboko Mining Limited & $19 / 03 / 2004$ & \\
\hline KAI & Kairos Minerals Limited & $7 / 04 / 1983$ & \\
\hline KAM & K2 Asset Management Holdings Limited & $29 / 11 / 2007$ & \\
\hline KAR & Karoon Gas Australia Ltd & $8 / 06 / 2004$ & \\
\hline KAS & Kasbah Resources Limited & $27 / 04 / 2007$ & \\
\hline KAT & Katana Capital Limited & $23 / 12 / 2005$ & \\
\hline KAZ & KAZ Group Limited & - & $20 / 07 / 2004$ \\
\hline KBC & Keybridge Capital Limited & $23 / 12 / 1999$ & \\
\hline KBL & KBL Mining Limited & $25 / 02 / 2010$ & \\
\hline KBS & Kimberley Securities Limited & - & $12 / 10 / 2005$ \\
\hline KBU & Kabuni Ltd & $1 / 11 / 2012$ & \\
\hline KCN & Kingsgate Consolidated Limited & $21 / 04 / 1988$ & \\
\hline KDL & Kimberley Diamonds Ltd & $24 / 02 / 2012$ & \\
\hline KDR & Kidman Resources Limited & $18 / 01 / 2011$ & \\
\hline KDS & Kids Campus Limited & $30 / 06 / 1974$ & $21 / 07 / 2006$ \\
\hline KEN & KUTh Energy Limited & $25 / 09 / 2007$ & $24 / 01 / 2014$ \\
\hline KEY & Key Petroleum Limited & $26 / 04 / 2007$ & \\
\hline KFE & Kogi Iron Limited & $16 / 01 / 1987$ & \\
\hline KFG & Kingform Health Hometextile Group Limited & $8 / 06 / 2012$ & \\
\hline KGD & Kula Gold Limited & $16 / 11 / 2010$ & \\
\hline KGL & KGL Resources Limited & $17 / 03 / 2005$ & \\
\hline
\end{tabular}




\begin{tabular}{|c|c|c|c|}
\hline ASX Code & Company Name & Listing Date & Delisting Date \\
\hline KGM & Kalnorth Gold Mines Limited & $20 / 01 / 2005$ & \\
\hline KHF & KH Foods Limited & $30 / 06 / 1962$ & $25 / 03 / 2009$ \\
\hline KIK & Kairiki Energy Limited & $10 / 11 / 1983$ & \\
\hline KIM & Kimberley Diamond Company NL & $4 / 01 / 1994$ & $29 / 01 / 2008$ \\
\hline KIN & Kin Mining NL & $2 / 10 / 2013$ & \\
\hline KIS & King Island Scheelilte Limited & $21 / 09 / 1967$ & \\
\hline KKL & Kollakorn Corporation Limited & $29 / 12 / 1986$ & \\
\hline KKO & Kinetiko Energy Limited & $21 / 07 / 2011$ & \\
\hline KKT & Konekt Limited & $4 / 06 / 1987$ & \\
\hline KLM & KLM Group Ltd & $23 / 11 / 1999$ & $4 / 02 / 2010$ \\
\hline KLR & Kaili Resources Limited & 6/03/1997 & \\
\hline KMC & Kalgoorlie Mining Company Ltd & $12 / 04 / 2000$ & $29 / 08 / 2013$ \\
\hline KMD & Kathmandu Holdings Limited & $13 / 11 / 2009$ & \\
\hline KME & Kip McGrath Education Centres Limited & $15 / 08 / 2003$ & \\
\hline KMR & Kumarina Resources Limited & $15 / 12 / 2011$ & $25 / 06 / 2013$ \\
\hline KNH & Koon Holdings Limited & $11 / 07 / 2003$ & \\
\hline KNL & Kibaran Resources Limited & $29 / 10 / 2010$ & \\
\hline KNM & Kneomedia Limited & $2 / 01 / 1992$ & \\
\hline KOR & Korab Resources Limited & $31 / 08 / 2005$ & \\
\hline KOV & Korvest Ltd & $17 / 09 / 1970$ & \\
\hline KPC & Kazakhstan Potash Corporation Limited & $15 / 12 / 2010$ & \\
\hline KPL & Kina Petroleum Limited & $19 / 12 / 2011$ & \\
\hline KPO & Kalina Power Limited & $18 / 01 / 1952$ & \\
\hline KPR & Kupang Resources Limited & $12 / 08 / 2003$ & $1 / 09 / 2015$ \\
\hline KPT & Kangaroo Island Plantation Timbers Ltd & $8 / 07 / 2002$ & \\
\hline KRC & King River Copper Limited & $21 / 09 / 2007$ & \\
\hline KRL & Kangaroo Resources Limited & $19 / 04 / 2007$ & \\
\hline KRM & Kingsrose Mining Limited & $7 / 12 / 2007$ & \\
\hline KRS & Kresta Holdings Limited & 6/01/1977 & \\
\hline KSC & K\&S Corporation Limited & $25 / 08 / 1971$ & \\
\hline KSN & Kingston Resources Limited & $5 / 03 / 1987$ & \\
\hline KTA & Krakatoa Resources Limited & $28 / 12 / 2012$ & \\
\hline KTE & K2 Energy Limited & $2 / 07 / 2004$ & \\
\hline KTL & KTL Technologies Limited & $16 / 07 / 1987$ & $30 / 08 / 2016$ \\
\hline KTO & K2 Energy Limited & $16 / 11 / 2006$ & $2 / 08 / 2007$ \\
\hline KYC & Keycorp Limited & $2 / 07 / 1987$ & $8 / 12 / 2010$ \\
\hline KZL & Kagara Limited & $22 / 12 / 1999$ & $11 / 08 / 2014$ \\
\hline LAA & Latam Autos Limited & $17 / 12 / 2014$ & \\
\hline LAK & Lake Technology Limited & - & $5 / 01 / 2005$ \\
\hline LAL & Lowan Australia Limited & - & $10 / 06 / 2005$ \\
\hline LAM & Laramide Resources Ltd & $30 / 04 / 2013$ & \\
\hline LAS & Lasseters Corporation Limited & $5 / 06 / 2000$ & $12 / 04 / 2016$ \\
\hline LAU & Lindsay Australia Limited & $5 / 07 / 1994$ & \\
\hline LBL & Laserbond Limited & $17 / 12 / 2007$ & \\
\hline LBT & LBT Innovations Limited & $31 / 07 / 2006$ & \\
\hline LCD & Latitude Consolidated Limited & $21 / 12 / 1999$ & \\
\hline LCE & London City Equities Limited & 6/03/1987 & \\
\hline LCG & Living Cities Development Group Limited & $31 / 07 / 2006$ & \\
\hline LCK & Leigh Creek Energy Limited & $17 / 03 / 2005$ & \\
\hline LCL & Lighting Corporation Limited & $15 / 03 / 2000$ & $25 / 02 / 2008$ \\
\hline LCM & Logicamms Limited & $11 / 12 / 2007$ & \\
\hline LCR & Laconia Resources Limited & $16 / 10 / 2009$ & \\
\hline LCT & Living Cell Technologies Limited & $1 / 09 / 2004$ & \\
\hline LCY & Legacy Iron Ore Limited & $8 / 07 / 2008$ & \\
\hline LDM & Land and Mineral Exploration Limited & $29 / 08 / 2003$ & $4 / 01 / 2016$ \\
\hline LDN & Linden \& Conway Limited & $24 / 08 / 1971$ & $23 / 06 / 2008$ \\
\hline LDW & Ludowici Limited & $28 / 07 / 1971$ & $9 / 07 / 2012$ \\
\hline
\end{tabular}




\begin{tabular}{|c|c|c|c|}
\hline ASX Code & Company Name & Listing Date & Delisting Date \\
\hline LEG & Legend Mining Limited & $25 / 08 / 1995$ & \\
\hline LEP & ALE Property Group & $12 / 11 / 2003$ & \\
\hline LER & Leaf Resources Ltd & $5 / 01 / 1999$ & \\
\hline LEX & Lefroy Exploration Limited & $5 / 09 / 1991$ & \\
\hline LFC & Life Corporation Ltd & $18 / 06 / 2004$ & \\
\hline LGD & Legend Corporation Limited & $16 / 03 / 2004$ & \\
\hline LGL & Lihir Gold Limited & 9/10/1995 & $1 / 10 / 2010$ \\
\hline LGM & Luiri Gold Limited & 19/11/2009 & \\
\hline LGO & Longreach Oil Limited & $1 / 12 / 1954$ & \\
\hline LGR & Lanka Graphite Limited & $3 / 11 / 1997$ & \\
\hline LHB & Lionhub Group Limited & $25 / 10 / 2006$ & \\
\hline LHC & Lifehealthcare Group Limited & $5 / 12 / 2013$ & \\
\hline LHD & Lochard Energy Group plc & $7 / 12 / 2007$ & $23 / 05 / 2013$ \\
\hline LHM & Land \& Homes Group Limited & $5 / 10 / 2004$ & \\
\hline LIC & Lifestyle Communities Limited & $15 / 12 / 1998$ & \\
\hline LIM & Lionore Mining International Limited & $8 / 08 / 2003$ & $5 / 06 / 2007$ \\
\hline LIN & Lindian Resources Limited & $2 / 03 / 2000$ & \\
\hline LIO & Lion Energy Limited & $15 / 02 / 1972$ & \\
\hline LIP & Lipa Pharmaceuticals Limited & $29 / 07 / 2004$ & $7 / 11 / 2007$ \\
\hline LIT & Lithium Australia NL & $30 / 10 / 2007$ & \\
\hline LKE & Lake Resources NL & $29 / 08 / 2001$ & \\
\hline LKO & Lakes Oil NL & $5 / 12 / 1985$ & \\
\hline LLA & Living and Leisure Australia Group & $14 / 05 / 1999$ & $19 / 04 / 2012$ \\
\hline LLC & LendLease Group & $31 / 01 / 1962$ & \\
\hline LLO & Lion One Metals Limited & $9 / 05 / 2006$ & \\
\hline LLP & Lend Lease Primelife Group & 6/06/1991 & $24 / 12 / 2009$ \\
\hline LMC & Lemarne Corporation Limited & $23 / 12 / 1971$ & $4 / 01 / 2016$ \\
\hline LME & L\&M Energy Limited & $11 / 01 / 2007$ & $7 / 02 / 2013$ \\
\hline LMG & Latrobe Magnesium Limited & $18 / 12 / 1986$ & \\
\hline LML & Lincoln Minerals Limited & $9 / 03 / 2007$ & \\
\hline LMR & Lemur Resources Limited & $24 / 08 / 2011$ & $2 / 09 / 2015$ \\
\hline LMW & Landmark White Limited & $5 / 12 / 2003$ & \\
\hline LNC & Linc Energy Ltd & $10 / 05 / 2006$ & $20 / 12 / 2013$ \\
\hline LNG & Liquefied Natural Gas Limited & $14 / 09 / 2004$ & \\
\hline LNN & Lion Nathan Limited & $5 / 06 / 2000$ & $27 / 10 / 2009$ \\
\hline LNR & Lonestar Resources Limited & $20 / 03 / 1997$ & $7 / 07 / 2016$ \\
\hline LNU & Linius Technologies Limited & $26 / 07 / 2011$ & \\
\hline LNY & Laneway Resources Ltd & $2 / 04 / 1987$ & \\
\hline LOK & LookSmart Limited & $25 / 02 / 2000$ & $1 / 10 / 2007$ \\
\hline LOM & Lucapa Diamond Company Limited & $19 / 12 / 2006$ & \\
\hline LOV & Lovisa Holdings Limited & $18 / 12 / 2014$ & \\
\hline LPE & Locality Planning Energy Holdings Limited & $11 / 10 / 2011$ & \\
\hline LRC & Laguna Resources NL & $16 / 05 / 1968$ & $16 / 02 / 2012$ \\
\hline LRF & LinQ Resources Fund & $20 / 01 / 2005$ & $12 / 04 / 2013$ \\
\hline LRL & Leyshon Resources Limited & $10 / 01 / 1985$ & \\
\hline LRS & Latin Resources Limited & $21 / 09 / 2010$ & \\
\hline LRX & Longreach Group Limited & $26 / 05 / 1994$ & $29 / 11 / 2006$ \\
\hline LSA & Lachlan Star Limited & $17 / 01 / 1983$ & \\
\hline LSG & Lion Selection Group Limited & $24 / 07 / 1997$ & $7 / 05 / 2007$ \\
\hline LSL & Luminus Systems Limited & $21 / 10 / 2004$ & $21 / 06 / 2012$ \\
\hline LSN & Lawson Gold Limited & $5 / 08 / 2010$ & \\
\hline LSR & Lodestar Minerals Limited & $21 / 12 / 2007$ & \\
\hline LST & Lion Selection Limited & $17 / 04 / 2007$ & $5 / 02 / 2010$ \\
\hline LSX & Lion Selection Group Limited & $13 / 03 / 2013$ & \\
\hline LTN & Lantern Hotel Group & $8 / 07 / 2004$ & \\
\hline LTR & Liontown Resources Limited & $27 / 12 / 2006$ & \\
\hline LTX & Lithex Resources Limited & $17 / 05 / 2011$ & \\
\hline
\end{tabular}




\begin{tabular}{|c|c|c|c|}
\hline ASX Code & Company Name & Listing Date & Delisting Date \\
\hline LVL & LV Living Limited & $8 / 07 / 1993$ & $30 / 08 / 2010$ \\
\hline LVR & Leviathan Resources Limited & $21 / 12 / 2004$ & $25 / 01 / 2007$ \\
\hline LVT & Livetiles Limited & $17 / 08 / 1999$ & \\
\hline LWB & Little World Beverages Limited & $10 / 11 / 2005$ & $10 / 10 / 2012$ \\
\hline LWP & LWP Technologies Limited & $31 / 08 / 2006$ & \\
\hline $\mathrm{LYC}$ & Lynas Corporation Limited & $11 / 09 / 1986$ & \\
\hline LYL & Lycopodium Limited & $17 / 12 / 2004$ & \\
\hline M7T & Mach7 Technologies Limited & $30 / 11 / 2005$ & \\
\hline MAC & Macro Corporation Limited & $6 / 08 / 1990$ & $1 / 09 / 2014$ \\
\hline MAD & Maverick Drilling and Exploration Limited & $8 / 09 / 2010$ & \\
\hline MAG & Magellan Petroleum Australia Limited & - & $3 / 07 / 2006$ \\
\hline MAH & Macmahon Holdings Limited & $1 / 12 / 1983$ & \\
\hline MAM & Aii S\&P/ASX 200 Metals and Mining & $12 / 04 / 2010$ & $13 / 06 / 2013$ \\
\hline MAN & Magna Mining NL & $13 / 03 / 1986$ & $1 / 09 / 2014$ \\
\hline MAQ & Macquarie Telecom Group Limited & $27 / 09 / 1999$ & \\
\hline MAR & Malachite Resources Limited & $26 / 11 / 2002$ & \\
\hline MAS & Mesa Minerals Limited & $21 / 11 / 1980$ & \\
\hline MAT & Matsa Resources Limited & $20 / 04 / 2005$ & \\
\hline MAU & Magnetic Resources NL & $5 / 04 / 2007$ & \\
\hline MAV & Mavuzi Resources Limited & $1 / 08 / 2007$ & $27 / 03 / 2008$ \\
\hline MAX & Millinium's Alternatives Fund & $20 / 12 / 2006$ & \\
\hline $\mathrm{MBC}$ & Malabar Coal Limited & $28 / 03 / 2013$ & \\
\hline MBD & MBD Corporation Limited & $14 / 12 / 2007$ & $1 / 09 / 2015$ \\
\hline MBE & Mobile Embrace Limited & $11 / 05 / 2000$ & \\
\hline MBF & MBf Carpenters Limited & - & $19 / 06 / 2006$ \\
\hline MBK & Metal Bank Limited & $2 / 03 / 2011$ & \\
\hline MBN & Mirabela Nickel Limited & $16 / 07 / 2004$ & $30 / 08 / 2016$ \\
\hline MBO & Mobilarm Limited & $21 / 09 / 2010$ & \\
\hline MBT & Mission NewEnergy Limited & $4 / 05 / 2006$ & \\
\hline $\mathrm{MCC}$ & Macarthur Coal Limited & $5 / 07 / 2001$ & $22 / 12 / 2011$ \\
\hline MCE & Matrix Composites \& Engineering Limited & $16 / 11 / 2009$ & \\
\hline MCG & Macquarie Communications Infrastructure Group & $13 / 08 / 2002$ & $24 / 07 / 2009$ \\
\hline $\mathrm{MCH}$ & Murchison Holdings Limited & $30 / 06 / 1975$ & \\
\hline MCK & MacarthurCook Limited & $11 / 04 / 1991$ & $4 / 09 / 2009$ \\
\hline $\mathrm{MCO}$ & Morning Star Gold NL & $16 / 02 / 1989$ & $4 / 01 / 2016$ \\
\hline MCP & McPherson's Limited & $5 / 12 / 1944$ & \\
\hline MCQ & Macquarie Capital Alliance Group & $8 / 04 / 2005$ & $13 / 10 / 2008$ \\
\hline MCR & Mincor Resources NL & $8 / 07 / 1997$ & \\
\hline MCS & Mcaleese Limited & $28 / 11 / 2013$ & \\
\hline MCT & Metalicity Limited & $21 / 12 / 2005$ & \\
\hline MCU & Mitchell Communication Group Limited & $10 / 03 / 2000$ & 24/11/2010 \\
\hline MCX & Mariner Corporation Limited & $20 / 06 / 1986$ & \\
\hline MCY & Mercury NZ Limited & $10 / 05 / 2013$ & \\
\hline MDD & Mandalong Resources Limtied & $7 / 12 / 2012$ & \\
\hline MDE & MedAire Inc & $20 / 01 / 2003$ & $18 / 08 / 2008$ \\
\hline MDG & Medtech Global Limited & $13 / 08 / 1987$ & \\
\hline MDI & Middle Island Resources Limited & $16 / 12 / 2010$ & \\
\hline MDL & Mineral Deposits Limited & $17 / 04 / 1997$ & \\
\hline MDO & Mindoro Resources Limited & $7 / 12 / 2010$ & $1 / 07 / 2014$ \\
\hline MDR & MedAdvisor Limited & $26 / 05 / 2011$ & \\
\hline MDV & Medivac Limited & $12 / 11 / 1993$ & \\
\hline MDX & Mindax Limited & $8 / 12 / 2004$ & \\
\hline MEB & Medibio Limited & $29 / 01 / 2001$ & \\
\hline MED & Merlin Diamonds Limited & $16 / 10 / 1979$ & \\
\hline MEG & MCM Entertainment Group Limited & $19 / 12 / 2007$ & $8 / 01 / 2013$ \\
\hline MEI & Meteoric Resources NL & $16 / 07 / 2004$ & \\
\hline MEL & Metgasco Limited & $23 / 12 / 2004$ & \\
\hline
\end{tabular}




\begin{tabular}{|c|c|c|c|}
\hline ASX Code & Company Name & Listing Date & Delisting Date \\
\hline MEM & Memphasys Limited & $14 / 05 / 2007$ & \\
\hline MEO & MEO Australia Limited & 27/03/1998 & \\
\hline MEP & Minotaur Exploration Limited & $24 / 02 / 2005$ & \\
\hline MES & Mesbon China Nylon Limited & $11 / 12 / 2007$ & $9 / 11 / 2012$ \\
\hline MEU & Marmota Energy Limited & $21 / 11 / 2007$ & \\
\hline MEY & Marenica Energy Ltd & $6 / 11 / 1980$ & \\
\hline MEZ & Meridian Energy Limited & $29 / 10 / 2013$ & \\
\hline MFF & Magellan Flagship Fund Limited & $19 / 12 / 2006$ & \\
\hline MFG & Magellan Financial Group Limited & $1 / 07 / 2004$ & \\
\hline MGI & Macquarie Goodman Industrial Trust & - & $9 / 02 / 2005$ \\
\hline MGL & Magontec Limited & $6 / 02 / 1986$ & \\
\hline MGM & Macquarie Goodman Management Ltd & - & $9 / 02 / 2005$ \\
\hline MGN & Magellan Petroleum Corporation & $28 / 04 / 2006$ & $2 / 04 / 2013$ \\
\hline MGO & Marengo Mining Limited & $13 / 11 / 2003$ & $17 / 01 / 2013$ \\
\hline MGP & Managed Accounts Holdings Limited & $25 / 06 / 2014$ & \\
\hline MGR & Mirvac Group & $18 / 06 / 1999$ & \\
\hline MGS & MGT Resources Limited & $9 / 01 / 2013$ & \\
\hline MGT & Magnetite Mines Limited & $6 / 04 / 2006$ & \\
\hline MGU & Magnum Mining and Exploration Limited & $23 / 04 / 1987$ & \\
\hline MGV & Musgrave Minerals Limited & $29 / 04 / 2011$ & \\
\hline MGX & Mount Gibson Iron Limited & $12 / 02 / 1976$ & \\
\hline MGZ & Medigard Limited & $6 / 02 / 2004$ & \\
\hline MHC & Manhattan Corporation Limited & $29 / 01 / 2008$ & \\
\hline MHI & Merchant House International Limited & $31 / 10 / 1994$ & \\
\hline MHM & MHM Metals Limited & $14 / 12 / 2007$ & \\
\hline MIA & MIA Group Limited & - & $7 / 10 / 2004$ \\
\hline MIC & Michelago Limited & $20 / 06 / 1996$ & $12 / 07 / 2007$ \\
\hline MIF & Macarthurcook Industrial Property Fund & $5 / 12 / 2007$ & $8 / 10 / 2010$ \\
\hline MIG & Migme Limited & $6 / 06 / 2001$ & \\
\hline MIH & MNC Media Investment Ltd & $13 / 09 / 2013$ & \\
\hline MII & Meridian Minerals Limited & $18 / 12 / 2007$ & $12 / 12 / 2011$ \\
\hline MIN & Mineral Resources Limited & $28 / 07 / 2006$ & \\
\hline MIO & Miclyn Express Offshore Limited & $26 / 03 / 2010$ & $18 / 12 / 2013$ \\
\hline MIR & Mirrabooka Investments Limited & $28 / 06 / 2001$ & \\
\hline MIS & Midwest Corporation Limited & $29 / 06 / 1989$ & $29 / 01 / 2009$ \\
\hline MIX & Mirvac Industrial Trust & $20 / 05 / 2005$ & $9 / 12 / 2014$ \\
\hline MIZ & Minera Gold Limited & $15 / 11 / 2006$ & \\
\hline MJC & Mejority Capital Limited & $21 / 12 / 2006$ & \\
\hline MKB & Moko Social Media Limited & $27 / 06 / 2007$ & \\
\hline MKE & Mako Hydrocarbons Limited & $1 / 07 / 1992$ & $4 / 04 / 2016$ \\
\hline MKO & Metaliko Resources Limited & $6 / 10 / 2010$ & \\
\hline MLA & Medical Australia Limited & $20 / 12 / 2004$ & \\
\hline MLB & Melbourne IT Limited & $14 / 12 / 1999$ & \\
\hline MLC & Mothercare Australia Limited & $24 / 08 / 1993$ & $30 / 08 / 2013$ \\
\hline MLD & Maca Limited & $3 / 11 / 2010$ & \\
\hline MLI & Mintails Limited & 28/07/1971 & \\
\hline MLM & Metallica Minerals Limited & $23 / 11 / 2004$ & \\
\hline MLS & Metals Australia Ltd & $23 / 01 / 1986$ & \\
\hline MLT & Milton Corporation Limited & $30 / 01 / 1962$ & \\
\hline MLX & Metals X Limited & $26 / 08 / 2004$ & \\
\hline MMC & Marengo Mining Limited & $8 / 01 / 2013$ & $3 / 06 / 2015$ \\
\hline MME & MTM Entertainment Trust & $12 / 06 / 1998$ & $27 / 09 / 2007$ \\
\hline MMI & Metro Mining Limited & $4 / 12 / 2009$ & \\
\hline MML & Medusa Mining Ltd & $23 / 12 / 2003$ & \\
\hline MMR & MEC Resources Limited & $29 / 05 / 2006$ & \\
\hline MMS & McMillan Shakespeare Limited & $15 / 03 / 2004$ & \\
\hline MMW & Magma Metals Limited & $2 / 06 / 2006$ & $29 / 06 / 2012$ \\
\hline
\end{tabular}




\begin{tabular}{|c|c|c|c|}
\hline ASX Code & Company Name & Listing Date & Delisting Date \\
\hline MMX & Murchison Metals Ltd & $1 / 04 / 2005$ & $28 / 07 / 2014$ \\
\hline MMZ & Mooter Media Limited & $26 / 06 / 2000$ & $5 / 02 / 2016$ \\
\hline MNA & Magnetica Limited & $16 / 06 / 1987$ & $15 / 10 / 2007$ \\
\hline MNB & Minbos Resources Limited & $18 / 10 / 2010$ & \\
\hline MNC & Metminco Limited & $2 / 10 / 2007$ & \\
\hline MND & Monadelphous Group Limited & $28 / 03 / 1991$ & \\
\hline MNE & Metallum Limited & $1 / 07 / 2011$ & \\
\hline MNF & MNF Group Limited & $18 / 05 / 2006$ & \\
\hline MNM & Mantle Mining Corporation Limited & $22 / 11 / 2006$ & \\
\hline MNQ & Minquest Limited & $1 / 05 / 2012$ & \\
\hline MNR & Minotaur Resources Ltd & - & $3 / 03 / 2005$ \\
\hline MNS & Magnis Resources Limited & $25 / 10 / 2005$ & \\
\hline MNW & Mint Payments Limited & $2 / 08 / 2007$ & \\
\hline MNY & Money3 Corporation Limited & $19 / 10 / 2006$ & \\
\hline MOC & Mortgage Choice Limited & $10 / 08 / 2004$ & \\
\hline MOD & MOD Resources Limited & $18 / 12 / 1986$ & \\
\hline MOE & Moto Goldmines Limited & $30 / 05 / 2005$ & $25 / 10 / 2006$ \\
\hline MOG & Moby Oil \& Gas Limited & $29 / 07 / 2004$ & $10 / 10 / 2013$ \\
\hline MOL & Moly Mines Limited & $11 / 03 / 2004$ & \\
\hline MOQ & MOQ Limited & $19 / 12 / 2000$ & \\
\hline MOS & Mosaic Oil NL & $23 / 02 / 1989$ & $21 / 10 / 2010$ \\
\hline MOT & Motopia Limited & $29 / 05 / 2002$ & \\
\hline MOX & Monax Mining Limited & $21 / 09 / 2005$ & \\
\hline MOY & Millennium Minerals Limited & $11 / 02 / 1993$ & \\
\hline MPA & Marine Produce Australia Limited & $20 / 10 / 2000$ & $22 / 12 / 2010$ \\
\hline MPB & Mackay Permanent Building Society Limited & $1 / 12 / 2000$ & $15 / 02 / 2008$ \\
\hline MPE & Magnum Gas \& Power Limited & $24 / 11 / 2004$ & \\
\hline MPG & Macquarie Private Capital Group & $22 / 03 / 2005$ & $12 / 06 / 2008$ \\
\hline MPH & Magna Pacific (Holdings) Limited & $20 / 04 / 1988$ & $31 / 08 / 2007$ \\
\hline MPL & Medibank Private Limited & $25 / 11 / 2014$ & \\
\hline MPM & MPI Mines Limited & - & $10 / 01 / 2005$ \\
\hline MPO & Molopo Energy Limited & 23/12/1986 & \\
\hline MPP & Metro Performance Glass Limited & $30 / 07 / 2014$ & \\
\hline MPR & Macquarie Prologis Trust & $26 / 06 / 2002$ & 16/07/2007 \\
\hline MPX & Mustera Property Group Limited & 28/11/2014 & \\
\hline MQA & Macquarie Atlas Roads Group & $25 / 01 / 2010$ & \\
\hline MQG & Macquarie Group Limited & $29 / 07 / 1996$ & \\
\hline MRC & Mineral Commodities Limited & $29 / 08 / 1970$ & \\
\hline MRD & Mount Ridley Mines Limited & $15 / 09 / 2004$ & \\
\hline MRE & Minara Resources Limited & 17/03/1994 & $27 / 10 / 2011$ \\
\hline MRI & MRI Holdings Limited & $25 / 08 / 1971$ & $30 / 08 / 2010$ \\
\hline MRM & MMA Offshore Limited & $21 / 06 / 1999$ & \\
\hline MRN & Macquarie Media Limited & $20 / 04 / 2005$ & \\
\hline MRP & Macphersons Resources Limited & $24 / 12 / 2010$ & \\
\hline MRQ & MRG Metals Limited & $8 / 06 / 2011$ & \\
\hline MRR & MinRex Resources Limited & $11 / 11 / 2011$ & \\
\hline MRT & Mariner Retirement Solutions Limited & $16 / 03 / 2000$ & $5 / 01 / 2007$ \\
\hline MRU & Mantra Resources Limited & $9 / 10 / 2006$ & $15 / 06 / 2011$ \\
\hline MRV & Moreton Resources Ltd & $9 / 02 / 1994$ & \\
\hline MRZ & Mirvac Real Estate Investment Trust & $16 / 12 / 1993$ & $11 / 12 / 2009$ \\
\hline MSA & MacathurCook Asian Real Estate Securities Fund & $11 / 04 / 2007$ & $16 / 04 / 2009$ \\
\hline MSB & Mesoblast Limited & $16 / 12 / 2004$ & \\
\hline MSF & MSF Sugar Limited & 24/08/1971 & $16 / 03 / 2012$ \\
\hline MSG & MCS Services Limited & $17 / 01 / 2012$ & \\
\hline MSI & Multistack International Limited & $11 / 02 / 1993$ & \\
\hline MSL & The MAC Services Group Limited & $12 / 04 / 2007$ & $31 / 12 / 2010$ \\
\hline MSM & MSM Corporation International Ltd & 20/11/1996 & \\
\hline
\end{tabular}




\begin{tabular}{|c|c|c|c|}
\hline ASX Code & Company Name & Listing Date & Delisting Date \\
\hline MSP & Maxsec Group Limited & $10 / 07 / 1986$ & \\
\hline MSR & Manas Resources Limited & $22 / 07 / 2008$ & \\
\hline MST & Metal Storm Limited & $8 / 07 / 1999$ & $30 / 08 / 2013$ \\
\hline MSV & Mitchell Services Limited & $2 / 08 / 2011$ & \\
\hline MSX & Mineral Securities Limited & $12 / 12 / 2000$ & $13 / 07 / 2007$ \\
\hline MTA & Metals of Africa Limited & $29 / 10 / 2012$ & \\
\hline MTB & Mount Burgess Mining NL & $5 / 09 / 1985$ & \\
\hline MTD & Metroland Australia Limited & $5 / 12 / 1985$ & $4 / 01 / 2016$ \\
\hline MTH & Mithril Resources Limited & $18 / 11 / 2002$ & \\
\hline MTL & Manalto Limited & $6 / 11 / 2003$ & \\
\hline MTM & Mareterram Limited & $7 / 04 / 2004$ & \\
\hline MTR & Mantra Group Limited & $20 / 06 / 2014$ & \\
\hline MTR & Meditech Research Limited & $21 / 01 / 1994$ & $17 / 08 / 2006$ \\
\hline MTS & Metcash Limited & $17 / 06 / 1994$ & \\
\hline MTU & M2 Group Ltd & $29 / 10 / 2004$ & $24 / 02 / 2016$ \\
\hline MTX & Metals Exploration Limited & $27 / 02 / 1992$ & $17 / 01 / 2007$ \\
\hline MUB & Mongolian Resource Corporation Ltd & $29 / 07 / 2008$ & $16 / 05 / 2016$ \\
\hline MUE & Multiplex European Property Fund & $3 / 07 / 2007$ & $18 / 09 / 2015$ \\
\hline MUM & Mount Magnet South Limited & $23 / 05 / 2007$ & \\
\hline MUS & Mustang Resources Limited & $10 / 05 / 2002$ & \\
\hline MVA & VanEck Vectors Australian Property ETF & $16 / 10 / 2013$ & \\
\hline MVB & VanEck Vectors Australian Banks ETF & $16 / 10 / 2013$ & \\
\hline MVE & Vaneck Vectors S\&P/ASX Midcap ETF & $16 / 10 / 2013$ & \\
\hline MVF & Monash IVF Group Limited & $26 / 06 / 2014$ & \\
\hline MVP & Medical Developments International Limited & $15 / 12 / 2003$ & \\
\hline MVR & VanEck Vectors Australian Resources ETF & $16 / 10 / 2013$ & \\
\hline MVT & Mercantile Investment Company Ltd & $5 / 04 / 2007$ & \\
\hline MVU & Matrix View & $5 / 08 / 2004$ & $12 / 01 / 2010$ \\
\hline MVW & VanEck Vectors Australian Equal Weight ETF & $6 / 03 / 2014$ & \\
\hline MWC & Media World Communications Limited & - & $29 / 08 / 2005$ \\
\hline MWE & Mawson West Limited & $5 / 09 / 1997$ & $17 / 06 / 2009$ \\
\hline MWG & Macquarie Winton Global Opportunities Trust & $8 / 12 / 2005$ & $2 / 01 / 2013$ \\
\hline MWR & MGM Wireless Ltd & $17 / 07 / 2001$ & \\
\hline MXC & MGC Pharmaceuticals Ltd & $21 / 12 / 2006$ & \\
\hline MXG & Multiplex Group & $2 / 12 / 2003$ & 19/12/2007 \\
\hline MXI & Maxitrans Industries Limited & $7 / 12 / 1994$ & \\
\hline MXQ & Max Trust & $16 / 09 / 2005$ & $4 / 02 / 2014$ \\
\hline MXR & Maximus Resources Limited & $26 / 10 / 2005$ & \\
\hline MXX & Mineral Securities Limited & $2 / 07 / 2007$ & $3 / 07 / 2009$ \\
\hline MYE & Mastermyne Group Limited & $7 / 05 / 2010$ & \\
\hline MYG & Mutiny Gold Limited & $10 / 07 / 2006$ & $17 / 02 / 2015$ \\
\hline MYO & MYOB Limited & $9 / 07 / 1999$ & $23 / 01 / 2009$ \\
\hline MYP & Mayne Pharma Limited & $21 / 11 / 2005$ & $5 / 02 / 2007$ \\
\hline MYR & Myer Holdings Limited & $2 / 11 / 2009$ & \\
\hline MYS & Mystate Limited & $10 / 09 / 2009$ & \\
\hline MYX & Mayne Pharma Group Limited & $29 / 06 / 2007$ & \\
\hline MZI & MZI Resources Limited & $26 / 08 / 2004$ & \\
\hline MZM & Montezuma Mining Company Limited & $9 / 11 / 2006$ & \\
\hline MZN & Marindi Metals Limited & $14 / 12 / 2006$ & \\
\hline MZU & Mazu Alliance Limited & $2 / 01 / 1998$ & $22 / 03 / 2016$ \\
\hline NAB & National Australia Bank Limited & $31 / 01 / 1962$ & \\
\hline NAC & Naos Absolute Opportunities Company Ltd & $12 / 11 / 2014$ & \\
\hline NAE & New Age Exploration Limited & $18 / 02 / 1994$ & \\
\hline NAG & Nagambie Resources Limited & $23 / 06 / 2006$ & \\
\hline NAM & Namoi Cotton Co-Operative Limited & $6 / 04 / 1998$ & \\
\hline NAN & Nanosonics Limited & $17 / 05 / 2007$ & \\
\hline NAV & Navigator Resources Ltd & $19 / 09 / 2003$ & \\
\hline
\end{tabular}




\begin{tabular}{|c|c|c|c|}
\hline ASX Code & Company Name & Listing Date & Delisting Date \\
\hline NBL & Noni B Limited & $11 / 05 / 2000$ & \\
\hline NBS & Nexbis Limited & $4 / 04 / 2000$ & $11 / 07 / 2012$ \\
\hline NCA & Neighborhood Cable Limited & - & $15 / 07 / 2005$ \\
\hline NCC & Naos Emerging Opportunities Company Limited & $26 / 02 / 2013$ & \\
\hline NCI & National Can Industries Limited & $12 / 07 / 1984$ & $13 / 06 / 2012$ \\
\hline NCK & Nick Scali Limited & $26 / 05 / 2004$ & \\
\hline $\mathrm{NCM}$ & Newcrest Mining Limited & $4 / 06 / 1987$ & \\
\hline $\mathrm{NCO}$ & Namibian Copper NL & $18 / 12 / 2007$ & \\
\hline NCR & Nucoal Resources Limited & $31 / 05 / 1996$ & \\
\hline NDO & Nido Petroleum Limited & $30 / 07 / 1999$ & \\
\hline NEA & Nearmap Ltd & $7 / 12 / 2000$ & \\
\hline NEC & Nine Entertainment Co. Holdings Limited & $6 / 12 / 2013$ & \\
\hline NEC & Northern Energy Corporation Limited & $30 / 07 / 1998$ & $24 / 10 / 2011$ \\
\hline NEL & Nova Energy Limited & $23 / 08 / 2005$ & $16 / 11 / 2007$ \\
\hline NEM & Newmont Mining Corporation & $25 / 02 / 2002$ & $17 / 02 / 2010$ \\
\hline NEN & Neon Capital Ltd & $13 / 03 / 1990$ & \\
\hline NER & Neo Resources Limited & $30 / 04 / 1987$ & $30 / 08 / 2013$ \\
\hline NEU & Neuren Pharmaceuticals Limited & $3 / 02 / 2005$ & \\
\hline NFD & National Foods Limited & - & $22 / 06 / 2005$ \\
\hline NFE & Northern Iron Limited & 13/12/2007 & \\
\hline NFK & Norfolk Group Limited & $27 / 07 / 2007$ & $6 / 08 / 2013$ \\
\hline NFO & Network Foods Limited & $5 / 01 / 1994$ & $9 / 02 / 2007$ \\
\hline NGE & New Guinea Energy Limited & 28/12/2007 & \\
\hline NGF & Norton Gold Fields Limited & $16 / 09 / 2005$ & $2 / 07 / 2015$ \\
\hline NGM & NGM Resources Limited & $3 / 05 / 2004$ & $17 / 12 / 2010$ \\
\hline NGX & Norseman Gold PLC & $25 / 06 / 2009$ & $1 / 09 / 2015$ \\
\hline NGY & NuEnergy Gas Limited & $15 / 01 / 1987$ & \\
\hline NHC & New Hope Corporation Limited & $16 / 09 / 2003$ & \\
\hline NHF & NIB Holdings Limited & $5 / 11 / 2007$ & \\
\hline NHH & Newhaven Hotels Limited & $16 / 01 / 1986$ & \\
\hline NHL & Nova Health Limited & - & $9 / 06 / 2005$ \\
\hline NHO & New Horizon Coal Ltd & $20 / 10 / 2010$ & \\
\hline NHR & National Hire Group Limited & $5 / 06 / 1997$ & $22 / 12 / 2011$ \\
\hline NIC & Nicu Metals Limited & - & $30 / 08 / 2004$ \\
\hline NIO & Nickelore Limited & $24 / 07 / 2000$ & \\
\hline NIU & Niuminco Group Limited & $10 / 12 / 1986$ & \\
\hline NKP & Nkwe Platinum Limited & $24 / 09 / 2003$ & \\
\hline NLG & National Leisure \& Gaming Limited & $14 / 12 / 2005$ & $30 / 08 / 2012$ \\
\hline NLX & Nylex Limited & $9 / 10 / 1987$ & $30 / 08 / 2010$ \\
\hline NME & Nex Metals Exploration Limited & $6 / 12 / 2007$ & \\
\hline NMG & Noble Mineral Resources Limited & $27 / 06 / 2008$ & \\
\hline NMI & Northern Mining Limited & $12 / 09 / 2006$ & \\
\hline NML & Navarre Minerals Limited & $31 / 03 / 2011$ & \\
\hline NMM & Noble Metals Limited & $2 / 09 / 2010$ & \\
\hline NMS & Neptune Marine Services Limited & $15 / 04 / 2004$ & \\
\hline NMT & Neometals Limited & $10 / 07 / 2002$ & \\
\hline NNG & Northern Gold NL & - & $16 / 01 / 2006$ \\
\hline NNW & 99 Wuxian Limited & $8 / 10 / 2013$ & \\
\hline NOC & Northern Crest Investments Limited & $9 / 05 / 2006$ & $30 / 08 / 2011$ \\
\hline NOE & Novarise Renewable Resources International Limited & $29 / 04 / 2010$ & \\
\hline NOL & National 1 Limited & - & $30 / 07 / 2004$ \\
\hline NOP & New Opportunity Limited & $27 / 06 / 2000$ & $29 / 01 / 2008$ \\
\hline NOR & Norwood Systems Limited & $27 / 06 / 1995$ & \\
\hline NPH & New Privateer Holdings Limited & $4 / 04 / 1985$ & $28 / 02 / 2008$ \\
\hline NPX & Nuplex Industries Limited & 25/11/1999 & 14/09/2016 \\
\hline NQM & North Queensland Metals Limited & $15 / 12 / 2006$ & $10 / 12 / 2010$ \\
\hline NRT & Novogen Limited & $1 / 09 / 1994$ & \\
\hline
\end{tabular}




\begin{tabular}{|c|c|c|c|}
\hline ASX Code & Company Name & Listing Date & Delisting Date \\
\hline NSE & New Standard Energy Limited & $15 / 08 / 2007$ & \\
\hline NSL & NSL Consolidated Limited & $25 / 02 / 1998$ & \\
\hline NSN & Natasa Mining Ltd & $6 / 09 / 1979$ & $30 / 06 / 2010$ \\
\hline NSR & National Storage REIT & $19 / 12 / 2013$ & \\
\hline NST & Northern Star Resources Ltd & $17 / 12 / 2003$ & \\
\hline NSX & NSX Limited & $13 / 01 / 2005$ & \\
\hline NTC & NetComm Wireless Limited & $21 / 12 / 1993$ & \\
\hline NTG & National Telecoms Limited & - & $3 / 03 / 2006$ \\
\hline NTL & New Talisman Gold Mines Limited & 28/05/1987 & \\
\hline NTM & Northern Manganese Limited & $26 / 09 / 2006$ & \\
\hline NTU & Northern Minerals Limited & $15 / 11 / 2006$ & \\
\hline NUF & Nufarm Limited & $10 / 11 / 1988$ & \\
\hline NUH & Nuheara Limited & $5 / 03 / 2010$ & \\
\hline NVE & Novera Energy Limited & - & $4 / 04 / 2006$ \\
\hline NVI & Nevada Iron Ltd & $12 / 06 / 2008$ & \\
\hline NVN & Novion Property Group & $7 / 04 / 1994$ & $15 / 06 / 2015$ \\
\hline NVO & Nvoi Ltd & $30 / 11 / 2010$ & \\
\hline NVS & Novus Petroleum Limited & - & $13 / 07 / 2004$ \\
\hline NVT & Navitas Limited & $15 / 12 / 2004$ & \\
\hline NWE & Norwest Energy NL & 19/09/1997 & \\
\hline NWF & Newfield Resources Limited & $8 / 06 / 2012$ & \\
\hline NWH & NRW Holdings Limited & $5 / 09 / 2007$ & \\
\hline NWR & Northwest Resources Limited & $6 / 12 / 2004$ & $1 / 09 / 2015$ \\
\hline NWS & News Corporation & $19 / 06 / 2013$ & \\
\hline NWT & Newsat Limited & 29/10/1987 & $1 / 09 / 2015$ \\
\hline NWZ & Newzulu Limited & $7 / 04 / 2000$ & \\
\hline NXM & Nexus Minerals Limited & $23 / 02 / 2007$ & \\
\hline NXR & Nemex Resources Limited & $2 / 05 / 2011$ & \\
\hline NXS & Nexus Energy Limited & $13 / 09 / 2000$ & $8 / 01 / 2015$ \\
\hline NXT & NEXTDC Limited & $13 / 12 / 2010$ & \\
\hline NYO & Nyota Minerals Limited & $1 / 12 / 2000$ & \\
\hline NZO & New Zealand Oil \& Gas Limited & 27/04/1989 & $1 / 06 / 2016$ \\
\hline OAK & Oaks Hotels \& Resorts Limited & $3 / 01 / 2006$ & $10 / 10 / 2011$ \\
\hline OAR & Oakdale Resources Limited & $17 / 12 / 1998$ & \\
\hline OBJ & OBJ Limited & $29 / 05 / 2000$ & \\
\hline OBL & Oil Basins Limited & $5 / 06 / 1987$ & \\
\hline OBS & Orbis Gold Limited & $22 / 08 / 2008$ & $17 / 03 / 2015$ \\
\hline $\mathrm{OCC}$ & Orthocell Limited & $12 / 08 / 2014$ & \\
\hline OCE & Ocean Capital Limited & $24 / 03 / 1988$ & $25 / 01 / 2013$ \\
\hline OCL & Objective Corporation Limited & $17 / 08 / 2000$ & \\
\hline $\mathrm{OCP}$ & Oceania Capital Partners Limited & $22 / 12 / 2004$ & \\
\hline $\mathrm{OCV}$ & Octaviar Limited & $12 / 10 / 1970$ & $1 / 09 / 2009$ \\
\hline ODG & Odyssey Gaming Limited & $9 / 04 / 1997$ & $27 / 07 / 2011$ \\
\hline ODN & Odin Energy Limited & $5 / 09 / 2007$ & \\
\hline ODY & Odyssey Energy Limited & $30 / 11 / 2005$ & \\
\hline OEC & Orbital Corporation Limited & $16 / 11 / 1988$ & \\
\hline OEG & Orpheus Energy Limited & $31 / 05 / 2007$ & \\
\hline OEL & Otto Energy Limited & $22 / 12 / 2004$ & \\
\hline OEQ & Orion Equities Limited & 19/11/1970 & \\
\hline OEX & Oilex Ltd & $24 / 10 / 2003$ & \\
\hline OFW & Oilfield Workplace Group Limited & $29 / 01 / 2013$ & \\
\hline OFX & OFX Group Limited & $11 / 10 / 2013$ & \\
\hline OGC & OceanaGold Corporation & $18 / 03 / 2004$ & \\
\hline OGH & Ochre Group Holdings Limited & $31 / 07 / 1979$ & \\
\hline OGL & OGL Resources Limited & $21 / 05 / 1987$ & $1 / 09 / 2015$ \\
\hline OGX & Orinoco Gold Limited & $5 / 09 / 2011$ & \\
\hline OGY & Orca Energy Limited & $23 / 07 / 1987$ & \\
\hline
\end{tabular}




\begin{tabular}{|c|c|c|c|}
\hline ASX Code & Company Name & Listing Date & Delisting Date \\
\hline OHE & Orion Health Group Limited & $26 / 11 / 2014$ & \\
\hline OIL & Optiscan Imaging Limited & $8 / 08 / 1997$ & \\
\hline OKJ & Oakajee Corporation Limited & $11 / 06 / 2008$ & \\
\hline OKN & Oakton Limited & $2 / 06 / 2000$ & $27 / 11 / 2014$ \\
\hline OKU & Oklo Resources Limited & $16 / 05 / 2007$ & \\
\hline OLH & Oldfields Holdings Limited & $15 / 06 / 1960$ & \\
\hline $\mathrm{OMC}$ & OmegaCorp Limited & $6 / 08 / 2004$ & 14/09/2007 \\
\hline $\mathrm{OMH}$ & OM Holdings Limited & 19/03/1998 & \\
\hline OML & Oohmedia Limited & $17 / 12 / 2014$ & \\
\hline OMP & OAMPS Limited & $26 / 10 / 1989$ & $16 / 11 / 2006$ \\
\hline OMT & Omni Market Tide Ltd & $14 / 12 / 2005$ & \\
\hline ONL & One North Entertainment Limited & $28 / 06 / 2000$ & $30 / 08 / 2012$ \\
\hline ONT & 1300 Smiles Limited & $11 / 03 / 2005$ & \\
\hline ONX & Onyx Property Group & - & $13 / 12 / 2004$ \\
\hline $\mathrm{OOH}$ & Oohmedia Group Limited & $28 / 07 / 2000$ & $16 / 03 / 2012$ \\
\hline OOK & Ookami Limited & $21 / 02 / 2000$ & \\
\hline $\mathrm{OOO}$ & $\begin{array}{l}\text { BetaShares Crude Oil Index ETF-Currency Hedged } \\
\text { (Synthetic) }\end{array}$ & $16 / 11 / 2011$ & \\
\hline OPG & Opus Group Limited & $10 / 04 / 2012$ & \\
\hline OPL & Orchard Petroleum Limited & $4 / 10 / 2000$ & $30 / 04 / 2007$ \\
\hline OPS & OPSM Group Limited & - & $18 / 02 / 2005$ \\
\hline OPT & Opthea Limited & $18 / 04 / 1991$ & \\
\hline ORA & Orora Limited & $18 / 12 / 2013$ & \\
\hline ORE & Orocobre Limited & $4 / 12 / 2007$ & \\
\hline ORG & Origin Energy Limited & $13 / 07 / 1961$ & \\
\hline ORH & ORH Limited & $5 / 09 / 1997$ & \\
\hline ORI & Orica Limited & $6 / 09 / 1938$ & \\
\hline ORL & OrotonGroup Limited & $18 / 06 / 1987$ & \\
\hline ORM & Orion Metals Limited & $3 / 01 / 2007$ & \\
\hline ORN & Orion Gold NL & $31 / 10 / 2003$ & \\
\hline ORR & Orecorp Limited & $4 / 08 / 2011$ & \\
\hline ORS & Octagonal Resources Limited & $5 / 01 / 2011$ & $24 / 02 / 2016$ \\
\hline OSH & Oil Search Limited & $1 / 12 / 1980$ & \\
\hline OSL & Oncosil Medical Limited & $15 / 08 / 2005$ & \\
\hline OSP & Osprey Medical Inc. & $2 / 05 / 2012$ & \\
\hline OTC & OTOC Limited & $25 / 06 / 2008$ & \\
\hline OTH & Onthehouse Holdings Limited & $3 / 06 / 2011$ & \\
\hline OTI & Oriental Technologies Investment Limited & $5 / 08 / 1993$ & \\
\hline OTL & Orion Telecommunications Limited & $5 / 10 / 2004$ & 19/12/2007 \\
\hline OTR & Onterran Limited & $31 / 10 / 2006$ & \\
\hline OTT & Open Telecommunications Limited & - & $31 / 05 / 2006$ \\
\hline OUM & Outback Metals Limited & $2 / 09 / 2008$ & $27 / 02 / 2015$ \\
\hline $\mathrm{OVH}$ & Onevue Holdings Limited & $25 / 07 / 2014$ & \\
\hline OVL & Oro Verde Limited & 10/12/1999 & \\
\hline OVR & Overland Resources Limited & $14 / 11 / 2006$ & \\
\hline $\mathrm{OXX}$ & Octanex N.L. & $6 / 11 / 2009$ & \\
\hline OZF & SPDR S\&P/ASX200 Financials ex-REIT Fund & $13 / 04 / 2011$ & \\
\hline OZG & Ozgrowth Limited & $2 / 01 / 2008$ & \\
\hline OZL & OZ Minerals Limited & $1 / 01 / 1972$ & \\
\hline OZR & SPDR S\&P/ASX200 Resources Fund & $13 / 04 / 2011$ & \\
\hline PAA & PharmAust Limited & $5 / 10 / 2001$ & \\
\hline PAB & Patrys Limited & $13 / 07 / 2007$ & \\
\hline PAC & Pacific Current Group Limited & $2 / 01 / 1992$ & \\
\hline PAF & PM Capital Asian Opportunities Fund Limited & $22 / 05 / 2014$ & \\
\hline PAG & PrimeAg Australia & $24 / 12 / 2007$ & $6 / 11 / 2013$ \\
\hline$\overline{\text { PAK }}$ & Pacific American Coal Limited & $20 / 12 / 2007$ & \\
\hline PAN & Panoramic Resources Limited & $12 / 09 / 2001$ & \\
\hline
\end{tabular}




\begin{tabular}{|c|c|c|c|}
\hline ASX Code & Company Name & Listing Date & Delisting Date \\
\hline PAO & Principal America Office Trust & - & $25 / 11 / 2004$ \\
\hline PAS & Pasminco Limited & - & $16 / 03 / 2006$ \\
\hline PAW & Pawnee Energy Limited & $10 / 12 / 2007$ & \\
\hline PAY & Payce Consolidated Limited & $19 / 07 / 1979$ & \\
\hline PBA & Peoplebank Australia Limited & $15 / 06 / 2005$ & $26 / 11 / 2009$ \\
\hline PBB & Pacifica Group Limited & $2 / 11 / 1989$ & $28 / 08 / 2009$ \\
\hline PBG & Pacific Brands Limited & $2 / 04 / 2004$ & $19 / 07 / 2016$ \\
\hline PBO & PanBio Limited & 9/04/2001 & $17 / 01 / 2008$ \\
\hline PBP & Probiotec Limited & $14 / 11 / 2006$ & \\
\hline PBS & Pioneer Permanent Building Society Limited & $14 / 08 / 2006$ & $11 / 05 / 2007$ \\
\hline PBT & Prana Biotechnology Limited & $28 / 03 / 2000$ & \\
\hline PCG & PCH Group Limited & $3 / 12 / 1986$ & $28 / 12 / 2007$ \\
\hline $\mathrm{PCH}$ & Property Connect Holdings Limited & $23 / 02 / 2000$ & \\
\hline PCL & Pancontinental Oil \& Gas NL & $11 / 09 / 1986$ & \\
\hline PCP & Paramount Mining Corporation Limited & $13 / 02 / 2004$ & $30 / 08 / 2016$ \\
\hline PDG & Placer Dome Inc. & - & $15 / 03 / 2006$ \\
\hline PDI & Predictive Discovery Ltd & $1 / 12 / 2010$ & \\
\hline PDL & Petra Diamonds Limited & $20 / 06 / 2005$ & $14 / 03 / 2007$ \\
\hline PDN & Paladin Energy Ltd & $29 / 03 / 1994$ & \\
\hline PDR & Port Douglas Reef Resorts Limited & $5 / 07 / 1994$ & $29 / 08 / 2006$ \\
\hline PDY & Padbury Mining Limited & $4 / 11 / 1993$ & \\
\hline PDZ & Prairie Mining Limited & $9 / 09 / 1993$ & \\
\hline PEA & Pacific Energy Limited & 2/04/1987 & \\
\hline PEC & Perpetual Resources Limited & $28 / 02 / 2013$ & \\
\hline PEG & Pixie Group Limited & $16 / 11 / 2007$ & \\
\hline PEH & Pacific Environment Limited & $11 / 02 / 2008$ & \\
\hline PEK & Peak Resources Limited & $29 / 11 / 2006$ & \\
\hline PEL & Pelican Resources Limited & $25 / 01 / 2002$ & \\
\hline PEM & Perilya Limited & 23/07/1987 & $20 / 12 / 2013$ \\
\hline PEN & Peninsula Energy Limited & $26 / 05 / 1994$ & \\
\hline PEO & People Telecom Limited & $9 / 01 / 1986$ & $1 / 05 / 2009$ \\
\hline PEP & Peplin Limited & $22 / 09 / 2000$ & $8 / 01 / 2008$ \\
\hline PES & Pure Energy Resources Limited & $12 / 09 / 2006$ & $3 / 06 / 2009$ \\
\hline PET & Peters MacGregor Investments Limited & $16 / 04 / 2004$ & $30 / 05 / 2013$ \\
\hline PEX & Peel Mining Limited & $17 / 05 / 2007$ & \\
\hline PFG & Prime Financial Group Limited & $7 / 12 / 2004$ & \\
\hline PFL & Patties Foods Limited & $6 / 11 / 2006$ & $28 / 09 / 2016$ \\
\hline PFM & Pacific Mining Limited & 2/08/1971 & \\
\hline PGC & Paragon Care Limited & $15 / 10 / 1999$ & \\
\hline PGF & PM Capital Global Opportunities Fund Limited & $12 / 12 / 2013$ & \\
\hline PGH & Pact Group Holdings Ltd & $17 / 12 / 2013$ & \\
\hline PGI & Panterra Gold Limited & $25 / 11 / 1993$ & \\
\hline PGM & Platina Resources Limited & $29 / 05 / 2006$ & \\
\hline PGO & Primary Gold Limited & $16 / 07 / 2007$ & \\
\hline PGR & The PAS Group Limited & $16 / 06 / 2014$ & \\
\hline PGS & Planet Gas Limited & $11 / 03 / 2004$ & \\
\hline PGY & Pilot Energy Limited & $20 / 04 / 2006$ & \\
\hline PHA & Public Holdings (Australia) Limited & $1 / 01 / 1970$ & \\
\hline PHG & Pulse Health Limited & $20 / 11 / 2003$ & \\
\hline PHI & Phileo Australia Limited & $2 / 01 / 1991$ & \\
\hline PHK & Phoslock Water Solutions Limited & $16 / 08 / 2002$ & \\
\hline PHL & Pearl Healthcare Limited & $4 / 03 / 1994$ & $2 / 07 / 2012$ \\
\hline PHY & Pacific Hydro Limited & - & $26 / 08 / 2005$ \\
\hline PIC & Perpetual Equity Investment Company Limited & $18 / 12 / 2014$ & \\
\hline PIH & Prime Infrastructure Group & $24 / 06 / 2002$ & $2 / 06 / 2011$ \\
\hline PIL & Peppermint Innovation Limited & $29 / 05 / 2008$ & \\
\hline $\mathrm{PIO}$ & Pioneer Resources Limited & $4 / 12 / 2003$ & \\
\hline
\end{tabular}




\begin{tabular}{|c|c|c|c|}
\hline ASX Code & Company Name & Listing Date & Delisting Date \\
\hline PIR & Papillon Resources Limited & $1 / 03 / 2007$ & $6 / 10 / 2014$ \\
\hline PKA & Plukka Limited & $28 / 01 / 2004$ & \\
\hline $\mathrm{PKO}$ & Peako Limited & $2 / 12 / 2009$ & \\
\hline PLA & Platinum Australia Limited & $9 / 10 / 2000$ & $1 / 09 / 2015$ \\
\hline PLB & Plan B Group Holdings Limited & $12 / 07 / 2007$ & $18 / 10 / 2012$ \\
\hline PLH & Plymouth Minerals Limited & $15 / 04 / 2011$ & \\
\hline PLI & Peplin Inc. & $9 / 10 / 2007$ & $30 / 11 / 2009$ \\
\hline PLN & Planar Semiconductor Inc. & - & $29 / 08 / 2005$ \\
\hline PLP & Platypus Minerals Ltd & $2 / 05 / 1994$ & \\
\hline PLS & Pilbara Minerals Limited & $19 / 09 / 2007$ & \\
\hline PLT & Polartechnics Limited & $8 / 05 / 1987$ & $30 / 08 / 2010$ \\
\hline PLV & Pluton Resources Limited & $15 / 12 / 2006$ & $30 / 08 / 2016$ \\
\hline PLW & Peter Lehmann Wines Limited & - & $12 / 10 / 2004$ \\
\hline PLY & Polymetals Mining Limited & $4 / 05 / 2011$ & $26 / 08 / 2013$ \\
\hline PMC & Platinum Capital Limited & $29 / 06 / 1994$ & \\
\hline PME & Pro Medicus Limited & $10 / 10 / 2000$ & \\
\hline PMG & Peppercorn Management Group & - & $31 / 12 / 2004$ \\
\hline PMH & PacMag Metals Limited & $23 / 08 / 1995$ & $5 / 07 / 2010$ \\
\hline PMM & Portman Limited & $31 / 05 / 1984$ & $10 / 12 / 2008$ \\
\hline PMN & Promina Group Limited & $12 / 05 / 2003$ & $28 / 06 / 2007$ \\
\hline PMP & PMP Limited & $13 / 02 / 1992$ & \\
\hline PMV & Premier Investments Limited & $15 / 12 / 1987$ & \\
\hline PMX & Palamedia Limited & $1 / 04 / 1996$ & $30 / 08 / 2011$ \\
\hline PMY & Pacifico Minerals Limited & $15 / 07 / 2004$ & \\
\hline PNA & PanAust Limited & $15 / 01 / 1987$ & $15 / 06 / 2015$ \\
\hline $\mathrm{PNC}$ & Pioneer Credit Limited & $1 / 05 / 2014$ & \\
\hline PNI & Pinnacle Investment Management Group Limited & $19 / 06 / 2007$ & \\
\hline PNL & Paringa Resources Limited & $20 / 12 / 2012$ & \\
\hline PNM & Pallane Medical Limited & $24 / 01 / 2005$ & $30 / 08 / 2011$ \\
\hline PNN & PepinNini Minerals Limited & $15 / 04 / 2005$ & \\
\hline PNO & PharmaNet Group Limited & 13/03/1987 & \\
\hline PNR & Pantoro Limited & $21 / 05 / 1987$ & \\
\hline PNV & Polynovo Limited & $26 / 11 / 1998$ & \\
\hline PNW & Pacific Star Network Limited & $28 / 11 / 2000$ & \\
\hline PNX & PNX Metals Limited & $12 / 02 / 2008$ & \\
\hline$\overline{\mathrm{POF}}$ & Principal Office Fund & - & $16 / 07 / 2004$ \\
\hline $\begin{array}{l}\mathrm{POH} \\
\end{array}$ & Phosphagenics Limited & $11 / 08 / 1993$ & \\
\hline POL & Polaris Metals NL & $16 / 04 / 2004$ & $11 / 03 / 2010$ \\
\hline POP & Primary Opinion Limited & $27 / 08 / 2004$ & \\
\hline POS & Poseidon Nickel Limited & $30 / 11 / 1993$ & \\
\hline POU & BetaShares British Pound ETF & $11 / 07 / 2011$ & \\
\hline POW & Protean Wave Energy Ltd & $21 / 12 / 2006$ & \\
\hline POZ & Phosphate Australia Limited & $1 / 07 / 2008$ & \\
\hline PPC & Peet Limited & $5 / 08 / 2004$ & \\
\hline PPG & PRO-PAC Packaging Limited & $27 / 04 / 2005$ & \\
\hline PPI & Pelorus Property Group Limited & $20 / 07 / 2006$ & $10 / 01 / 2011$ \\
\hline PPK & PPK Group Limited & 21/12/1994 & \\
\hline PPM & Primero Mining Corp. & $24 / 05 / 2013$ & $31 / 12 / 2013$ \\
\hline PPN & Planet Platinum Limited & $1 / 05 / 2003$ & $9 / 03 / 2016$ \\
\hline PPP & Pan Pacific Petroleum NL & $9 / 04 / 1983$ & \\
\hline PPR & Promentum Limited & $17 / 03 / 1989$ & $4 / 05 / 2007$ \\
\hline PPS & Praemium Limited & $11 / 05 / 2006$ & \\
\hline PPT & Perpetual Limited & $2 / 11 / 1988$ & \\
\hline PPY & Papyrus Australia Limited & $15 / 04 / 2005$ & \\
\hline PRC & Pike River Coal Limited & $25 / 07 / 2007$ & $30 / 08 / 2011$ \\
\hline PRG & Programmed Maintenance Services Ltd & $1 / 10 / 1999$ & \\
\hline $\mathrm{PRH}$ & Phillips River Mining Limited & $28 / 01 / 1993$ & $23 / 05 / 2016$ \\
\hline
\end{tabular}




\begin{tabular}{|c|c|c|c|}
\hline ASX Code & Company Name & Listing Date & Delisting Date \\
\hline PRK & Patrick Corporation Limited & - & $3 / 07 / 2006$ \\
\hline PRL & Petrel Energy Limited & $12 / 12 / 2007$ & \\
\hline PRM & Plentex Limited & $19 / 05 / 1983$ & $4 / 01 / 2016$ \\
\hline PRN & Pelsart Resources NL & $30 / 06 / 1981$ & $25 / 09 / 2008$ \\
\hline PRO & Prophecy International Holdings Limited & $22 / 04 / 1998$ & \\
\hline PRR & Prima BioMed Limited & $23 / 06 / 1988$ & \\
\hline PRT & Prime Media Group Limited & $15 / 10 / 1970$ & \\
\hline PRU & Perseus Mining Limited & $22 / 09 / 2004$ & \\
\hline PRV & Premium Investors Limited & $27 / 11 / 2003$ & $4 / 01 / 2013$ \\
\hline PRW & Proto Resources \& Investments Ltd & $29 / 11 / 2006$ & \\
\hline PRY & Primary Health Care Limited & $3 / 07 / 1998$ & \\
\hline PSA & Petsec Energy Limited & $1 / 12 / 1980$ & \\
\hline PSC & Prospect Resources Limited & $4 / 12 / 2009$ & \\
\hline PSD & pSivida Limited & 17/09/1987 & $20 / 06 / 2008$ \\
\hline PSH & Penrice Soda Holdings Limited & $4 / 07 / 2005$ & $1 / 09 / 2014$ \\
\hline PSI & Pacific Strategic Investments Limited & - & $31 / 03 / 2005$ \\
\hline PSM & Peninsula Mines Limited & $15 / 08 / 2007$ & \\
\hline PSN & Peninsular \& Oriental Steam Navigation Company & - & $9 / 03 / 2006$ \\
\hline PSP & Prosperity Resources Limited & $24 / 11 / 2003$ & \\
\hline PSQ & Pacific Smiles Group Limited & $21 / 11 / 2014$ & \\
\hline PST & Pearlstreet Limited & $23 / 02 / 2007$ & $17 / 12 / 2009$ \\
\hline PSV & Perseverance Corporation Limited & $2 / 04 / 1987$ & $26 / 03 / 2008$ \\
\hline PSY & Panorama Synergy Ltd & $11 / 05 / 2001$ & \\
\hline PSZ & PS\&C Limited & $2 / 12 / 2013$ & \\
\hline PTB & PTB Group Limited & $18 / 12 / 2006$ & \\
\hline PTL & Pental Limited & $29 / 08 / 2000$ & \\
\hline PTM & Platinum Asset Management Limited & $23 / 05 / 2007$ & \\
\hline PTN & Prime Retirement and Aged Care Property Trust & $3 / 08 / 2007$ & $30 / 08 / 2012$ \\
\hline PTR & Petratherm Ltd & $27 / 07 / 2004$ & \\
\hline PTX & Prescient Therapeutics Limited & $2 / 01 / 1992$ & \\
\hline PUN & Pegasus Metals Limited & $7 / 03 / 2007$ & \\
\hline PVA & pSivida Corp & $12 / 06 / 2008$ & \\
\hline PVD & Pura Vida Energy NL & $14 / 02 / 2012$ & \\
\hline PVE & Po Valley Energy Limited & $14 / 12 / 2004$ & \\
\hline PVM & PMI Gold Corporation & $21 / 12 / 2010$ & $11 / 02 / 2014$ \\
\hline PVT & Provet Holdings Limited & $15 / 03 / 2010$ & $24 / 01 / 2011$ \\
\hline PWK & Pipe Networks Limited & $17 / 05 / 2005$ & $14 / 04 / 2010$ \\
\hline PWN & Potash West NL & $13 / 05 / 2011$ & \\
\hline PWT & PowerTel Limited & $5 / 06 / 1986$ & $22 / 05 / 2007$ \\
\hline PWW & Power Resources Limited & $19 / 02 / 2008$ & \\
\hline PXG & Phoenix Gold Limited & $22 / 12 / 2010$ & $7 / 01 / 2016$ \\
\hline PXS & Pharmaxis Ltd & $10 / 11 / 2003$ & \\
\hline PYC & Phylogica Limited & $30 / 03 / 2005$ & \\
\hline PZC & Pan Asia Corporation Limited & $21 / 12 / 2001$ & \\
\hline QAG & BetaShares Agriculture ETF-Currency Hedged (Synthetic) & $2 / 12 / 2011$ & \\
\hline QAN & Qantas Airways Limited & $31 / 07 / 1995$ & \\
\hline QAU & Betashares Gold Bullion ETF (A \$ Hedged) & $4 / 05 / 2011$ & \\
\hline QBE & QBE Insurance Group Limited & $30 / 06 / 1973$ & \\
\hline QBL & Queensland Bauxite Limited & $14 / 01 / 2008$ & \\
\hline $\mathrm{QCB}$ & $\begin{array}{l}\text { BetaShares Commodities Basket ETF-Currency Hedged } \\
\text { (Synthetic) }\end{array}$ & $15 / 12 / 2011$ & \\
\hline$\overline{\mathrm{QCH}}$ & Queensland Cotton Holdings Limited & $2 / 07 / 1992$ & $7 / 08 / 2007$ \\
\hline QFN & Betashares S\&P/ASX 200 Financials Sector ETF & $15 / 12 / 2010$ & \\
\hline QFX & Quickflix Limited & $27 / 06 / 2005$ & \\
\hline QGC & Queensland Gas Company Limited & $28 / 08 / 2000$ & $3 / 04 / 2009$ \\
\hline QHL & Quickstep Holdings Limited & $17 / 10 / 2005$ & \\
\hline QIL & Quoin (Int) Limited & $4 / 05 / 1994$ & $23 / 09 / 2013$ \\
\hline
\end{tabular}




\begin{tabular}{|c|c|c|c|}
\hline ASX Code & Company Name & Listing Date & Delisting Date \\
\hline QMG & Quay Magnesium Limited & $29 / 09 / 2004$ & $30 / 08 / 2012$ \\
\hline QML & QMASTOR Limited & $14 / 12 / 2001$ & $16 / 01 / 2012$ \\
\hline QMN & Queensland Mining Corporation Limited & $2 / 10 / 2008$ & \\
\hline QMT & QM Technologies Limited & $17 / 11 / 2005$ & $1 / 05 / 2008$ \\
\hline QNL & Quest Minerals Limited & $13 / 02 / 1995$ & \\
\hline $\mathrm{QOZ}$ & Betashares FTSE RAFI Australia 200 ETF & $11 / 07 / 2013$ & \\
\hline QRE & Betashares S\&P/ASX 200 Resources Sector ETF & $16 / 12 / 2010$ & \\
\hline QRX & QRxPharma Limited & $25 / 05 / 2007$ & \\
\hline QSS & Questus Limited & $7 / 08 / 2003$ & \\
\hline QST & Quest Investments Limited & $1 / 01 / 1972$ & \\
\hline QTG & Q Technology Group Limited & $2 / 12 / 1987$ & \\
\hline QTK & Quiktrak Networks Limited & $15 / 05 / 1969$ & $17 / 09 / 2008$ \\
\hline QTM & Quantum Energy Limited & $8 / 09 / 1989$ & \\
\hline QUB & Qube Holdings Limited & $4 / 01 / 2007$ & \\
\hline QUE & Queste Communications Limited & $11 / 11 / 1998$ & \\
\hline QUR & Quantum Resources Limited & $11 / 06 / 1987$ & \\
\hline QUS & Betashares FTSE RAFI U.S. 1000 ETF & $24 / 12 / 2014$ & \\
\hline QVE & QV Equities Limited & $22 / 08 / 2014$ & \\
\hline R3D & Redchip International Limited & $17 / 12 / 2004$ & \\
\hline RAF & Raffles Capital Limited & $2 / 01 / 1992$ & \\
\hline RAP & ResApp Health Limited & $12 / 01 / 2005$ & \\
\hline RAT & Rubicon America Trust & $6 / 12 / 2004$ & $23 / 12 / 2009$ \\
\hline RAW & Rawson Resources Limited & $22 / 09 / 2005$ & \\
\hline RBL & Rib Loc Group Limited & - & $25 / 08 / 2004$ \\
\hline RBR & RBR Group Limited & $2 / 02 / 2007$ & \\
\hline RBS & Roberts Limited & 17/06/1949 & $27 / 06 / 2006$ \\
\hline RBV & Rabinov Property Trust & $12 / 08 / 2003$ & $8 / 08 / 2011$ \\
\hline RBX & Resource Base Limited & $23 / 11 / 2007$ & \\
\hline RBY & Rockeby Biomed Limited & $23 / 12 / 2003$ & $27 / 01 / 2010$ \\
\hline RCA & Raw Capital Partners Limited & $28 / 06 / 2000$ & $1 / 09 / 2009$ \\
\hline $\mathrm{RCB}$ & Russell Australian Select Corporate Bond ETF & $13 / 03 / 2012$ & \\
\hline $\mathrm{RCF}$ & Redcliffe Resources Limited & 23/02/1989 & $14 / 04 / 2016$ \\
\hline RCG & RCG Corporation Limited & $7 / 07 / 2004$ & \\
\hline RCI & Rocklands Richfield Limited & $1 / 11 / 2000$ & $16 / 10 / 2012$ \\
\hline RCL & Repco Corporation Limited & $20 / 11 / 2003$ & $3 / 05 / 2007$ \\
\hline $\mathrm{RCO}$ & Royalco Resources Limited & $29 / 06 / 2006$ & \\
\hline RCP & Redbank Copper Limited & $22 / 12 / 1993$ & \\
\hline RCR & RCR Tomlinson Limited & $15 / 11 / 1996$ & \\
\hline RCT & Reef Casino Trust & $16 / 11 / 1995$ & \\
\hline $\mathrm{RCU}$ & Real Estate Capital Partners USA Property Trust & $23 / 09 / 2005$ & \\
\hline RCY & Rivercity Motorway Group & $3 / 08 / 2006$ & $4 / 01 / 2016$ \\
\hline RDF & Redflex Holdings Limited & $20 / 01 / 1997$ & \\
\hline RDG & Resource Development Group Limited & $19 / 05 / 2011$ & \\
\hline RDH & RedHill Education Limited & $21 / 09 / 2010$ & \\
\hline RDM & Red Metal Limited & $27 / 10 / 2003$ & \\
\hline RDS & Redstone Resources Limited & $3 / 08 / 2006$ & \\
\hline RDV & Russell High Dividend Australian Shares ETF & $14 / 05 / 2010$ & \\
\hline REA & REA Group Ltd & $1 / 12 / 1999$ & \\
\hline REB & Rebel Sport Limited & $30 / 11 / 1993$ & $5 / 04 / 2007$ \\
\hline REC & Recall Holdings Limited & $10 / 12 / 2013$ & $4 / 05 / 2016$ \\
\hline RED & Red 5 Limited & $16 / 10 / 1996$ & \\
\hline REF & Reverse Corp Limited & $16 / 12 / 2005$ & \\
\hline REG & Regis Healthcare Limited & $7 / 10 / 2014$ & \\
\hline REG & RG Capital Radio Limited & - & $13 / 09 / 2004$ \\
\hline REH & Reece Limited & $31 / 01 / 1978$ & \\
\hline REM & Reliance Mining Limited & - & $22 / 04 / 2005$ \\
\hline RER & Regal Resources Limited & $28 / 06 / 2005$ & \\
\hline
\end{tabular}




\begin{tabular}{|c|c|c|c|}
\hline ASX Code & Company Name & Listing Date & Delisting Date \\
\hline RES & Resource Generation Limited & $31 / 10 / 2003$ & \\
\hline REU & Rubicon Europe Trust Group & $9 / 12 / 2005$ & 23/12/2009 \\
\hline REW & The Rewards Factory Limited & $30 / 06 / 2008$ & $1 / 09 / 2009$ \\
\hline REX & Regional Express Holdings Limited & $9 / 11 / 2005$ & \\
\hline REY & Rey Resources Limited & $5 / 06 / 2006$ & \\
\hline REZ & Resources \& Energy Group Limited & $11 / 03 / 2005$ & \\
\hline RFF & Rural Funds Group & $14 / 02 / 2014$ & \\
\hline RFG & Retail Food Group Limited & $22 / 06 / 2006$ & \\
\hline RFL & Rubik Financial Limited & $23 / 02 / 2000$ & \\
\hline RFT & Rectifier Technologies Ltd & $22 / 09 / 1994$ & \\
\hline RFV & Rift Valley Resources Limited & 19/05/2011 & $2 / 07 / 2012$ \\
\hline RFX & Redflow Limited & $14 / 12 / 2010$ & \\
\hline RGB & Russell Australian Government Bond ETF & $13 / 03 / 2012$ & \\
\hline RGP & Refresh Group Limited & $29 / 03 / 2006$ & \\
\hline RGS & Regeneus Ltd & 19/09/2013 & \\
\hline RGU & Regalpoint Resources Limited & $30 / 05 / 2011$ & \\
\hline RHC & Ramsay Health Care Limited & 24/09/1997 & \\
\hline RHD & Ross Human Directions Limited & $8 / 09 / 2000$ & $6 / 05 / 2011$ \\
\hline RHG & RHG Limited & $27 / 07 / 2007$ & $14 / 01 / 2014$ \\
\hline RHI & Red Hill Iron Limited & $14 / 02 / 2006$ & \\
\hline RHL & Ruralco Holdings Limited & $30 / 03 / 1988$ & \\
\hline RHP & Rhipe Limited & $17 / 03 / 2005$ & \\
\hline RHS & Reproductive Health Science Limited & $5 / 03 / 1987$ & \\
\hline RHT & Resonance Health Limited & $2 / 01 / 1992$ & \\
\hline $\mathrm{RIC}$ & Ridley Corporation Limited & $13 / 08 / 1987$ & \\
\hline RIE & Riedel Resources Limited & $31 / 01 / 2011$ & \\
\hline RIG & Rongtai International Group Holdings Limited & $30 / 12 / 2010$ & $1 / 09 / 2015$ \\
\hline RIL & Riddock International Limited & 19/02/1987 & $1 / 03 / 2016$ \\
\hline RIM & Rimfire Pacific Mining NL & 4/03/1997 & \\
\hline RIN & Rinker Group Limited & $31 / 03 / 2003$ & $7 / 08 / 2007$ \\
\hline RIO & Rio Tinto Limited & $1 / 01 / 1961$ & \\
\hline RIS & Richfield International Limited & 23/03/2006 & $30 / 09 / 2016$ \\
\hline RIV & Riversdale Mining Limited & $24 / 01 / 1986$ & 7/07/2011 \\
\hline RJT & Rubicon Japanese Trust & $31 / 10 / 2006$ & $23 / 12 / 2009$ \\
\hline RKN & Reckon Limited & 19/07/1999 & \\
\hline RLC & Reedy Lagoon Corporation Limited & $22 / 06 / 2007$ & \\
\hline RLE & Real Energy Corporation Limited & $12 / 12 / 2013$ & \\
\hline RLG & RCL Group & $30 / 06 / 2006$ & $30 / 08 / 2012$ \\
\hline RMA & Reeltime Media Limited & $8 / 09 / 2000$ & $4 / 01 / 2016$ \\
\hline RMD & ResMed Inc. & $25 / 11 / 1999$ & \\
\hline RMG & RMG Limited & 19/04/1996 & \\
\hline RMI & Resource Mining Corporation Limited & $14 / 02 / 1985$ & \\
\hline RML & Rusina Mining NL & 24/12/1987 & $7 / 07 / 2010$ \\
\hline RMP & Red Emperor Resources NL & $16 / 08 / 2007$ & \\
\hline RMR & Ram Resources Limited & $21 / 09 / 2005$ & \\
\hline RMS & Ramelius Resources Limited & $31 / 03 / 2003$ & \\
\hline RMT & RMA Energy Limited & $7 / 06 / 2007$ & \\
\hline RMX & Red Mountain Mining Limited & $1 / 09 / 2011$ & \\
\hline $\mathrm{RNC}$ & Real Estate Corp Limited & $12 / 12 / 2005$ & $17 / 04 / 2014$ \\
\hline RND & Rand Mining Limited & $15 / 08 / 1985$ & \\
\hline RNI & RNI NL & $15 / 03 / 2001$ & \\
\hline RNL & Rision Limited & $21 / 02 / 2002$ & \\
\hline RNO & Rhinomed Limited & $21 / 09 / 2007$ & \\
\hline RNS & Renaissance Minerals Limited & $11 / 06 / 2010$ & \\
\hline RNT & Rent.com.au Limited & $26 / 05 / 1994$ & \\
\hline RNU & Renascor Resources Limited & $15 / 12 / 2010$ & \\
\hline RNY & RNY Property Trust & $26 / 09 / 2005$ & \\
\hline
\end{tabular}




\begin{tabular}{|c|c|c|c|}
\hline ASX Code & Company Name & Listing Date & Delisting Date \\
\hline ROC & Roc Oil Company Limited & $5 / 08 / 1999$ & $2 / 02 / 2015$ \\
\hline ROG & Red Sky Energy Limited & $23 / 05 / 2007$ & \\
\hline ROK & The Rock Building Society Limited & $10 / 12 / 1992$ & $20 / 12 / 2011$ \\
\hline ROL & Robust Resources Limited & $26 / 02 / 2007$ & $18 / 11 / 2014$ \\
\hline RPD & RP Data Limited & - & $28 / 09 / 2004$ \\
\hline RPF & Redcape Property Group & $2 / 08 / 2007$ & $11 / 05 / 2012$ \\
\hline RPG & Raptis Group Limited & $17 / 07 / 1986$ & \\
\hline RPM & Roma Petroleum NL & $10 / 01 / 1995$ & $30 / 01 / 2009$ \\
\hline RPT & Redport Limited & 28/11/1995 & $22 / 01 / 2007$ \\
\hline RPX & RP Data Ltd & $15 / 12 / 2006$ & $13 / 05 / 2011$ \\
\hline RQL & Resource Equipment Ltd & $16 / 05 / 2002$ & $18 / 05 / 2015$ \\
\hline RRL & Regis Resources Limited & $19 / 02 / 1987$ & \\
\hline RRP & Realm Resources Limited & $16 / 11 / 1989$ & \\
\hline RRS & Range Resources Limited & 24/01/1985 & \\
\hline RRT & Record Realty & $20 / 12 / 2002$ & $1 / 09 / 2009$ \\
\hline RSG & Resolute Mining Limited & $11 / 01 / 1979$ & \\
\hline RSH & Respiri Limited & $14 / 07 / 2000$ & \\
\hline RSM & Russell Australian Semi-Government Bond ETF & $13 / 03 / 2012$ & \\
\hline RSP & Resource Pacific Holdings Limited & $29 / 11 / 2004$ & $28 / 04 / 2008$ \\
\hline RSR & Aii S\&P/ASX 200 Resources & $25 / 03 / 2010$ & $13 / 06 / 2013$ \\
\hline RTA & Rutila Resources Limited & $23 / 09 / 2010$ & $1 / 09 / 2015$ \\
\hline RTG & RTG Mining Inc. & $5 / 06 / 2014$ & \\
\hline RTR & Rumble Resources Limited & $1 / 07 / 2011$ & \\
\hline RTS & Retail Technology \& Services Limited & - & $12 / 08 / 2005$ \\
\hline RUB & Rubicor Group Limited & $15 / 06 / 2007$ & \\
\hline RUL & Runge Pincock Minarco Limited & $27 / 05 / 2008$ & \\
\hline RUM & Rum Jungle Resources Ltd & $21 / 11 / 2007$ & \\
\hline RUP & Rural Press Limited & $16 / 03 / 1989$ & 15/05/2007 \\
\hline RVA & Reva Medical Inc & $23 / 12 / 2010$ & \\
\hline RVL & Russell Australian Value ETF & $18 / 03 / 2011$ & \\
\hline RVR & Red River Resources Limited & $15 / 07 / 2005$ & \\
\hline RVY & Rift Valley Resources Limited & $21 / 12 / 2007$ & \\
\hline RWD & Reward Minerals Ltd & $12 / 12 / 1991$ & \\
\hline RWH & Royal Wolf Holdings Ltd & $31 / 05 / 2011$ & \\
\hline RXH & Rewardle Holdings Limited & $7 / 10 / 2014$ & \\
\hline RXL & Rox Resources Limited & $27 / 04 / 2004$ & \\
\hline RXM & Rex Minerals Limited & $20 / 09 / 2007$ & \\
\hline RXP & RXP Services Limited & 28/11/2011 & \\
\hline RZR & Razor Risk Technologies Limited & $3 / 12 / 1999$ & $29 / 03 / 2012$ \\
\hline SAI & SAI Global Limited & $17 / 12 / 2003$ & \\
\hline SAM & SIV Asset Management Limited & $13 / 07 / 2010$ & \\
\hline SAN & Sagalio Energy Limited & $15 / 12 / 2011$ & \\
\hline SAO & Sino Australia Oil \& Gas Limited & $12 / 12 / 2013$ & $30 / 08 / 2016$ \\
\hline SAP & Sabina Corporation Limited & $18 / 11 / 1988$ & $1 / 09 / 2015$ \\
\hline SAQ & Sydney Attractions Group Limited & $10 / 06 / 1993$ & $2 / 05 / 2008$ \\
\hline SAR & Saracen Mineral Holdings Limited & $2 / 01 / 1992$ & \\
\hline SAS & Sky and Space Global Ltd & $22 / 05 / 2006$ & \\
\hline SAU & Southern Gold Limited & $21 / 04 / 2005$ & \\
\hline SAX & Stadium Australia Group & $11 / 04 / 1997$ & $29 / 03 / 2007$ \\
\hline SAY & South America Iron \& Steel Corporation Limited & $27 / 05 / 1997$ & \\
\hline SBB & Sunbridge Group Limited & $27 / 11 / 2013$ & \\
\hline SBC & Southern Cross Broadcasting Ltd & $20 / 08 / 1987$ & $12 / 11 / 2007$ \\
\hline SBI & Sterling Plantations Limited & $25 / 09 / 2006$ & \\
\hline SBL & Signature Metals Limited & $19 / 01 / 2004$ & $4 / 05 / 2015$ \\
\hline SBM & St Barbara Limited & $15 / 05 / 1969$ & \\
\hline SBR & Sabre Resources Limited & $17 / 12 / 1987$ & \\
\hline SBS & Sub-Sahara Resources NL & $30 / 03 / 1994$ & $18 / 12 / 2009$ \\
\hline
\end{tabular}




\begin{tabular}{|c|c|c|c|}
\hline ASX Code & Company Name & Listing Date & Delisting Date \\
\hline SBU & Siburan Resources Limited & $18 / 05 / 2010$ & \\
\hline SCB & Scarborough Equities Limited & $5 / 08 / 1994$ & $16 / 03 / 2009$ \\
\hline SCC & Scott Corporation Limited & 10/01/1994 & $8 / 04 / 2014$ \\
\hline SCD & Scantech Limited & $8 / 10 / 1987$ & $20 / 10 / 2015$ \\
\hline SCF & Southern Cross FLIERS Trust & $13 / 08 / 2002$ & $22 / 12 / 2006$ \\
\hline SCG & Scentre Group & $13 / 12 / 2010$ & \\
\hline SCI & Silver City Minerals Limited & $6 / 07 / 2011$ & \\
\hline SCP & Shopping Centres Australasia Property Group & $26 / 11 / 2012$ & \\
\hline$\overline{S C R}$ & Scandinavian Resources Limited & $21 / 04 / 2010$ & $13 / 06 / 2012$ \\
\hline SCU & Stemcell United Limited & $13 / 06 / 2000$ & \\
\hline SDA & Speedcast International Limited & $12 / 08 / 2014$ & \\
\hline SDF & Steadfast Group Limited & $2 / 08 / 2013$ & \\
\hline$\overline{\text { SDG }}$ & Sunland Group Limited & $28 / 02 / 1995$ & \\
\hline SDI & SDI Limited & $7 / 11 / 1985$ & \\
\hline SDL & Sundance Resources Limited & 9/12/1993 & \\
\hline SDM & Sedgman Limited & $8 / 06 / 2006$ & $18 / 04 / 2016$ \\
\hline SDR & Sundowner Motor Inns Limited & $6 / 11 / 1997$ & $16 / 05 / 2007$ \\
\hline SDS & SDS Corporation Limited & - & $9 / 06 / 2006$ \\
\hline SDT & Shandong Tianye Australia Limited & 20/07/1970 & $30 / 08 / 2013$ \\
\hline SEA & Sundance Energy Australia Limited & $28 / 04 / 2005$ & \\
\hline SED & Sedimentary Holdings Limited & $8 / 04 / 1983$ & $15 / 12 / 2006$ \\
\hline SEG & Segue Resources Limited & $28 / 10 / 2005$ & \\
\hline SEH & Sino Gas \& Energy Holdings Limited & $15 / 09 / 2009$ & \\
\hline SEK & SEEK Limited & $19 / 04 / 2005$ & \\
\hline SEL & S8 Limited & $21 / 12 / 2001$ & $29 / 01 / 2007$ \\
\hline SEM & Select Managed Funds Limited & $30 / 03 / 2005$ & $27 / 06 / 2006$ \\
\hline SEN & Senetas Corporation Limited & $21 / 06 / 1984$ & \\
\hline SEQ & Sequoia Financial Group Ltd & $19 / 09 / 2000$ & \\
\hline SER & Strategic Energy Resources Limited & $15 / 08 / 1991$ & \\
\hline SES & SECOS Group Ltd & $21 / 11 / 1996$ & \\
\hline SFC & Schaffer Corporation Limited & $1 / 12 / 1964$ & \\
\hline SFE & SFE Corporation Limited & $16 / 04 / 2002$ & $26 / 07 / 2006$ \\
\hline$\overline{\mathrm{SFG}}$ & Seafarms Group Limited & $14 / 06 / 1990$ & \\
\hline SFH & Specialty Fashion Group Limited & $28 / 05 / 1998$ & \\
\hline SFI & Spookfish Limited & $19 / 04 / 2007$ & \\
\hline SFR & Sandfire Resources NL & $4 / 03 / 2004$ & \\
\hline SFW & SFG Australia Limited & $19 / 12 / 1986$ & $22 / 08 / 2014$ \\
\hline SFX & Sheffield Resources Limited & $15 / 12 / 2010$ & \\
\hline SFY & SPDR S\&P/ASX 50 Fund & $28 / 08 / 2001$ & \\
\hline SGB & St. George Bank Limited & $2 / 07 / 1992$ & $3 / 04 / 2009$ \\
\hline SGC & Sacgasco Limited & $11 / 10 / 2005$ & \\
\hline SGF & Sg Fleet Group Limited & $4 / 03 / 2014$ & \\
\hline SGH & Slater \& Gordon Limited & $21 / 05 / 2007$ & \\
\hline SGI & Signature Capital Investments Limited & $6 / 12 / 2005$ & $10 / 07 / 2013$ \\
\hline SGL & Sydney Gas Ltd & $27 / 06 / 1996$ & 9/04/2009 \\
\hline SGM & Sims Metal Management Limited & 14/11/1991 & \\
\hline SGO & Stream Group Limited & $31 / 10 / 1986$ & \\
\hline SGP & Stockland & $12 / 02 / 1988$ & \\
\hline SGQ & St George Mining Limited & $16 / 11 / 2010$ & \\
\hline SGR & The Star Entertainment Group Limited & 6/06/2011 & \\
\hline SGS & Stargames Limited & - & $10 / 03 / 2006$ \\
\hline SGT & Singapore Telecommunications Limited & $10 / 09 / 2001$ & $8 / 06 / 2015$ \\
\hline SGU & Success Global Media Limited & $23 / 01 / 2006$ & \\
\hline SGW & Sons of Gwalia Limited & $5 / 05 / 1983$ & 29/08/2006 \\
\hline SGX & Sino Gold Mining Limited & $3 / 12 / 2002$ & $16 / 12 / 2009$ \\
\hline SGZ & Scotgold Resources Limited & $15 / 01 / 2008$ & \\
\hline SHA & Shannon Resources Limited & $17 / 07 / 2006$ & $14 / 05 / 2007$ \\
\hline
\end{tabular}




\begin{tabular}{|c|c|c|c|}
\hline ASX Code & Company Name & Listing Date & Delisting Date \\
\hline SHC & Sunshine Heart, Inc & $28 / 09 / 2004$ & $7 / 05 / 2013$ \\
\hline SHD & Sherwin Iron Limited & $11 / 12 / 1986$ & $1 / 09 / 2014$ \\
\hline SHG & Sunshine Gas Limited & $3 / 07 / 2002$ & $30 / 01 / 2009$ \\
\hline SHH & Shree Minerals Limited & $18 / 02 / 2010$ & \\
\hline SHJ & Shine Corporate Ltd & $15 / 05 / 2013$ & \\
\hline SHK & Stone Resources Australia Limited & $5 / 12 / 2003$ & \\
\hline SHL & Sonic Healthcare Limited & $30 / 04 / 1987$ & \\
\hline SHR & John Shearer (Holdings) Limited & $30 / 06 / 1972$ & $29 / 10 / 2015$ \\
\hline SHU & Shenhua International Limited & $30 / 07 / 2009$ & \\
\hline SHV & Select Harvests Limited & $22 / 12 / 1983$ & \\
\hline SHX & Shield Mining Limited & $27 / 09 / 2006$ & 17/09/2010 \\
\hline SIB & Siberia Mining Corporation Limited & - & $5 / 05 / 2006$ \\
\hline SIE & SciGen Limited & $15 / 11 / 2002$ & \\
\hline SIG & Sigma Company Limited & - & $19 / 12 / 2005$ \\
\hline SIH & Sihayo Gold Limited & $31 / 03 / 1988$ & \\
\hline SIO & Simonds Group Limited & $17 / 11 / 2014$ & \\
\hline SIP & Sigma Pharmaceuticals Limited & $30 / 10 / 2002$ & \\
\hline SIQ & Smartgroup Corporation Ltd & $2 / 07 / 2014$ & \\
\hline$\overline{\text { SIR }}$ & Sirius Resources NL & $24 / 07 / 1986$ & $24 / 09 / 2015$ \\
\hline SIT & Site Group International Limited & $21 / 08 / 1987$ & \\
\hline SIV & Silver Chef Limited & $31 / 05 / 2005$ & \\
\hline SIX & Sprintex Limited & $29 / 05 / 2008$ & \\
\hline SKC & SkyCity Entertainment Group Limited & $29 / 03 / 1999$ & \\
\hline SKD & Stockford Limited & $28 / 11 / 2000$ & $29 / 08 / 2006$ \\
\hline SKE & Skilled Group Limited & $27 / 07 / 1994$ & $20 / 10 / 2015$ \\
\hline SKF & Skyfii Ltd & $17 / 12 / 1999$ & \\
\hline SKI & Spark Infrastructure Group & $16 / 12 / 2005$ & \\
\hline SKP & Skyland Petroleum Limited & $23 / 05 / 1997$ & $1 / 09 / 2016$ \\
\hline SKS & Stokes Limited & $1 / 01 / 1979$ & \\
\hline SKT & Sky Network Television Limited & $11 / 04 / 2000$ & \\
\hline SKW & Skywest Limited & $23 / 11 / 2004$ & $8 / 09 / 2006$ \\
\hline SLA & Solagran Limited & $22 / 12 / 1983$ & $4 / 01 / 2016$ \\
\hline SLC & SoftLaw Corporation Limited & - & $28 / 02 / 2005$ \\
\hline SLE & Sino-Excel Energy Limited & $2 / 08 / 2000$ & $30 / 08 / 2016$ \\
\hline SLF & SPDR S\&P/ASX 200 Listed Property Fund & $18 / 02 / 2002$ & \\
\hline SLK & Sealink Travel Group Limited & $16 / 10 / 2013$ & \\
\hline SLM & Salmat Limited & $2 / 12 / 2002$ & \\
\hline SLN & Selwyn Mines Limited & $30 / 06 / 2000$ & $29 / 08 / 2007$ \\
\hline SLP & Sylvania Platinum Limited & $10 / 03 / 2011$ & $27 / 04 / 2012$ \\
\hline SLR & Silver Lake Resources Limited & $14 / 11 / 2007$ & \\
\hline SLX & Silex Systems Limited & $7 / 05 / 1998$ & \\
\hline SMA & SmartTrans Holdings Limited & $22 / 03 / 1984$ & \\
\hline SMC & Strategic Minerals Corporation NL & $1 / 10 / 1981$ & \\
\hline SMD & Syndicated Metals Limited & $13 / 12 / 2007$ & \\
\hline SMI & Santana Minerals Limited & $31 / 05 / 2013$ & \\
\hline SMM & Summit Resources Limited & $7 / 04 / 1994$ & \\
\hline SMN & Structural Monitoring Systems Plc & $16 / 08 / 2004$ & \\
\hline SMP & Smartpay Holdings Limited & $4 / 09 / 2013$ & \\
\hline SMR & Stanmore Coal Limited & $9 / 12 / 2009$ & \\
\hline SMX & SMS Management and Technology Limited & $12 / 12 / 1986$ & \\
\hline SNC & Sandon Capital Investments Limited & $23 / 12 / 2013$ & \\
\hline SND & Saunders International Limited & $5 / 12 / 2007$ & \\
\hline SNE & Somerton Energy Limited & $9 / 02 / 2001$ & $29 / 06 / 2012$ \\
\hline SNL & Supply Network Limited & $10 / 09 / 1987$ & \\
\hline SNN & Sonnet Corporation Limited & $25 / 01 / 1990$ & $21 / 08 / 2007$ \\
\hline SNR & Synergy Plus Limited & $6 / 12 / 2000$ & $5 / 04 / 2016$ \\
\hline SNV & Sinovus Mining Limited & $11 / 12 / 2007$ & \\
\hline
\end{tabular}




\begin{tabular}{|c|c|c|c|}
\hline ASX Code & Company Name & Listing Date & Delisting Date \\
\hline SNZ & Summerset Group Holdings Limited & $18 / 07 / 2013$ & \\
\hline $\mathrm{SO} 4$ & Salt Lake Potash Limited & $3 / 11 / 2006$ & \\
\hline SOC & Sovereign Gold Company Limited & $3 / 12 / 2010$ & \\
\hline SOE & Souls Private Equity Limited & $16 / 12 / 2004$ & $10 / 01 / 2012$ \\
\hline SOF & Sofcom Limited & $19 / 09 / 2000$ & $4 / 01 / 2016$ \\
\hline $\mathrm{SOH}$ & Solution 6 Holdings Limited & - & $27 / 08 / 2004$ \\
\hline SOL & Washington H. Soul Pattinson and Co. Limited & $21 / 01 / 1903$ & \\
\hline SOM & SomnoMed Limited & $27 / 08 / 2004$ & \\
\hline SOP & SML Corporation Limited & 14/05/1987 & \\
\hline SOR & Strategic Elements Limited & $11 / 08 / 2011$ & \\
\hline SP1 & Search Party Group Ltd & $9 / 04 / 2010$ & \\
\hline SPB & South Pacific Resources Limited & $27 / 02 / 1997$ & \\
\hline SPC & SPC Ardmona Limited & - & $25 / 02 / 2005$ \\
\hline SPD & Strategic Pooled Development Limited & $7 / 02 / 1994$ & $27 / 07 / 2012$ \\
\hline SPH & Sphere Minerals Limited & $3 / 12 / 1986$ & $9 / 06 / 2016$ \\
\hline SPI & Spitfire Materials Ltd & $12 / 12 / 2007$ & \\
\hline SPK & Spark New Zealand Limited & 22/08/1991 & \\
\hline SPL & Starpharma Holdings Limited & $28 / 09 / 2000$ & \\
\hline SPO & Spotless Group Holdings Limited & $23 / 05 / 2014$ & \\
\hline SPQ & Superior Resources Limited & $12 / 11 / 2007$ & \\
\hline SPR & S8 Property Trust & $12 / 07 / 2005$ & $6 / 07 / 2007$ \\
\hline SPT & Spotless Group Limited & $1 / 01 / 1974$ & $20 / 08 / 2012$ \\
\hline SPX & Spectrum Rare Earths Limited & $26 / 04 / 2007$ & \\
\hline SPY & SPDR S\&P 500 ETF Trust & $13 / 10 / 2014$ & \\
\hline SPZ & Smart Parking Limited & $9 / 01 / 2007$ & \\
\hline SRA & Strathfield Group Limited & $6 / 07 / 1998$ & $30 / 08 / 2013$ \\
\hline SRB & Scarborough Minerals Plc & 19/05/2006 & $31 / 07 / 2007$ \\
\hline SRE & Stirling Resources Limited & 24/08/1971 & $14 / 04 / 2014$ \\
\hline SRF & Surfstitch Group Limited & $16 / 12 / 2014$ & \\
\hline$\overline{\text { SRG }}$ & SRG Limited & $9 / 04 / 1987$ & \\
\hline SRG & Sydney Roads Group & $31 / 07 / 2006$ & $28 / 06 / 2007$ \\
\hline SRH & Saferoads Holdings Limited & $15 / 12 / 2005$ & \\
\hline SRI & Sipa Resources Limited & $23 / 08 / 1990$ & \\
\hline SRK & Strike Resources Limited & $7 / 03 / 2000$ & \\
\hline SRM & Sierra Mining & $13 / 12 / 2006$ & $5 / 06 / 2014$ \\
\hline SRO & ShareRoot Ltd & $7 / 03 / 1996$ & \\
\hline SRP & Southcorp Limited & - & $17 / 06 / 2005$ \\
\hline SRR & Shaw River Manganese Limited & $22 / 12 / 2006$ & \\
\hline SRS & Spicers Limited & $17 / 04 / 2000$ & \\
\hline SRV & Servcorp Limited & $6 / 12 / 1999$ & \\
\hline SRX & Sirtex Medical Limited & $24 / 08 / 2000$ & \\
\hline SRZ & Stellar Resources Limited & $28 / 04 / 2005$ & \\
\hline SSI & Sino Strategic International Limited & 22/05/1987 & $1 / 09 / 2015$ \\
\hline SSL & Sietel Limited & $1 / 01 / 1974$ & \\
\hline SSM & Service Stream Limited & $9 / 12 / 2004$ & \\
\hline SSN & Samson Oil \& Gas Limited & $6 / 04 / 1983$ & \\
\hline SSO & SPDR S\&P/ASX Small Ordinaries Fund & $13 / 04 / 2011$ & \\
\hline SST & Steamships Trading Company Limited & $30 / 06 / 1962$ & \\
\hline SSX & Smorgon Steel Group Limited & $3 / 02 / 1999$ & $20 / 08 / 2007$ \\
\hline ST1 & Spirit Telecom Limited & $22 / 10 / 1999$ & \\
\hline STA & Strandline Resources Limited & $17 / 05 / 2000$ & \\
\hline STC & Asset Realisation Company Plc & $16 / 04 / 2007$ & $18 / 09 / 2009$ \\
\hline STG & Staging Connections Group Limited & $2 / 09 / 1999$ & $12 / 12 / 2011$ \\
\hline STI & Stirling Products Limited & 24/07/1997 & 23/03/2016 \\
\hline STL & StarGroup Limited & $22 / 04 / 1994$ & \\
\hline STO & Santos Limited & $12 / 10 / 1954$ & \\
\hline STP & Sterihealth Limited & $29 / 11 / 1993$ & $7 / 07 / 2014$ \\
\hline
\end{tabular}




\begin{tabular}{|c|c|c|c|}
\hline ASX Code & Company Name & Listing Date & Delisting Date \\
\hline STR & Service Stream Ltd & $29 / 05 / 1987$ & $9 / 01 / 2007$ \\
\hline STU & Stuart Petroleum Limited & $16 / 07 / 1993$ & $1 / 06 / 2011$ \\
\hline STV & Sunraysia Television Limited & $1 / 01 / 1974$ & $21 / 12 / 2007$ \\
\hline STW & SPDR S\&P/ASX 200 Fund & $27 / 08 / 2001$ & \\
\hline STX & Strike Energy Limited & $5 / 08 / 2004$ & \\
\hline SUD & Suda Ltd & $24 / 01 / 2002$ & \\
\hline SUH & Southern Hemisphere Mining Limited & $5 / 01 / 2010$ & \\
\hline SUL & Super Retail Group Limited & $6 / 07 / 2004$ & \\
\hline SUM & Sumatra Copper \& Gold PLC & $26 / 10 / 2009$ & \\
\hline SUN & Suncorp Group Limited & $7 / 07 / 1988$ & \\
\hline SUR & Sun Resources NL & $10 / 08 / 1993$ & \\
\hline SVA & Simavita Limited & $20 / 02 / 2014$ & \\
\hline SVL & Silver Mines Limited & $19 / 01 / 2007$ & \\
\hline SVM & Sovereign Metals Limited & $22 / 01 / 2007$ & \\
\hline SVS & Sunvest Corporation Limited & $20 / 08 / 1987$ & \\
\hline SVW & Seven Group Holdings Limited & $6 / 08 / 1993$ & \\
\hline SVY & Stavely Minerals Limited & $7 / 05 / 2014$ & \\
\hline SW1 & Swift Networks Group Limited & $23 / 03 / 1995$ & \\
\hline SWE & Swala Energy Limited & $18 / 04 / 2013$ & \\
\hline SWJ & Stonewall Resources Limited & $7 / 04 / 2011$ & \\
\hline SWK & Swick Mining Services Ltd & $1 / 11 / 2006$ & \\
\hline SWL & Seymour Whyte Limited & $31 / 05 / 2010$ & \\
\hline SWM & Seven West Media Limited & 9/01/1992 & \\
\hline SXA & Strata-X Energy Limited & $12 / 03 / 2013$ & \\
\hline SXE & Southern Cross Electrical Engineering Ltd & $28 / 11 / 2007$ & \\
\hline SXI & South East Asia Resources Limited & $7 / 12 / 1994$ & \\
\hline SXL & Southern Cross Media Group Limited & $17 / 11 / 2005$ & \\
\hline SXM & SA Metals Limited & $28 / 11 / 2000$ & $10 / 09 / 2009$ \\
\hline SXP & SAPEX Limited & $25 / 05 / 2007$ & $24 / 10 / 2008$ \\
\hline SXR & Skywest Airlines Ltd & $8 / 12 / 2009$ & $12 / 04 / 2013$ \\
\hline SXS & Solimar Energy Limited & $14 / 09 / 2005$ & $30 / 08 / 2016$ \\
\hline SXX & Southern Cross Exploration N.L. & $6 / 04 / 1983$ & \\
\hline SXY & Senex Energy Limited & $18 / 06 / 1984$ & \\
\hline SYA & Sayona Mining Limited & $30 / 03 / 2004$ & \\
\hline SYB & Symbion Health Limited & $30 / 06 / 1962$ & $8 / 05 / 2008$ \\
\hline SYD & Sydney Airport & $2 / 04 / 2002$ & \\
\hline SYI & SPDR MSCI Australia Select High Dividend Yield Fund & $29 / 09 / 2010$ & \\
\hline SYL & Sylvastate Limited & $28 / 07 / 1971$ & $16 / 05 / 2011$ \\
\hline SYR & Syrah Resources Limited & $11 / 09 / 2007$ & \\
\hline SYS & Syngas Limited & $26 / 08 / 1998$ & \\
\hline SYT & Syntonic Limited & $12 / 06 / 2007$ & \\
\hline SZG & Subzero Group Limited & $12 / 03 / 1987$ & \\
\hline T3D & 333D Limited & $27 / 12 / 2006$ & \\
\hline TAB & TAB Limited & - & $30 / 08 / 2004$ \\
\hline TAG & Tag Pacific Limited & $1 / 01 / 1974$ & \\
\hline TAH & Tabcorp Holdings Limited & $26 / 08 / 1994$ & \\
\hline TAL & Tower Australia Group Limited & $21 / 11 / 2006$ & $12 / 05 / 2011$ \\
\hline TAM & Tanami Gold NL & $11 / 12 / 1986$ & \\
\hline TAN & Tandou Limited & $26 / 11 / 1987$ & $14 / 08 / 2015$ \\
\hline TAP & Tap Oil Limited & 17/09/1996 & \\
\hline TAR & Taruga Gold Limited & $7 / 02 / 2012$ & \\
\hline TAS & Tasman Resources Limited & $18 / 12 / 2001$ & \\
\hline TAT & Tat Hong Holdings Limited & - & $24 / 11 / 2005$ \\
\hline TAU & Trustees Australia Limited & $23 / 02 / 1989$ & \\
\hline TAW & Tawana Resources NL & $19 / 04 / 2001$ & \\
\hline TBC & Tribeca Learning Limited & - & 23/05/2006 \\
\hline TBG & Tutt Bryant Group Limited & $15 / 12 / 2005$ & $14 / 10 / 2010$ \\
\hline
\end{tabular}




\begin{tabular}{|c|c|c|c|}
\hline ASX Code & Company Name & Listing Date & Delisting Date \\
\hline TBR & Tribune Resources Limited & $4 / 05 / 1989$ & \\
\hline TCL & Transurban Group & $15 / 03 / 1996$ & \\
\hline TCM & Tiaro Coal Limited & $3 / 03 / 2008$ & $1 / 09 / 2015$ \\
\hline TCN & Techniche Limited & $16 / 04 / 1987$ & \\
\hline TCO & Transmetro Corporation Limited & 17/09/1987 & \\
\hline TDL & TBG Diagnostics Limited & $22 / 12 / 1995$ & \\
\hline TDO & 3D Oil Limited & $22 / 05 / 2007$ & \\
\hline TEA & Terra Gold Mining Limited & - & $30 / 12 / 2005$ \\
\hline TEG & Triangle Energy (Global) Limited & $6 / 01 / 2006$ & \\
\hline TEM & Tempo Services Limited & - & $13 / 04 / 2005$ \\
\hline TEN & Ten Network Holdings Limited & $31 / 03 / 1998$ & \\
\hline TEO & Telesso Technologies Limited & $20 / 08 / 1996$ & $4 / 01 / 2016$ \\
\hline TER & TerraCom Limited & $22 / 07 / 2010$ & \\
\hline TER & Terrain Australia Limited & - & $10 / 01 / 2005$ \\
\hline TEX & Target Energy Limited & $27 / 11 / 2006$ & \\
\hline TFC & TFS Corporation Limited & $21 / 12 / 2004$ & \\
\hline TFL & TasFoods Limited & 16/12/1999 & \\
\hline TFS & Tranzact Financial Services Limited & $26 / 05 / 2000$ & $14 / 02 / 2014$ \\
\hline TGA & Thorn Group Limited & $13 / 12 / 2006$ & \\
\hline TGG & Templeton Global Growth Fund Limited & 28/05/1987 & \\
\hline TGN & Tungsten Mining NL & $17 / 12 / 2012$ & \\
\hline TGP & 360 Capital Group & $26 / 07 / 2005$ & \\
\hline TGR & Tassal Group Limited & $12 / 11 / 2003$ & \\
\hline TGS & Tiger Resources Limited & $8 / 05 / 1997$ & \\
\hline TGZ & Teranga Gold Corporation & $10 / 12 / 2010$ & \\
\hline THD & Thred Limited & $12 / 11 / 2009$ & \\
\hline THG & Thakral Holdings Limited & $17 / 06 / 1994$ & $19 / 10 / 2012$ \\
\hline THO & THO Services Limited & $30 / 05 / 1996$ & $30 / 08 / 2016$ \\
\hline THR & Thor Mining Plc & $27 / 09 / 2006$ & \\
\hline THX & Thundelarra Limited & $22 / 03 / 2001$ & \\
\hline TIA & Tian An Australia Limited & $11 / 12 / 1985$ & \\
\hline TIF & Technology Investment Fund & - & $29 / 07 / 2005$ \\
\hline TIG & Tigers Realm Coal Limited & $29 / 08 / 2011$ & \\
\hline TIM & Timbercorp Limited & $30 / 05 / 1996$ & $30 / 08 / 2011$ \\
\hline TIR & Titan Resources Limited & $1 / 06 / 1987$ & $14 / 09 / 2006$ \\
\hline TIX & 360 Capital Industrial Fund & $13 / 12 / 2012$ & \\
\hline TKA & Tusker Gold Limited & $16 / 12 / 2009$ & $23 / 05 / 2010$ \\
\hline TKF & TikForce Limited & $15 / 12 / 2006$ & \\
\hline TKG & Takoradi Limited & $15 / 10 / 1987$ & \\
\hline TKL & Traka Resources Limited & $20 / 11 / 2003$ & \\
\hline TKR & Triako Resources Limited & $29 / 02 / 1984$ & $18 / 10 / 2006$ \\
\hline TLC & Tourism, Hotels \& Leisure Limited & $15 / 08 / 1985$ & $3 / 10 / 2006$ \\
\hline TLG & Talga Resources Ltd & $9 / 07 / 2010$ & \\
\hline TLM & Talisman Mining Limited & $25 / 11 / 2005$ & \\
\hline TLS & Telstra Corporation Limited & 17/11/1997 & \\
\hline TLT & Tourism \& Leisure Trust & $22 / 05 / 1997$ & $1 / 02 / 2007$ \\
\hline TLZ & Telezon Limited & 17/04/1986 & 24/11/2011 \\
\hline TMA & TMA Group of Companies Limited & $19 / 12 / 1991$ & $1 / 11 / 2011$ \\
\hline TME & Trade Me Group Limited & $13 / 12 / 2011$ & \\
\hline TMK & Tamaska Oil \& Gas Ltd & $10 / 07 / 2008$ & \\
\hline TMM & Tasmania Mines Limited & $13 / 11 / 1969$ & \\
\hline TMP & Tech Mpire Limited & $18 / 12 / 2012$ & \\
\hline TMR & Tamaya Resources Limited & $31 / 01 / 2001$ & $30 / 08 / 2010$ \\
\hline TMX & Terrain Minerals Limited & $23 / 03 / 2006$ & \\
\hline TMZ & Thomson Resources Limited & $17 / 12 / 2010$ & \\
\hline TND & Top End Minerals Limited & $3 / 12 / 2007$ & \\
\hline TNE & Technology One Limited & $8 / 12 / 1999$ & \\
\hline
\end{tabular}




\begin{tabular}{|c|c|c|c|}
\hline ASX Code & Company Name & Listing Date & Delisting Date \\
\hline TNG & TNG Limited & $18 / 02 / 1971$ & \\
\hline TNK & Think Childcare Limited & $24 / 10 / 2014$ & \\
\hline TNN & Tenon Limited & - & $2 / 10 / 2004$ \\
\hline TNP & Triple Energy Limited & $14 / 12 / 2005$ & \\
\hline TNR & Torian Resources Limited & $1 / 10 / 1982$ & \\
\hline TNS & Transonic Travel Limited & - & $20 / 07 / 2006$ \\
\hline TOE & Toro Energy Limited & $24 / 03 / 2006$ & \\
\hline TOF & 360 Capital Office Fund & $24 / 04 / 2014$ & \\
\hline TOL & Toll Holdings Limited & $12 / 10 / 1993$ & $1 / 06 / 2015$ \\
\hline TOM & Tomizone Limited & $1 / 01 / 1974$ & \\
\hline TON & Triton Minerals Ltd & $14 / 08 / 2009$ & \\
\hline TOP & Thorney Opportunities Ltd & $22 / 03 / 2000$ & \\
\hline TOR & Ticor Limited & - & $24 / 11 / 2005$ \\
\hline TOU & Tlou Energy Limited & 9/04/2013 & \\
\hline TOX & Tox Free Solutions Limited & $29 / 09 / 2000$ & \\
\hline TPC & TPC Consolidated Limited & $16 / 07 / 2007$ & \\
\hline TPD & Talon Petroleum Limited & $28 / 02 / 2013$ & \\
\hline TPF & Timbercorp Primary Infrastructure Fund & $14 / 12 / 2006$ & $30 / 08 / 2010$ \\
\hline TPM & TPG Telecom Limited & $10 / 05 / 2001$ & \\
\hline TPO & Tian Poh Resources Limited & $11 / 11 / 2014$ & \\
\hline TPP & Tempo Australia Ltd & $1 / 01 / 1969$ & \\
\hline TPR & Timpetra Resources Limited & $30 / 03 / 2011$ & \\
\hline TPS & Threat Protect Australia Limited & $10 / 03 / 1994$ & \\
\hline TPX & Tasmanian Perpetual Trustees Limited & $10 / 10 / 1986$ & $25 / 09 / 2009$ \\
\hline TQH & 3Q Holdings Limited & $11 / 04 / 2000$ & $13 / 02 / 2013$ \\
\hline TRF & Trafford Resources Limited & $19 / 06 / 2006$ & $22 / 05 / 2015$ \\
\hline TRL & Tanga Resources Limited & $9 / 03 / 2011$ & \\
\hline TRM & Truscott Mining Corporation Limited & $18 / 04 / 2007$ & \\
\hline TRO & Triausmin Limited & $9 / 01 / 2004$ & $21 / 08 / 2014$ \\
\hline TRS & The Reject Shop Limited & $1 / 06 / 2004$ & \\
\hline TRU & The Trust Company Limited & $3 / 11 / 1983$ & $20 / 12 / 2013$ \\
\hline TRY & Troy Resources Limited & $5 / 03 / 1987$ & \\
\hline TSI & Transfield Services Infrastructure Fund & $12 / 06 / 2007$ & $11 / 07 / 2011$ \\
\hline TSM & ThinkSmart Limited & $4 / 06 / 2007$ & \\
\hline TSN & Transaction Solutions International Limited & $8 / 08 / 2001$ & \\
\hline TSO & Tishman Speyer Office Fund & $1 / 12 / 2004$ & $29 / 06 / 2012$ \\
\hline TSV & Transerv Energy Limited & $10 / 03 / 2004$ & \\
\hline TTA & TTA Holdings Limited & $16 / 02 / 2006$ & \\
\hline TTE & TTE Petroleum Limited & $21 / 03 / 2005$ & \\
\hline TTH & Tooth \& Company Limited & $5 / 07 / 1961$ & $12 / 02 / 2010$ \\
\hline TTI & Traffic Technologies Ltd & $17 / 02 / 1998$ & \\
\hline TTL & Transcendence Technologies Limited & $21 / 03 / 2003$ & \\
\hline TTS & Tatts Group Limited & $7 / 07 / 2005$ & \\
\hline TTT & Tritton Resources Limited & $3 / 12 / 2003$ & $25 / 08 / 2006$ \\
\hline TTW & Toptung Ltd & $15 / 11 / 2007$ & \\
\hline TTY & Territory Resources Limited & $11 / 03 / 2005$ & $30 / 08 / 2011$ \\
\hline TUP & TTG Fintech Limited & $27 / 11 / 2012$ & \\
\hline TV2 & TV2U International Limited & $7 / 06 / 2007$ & \\
\hline TVL & Travel.com.au Limited & $12 / 05 / 1999$ & $18 / 02 / 2008$ \\
\hline TWD & Tamawood Limited & $14 / 08 / 2000$ & \\
\hline TWE & Treasury Wine Estates Limited & $10 / 05 / 2011$ & \\
\hline TWH & TW Holdings Limited & $18 / 12 / 1986$ & \\
\hline TWO & Talent2 International Limited & $7 / 05 / 1970$ & $17 / 09 / 2012$ \\
\hline TWR & Tower Limited & $28 / 09 / 1999$ & \\
\hline TWT & TWT Group Limited & $12 / 04 / 2007$ & $1 / 09 / 2015$ \\
\hline TXN & Texon Petroleum Limited & $15 / 05 / 2007$ & $13 / 03 / 2013$ \\
\hline TYC & Tethyan Copper Company Limited & - & $19 / 05 / 2006$ \\
\hline
\end{tabular}




\begin{tabular}{|c|c|c|c|}
\hline ASX Code & Company Name & Listing Date & Delisting Date \\
\hline TYK & Tychean Resources Ltd & $31 / 10 / 2006$ & \\
\hline TYO & Treyo Leisure And Entertainment Limited & $8 / 01 / 2009$ & $23 / 12 / 2015$ \\
\hline TYX & Tyranna Resources Limited & $11 / 07 / 2007$ & \\
\hline TZL & TZ Limited & $2 / 01 / 1992$ & \\
\hline TZN & Terramin Australia Limited & $23 / 12 / 2003$ & \\
\hline UBI & Universal Biosensors, Inc. & $13 / 12 / 2006$ & \\
\hline UBN & Urbanise.Com Limited & $22 / 09 / 2014$ & \\
\hline UCL & UCL Resources Limited & $15 / 12 / 1983$ & $30 / 08 / 2013$ \\
\hline $\mathrm{UCM}$ & USCOM Ltd & $10 / 12 / 2003$ & \\
\hline UCW & UCW Limited & $25 / 06 / 2004$ & \\
\hline UEC & Uecomm Limited & - & $19 / 08 / 2004$ \\
\hline UEQ & Uranium Equities Limited & $3 / 10 / 2000$ & \\
\hline UGL & UGL Limited & 19/12/1994 & \\
\hline UIL & UIL Energy Ltd & $6 / 11 / 2014$ & \\
\hline UKL & Uranium King Limited & $4 / 09 / 2006$ & $5 / 11 / 2008$ \\
\hline ULT & Ultrapay Limited & $8 / 03 / 1994$ & $26 / 04 / 2013$ \\
\hline UMC & United Minerals Corporation NL & $9 / 12 / 2004$ & $22 / 02 / 2010$ \\
\hline$\overline{\mathrm{UML}}$ & Unity Mining Limited & $1 / 11 / 1985$ & $7 / 06 / 2016$ \\
\hline UMS & UCMS Group Limited & $21 / 06 / 2007$ & $18 / 08 / 2009$ \\
\hline UND & U \& D Coal Limited & $19 / 02 / 2014$ & $18 / 06 / 2015$ \\
\hline UNS & Unilife Corporation & 26/06/1986 & \\
\hline UNV & Universal Coal Plc & $10 / 12 / 2010$ & \\
\hline UNW & Unwired Group Limited & $5 / 08 / 1994$ & $7 / 05 / 2008$ \\
\hline UOS & United Overseas Australia Limited & $17 / 02 / 1988$ & \\
\hline UPG & Unity Pacific Group & $14 / 12 / 2004$ & \\
\hline URF & US Masters Residential Property Fund & 23/07/2012 & \\
\hline USA & UraniumSA Limited & $18 / 10 / 2006$ & \\
\hline USD & Betashares U.S. Dollar ETF & $1 / 02 / 2011$ & \\
\hline USF & US Select Private Opportunities Fund & $14 / 08 / 2012$ & \\
\hline USG & US Select Private Opportunities Fund II & $9 / 04 / 2013$ & \\
\hline UTB & UNiTAB Limited & 16/11/1999 & $17 / 11 / 2006$ \\
\hline UUL & Ultima United Limited & $1 / 06 / 2007$ & \\
\hline UXA & UXA Resources Ltd & $22 / 11 / 2005$ & $29 / 03 / 2016$ \\
\hline UXC & UXC Limited & 19/02/1997 & $1 / 03 / 2016$ \\
\hline VAF & Vanguard Australian Fixed Interest Index ETF & $31 / 10 / 2012$ & \\
\hline VAH & Virgin Australia Holdings Limited & $8 / 12 / 2003$ & \\
\hline VAN & Vango Mining Limited & $8 / 03 / 2005$ & \\
\hline VAP & Vanguard Australian Property Securities Index ETF & $15 / 10 / 2010$ & \\
\hline VAR & Variscan Mines Limited & $20 / 08 / 1987$ & \\
\hline VAS & Vanguard Australian Shares Index & $8 / 05 / 2009$ & \\
\hline VCM & VeCommerce Limited & $1 / 10 / 1987$ & $5 / 12 / 2006$ \\
\hline $\mathrm{VCN}$ & Vulcan Resources Limited & $10 / 09 / 2002$ & $23 / 02 / 2010$ \\
\hline VCR & Ventracor Limited & 17/08/1993 & $1 / 09 / 2009$ \\
\hline $\mathrm{VCX}$ & Vicinity Centres & $5 / 12 / 2011$ & \\
\hline VEA & Veda Advantage Limited & $30 / 09 / 1998$ & $12 / 07 / 2007$ \\
\hline VEC & Vector Resources Limited & $17 / 12 / 2007$ & \\
\hline VED & Veda Group Limited & $5 / 12 / 2013$ & 29/02/2016 \\
\hline VEI & Vision Eye Institute Limited & $17 / 12 / 2004$ & $16 / 12 / 2015$ \\
\hline VEL & Vealls Limited & $1 / 12 / 1970$ & \\
\hline VES & Vesture Limited & 19/12/1994 & 26/08/2013 \\
\hline VET & Vocation Limited & $9 / 12 / 2013$ & $30 / 08 / 2016$ \\
\hline VEU & Vanguard All-World ex-US Shares Index ETF & $8 / 05 / 2009$ & \\
\hline VGB & Vanguard Australian Government Bond Index ETF & $30 / 04 / 2012$ & \\
\hline VGE & Vanguard FTSE Emerging Markets Shares ETF & $21 / 11 / 2013$ & \\
\hline VGL & Vista Group International Limited & $11 / 08 / 2014$ & \\
\hline VGL & Volante Group Limited & - & $10 / 05 / 2006$ \\
\hline VGO & Vantage Goldfields Limited & $27 / 05 / 2010$ & $20 / 01 / 2015$ \\
\hline
\end{tabular}




\begin{tabular}{|c|c|c|c|}
\hline ASX Code & Company Name & Listing Date & Delisting Date \\
\hline VGR & Voyager Global Group Ltd & $21 / 11 / 2007$ & \\
\hline VGS & Vanguard MSCI Index International Shares ETF & $20 / 11 / 2014$ & \\
\hline VHY & Vanguard Australian Shares High Yield ETF & $26 / 05 / 2011$ & \\
\hline VIA & Viagold Capital Limited & $9 / 02 / 1996$ & \\
\hline VIC & Victory Mines Limited & 9/10/2012 & \\
\hline VID & Videlli Limited & $18 / 04 / 1991$ & $30 / 06 / 2009$ \\
\hline VIE & Viento Group Limited & $16 / 05 / 1991$ & \\
\hline VIG & Victor Group Holdings Limited & 9/05/2014 & \\
\hline VII & Vietnam Industrial Investments Limited & $18 / 09 / 1995$ & \\
\hline VIP & Voltage IP Limited & $5 / 02 / 1997$ & \\
\hline VIR & Viridis Clean Energy & $14 / 09 / 2005$ & $30 / 08 / 2011$ \\
\hline VKA & Viking Mines Limited & $12 / 05 / 2010$ & \\
\hline VKI & Viking Industries Limited & $31 / 12 / 1984$ & $27 / 02 / 2008$ \\
\hline VLA & Viralytics Limited & $15 / 10 / 1986$ & \\
\hline VLC & Vanguard MSCI Australian Large Companies Index ETF & $26 / 05 / 2011$ & \\
\hline VLT & Vault Intelligence Limited & $7 / 04 / 2011$ & \\
\hline VLW & Villa World Limited & $14 / 11 / 2003$ & \\
\hline VMC & Venus Metals Corporation Limited & $12 / 06 / 2007$ & \\
\hline VMG & VDM Group Limited & $15 / 02 / 2006$ & \\
\hline VML & Vital Metals Limited & $11 / 10 / 2005$ & \\
\hline VMS & Venture Minerals Limited & $22 / 09 / 2006$ & \\
\hline VMT & Vmoto Limited & $31 / 01 / 2002$ & \\
\hline VMX & Valmec Limited & $13 / 01 / 2005$ & \\
\hline VMY & Vimy Resources Limited & $23 / 05 / 2008$ & \\
\hline VNX & Vonex Limited & $9 / 07 / 1996$ & $12 / 04 / 2016$ \\
\hline VOC & Vocus Communications Limited & 10/06/1999 & \\
\hline VOF & Valad Opportunity Fund No 11 & $6 / 07 / 2004$ & 13/07/2007 \\
\hline VOL & Volant Petroleum Limited & $23 / 06 / 1987$ & $25 / 10 / 2007$ \\
\hline VOY & Voyager Energy Limited & - & $29 / 09 / 2005$ \\
\hline $\mathrm{VPC}$ & Velpic Limited & $28 / 07 / 2011$ & \\
\hline VPG & Valad Property Group & $13 / 12 / 2002$ & $31 / 08 / 2011$ \\
\hline VRC & Volt Resources Limited & $19 / 12 / 2003$ & \\
\hline VRI & Veriluma Limited & $17 / 12 / 2010$ & \\
\hline VRL & Village Roadshow Limited & $26 / 02 / 1987$ & \\
\hline VRT & Virtus Health Limited & $11 / 06 / 2013$ & \\
\hline VRX & Ventnor Resources Ltd & $24 / 02 / 2011$ & \\
\hline VSC & Vita Life Sciences Limited & $23 / 08 / 2007$ & \\
\hline VSG & Visiomed Group Limited & $1 / 06 / 1987$ & $5 / 03 / 2008$ \\
\hline VSL & Vision Systems Limited & $14 / 11 / 1985$ & $23 / 02 / 2007$ \\
\hline $\mathrm{VSO}$ & Vanguard MSCI Australian Small Companies Index ETF & $26 / 05 / 2011$ & \\
\hline VTA & Viterra Inc & $14 / 09 / 2009$ & 19/12/2012 \\
\hline VTG & Vita Group Limited & $2 / 11 / 2005$ & \\
\hline VTI & Virotec International Limited & - & $30 / 12 / 2005$ \\
\hline VTM & Volta Mining Limited & 19/10/2011 & \\
\hline VTS & Vanguard US Total Market Shares Index ETF & $8 / 05 / 2009$ & \\
\hline VUL & Valhalla Uranium Limited & $19 / 12 / 2005$ & $24 / 11 / 2006$ \\
\hline VWD & Villa World Ltd & $9 / 04 / 1986$ & $21 / 12 / 2006$ \\
\hline VXL & Valence Industries Limited & $6 / 01 / 2014$ & \\
\hline VXR & Venturex Resources Limited & $27 / 04 / 2007$ & \\
\hline VXS & Voxson Limited & $17 / 12 / 1999$ & $15 / 12 / 2006$ \\
\hline WAA & WAM Active Limited & $11 / 01 / 2008$ & \\
\hline WAF & West African Resources Limited & $11 / 06 / 2010$ & \\
\hline WAK & Wakenby Limited & $17 / 08 / 2007$ & \\
\hline WAL & Wavenet International Limited & $17 / 03 / 2000$ & \\
\hline WAM & WAM Capital Limited & $12 / 08 / 1999$ & \\
\hline WAT & Waterco Limited & 23/03/1989 & \\
\hline WAX & WAM Research Limited & $19 / 08 / 2003$ & \\
\hline
\end{tabular}




\begin{tabular}{|c|c|c|c|}
\hline ASX Code & Company Name & Listing Date & Delisting Date \\
\hline WBA & Webster Limited & $1 / 01 / 1974$ & \\
\hline WBC & Westpac Banking Corporation & $31 / 01 / 1962$ & \\
\hline WBT & Weebit Nano Ltd & $21 / 12 / 2010$ & \\
\hline WCB & Warrnambool Cheese and Butter Factory Company & $27 / 05 / 2004$ & \\
\hline WCC & The Waterberg Coal Company Limited & $17 / 06 / 2002$ & $30 / 08 / 2016$ \\
\hline WCG & Webcentral Group Limited & $7 / 07 / 1994$ & $25 / 09 / 2006$ \\
\hline WCI & Waratah Coal Inc & $10 / 11 / 2008$ & $1 / 04 / 2009$ \\
\hline WCL & Westside Corporation Limited & $10 / 01 / 2007$ & $19 / 09 / 2014$ \\
\hline WCN & White Cliff Minerals Limited & $14 / 12 / 2007$ & \\
\hline WCP & WCP Resources Limited & $28 / 03 / 1983$ & \\
\hline WCU & White Canyon Uranium Limited & $3 / 03 / 2008$ & $8 / 07 / 2011$ \\
\hline WDC & WESTFIELD GROUP & $5 / 07 / 2004$ & \\
\hline WDP & Wadepack Limited & - & $28 / 02 / 2005$ \\
\hline WDR & Western Desert Resources Limited & $23 / 07 / 2007$ & $1 / 09 / 2015$ \\
\hline WDS & WDS Limited & $7 / 12 / 2006$ & $30 / 08 / 2016$ \\
\hline WEB & Webjet Limited & $18 / 12 / 1997$ & \\
\hline WEC & White Energy Company Limited & 23/07/1999 & \\
\hline WEL & Winchester Energy Limited & $23 / 09 / 2014$ & \\
\hline WES & Wesfarmers Limited & $15 / 11 / 1984$ & \\
\hline WFA & Westfield America Trust & $3 / 07 / 1996$ & $12 / 08 / 2004$ \\
\hline WFD & Westfield Corporation & $25 / 06 / 2014$ & \\
\hline WFE & Winmar Resources Limited & $14 / 12 / 1999$ & \\
\hline WFL & Willmott Forests Limited & $20 / 12 / 2000$ & $30 / 08 / 2011$ \\
\hline WFT & Westfield Trust & $5 / 08 / 1982$ & $2 / 07 / 2004$ \\
\hline WGL & Wangle Technologies Limited & $10 / 08 / 2004$ & \\
\hline WGO & Waratah Resources Limited & $17 / 07 / 2008$ & \\
\hline WGR & Westgold Resources Limited & $29 / 09 / 1988$ & $22 / 10 / 2012$ \\
\hline WHC & Whitehaven Coal Limited & $1 / 06 / 2007$ & \\
\hline WHF & Whitefield Limited & $2 / 08 / 1971$ & \\
\hline WHN & WHL Energy Limited & $10 / 09 / 2007$ & \\
\hline WHS & Warehouse Group Limited (The) & $20 / 11 / 2000$ & $2 / 07 / 2012$ \\
\hline WIC & Westoz Investment Company Limited & $16 / 09 / 2009$ & \\
\hline WIN & Windward Resources Ltd & $28 / 11 / 2012$ & \\
\hline WKT & Walkabout Resources Ltd & $7 / 11 / 2006$ & \\
\hline WLC & Wollongong Coal Limited & $10 / 07 / 2007$ & \\
\hline WLF & Wolf Minerals Limited & $15 / 02 / 2007$ & \\
\hline WLL & Wellcom Group Limited & $22 / 07 / 2005$ & \\
\hline WMK & Watermark Market Neutral Fund Limited & $18 / 07 / 2013$ & \\
\hline WMN & Western Mining Network Limited & $4 / 03 / 2011$ & \\
\hline WMR & WMC Resources Limited & - & $29 / 06 / 2005$ \\
\hline WNR & Wingara Ag Ltd & $31 / 01 / 1990$ & \\
\hline WNS & World.Net Services Limited & $24 / 08 / 2000$ & \\
\hline WNZ & Waste Management NZ Limited & - & $27 / 06 / 2006$ \\
\hline WOF & Wolf Petroleum Ltd & $8 / 03 / 2006$ & \\
\hline WOR & WorleyParsons Limited & $28 / 11 / 2002$ & \\
\hline WOT & Westpac Office Trust & $7 / 08 / 2003$ & $5 / 08 / 2010$ \\
\hline WOW & Woolworths Limited & $23 / 07 / 1993$ & \\
\hline WPG & WPG Resources Ltd & $23 / 08 / 2005$ & \\
\hline WPL & Woodside Petroleum Limited & 18/11/1971 & \\
\hline WPP & WPP AUNZ Ltd & 14/01/1994 & \\
\hline WRG & Water Resources Group Limited & $30 / 12 / 2010$ & \\
\hline WRK & Warwick Resources Limited & $13 / 02 / 2007$ & $21 / 12 / 2009$ \\
\hline WRM & White Rock Minerals Ltd & $8 / 10 / 2010$ & \\
\hline WRR & World Reach Limited & $6 / 11 / 1986$ & \\
\hline WSA & Western Areas Limited & $28 / 07 / 2000$ & \\
\hline WSF & Westfield Holdings Limited & $28 / 09 / 1961$ & $2 / 07 / 2004$ \\
\hline WSG & Wolfstrike Rentals Group Limited & $11 / 11 / 2004$ & \\
\hline
\end{tabular}




\begin{tabular}{|c|c|c|c|}
\hline ASX Code & Company Name & Listing Date & Delisting Date \\
\hline WSI & WestStar Industrial Limited & $7 / 06 / 2006$ & \\
\hline WTF & Wotif.com Holdings Limited & $2 / 06 / 2006$ & $18 / 11 / 2014$ \\
\hline WTP & Watpac Limited & $29 / 08 / 1985$ & \\
\hline WTR & World Titanium Resources Limited & $28 / 12 / 2006$ & \\
\hline WVL & Windimurra Vanadium Limited & $22 / 12 / 1988$ & \\
\hline WWA & Wridgways Australia Limited & 19/05/1999 & $21 / 12 / 2010$ \\
\hline WWI & West Wits Mining Limited & 19/12/2007 & \\
\hline WYL & Wattyl Limited & $30 / 06 / 1962$ & $22 / 09 / 2010$ \\
\hline XAM & Xanadu Mines Limited & $21 / 12 / 2010$ & \\
\hline XCD & Xceed Resources Limited & 28/03/1996 & $27 / 02 / 2014$ \\
\hline $\mathrm{XF} 1$ & Xref Limited & $18 / 04 / 2007$ & \\
\hline XPE & Xped Limited & $22 / 06 / 2007$ & \\
\hline XRF & XRF Scientific Limited & $31 / 10 / 2006$ & \\
\hline XRO & Xero Limited & $8 / 11 / 2012$ & \\
\hline XST & Xstate Resources Limited & $14 / 01 / 1988$ & \\
\hline XTD & XTD Limited & $9 / 05 / 2011$ & \\
\hline XTE & XTEK Ltd & $15 / 09 / 2005$ & \\
\hline XTV & XTV Networks Ltd & $19 / 06 / 2007$ & \\
\hline $\mathrm{XXL}$ & XiaoXiao Education Limited & $2 / 02 / 2010$ & $4 / 01 / 2016$ \\
\hline YAL & Yancoal Australia Limited & $28 / 06 / 2012$ & \\
\hline YBR & Yellow Brick Road Holdings Limited & $31 / 01 / 2008$ & \\
\hline YHL & Yanghao International Limited & $20 / 03 / 2009$ & \\
\hline YNB & Yonder and Beyond Group Limited & $10 / 08 / 2011$ & \\
\hline YOJ & Yojee Limited & $2 / 12 / 2010$ & \\
\hline YOW & Yowie Group Ltd & $15 / 09 / 1999$ & \\
\hline YPB & YPB Group Ltd & $7 / 03 / 2005$ & \\
\hline YSF & Yield Strategies Fund (The) & - & $13 / 08 / 2004$ \\
\hline YSL & York Group Limited & - & $28 / 02 / 2005$ \\
\hline ZAM & Zamanco Minerals Limited & $31 / 10 / 2007$ & \\
\hline ZBB & ZBB Energy Corporation & $30 / 03 / 2005$ & $9 / 08 / 2007$ \\
\hline ZDX & Zedex Minerals Limited & $23 / 05 / 2006$ & $28 / 04 / 2010$ \\
\hline ZEL & Z Energy Limited & $19 / 08 / 2013$ & \\
\hline$\overline{Z E R}$ & Zeta Resources Limited & $12 / 06 / 2013$ & \\
\hline$\overline{\mathrm{ZEU}}$ & Zeus Resources Limited & $21 / 01 / 2013$ & \\
\hline ZFX & Zinifex Limited & $5 / 04 / 2004$ & $2 / 07 / 2008$ \\
\hline ZGL & Zicom Group Limited & $30 / 06 / 1977$ & \\
\hline ZGM & Zamia Metals Limited & $10 / 01 / 2007$ & \\
\hline ZHE & Zheng He Global Capital Limited & $24 / 09 / 2010$ & $30 / 08 / 2012$ \\
\hline ZIM & Zimplats Holdings Limited & $15 / 10 / 1998$ & \\
\hline ZIP & ZipTel Limited & $15 / 06 / 2004$ & \\
\hline ZMI & Zinc of Ireland NL & $18 / 09 / 2007$ & \\
\hline ZML & Zipmoney Ltd & $9 / 12 / 2009$ & \\
\hline $\mathrm{ZNC}$ & Zenith Minerals Limited & $29 / 05 / 2007$ & \\
\hline ZRL & Zambezi Resources Limited & $5 / 07 / 2007$ & \\
\hline ZTA & Zeta Petroleum Plc & $23 / 05 / 2012$ & \\
\hline ZTL & Zenyth Therapeutics Limited & $5 / 12 / 1996$ & $17 / 11 / 2006$ \\
\hline ZYB & Zyber Holdings Ltd & $1 / 12 / 2009$ & \\
\hline$\overline{\mathrm{ZYL}}$ & ZYL Limited & $1 / 12 / 1980$ & \\
\hline
\end{tabular}


APPENDIX II: ANNOUNCEMENT DISTRIBUTION OF EACH CATEGORY OVER THE STUDY PERIOD

\begin{tabular}{|c|c|c|c|c|c|c|c|c|c|c|c|c|}
\hline $\begin{array}{l}\text { Takeover/Scheme } \\
\text { Announcements }\end{array}$ & 2004 & 2005 & 2006 & 2007 & 2008 & 2009 & 2010 & 2011 & 2012 & 2013 & 2014 & Total \\
\hline Pre trading & 107 & 217 & 538 & 563 & 435 & 395 & 423 & 510 & 421 & 319 & 321 & 4,249 \\
\hline $10.00-11.00^{*}$ & 53 & 127 & 254 & 308 & 249 & 184 & 182 & 177 & 214 & 182 & 156 & 2,086 \\
\hline 11.00-12.00* & 34 & 79 & 153 & 182 & 164 & 132 & 102 & 93 & 96 & 92 & 81 & 1,208 \\
\hline $12.00-13.00^{*}$ & 44 & 92 & 167 & 225 & 118 & 135 & 137 & 125 & 107 & 96 & 92 & 1,338 \\
\hline $13.00-14.00^{*}$ & 37 & 73 & 115 & 134 & 124 & 128 & 123 & 101 & 108 & 84 & 88 & 1,115 \\
\hline $14.00-15.00^{*}$ & 48 & 106 & 147 & 179 & 171 & 112 & 121 & 114 & 120 & 86 & 63 & 1,267 \\
\hline $15.00-16.00$ & 76 & 127 & 216 & 186 & 172 & 166 & 152 & 158 & 134 & 100 & 90 & 1,577 \\
\hline Post trading & 301 & 464 & 757 & 762 & 621 & 550 & 602 & 478 & 445 & 376 & 379 & 5,735 \\
\hline Weekends & 0 & 0 & 0 & 0 & 1 & 0 & 0 & 0 & 0 & 0 & 0 & 1 \\
\hline Trading hours & 292 & 604 & 1,052 & 1,214 & 998 & 857 & 817 & 768 & 779 & 640 & 570 & 8,591 \\
\hline \% Each Year & $41.71 \%$ & $47.00 \%$ & $44.82 \%$ & $47.81 \%$ & $48.56 \%$ & $47.56 \%$ & $44.35 \%$ & $43.74 \%$ & $47.36 \%$ & $47.94 \%$ & $44.88 \%$ & $46.25 \%$ \\
\hline Off-trading hours & 408 & 681 & 1,295 & 1,325 & 1,057 & 945 & 1,025 & 988 & 866 & 695 & 700 & 9,985 \\
\hline \% Each Year & $58.29 \%$ & $53.00 \%$ & $55.18 \%$ & $52.19 \%$ & $51.44 \%$ & $52.44 \%$ & $55.65 \%$ & $56.26 \%$ & $52.64 \%$ & $52.06 \%$ & $55.12 \%$ & $53.75 \%$ \\
\hline Total & 700 & 1,285 & 2,347 & 2,539 & 2,055 & 1,802 & 1,842 & 1,756 & 1,645 & 1,335 & 1,270 & 18,576 \\
\hline \% Each Category & $3.77 \%$ & $6.92 \%$ & $12.63 \%$ & $13.67 \%$ & $11.06 \%$ & $9.70 \%$ & $9.92 \%$ & $9.45 \%$ & $8.86 \%$ & $7.19 \%$ & $6.84 \%$ & $100.00 \%$ \\
\hline $\begin{array}{l}\text { Market-sensitive } \\
\text { Takeover/Scheme } \\
\text { Announcements }\end{array}$ & 2004 & 2005 & 2006 & 2007 & 2008 & 2009 & 2010 & 2011 & 2012 & 2013 & 2014 & Total \\
\hline Pre trading & 62 & 116 & 211 & 283 & 169 & 212 & 236 & 256 & 236 & 165 & 185 & 2,131 \\
\hline $10.00-11.00^{*}$ & 26 & 51 & 105 & 153 & 125 & 89 & 100 & 99 & 121 & 101 & 90 & 1,060 \\
\hline 11.00-12.00* & 14 & 43 & 58 & 77 & 63 & 64 & 38 & 43 & 39 & 41 & 38 & 518 \\
\hline $12.00-13.00^{*}$ & 14 & 43 & 72 & 88 & 44 & 52 & 53 & 56 & 55 & 47 & 53 & 577 \\
\hline $13.00-14.00^{*}$ & 18 & 38 & 49 & 61 & 51 & 56 & 57 & 47 & 45 & 38 & 46 & 506 \\
\hline $14.00-15.00^{*}$ & 19 & 51 & 57 & 80 & 82 & 51 & 59 & 56 & 65 & 35 & 32 & 587 \\
\hline $15.00-16.00$ & 32 & 57 & 82 & 80 & 71 & 64 & 57 & 79 & 68 & 45 & 40 & 675 \\
\hline Post trading & 120 & 217 & 310 & 343 & 300 & 250 & 269 & 212 & 214 & 184 & 186 & 2,605 \\
\hline Weekends & 0 & 0 & 0 & 0 & 1 & 0 & 0 & 0 & 0 & 0 & 0 & 1 \\
\hline Trading hours & 123 & 283 & 423 & 539 & 436 & 376 & 364 & 380 & 393 & 307 & 299 & 3,923 \\
\hline \% Each Year & $40.33 \%$ & $45.94 \%$ & $44.81 \%$ & $46.27 \%$ & $48.12 \%$ & $44.87 \%$ & $41.89 \%$ & $44.81 \%$ & $46.62 \%$ & $46.80 \%$ & $44.63 \%$ & $45.30 \%$ \\
\hline Off-trading hours & 182 & 333 & 521 & 626 & 470 & 462 & 505 & 468 & 450 & 349 & 371 & 4,737 \\
\hline \% Each Year & $59.67 \%$ & $54.06 \%$ & $55.19 \%$ & $53.73 \%$ & $51.88 \%$ & $55.13 \%$ & $58.11 \%$ & $55.19 \%$ & $53.38 \%$ & $53.20 \%$ & $55.37 \%$ & $54.70 \%$ \\
\hline Total & 305 & 616 & 944 & 1,165 & 906 & 838 & 869 & 848 & 843 & 656 & 670 & 8,660 \\
\hline \% Each Category & $43.57 \%$ & $47.94 \%$ & $40.22 \%$ & $45.88 \%$ & $44.09 \%$ & $46.50 \%$ & $47.18 \%$ & $48.29 \%$ & $51.25 \%$ & $49.14 \%$ & $52.76 \%$ & $46.62 \%$ \\
\hline \% Total MS & $3.52 \%$ & $7.11 \%$ & $10.90 \%$ & $13.45 \%$ & $10.46 \%$ & $9.68 \%$ & $10.03 \%$ & $9.79 \%$ & $9.73 \%$ & $7.58 \%$ & $7.74 \%$ & $100.00 \%$ \\
\hline
\end{tabular}


Appendix II cont.

\begin{tabular}{|c|c|c|c|c|c|c|c|c|c|c|c|c|}
\hline Security Holder Details & 2004 & 2005 & 2006 & 2007 & 2008 & 2009 & 2010 & 2011 & 2012 & 2013 & 2014 & Total \\
\hline Pre trading & 1,412 & 3,007 & 4,273 & 4,703 & 5,007 & 3,998 & 4,594 & 5,137 & 4,685 & 4,477 & 4,723 & 46,016 \\
\hline $10.00-11.00^{*}$ & 732 & 1,401 & 1,642 & 1,838 & 2,086 & 1,652 & 1,429 & 1,511 & 1,332 & 1,340 & 1,456 & 16,419 \\
\hline $11.00-12.00^{*}$ & 656 & 1,407 & 1,649 & 1,923 & 2,026 & 1,742 & 1,556 & 1,509 & 1,451 & 1,419 & 1,355 & 16,693 \\
\hline $12.00-13.00^{*}$ & 696 & 1,409 & 1,588 & 1,811 & 1,992 & 1,570 & 1,563 & 1,561 & 1,378 & 1,355 & 1,464 & 16,387 \\
\hline 13.00-14.00* & 572 & 1,115 & 1,270 & 1,510 & 1,680 & 1,446 & 1,372 & 1,440 & 1,357 & 1,238 & 1,228 & 14,228 \\
\hline $14.00-15.00^{*}$ & 809 & 1,642 & 1,819 & 1,969 & 2,132 & 1,901 & 1,758 & 1,885 & 1,767 & 1,592 & 1,799 & 19,073 \\
\hline $15.00-16.00$ & 980 & 2,108 & 2,374 & 2,653 & 2,722 & 2,519 & 2,191 & 2,248 & 2,141 & 2,076 & 2,020 & 24,032 \\
\hline Post trading & 3,432 & 6,566 & 7,136 & 8,529 & 8,953 & 8,020 & 7,857 & 8,108 & 7,507 & 7,797 & 7,698 & 81,603 \\
\hline Weekends & 0 & 0 & 0 & 2 & 1 & 0 & 0 & 0 & 0 & 0 & 0 & 3 \\
\hline Trading hours & 4,445 & 9,082 & 10,342 & 11,704 & 12,638 & 10,830 & 9,869 & 10,154 & 9,426 & 9,020 & 9,322 & 106,832 \\
\hline \% Each Year & $47.85 \%$ & $48.68 \%$ & $47.55 \%$ & $46.93 \%$ & $47.51 \%$ & $47.40 \%$ & $44.22 \%$ & $43.40 \%$ & $43.60 \%$ & $42.36 \%$ & $42.87 \%$ & $45.57 \%$ \\
\hline Off-trading hours & 4,844 & 9,573 & 11,409 & 13,234 & 13,961 & 12,018 & 12,451 & 13,245 & 12,192 & 12,274 & 12,421 & 127,622 \\
\hline \% Each Year & $52.15 \%$ & $51.32 \%$ & $52.45 \%$ & $53.07 \%$ & $52.49 \%$ & $52.60 \%$ & $55.78 \%$ & $56.60 \%$ & $56.40 \%$ & $57.64 \%$ & $57.13 \%$ & $54.43 \%$ \\
\hline Total & 9,289 & 18,655 & 21,751 & 24,938 & 26,599 & 22,848 & 22,320 & 23,399 & 21,618 & 21,294 & 21,743 & 234,454 \\
\hline \% Each Category & $3.96 \%$ & $7.96 \%$ & $9.28 \%$ & $10.64 \%$ & $11.35 \%$ & $9.75 \%$ & $9.52 \%$ & $9.98 \%$ & $9.22 \%$ & $9.08 \%$ & $9.27 \%$ & $100.00 \%$ \\
\hline $\begin{array}{c}\text { Market-sensitive } \\
\text { Security Holder Details }\end{array}$ & 2004 & 2005 & 2006 & 2007 & 2008 & 2009 & 2010 & 2011 & 2012 & 2013 & 2014 & Total \\
\hline Pre trading & 9 & 16 & 30 & 46 & 109 & 53 & 65 & 72 & 73 & 84 & 130 & 687 \\
\hline 10.00-11.00* & 6 & 7 & 19 & 31 & 58 & 31 & 26 & 21 & 32 & 25 & 33 & 289 \\
\hline $11.00-12.00^{*}$ & 2 & 3 & 8 & 17 & 26 & 15 & 11 & 11 & 8 & 9 & 3 & 113 \\
\hline $12.00-13.00^{*}$ & 1 & 3 & 4 & 19 & 34 & 4 & 6 & 11 & 8 & 13 & 19 & 122 \\
\hline $13.00-14.00^{*}$ & 3 & 5 & 3 & 9 & 29 & 9 & 7 & 10 & 7 & 7 & 10 & 99 \\
\hline $14.00-15.00^{*}$ & 2 & 3 & 5 & 13 & 26 & 7 & 10 & 11 & 6 & 7 & 10 & 100 \\
\hline $15.00-16.00$ & 2 & 5 & 4 & 13 & 24 & 13 & 8 & 10 & 6 & 6 & 7 & 98 \\
\hline Post trading & 4 & 17 & 19 & 39 & 103 & 23 & 13 & 21 & 20 & 25 & 32 & 316 \\
\hline Weekends & 0 & 0 & 0 & 0 & 0 & 0 & 0 & 0 & 0 & 0 & 0 & 0 \\
\hline Trading hours & 16 & 26 & 43 & 102 & 197 & 79 & 68 & 74 & 67 & 67 & 82 & 821 \\
\hline \% Each Year & $55.17 \%$ & $44.07 \%$ & $46.74 \%$ & $54.55 \%$ & $48.17 \%$ & $50.97 \%$ & $46.58 \%$ & $44.31 \%$ & $41.88 \%$ & $38.07 \%$ & $33.61 \%$ & $45.01 \%$ \\
\hline Off-trading hours & 13 & 33 & 49 & 85 & 212 & 76 & 78 & 93 & 93 & 109 & 162 & 1,003 \\
\hline \% Each Year & $44.83 \%$ & $55.93 \%$ & $53.26 \%$ & $45.45 \%$ & $51.83 \%$ & $49.03 \%$ & $53.42 \%$ & $55.69 \%$ & $58.13 \%$ & $61.93 \%$ & $66.39 \%$ & $54.99 \%$ \\
\hline Total & 29 & 59 & 92 & 187 & 409 & 155 & 146 & 167 & 160 & 176 & 244 & 1,824 \\
\hline \% Each Category & $0.31 \%$ & $0.32 \%$ & $0.42 \%$ & $0.75 \%$ & $1.54 \%$ & $0.68 \%$ & $0.65 \%$ & $0.71 \%$ & $0.74 \%$ & $0.83 \%$ & $1.12 \%$ & $0.78 \%$ \\
\hline$\%$ Total MS & $1.59 \%$ & $3.23 \%$ & $5.04 \%$ & $10.25 \%$ & $22.42 \%$ & $8.50 \%$ & $8.00 \%$ & $9.16 \%$ & $8.77 \%$ & $9.65 \%$ & $13.38 \%$ & $100.00 \%$ \\
\hline
\end{tabular}


Appendix II cont.

\begin{tabular}{|c|c|c|c|c|c|c|c|c|c|c|c|c|}
\hline Periodic Reports & 2004 & 2005 & 2006 & 2007 & 2008 & 2009 & 2010 & 2011 & 2012 & 2013 & 2014 & Total \\
\hline Pre trading & 1,604 & 3,385 & 3,623 & 4,029 & 4,478 & 4,103 & 4,329 & 4,624 & 4,917 & 5,151 & 6,117 & 46,360 \\
\hline $10.00-11.00^{*}$ & 902 & 1,787 & 1,682 & 1,735 & 1,902 & 1,577 & 1,368 & 1,211 & 1,243 & 1,048 & 1,173 & 15,628 \\
\hline $11.00-12.00^{*}$ & 515 & 1,001 & 1,319 & 1,308 & 1,428 & 1,512 & 1,309 & 1,145 & 947 & 866 & 817 & 12,167 \\
\hline $12.00-13.00^{*}$ & 429 & 861 & 1,118 & 1,158 & 1,183 & 943 & 954 & 958 & 827 & 726 & 732 & 9,889 \\
\hline $13.00-14.00^{*}$ & 349 & 651 & 729 & 876 & 855 & 808 & 801 & 755 & 745 & 669 & 605 & 7,843 \\
\hline $14.00-15.00^{*}$ & 414 & 856 & 916 & 942 & 950 & 1,047 & 1,001 & 940 & 953 & 771 & 722 & 9,512 \\
\hline $15.00-16.00$ & 540 & 1,005 & 1,067 & 1,225 & 1,221 & 1,135 & 1,082 & 1,223 & 1,033 & 951 & 964 & 11,446 \\
\hline Post trading & 2,091 & 4,057 & 4,042 & 4,159 & 4,152 & 4,272 & 4,197 & 4,873 & 4,788 & 5,618 & 4,434 & 46,683 \\
\hline Weekends & 0 & 0 & 0 & 1 & 1 & 0 & 0 & 0 & 0 & 0 & 0 & 2 \\
\hline Trading hours & 3,149 & 6,161 & 6,831 & 7,244 & 7,539 & 7,022 & 6,515 & 6,232 & 5,748 & 5,031 & 5,013 & 66,485 \\
\hline \% Each Year & $46.01 \%$ & $45.29 \%$ & $47.12 \%$ & $46.94 \%$ & $46.62 \%$ & $45.61 \%$ & $43.31 \%$ & $39.62 \%$ & $37.20 \%$ & $31.84 \%$ & $32.21 \%$ & $41.68 \%$ \\
\hline Off-trading hours & 3,695 & 7,442 & 7,665 & 8,189 & 8,631 & 8,375 & 8,526 & 9,497 & 9,705 & 10,769 & 10,551 & 93,045 \\
\hline \% Each Year & $53.99 \%$ & $54.71 \%$ & $52.88 \%$ & $53.06 \%$ & $53.38 \%$ & $54.39 \%$ & $56.69 \%$ & $60.38 \%$ & $62.80 \%$ & $68.16 \%$ & $67.79 \%$ & $58.32 \%$ \\
\hline Total & 6,844 & 13,603 & 14,496 & 15,433 & 16,170 & 15,397 & 15,041 & 15,729 & 15,453 & 15,800 & 15,564 & 159,530 \\
\hline \% Each Category & $4.29 \%$ & $8.53 \%$ & $9.09 \%$ & $9.67 \%$ & $10.14 \%$ & $9.65 \%$ & $9.43 \%$ & $9.86 \%$ & $9.69 \%$ & $9.90 \%$ & $9.76 \%$ & $100.00 \%$ \\
\hline $\begin{array}{l}\text { Market-sensitive } \\
\text { Periodic Reports }\end{array}$ & 2004 & 2005 & 2006 & 2007 & 2008 & 2009 & 2010 & 2011 & 2012 & 2013 & 2014 & Total \\
\hline Pre trading & 462 & 1,032 & 1,189 & 1,313 & 1,410 & 1,431 & 1,490 & 1,568 & 1,604 & 1,598 & 1,830 & 14,927 \\
\hline $10.00-11.00^{*}$ & 163 & 354 & 423 & 519 & 486 & 449 & 410 & 372 & 409 & 338 & 332 & 4,255 \\
\hline $11.00-12.00^{*}$ & 121 & 253 & 260 & 264 & 243 & 265 & 255 & 203 & 227 & 182 & 199 & 2,472 \\
\hline $12.00-13.00^{*}$ & 107 & 238 & 273 & 235 & 287 & 257 & 218 & 221 & 218 & 188 & 170 & 2,412 \\
\hline $13.00-14.00 *$ & 97 & 205 & 226 & 214 & 235 & 206 & 210 & 169 & 173 & 161 & 157 & 2,053 \\
\hline $14.00-15.00^{*}$ & 115 & 218 & 255 & 256 & 234 & 278 & 251 & 239 & 200 & 173 & 170 & 2,389 \\
\hline $15.00-16.00$ & 146 & 312 & 274 & 351 & 327 & 306 & 252 & 282 & 250 & 232 & 229 & 2,961 \\
\hline Post trading & 446 & 1,053 & 936 & 977 & 973 & 978 & 880 & 852 & 847 & 791 & 782 & 9,515 \\
\hline Weekends & 0 & 0 & 0 & 1 & 0 & 0 & 0 & 0 & 0 & 0 & 0 & 1 \\
\hline Trading hours & 749 & 1,580 & 1,711 & 1,839 & 1,812 & 1,761 & 1,596 & 1,486 & 1,477 & 1,274 & 1,257 & 16,542 \\
\hline \% Each Year & $45.20 \%$ & $43.11 \%$ & $44.60 \%$ & $44.53 \%$ & $43.19 \%$ & $42.23 \%$ & $40.24 \%$ & $38.04 \%$ & $37.60 \%$ & $34.78 \%$ & $32.49 \%$ & $40.36 \%$ \\
\hline Off-trading hours & 908 & 2,085 & 2,125 & 2,291 & 2,383 & 2,409 & 2,370 & 2,420 & 2,451 & 2,389 & 2,612 & 24,443 \\
\hline \% Each Year & $54.80 \%$ & $56.89 \%$ & $55.40 \%$ & $55.47 \%$ & $56.81 \%$ & $57.77 \%$ & $59.76 \%$ & $61.96 \%$ & $62.40 \%$ & $65.22 \%$ & $67.51 \%$ & $59.64 \%$ \\
\hline Total & 1,657 & 3,665 & 3,836 & 4,130 & 4,195 & 4,170 & 3,966 & 3,906 & 3,928 & 3,663 & 3,869 & 40,985 \\
\hline \% Each Category & $24.21 \%$ & $26.94 \%$ & $26.46 \%$ & $26.76 \%$ & $25.94 \%$ & $27.08 \%$ & $26.37 \%$ & $24.83 \%$ & $25.42 \%$ & $23.18 \%$ & $24.86 \%$ & $25.69 \%$ \\
\hline \% Total MS & $4.04 \%$ & $8.94 \%$ & $9.36 \%$ & $10.08 \%$ & $10.24 \%$ & $10.17 \%$ & $9.68 \%$ & $9.53 \%$ & $9.58 \%$ & $8.94 \%$ & $9.44 \%$ & $100.00 \%$ \\
\hline
\end{tabular}


Appendix II cont.

\begin{tabular}{|c|c|c|c|c|c|c|c|c|c|c|c|c|}
\hline $\begin{array}{c}\text { Quarterly } \\
\text { Activities Report }\end{array}$ & 2004 & 2005 & 2006 & 2007 & 2008 & 2009 & 2010 & 2011 & 2012 & 2013 & 2014 & Total \\
\hline Pre trading & 118 & 248 & 342 & 494 & 597 & 600 & 649 & 816 & 956 & 1,009 & 1,008 & 6,837 \\
\hline $10.00-11.00^{*}$ & 87 & 188 & 211 & 267 & 309 & 262 & 286 & 326 & 302 & 311 & 277 & 2,826 \\
\hline 11.00-12.00* & 85 & 177 & 203 & 298 & 331 & 272 & 290 & 293 & 264 & 292 & 285 & 2,790 \\
\hline $12.00-13.00^{*}$ & 85 & 181 & 202 & 252 & 330 & 309 & 310 & 315 & 301 & 277 & 323 & 2,885 \\
\hline $13.00-14.00^{*}$ & 75 & 166 & 183 & 245 & 244 & 300 & 293 & 309 & 291 & 280 & 291 & 2,677 \\
\hline $14.00-15.00^{*}$ & 77 & 214 & 217 & 257 & 307 & 303 & 297 & 285 & 324 & 320 & 339 & 2,940 \\
\hline $15.00-16.00$ & 91 & 223 & 245 & 251 & 267 & 305 & 350 & 312 & 355 & 329 & 339 & 3,067 \\
\hline Post trading & 283 & 651 & 741 & 755 & 940 & 974 & 963 & 1,131 & 1,146 & 1,189 & 1,174 & 9,947 \\
\hline Weekends & 0 & 0 & 0 & 1 & 0 & 0 & 0 & 0 & 0 & 0 & 0 & 1 \\
\hline Trading hours & 500 & 1,149 & 1,261 & 1,570 & 1,788 & 1,751 & 1,826 & 1,840 & 1,837 & 1,809 & 1,854 & 17,185 \\
\hline \% Each Year & $55.49 \%$ & $56.10 \%$ & $53.80 \%$ & $55.67 \%$ & $53.77 \%$ & $52.66 \%$ & $53.11 \%$ & $48.59 \%$ & $46.64 \%$ & $45.15 \%$ & $45.94 \%$ & $50.59 \%$ \\
\hline Off-trading hours & 401 & 899 & 1,083 & 1,250 & 1,537 & 1,574 & 1,612 & 1,947 & 2,102 & 2,198 & 2,182 & 16,785 \\
\hline \% Each Year & $44.51 \%$ & $43.90 \%$ & $46.20 \%$ & $44.33 \%$ & $46.23 \%$ & $47.34 \%$ & $46.89 \%$ & $51.41 \%$ & $53.36 \%$ & $54.85 \%$ & $54.06 \%$ & $49.41 \%$ \\
\hline Total & 901 & 2,048 & 2,344 & 2,820 & 3,325 & 3,325 & 3,438 & 3,787 & 3,939 & 4,007 & 4,036 & 33,970 \\
\hline \% Each Category & $2.65 \%$ & $6.03 \%$ & $6.90 \%$ & $8.30 \%$ & $9.79 \%$ & $9.79 \%$ & $10.12 \%$ & $11.15 \%$ & $11.60 \%$ & $11.80 \%$ & $11.88 \%$ & $100.00 \%$ \\
\hline $\begin{array}{c}\text { Market-sensitive Quarterly } \\
\text { Activities Report }\end{array}$ & 2004 & 2005 & 2006 & 2007 & 2008 & 2009 & 2010 & 2011 & 2012 & 2013 & 2014 & Total \\
\hline Pre trading & 112 & 235 & 322 & 472 & 563 & 557 & 603 & 774 & 894 & 922 & 929 & 6,383 \\
\hline $10.00-11.00^{*}$ & 84 & 182 & 202 & 250 & 291 & 245 & 270 & 309 & 290 & 295 & 251 & 2,669 \\
\hline $11.00-12.00^{*}$ & 83 & 168 & 196 & 282 & 313 & 259 & 287 & 277 & 254 & 276 & 260 & 2,655 \\
\hline 12.00-13.00* & 85 & 175 & 191 & 239 & 316 & 290 & 295 & 299 & 286 & 261 & 294 & 2,731 \\
\hline $13.00-14.00^{*}$ & 74 & 159 & 179 & 236 & 232 & 282 & 285 & 296 & 279 & 273 & 271 & 2,566 \\
\hline $14.00-15.00 *$ & 75 & 208 & 213 & 241 & 295 & 282 & 285 & 277 & 311 & 308 & 306 & 2,801 \\
\hline $15.00-16.00$ & 90 & 216 & 238 & 245 & 248 & 289 & 338 & 302 & 340 & 311 & 310 & 2,927 \\
\hline Post trading & 267 & 625 & 702 & 712 & 878 & 917 & 912 & 1,081 & 1,086 & 1,110 & 1,051 & 9,341 \\
\hline Weekends & 0 & 0 & 0 & 1 & 0 & 0 & 0 & 0 & 0 & 0 & 0 & 1 \\
\hline Trading hours & 491 & 1,108 & 1,219 & 1,493 & 1,695 & 1,647 & 1,760 & 1,760 & 1,760 & 1,724 & 1,692 & 16,349 \\
\hline \% Each Year & $56.44 \%$ & $56.30 \%$ & $54.35 \%$ & $55.75 \%$ & $54.05 \%$ & $52.77 \%$ & $53.74 \%$ & $48.69 \%$ & $47.06 \%$ & $45.90 \%$ & $46.08 \%$ & $50.97 \%$ \\
\hline Off-trading hours & 379 & 860 & 1,024 & 1,185 & 1,441 & 1,474 & 1,515 & 1,855 & 1,980 & 2,032 & 1,980 & 15,725 \\
\hline \% Each Year & $43.56 \%$ & $43.70 \%$ & $45.65 \%$ & $44.25 \%$ & $45.95 \%$ & $47.23 \%$ & $46.26 \%$ & $51.31 \%$ & $52.94 \%$ & $54.10 \%$ & $53.92 \%$ & $49.03 \%$ \\
\hline Total & 870 & 1,968 & 2,243 & 2,678 & 3,136 & 3,121 & 3,275 & 3,615 & 3,740 & 3,756 & 3,672 & 32,074 \\
\hline \% Each Category & $96.56 \%$ & $96.09 \%$ & $95.69 \%$ & $94.96 \%$ & $94.32 \%$ & $93.86 \%$ & $95.26 \%$ & $95.46 \%$ & $94.95 \%$ & $93.74 \%$ & $90.98 \%$ & $94.42 \%$ \\
\hline \% Total MS & $2.71 \%$ & $6.14 \%$ & $6.99 \%$ & $8.35 \%$ & $9.78 \%$ & $9.73 \%$ & $10.21 \%$ & $11.27 \%$ & $11.66 \%$ & $11.71 \%$ & $11.45 \%$ & $100.00 \%$ \\
\hline
\end{tabular}


Appendix II cont.

\begin{tabular}{|c|c|c|c|c|c|c|c|c|c|c|c|c|}
\hline $\begin{array}{c}\text { Quarterly } \\
\text { Cash Flow Report }\end{array}$ & 2004 & 2005 & 2006 & 2007 & 2008 & 2009 & 2010 & 2011 & 2012 & 2013 & 2014 & Total \\
\hline Pre trading & 34 & 55 & 94 & 152 & 190 & 190 & 238 & 310 & 366 & 378 & 378 & 2,385 \\
\hline $10.00-11.00^{*}$ & 19 & 38 & 40 & 71 & 89 & 86 & 102 & 121 & 137 & 125 & 104 & 932 \\
\hline $11.00-12.00^{*}$ & 30 & 37 & 49 & 107 & 141 & 99 & 137 & 127 & 141 & 138 & 125 & 1,131 \\
\hline $12.00-13.00^{*}$ & 23 & 50 & 69 & 105 & 155 & 120 & 157 & 147 & 140 & 119 & 139 & 1,224 \\
\hline $13.00-14.00^{*}$ & 21 & 55 & 61 & 91 & 124 & 129 & 145 & 135 & 149 & 149 & 148 & 1,207 \\
\hline $14.00-15.00^{*}$ & 21 & 70 & 82 & 88 & 108 & 141 & 134 & 157 & 168 & 128 & 153 & 1,250 \\
\hline $15.00-16.00$ & 29 & 75 & 85 & 95 & 126 & 128 & 181 & 151 & 169 & 154 & 158 & 1,351 \\
\hline Post trading & 108 & 258 & 308 & 357 & 443 & 497 & 577 & 617 & 595 & 603 & 544 & 4,907 \\
\hline Weekends & 0 & 0 & 0 & 0 & 0 & 0 & 0 & 0 & 0 & 0 & 0 & 0 \\
\hline Trading hours & 143 & 325 & 386 & 557 & 743 & 703 & 856 & 838 & 904 & 813 & 827 & 7,095 \\
\hline \% Each Year & $50.18 \%$ & $50.94 \%$ & $48.98 \%$ & $52.25 \%$ & $54.00 \%$ & $50.58 \%$ & $51.23 \%$ & $47.48 \%$ & $48.47 \%$ & $45.32 \%$ & $47.28 \%$ & $49.32 \%$ \\
\hline Off-trading hours & 142 & 313 & 402 & 509 & 633 & 687 & 815 & 927 & 961 & 981 & 922 & 7,292 \\
\hline \% Each Year & $49.82 \%$ & $49.06 \%$ & $51.02 \%$ & $47.75 \%$ & $46.00 \%$ & $49.42 \%$ & $48.77 \%$ & $52.52 \%$ & $51.53 \%$ & $54.68 \%$ & $52.72 \%$ & $50.68 \%$ \\
\hline Total & 285 & 638 & 788 & 1,066 & 1,376 & 1,390 & 1,671 & 1,765 & 1,865 & 1,794 & 1,749 & 14,387 \\
\hline \% Each Category & $1.98 \%$ & $4.43 \%$ & $5.48 \%$ & $7.41 \%$ & $9.56 \%$ & $9.66 \%$ & $11.61 \%$ & $12.27 \%$ & $12.96 \%$ & $12.47 \%$ & $12.16 \%$ & $100.00 \%$ \\
\hline $\begin{array}{l}\text { Market-sensitive Quarterly } \\
\text { Cash Flow Report }\end{array}$ & 2004 & 2005 & 2006 & 2007 & 2008 & 2009 & 2010 & 2011 & 2012 & 2013 & 2014 & Total \\
\hline Pre trading & 33 & 53 & 90 & 141 & 184 & 183 & 229 & 298 & 354 & 361 & 369 & 2,295 \\
\hline $10.00-11.00^{*}$ & 17 & 37 & 40 & 68 & 84 & 77 & 96 & 120 & 134 & 119 & 97 & 889 \\
\hline $11.00-12.00^{*}$ & 29 & 37 & 46 & 106 & 139 & 98 & 126 & 122 & 138 & 133 & 117 & 1,091 \\
\hline 12.00-13.00* & 23 & 50 & 68 & 100 & 154 & 115 & 147 & 144 & 131 & 114 & 127 & 1,173 \\
\hline $13.00-14.00^{*}$ & 21 & 53 & 60 & 89 & 122 & 129 & 133 & 130 & 145 & 146 & 144 & 1,172 \\
\hline $14.00-15.00^{*}$ & 21 & 69 & 81 & 84 & 106 & 134 & 127 & 147 & 165 & 120 & 145 & 1,199 \\
\hline $15.00-16.00$ & 29 & 72 & 83 & 94 & 120 & 123 & 170 & 147 & 162 & 146 & 150 & 1,296 \\
\hline Post trading & 101 & 249 & 299 & 337 & 426 & 476 & 554 & 588 & 564 & 559 & 491 & 4,644 \\
\hline Weekends & 0 & 0 & 0 & 0 & 0 & 0 & 0 & 0 & 0 & 0 & 0 & 0 \\
\hline Trading hours & 140 & 318 & 378 & 541 & 725 & 676 & 799 & 810 & 875 & 778 & 780 & 6,820 \\
\hline \% Each Year & $51.09 \%$ & $51.29 \%$ & $49.28 \%$ & $53.09 \%$ & $54.31 \%$ & $50.64 \%$ & $50.51 \%$ & $47.76 \%$ & $48.80 \%$ & $45.82 \%$ & $47.56 \%$ & $49.57 \%$ \\
\hline Off-trading hours & 134 & 302 & 389 & 478 & 610 & 659 & 783 & 886 & 918 & 920 & 860 & 6,939 \\
\hline \% Each Year & $48.91 \%$ & $48.71 \%$ & $50.72 \%$ & $46.91 \%$ & $45.69 \%$ & $49.36 \%$ & $49.49 \%$ & $52.24 \%$ & $51.20 \%$ & $54.18 \%$ & $52.44 \%$ & $50.43 \%$ \\
\hline Total & 274 & 620 & 767 & 1,019 & 1,335 & 1,335 & 1,582 & 1,696 & 1,793 & 1,698 & 1,640 & 13,759 \\
\hline$\%$ Each Category & $96.14 \%$ & $97.18 \%$ & $97.34 \%$ & $95.59 \%$ & $97.02 \%$ & $96.04 \%$ & $94.67 \%$ & $96.09 \%$ & $96.14 \%$ & $94.65 \%$ & $93.77 \%$ & $95.63 \%$ \\
\hline \% Total MS & $1.99 \%$ & $4.51 \%$ & $5.57 \%$ & $7.41 \%$ & $9.70 \%$ & $9.70 \%$ & $11.50 \%$ & $12.33 \%$ & $13.03 \%$ & $12.34 \%$ & $11.92 \%$ & $100.00 \%$ \\
\hline
\end{tabular}


Appendix II cont.

\begin{tabular}{|c|c|c|c|c|c|c|c|c|c|c|c|c|}
\hline Issued Capital & 2004 & 2005 & 2006 & 2007 & 2008 & 2009 & 2010 & 2011 & 2012 & 2013 & 2014 & Total \\
\hline Pre trading & 1,731 & 4,037 & 4,699 & 5,850 & 7,045 & 6,797 & 6,071 & 7,026 & 7,034 & 6,009 & 6,155 & 62,454 \\
\hline $10.00-11.00^{*}$ & 722 & 1,606 & 1,818 & 2,273 & 1,861 & 1,959 & 1,786 & 1,807 & 1,641 & 1,467 & 1,486 & 18,426 \\
\hline $11.00-12.00^{*}$ & 776 & 1,507 & 1,719 & 2,028 & 1,553 & 1,653 & 1,434 & 1,474 & 1,450 & 1,182 & 1,186 & 15,962 \\
\hline $12.00-13.00^{*}$ & 708 & 1,443 & 1,567 & 1,859 & 1,517 & 1,648 & 1,424 & 1,409 & 1,367 & 1,218 & 1,156 & 15,316 \\
\hline $13.00-14.00^{*}$ & 527 & 1,177 & 1,448 & 1,586 & 1,225 & 1,384 & 1,363 & 1,254 & 1,162 & 1,042 & 1,017 & 13,185 \\
\hline $14.00-15.00^{*}$ & 703 & 1,335 & 1,530 & 1,860 & 1,454 & 1,531 & 1,611 & 1,587 & 1,376 & 1,218 & 1,272 & 15,477 \\
\hline $15.00-16.00$ & 892 & 1,683 & 1,868 & 2,215 & 1,831 & 1,867 & 1,893 & 1,889 & 1,662 & 1,458 & 1,586 & 18,844 \\
\hline Post trading & 3,051 & 5,632 & 6,193 & 7,466 & 6,480 & 6,859 & 7,207 & 7,638 & 6,729 & 5,745 & 5,541 & 68,541 \\
\hline Weekends & 0 & 0 & 0 & 0 & 3 & 0 & 0 & 0 & 0 & 0 & 0 & 3 \\
\hline Trading hours & 4,328 & 8,751 & 9,950 & 11,821 & 9,441 & 10,042 & 9,511 & 9,420 & 8,658 & 7,585 & 7,703 & 97,210 \\
\hline \% Each Year & $47.51 \%$ & $47.51 \%$ & $47.74 \%$ & $47.03 \%$ & $41.10 \%$ & $42.37 \%$ & $41.74 \%$ & $39.11 \%$ & $38.62 \%$ & $39.22 \%$ & $39.71 \%$ & $42.60 \%$ \\
\hline Off-trading hours & 4,782 & 9,669 & 10,892 & 13,316 & 13,528 & 13,656 & 13,278 & 14,664 & 13,763 & 11,754 & 11,696 & 130,998 \\
\hline$\%$ Each Year & $52.49 \%$ & $52.49 \%$ & $52.26 \%$ & $52.97 \%$ & $58.90 \%$ & $57.63 \%$ & $58.26 \%$ & $60.89 \%$ & $61.38 \%$ & $60.78 \%$ & $60.29 \%$ & $57.40 \%$ \\
\hline Total & 9,110 & 18,420 & 20,842 & 25,137 & 22,969 & 23,698 & 22,789 & 24,084 & 22,421 & 19,339 & 19,399 & 228,208 \\
\hline \% Each Category & $3.99 \%$ & $8.07 \%$ & $9.13 \%$ & $11.01 \%$ & $10.06 \%$ & $10.38 \%$ & $9.99 \%$ & $10.55 \%$ & $9.82 \%$ & $8.47 \%$ & $8.50 \%$ & $100.00 \%$ \\
\hline $\begin{array}{c}\text { Market-sensitive } \\
\text { Issued Capital }\end{array}$ & 2004 & 2005 & 2006 & 2007 & 2008 & 2009 & 2010 & 2011 & 2012 & 2013 & 2014 & Total \\
\hline Pre trading & 233 & 440 & 621 & 795 & 715 & 1,057 & 1,028 & 1,084 & 1,182 & 1,182 & 1,259 & 9,596 \\
\hline $10.00-11.00^{*}$ & 114 & 229 & 269 & 388 & 340 & 437 & 395 & 353 & 305 & 302 & 310 & 3,442 \\
\hline $11.00-12.00^{*}$ & 57 & 127 & 158 & 193 & 157 & 207 & 144 & 134 & 146 & 99 & 129 & 1,551 \\
\hline $12.00-13.00^{*}$ & 58 & 126 & 125 & 162 & 150 & 188 & 153 & 120 & 114 & 106 & 97 & 1,399 \\
\hline $13.00-14.00^{*}$ & 47 & 101 & 114 & 122 & 115 & 150 & 124 & 116 & 109 & 102 & 102 & 1,202 \\
\hline $14.00-15.00^{*}$ & 57 & 112 & 106 & 166 & 137 & 158 & 153 & 116 & 136 & 116 & 116 & 1,373 \\
\hline $15.00-16.00$ & 76 & 146 & 155 & 165 & 164 & 204 & 150 & 165 & 145 & 130 & 131 & 1,631 \\
\hline Post trading & 253 & 512 & 529 & 605 & 557 & 773 & 620 & 563 & 553 & 516 & 566 & 6,047 \\
\hline Weekends & 0 & 0 & 0 & 0 & 1 & 0 & 0 & 0 & 0 & 0 & 0 & 1 \\
\hline Trading hours & 409 & 841 & 927 & 1,196 & 1,063 & 1,344 & 1,119 & 1,004 & 955 & 855 & 885 & 10,598 \\
\hline \% Each Year & $45.70 \%$ & $46.90 \%$ & $44.63 \%$ & $46.07 \%$ & $45.51 \%$ & $42.34 \%$ & $40.44 \%$ & $37.87 \%$ & $35.50 \%$ & $33.49 \%$ & $32.66 \%$ & $40.39 \%$ \\
\hline Off-trading hours & 486 & 952 & 1,150 & 1,400 & 1,273 & 1,830 & 1,648 & 1,647 & 1,735 & 1,698 & 1,825 & 15,644 \\
\hline \% Each Year & $54.30 \%$ & $53.10 \%$ & $55.37 \%$ & $53.93 \%$ & $54.49 \%$ & $57.66 \%$ & $59.56 \%$ & $62.13 \%$ & $64.50 \%$ & $66.51 \%$ & $67.34 \%$ & $59.61 \%$ \\
\hline Total & 895 & 1,793 & 2,077 & 2,596 & 2,336 & 3,174 & 2,767 & 2,651 & 2,690 & 2,553 & 2,710 & 26,242 \\
\hline \% Each Category & $9.82 \%$ & $9.73 \%$ & $9.97 \%$ & $10.33 \%$ & $10.17 \%$ & $13.39 \%$ & $12.14 \%$ & $11.01 \%$ & $12.00 \%$ & $13.20 \%$ & $13.97 \%$ & $11.50 \%$ \\
\hline \% Total MS & $3.41 \%$ & $6.83 \%$ & $7.91 \%$ & $9.89 \%$ & $8.90 \%$ & $12.10 \%$ & $10.54 \%$ & $10.10 \%$ & $10.25 \%$ & $9.73 \%$ & $10.33 \%$ & $100.00 \%$ \\
\hline
\end{tabular}


Appendix II cont.

\begin{tabular}{|c|c|c|c|c|c|c|c|c|c|c|c|c|}
\hline $\begin{array}{c}\text { Asset Acquisition } \\
\text { \& Disposal }\end{array}$ & 2004 & 2005 & 2006 & 2007 & 2008 & 2009 & 2010 & 2011 & 2012 & 2013 & 2014 & Total \\
\hline Pre trading & 257 & 613 & 707 & 1011 & 755 & 654 & 838 & 892 & 843 & 759 & 886 & 8,215 \\
\hline $10.00-11.00^{*}$ & 142 & 297 & 366 & 546 & 413 & 342 & 340 & 363 & 301 & 232 & 241 & 3,583 \\
\hline $11.00-12.00^{*}$ & 99 & 215 & 244 & 368 & 264 & 215 & 217 & 199 & 167 & 133 & 142 & 2,263 \\
\hline $12.00-13.00^{*}$ & 88 & 194 & 188 & 270 & 234 & 180 & 192 & 190 & 174 & 116 & 149 & 1,975 \\
\hline $13.00-14.00^{*}$ & 72 & 146 & 135 & 222 & 171 & 131 & 144 & 128 & 134 & 125 & 124 & 1,532 \\
\hline $14.00-15.00^{*}$ & 105 & 164 & 216 & 218 & 166 & 170 & 160 & 177 & 138 & 112 & 130 & 1,756 \\
\hline $15.00-16.00$ & 84 & 164 & 181 & 242 & 199 & 162 & 130 & 180 & 135 & 136 & 131 & 1,744 \\
\hline Post trading & 235 & 454 & 385 & 463 & 408 & 399 & 394 & 381 & 367 & 302 & 348 & 4,136 \\
\hline Weekends & 0 & 0 & 0 & 0 & 0 & 0 & 0 & 0 & 0 & 0 & 0 & 0 \\
\hline Trading hours & 590 & 1180 & 1330 & 1866 & 1447 & 1200 & 1183 & 1237 & 1049 & 854 & 917 & 12,853 \\
\hline \% Each Year & $54.53 \%$ & $52.51 \%$ & $54.91 \%$ & $55.87 \%$ & $55.44 \%$ & $53.26 \%$ & $48.99 \%$ & $49.28 \%$ & $46.44 \%$ & $44.60 \%$ & $42.63 \%$ & $51.00 \%$ \\
\hline Off-trading hours & 492 & 1,067 & 1,092 & 1,474 & 1,163 & 1,053 & 1,232 & 1,273 & 1,210 & 1,061 & 1,234 & 12,351 \\
\hline$\%$ Each Year & $45.47 \%$ & $47.49 \%$ & $45.09 \%$ & $44.13 \%$ & $44.56 \%$ & $46.74 \%$ & $51.01 \%$ & $50.72 \%$ & $53.56 \%$ & $55.40 \%$ & $57.37 \%$ & $49.00 \%$ \\
\hline Total & 1,082 & 2,247 & 2,422 & 3,340 & 2,610 & 2,253 & 2,415 & 2,510 & 2,259 & 1,915 & 2,151 & 25,204 \\
\hline \% Each Category & $4.29 \%$ & $8.92 \%$ & $9.61 \%$ & $13.25 \%$ & $10.36 \%$ & $8.94 \%$ & $9.58 \%$ & $9.96 \%$ & $8.96 \%$ & $7.60 \%$ & $8.53 \%$ & $100.00 \%$ \\
\hline $\begin{array}{l}\text { Market-sensitive Asset } \\
\text { Acquisition \& Disposal }\end{array}$ & 2004 & 2005 & 2006 & 2007 & 2008 & 2009 & 2010 & 2011 & 2012 & 2013 & 2014 & Total \\
\hline Pre trading & 175 & 435 & 525 & 777 & 581 & 498 & 642 & 700 & 653 & 591 & 665 & 6,242 \\
\hline $10.00-11.00^{*}$ & 98 & 212 & 268 & 432 & 324 & 263 & 263 & 273 & 225 & 164 & 155 & 2,677 \\
\hline $11.00-12.00^{*}$ & 68 & 153 & 177 & 297 & 203 & 153 & 156 & 148 & 117 & 97 & 86 & 1,655 \\
\hline 12.00-13.00* & 56 & 112 & 130 & 216 & 174 & 109 & 138 & 132 & 118 & 73 & 91 & 1,349 \\
\hline $13.00-14.00^{*}$ & 41 & 90 & 88 & 146 & 119 & 83 & 88 & 89 & 88 & 72 & 76 & 980 \\
\hline $14.00-15.00^{*}$ & 58 & 99 & 138 & 148 & 121 & 109 & 99 & 122 & 79 & 65 & 72 & 1,110 \\
\hline $15.00-16.00$ & 47 & 92 & 105 & 172 & 131 & 105 & 74 & 110 & 76 & 80 & 60 & 1,052 \\
\hline Post trading & 127 & 255 & 240 & 300 & 268 & 239 & 246 & 201 & 214 & 177 & 157 & 2,424 \\
\hline Weekends & 0 & 0 & 0 & 0 & 0 & 0 & 0 & 0 & 0 & 0 & 0 & 0 \\
\hline Trading hours & 368 & 758 & 906 & 1411 & 1072 & 822 & 818 & 874 & 703 & 551 & 540 & 8,823 \\
\hline$\%$ Each Year & $54.93 \%$ & $52.35 \%$ & $54.22 \%$ & $56.71 \%$ & $55.80 \%$ & $52.73 \%$ & $47.95 \%$ & $49.24 \%$ & $44.78 \%$ & $41.77 \%$ & $39.65 \%$ & $50.45 \%$ \\
\hline Off-trading hours & 302 & 690 & 765 & 1,077 & 849 & 737 & 888 & 901 & 867 & 768 & 822 & 8,666 \\
\hline$\%$ Each Year & $45.07 \%$ & $47.65 \%$ & $45.78 \%$ & $43.29 \%$ & $44.20 \%$ & $47.27 \%$ & $52.05 \%$ & $50.76 \%$ & $55.22 \%$ & $58.23 \%$ & $60.35 \%$ & $49.55 \%$ \\
\hline Total & 670 & 1,448 & 1,671 & 2,488 & 1,921 & 1,559 & 1,706 & 1,775 & 1,570 & 1,319 & 1,362 & 17,489 \\
\hline$\%$ Each Category & $61.92 \%$ & $64.44 \%$ & $68.99 \%$ & $74.49 \%$ & $73.60 \%$ & $69.20 \%$ & $70.64 \%$ & $70.72 \%$ & $69.50 \%$ & $68.88 \%$ & $63.32 \%$ & $69.39 \%$ \\
\hline \% Total MS & $3.83 \%$ & $8.28 \%$ & $9.55 \%$ & $14.23 \%$ & $10.98 \%$ & $8.91 \%$ & $9.75 \%$ & $10.15 \%$ & $8.98 \%$ & $7.54 \%$ & $7.79 \%$ & $100.00 \%$ \\
\hline
\end{tabular}


Appendix II cont.

\begin{tabular}{|c|c|c|c|c|c|c|c|c|c|c|c|c|}
\hline Notice of Meeting & 2004 & 2005 & 2006 & 2007 & 2008 & 2009 & 2010 & 2011 & 2012 & 2013 & 2014 & Total \\
\hline Pre trading & 311 & 544 & 623 & 798 & 729 & 810 & 760 & 804 & 822 & 849 & 870 & 7,920 \\
\hline $10.00-11.00^{*}$ & 245 & 330 & 354 & 401 & 377 & 381 & 340 & 371 & 351 & 357 & 347 & 3,854 \\
\hline $11.00-12.00^{*}$ & 277 & 386 & 438 & 522 & 475 & 459 & 441 & 476 & 472 & 474 & 448 & 4,868 \\
\hline $12.00-13.00^{*}$ & 322 & 457 & 478 & 569 & 590 & 624 & 649 & 609 & 589 & 594 & 589 & 6,070 \\
\hline $13.00-14.00^{*}$ & 263 & 419 & 477 & 636 & 567 & 589 & 575 & 588 & 540 & 552 & 543 & 5,749 \\
\hline $14.00-15.00^{*}$ & 378 & 468 & 556 & 608 & 616 & 612 & 681 & 690 & 642 & 618 & 611 & 6,480 \\
\hline $15.00-16.00$ & 356 & 604 & 616 & 612 & 636 & 716 & 672 & 752 & 681 & 629 & 643 & 6,917 \\
\hline Post trading & 1,030 & 1,563 & 1,547 & 1,727 & 1,784 & 1,931 & 1,844 & 1,942 & 1,880 & 1,703 & 1,810 & 18,761 \\
\hline Weekends & 0 & 0 & 0 & 0 & 0 & 0 & 0 & 0 & 0 & 0 & 0 & 0 \\
\hline Trading hours & 1,841 & 2,664 & 2,919 & 3,348 & 3,261 & 3,381 & 3,358 & 3,486 & 3,275 & 3,224 & 3,181 & 33,938 \\
\hline \% Each Year & $57.86 \%$ & $55.84 \%$ & $57.36 \%$ & $57.01 \%$ & $56.48 \%$ & $55.23 \%$ & $56.32 \%$ & $55.94 \%$ & $54.79 \%$ & $55.82 \%$ & $54.27 \%$ & $55.99 \%$ \\
\hline Off-trading hours & 1,341 & 2,107 & 2,170 & 2,525 & 2,513 & 2,741 & 2,604 & 2,746 & 2,702 & 2,552 & 2,680 & 26,681 \\
\hline \% Each Year & $42.14 \%$ & $44.16 \%$ & $42.64 \%$ & $42.99 \%$ & $43.52 \%$ & $44.77 \%$ & $43.68 \%$ & $44.06 \%$ & $45.21 \%$ & $44.18 \%$ & $45.73 \%$ & $44.01 \%$ \\
\hline Total & 3,182 & 4,771 & 5,089 & 5,873 & 5,774 & 6,122 & 5,962 & 6,232 & 5,977 & 5,776 & 5,861 & 60,619 \\
\hline$\%$ Each Category & $5.25 \%$ & $7.87 \%$ & $8.40 \%$ & $9.69 \%$ & $9.53 \%$ & $10.10 \%$ & $9.84 \%$ & $10.28 \%$ & $9.86 \%$ & $9.53 \%$ & $9.67 \%$ & $100.00 \%$ \\
\hline $\begin{array}{l}\text { Market-sensitive } \\
\text { Notice of Meeting }\end{array}$ & 2004 & 2005 & 2006 & 2007 & 2008 & 2009 & 2010 & 2011 & 2012 & 2013 & 2014 & Total \\
\hline Pre trading & 4 & 4 & 6 & 4 & 4 & 15 & 6 & 8 & 13 & 9 & 10 & 83 \\
\hline $10.00-11.00^{*}$ & 1 & 7 & 4 & 6 & 7 & 5 & 10 & 8 & 5 & 3 & 0 & 56 \\
\hline 11.00-12.00* & 1 & 1 & 4 & 8 & 4 & 5 & 1 & 4 & 3 & 4 & 3 & 38 \\
\hline $12.00-13.00^{*}$ & 3 & 2 & 0 & 6 & 4 & 2 & 5 & 6 & 1 & 5 & 2 & 36 \\
\hline 13.00-14.00* & 1 & 2 & 5 & 4 & 3 & 7 & 9 & 3 & 2 & 4 & 3 & 43 \\
\hline $14.00-15.00^{*}$ & 2 & 1 & 2 & 5 & 3 & 4 & 7 & 6 & 6 & 1 & 1 & 38 \\
\hline $15.00-16.00$ & 4 & 1 & 2 & 2 & 2 & 8 & 5 & 4 & 2 & 1 & 5 & 36 \\
\hline Post trading & 3 & 5 & 7 & 12 & 13 & 8 & 7 & 10 & 12 & 10 & 13 & 100 \\
\hline Weekends & 0 & 0 & 0 & 0 & 0 & 0 & 0 & 0 & 0 & 0 & 0 & 0 \\
\hline Trading hours & 12 & 14 & 17 & 31 & 23 & 31 & 37 & 31 & 19 & 18 & 14 & 247 \\
\hline \% Each Year & $63.16 \%$ & $60.87 \%$ & $56.67 \%$ & $65.96 \%$ & $57.50 \%$ & $57.41 \%$ & $74.00 \%$ & $63.27 \%$ & $43.18 \%$ & $48.65 \%$ & $37.84 \%$ & $57.44 \%$ \\
\hline Off-trading hours & 7 & 9 & 13 & 16 & 17 & 23 & 13 & 18 & 25 & 19 & 23 & 183 \\
\hline \% Each Year & $36.84 \%$ & $39.13 \%$ & $43.33 \%$ & $34.04 \%$ & $42.50 \%$ & $42.59 \%$ & $26.00 \%$ & $36.73 \%$ & $56.82 \%$ & $51.35 \%$ & $62.16 \%$ & $42.56 \%$ \\
\hline Total & 19 & 23 & 30 & 47 & 40 & 54 & 50 & 49 & 44 & 37 & 37 & 430 \\
\hline \% Each Category & $0.60 \%$ & $0.48 \%$ & $0.59 \%$ & $0.80 \%$ & $0.69 \%$ & $0.88 \%$ & $0.84 \%$ & $0.79 \%$ & $0.74 \%$ & $0.64 \%$ & $0.63 \%$ & $0.71 \%$ \\
\hline$\%$ Total MS & $4.42 \%$ & $5.35 \%$ & $6.98 \%$ & $10.93 \%$ & $9.30 \%$ & $12.56 \%$ & $11.63 \%$ & $11.40 \%$ & $10.23 \%$ & $8.60 \%$ & $8.60 \%$ & $100.00 \%$ \\
\hline
\end{tabular}


Appendix II cont.

\begin{tabular}{|c|c|c|c|c|c|c|c|c|c|c|c|c|}
\hline ASX Announcement & 2004 & 2005 & 2006 & 2007 & 2008 & 2009 & 2010 & 2011 & 2012 & 2013 & 2014 & Total \\
\hline Pre trading & 273 & 545 & 663 & 908 & 791 & 1,227 & 1,178 & 1,175 & 1,317 & 1,392 & 1,480 & 10,949 \\
\hline $10.00-11.00^{*}$ & 208 & 335 & 452 & 658 & 668 & 620 & 661 & 548 & 544 & 498 & 583 & 5,775 \\
\hline 11.00-12.00* & 120 & 144 & 201 & 272 & 213 & 235 & 257 & 238 & 218 & 179 & 241 & 2,318 \\
\hline 12.00-13.00* & 86 & 137 & 172 & 194 & 203 & 167 & 195 & 207 & 178 & 154 & 182 & 1,875 \\
\hline $13.00-14.00^{*}$ & 62 & 112 & 147 & 201 & 155 & 152 & 210 & 165 & 160 & 132 & 135 & 1,631 \\
\hline $14.00-15.00^{*}$ & 86 & 160 & 186 & 226 & 140 & 153 & 185 & 179 & 138 & 144 & 132 & 1,729 \\
\hline $15.00-16.00$ & 89 & 121 & 176 & 232 & 136 & 134 & 161 & 167 & 140 & 123 & 163 & 1,642 \\
\hline Post trading & 378 & 663 & 781 & 911 & 614 & 762 & 777 & 818 & 554 & 448 & 424 & 7,130 \\
\hline Weekends & 0 & 0 & 0 & 0 & 0 & 0 & 0 & 0 & 0 & 0 & 0 & 0 \\
\hline Trading hours & 651 & 1,009 & 1,334 & 1,783 & 1,515 & 1,461 & 1,669 & 1,504 & 1,378 & 1,230 & 1,436 & 14,970 \\
\hline$\%$ Each Year & $50.00 \%$ & $45.51 \%$ & $48.02 \%$ & $49.50 \%$ & $51.88 \%$ & $42.35 \%$ & $46.05 \%$ & $43.01 \%$ & $42.41 \%$ & $40.07 \%$ & $42.99 \%$ & $45.30 \%$ \\
\hline Off-trading hours & 651 & 1,208 & 1,444 & 1,819 & 1,405 & 1,989 & 1,955 & 1,993 & 1,871 & 1,840 & 1,904 & 18,079 \\
\hline \% Each Year & $50.00 \%$ & $54.49 \%$ & $51.98 \%$ & $50.50 \%$ & $48.12 \%$ & $57.65 \%$ & $53.95 \%$ & $56.99 \%$ & $57.59 \%$ & $59.93 \%$ & $57.01 \%$ & $54.70 \%$ \\
\hline Total & 1,302 & 2,217 & 2,778 & 3,602 & 2,920 & 3,450 & 3,624 & 3,497 & 3,249 & 3,070 & 3,340 & 33,049 \\
\hline \% Each Category & $3.94 \%$ & $6.71 \%$ & $8.41 \%$ & $10.90 \%$ & $8.84 \%$ & $10.44 \%$ & $10.97 \%$ & $10.58 \%$ & $9.83 \%$ & $9.29 \%$ & $10.11 \%$ & $100.00 \%$ \\
\hline $\begin{array}{c}\text { Market-sensitive } \\
\text { ASX Announcement }\end{array}$ & 2004 & 2005 & 2006 & 2007 & 2008 & 2009 & 2010 & 2011 & 2012 & 2013 & 2014 & Total \\
\hline Pre trading & 242 & 465 & 589 & 799 & 683 & 1,133 & 1,080 & 1,076 & 1,192 & 1,198 & 1,298 & 9,755 \\
\hline $10.00-11.00^{*}$ & 162 & 256 & 344 & 502 & 473 & 475 & 521 & 415 & 397 & 335 & 423 & 4,303 \\
\hline $11.00-12.00^{*}$ & 70 & 85 & 123 & 181 & 132 & 157 & 161 & 129 & 137 & 101 & 144 & 1,420 \\
\hline $12.00-13.00^{*}$ & 60 & 89 & 116 & 123 & 132 & 107 & 123 & 122 & 107 & 87 & 103 & 1,169 \\
\hline $13.00-14.00^{*}$ & 43 & 78 & 90 & 134 & 99 & 100 & 134 & 102 & 85 & 80 & 86 & 1,031 \\
\hline $14.00-15.00 *$ & 47 & 81 & 108 & 137 & 86 & 96 & 103 & 106 & 85 & 79 & 81 & 1,009 \\
\hline $15.00-16.00$ & 55 & 73 & 108 & 127 & 82 & 88 & 103 & 98 & 74 & 66 & 81 & 955 \\
\hline Post trading & 191 & 270 & 358 & 438 & 258 & 401 & 382 & 365 & 280 & 278 & 247 & 3,468 \\
\hline Weekends & 0 & 0 & 0 & 0 & 0 & 0 & 0 & 0 & 0 & 0 & 0 & 0 \\
\hline Trading hours & 437 & 662 & 889 & 1,204 & 1,004 & 1,023 & 1,145 & 972 & 885 & 748 & 918 & 9,887 \\
\hline \% Each Year & $50.23 \%$ & $47.39 \%$ & $48.42 \%$ & $49.32 \%$ & $51.62 \%$ & $40.01 \%$ & $43.92 \%$ & $40.28 \%$ & $37.55 \%$ & $33.63 \%$ & $37.27 \%$ & $42.78 \%$ \\
\hline Off-trading hours & 433 & 735 & 947 & 1,237 & 941 & 1,534 & 1,462 & 1,441 & 1,472 & 1,476 & 1,545 & 13,223 \\
\hline \% Each Year & $49.77 \%$ & $52.61 \%$ & $51.58 \%$ & $50.68 \%$ & $48.38 \%$ & $59.99 \%$ & $56.08 \%$ & $59.72 \%$ & $62.45 \%$ & $66.37 \%$ & $62.73 \%$ & $57.22 \%$ \\
\hline Total & 870 & 1,397 & 1,836 & 2,441 & 1,945 & 2,557 & 2,607 & 2,413 & 2,357 & 2,224 & 2,463 & 23,110 \\
\hline \% Each Category & $66.82 \%$ & $63.01 \%$ & $66.09 \%$ & $67.77 \%$ & $66.61 \%$ & $74.12 \%$ & $71.94 \%$ & $69.00 \%$ & $72.55 \%$ & $72.44 \%$ & $73.74 \%$ & $69.93 \%$ \\
\hline$\%$ Total MS & $3.76 \%$ & $6.05 \%$ & $7.94 \%$ & $10.56 \%$ & $8.42 \%$ & $11.06 \%$ & $11.28 \%$ & $10.44 \%$ & $10.20 \%$ & $9.62 \%$ & $10.66 \%$ & $100.00 \%$ \\
\hline
\end{tabular}


Appendix II cont.

\begin{tabular}{|c|c|c|c|c|c|c|c|c|c|c|c|c|}
\hline $\begin{array}{c}\text { Distribution } \\
\text { Announcement }\end{array}$ & 2004 & 2005 & 2006 & 2007 & 2008 & 2009 & 2010 & 2011 & 2012 & 2013 & 2014 & Total \\
\hline Pre trading & 96 & 175 & 231 & 309 & 393 & 324 & 315 & 391 & 497 & 794 & 867 & 4,392 \\
\hline $10.00-11.00^{*}$ & 51 & 114 & 144 & 153 & 179 & 178 & 145 & 150 & 240 & 200 & 232 & 1,786 \\
\hline $11.00-12.00^{*}$ & 45 & 101 & 118 & 146 & 188 & 155 & 151 & 177 & 199 & 211 & 195 & 1,686 \\
\hline $12.00-13.00^{*}$ & 31 & 88 & 121 & 155 & 164 & 111 & 151 & 141 & 171 & 203 & 185 & 1,521 \\
\hline $13.00-14.00^{*}$ & 24 & 62 & 71 & 94 & 128 & 102 & 99 & 97 & 118 & 142 & 157 & 1,094 \\
\hline $14.00-15.00^{*}$ & 44 & 112 & 123 & 137 & 201 & 166 & 198 & 182 & 182 & 281 & 282 & 1,908 \\
\hline $15.00-16.00$ & 59 & 126 & 149 & 170 & 226 & 200 & 214 & 212 & 226 & 238 & 327 & 2,147 \\
\hline Post trading & 107 & 313 & 287 & 376 & 402 & 349 & 333 & 362 & 415 & 472 & 635 & 4,051 \\
\hline Weekends & 0 & 0 & 0 & 14 & 0 & 13 & 3 & 4 & 14 & 0 & 0 & 48 \\
\hline Trading hours & 254 & 603 & 726 & 855 & 1,086 & 912 & 958 & 959 & 1,136 & 1,275 & 1,378 & 10,142 \\
\hline \% Each Year & $55.58 \%$ & $55.27 \%$ & $58.36 \%$ & $55.02 \%$ & $57.74 \%$ & $57.07 \%$ & $59.54 \%$ & $55.89 \%$ & $55.09 \%$ & $50.18 \%$ & $47.85 \%$ & $54.43 \%$ \\
\hline Off-trading hours & 203 & 488 & 518 & 699 & 795 & 686 & 651 & 757 & 926 & 1,266 & 1,502 & 8,491 \\
\hline$\%$ Each Year & $44.42 \%$ & $44.73 \%$ & $41.64 \%$ & $44.98 \%$ & $42.26 \%$ & $42.93 \%$ & $40.46 \%$ & $44.11 \%$ & $44.91 \%$ & $49.82 \%$ & $52.15 \%$ & $45.57 \%$ \\
\hline Total & 457 & 1,091 & 1,244 & 1,554 & 1,881 & 1,598 & 1,609 & 1,716 & 2,062 & 2,541 & 2,880 & 18,633 \\
\hline \% Each Category & $2.45 \%$ & $5.86 \%$ & $6.68 \%$ & $8.34 \%$ & $10.09 \%$ & $8.58 \%$ & $8.64 \%$ & $9.21 \%$ & $11.07 \%$ & $13.64 \%$ & $15.46 \%$ & $100.00 \%$ \\
\hline $\begin{array}{c}\text { Market-sensitive Distribution } \\
\text { Announcement }\end{array}$ & 2004 & 2005 & 2006 & 2007 & 2008 & 2009 & 2010 & 2011 & 2012 & 2013 & 2014 & Total \\
\hline Pre trading & 36 & 81 & 100 & 128 & 139 & 109 & 111 & 149 & 234 & 392 & 387 & 1,866 \\
\hline $10.00-11.00^{*}$ & 21 & 54 & 73 & 66 & 62 & 49 & 45 & 65 & 70 & 57 & 71 & 633 \\
\hline 11.00-12.00* & 20 & 43 & 50 & 42 & 43 & 34 & 35 & 54 & 50 & 32 & 33 & 436 \\
\hline 12.00-13.00* & 13 & 33 & 41 & 64 & 47 & 30 & 35 & 29 & 38 & 32 & 45 & 407 \\
\hline $13.00-14.00^{*}$ & 10 & 28 & 33 & 47 & 36 & 30 & 18 & 35 & 18 & 29 & 29 & 313 \\
\hline $14.00-15.00^{*}$ & 15 & 38 & 60 & 38 & 42 & 26 & 35 & 34 & 22 & 39 & 26 & 375 \\
\hline $15.00-16.00$ & 27 & 52 & 67 & 63 & 70 & 39 & 38 & 40 & 33 & 39 & 47 & 515 \\
\hline Post trading & 47 & 129 & 133 & 151 & 106 & 89 & 84 & 104 & 109 & 92 & 113 & 1,157 \\
\hline Weekends & 0 & 0 & 0 & 0 & 0 & 4 & 0 & 2 & 9 & 0 & 0 & 15 \\
\hline Trading hours & 106 & 248 & 324 & 320 & 300 & 208 & 206 & 257 & 231 & 228 & 251 & 2,679 \\
\hline \% Each Year & $56.08 \%$ & $54.15 \%$ & $58.17 \%$ & $53.42 \%$ & $55.05 \%$ & $50.73 \%$ & $51.37 \%$ & $50.20 \%$ & $39.62 \%$ & $32.02 \%$ & $33.42 \%$ & $46.86 \%$ \\
\hline Off-trading hours & 83 & 210 & 233 & 279 & 245 & 202 & 195 & 255 & 352 & 484 & 500 & 3,038 \\
\hline \% Each Year & $43.92 \%$ & $45.85 \%$ & $41.83 \%$ & $46.58 \%$ & $44.95 \%$ & $49.27 \%$ & $48.63 \%$ & $49.80 \%$ & $60.38 \%$ & $67.98 \%$ & $66.58 \%$ & $53.14 \%$ \\
\hline Total & 189 & 458 & 557 & 599 & 545 & 410 & 401 & 512 & 583 & 712 & 751 & 5,717 \\
\hline \% Each Category & $41.36 \%$ & $41.98 \%$ & $44.77 \%$ & $38.55 \%$ & $28.97 \%$ & $25.66 \%$ & $24.92 \%$ & $29.84 \%$ & $28.27 \%$ & $28.02 \%$ & $26.08 \%$ & $30.68 \%$ \\
\hline$\%$ Total MS & $3.31 \%$ & $8.01 \%$ & $9.74 \%$ & $10.48 \%$ & $9.53 \%$ & $7.17 \%$ & $7.01 \%$ & $8.96 \%$ & $10.20 \%$ & $12.45 \%$ & $13.14 \%$ & $100.00 \%$ \\
\hline
\end{tabular}


Appendix II cont.

\begin{tabular}{|c|c|c|c|c|c|c|c|c|c|c|c|c|}
\hline Progress Report & 2004 & 2005 & 2006 & 2007 & 2008 & 2009 & 2010 & 2011 & 2012 & 2013 & 2014 & Total \\
\hline Pre trading & 864 & 2,008 & 2,793 & 3,349 & 4,164 & 3,920 & 5,502 & 6,353 & 6,339 & 5,916 & 5,773 & 46,981 \\
\hline $10.00-11.00^{*}$ & 889 & 1,773 & 2,174 & 2,730 & 3,013 & 2,293 & 2,773 & 2,761 & 2,567 & 2,109 & 1,894 & 24,976 \\
\hline $11.00-12.00^{*}$ & 681 & 1,436 & 1,774 & 2,072 & 2,238 & 1,665 & 1,915 & 1,865 & 1,626 & 1,346 & 1,155 & 17,773 \\
\hline $12.00-13.00^{*}$ & 557 & 1,203 & 1,393 & 1,408 & 1,709 & 1,346 & 1,593 & 1,612 & 1,355 & 1,136 & 968 & 14,280 \\
\hline $13.00-14.00^{*}$ & 404 & 802 & 968 & 1,150 & 1,233 & 1,019 & 1,203 & 1,142 & 945 & 854 & 662 & 10,382 \\
\hline $14.00-15.00^{*}$ & 411 & 813 & 893 & 1,062 & 1,141 & 1,024 & 1,074 & 970 & 838 & 746 & 629 & 9,601 \\
\hline $15.00-16.00$ & 326 & 639 & 718 & 901 & 1,081 & 869 & 948 & 846 & 686 & 643 & 559 & 8,216 \\
\hline Post trading & 458 & 1,023 & 1,253 & 1,533 & 1,876 & 1,699 & 1,741 & 1,678 & 1,493 & 1,369 & 1,263 & 15,386 \\
\hline Weekends & 0 & 0 & 0 & 0 & 2 & 0 & 0 & 0 & 0 & 0 & 0 & 2 \\
\hline Trading hours & 3,268 & 6,666 & 7,920 & 9,323 & 10,415 & 8,216 & 9,506 & 9,196 & 8,017 & 6,834 & 5,867 & 85,228 \\
\hline \% Each Year & $71.20 \%$ & $68.74 \%$ & $66.19 \%$ & $65.63 \%$ & $63.29 \%$ & $59.39 \%$ & $56.76 \%$ & $53.38 \%$ & $50.58 \%$ & $48.40 \%$ & $45.47 \%$ & $57.74 \%$ \\
\hline Off-trading hours & 1,322 & 3,031 & 4,046 & 4,882 & 6,042 & 5,619 & 7,243 & 8,031 & 7,832 & 7,285 & 7,036 & 62,369 \\
\hline$\%$ Each Year & $28.80 \%$ & $31.26 \%$ & $33.81 \%$ & $34.37 \%$ & $36.71 \%$ & $40.61 \%$ & $43.24 \%$ & $46.62 \%$ & $49.42 \%$ & $51.60 \%$ & $54.53 \%$ & $42.26 \%$ \\
\hline Total & 4,590 & 9,697 & 11,966 & 14,205 & 16,457 & 13,835 & 16,749 & 17,227 & 15,849 & 14,119 & 12,903 & 147,597 \\
\hline \% Each Category & $3.11 \%$ & $6.57 \%$ & $8.11 \%$ & $9.62 \%$ & $11.15 \%$ & $9.37 \%$ & $11.35 \%$ & $11.67 \%$ & $10.74 \%$ & $9.57 \%$ & $8.74 \%$ & $100.00 \%$ \\
\hline $\begin{array}{l}\text { Market-sensitive } \\
\text { Progress Report }\end{array}$ & 2004 & 2005 & 2006 & 2007 & 2008 & 2009 & 2010 & 2011 & 2012 & 2013 & 2014 & Total \\
\hline Pre trading & 521 & 1,306 & 1,973 & 2,318 & 2,691 & 2,511 & 3,609 & 4,363 & 4,432 & 4,108 & 3,925 & 31,757 \\
\hline $10.00-11.00^{*}$ & 509 & 1,136 & 1,465 & 1,990 & 2,078 & 1,459 & 1,826 & 1,839 & 1,699 & 1,381 & 1,252 & 16,634 \\
\hline $11.00-12.00^{*}$ & 400 & 878 & 1,143 & 1,374 & 1,364 & 941 & 1,200 & 1,154 & 960 & 785 & 685 & 10,884 \\
\hline $12.00-13.00^{*}$ & 313 & 735 & 885 & 911 & 959 & 736 & 900 & 954 & 791 & 616 & 519 & 8,319 \\
\hline $13.00-14.00 *$ & 195 & 476 & 630 & 719 & 705 & 553 & 683 & 670 & 508 & 486 & 350 & 5,975 \\
\hline $14.00-15.00^{*}$ & 195 & 453 & 563 & 634 & 626 & 510 & 558 & 514 & 427 & 398 & 333 & 5,211 \\
\hline $15.00-16.00$ & 166 & 337 & 417 & 508 & 545 & 405 & 480 & 436 & 332 & 306 & 267 & 4,199 \\
\hline Post trading & 229 & 519 & 690 & 788 & 821 & 724 & 762 & 745 & 675 & 643 & 546 & 7,142 \\
\hline Weekends & 0 & 0 & 0 & 0 & 2 & 0 & 0 & 0 & 0 & 0 & 0 & 2 \\
\hline Trading hours & 1,778 & 4,015 & 5,103 & 6,136 & 6,277 & 4,604 & 5,647 & 5,567 & 4,717 & 3,972 & 3,406 & 51,222 \\
\hline \% Each Year & $70.33 \%$ & $68.75 \%$ & $65.71 \%$ & $66.39 \%$ & $64.11 \%$ & $58.73 \%$ & $56.37 \%$ & $52.15 \%$ & $48.02 \%$ & $45.53 \%$ & $43.24 \%$ & $56.84 \%$ \\
\hline Off-trading hours & 750 & 1,825 & 2,663 & 3,106 & 3,514 & 3,235 & 4,371 & 5,108 & 5,107 & 4,751 & 4,471 & 38,901 \\
\hline$\%$ Each Year & $29.67 \%$ & $31.25 \%$ & $34.29 \%$ & $33.61 \%$ & $35.89 \%$ & $41.27 \%$ & $43.63 \%$ & $47.85 \%$ & $51.98 \%$ & $54.47 \%$ & $56.76 \%$ & $43.16 \%$ \\
\hline Total & 2,528 & 5,840 & 7,766 & 9,242 & 9,791 & 7,839 & 10,018 & 10,675 & 9,824 & 8,723 & 7,877 & 90,123 \\
\hline \% Each Category & $55.08 \%$ & $60.22 \%$ & $64.90 \%$ & $65.06 \%$ & $59.49 \%$ & $56.66 \%$ & $59.81 \%$ & $61.97 \%$ & $61.98 \%$ & $61.78 \%$ & $61.05 \%$ & $61.06 \%$ \\
\hline \% Total MS & $2.81 \%$ & $6.48 \%$ & $8.62 \%$ & $10.25 \%$ & $10.86 \%$ & $8.70 \%$ & $11.12 \%$ & $11.84 \%$ & $10.90 \%$ & $9.68 \%$ & $8.74 \%$ & $100.00 \%$ \\
\hline
\end{tabular}


Appendix II cont.

\begin{tabular}{|c|c|c|c|c|c|c|c|c|c|c|c|c|}
\hline Company Administration & 2004 & 2005 & 2006 & 2007 & 2008 & 2009 & 2010 & 2011 & 2012 & 2013 & 2014 & Total \\
\hline Pre trading & 317 & 731 & 834 & 935 & 1,065 & 943 & 1,106 & 1,293 & 1,352 & 1,519 & 1,507 & 11,602 \\
\hline $10.00-11.00^{*}$ & 241 & 457 & 484 & 621 & 499 & 422 & 523 & 490 & 416 & 507 & 460 & 5,120 \\
\hline $11.00-12.00^{*}$ & 209 & 379 & 454 & 548 & 430 & 360 & 391 & 438 & 376 & 434 & 386 & 4,405 \\
\hline 12.00-13.00* & 169 & 343 & 399 & 480 & 413 & 361 & 423 & 417 & 398 & 393 & 386 & 4,182 \\
\hline $13.00-14.00^{*}$ & 147 & 298 & 324 & 401 & 342 & 328 & 337 & 375 & 329 & 319 & 344 & 3,544 \\
\hline $14.00-15.00^{*}$ & 168 & 368 & 420 & 482 & 390 & 387 & 429 & 429 & 383 & 407 & 362 & 4,225 \\
\hline $15.00-16.00$ & 184 & 414 & 465 & 503 & 466 & 461 & 476 & 477 & 414 & 414 & 456 & 4,730 \\
\hline Post trading & 519 & 1,204 & 1,328 & 1,389 & 1,295 & 1,335 & 1,290 & 1,488 & 1,303 & 1,276 & 1,357 & 13,784 \\
\hline Weekends & 0 & 0 & 0 & 0 & 0 & 0 & 0 & 0 & 0 & 0 & 0 & 0 \\
\hline Trading hours & 1,118 & 2,259 & 2,546 & 3,035 & 2,540 & 2,319 & 2,579 & 2,626 & 2,316 & 2,474 & 2,394 & 26,206 \\
\hline \% Each Year & $57.22 \%$ & $53.86 \%$ & $54.08 \%$ & $56.63 \%$ & $51.84 \%$ & $50.45 \%$ & $51.84 \%$ & $48.57 \%$ & $46.59 \%$ & $46.95 \%$ & $45.53 \%$ & $50.79 \%$ \\
\hline Off-trading hours & 836 & 1,935 & 2,162 & 2,324 & 2,360 & 2,278 & 2,396 & 2,781 & 2,655 & 2,795 & 2,864 & 25,386 \\
\hline \% Each Year & $42.78 \%$ & $46.14 \%$ & $45.92 \%$ & $43.37 \%$ & $48.16 \%$ & $49.55 \%$ & $48.16 \%$ & $51.43 \%$ & $53.41 \%$ & $53.05 \%$ & $54.47 \%$ & $49.21 \%$ \\
\hline Total & 1,954 & 4,194 & 4,708 & 5,359 & 4,900 & 4,597 & 4,975 & 5,407 & 4,971 & 5,269 & 5,258 & 51,592 \\
\hline$\%$ Each Category & $3.79 \%$ & $8.13 \%$ & $9.13 \%$ & $10.39 \%$ & $9.50 \%$ & $8.91 \%$ & $9.64 \%$ & $10.48 \%$ & $9.64 \%$ & $10.21 \%$ & $10.19 \%$ & $100.00 \%$ \\
\hline $\begin{array}{c}\text { Market-sensitive } \\
\text { Company Administration }\end{array}$ & 2004 & 2005 & 2006 & 2007 & 2008 & 2009 & 2010 & 2011 & 2012 & 2013 & 2014 & Total \\
\hline Pre trading & 13 & 20 & 21 & 32 & 32 & 31 & 150 & 216 & 255 & 231 & 208 & 1,209 \\
\hline 10.00-11.00* & 10 & 8 & 7 & 14 & 15 & 9 & 59 & 71 & 60 & 65 & 63 & 381 \\
\hline $11.00-12.00^{*}$ & 5 & 3 & 3 & 8 & 5 & 2 & 24 & 36 & 38 & 32 & 36 & 192 \\
\hline $12.00-13.00^{*}$ & 5 & 5 & 9 & 1 & 7 & 8 & 35 & 47 & 48 & 42 & 33 & 240 \\
\hline $13.00-14.00 *$ & 1 & 8 & 4 & 4 & 1 & 5 & 28 & 32 & 32 & 33 & 28 & 176 \\
\hline $14.00-15.00 *$ & 2 & 3 & 6 & 2 & 2 & 4 & 23 & 33 & 37 & 33 & 26 & 171 \\
\hline $15.00-16.00$ & 1 & 3 & 3 & 2 & 1 & 3 & 22 & 38 & 43 & 34 & 34 & 184 \\
\hline Post trading & 15 & 18 & 11 & 11 & 13 & 19 & 80 & 117 & 133 & 112 & 102 & 631 \\
\hline Weekends & 0 & 0 & 0 & 0 & 0 & 0 & 0 & 0 & 0 & 0 & 0 & 0 \\
\hline Trading hours & 24 & 30 & 32 & 31 & 31 & 31 & 191 & 257 & 258 & 239 & 220 & 1,344 \\
\hline \% Each Year & $46.15 \%$ & $44.12 \%$ & $50.00 \%$ & $41.89 \%$ & $40.79 \%$ & $38.27 \%$ & $45.37 \%$ & $43.56 \%$ & $39.94 \%$ & $41.07 \%$ & $41.51 \%$ & $42.21 \%$ \\
\hline Off-trading hours & 28 & 38 & 32 & 43 & 45 & 50 & 230 & 333 & 388 & 343 & 310 & 1,840 \\
\hline$\%$ Each Year & $53.85 \%$ & $55.88 \%$ & $50.00 \%$ & $58.11 \%$ & $59.21 \%$ & $61.73 \%$ & $54.63 \%$ & $56.44 \%$ & $60.06 \%$ & $58.93 \%$ & $58.49 \%$ & $57.79 \%$ \\
\hline Total & 52 & 68 & 64 & 74 & 76 & 81 & 421 & 590 & 646 & 582 & 530 & 3,184 \\
\hline \% Each Category & $2.66 \%$ & $1.62 \%$ & $1.36 \%$ & $1.38 \%$ & $1.55 \%$ & $1.76 \%$ & $8.46 \%$ & $10.91 \%$ & $13.00 \%$ & $11.05 \%$ & $10.08 \%$ & $6.17 \%$ \\
\hline \% Total MS & $1.63 \%$ & $2.14 \%$ & $2.01 \%$ & $2.32 \%$ & $2.39 \%$ & $2.54 \%$ & $13.22 \%$ & $18.53 \%$ & $20.29 \%$ & $18.28 \%$ & $16.65 \%$ & $100.00 \%$ \\
\hline
\end{tabular}


Appendix II cont.

\begin{tabular}{|c|c|c|c|c|c|c|c|c|c|c|c|c|}
\hline Notice of Call & 2004 & 2005 & 2006 & 2007 & 2008 & 2009 & 2010 & 2011 & 2012 & 2013 & 2014 & Total \\
\hline Pre trading & 0 & 0 & 3 & 1 & 1 & 1 & 1 & 1 & 0 & 2 & 1 & 11 \\
\hline $10.00-11.00^{*}$ & 0 & 0 & 1 & 0 & 1 & 1 & 0 & 0 & 0 & 0 & 0 & 3 \\
\hline $11.00-12.00^{*}$ & 0 & 0 & 1 & 1 & 0 & 0 & 0 & 0 & 0 & 0 & 0 & 2 \\
\hline $12.00-13.00^{*}$ & 0 & 0 & 5 & 0 & 0 & 0 & 0 & 0 & 0 & 0 & 0 & 5 \\
\hline $13.00-14.00^{*}$ & 0 & 0 & 0 & 1 & 0 & 0 & 0 & 0 & 0 & 0 & 0 & 1 \\
\hline $14.00-15.00^{*}$ & 0 & 0 & 0 & 1 & 0 & 1 & 0 & 1 & 0 & 0 & 0 & 3 \\
\hline $15.00-16.00$ & 0 & 0 & 0 & 2 & 1 & 0 & 0 & 1 & 0 & 0 & 0 & 4 \\
\hline Post trading & 0 & 0 & 0 & 2 & 4 & 7 & 2 & 0 & 1 & 2 & 0 & 18 \\
\hline Weekends & 0 & 0 & 0 & 0 & 0 & 0 & 0 & 0 & 0 & 0 & 0 & 0 \\
\hline Trading hours & 0 & 0 & 7 & 5 & 2 & 2 & 0 & 2 & 0 & 0 & 0 & 18 \\
\hline \% Each Year & - & - & $70.00 \%$ & $62.50 \%$ & $28.57 \%$ & $20.00 \%$ & $0.00 \%$ & $66.67 \%$ & $0.00 \%$ & $0.00 \%$ & $0.00 \%$ & $38.30 \%$ \\
\hline Off-trading hours & 0 & 0 & 3 & 3 & 5 & 8 & 3 & 1 & 1 & 4 & 1 & 29 \\
\hline \% Each Year & - & - & $30.00 \%$ & $37.50 \%$ & $71.43 \%$ & $80.00 \%$ & $100.00 \%$ & $33.33 \%$ & $100.00 \%$ & $100.00 \%$ & $100.00 \%$ & $61.70 \%$ \\
\hline Total & 0 & 0 & 10 & 8 & 7 & 10 & 3 & 3 & 1 & 4 & 1 & 47 \\
\hline$\%$ Each Category & $0.00 \%$ & $0.00 \%$ & $21.28 \%$ & $17.02 \%$ & $14.89 \%$ & $21.28 \%$ & $6.38 \%$ & $6.38 \%$ & $2.13 \%$ & $8.51 \%$ & $2.13 \%$ & $100.00 \%$ \\
\hline $\begin{array}{c}\text { Market-sensitive } \\
\text { Notice of Call }\end{array}$ & 2004 & 2005 & 2006 & 2007 & 2008 & 2009 & 2010 & 2011 & 2012 & 2013 & 2014 & Total \\
\hline Pre trading & 0 & 0 & 0 & 0 & 1 & 1 & 0 & 1 & 0 & 1 & 0 & 4 \\
\hline $10.00-11.00^{*}$ & 0 & 0 & 0 & 0 & 0 & 0 & 0 & 0 & 0 & 0 & 0 & 0 \\
\hline $11.00-12.00^{*}$ & 0 & 0 & 0 & 0 & 0 & 0 & 0 & 0 & 0 & 0 & 0 & 0 \\
\hline $12.00-13.00^{*}$ & 0 & 0 & 0 & 0 & 0 & 0 & 0 & 0 & 0 & 0 & 0 & 0 \\
\hline $13.00-14.00^{*}$ & 0 & 0 & 0 & 0 & 0 & 0 & 0 & 0 & 0 & 0 & 0 & 0 \\
\hline $14.00-15.00^{*}$ & 0 & 0 & 0 & 0 & 0 & 0 & 0 & 1 & 0 & 0 & 0 & 1 \\
\hline $15.00-16.00$ & 0 & 0 & 0 & 0 & 0 & 0 & 0 & 0 & 0 & 0 & 0 & 0 \\
\hline Post trading & 0 & 0 & 0 & 0 & 1 & 3 & 0 & 0 & 0 & 0 & 0 & 4 \\
\hline Weekends & 0 & 0 & 0 & 0 & 0 & 0 & 0 & 0 & 0 & 0 & 0 & 0 \\
\hline Trading hours & 0 & 0 & 0 & 0 & 0 & 0 & 0 & 1 & 0 & 0 & 0 & 1 \\
\hline \% Each Year & - & - & - & - & $0.00 \%$ & $0.00 \%$ & - & $50.00 \%$ & - & $0.00 \%$ & - & $11.11 \%$ \\
\hline Off-trading hours & 0 & 0 & 0 & 0 & 2 & 4 & 0 & 1 & 0 & 1 & 0 & 8 \\
\hline \% Each Year & - & - & - & - & $100.00 \%$ & $100.00 \%$ & - & $50.00 \%$ & - & $100.00 \%$ & - & $88.89 \%$ \\
\hline Total & 0 & 0 & 0 & 0 & 2 & 4 & 0 & 2 & 0 & 1 & 0 & 9 \\
\hline \% Each Category & - & - & $0.00 \%$ & $0.00 \%$ & $28.57 \%$ & $40.00 \%$ & $0.00 \%$ & $66.67 \%$ & $0.00 \%$ & $25.00 \%$ & $0.00 \%$ & $19.15 \%$ \\
\hline \% Total MS & $0.00 \%$ & $0.00 \%$ & $0.00 \%$ & $0.00 \%$ & $22.22 \%$ & $44.44 \%$ & $0.00 \%$ & $22.22 \%$ & $0.00 \%$ & $11.11 \%$ & $0.00 \%$ & $100.00 \%$ \\
\hline
\end{tabular}


Appendix II cont.

\begin{tabular}{|c|c|c|c|c|c|c|c|c|c|c|c|c|}
\hline Other & 2004 & 2005 & 2006 & 2007 & 2008 & 2009 & 2010 & 2011 & 2012 & 2013 & 2014 & Total \\
\hline Pre trading & 720 & 1,359 & 1,112 & 946 & 636 & 538 & 805 & 704 & 363 & 196 & 195 & 7,574 \\
\hline $10.00-11.00^{*}$ & 261 & 521 & 427 & 351 & 80 & 66 & 201 & 93 & 55 & 89 & 102 & 2,246 \\
\hline $11.00-12.00^{*}$ & 273 & 424 & 322 & 316 & 75 & 23 & 178 & 92 & 37 & 70 & 63 & 1,873 \\
\hline $12.00-13.00^{*}$ & 226 & 376 & 390 & 298 & 48 & 31 & 184 & 68 & 42 & 64 & 56 & 1,783 \\
\hline $13.00-14.00^{*}$ & 166 & 299 & 267 & 270 & 48 & 17 & 185 & 46 & 38 & 51 & 53 & 1,440 \\
\hline $14.00-15.00^{*}$ & 172 & 305 & 209 & 169 & 43 & 30 & 183 & 77 & 32 & 59 & 50 & 1,329 \\
\hline $15.00-16.00$ & 187 & 323 & 182 & 169 & 48 & 27 & 209 & 79 & 41 & 44 & 54 & 1,363 \\
\hline Post trading & 562 & 872 & 640 & 532 & 94 & 53 & 458 & 276 & 103 & 168 & 165 & 3,923 \\
\hline Weekends & 0 & 22 & 13 & 50 & 28 & 40 & 4 & 9 & 27 & 1 & 1 & 195 \\
\hline Trading hours & 1,285 & 2,248 & 1,797 & 1,573 & 342 & 194 & 1,140 & 455 & 245 & 377 & 378 & 10,034 \\
\hline \% Each Year & $50.06 \%$ & $49.94 \%$ & $50.45 \%$ & $50.73 \%$ & $31.09 \%$ & $23.52 \%$ & $47.36 \%$ & $31.51 \%$ & $33.20 \%$ & $50.81 \%$ & $51.15 \%$ & $46.18 \%$ \\
\hline Off-trading hours & 1,282 & 2,253 & 1,765 & 1,528 & 758 & 631 & 1,267 & 989 & 493 & 365 & 361 & 11,692 \\
\hline$\%$ Each Year & $49.94 \%$ & $50.06 \%$ & $49.55 \%$ & $49.27 \%$ & $68.91 \%$ & $76.48 \%$ & $52.64 \%$ & $68.49 \%$ & $66.80 \%$ & $49.19 \%$ & $48.85 \%$ & $53.82 \%$ \\
\hline Total & 2,567 & 4,501 & 3,562 & 3,101 & 1,100 & 825 & 2,407 & 1,444 & 738 & 742 & 739 & 21,726 \\
\hline \% Each Category & $11.82 \%$ & $20.72 \%$ & $16.40 \%$ & $14.27 \%$ & $5.06 \%$ & $3.80 \%$ & $11.08 \%$ & $6.65 \%$ & $3.40 \%$ & $3.42 \%$ & $3.40 \%$ & $100.00 \%$ \\
\hline $\begin{array}{c}\text { Market-sensitive } \\
\text { Other }\end{array}$ & 2004 & 2005 & 2006 & 2007 & 2008 & 2009 & 2010 & 2011 & 2012 & 2013 & 2014 & Total \\
\hline Pre trading & 132 & 206 & 97 & 71 & 58 & 29 & 25 & 22 & 31 & 63 & 55 & 789 \\
\hline $10.00-11.00^{*}$ & 70 & 125 & 70 & 60 & 41 & 47 & 10 & 15 & 19 & 27 & 31 & 515 \\
\hline $11.00-12.00^{*}$ & 71 & 71 & 46 & 41 & 46 & 15 & 5 & 13 & 10 & 10 & 13 & 341 \\
\hline $12.00-13.00^{*}$ & 44 & 69 & 47 & 30 & 22 & 16 & 9 & 12 & 14 & 13 & 15 & 291 \\
\hline $13.00-14.00^{*}$ & 29 & 59 & 33 & 14 & 27 & 6 & 4 & 2 & 10 & 6 & 10 & 200 \\
\hline $14.00-15.00^{*}$ & 40 & 82 & 36 & 26 & 24 & 18 & 2 & 6 & 4 & 13 & 11 & 262 \\
\hline $15.00-16.00$ & 52 & 63 & 17 & 11 & 24 & 10 & 6 & 5 & 6 & 11 & 7 & 212 \\
\hline Post trading & 81 & 101 & 51 & 36 & 24 & 13 & 13 & 14 & 15 & 35 & 32 & 415 \\
\hline Weekends & 0 & 1 & 0 & 17 & 0 & 8 & 0 & 0 & 11 & 1 & 1 & 39 \\
\hline Trading hours & 306 & 469 & 249 & 182 & 184 & 112 & 36 & 53 & 63 & 80 & 87 & 1,821 \\
\hline \% Each Year & $58.96 \%$ & $60.36 \%$ & $62.72 \%$ & $59.48 \%$ & $69.17 \%$ & $69.14 \%$ & $48.65 \%$ & $59.55 \%$ & $52.50 \%$ & $44.69 \%$ & $49.71 \%$ & $59.43 \%$ \\
\hline Off-trading hours & 213 & 308 & 148 & 124 & 82 & 50 & 38 & 36 & 57 & 99 & 88 & 1,243 \\
\hline \% Each Year & $41.04 \%$ & $39.64 \%$ & $37.28 \%$ & $40.52 \%$ & $30.83 \%$ & $30.86 \%$ & $51.35 \%$ & $40.45 \%$ & $47.50 \%$ & $55.31 \%$ & $50.29 \%$ & $40.57 \%$ \\
\hline Total & 519 & 777 & 397 & 306 & 266 & 162 & 74 & 89 & 120 & 179 & 175 & 3,064 \\
\hline \% Each Category & $20.22 \%$ & $17.26 \%$ & $11.15 \%$ & $9.87 \%$ & $24.18 \%$ & $19.64 \%$ & $3.07 \%$ & $6.16 \%$ & $16.26 \%$ & $24.12 \%$ & $23.68 \%$ & $14.10 \%$ \\
\hline$\%$ Total MS & $16.94 \%$ & $25.36 \%$ & $12.96 \%$ & $9.99 \%$ & $8.68 \%$ & $5.29 \%$ & $2.42 \%$ & $2.90 \%$ & $3.92 \%$ & $5.84 \%$ & $5.71 \%$ & $100.00 \%$ \\
\hline
\end{tabular}


Appendix II cont.

\begin{tabular}{|c|c|c|c|c|c|c|c|c|c|c|c|c|}
\hline Chairman's Address & 2004 & 2005 & 2006 & 2007 & 2008 & 2009 & 2010 & 2011 & 2012 & 2013 & 2014 & Total \\
\hline Pre trading & 202 & 301 & 363 & 387 & 424 & 381 & 368 & 431 & 374 & 394 & 408 & 4,033 \\
\hline $10.00-11.00^{*}$ & 207 & 312 & 313 & 319 & 374 & 333 & 325 & 387 & 303 & 306 & 343 & 3,522 \\
\hline $11.00-12.00 *$ & 129 & 164 & 189 & 250 & 294 & 257 & 244 & 250 & 208 & 216 & 209 & 2,410 \\
\hline 12.00-13.00* & 91 & 127 & 137 & 130 & 186 & 187 & 186 & 179 & 187 & 171 & 169 & 1,750 \\
\hline $13.00-14.00^{*}$ & 79 & 131 & 113 & 138 & 162 & 148 & 157 & 182 & 143 & 148 & 139 & 1,540 \\
\hline $14.00-15.00^{*}$ & 88 & 140 & 154 & 154 & 166 & 148 & 133 & 135 & 116 & 98 & 90 & 1,422 \\
\hline $15.00-16.00$ & 61 & 100 & 80 & 85 & 145 & 100 & 108 & 88 & 66 & 55 & 80 & 968 \\
\hline Post trading & 84 & 184 & 128 & 167 & 216 & 181 & 189 & 191 & 148 & 120 & 94 & 1,702 \\
\hline Weekends & 0 & 0 & 0 & 0 & 0 & 0 & 0 & 0 & 0 & 0 & 0 & 0 \\
\hline Trading hours & 655 & 974 & 986 & 1,076 & 1,327 & 1,173 & 1,153 & 1,221 & 1,023 & 994 & 1,030 & 11,612 \\
\hline \% Each Year & $69.61 \%$ & $66.76 \%$ & $66.76 \%$ & $66.01 \%$ & $67.46 \%$ & $67.61 \%$ & $67.43 \%$ & $66.25 \%$ & $66.21 \%$ & $65.92 \%$ & $67.23 \%$ & $66.94 \%$ \\
\hline Off-trading hours & 286 & 485 & 491 & 554 & 640 & 562 & 557 & 622 & 522 & 514 & 502 & 5,735 \\
\hline$\%$ Each Year & $30.39 \%$ & $33.24 \%$ & $33.24 \%$ & $33.99 \%$ & $32.54 \%$ & $32.39 \%$ & $32.57 \%$ & $33.75 \%$ & $33.79 \%$ & $34.08 \%$ & $32.77 \%$ & $33.06 \%$ \\
\hline Total & 941 & 1,459 & 1,477 & 1,630 & 1,967 & 1,735 & 1,710 & 1,843 & 1,545 & 1,508 & 1,532 & 17,347 \\
\hline \% Each Category & $5.42 \%$ & $8.41 \%$ & $8.51 \%$ & $9.40 \%$ & $11.34 \%$ & $10.00 \%$ & $9.86 \%$ & $10.62 \%$ & $8.91 \%$ & $8.69 \%$ & $8.83 \%$ & $100.00 \%$ \\
\hline $\begin{array}{c}\text { Market-sensitive } \\
\text { Chairman's Address }\end{array}$ & 2004 & 2005 & 2006 & 2007 & 2008 & 2009 & 2010 & 2011 & 2012 & 2013 & 2014 & Total \\
\hline Pre trading & 24 & 22 & 28 & 29 & 45 & 25 & 27 & 47 & 29 & 27 & 14 & 317 \\
\hline $10.00-11.00^{*}$ & 34 & 43 & 33 & 31 & 57 & 29 & 25 & 43 & 36 & 16 & 8 & 355 \\
\hline $11.00-12.00^{*}$ & 16 & 16 & 15 & 36 & 39 & 13 & 16 & 14 & 9 & 3 & 2 & 179 \\
\hline $12.00-13.00^{*}$ & 5 & 10 & 9 & 8 & 10 & 12 & 8 & 12 & 11 & 6 & 0 & 91 \\
\hline $13.00-14.00^{*}$ & 10 & 10 & 9 & 9 & 11 & 11 & 9 & 13 & 10 & 6 & 3 & 101 \\
\hline $14.00-15.00^{*}$ & 13 & 19 & 14 & 19 & 12 & 6 & 10 & 11 & 7 & 4 & 1 & 116 \\
\hline $15.00-16.00$ & 1 & 6 & 5 & 7 & 8 & 6 & 6 & 7 & 6 & 2 & 1 & 55 \\
\hline Post trading & 3 & 9 & 7 & 10 & 10 & 9 & 8 & 8 & 5 & 3 & 1 & 73 \\
\hline Weekends & 0 & 0 & 0 & 0 & 0 & 0 & 0 & 0 & 0 & 0 & 0 & 0 \\
\hline Trading hours & 79 & 104 & 85 & 110 & 137 & 77 & 74 & 100 & 79 & 37 & 15 & 897 \\
\hline \% Each Year & $74.53 \%$ & $77.04 \%$ & $70.83 \%$ & $73.83 \%$ & $71.35 \%$ & $69.37 \%$ & $67.89 \%$ & $64.52 \%$ & $69.91 \%$ & $55.22 \%$ & $50.00 \%$ & $69.70 \%$ \\
\hline Off-trading hours & 27 & 31 & 35 & 39 & 55 & 34 & 35 & 55 & 34 & 30 & 15 & 390 \\
\hline \% Each Year & $25.47 \%$ & $22.96 \%$ & $29.17 \%$ & $26.17 \%$ & $28.65 \%$ & $30.63 \%$ & $32.11 \%$ & $35.48 \%$ & $30.09 \%$ & $44.78 \%$ & $50.00 \%$ & $30.30 \%$ \\
\hline Total & 106 & 135 & 120 & 149 & 192 & 111 & 109 & 155 & 113 & 67 & 30 & 1,287 \\
\hline \% Each Category & $11.26 \%$ & $9.25 \%$ & $8.12 \%$ & $9.14 \%$ & $9.76 \%$ & $6.40 \%$ & $6.37 \%$ & $8.41 \%$ & $7.31 \%$ & $4.44 \%$ & $1.96 \%$ & $7.42 \%$ \\
\hline \% Total MS & $8.24 \%$ & $10.49 \%$ & $9.32 \%$ & $11.58 \%$ & $14.92 \%$ & $8.62 \%$ & $8.47 \%$ & $12.04 \%$ & $8.78 \%$ & $5.21 \%$ & $2.33 \%$ & $100.00 \%$ \\
\hline
\end{tabular}


Appendix II cont.

\begin{tabular}{|c|c|c|c|c|c|c|c|c|c|c|c|c|}
\hline Letter to Shareholders & 2004 & 2005 & 2006 & 2007 & 2008 & 2009 & 2010 & 2011 & 2012 & 2013 & 2014 & Total \\
\hline Pre trading & 44 & 72 & 71 & 93 & 73 & 64 & 39 & 51 & 43 & 58 & 35 & 643 \\
\hline $10.00-11.00^{*}$ & 31 & 60 & 47 & 64 & 39 & 17 & 20 & 15 & 13 & 23 & 12 & 341 \\
\hline $11.00-12.00^{*}$ & 28 & 36 & 40 & 72 & 26 & 18 & 21 & 11 & 18 & 16 & 22 & 308 \\
\hline $12.00-13.00^{*}$ & 33 & 49 & 52 & 60 & 29 & 17 & 23 & 14 & 12 & 19 & 17 & 325 \\
\hline $13.00-14.00^{*}$ & 25 & 33 & 42 & 42 & 25 & 16 & 13 & 13 & 4 & 21 & 12 & 246 \\
\hline $14.00-15.00^{*}$ & 15 & 51 & 38 & 37 & 27 & 14 & 20 & 13 & 16 & 15 & 14 & 260 \\
\hline $15.00-16.00$ & 31 & 51 & 38 & 62 & 30 & 26 & 22 & 21 & 16 & 22 & 25 & 344 \\
\hline Post trading & 96 & 190 & 198 & 207 & 93 & 79 & 76 & 45 & 57 & 69 & 46 & 1,156 \\
\hline Weekends & 0 & 0 & 0 & 0 & 0 & 0 & 0 & 0 & 0 & 0 & 0 & 0 \\
\hline Trading hours & 163 & 280 & 257 & 337 & 176 & 108 & 119 & 87 & 79 & 116 & 102 & 1,824 \\
\hline \% Each Year & $53.80 \%$ & $51.66 \%$ & $48.86 \%$ & $52.90 \%$ & $51.46 \%$ & $43.03 \%$ & $50.85 \%$ & $47.54 \%$ & $44.13 \%$ & $47.74 \%$ & $55.74 \%$ & $50.35 \%$ \\
\hline Off-trading hours & 140 & 262 & 269 & 300 & 166 & 143 & 115 & 96 & 100 & 127 & 81 & 1,799 \\
\hline \% Each Year & $46.20 \%$ & $48.34 \%$ & $51.14 \%$ & $47.10 \%$ & $48.54 \%$ & $56.97 \%$ & $49.15 \%$ & $52.46 \%$ & $55.87 \%$ & $52.26 \%$ & $44.26 \%$ & $49.65 \%$ \\
\hline Total & 303 & 542 & 526 & 637 & 342 & 251 & 234 & 183 & 179 & 243 & 183 & 3,623 \\
\hline \% Each Category & $8.36 \%$ & $14.96 \%$ & $14.52 \%$ & $17.58 \%$ & $9.44 \%$ & $6.93 \%$ & $6.46 \%$ & $5.05 \%$ & $4.94 \%$ & $6.71 \%$ & $5.05 \%$ & $100.00 \%$ \\
\hline $\begin{array}{c}\text { Market-sensitive } \\
\text { Letter to Shareholders }\end{array}$ & 2004 & 2005 & 2006 & 2007 & 2008 & 2009 & 2010 & 2011 & 2012 & 2013 & 2014 & Total \\
\hline Pre trading & 0 & 0 & 1 & 0 & 0 & 0 & 2 & 0 & 2 & 1 & 1 & 7 \\
\hline $10.00-11.00^{*}$ & 0 & 0 & 1 & 2 & 2 & 0 & 1 & 0 & 0 & 0 & 1 & 7 \\
\hline $11.00-12.00^{*}$ & 0 & 0 & 0 & 0 & 0 & 0 & 1 & 1 & 0 & 1 & 1 & 4 \\
\hline $12.00-13.00^{*}$ & 0 & 1 & 0 & 1 & 1 & 0 & 2 & 1 & 0 & 0 & 1 & 7 \\
\hline 13.00-14.00* & 0 & 0 & 0 & 0 & 1 & 0 & 0 & 0 & 0 & 0 & 0 & 1 \\
\hline $14.00-15.00^{*}$ & 0 & 0 & 3 & 0 & 0 & 0 & 0 & 0 & 0 & 0 & 0 & 3 \\
\hline $15.00-16.00$ & 0 & 0 & 0 & 2 & 0 & 0 & 0 & 1 & 0 & 0 & 1 & 4 \\
\hline Post trading & 1 & 2 & 2 & 1 & 1 & 1 & 0 & 1 & 1 & 0 & 1 & 11 \\
\hline Weekends & 0 & 0 & 0 & 0 & 0 & 0 & 0 & 0 & 0 & 0 & 0 & 0 \\
\hline Trading hours & 0 & 1 & 4 & 5 & 4 & 0 & 4 & 3 & 0 & 1 & 4 & 26 \\
\hline \% Each Year & $0.00 \%$ & $33.33 \%$ & $57.14 \%$ & $83.33 \%$ & $80.00 \%$ & $0.00 \%$ & $66.67 \%$ & $75.00 \%$ & $0.00 \%$ & $50.00 \%$ & $66.67 \%$ & $59.09 \%$ \\
\hline Off-trading hours & 1 & 2 & 3 & 1 & 1 & 1 & 2 & 1 & 3 & 1 & 2 & 18 \\
\hline \% Each Year & $100.00 \%$ & $66.67 \%$ & $42.86 \%$ & $16.67 \%$ & $20.00 \%$ & $100.00 \%$ & $33.33 \%$ & $25.00 \%$ & $100.00 \%$ & $50.00 \%$ & $33.33 \%$ & $40.91 \%$ \\
\hline Total & 1 & 3 & 7 & 6 & 5 & 1 & 6 & 4 & 3 & 2 & 6 & 44 \\
\hline$\%$ Each Category & $0.33 \%$ & $0.55 \%$ & $1.33 \%$ & $0.94 \%$ & $1.46 \%$ & $0.40 \%$ & $2.56 \%$ & $2.19 \%$ & $1.68 \%$ & $0.82 \%$ & $3.28 \%$ & $1.21 \%$ \\
\hline \% Total MS & $2.27 \%$ & $6.82 \%$ & $15.91 \%$ & $13.64 \%$ & $11.36 \%$ & $2.27 \%$ & $13.64 \%$ & $9.09 \%$ & $6.82 \%$ & $4.55 \%$ & $13.64 \%$ & $100.00 \%$ \\
\hline
\end{tabular}


Appendix II cont.

\begin{tabular}{|c|c|c|c|c|c|c|c|c|c|c|c|c|}
\hline ASX Query & 2004 & 2005 & 2006 & 2007 & 2008 & 2009 & 2010 & 2011 & 2012 & 2013 & 2014 & Total \\
\hline Pre trading & 30 & 93 & 170 & 152 & 183 & 325 & 210 & 202 & 212 & 156 & 173 & 1,906 \\
\hline $10.00-11.00^{*}$ & 10 & 39 & 57 & 86 & 98 & 141 & 82 & 62 & 40 & 34 & 33 & 682 \\
\hline $11.00-12.00^{*}$ & 10 & 13 & 10 & 17 & 27 & 63 & 44 & 38 & 34 & 25 & 22 & 303 \\
\hline $12.00-13.00^{*}$ & 8 & 14 & 28 & 23 & 46 & 71 & 56 & 49 & 54 & 41 & 36 & 426 \\
\hline $13.00-14.00^{*}$ & 8 & 20 & 42 & 44 & 64 & 123 & 102 & 70 & 63 & 58 & 64 & 658 \\
\hline $14.00-15.00^{*}$ & 12 & 32 & 45 & 64 & 59 & 103 & 84 & 80 & 68 & 74 & 83 & 704 \\
\hline $15.00-16.00$ & 10 & 27 & 60 & 39 & 51 & 104 & 77 & 70 & 82 & 86 & 71 & 677 \\
\hline Post trading & 68 & 138 & 160 & 176 & 195 & 387 & 303 & 266 & 258 & 204 & 209 & 2,364 \\
\hline Weekends & 0 & 0 & 0 & 0 & 0 & 0 & 0 & 0 & 0 & 0 & 0 & 0 \\
\hline Trading hours & 58 & 145 & 242 & 273 & 345 & 605 & 445 & 369 & 341 & 318 & 309 & 3,450 \\
\hline \% Each Year & $37.18 \%$ & $38.56 \%$ & $42.31 \%$ & $45.42 \%$ & $47.72 \%$ & $45.94 \%$ & $46.45 \%$ & $44.09 \%$ & $42.05 \%$ & $46.90 \%$ & $44.72 \%$ & $44.69 \%$ \\
\hline Off-trading hours & 98 & 231 & 330 & 328 & 378 & 712 & 513 & 468 & 470 & 360 & 382 & 4,270 \\
\hline \% Each Year & $62.82 \%$ & $61.44 \%$ & $57.69 \%$ & $54.58 \%$ & $52.28 \%$ & $54.06 \%$ & $53.55 \%$ & $55.91 \%$ & $57.95 \%$ & $53.10 \%$ & $55.28 \%$ & $55.31 \%$ \\
\hline Total & 156 & 376 & 572 & 601 & 723 & 1,317 & 958 & 837 & 811 & 678 & 691 & 7,720 \\
\hline \% Each Category & $2.02 \%$ & $4.87 \%$ & $7.41 \%$ & $7.78 \%$ & $9.37 \%$ & $17.06 \%$ & $12.41 \%$ & $10.84 \%$ & $10.51 \%$ & $8.78 \%$ & $8.95 \%$ & $100.00 \%$ \\
\hline $\begin{array}{c}\text { Market-sensitive } \\
\text { ASX Query }\end{array}$ & 2004 & 2005 & 2006 & 2007 & 2008 & 2009 & 2010 & 2011 & 2012 & 2013 & 2014 & Total \\
\hline Pre trading & 25 & 88 & 167 & 152 & 130 & 242 & 156 & 159 & 148 & 128 & 142 & 1,537 \\
\hline $10.00-11.00^{*}$ & 6 & 32 & 52 & 82 & 66 & 87 & 64 & 33 & 27 & 21 & 23 & 493 \\
\hline $11.00-12.00^{*}$ & 7 & 11 & 9 & 17 & 10 & 30 & 30 & 22 & 18 & 13 & 17 & 184 \\
\hline $12.00-13.00^{*}$ & 5 & 13 & 28 & 23 & 25 & 42 & 43 & 31 & 33 & 22 & 31 & 296 \\
\hline $13.00-14.00^{*}$ & 3 & 17 & 40 & 43 & 45 & 90 & 80 & 49 & 38 & 46 & 56 & 507 \\
\hline $14.00-15.00 *$ & 9 & 30 & 44 & 62 & 45 & 73 & 63 & 48 & 39 & 58 & 76 & 547 \\
\hline $15.00-16.00$ & 5 & 24 & 57 & 38 & 34 & 78 & 51 & 43 & 47 & 64 & 53 & 494 \\
\hline Post trading & 50 & 122 & 154 & 167 & 111 & 239 & 215 & 177 & 163 & 131 & 156 & 1,685 \\
\hline Weekends & 0 & 0 & 0 & 0 & 0 & 0 & 0 & 0 & 0 & 0 & 0 & 0 \\
\hline Trading hours & 35 & 127 & 230 & 265 & 225 & 400 & 331 & 226 & 202 & 224 & 256 & 2,521 \\
\hline \% Each Year & $31.82 \%$ & $37.69 \%$ & $41.74 \%$ & $45.38 \%$ & $48.28 \%$ & $45.40 \%$ & $47.15 \%$ & $40.21 \%$ & $39.38 \%$ & $46.38 \%$ & $46.21 \%$ & $43.90 \%$ \\
\hline Off-trading hours & 75 & 210 & 321 & 319 & 241 & 481 & 371 & 336 & 311 & 259 & 298 & 3,222 \\
\hline \% Each Year & $68.18 \%$ & $62.31 \%$ & $58.26 \%$ & $54.62 \%$ & $51.72 \%$ & $54.60 \%$ & $52.85 \%$ & $59.79 \%$ & $60.62 \%$ & $53.62 \%$ & $53.79 \%$ & $56.10 \%$ \\
\hline Total & 110 & 337 & 551 & 584 & 466 & 881 & 702 & 562 & 513 & 483 & 554 & 5,743 \\
\hline \% Each Category & $70.51 \%$ & $89.63 \%$ & $96.33 \%$ & $97.17 \%$ & $64.45 \%$ & $66.89 \%$ & $73.28 \%$ & $67.14 \%$ & $63.26 \%$ & $71.24 \%$ & $80.17 \%$ & $74.39 \%$ \\
\hline$\%$ Total MS & $1.92 \%$ & $5.87 \%$ & $9.59 \%$ & $10.17 \%$ & $8.11 \%$ & $15.34 \%$ & $12.22 \%$ & $9.79 \%$ & $8.93 \%$ & $8.41 \%$ & $9.65 \%$ & $100.00 \%$ \\
\hline
\end{tabular}


Appendix II cont.

\begin{tabular}{|c|c|c|c|c|c|c|c|c|c|c|c|c|}
\hline Structured Products & 2004 & 2005 & 2006 & 2007 & 2008 & 2009 & 2010 & 2011 & 2012 & 2013 & 2014 & Total \\
\hline Pre trading & 285 & 497 & 637 & 617 & 1,120 & 1,122 & 828 & 982 & 2,099 & 2,640 & 2,218 & 13,045 \\
\hline $10.00-11.00^{*}$ & 169 & 357 & 325 & 360 & 440 & 412 & 296 & 655 & 765 & 561 & 495 & 4,835 \\
\hline $11.00-12.00^{*}$ & 162 & 340 & 348 & 409 & 439 & 278 & 193 & 386 & 418 & 424 & 312 & 3,709 \\
\hline 12.00-13.00* & 152 & 242 & 287 & 325 & 287 & 217 & 150 & 297 & 358 & 312 & 155 & 2,782 \\
\hline $13.00-14.00^{*}$ & 65 & 110 & 163 & 222 & 221 & 127 & 84 & 167 & 230 & 270 & 121 & 1,780 \\
\hline $14.00-15.00^{*}$ & 90 & 289 & 299 & 337 & 483 & 224 & 165 & 256 & 363 & 326 & 297 & 3,129 \\
\hline $15.00-16.00$ & 189 & 447 & 438 & 495 & 463 & 323 & 250 & 450 & 540 & 509 & 307 & 4,411 \\
\hline Post trading & 428 & 1,128 & 1,360 & 1,307 & 979 & 998 & 1,125 & 2,200 & 3,028 & 3,251 & 1,860 & 17,664 \\
\hline Weekends & 0 & 0 & 0 & 0 & 0 & 0 & 0 & 0 & 0 & 0 & 0 & 0 \\
\hline Trading hours & 827 & 1,785 & 1,860 & 2,148 & 2,333 & 1,581 & 1,138 & 2,211 & 2,674 & 2,402 & 1,687 & 20,646 \\
\hline \% Each Year & $53.70 \%$ & $52.35 \%$ & $48.22 \%$ & $52.75 \%$ & $52.64 \%$ & $42.72 \%$ & $36.82 \%$ & $41.00 \%$ & $34.28 \%$ & $28.96 \%$ & $29.26 \%$ & $40.20 \%$ \\
\hline Off-trading hours & 713 & 1,625 & 1,997 & 1,924 & 2,099 & 2,120 & 1,953 & 3,182 & 5,127 & 5,891 & 4,078 & 30,709 \\
\hline \% Each Year & $46.30 \%$ & $47.65 \%$ & $51.78 \%$ & $47.25 \%$ & $47.36 \%$ & $57.28 \%$ & $63.18 \%$ & $59.00 \%$ & $65.72 \%$ & $71.04 \%$ & $70.74 \%$ & $59.80 \%$ \\
\hline Total & 1,540 & 3,410 & 3,857 & 4,072 & 4,432 & 3,701 & 3,091 & 5,393 & 7,801 & 8,293 & 5,765 & 51,355 \\
\hline \% Each Category & $3.00 \%$ & $6.64 \%$ & $7.51 \%$ & $7.93 \%$ & $8.63 \%$ & $7.21 \%$ & $6.02 \%$ & $10.50 \%$ & $15.19 \%$ & $16.15 \%$ & $11.23 \%$ & $100.00 \%$ \\
\hline $\begin{array}{c}\text { Market-sensitive } \\
\text { Structured Products }\end{array}$ & 2004 & 2005 & 2006 & 2007 & 2008 & 2009 & 2010 & 2011 & 2012 & 2013 & 2014 & Total \\
\hline Pre trading & 0 & 0 & 0 & 0 & 0 & 0 & 0 & 0 & 1 & 0 & 0 & 1 \\
\hline $10.00-11.00^{*}$ & 0 & 0 & 0 & 0 & 0 & 0 & 0 & 0 & 0 & 0 & 1 & 1 \\
\hline $11.00-12.00^{*}$ & 0 & 0 & 0 & 0 & 0 & 0 & 0 & 0 & 0 & 0 & 0 & 0 \\
\hline $12.00-13.00^{*}$ & 0 & 0 & 0 & 0 & 0 & 0 & 0 & 0 & 0 & 0 & 0 & 0 \\
\hline $13.00-14.00^{*}$ & 0 & 0 & 0 & 0 & 0 & 0 & 0 & 0 & 0 & 0 & 0 & 0 \\
\hline $14.00-15.00^{*}$ & 0 & 0 & 0 & 0 & 0 & 0 & 0 & 0 & 0 & 0 & 0 & 0 \\
\hline $15.00-16.00$ & 0 & 0 & 0 & 1 & 0 & 0 & 0 & 0 & 0 & 0 & 0 & 1 \\
\hline Post trading & 0 & 0 & 0 & 0 & 0 & 0 & 0 & 0 & 0 & 0 & 0 & 0 \\
\hline Weekends & 0 & 0 & 0 & 0 & 0 & 0 & 0 & 0 & 0 & 0 & 0 & 0 \\
\hline Trading hours & 0 & 0 & 0 & 1 & 0 & 0 & 0 & 0 & 0 & 0 & 1 & 2 \\
\hline$\%$ Each Year & - & - & - & $100.00 \%$ & - & - & - & - & $0.00 \%$ & - & $100.00 \%$ & $66.67 \%$ \\
\hline Off-trading hours & 0 & 0 & 0 & 0 & 0 & 0 & 0 & 0 & 1 & 0 & 0 & 1 \\
\hline \% Each Year & - & - & - & $0.00 \%$ & - & - & - & - & $100.00 \%$ & - & $0.00 \%$ & $33.33 \%$ \\
\hline Total & 0 & 0 & 0 & 1 & 0 & 0 & 0 & 0 & 1 & 0 & 1 & 3 \\
\hline \% Each Category & $0.00 \%$ & $0.00 \%$ & $0.00 \%$ & $0.02 \%$ & $0.00 \%$ & $0.00 \%$ & $0.00 \%$ & $0.00 \%$ & $0.01 \%$ & $0.00 \%$ & $0.02 \%$ & $0.01 \%$ \\
\hline \% Total MS & $0.00 \%$ & $0.00 \%$ & $0.00 \%$ & $33.33 \%$ & $0.00 \%$ & $0.00 \%$ & $0.00 \%$ & $0.00 \%$ & $33.33 \%$ & $0.00 \%$ & $33.33 \%$ & $100.00 \%$ \\
\hline
\end{tabular}


Appendix II cont.

\begin{tabular}{|c|c|c|c|c|c|c|c|c|c|c|c|c|}
\hline CTE Quarterly Reports & 2004 & 2005 & 2006 & 2007 & 2008 & 2009 & 2010 & 2011 & 2012 & 2013 & 2014 & Total \\
\hline Pre trading & 62 & 170 & 215 & 249 & 278 & 268 & 216 & 221 & 236 & 215 & 249 & 2,379 \\
\hline $10.00-11.00^{*}$ & 38 & 70 & 106 & 109 & 119 & 99 & 95 & 112 & 104 & 85 & 83 & 1,020 \\
\hline 11.00-12.00* & 41 & 101 & 95 & 106 & 109 & 102 & 87 & 72 & 77 & 63 & 77 & 930 \\
\hline 12.00-13.00* & 35 & 98 & 114 & 131 & 120 & 118 & 108 & 96 & 84 & 95 & 94 & 1,093 \\
\hline $13.00-14.00^{*}$ & 45 & 93 & 113 & 105 & 135 & 94 & 111 & 86 & 112 & 84 & 90 & 1,068 \\
\hline $14.00-15.00^{*}$ & 54 & 134 & 144 & 153 & 160 & 160 & 138 & 122 & 99 & 92 & 85 & 1,341 \\
\hline $15.00-16.00$ & 75 & 160 & 152 & 162 & 150 & 164 & 116 & 145 & 120 & 103 & 109 & 1,456 \\
\hline Post trading & 211 & 512 & 517 & 551 & 553 & 492 & 471 & 452 & 393 & 374 & 317 & 4,843 \\
\hline Weekends & 0 & 0 & 0 & 0 & 0 & 0 & 0 & 0 & 0 & 0 & 0 & 0 \\
\hline Trading hours & 288 & 656 & 724 & 766 & 793 & 737 & 655 & 633 & 596 & 522 & 538 & 6,908 \\
\hline \% Each Year & $51.34 \%$ & $49.03 \%$ & $49.73 \%$ & $48.91 \%$ & $48.83 \%$ & $49.23 \%$ & $48.81 \%$ & $48.47 \%$ & $48.65 \%$ & $46.98 \%$ & $48.73 \%$ & $48.89 \%$ \\
\hline Off-trading hours & 273 & 682 & 732 & 800 & 831 & 760 & 687 & 673 & 629 & 589 & 566 & 7,222 \\
\hline$\%$ Each Year & $48.66 \%$ & $50.97 \%$ & $50.27 \%$ & $51.09 \%$ & $51.17 \%$ & $50.77 \%$ & $51.19 \%$ & $51.53 \%$ & $51.35 \%$ & $53.02 \%$ & $51.27 \%$ & $51.11 \%$ \\
\hline Total & 561 & 1,338 & 1,456 & 1,566 & 1,624 & 1,497 & 1,342 & 1,306 & 1,225 & 1,111 & 1,104 & 14,130 \\
\hline \% Each Category & $3.97 \%$ & $9.47 \%$ & $10.30 \%$ & $11.08 \%$ & $11.49 \%$ & $10.59 \%$ & $9.50 \%$ & $9.24 \%$ & $8.67 \%$ & $7.86 \%$ & $7.81 \%$ & $100.00 \%$ \\
\hline $\begin{array}{c}\text { Market-sensitive CTE } \\
\text { Quarterly Reports }\end{array}$ & 2004 & 2005 & 2006 & 2007 & 2008 & 2009 & 2010 & 2011 & 2012 & 2013 & 2014 & Total \\
\hline Pre trading & 51 & 151 & 185 & 219 & 246 & 231 & 185 & 183 & 203 & 186 & 218 & 2,058 \\
\hline $10.00-11.00^{*}$ & 33 & 65 & 100 & 99 & 113 & 93 & 87 & 100 & 90 & 77 & 72 & 929 \\
\hline 11.00-12.00* & 37 & 94 & 87 & 100 & 99 & 94 & 75 & 58 & 68 & 49 & 70 & 831 \\
\hline $12.00-13.00^{*}$ & 33 & 91 & 110 & 117 & 108 & 107 & 100 & 87 & 71 & 78 & 75 & 977 \\
\hline $13.00-14.00^{*}$ & 41 & 87 & 105 & 91 & 118 & 83 & 97 & 79 & 99 & 71 & 77 & 948 \\
\hline $14.00-15.00 *$ & 48 & 121 & 126 & 143 & 152 & 133 & 116 & 95 & 73 & 78 & 73 & 1,158 \\
\hline $15.00-16.00$ & 74 & 144 & 140 & 142 & 138 & 143 & 104 & 129 & 104 & 90 & 88 & 1,296 \\
\hline Post trading & 193 & 466 & 465 & 497 & 500 & 433 & 409 & 399 & 342 & 310 & 269 & 4,283 \\
\hline Weekends & 0 & 0 & 0 & 0 & 0 & 0 & 0 & 0 & 0 & 0 & 0 & 0 \\
\hline Trading hours & 266 & 602 & 668 & 692 & 728 & 653 & 579 & 548 & 505 & 443 & 455 & 6,139 \\
\hline \% Each Year & $52.16 \%$ & $49.38 \%$ & $50.68 \%$ & $49.15 \%$ & $49.39 \%$ & $49.58 \%$ & $49.36 \%$ & $48.50 \%$ & $48.10 \%$ & $47.18 \%$ & $48.30 \%$ & $49.19 \%$ \\
\hline Off-trading hours & 244 & 617 & 650 & 716 & 746 & 664 & 594 & 582 & 545 & 496 & 487 & 6,341 \\
\hline \% Each Year & $47.84 \%$ & $50.62 \%$ & $49.32 \%$ & $50.85 \%$ & $50.61 \%$ & $50.42 \%$ & $50.64 \%$ & $51.50 \%$ & $51.90 \%$ & $52.82 \%$ & $51.70 \%$ & $50.81 \%$ \\
\hline Total & 510 & 1,219 & 1,318 & 1,408 & 1,474 & 1,317 & 1,173 & 1,130 & 1,050 & 939 & 942 & 12,480 \\
\hline \% Each Category & $90.91 \%$ & $91.11 \%$ & $90.52 \%$ & $89.91 \%$ & $90.76 \%$ & $87.98 \%$ & $87.41 \%$ & $86.52 \%$ & $85.71 \%$ & $84.52 \%$ & $85.33 \%$ & $88.32 \%$ \\
\hline$\%$ Total MS & $4.09 \%$ & $9.77 \%$ & $10.56 \%$ & $11.28 \%$ & $11.81 \%$ & $10.55 \%$ & $9.40 \%$ & $9.05 \%$ & $8.41 \%$ & $7.52 \%$ & $7.55 \%$ & $100.00 \%$ \\
\hline
\end{tabular}

Note: * excluding the minute in the time window. Pre trading: 00.00-9.59; Post trading: 16.01-23.59. The announcement release time is rounded up to one minute. 
APPENDIX III: THE T-TEST RESULTS OF NON-MARKET-SENSITIVE ANNOUNCEMENTS

\begin{tabular}{|c|c|c|c|c|c|c|c|c|}
\hline Main Category & CAR10 & CAR15 & CAR30 & CAR60 & CAR90 & CAR120 & CAR150 & CAR180 \\
\hline $\mathbf{N}$ & 3,083 & 3,023 & 2,855 & 2,510 & 2,218 & 1,978 & 1,746 & 1,538 \\
\hline Takeover/Scheme Announcements & $0.025 \% *$ & $0.064 \% * * *$ & $0.090 \% * *$ & $0.219 \% * * *$ & $0.180 \% * *$ & $0.179 \% * *$ & $0.178 \% * *$ & $0.127 \% *$ \\
\hline$t$-value & 1.69 & 3.16 & 2.40 & 3.85 & 2.44 & 2.36 & 2.18 & 1.66 \\
\hline $\mathbf{N}$ & 71,861 & 70,078 & 65,413 & 56,486 & 48,419 & 41,731 & 36,212 & 31,353 \\
\hline Security Holder Details & $0.020 \% * * *$ & $0.034 \% * * *$ & $0.055 \% * * *$ & $0.101 \% * * *$ & $0.128 \% * * *$ & $0.144 \% * * *$ & $0.142 \% * * *$ & $0.121 \% * * *$ \\
\hline$t$-value & 3.95 & 6.08 & 7.41 & 9.15 & 9.42 & 9.03 & 7.58 & 6.00 \\
\hline $\mathbf{N}$ & 23,789 & 23,319 & 22,071 & 19,575 & 17,422 & 15,466 & 13,819 & 12,188 \\
\hline Periodic Reports & $0.049 \% * * *$ & $0.066 \% * * *$ & $0.073 \% * * *$ & $0.091 \% * * *$ & $0.101 \% * * *$ & $0.112 \% * * *$ & $0.105 \% * * *$ & $0.113 \% * * *$ \\
\hline$t$-value & 6.40 & 6.95 & 5.48 & 4.99 & 4.93 & 4.74 & 3.97 & 3.95 \\
\hline $\mathbf{N}$ & 405 & 404 & 389 & 360 & 329 & 290 & 268 & 235 \\
\hline Quarterly Activities Report & $0.000 \%$ & $0.001 \%$ & $-0.005 \%$ & $-0.101 \%$ & $-0.094 \%$ & $-0.174 \%$ & $-0.251 \%$ & $-0.268 \%$ \\
\hline$t$-value & -0.01 & 0.02 & -0.06 & -0.78 & -0.57 & -0.86 & -1.02 & -0.97 \\
\hline $\mathbf{N}$ & 85 & 79 & 74 & 63 & 53 & 45 & 40 & 29 \\
\hline Quarterly Cash Flow Report & $0.000 \%$ & $-0.006 \%$ & $-0.073 \%$ & $0.308 \%$ & $0.134 \%$ & $0.284 \%$ & $-0.190 \%$ & $0.327 \%$ \\
\hline$t$-value & -0.01 & -0.06 & -0.27 & 1.07 & 0.36 & 0.67 & -0.32 & 0.35 \\
\hline $\mathbf{N}$ & 55,759 & 54,566 & 51,288 & 45,309 & 39,978 & 35,076 & 30,774 & 26,524 \\
\hline Issued Capital & $-0.017 \% * * *$ & $-0.019 \% * * *$ & $-0.037 \% * * *$ & $-0.039 \% * * *$ & $-0.077 \% * * *$ & $-0.078 \% * * *$ & $-0.060 \% * * *$ & $-0.083 \% * * *$ \\
\hline$t$-value & -2.82 & -2.65 & -3.73 & -3.06 & -5.01 & -4.49 & -3.05 & -3.89 \\
\hline $\mathbf{N}$ & 2,259 & 2,219 & 2,087 & 1,884 & 1,667 & 1,476 & 1,307 & 1,144 \\
\hline Asset Acquisition \& Disposal & $0.203 \% * * *$ & $0.215 \% * * *$ & $0.390 \% * * *$ & $0.422 \% * * *$ & $0.388 \% * * *$ & $0.368 \% * * *$ & $0.384 \% * * *$ & $0.388 \% * * *$ \\
\hline$t$-value & 4.03 & 3.76 & 4.42 & 4.44 & 3.68 & 3.37 & 3.07 & 2.88 \\
\hline $\mathbf{N}$ & 20,521 & 20,122 & 18,900 & 16,531 & 14,236 & 12,128 & 10,182 & 8,332 \\
\hline Notice of Meeting & $0.004 \%$ & $-0.004 \%$ & $0.009 \%$ & $0.003 \%$ & $0.022 \%$ & $0.013 \%$ & $0.022 \%$ & $-0.012 \%$ \\
\hline$t$-value & 0.38 & -0.33 & 0.53 & 0.13 & 0.80 & 0.40 & 0.56 & -0.28 \\
\hline $\mathbf{N}$ & 978 & 956 & 910 & 832 & 748 & 664 & 588 & 494 \\
\hline ASX Announcement & $0.018 \%$ & $0.008 \%$ & $-0.033 \%$ & $-0.091 \%$ & $-0.097 \%$ & $-0.043 \%$ & $0.022 \%$ & $-0.038 \%$ \\
\hline$t$-value & 0.71 & 0.27 & -0.68 & -1.42 & -1.20 & -0.44 & 0.18 & -0.29 \\
\hline
\end{tabular}




\begin{tabular}{|c|c|c|c|c|c|c|c|c|}
\hline Main Category & CAR10 & CAR15 & CAR30 & CAR60 & CAR90 & CAR120 & CAR150 & CAR180 \\
\hline $\mathbf{N}$ & 3,295 & 3,232 & 3,061 & 2,699 & 2,375 & 2,086 & 1,851 & 1,674 \\
\hline Distribution Announcement & $0.013 \%$ & $0.026 \% * *$ & $0.029 \% *$ & $0.041 \% *$ & $0.032 \%$ & $0.057 \% * *$ & $0.047 \%$ & $0.047 \%$ \\
\hline$t$-value & 1.26 & 2.00 & 1.91 & 1.96 & 1.17 & 1.96 & 1.36 & 1.28 \\
\hline $\mathbf{N}$ & 24,093 & 23,864 & 23,071 & 21,427 & 19,691 & 17,891 & 16,233 & 14,447 \\
\hline Progress Report & $0.174 \% * * *$ & $0.209 \% * * *$ & $0.307 \% * * *$ & $0.415 \% * * *$ & $0.480 \% * * *$ & $0.497 \% * * *$ & $0.505 \% * * *$ & $0.542 \% * * *$ \\
\hline$t$-value & 13.16 & 13.73 & 15.59 & 16.67 & 17.06 & 15.60 & 14.07 & 13.58 \\
\hline $\mathbf{N}$ & 13,017 & 12,765 & 12,083 & 10,803 & 9,548 & 8,399 & 7,418 & 6,455 \\
\hline Company Administration & $0.028 \% *$ & $0.022 \%$ & $0.050 \% * *$ & $0.090 \% * * *$ & $0.052 \%$ & $0.084 \% * *$ & $0.066 \%$ & $0.070 \%$ \\
\hline$t$-value & 1.84 & 1.32 & 2.35 & 3.02 & 1.51 & 2.10 & 1.46 & 1.38 \\
\hline $\mathbf{N}$ & 9 & 9 & 8 & 7 & 7 & 7 & 7 & 7 \\
\hline Notice of Call & $0.545 \%$ & $0.544 \%$ & $0.550 \%$ & $0.970 \%$ & $0.838 \%$ & $0.856 \%$ & $0.886 \%$ & $-0.168 \%$ \\
\hline$t$-value & 0.94 & 0.94 & 0.86 & 1.39 & 1.16 & 1.13 & 1.13 & -0.38 \\
\hline $\mathbf{N}$ & 5,402 & 5,322 & 5,123 & 4,673 & 4,265 & 3,896 & 3,469 & 2,958 \\
\hline Other & $0.112 \% * * *$ & $0.155 \% * * *$ & $0.213 \% * * *$ & $0.250 \% * * *$ & $0.299 \% * * *$ & $0.336 \% * * *$ & $0.393 \% * * *$ & $0.336 \% * * *$ \\
\hline$t$-value & 5.36 & 6.18 & 6.83 & 5.83 & 5.91 & 5.95 & 6.11 & 4.62 \\
\hline $\mathbf{N}$ & 7,112 & 7,054 & 6,868 & 6,508 & 6,033 & 5,494 & 4,940 & 4,398 \\
\hline Chairman's Address & $0.054 \% * * *$ & $0.066 \% * * *$ & $0.064 \% * * *$ & $0.082 \% * *$ & $0.101 \% * *$ & $0.106 \% * *$ & $0.184 \% * * *$ & $0.186 \% * * *$ \\
\hline$t$-value & 3.76 & 3.54 & 2.60 & 2.37 & 2.53 & 2.36 & 3.65 & 3.26 \\
\hline $\mathbf{N}$ & 1,182 & 1,150 & 1,105 & 954 & 859 & 763 & 672 & 589 \\
\hline Letter to Shareholders & $0.062 \% *$ & $0.081 \% * *$ & $0.161 \% * * *$ & $0.110 \%$ & $0.226 \% * *$ & $0.307 \% * * *$ & $0.453 \% * * *$ & $0.118 \%$ \\
\hline$t$-value & 1.90 & 2.11 & 2.75 & 1.49 & 2.40 & 2.84 & 3.50 & 0.64 \\
\hline $\mathbf{N}$ & 474 & 465 & 439 & 387 & 344 & 291 & 242 & 192 \\
\hline ASX Query & $-0.027 \%$ & $-0.019 \%$ & $-0.012 \%$ & $-0.273 \% * *$ & $-0.200 \%$ & $-0.468 \% *$ & $-0.461 \%$ & $-0.450 \%$ \\
\hline$t$-value & -0.47 & -0.26 & -0.13 & -2.19 & -1.36 & -1.90 & -1.58 & -1.36 \\
\hline $\mathbf{N}$ & 3,217 & 3,122 & 2,874 & 2,493 & 2,124 & 1,870 & 1,632 & 1,491 \\
\hline Structured Products & $-0.001 \%$ & $0.000 \%$ & $-0.007 \%$ & $-0.023 \% * * *$ & $-0.024 \% * *$ & $-0.026 \% * *$ & $-0.028 \% * *$ & $-0.045 \% * * *$ \\
\hline$t$-value & -0.16 & -0.09 & -1.27 & -2.98 & -2.22 & -2.03 & -1.97 & -2.68 \\
\hline $\mathbf{N}$ & 102 & 101 & 90 & 80 & 64 & 51 & 42 & 36 \\
\hline CTE Quarterly Reports & $0.483 \%$ & $0.431 \%$ & $0.763 \% *$ & $0.924 \% *$ & $1.920 \% * * *$ & $0.928 \%$ & $1.330 \%$ & $1.310 \%$ \\
\hline$t$-value & 1.40 & 1.22 & 1.82 & 1.69 & 2.91 & 1.35 & 1.58 & 1.36 \\
\hline
\end{tabular}


Appendix III cont.

\begin{tabular}{|c|c|c|c|c|c|c|c|c|}
\hline Main Category & $\Delta$ CAR10 & $\Delta$ CAR15 & $\Delta$ CAR30 & $\Delta$ CAR60 & $\Delta$ CAR90 & $\Delta$ CAR120 & $\Delta$ CAR150 & $\Delta$ CAR180 \\
\hline $\mathbf{N}$ & 2,907 & 2,786 & 2,449 & 1,826 & 1,313 & 829 & 345 & 1 \\
\hline Takeover/Scheme Announcements & $0.030 \%$ & $0.068 \% * *$ & $0.051 \%$ & $0.199 \% * * *$ & $0.226 \% * *$ & $0.223 \%$ & $0.358 \%$ & $-6.260 \%$ \\
\hline$t$-value & 1.47 & 2.39 & 1.02 & 2.63 & 2.23 & 1.64 & 1.5 & . \\
\hline $\mathbf{N}$ & 69,348 & 66,416 & 58,701 & 43,888 & 29,940 & 17,625 & 7,392 & 19 \\
\hline Security Holder Details & $0.015 \% * *$ & $0.026 \% * * *$ & $0.063 \% * * *$ & $0.112 \% * * *$ & $0.126 \% * * *$ & $0.115 \% * * *$ & $0.273 \% * * *$ & $2.300 \% * * *$ \\
\hline$t$-value & 2.09 & 3.1 & 5.44 & 5.92 & 4.79 & 2.65 & 4.33 & 3.17 \\
\hline $\mathbf{N}$ & 22,470 & 21,427 & 18,629 & 13,607 & 9,310 & 5,476 & 2,319 & 8 \\
\hline Periodic Reports & $0.049 \% * * *$ & $0.069 \% * * *$ & $0.076 \% * * *$ & $0.112 \% * * *$ & $0.110 \% * * *$ & $0.128 \% * *$ & $0.143 \%$ & $-1.800 \%$ \\
\hline$t$-value & 4.9 & 5.58 & 4.2 & 3.89 & 2.85 & 2.16 & 1.47 & -1.04 \\
\hline $\mathbf{N}$ & 386 & 379 & 334 & 254 & 186 & 108 & 44 & 0 \\
\hline Quarterly Activities Report & $-0.108 \%$ & $-0.074 \%$ & $-0.160 \%$ & $-0.362 \% *$ & $-0.462 \%$ & $-0.589 \%$ & $0.359 \%$ & . \\
\hline$t$-value & -1.04 & -0.75 & -1.29 & -1.7 & -1.6 & -1.1 & 0.4 & . \\
\hline $\mathbf{N}$ & 83 & 76 & 69 & 54 & 41 & 24 & 13 & 0 \\
\hline Quarterly Cash Flow Report & $-0.006 \%$ & $-0.092 \%$ & $0.390 \%$ & $0.752 \%$ & $0.986 \%$ & $2.300 \% *$ & $3.480 \%$ & . \\
\hline$t$-value & -0.02 & -0.37 & 1 & 1.54 & 1.41 & 1.89 & 1.7 & . \\
\hline $\mathbf{N}$ & 53,523 & 51,396 & 45,458 & 34,577 & 24,462 & 14,864 & 6,580 & 15 \\
\hline Issued Capital & $-0.007 \%$ & $-0.011 \%$ & $-0.019 \%$ & $-0.004 \%$ & $0.018 \%$ & $-0.014 \%$ & $0.046 \%$ & $0.167 \%$ \\
\hline$t$-value & -0.9 & -1.1 & -1.44 & -0.23 & 0.66 & -0.35 & 0.72 & 0.13 \\
\hline $\mathbf{N}$ & 2,135 & 2,028 & 1,763 & 1,329 & 951 & 590 & 258 & 1 \\
\hline Asset Acquisition \& Disposal & $0.134 \% * *$ & $0.108 \% *$ & $0.307 \% * * *$ & $0.247 \% * *$ & $0.083 \%$ & $-0.056 \%$ & $-0.205 \%$ & $1.400 \%$ \\
\hline$t$-value & 2.53 & 1.73 & 3.09 & 2.14 & 0.59 & -0.29 & -0.78 & . \\
\hline $\mathbf{N}$ & 19,843 & 19,227 & 17,257 & 13,370 & 9,618 & 5,960 & 2,581 & 8 \\
\hline Notice of Meeting & $0.004 \%$ & $-0.002 \%$ & $-0.012 \%$ & $-0.042 \%$ & $-0.049 \%$ & $-0.111 \%$ & $-0.097 \%$ & $3.050 \%$ \\
\hline$t$-value & 0.30 & -0.11 & -0.55 & -1.17 & -1.02 & -1.61 & -0.80 & 1.17 \\
\hline $\mathbf{N}$ & 960 & 924 & 837 & 675 & 473 & 298 & 144 & 0 \\
\hline ASX Announcement & $0.017 \%$ & $0.017 \%$ & $-0.048 \%$ & $-0.290 \% *$ & $-0.895 \% * * *$ & $-0.918 \%$ & $-0.146 \%$ & . \\
\hline$t$-value & 0.49 & 0.39 & -0.62 & -1.93 & -3.33 & -1.51 & -0.17 & . \\
\hline $\mathbf{N}$ & 3,158 & 3,042 & 2,727 & 2,066 & 1,438 & 848 & 353 & 0 \\
\hline Distribution Announcement & $0.038 \% * *$ & $0.044 \% * *$ & $0.048 \% * *$ & $0.047 \%$ & $0.016 \%$ & $0.038 \%$ & $-0.022 \%$ & . \\
\hline$t$-value & 2.58 & 2.38 & 1.96 & 1.33 & 0.33 & 0.57 & -0.17 & . \\
\hline
\end{tabular}




\begin{tabular}{|c|c|c|c|c|c|c|c|c|}
\hline Main Category & $\triangle$ CAR10 & $\triangle \mathrm{CAR} 15$ & $\triangle$ CAR30 & $\triangle$ CAR60 & $\Delta$ CAR90 & $\triangle$ CAR120 & $\triangle$ CAR150 & $\Delta$ CAR180 \\
\hline $\mathbf{N}$ & 22,723 & 21,914 & 19,611 & 15,122 & 10,698 & 6,543 & 2,870 & 7 \\
\hline Progress Report & $0.181 \% * * *$ & $0.208 \% * * *$ & $0.291 \% * * *$ & $0.415 \% * * *$ & $0.497 \% * * *$ & $0.516 \% * * *$ & $0.562 \% * * *$ & $0.532 \%$ \\
\hline$t$-value & 10.42 & 10.29 & 10.89 & 10.79 & 9.76 & 7.21 & 4.43 & 0.40 \\
\hline $\mathbf{N}$ & 12,354 & 11,851 & 10,514 & 7,923 & 5,508 & 3,354 & 1,427 & 8 \\
\hline Company Administration & $0.022 \%$ & $0.033 \%$ & $0.046 \%$ & $0.064 \%$ & $0.018 \%$ & $0.031 \%$ & $-0.088 \%$ & $1.070 \%$ \\
\hline$t$-value & 1.10 & 1.47 & 1.55 & 1.41 & 0.29 & 0.34 & -0.54 & 0.59 \\
\hline $\mathbf{N}$ & 9 & 8 & 7 & 6 & 4 & 1 & 0 & 0 \\
\hline Notice of Call & $0.693 \%$ & $0.924 \%$ & $1.550 \%$ & $1.930 \%$ & $0.474 \%$ & $0.406 \%$ & . & . \\
\hline$t$-value & 0.95 & 1.12 & 1.07 & 1.23 & 5.57 & . & . & . \\
\hline $\mathbf{N}$ & 5,092 & 4,923 & 4,428 & 3,409 & 2,516 & 1,638 & 790 & 2 \\
\hline Other & $0.087 \% * * *$ & $0.127 \% * * *$ & $0.196 \% * * *$ & $0.259 \% * * *$ & $0.351 \% * * *$ & $0.433 \% * * *$ & $0.590 \% * * *$ & $-3.310 \%$ \\
\hline$t$-value & 3.49 & 4.35 & 4.79 & 4.32 & 4.38 & 3.96 & 3.66 & -1.04 \\
\hline $\mathbf{N}$ & 6,642 & 6,406 & 5,723 & 4,213 & 2,961 & 1,866 & 817 & 3 \\
\hline Chairman's Address & $0.053 \% * *$ & $0.058 \% * *$ & $0.045 \%$ & $-0.042 \%$ & $-0.046 \%$ & $-0.006 \%$ & $0.160 \%$ & $-2.880 \%$ \\
\hline$t$-value & 2.50 & 2.23 & 1.15 & -0.67 & -0.60 & -0.06 & 1.00 & -2.12 \\
\hline $\mathbf{N}$ & 1,130 & 1,074 & 960 & 723 & 516 & 313 & 129 & 0 \\
\hline Letter to Shareholders & $0.099 \% * *$ & $0.128 \% * *$ & $0.177 \% * *$ & $0.109 \%$ & $0.393 \% * *$ & $0.306 \%$ & $0.935 \% * *$ & . \\
\hline$t$-value & 2.00 & 2.09 & 2.03 & 0.99 & 2.44 & 1.46 & 2.35 & . \\
\hline $\mathbf{N}$ & 452 & 433 & 379 & 280 & 205 & 131 & 60 & 0 \\
\hline ASX Query & $0.005 \%$ & $-0.015 \%$ & $0.045 \%$ & $-0.230 \%$ & $-0.050 \%$ & $-0.734 \%$ & $-1.200 \%$ & . \\
\hline$t$-value & 0.06 & -0.14 & 0.30 & -0.97 & -0.15 & -1.45 & -1.43 & . \\
\hline $\mathbf{N}$ & 3,112 & 2,976 & 2,580 & 1,907 & 1,294 & 746 & 273 & 0 \\
\hline Structured Products & $-0.005 \%$ & $-0.003 \%$ & $-0.007 \%$ & $-0.013 \%$ & $-0.034 \% * *$ & $-0.088 \% * * *$ & $-0.047 \%$ & . \\
\hline$t$-value & -0.98 & -0.50 & -0.94 & -1.21 & -2.08 & -3.59 & -0.90 & . \\
\hline $\mathbf{N}$ & 96 & 94 & 80 & 63 & 39 & 24 & 10 & 0 \\
\hline CTE Quarterly Reports & $0.348 \%$ & $0.278 \%$ & $0.490 \%$ & $0.527 \%$ & $1.560 \% *$ & $-0.203 \%$ & $0.306 \%$ & . \\
\hline$t$-value & 0.93 & 0.71 & 1.04 & 0.85 & 1.85 & -0.46 & 0.50 & . \\
\hline
\end{tabular}


Appendix III cont.

\begin{tabular}{|c|c|c|c|c|c|c|c|c|}
\hline Main Category & ATV10 & ATV 15 & ATV 30 & ATV 60 & ATV 90 & ATV 120 & ATV 150 & ATV 180 \\
\hline $\mathbf{N}$ & 3,957 & 3,789 & 3,345 & 2,576 & 1,894 & 1,284 & 626 & 10 \\
\hline Takeover/Scheme Announcements & 10.915 & -19.598 & -13.901 & -41.366 & -23.616 & -115.530 & -90.063 & -141.737 \\
\hline$t$-value & 1.36 & -0.70 & -0.43 & -0.79 & -0.36 & -1.15 & -0.48 & -1.53 \\
\hline $\mathbf{N}$ & 95,776 & 92,441 & 82,928 & 64,383 & 46,712 & 29,756 & 14,219 & 234 \\
\hline Security Holder Details & $6.523 * *$ & $4.667 * *$ & $10.145^{* * *}$ & 3.571 & $-27.090 * * *$ & $-77.059 * * *$ & $-124.718 * * *$ & -147.270 \\
\hline$t$-value & 3.34 & 2.19 & 2.81 & 0.65 & -3.47 & -6.52 & -6.83 & -1.61 \\
\hline $\mathbf{N}$ & 38,795 & 37,141 & 33,090 & 25,687 & 18,107 & 11,415 & 5,402 & 86 \\
\hline Periodic Reports & $6.243 * * *$ & $13.169 * * *$ & $30.062 * * *$ & $43.520 * * *$ & $37.603 * * *$ & -6.790 & -21.635 & -0.812 \\
\hline$t$-value & 4.42 & 7.03 & 6.66 & 5.67 & 3.08 & -0.57 & -1.22 & -0.01 \\
\hline $\mathbf{N}$ & 694 & 668 & 604 & 475 & 356 & 231 & 118 & 1 \\
\hline Quarterly Activities Report & -6.993 & -14.835 & -13.720 & -40.586 & -28.130 & -52.720 & -39.863 & 0.000 \\
\hline$t$-value & -0.69 & -0.98 & -0.77 & -1.19 & -1.04 & -1.29 & -0.80 & 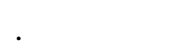 \\
\hline $\mathbf{N}$ & 214 & 202 & 178 & 142 & 103 & 64 & 37 & 0 \\
\hline Quarterly Cash Flow Report & 3.450 & -42.984 & -107.791 & -18.041 & 14.666 & -33.027 & -114.057 & . \\
\hline$t$-value & 1.34 & -0.92 & -1.13 & -0.82 & 0.59 & -0.82 & -0.98 & . \\
\hline $\mathbf{N}$ & 73,815 & 71,084 & 63,723 & 50,226 & 37,166 & 24,151 & 11,809 & 197 \\
\hline Issued Capital & $9.268 * * *$ & $14.040 * * *$ & $22.339 * * *$ & $29.757 * * *$ & 15.195 & $-38.384 * * *$ & $-74.982 * * *$ & -40.744 \\
\hline$t$-value & 3.56 & 4.86 & 5.30 & 4.17 & 1.49 & -2.48 & -3.15 & -0.57 \\
\hline $\mathbf{N}$ & 3,303 & 3,156 & 2,789 & 2,188 & 1,620 & 1,093 & 560 & 7 \\
\hline Asset Acquisition \& Disposal & $25.636 * * *$ & $35.936 * * *$ & $88.628 * * *$ & $146.577 * * *$ & $118.944 * * *$ & 82.814 & 21.604 & 133.360 \\
\hline$t$-value & 3.82 & 3.84 & 3.92 & 4.29 & 2.66 & 1.38 & 0.21 & 1.15 \\
\hline $\mathbf{N}$ & 30,771 & 29,894 & 27,360 & 22,358 & 17,140 & 11,461 & 5,808 & 80 \\
\hline Notice of Meeting & 2.511 & 4.247 & 4.860 & 2.061 & -4.200 & $-41.061 * * *$ & $-47.338 * * *$ & -99.083 \\
\hline$t$-value & 1.02 & 1.52 & 1.26 & 0.32 & -0.46 & -3.43 & -2.61 & -1.45 \\
\hline $\mathbf{N}$ & 2,460 & 2,324 & 1,954 & 1,442 & 1,028 & 647 & 352 & 8 \\
\hline ASX Announcement & 2.009 & 5.039 & -11.693 & $-130.761 * *$ & $-285.628 * * *$ & $-312.140^{*}$ & -139.068 & -374.468 \\
\hline$t$-value & 0.55 & 0.82 & -0.57 & -2.46 & -3.38 & -1.83 & -0.51 & -1.08 \\
\hline $\mathbf{N}$ & 3,986 & 3,846 & 3,445 & 2,708 & 1,933 & 1,190 & 537 & 4 \\
\hline Distribution Announcement & $7.706^{*}$ & $12.642 * *$ & $16.615 * * *$ & $19.659 * *$ & -19.452 & $-50.314 * *$ & $-68.472 *$ & 3.883 \\
\hline$t$-value & 1.79 & 2.53 & 2.62 & 2.04 & -1.11 & -2.23 & -1.89 & 0.24 \\
\hline
\end{tabular}




\begin{tabular}{|c|c|c|c|c|c|c|c|c|}
\hline Main Category & ATV10 & ATV 15 & ATV 30 & ATV 60 & ATV 90 & ATV 120 & ATV 150 & ATV 180 \\
\hline $\mathbf{N}$ & 30,410 & 29,357 & 26,548 & 20,969 & 15,304 & 10,080 & 4,876 & 78 \\
\hline Progress Report & $39.575 * * *$ & $62.771 * * *$ & $100.973^{* * *}$ & $129.725 * * *$ & $150.331 * *$ & $217.258 *$ & 375.447 & -752.477 \\
\hline$t$-value & 8.26 & 8.16 & 6.14 & 3.81 & 2.33 & 1.82 & 1.25 & -1.38 \\
\hline $\mathbf{N}$ & 19,654 & 18,912 & 16,993 & 13,360 & 9,822 & 6,340 & 3,099 & 66 \\
\hline Company Administration & $7.711 * * *$ & $11.788 * * *$ & $30.072 * * *$ & $48.751 * * *$ & 21.603 & -20.880 & -50.216 & 67.235 \\
\hline$t$-value & 2.62 & 3.33 & 5.20 & 4.38 & 1.30 & -1.06 & -1.57 & 1.11 \\
\hline $\mathbf{N}$ & 14 & 13 & 11 & 9 & 6 & 5 & 1 & 0 \\
\hline Notice of Call & -19.457 & -13.600 & -25.104 & -33.895 & -1.606 & -2.655 & 32.686 & . \\
\hline$t$-value & -1.16 & -1.29 & -1.19 & -1.03 & -0.20 & -0.18 & . & . \\
\hline $\mathbf{N}$ & 7,003 & 6,769 & 6,140 & 4,876 & 3,700 & 2,540 & 1,314 & 25 \\
\hline Other & $27.899 * * *$ & $45.507 * * *$ & $52.642 * * *$ & $85.023 * * *$ & 70.466 & 64.523 & 164.514 & 5066.116 \\
\hline$t$-value & 3.33 & 4.14 & 3.43 & 3.08 & 1.52 & 1.08 & 1.33 & 0.79 \\
\hline $\mathbf{N}$ & 9,774 & 9,440 & 8,535 & 6,568 & 4,670 & 3,098 & 1,541 & 27 \\
\hline Chairman's Address & $20.514 * * *$ & $28.184 * * *$ & $45.569 * * *$ & $77.020 * * *$ & $102.363 * * *$ & 59.106 & 20.964 & -436.996 \\
\hline$t$-value & 4.26 & 3.51 & 4.39 & 5.45 & 3.80 & 1.55 & 0.32 & -1.19 \\
\hline $\mathbf{N}$ & 1,592 & 1,537 & 1,379 & 1,077 & 812 & 545 & 263 & 6 \\
\hline Letter to Shareholders & $31.081 * * *$ & $33.117 * * *$ & $30.354 * *$ & 32.660 & 52.620 & 43.836 & -9.995 & 61.373 \\
\hline$t$-value & 4.34 & 3.99 & 2.31 & 1.39 & 0.96 & 1.10 & -0.13 & 1.33 \\
\hline $\mathbf{N}$ & 795 & 764 & 684 & 525 & 404 & 274 & 148 & 3 \\
\hline ASX Query & 14.604 & 23.886 & 23.726 & 107.841 & -151.904 & -283.278 & -790.971 & 133.100 \\
\hline$t$-value & 1.18 & 1.54 & 0.74 & 0.68 & -1.24 & -1.04 & -1.37 & 1.57 \\
\hline $\mathbf{N}$ & 3,308 & 3,168 & 2,759 & 2,058 & 1,428 & 841 & 321 & 1 \\
\hline Structured Products & $3.383^{*}$ & $3.875^{*}$ & 3.375 & $19.566 * *$ & $-54.149 * * *$ & $-134.840 * * *$ & $-271.572 * * *$ & -467.660 \\
\hline$t$-value & 1.85 & 1.82 & 1.02 & 2.58 & -3.48 & -5.83 & -5.63 & . \\
\hline $\mathbf{N}$ & 445 & 432 & 384 & 317 & 215 & 141 & 74 & 1 \\
\hline CTE Quarterly Reports & -9.537 & -12.128 & 5.277 & 7.495 & 76.077 & 50.482 & 64.361 & 44.984 \\
\hline$t$-value & -0.42 & -0.51 & 0.16 & 0.13 & 1.34 & 1.57 & 1.12 & \\
\hline
\end{tabular}


APPENDIX IV: THE CORRELATION MATRIX FOR VARIABLES IN IMMEDIATE MARKET REACTION TEST MODELS

\begin{tabular}{|c|c|c|c|c|c|c|c|c|c|c|c|}
\hline & CAR10 & CAR15 & CAR30 & CAR60 & CAR90 & CAR120 & CAR150 & CAR180 & ATV10 & ATV15 & ATV30 \\
\hline \multicolumn{12}{|l|}{ Before the GFC: } \\
\hline Takeover/Scheme Announcements & $-0.027^{* * *}$ & $-0.027^{* * *}$ & $-0.026^{* * *}$ & $-0.021^{* * *}$ & $-0.017^{* *}$ & -0.012 & $-0.016^{* *}$ & $-0.015^{*}$ & $-0.011^{* *}$ & $-0.011^{*}$ & -0.011 \\
\hline Security Holder Details & -0.004 & -0.002 & -0.003 & 0.002 & -0.001 & -0.003 & 0.006 & 0.005 & -0.004 & -0.007 & $-0.019^{* * *}$ \\
\hline Periodic Reports & $-0.044^{* * *}$ & $-0.044^{* * *}$ & $-0.040^{* * * *}$ & $-0.038^{* * *}$ & $-0.041^{* * *}$ & $-0.038^{* * *}$ & $-0.040^{* * *}$ & $-0.042^{* * *}$ & $-0.023^{* * *}$ & $-0.030^{* * * *}$ & $-0.025^{* * *}$ \\
\hline Issued Capital & $-0.047^{* * *}$ & $-0.050^{* * *}$ & $-0.039^{* * *}$ & $-0.036^{* * *}$ & $-0.036^{* * *}$ & $-0.037^{* * *}$ & $-0.032^{* * *}$ & $-0.030^{* * *}$ & -0.005 & 0.000 & -0.008 \\
\hline Asset Acquisition \& Disposal & -0.007 & 0.001 & 0.008 & 0.009 & 0.009 & 0.011 & 0.012 & 0.005 & $-0.014^{* *}$ & $-0.011^{*}$ & $-0.013^{*}$ \\
\hline Notice of Meeting & $-0.011^{*}$ & $-0.011^{*}$ & -0.008 & -0.010 & -0.009 & -0.009 & -0.008 & -0.009 & -0.008 & -0.006 & -0.004 \\
\hline ASX Announcement & $-0.036^{* * *}$ & $-0.039^{* * *}$ & $-0.034^{* * *}$ & $-0.031^{* * *}$ & $-0.023^{* * *}$ & $-0.024^{* * *}$ & $-0.023^{* * *}$ & $-0.019^{* * *}$ & $-0.021^{* * *}$ & $-0.025^{\text {*** }}$ & $-0.033^{* * *}$ \\
\hline Distribution Announcement & $-0.023^{* * *}$ & $-0.025^{* * *}$ & $-0.019^{* * *}$ & $-0.013^{* *}$ & -0.011 & -0.011 & -0.010 & -0.013 & $-0.018^{* * *}$ & $-0.017^{* * *}$ & $-0.017^{* *}$ \\
\hline Progress Reports & $0.187^{* * * *}$ & $0.187^{* * *}$ & $0.164^{* * *}$ & $0.146^{* * *}$ & $0.133^{* * *}$ & $0.124^{* * *}$ & $0.119^{* * *}$ & $0.117^{* * *}$ & $0.087^{* * *}$ & $0.086^{* * *}$ & $0.090^{* * *}$ \\
\hline Company Administration & -0.007 & -0.003 & -0.004 & -0.004 & -0.004 & -0.009 & -0.007 & -0.008 & -0.003 & 0.007 & 0.007 \\
\hline Other & $-0.016^{* *}$ & -0.008 & -0.008 & -0.007 & -0.006 & -0.005 & -0.007 & -0.002 & -0.009 & -0.005 & -0.006 \\
\hline Chairman's Address & $-0.023^{* * *}$ & $-0.020^{* * *}$ & $-0.022^{* * *}$ & $-0.019^{* * *}$ & $-0.017^{* *}$ & $-0.018^{* *}$ & $-0.015^{* *}$ & $-0.015^{*}$ & -0.010 & -0.010 & -0.010 \\
\hline Letter to Shareholders & -0.003 & 0.004 & 0.009 & 0.005 & 0.009 & $0.019^{* * *}$ & $0.016^{* * *}$ & $0.015^{*}$ & 0.009 & $0.013^{* *}$ & $0.018^{* * * *}$ \\
\hline ASX Query & $-0.060^{* * * *}$ & $-0.068^{* * * *}$ & $-0.067^{* * * *}$ & $-0.063^{* * *}$ & $-0.055^{* * * *}$ & $-0.060^{* * *}$ & -0.053 & -0.056 & -0.003 & -0.005 & -0.001 \\
\hline Structured Products & -0.002 & -0.002 & & & & & & & -0.001 & -0.001 & \\
\hline CTE Quarterly Reports & $-0.042^{* * * *}$ & $-0.044^{* * *}$ & $-0.036^{* * *}$ & $-0.027^{* * *}$ & $-0.026^{* * *}$ & $-0.016^{* *}$ & $-0.017^{* *}$ & $-0.020^{* *}$ & $-0.020^{* * *}$ & $-0.020^{* * * *}$ & $-0.021^{* * *}$ \\
\hline Bid-Ask Spread & $-0.016^{* *}$ & $-0.021^{* * *}$ & $-0.017^{* * *}$ & $-0.012^{* *}$ & -0.010 & -0.006 & -0.010 & -0.015 & $-0.027^{* * *}$ & $-0.035^{* * *}$ & $-0.036^{* * *}$ \\
\hline Size & $-0.106^{* * *}$ & $-0.109^{* * * *}$ & $-0.105^{* * *}$ & $-0.103^{* * *}$ & $-0.100^{* * * *}$ & $-0.101^{* * *}$ & $-0.106^{* * *}$ & $-0.104^{* * *}$ & $-0.060^{* * *}$ & $-0.063^{* * * *}$ & $-0.065^{* * *}$ \\
\hline Book-to-Market Ratio & 0.000 & 0.000 & 0.000 & 0.011 & 0.010 & 0.007 & 0.013 & 0.013 & & -0.005 & -0.005 \\
\hline Leverage & $0.015^{* *}$ & $0.032^{* * *}$ & $0.031^{* * *}$ & $0.022^{* * * *}$ & $0.030^{* * *}$ & $0.022^{* * *}$ & $0.021^{* * *}$ & $0.020^{* *}$ & -0.001 & 0.008 & 0.004 \\
\hline ROA & $-0.022^{* * *}$ & $-0.027^{* * *}$ & $-0.030^{* * *}$ & $-0.032^{* * *}$ & $-0.041^{* * *}$ & $-0.042^{* * *}$ & $-0.050^{* * *}$ & $-0.052^{* * *}$ & $-0.012^{*}$ & -0.010 & -0.010 \\
\hline Resource Sector & $0.042^{* * * *}$ & $0.032^{* * *}$ & $0.020^{* * *}$ & $0.014^{* *}$ & $0.014^{* *}$ & 0.005 & 0.008 & $0.014^{*}$ & $0.045^{* * * *}$ & $0.048^{* * *}$ & $0.042^{* * *}$ \\
\hline Financial Sector & $-0.053^{* * *}$ & $-0.054^{* * *}$ & $-0.047^{* * *}$ & $-0.036^{* * *}$ & $-0.032^{* * * *}$ & $-0.031^{* * *}$ & $-0.031^{* * *}$ & $-0.032^{* * *}$ & $-0.033^{* * *}$ & $-0.034^{* * *}$ & $-0.035^{* * *}$ \\
\hline \multicolumn{12}{|l|}{ During the GFC: } \\
\hline Takeover/Scheme Announcements & $-0.016^{* *}$ & $-0.017^{* * *}$ & $-0.012^{*}$ & -0.008 & -0.004 & -0.003 & 0.000 & 0.001 & -0.006 & $-0.013^{*}$ & $-0.013^{*}$ \\
\hline Security Holder Details & -0.004 & 0.000 & 0.006 & 0.000 & -0.005 & -0.002 & -0.016 & -0.013 & -0.007 & -0.009 & -0.009 \\
\hline Periodic Reports & $-0.029^{* * *}$ & $-0.039^{* * *}$ & $-0.034^{* * *}$ & $-0.026^{* * *}$ & $-0.022^{* * *}$ & $-0.022^{* * * *}$ & $-0.024^{* * *}$ & $-0.030^{* *}$ & $-0.014^{* *}$ & $-0.020^{\text {**** }}$ & $-0.020^{* * *}$ \\
\hline Issued Capital & $-0.017^{* * *}$ & $-0.021^{* * *}$ & $-0.018^{* * *}$ & $-0.015^{* *}$ & $-0.013^{*}$ & -0.007 & -0.006 & $-0.024^{* * *}$ & 0.000 & 0.001 & 0.005 \\
\hline Asset Acquisition \& Disposal & $0.014^{* *}$ & $0.026^{* * *}$ & $0.035^{* * *}$ & $0.039^{* * *}$ & $0.031^{* * *}$ & $0.028^{* * *}$ & $0.026^{* * *}$ & $0.029^{* * *}$ & $0.013^{* *}$ & $0.010^{* *}$ & $0.014^{* *}$ \\
\hline Notice of Meeting & -0.005 & -0.003 & -0.004 & -0.007 & -0.007 & -0.007 & -0.003 & -0.002 & -0.003 & -0.004 & -0.004 \\
\hline ASX Announcement & $-0.029^{* * * *}$ & $-0.036^{* * *}$ & $-0.036^{* * *}$ & $-0.041^{* * * *}$ & $-0.040^{* * * *}$ & $-0.036^{* * *}$ & $-0.036^{* * *}$ & $-0.034^{* * *}$ & $-0.013^{* *}$ & $-0.026^{* * * *}$ & $-0.030^{* * *}$ \\
\hline Distribution Announcement & $-0.016^{* *}$ & $-0.016^{* *}$ & -0.009 & -0.007 & -0.007 & -0.004 & -0.004 & 0.004 & -0.010 & $-0.014^{* *}$ & $-0.011^{*}$ \\
\hline Progress Reports & $0.120^{* * *}$ & $0.144^{* * *}$ & $0.125^{* * *}$ & $0.110^{* * *}$ & $0.095^{* * *}$ & $0.088^{* * *}$ & $0.082^{* * *}$ & $0.084^{* * *}$ & $0.052^{* * *}$ & $0.079^{* * *}$ & $0.075^{* * *}$ \\
\hline
\end{tabular}




\begin{tabular}{|c|c|c|c|c|c|c|c|c|c|c|c|}
\hline & CAR10 & CAR15 & CAR30 & CAR60 & CAR90 & CAR120 & CAR150 & CAR180 & ATV10 & ATV15 & ATV30 \\
\hline Company Administration & -0.006 & $-0.015^{* *}$ & $-0.013^{* *}$ & -0.010 & -0.005 & -0.005 & -0.007 & -0.005 & -0.005 & -0.004 & -0.003 \\
\hline Other & $-0.014^{* *}$ & $-0.016^{* *}$ & $-0.014^{* *}$ & -0.009 & -0.006 & -0.004 & -0.007 & -0.007 & -0.010 & $-0.011^{*}$ & -0.010 \\
\hline Chairman's Address & -0.010 & $-0.013^{* *}$ & $-0.016^{* *}$ & $-0.012^{*}$ & -0.008 & -0.009 & -0.006 & -0.003 & -0.006 & -0.010 & -0.010 \\
\hline Letter to Shareholders & 0.007 & -0.005 & $-0.012^{* *}$ & $-0.016^{* *}$ & $-0.016^{* *}$ & $-0.020^{* * *}$ & -0.021 & -0.004 & 0.007 & 0.006 & 0.005 \\
\hline ASX Query & $-0.048^{* * *}$ & $-0.059^{* * *}$ & $-0.058^{* * *}$ & $-0.052^{* * *}$ & $-0.049^{* * *}$ & $-0.044^{* * *}$ & $-0.041^{* * *}$ & $-0.036^{* * *}$ & $-0.020^{* * *}$ & -0.009 & -0.001 \\
\hline \multicolumn{12}{|l|}{ Structured Products } \\
\hline CTE Quarterly Reports & $-0.024^{* * *}$ & $-0.032^{* * *}$ & $-0.029^{* * *}$ & $-0.029^{* * *}$ & $-0.015^{* *}$ & $-0.013^{*}$ & -0.011 & -0.013 & -0.011 & $-0.016^{* *}$ & $-0.017^{* *}$ \\
\hline Bid-Ask Spread & -0.003 & -0.004 & -0.008 & 0.002 & -0.001 & 0.002 & -0.002 & -0.004 & -0.003 & -0.005 & $-0.027^{* * *}$ \\
\hline Size & $-0.061^{* * *}$ & $-0.079^{* * *}$ & $-0.072^{* * * *}$ & $-0.069^{* * *}$ & $-0.071^{* * *}$ & $-0.072^{* * *}$ & $-0.073^{* * *}$ & $-0.079^{* * *}$ & $-0.035^{* * *}$ & $-0.045^{* * *}$ & $-0.043^{* * *}$ \\
\hline Book-to-Market Ratio & -0.009 & -0.005 & 0.002 & 0.008 & 0.005 & 0.004 & -0.001 & -0.001 & 0.001 & -0.002 & -0.002 \\
\hline Leverage & 0.003 & 0.003 & 0.000 & -0.002 & -0.001 & -0.003 & -0.002 & -0.004 & 0.001 & 0.000 & 0.000 \\
\hline $\mathrm{ROA}$ & -0.007 & -0.005 & -0.004 & -0.005 & -0.010 & -0.010 & -0.011 & -0.011 & -0.003 & -0.004 & -0.005 \\
\hline Resource Sector & $0.024^{* * *}$ & $0.028^{* * *}$ & $0.022^{* * * *}$ & 0.011 & 0.004 & 0.001 & 0.001 & 0.010 & $0.020^{* * *}$ & $0.028^{* * *}$ & $0.023^{* * *}$ \\
\hline Financial Sector & $-0.028^{* * *}$ & $-0.033^{* * *}$ & $-0.029^{* * * *}$ & $-0.024^{* * *}$ & $-0.029^{* * * *}$ & $-0.033^{* * *}$ & $-0.034^{* * *}$ & $-0.036^{* * *}$ & $-0.017^{* * *}$ & $-0.019^{* * *}$ & $-0.016^{* *}$ \\
\hline \multicolumn{12}{|l|}{ After the GFC: } \\
\hline Takeover/Scheme Announcements & -0.010 & -0.008 & -0.007 & -0.003 & 0.003 & 0.006 & 0.006 & 0.006 & -0.006 & -0.008 & -0.004 \\
\hline Security Holder Details & -0.001 & -0.003 & -0.003 & -0.004 & -0.005 & -0.001 & -0.003 & 0.003 & -0.009 & -0.007 & -0.008 \\
\hline Periodic Reports & $-0.023^{* * *}$ & $-0.025^{* * *}$ & $-0.024^{* * *}$ & $-0.027^{* * *}$ & $-0.024^{* * *}$ & $-0.024^{* * *}$ & $-0.023^{* * *}$ & -0.014 & -0.010 & $-0.017^{* *}$ & $-0.015^{* *}$ \\
\hline Issued Capital & $-0.018^{* * *}$ & $-0.029^{* * *}$ & $-0.029^{* * *}$ & $-0.027^{* * *}$ & $-0.027^{* * *}$ & $-0.025^{* * *}$ & $-0.024^{* * *}$ & $-0.018^{* *}$ & 0.005 & 0.000 & 0.000 \\
\hline Asset Acquisition \& Disposal & $0.014^{* *}$ & $0.020^{* * *}$ & $0.023^{* * *}$ & $0.015^{* *}$ & $0.014^{*}$ & $0.018^{* *}$ & $0.017^{* * *}$ & $0.017^{* *}$ & -0.004 & -0.002 & -0.001 \\
\hline Notice of Meeting & 0.003 & 0.001 & 0.001 & 0.000 & 0.002 & 0.001 & -0.001 & 0.001 & 0.010 & 0.008 & $0.015^{* *}$ \\
\hline ASX Announcement & $-0.023^{* * *}$ & $-0.027^{* * *}$ & $-0.025^{* * *}$ & $-0.024^{* * *}$ & $-0.023^{* * *}$ & $-0.021^{* * *}$ & $-0.019^{* *}$ & $-0.019^{* *}$ & $-0.017^{* *}$ & $-0.026^{* * *}$ & $-0.027^{* * *}$ \\
\hline Distribution Announcement & -0.007 & $-0.013^{* *}$ & -0.010 & -0.007 & -0.002 & -0.004 & -0.002 & -0.002 & -0.011 & -0.010 & -0.011 \\
\hline Progress Reports & $0.108^{* * *}$ & $0.130^{* * *}$ & $0.121^{* * *}$ & $0.117^{* * *}$ & $0.107^{* * *}$ & $0.100^{* * *}$ & $0.092^{* * *}$ & $0.085^{\text {*** }}$ & $0.059^{* * *}$ & $0.077^{* * *}$ & $0.073^{* * *}$ \\
\hline Company Administration & $-0.018^{* * *}$ & $-0.020^{* * *}$ & $-0.018^{* * *}$ & -0.009 & -0.006 & -0.002 & -0.006 & -0.012 & -0.007 & -0.011 & -0.013 \\
\hline Other & -0.008 & -0.009 & -0.006 & -0.003 & -0.003 & -0.004 & -0.004 & -0.004 & -0.001 & -0.001 & -0.003 \\
\hline Chairman's Address & -0.006 & -0.008 & -0.006 & -0.007 & -0.008 & -0.005 & -0.012 & $-0.015^{*}$ & -0.005 & -0.004 & 0.000 \\
\hline Letter to Shareholders & -0.001 & -0.002 & -0.002 & -0.002 & -0.002 & -0.002 & 0.000 & 0.000 & -0.001 & -0.001 & -0.001 \\
\hline ASX Query & $-0.038^{* * *}$ & $-0.050^{* * *}$ & $-0.047^{* * *}$ & $-0.047^{* * *}$ & $-0.053^{* * *}$ & $-0.055^{* * *}$ & $-0.036^{* * *}$ & $-0.017^{*}$ & -0.006 & -0.003 & -0.004 \\
\hline \multicolumn{12}{|l|}{ Structured Products } \\
\hline CTE Quarterly Reports & $-0.029^{* * *}$ & $-0.027^{* * *}$ & $-0.025^{* * *}$ & $-0.023^{* * *}$ & $-0.024^{* * *}$ & $-0.022^{* * *}$ & $-0.025^{* * *}$ & $-0.022^{* *}$ & -0.006 & $-0.013^{* *}$ & $-0.013^{*}$ \\
\hline Bid-Ask Spread & $-0.012^{*}$ & $-0.015^{* *}$ & $-0.011^{*}$ & -0.005 & -0.008 & -0.013 & -0.012 & -0.006 & $-0.013^{*}$ & $-0.017^{* *}$ & $-0.016^{* *}$ \\
\hline Size & $-0.057^{* * * *}$ & $-0.063^{* * *}$ & $-0.061^{* * *}$ & $-0.059^{* * *}$ & $-0.058^{* * *}$ & $-0.048^{* * *}$ & $-0.048^{* * * *}$ & $-0.045^{* * *}$ & $-0.025^{* * *}$ & $-0.031^{* * *}$ & $-0.023^{* * *}$ \\
\hline Book-to-Market Ratio & 0.003 & 0.000 & 0.000 & 0.003 & 0.005 & 0.007 & $0.017^{* *}$ & $0.019^{* *}$ & -0.006 & $0.016^{* *}$ & 0.009 \\
\hline Leverage & -0.001 & 0.000 & -0.002 & -0.004 & -0.008 & -0.009 & -0.007 & -0.011 & -0.003 & -0.003 & -0.004 \\
\hline ROA & $-0.022^{* * *}$ & $-0.037^{* * *}$ & $-0.026^{* * *}$ & $-0.018^{* *}$ & $-0.019^{* *}$ & $-0.018^{* *}$ & 0.000 & 0.001 & $-0.012^{*}$ & $-0.015^{* *}$ & -0.007 \\
\hline Resource Sector & $0.017^{* *}$ & $0.024^{* * *}$ & $0.017^{* *}$ & 0.011 & 0.008 & -0.002 & 0.006 & -0.008 & 0.002 & $0.011^{*}$ & 0.009 \\
\hline Financial Sector & $-0.024^{* * *}$ & $-0.030^{* * *}$ & $-0.028^{* * *}$ & $-0.024^{* * *}$ & $-0.021^{* * *}$ & $-0.017^{* *}$ & $-0.016^{*}$ & $-0.015^{*}$ & $-0.021^{* * *}$ & $-0.025^{* * *}$ & $-0.024^{* * *}$ \\
\hline
\end{tabular}




\section{Before the GFC:}

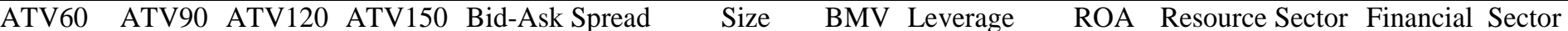

Takeover/Scheme Announcements

Security Holder Details

Periodic Reports

$\begin{array}{llll}-0.006 & -0.001 & 0.004 & -0.01\end{array}$

$-0.021^{* * *}-0.003 \quad-0.007 \quad-0.011$

Issued Capital

$-0.013^{*}-0.011-0.016-0.013$

Asset Acquisition \& Disposal

$\begin{array}{rrrr}0.000 & -0.006 & -0.009 & -0.011\end{array}$

$\begin{array}{llll}-0.013^{*} & -0.010 & -0.012 & -0.008\end{array}$

Notice of Meeting

ASX Announcement

Distribution Announcement

$\begin{array}{llll}-0.003 & -0.003 & -0.003 & -0.001\end{array}$

Progress Reports

$-0.065^{* * *}-0.060^{* * * *}-0.045^{* * *}-0.052^{* * *}$

Company Administration

$\begin{array}{llll}-0.014 & -0.014 & -0.014 & -0.014\end{array}$

Othe

$0.079^{* * *} \quad 0.073^{* * *} \quad 0.073^{* * *} \quad 0.087^{* * *}$

$\begin{array}{llll}0.002 & 0.003 & 0.006 & -0.026\end{array}$

$\begin{array}{llll}-0.010 & -0.011 & -0.001 & -0.001\end{array}$

$\begin{array}{llll}-0.005 & -0.003 & -0.003 & -0.002\end{array}$

$\begin{array}{llll}-0.002 & -0.001 & -0.001 & -0.001\end{array}$

Letter to Shareholders

ASX Query

Structured Products

CTE Quarterly Reports

Bid-Ask Spread

Size

Book-to-Market Ratio

Leverage

ROA

Resource Sector

$\begin{array}{llll}0.011 & -0.005 & -0.011 & -0.027\end{array}$

Financial Sector

$-0.020^{* * *}-0.015^{*} \quad-0.011 \quad-0.015$

$-0.034^{* * *}-0.026^{* * *}-0.020^{*}-0.014$

$\begin{array}{rrrr}-0.046^{* * *} & -0.043^{* * *} & -0.048^{* * *} & -0.043^{* * *} \\ -0.007 & -0.005 & -0.009 & -0.018\end{array}$

$\begin{array}{rrrr}-0.007 & -0.005 & -0.009 & -0.018 \\ 0.034^{* * * *} & 0.034^{* * * *} & 0.002 & -0.028\end{array}$

$\begin{array}{llll}-0.003 & 0.002 & 0.001 & -0.075^{\text {*** }}\end{array}$

$\begin{array}{rrrr}-0.003 & 0.002 & 0.001 & -0.075^{* *} \\ 0.037^{* * *} & 0.028^{* * *} & 0.025^{* *} & 0.013\end{array}$

$-0.030^{* * *}-0.026^{* * *}-0.025^{* *}-0.022$

$0.0070 .099^{* * *}-0.027^{\text {**** }}$

$\begin{array}{lll}-0.003 & 0.028^{* * *} & -0.001\end{array}$

$0.112^{* * *} \quad 0.149^{* * *} \quad 0.003$

$0.003-0.017^{* * *} \quad 0.019^{* * *}$

$0.004 \quad 0.092^{* * *} 0.018^{* * *}$

$\begin{array}{lll}0.006 & 0.021^{* * * *} \quad 0.008\end{array}$

$0.008-0.037^{* * *}-0.025^{* * *}$

$0.025^{* * *} \quad 0.121^{* * *} \quad 0.058^{* * *}$

$-0.071^{* * *}-0.090^{* * * *}-0.024^{* * *}$

$\begin{array}{lll}-0.001 & 0.042^{* * *} & -0.008\end{array}$

$0.0050 .109^{* * *}-0.019^{* * * *}$

$0.020^{* * *} \quad 0.079^{* * *} \quad-0.008$

$\begin{array}{lll}-0.003 & -0.009 & 0.001\end{array}$

$-0.007-0.049^{* * *}-0.014^{* *}$

$0.007 \quad-0.002$

$-0.007-0.140^{* * *} \quad 0.013^{* *}$

$0.097^{* * *}$

$-0.011-0.166^{* * *}$

$-0.003-0.001-0.021^{* * * *}$

$0.030^{* * *} \quad 0.177^{* * * *} \quad 0.077^{* * *}$

$-0.093^{* * * *}-0.239^{* * *}-0.034^{* * *}-0.018^{* *}$

$\begin{array}{lllll}0.076^{* * *} & 0.259^{* * *} & 0.103^{* * *} & 0.019^{* * *} & 0.071^{* *}\end{array}$

\section{During the GFC:}

Takeover/Scheme Announcements

Security Holder Details

Periodic Reports

Issued Capital

Asset Acquisition \& Disposal

Notice of Meeting

ASX Announcement

Distribution Announcement

Progress Reports

Company Administration

Other

Chairman's Address
$-0.014^{* *}-0.016^{* *} \quad-0.013 \quad-0.013$

$\begin{array}{llll}-0.006 & -0.010 & -0.006 & -0.006\end{array}$

$\begin{array}{rrrr}-0.023^{* * *} & -0.018^{* *} & -0.020 & -0.016\end{array}$

$\begin{array}{llll}0.007 & 0.008 & -0.002 & 0.013\end{array}$

$\begin{array}{llll}0.016^{* *} & 0.009 & 0.015 & 0.016\end{array}$

$\begin{array}{llll}-0.003 & -0.003 & -0.003 & -0.003\end{array}$

$-0.041^{* * *}-0.043^{* * *}-0.046^{* * *}-0.052^{* * *}$

$\begin{array}{llll}-0.009 & -0.012 & -0.009 & -0.004\end{array}$

$\begin{array}{llll}0.075^{* * *} & 0.083^{* * *} & 0.091^{* * *} & 0.081^{* * *}\end{array}$

$\begin{array}{llll}0.001 & -0.001 & 0.004 & 0.011\end{array}$

$\begin{array}{llll}-0.005 & -0.005 & -0.008 & -0.001\end{array}$

$\begin{array}{llll}-0.009 & -0.007 & -0.006 & -0.006\end{array}$
$-0.0020 .050^{* * *} \quad 0.018^{* * *}$

$\begin{array}{lll}0.002 & 0.003 & 0.000\end{array}$

$0.004-0.017^{* * *} \quad 0.004$

$0.0050 .060^{\text {*** }} 0.017^{* *}$

$\begin{array}{lll}-0.002 & -0.001 & -0.005\end{array}$

$\begin{array}{lll}-0.005 & -0.050^{* * *} \quad-0.007\end{array}$

$0.023^{* * *} \quad 0.094^{* * *} \quad 0.034^{* * *}$

$-0.049^{* * *} \quad-0.004-0.042^{* * *}$

$\begin{array}{lll}-0.007 & 0.029^{* * *} & 0.029^{* * * *} \\ 0.006 & 0.083 & -0.011^{*}\end{array}$

$0.006 \quad 0.083^{* * *}-0.011$

$0.027^{* * *} \quad 0.061^{* * *} \quad 0.022^{* * * *}$
$0.092^{* * *} \quad 0.149^{* * *} \quad 0.029^{* * *}$

$\begin{array}{rr}-0.002 & 0.022^{* * *} \\ -0.001 & 0.000 \\ -0.006 & 0.054^{* * *} \\ 0.014^{* *} & -0.018^{* * *} \\ -0.004 & 0.021^{* * *} \\ 0.000 & 0.007 \\ -0.003 & -0.017^{* * *} \\ -0.002 & 0.042^{* * *} \\ 0.002 & -0.018^{* * *} \\ -0.001 & 0.003 \\ -0.002 & 0.020^{* * *} \\ -0.001 & 0.024^{* * *} \\ 0.000 & -0.008 \\ 0.016^{* *} & -0.009 \\ 0.000 & 0.003 \\ 0.009 & -0.070^{* * *}\end{array}$

$-0.041^{* * *}$

$-0.016^{* *}$

$-0.317^{* * *}$

$-0.038^{* * *}$

$-0.132^{* * *}$

$-0.014^{* * *}$

$-0.017^{* * *}$

$-0.157^{* * *}$

$0.292^{* * * *}$

$-0.038^{* * *}$

$-0.085^{* * *}$

$-0.081^{* * *}$

$-0.006$

0.009

0.005

$-0.185^{* * *}$

$-0.001$

0.005
$0.155^{* * *}$

$0.155^{* * *}$

$0.141^{* * * *}$

0.006

$-0.015^{* * *}$

$0.272^{* * * *}$

$-0.196^{* * *}$

$0.019^{* * *}$

$0.015^{* *}$

$0.020^{* * * *}$

0.003

$-0.032^{* * *}$

$-0.002$

$-0.016^{* * *}$

$0.071^{* * *} \quad-0.403^{* * *}$

$-0.403$

$-0.001-0.005$
$0.000-0.002$

$-0.035^{* * *}$

$-0.017^{* * * *}$

$0.0090 .018^{\text {**** }}$

$-0.351^{* * *}$

$-0.004 \quad 0.001$

$-0.057^{* * *}$

$-0.002 \quad 0.006$

$0.000 \quad 0.000$

$-0.002 \quad-0.002$

$-0.001 \quad 0.009$

$0.005-0.003$

$\begin{array}{ll}-0.001 & 0.002\end{array}$

$-0.001 \quad 0.006$

$0.000 \quad 0.007$

$-0.019^{* * *}$

0.006

$-0.160^{* * *}$

$0.239^{* * *}$

$-0.050^{* * *}$

$-0.040^{* * *}$

$-0.093^{* * *}$

$0.037^{\text {*** }}$

0.013

$0.197^{\text {*** }}$

$0.061^{\text {*** }}$

$0.078^{* * *}$

$0.019^{\text {*** }}$

-0.010
$0.253^{* * *}$

$-0.166^{* * *}$

$-0.010$ $0.033^{* * *}$ 


\begin{tabular}{|c|c|c|c|c|c|c|c|c|c|c|c|}
\hline & ATV60 & ATV90 & ATV120 & ATV150 & Bid-Ask Spread & Size & BMV & Leverage & ROA & Resource Sector & Financial Sector \\
\hline Letter to Shareholders & 0.004 & 0.004 & 0.005 & 0.008 & -0.002 & -0.001 & 0.003 & 0.000 & 0.001 & $-0.012^{*}$ & 0.008 \\
\hline ASX Query & -0.004 & $-0.021^{* *}$ & $-0.034^{* * *}$ & $-0.044^{* * *}$ & -0.008 & $-0.021^{* * *}$ & 0.003 & -0.002 & 0.000 & 0.004 & -0.001 \\
\hline \multicolumn{12}{|l|}{ Structured Products } \\
\hline CTE Quarterly Reports & $-0.015^{* *}$ & $-0.018^{* *}$ & $-0.019^{*}$ & -0.021 & -0.007 & $-0.120^{* * *}$ & $-0.032^{* * *}$ & -0.002 & $-0.051^{* * *}$ & $-0.202^{* * *}$ & $-0.019^{* * *}$ \\
\hline Bid-Ask Spread & $-0.021^{* * *}$ & $-0.020^{* *}$ & $-0.034^{* * *}$ & $-0.034^{* *}$ & & & & & & & \\
\hline Size & $-0.040^{* * *}$ & $-0.041^{* * *}$ & $-0.051^{* * *}$ & $-0.058^{* * *}$ & $0.097^{* * *}$ & 1 & & & & & \\
\hline Book-to-Market Ratio & -0.001 & -0.007 & -0.007 & -0.006 & -0.011 & $-0.169^{* * *}$ & 1 & & & & \\
\hline Leverage & 0.000 & -0.001 & -0.004 & -0.003 & -0.003 & $0.045^{* * *}$ & $0.042^{* * *}$ & 1 & & & \\
\hline ROA & -0.004 & -0.007 & -0.007 & -0.005 & $0.030^{* * *}$ & $0.054^{* * *}$ & $0.016^{* *}$ & 0.003 & 1 & & \\
\hline Resource Sector & $0.028^{* * *}$ & $0.034^{* * *}$ & $0.036^{* * *}$ & $0.026^{*}$ & $-0.093^{* * *}$ & $-0.196^{* * *}$ & $-0.036^{* * *}$ & $-0.018^{* * *}$ & 0.004 & 1 & \\
\hline Financial Sector & $-0.017^{* *}$ & $-0.023^{* *}$ & $-0.028^{* *}$ & $-0.032^{* *}$ & $0.076^{* * *}$ & $0.215^{* * *}$ & $0.096^{* * *}$ & $0.039^{* * *}$ & $0.018^{* * *}$ & $-0.438^{* * *}$ & 1 \\
\hline \multicolumn{12}{|l|}{ After the GFC: } \\
\hline Takeover/Scheme Announcements & -0.003 & 0.001 & 0.004 & 0.003 & 0.000 & $0.043^{* * *}$ & -0.001 & 0.004 & 0.005 & $-0.026^{* * *}$ & $0.031^{* * *}$ \\
\hline Security Holder Details & -0.007 & -0.003 & -0.001 & -0.003 & -0.004 & 0.008 & 0.001 & -0.002 & 0.002 & -0.005 & -0.004 \\
\hline Periodic Reports & -0.012 & -0.008 & 0.000 & 0.024 & $0.110^{* * *}$ & $0.175^{* * *}$ & 0.010 & $0.022^{* * *}$ & $0.021^{* * *}$ & $-0.369^{* * *}$ & $0.179^{* * *}$ \\
\hline Issued Capital & 0.004 & 0.007 & 0.008 & 0.014 & 0.004 & $-0.024^{* * *}$ & 0.005 & $0.017^{* *}$ & -0.003 & $-0.059^{* * *}$ & $0.070^{* * *}$ \\
\hline Asset Acquisition \& Disposal & -0.002 & -0.004 & -0.001 & 0.009 & 0.002 & $0.055^{* * *}$ & -0.001 & 0.002 & 0.009 & $-0.076^{* * *}$ & $0.076^{* * *}$ \\
\hline Notice of Meeting & $0.014^{*}$ & -0.002 & -0.005 & -0.001 & -0.001 & 0.000 & 0.001 & 0.001 & 0.001 & $-0.011^{*}$ & $0.017^{* * *}$ \\
\hline ASX Announcement & $-0.032^{* * *}$ & $-0.046^{* * *}$ & $-0.041^{* * *}$ & $-0.060^{* * *}$ & -0.007 & $-0.031^{* * *}$ & -0.004 & 0.008 & 0.001 & 0.000 & -0.006 \\
\hline Distribution Announcement & -0.008 & -0.007 & -0.007 & -0.003 & $0.026^{* * *}$ & $0.088^{* * *}$ & $0.018^{* * *}$ & 0.007 & $0.013^{*}$ & $-0.182^{* * *}$ & $0.302^{* * *}$ \\
\hline Progress Reports & $0.068^{* * *}$ & $0.067^{* * * *}$ & $0.057^{* * * *}$ & $0.027^{*}$ & $-0.050^{* * *}$ & 0.006 & $-0.036^{* * * *}$ & $-0.018^{* * *}$ & $-0.019^{* * *}$ & $0.228^{* * *}$ & $-0.165^{* * *}$ \\
\hline Company Administration & $-0.014^{*}$ & -0.010 & -0.007 & -0.008 & -0.003 & $0.040^{* * * *}$ & 0.007 & -0.001 & 0.008 & $-0.048^{* * *}$ & $0.011^{*}$ \\
\hline \multicolumn{12}{|l|}{ Notice of Call } \\
\hline Other & 0.000 & 0.003 & -0.003 & 0.000 & -0.004 & $0.039^{* * *}$ & 0.003 & 0.004 & 0.002 & $-0.017^{* *}$ & 0.003 \\
\hline Chairman's Address & -0.001 & 0.002 & -0.002 & -0.001 & 0.011 & $0.059^{* * *}$ & 0.010 & 0.003 & 0.007 & $-0.076^{* * *}$ & 0.006 \\
\hline Letter to Shareholders & -0.002 & & & & 0.000 & 0.004 & 0.002 & 0.000 & 0.001 & 0.000 & 0.012 \\
\hline ASX Query & $-0.015^{*}$ & $-0.020^{* *}$ & $-0.025^{* *}$ & $-0.048^{* * *}$ & -0.011 & $-0.057^{* * *}$ & $0.017^{* *}$ & 0.006 & -0.004 & 0.009 & $-0.026^{* * *}$ \\
\hline \multicolumn{12}{|l|}{ Structured Products } \\
\hline CTE Quarterly Reports & $-0.014^{*}$ & -0.013 & -0.010 & -0.002 & -0.004 & $-0.103^{* * *}$ & $-0.053^{* * *}$ & 0.010 & $-0.021^{* * * *}$ & $-0.213^{* * *}$ & -0.005 \\
\hline Bid-Ask Spread & -0.014 & -0.019 & -0.011 & -0.005 & & & & & & & \\
\hline Size & -0.013 & -0.010 & -0.005 & -0.001 & $0.047^{* * *}$ & 1 & & & & & \\
\hline Book-to-Market Ratio & 0.002 & 0.005 & -0.012 & 0.002 & $-0.025^{* * *}$ & $-0.206^{* * *}$ & 1 & & & & \\
\hline Leverage & -0.003 & -0.002 & -0.001 & 0.002 & 0.001 & $0.018^{* * *}$ & 0.010 & 1 & & & \\
\hline ROA & -0.003 & -0.001 & -0.004 & 0.018 & 0.009 & $0.074^{* * *}$ & $0.060^{* * *}$ & 0.002 & 1 & & \\
\hline Resource Sector & $0.013^{*}$ & 0.015 & 0.012 & -0.010 & $-0.093^{* * *}$ & $-0.200^{* * *}$ & $0.062^{* * *}$ & $-0.045^{* * *}$ & 0.007 & 1 & \\
\hline Financial Sector & $-0.018^{* *}$ & $-0.017^{*}$ & -0.014 & -0.007 & $0.096^{* * *}$ & $0.179^{\text {**** }}$ & $0.049^{* * *}$ & $0.033^{* * *}$ & $0.022^{* * *}$ & $-0.412^{* * *}$ & 1 \\
\hline
\end{tabular}

Note: No valid observations for Notice of Call before and during the GFC. There are eight time weighted Bid-Ask Spreads to match with CARs/ATVs over different time windows; due to the thesis word limit (max. 100,000), the table just shows the correlation between independent/control variables and the Bid-Ask Spread over [0, 90 min]. 
APPENDIX V: STATISTIC SUMMARY OF MARKET-SENSITIVE

ANNOUNCEMENTS RELEASED AT DIFFERENT TIMES

\begin{tabular}{|c|c|c|c|c|c|c|c|}
\hline & Mean & $\begin{array}{l}10.00- \\
11.00\end{array}$ & $\begin{array}{l}11.00- \\
12.00\end{array}$ & $\begin{array}{l}12.00- \\
13.00\end{array}$ & $\begin{array}{l}13.00- \\
14.00\end{array}$ & $\begin{array}{l}14.00- \\
15.00\end{array}$ & $\begin{array}{l}15.00- \\
16.00\end{array}$ \\
\hline \multicolumn{8}{|l|}{ Before the GFC } \\
\hline CAR10min & 0.0070 & 0.0092 & 0.0087 & 0.0078 & 0.0061 & 0.0050 & 0.0033 \\
\hline CAR15min & 0.0091 & 0.0113 & 0.0110 & 0.0104 & 0.0085 & 0.0063 & 0.0047 \\
\hline CAR30min & 0.0102 & 0.0116 & 0.0119 & 0.0115 & 0.0089 & 0.0070 & 0.0085 \\
\hline CAR60min & 0.0106 & 0.0112 & 0.0119 & 0.0121 & 0.0100 & 0.0070 & \\
\hline CAR90min & 0.0110 & 0.0110 & 0.0122 & 0.0126 & 0.0101 & 0.0064 & \\
\hline CAR120min & 0.0111 & 0.0104 & 0.0121 & 0.0120 & 0.0094 & & \\
\hline CAR150min & 0.0114 & 0.0109 & 0.0122 & 0.0119 & 0.0094 & & \\
\hline$\triangle \mathrm{CAR} 10 \mathrm{~min}$ & 0.0070 & 0.0093 & 0.0088 & 0.0082 & 0.0062 & 0.0047 & 0.0033 \\
\hline$\Delta$ CAR15min & 0.0090 & 0.0112 & 0.0112 & 0.0106 & 0.0084 & 0.0062 & 0.0046 \\
\hline$\triangle \mathrm{CAR} 30 \mathrm{~min}$ & 0.0101 & 0.0118 & 0.0122 & 0.0117 & 0.0089 & 0.0069 & 0.0079 \\
\hline$\triangle \mathrm{CAR} 60 \mathrm{~min}$ & 0.0102 & & 0.0124 & 0.0115 & 0.0103 & 0.0060 & \\
\hline$\triangle \mathrm{CAR} 90 \mathrm{~min}$ & 0.0105 & & 0.0129 & 0.0117 & 0.0108 & 0.0058 & \\
\hline$\triangle \mathrm{CAR} 120 \mathrm{~min}$ & 0.0099 & & & 0.0100 & 0.0097 & & \\
\hline$\triangle \mathrm{CAR} 150 \mathrm{~min}$ & 0.0084 & & & 0.0075 & 0.0091 & & \\
\hline ATV10min & 0.1234 & 0.1511 & 0.1371 & 0.1265 & 0.1345 & 0.0900 & 0.0867 \\
\hline ATV15min & 0.2556 & 0.3086 & 0.2643 & 0.2579 & 0.2557 & 0.2215 & 0.2075 \\
\hline ATV30min & 0.4053 & 0.5065 & 0.4092 & 0.4106 & 0.4105 & 0.3366 & 0.3751 \\
\hline ATV60min & 0.5090 & & 0.4473 & 0.5364 & 0.5515 & 0.5154 & \\
\hline ATV90min & 0.5873 & & 0.3062 & 0.6170 & 0.6760 & 0.7023 & \\
\hline ATV120min & 0.6606 & & & 0.5731 & 0.7603 & & \\
\hline ATV150min & 0.5702 & & & 0.4167 & 0.7370 & & \\
\hline \multicolumn{8}{|l|}{ During the GFC } \\
\hline CAR10min & 0.0053 & 0.0068 & 0.0065 & 0.0056 & 0.0052 & 0.0035 & 0.0034 \\
\hline CAR15min & 0.0097 & 0.0117 & 0.0118 & 0.0108 & 0.0093 & 0.0069 & 0.0060 \\
\hline CAR30min & 0.0106 & 0.0118 & 0.0118 & 0.0118 & 0.0109 & 0.0075 & 0.0071 \\
\hline CAR60min & 0.0111 & 0.0113 & 0.0126 & 0.0118 & 0.0116 & 0.0079 & \\
\hline CAR90min & 0.0119 & 0.0124 & 0.0134 & 0.0124 & 0.0111 & 0.0073 & \\
\hline CAR120min & 0.0124 & 0.0130 & 0.0137 & 0.0121 & 0.0104 & & \\
\hline CAR150min & 0.0123 & 0.0122 & 0.0132 & 0.0116 & 0.0117 & & \\
\hline$\triangle \mathrm{CAR} 10 \mathrm{~min}$ & 0.0053 & 0.0072 & 0.0068 & 0.0055 & 0.0051 & 0.0031 & 0.0032 \\
\hline$\triangle \mathrm{CAR} 15 \mathrm{~min}$ & 0.0096 & 0.0129 & 0.0120 & 0.0106 & 0.0091 & 0.0065 & 0.0053 \\
\hline$\triangle \mathrm{CAR} 30 \mathrm{~min}$ & 0.0100 & 0.0128 & 0.0123 & 0.0108 & 0.0106 & 0.0067 & 0.0056 \\
\hline$\triangle \mathrm{CAR} 60 \mathrm{~min}$ & 0.0102 & & 0.0125 & 0.0109 & 0.0108 & 0.0065 & \\
\hline$\triangle$ CAR90min & 0.0105 & & 0.0135 & 0.0105 & 0.0112 & 0.0070 & \\
\hline$\triangle \mathrm{CAR} 120 \mathrm{~min}$ & 0.0095 & & & 0.0098 & 0.0090 & & \\
\hline$\triangle \mathrm{CAR} 150 \mathrm{~min}$ & 0.0090 & & & 0.0076 & 0.0101 & & \\
\hline ATV10min & 0.1011 & 0.0794 & 0.1443 & 0.1141 & 0.1083 & 0.0606 & 0.0898 \\
\hline ATV15min & 0.3219 & 0.3255 & 0.4437 & 0.3190 & 0.3500 & 0.2375 & 0.2007 \\
\hline ATV30min & 0.5486 & 0.4431 & 0.7062 & 0.5384 & 0.5760 & 0.4542 & 0.4574 \\
\hline ATV60min & 0.7716 & & 0.8247 & 0.7284 & 0.7654 & 0.7407 & \\
\hline ATV90min & 0.7850 & & 0.5881 & 0.7872 & 0.8742 & 0.8357 & \\
\hline ATV120min & 0.7995 & & & 0.6624 & 0.9587 & & \\
\hline ATV150min & 0.9063 & & & 0.8899 & 0.9244 & & \\
\hline \multicolumn{8}{|l|}{ After the GFC } \\
\hline CAR10min & 0.0042 & 0.0068 & 0.0044 & 0.0043 & 0.0031 & 0.0038 & 0.0017 \\
\hline CAR15min & 0.0065 & 0.0101 & 0.0069 & 0.0067 & 0.0052 & 0.0057 & 0.0023 \\
\hline CAR30min & 0.0075 & 0.0107 & 0.0082 & 0.0078 & 0.0057 & 0.0064 & 0.0039 \\
\hline CAR60min & 0.0081 & 0.0107 & 0.0085 & 0.0076 & 0.0064 & 0.0069 & \\
\hline CAR90min & 0.0080 & 0.0098 & 0.0089 & 0.0076 & 0.0059 & 0.0061 & \\
\hline CAR120min & 0.0079 & 0.0098 & 0.0085 & 0.0070 & 0.0058 & & \\
\hline CAR150min & 0.0083 & 0.0099 & 0.0081 & 0.0071 & 0.0077 & & \\
\hline$\triangle \mathrm{CAR} 10 \mathrm{~min}$ & 0.0041 & 0.0071 & 0.0042 & 0.0042 & 0.0031 & 0.0038 & 0.0015 \\
\hline
\end{tabular}




\begin{tabular}{|c|c|c|c|c|c|c|c|}
\hline & Mean & $\begin{array}{l}10.00- \\
11.00\end{array}$ & $\begin{array}{l}11.00- \\
12.00\end{array}$ & $\begin{array}{l}12.00- \\
13.00\end{array}$ & \begin{tabular}{|l|}
$13.00-$ \\
14.00 \\
\end{tabular} & \begin{tabular}{|l|}
$14.00-$ \\
15.00 \\
\end{tabular} & $\begin{array}{l}15.00- \\
16.00\end{array}$ \\
\hline$\Delta$ CAR15min & 0.0062 & 0.0105 & 0.0067 & 0.0067 & 0.0052 & 0.0057 & 0.0021 \\
\hline$\triangle \mathrm{CAR} 30 \mathrm{~min}$ & 0.0072 & 0.0119 & 0.0082 & 0.0077 & 0.0056 & 0.0066 & 0.0042 \\
\hline$\triangle$ CAR60min & 0.0077 & & 0.0094 & 0.0081 & 0.0062 & 0.0072 & \\
\hline$\Delta$ CAR90min & 0.0073 & & 0.0086 & 0.0077 & 0.0063 & 0.0070 & \\
\hline$\Delta$ CAR120min & 0.0064 & & & 0.0069 & 0.0056 & & \\
\hline$\Delta$ CAR150min & 0.0051 & & & 0.0039 & 0.0062 & & \\
\hline ATV10min & 0.0979 & 0.1010 & 0.1305 & 0.1272 & 0.0722 & 0.0937 & 0.0440 \\
\hline ATV15min & 0.2572 & 0.2893 & 0.3251 & 0.2652 & 0.2270 & 0.2477 & 0.1536 \\
\hline ATV30min & 0.4116 & 0.4186 & 0.4872 & 0.4065 & 0.4018 & 0.4181 & 0.2546 \\
\hline ATV60min & 0.5875 & & 0.5163 & 0.5112 & 0.6545 & 0.6991 & \\
\hline ATV90min & 0.5955 & & 0.3575 & 0.5345 & 0.7434 & 0.7134 & \\
\hline ATV120min & 0.5993 & & & 0.4264 & 0.8031 & & \\
\hline ATV150min & 0.2813 & & & 0.0792 & 0.4981 & & \\
\hline
\end{tabular}




\section{APPENDIX VI: ROBUSTNESS TESTS ON THE TIMELINESS OF PRICE DISCOVERY}

The Impact of Announcement and Periodic/Non-periodic Report Frequency on the Timeliness of Price Discovery

\begin{tabular}{|c|c|c|c|c|c|c|c|c|c|c|c|c|}
\hline \multirow{2}{*}{ Variable } & \multicolumn{6}{|c|}{ Total Announcements } & \multicolumn{6}{|c|}{ Periodic/Non-Periodic Reports } \\
\hline & Coeff & $t$-stat & VIF & Coeff & $t$-stat & VIF & Coeff & $t$-stat & VIF & Coeff & $t$-stat & VIF \\
\hline Intercept & $0.016780 * * *$ & 10.87 & 0.00 & $0.014330 * * *$ & 9.29 & 0.00 & $0.01746 * * *$ & 11.28 & 0.00 & $0.01425^{* * *}$ & 9.14 & 0.00 \\
\hline All Announcements & $0.000041 * * *$ & 6.78 & 1.16 & & & & & & & & & \\
\hline All Announcements - MS & & & & $0.000415^{* * *}$ & 14.17 & 1.33 & & & & & & \\
\hline Periodic Reports & & & & & & & $-0.000072 * * *$ & -2.98 & 1.17 & & & \\
\hline Non-Periodic Reports & & & & & & & $0.000050 * * *$ & 7.89 & 1.14 & & & \\
\hline Periodic Reports - MS & & & & & & & & & & $0.000466^{* * *}$ & 3.14 & 1.66 \\
\hline Non-Periodic Reports - MS & & & & & & & & & & $0.000414 * * *$ & 14.02 & 1.49 \\
\hline Stock Return Volatility & $0.206280 * * *$ & 19.46 & 2.15 & $0.196330 * * *$ & 18.62 & 2.15 & $0.202680 * * *$ & 19.10 & 2.16 & $0.196220 * * *$ & 18.60 & 2.16 \\
\hline Well-performing Company & $-0.002180 * * *$ & -4.05 & 1.03 & $-0.001980 * * *$ & -3.70 & 1.03 & $-0.002230 * * *$ & -4.14 & 1.03 & $-0.001970 * * *$ & -3.67 & 1.03 \\
\hline Size & $-0.000365 * *$ & -2.00 & 2.17 & $-0.000371 * *$ & -2.12 & 2.02 & $-0.000351 *$ & -1.92 & 2.17 & $-0.000390 * *$ & -2.13 & 2.22 \\
\hline Book-to-Market Ratio & 0.000095 & 0.63 & 1.05 & 0.000164 & 1.11 & 1.05 & 0.000098 & 0.66 & 1.05 & 0.000163 & 1.09 & 1.05 \\
\hline Leverage & 0.000007 & 1.37 & 1.00 & 0.000006 & 1.16 & 1.00 & 0.000007 & 1.37 & 1.00 & 0.000006 & 1.16 & 1.00 \\
\hline ROA & 0.000028 & 0.24 & 1.03 & 0.000077 & 0.66 & 1.03 & 0.000030 & 0.25 & 1.03 & 0.000077 & 0.66 & 1.03 \\
\hline Resource Sector & $0.003530 * * *$ & 5.90 & 1.26 & 0.000280 & 0.43 & 1.49 & $0.003130 * * *$ & 5.19 & 1.28 & 0.000406 & 0.55 & 1.96 \\
\hline Financial Sector & $-0.005930 * * *$ & -7.13 & 1.23 & $-0.004500 * * *$ & -5.48 & 1.22 & $-0.005110 * * *$ & -6.02 & 1.28 & $-0.004500 * * *$ & -5.48 & 1.22 \\
\hline Year Control & $\mathrm{Y}$ & & & $\mathrm{Y}$ & & & $\mathrm{Y}$ & & & $\mathrm{Y}$ & & \\
\hline $\mathbf{N}$ & 10,834 & & & 10,834 & & & 10,834 & & & 10,834 & & \\
\hline F Value & 79.13 & & & 88.83 & & & 76.36 & & & 84.15 & & \\
\hline R-Square & $11.64 \%$ & & & $12.88 \%$ & & & $11.83 \%$ & & & $12.88 \%$ & & \\
\hline Adj R-Sq & $11.49 \%$ & & & $12.74 \%$ & & & $11.67 \%$ & & & $12.73 \%$ & & \\
\hline
\end{tabular}

$*, * *$ and $* * *$ denote significance at the $10 \%, 5 \%$ and $1 \%$ levels respectively (two-tailed). 
The Impact of Different Non-periodic Announcement Frequency on the Timeliness of Price Discovery

\begin{tabular}{|c|c|c|c|c|c|c|}
\hline \multirow{2}{*}{ Variable } & \multicolumn{3}{|c|}{ Announcement Frequency of Each Category } & \multicolumn{3}{|c|}{ Marke-sensitive Announcement Frequency of Each Category } \\
\hline & Coeff & $t$-stat & VIF & Coeff & $t$-stat & VIF \\
\hline Intercept & $0.013160 * * *$ & 8.18 & 0.00 & $0.015400 * * *$ & 9.90 & 0.00 \\
\hline Takeover/Scheme Announcements & -0.000007 & -0.09 & 1.09 & 0.000109 & 0.76 & 1.03 \\
\hline Security Holder Details & $0.000123 * * *$ & 4.40 & 1.51 & $-0.001130 *$ & -1.65 & 1.04 \\
\hline Quarterly Activities Report & 0.000340 & 1.27 & 4.71 & 0.000145 & 0.48 & 5.26 \\
\hline Quarterly Cash Flow Report & -0.000332 & -1.57 & 1.48 & -0.000290 & -1.33 & 1.49 \\
\hline Issued Capital & $-0.000026^{*}$ & -1.65 & 1.19 & $0.000368 * *$ & 2.21 & 1.24 \\
\hline Asset Acquisition \& Disposal & 0.000018 & 0.14 & 1.13 & -0.000014 & -0.08 & 1.11 \\
\hline Notice of Meeting & $0.000872 * * *$ & 5.43 & 1.22 & $0.004460 * * *$ & 2.80 & 1.02 \\
\hline ASX Announcement & $0.002030 * * *$ & 15.19 & 1.25 & $0.002370 * * *$ & 14.87 & 1.29 \\
\hline Distribution Announcement & $-0.000582 * * *$ & -3.59 & 1.51 & $-0.000718 *$ & -1.88 & 1.30 \\
\hline Progress Reports & $0.000058 * *$ & 2.17 & 1.45 & $0.000136 * * *$ & 3.03 & 1.53 \\
\hline Company Administration & $0.000234 *$ & 1.90 & 1.25 & $0.001740 * * *$ & 3.20 & 1.12 \\
\hline Notice of Call & -0.001450 & -0.40 & 1.00 & 0.003810 & 0.37 & 1.00 \\
\hline Other & 0.000100 & 0.61 & 1.46 & $0.000692 *$ & 1.69 & 1.13 \\
\hline Chairman's Address & 0.000132 & 0.43 & 1.18 & 0.000421 & 0.44 & 1.08 \\
\hline Letter to Shareholders & -0.000319 & -0.63 & 1.12 & 0.005980 & 1.06 & 1.00 \\
\hline ASX Query & $0.003340 * * *$ & 9.94 & 1.13 & $0.004170 * * *$ & 11.00 & 1.11 \\
\hline Structured Products & -0.000004 & -0.40 & 1.12 & 0.039320 & 1.45 & 1.00 \\
\hline CTE Quarterly Reports & -0.000037 & -0.18 & 1.48 & -0.000087 & -0.39 & 1.51 \\
\hline Stock Return Volatility & $0.173120 * * *$ & 16.17 & 2.30 & $0.177240 * * *$ & 16.55 & 2.29 \\
\hline Well-performing Company & $-0.002100 * * *$ & -3.97 & 1.04 & $-0.002060 * * *$ & -3.89 & 1.04 \\
\hline Size & $-0.000476 * *$ & -2.35 & 2.80 & $-0.000363 * *$ & -1.98 & 2.28 \\
\hline Book-to-Market Ratio & 0.000228 & 1.55 & 1.06 & $0.000266 *$ & 1.81 & 1.06 \\
\hline Leverage & 0.000008 & 1.59 & 1.00 & 0.000008 & 1.49 & 1.00 \\
\hline ROA & 0.000110 & 0.95 & 1.03 & 0.000114 & 0.99 & 1.03 \\
\hline Resource Sector & 0.000360 & 0.35 & 3.87 & 0.000526 & 0.50 & 4.04 \\
\hline Financial Sector & $-0.002870 * * *$ & -3.27 & 1.45 & $-0.003540 * * *$ & -4.02 & 1.45 \\
\hline Year Control & $\mathrm{Y}$ & & & $\mathrm{Y}$ & & \\
\hline $\mathbf{N}$ & 10,834 & & & 10,834 & & \\
\hline F Value & 59.57 & & & 57.42 & & \\
\hline R-Square & $16.18 \%$ & & & $15.69 \%$ & & \\
\hline Adj R-Sq & $15.91 \%$ & & & $15.42 \%$ & & \\
\hline
\end{tabular}

